\title{
IMPROVEMENT OF IN VITRO FOOD ALLERGY DIAGNOSTICS BY IDENTIFYING UNIQUE ANTIBODY TRAITS
}

\section{Anna Ehlers}

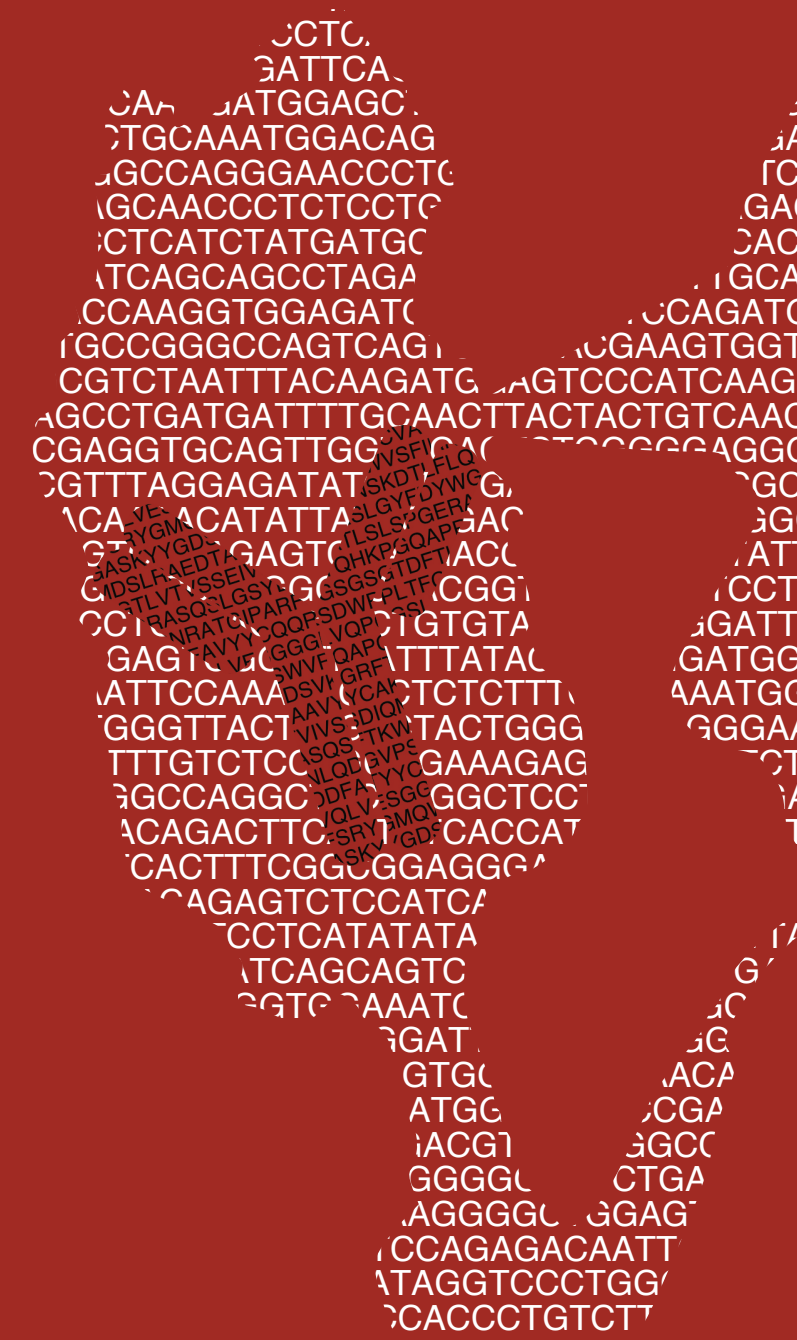

ICTC. TGCAAATGGACAG GCCAGGGAACCCTC

¿TCCTCA ICCATCAGC viGACCAAGGTGGAAn . I I GTCCTGTGCAGCCTCTGGAT । iGGTCTCAGGCATCAGTGGCAGTGGA, , GCCAAAAACATCCTGTTTCTGCAAATGGCC AGACTATGACGAGGTCGAGATTATGGACGTCTGC: जTCTGGGGGAGGCATGGTCCAGCCTGGGGGGTCCC .TGCAGTGGGTU,

¿ACTCCGTG/ „ACACGGCTG CCAGGAAATTG GAGTCTTGGCAGC

JACTGGCATCCCAGCB

GCAGTTTATTACTGTCAGCAGCGTAGE CAGGCAAGGGGCTGGAl MTCTCCAGAGACAATTCI TVEATEAS ECTGGGTT, NCSTTTGTL IGGCCA IACAGA TCACTT CCAAGACAGAG
CACCTC 


\section{IMPROVEMENT OF IN VITRO FOOD ALLERGY DIAGNOSTICS BY IDENTIFYING UNIQUE ANTIBODY TRAITS}


Improvement of in vitro food allergy diagnostics by identifying unique antibody traits Thesis with a summary in Dutch and German, Utrecht University (C) Anna Ehlers, 2021

The copyrights of published articles have been transferred to the respective journals. All rights are reserved. No part of this thesis may be reproduced, stored in a retrieval system or transmitted in any other form or by any means, without permission of the author.

ISBN: 978-94-6416-368-1

DOI: https://doi.org/10.33540/190

Cover and layout design: Michael Drefahl

Printed and published by: Ridderprint, www.ridderprint.nl 


\section{Improvement of in vitro food allergy \\ diagnostics by identifying unique antibody \\ traits}

Verbetering van in vitro diagnostiek naar voedselallergie door het identificeren van unieke antilichaam kenmerken

(met een samenvatting in het Nederlands)

Optimierung der in vitro Nahrungsmittelallergie-Diagnostik durch die Identifizierung von einzigartigen Antikörper-Merkmalen

(mit einer Zusammenfassung in deutscher Sprache)

Proefschrift

ter verkrijging van de graad van doctor aan de

Universiteit Utrecht

op gezag van de

rector magnificus, prof. dr. H.R.B.M. Kummeling,

ingevolge het besluit van het college voor promoties

in het openbaar te verdedigen op

donderdag 18 februari 2021

des ochtends te 10.30 uur

door

\section{Anna Maria Ehlers}

geboren op 11 december 1990

te Itzehoe, Duitsland 


\section{Promotor}

Prof. dr. A.C. Knulst

\section{Copromotor}

Dr. H.G. Otten 


\section{Table of contents}

$\begin{array}{lll}\text { Chapter } 1 & \text { General introduction } & 7\end{array}$

\section{Part I Preventing „false-negative“ slgE testing}

Chapter 2 IgE-binding to vicilin-like antimicrobial peptides is associated with systemic reactions to macadamia nut

Chapter 3 Sesame oleosins are minor allergens

\section{Part II Preventing „false-positive“ slgE testing}

Chapter 4 Detection of specific lgE against linear epitopes from Gal d 1 has additional value in diagnosing hen's egg allergy in adults

Chapter 5 2S protein Ara h 7.0201 has unique epitopes compared to other Ara h 7 isoforms and is comparable to $2 S$ proteins Ara h 2 and 6 in basophil degranulation capacity

Chapter 6 Ara h 7 isoforms share many linear epitopes: Are 3D epitopes crucial to elucidate divergent abilities?

Chapter 7 Can alternative epitope mapping approaches increase the impact of B-cell epitopes in food allergy diagnostics?

Chapter 8 Comparison of two strategies to generate specific human monoclonal antibodies: Which method to choose for which purpose?

Chapter 9 Distinction between peanut allergy and tolerance by characterization of B-cell receptor repertoires

Chapter 10 General discussion

Chapter 11 Nederlandse samenvatting

Chapter 12 Zusammenfassung in deutscher Sprache

Chapter 13 Appendices

Abbreviations

Dankwoord

List of publications 
MIUTCT

AGCCTCTGGA7

CCTGTTTCTGCAAA

iGCGTGGTCCAGCCTC

AGGGCCGATTCACCATL

AATTGTGTTGACACAGTCTCCAGCCALU

UTGGCATCCCAGCCAGGTTCAGTGGCAGTGGGTC

$\checkmark$ CAGATGACCCAGTCTCCTTCCACCCTGGCTGCATCTGTAGL

AGTCCCATCAAGGTTCAGCGGCAGTGGGTCTGGGACAGAGTTCACL

GAGTCTGGGGGAGGCTTGGTACAGCCGGGGGGGTCCCTGAGACTGTCL

TATCGAGACTCTGTGAAGGGCCGCTTCACCATCTCCAGAGACAATGCCAAAAAC ¿AGGGGACCACGGTCATCGTCTCCTCAGCAGGTGCAGCTGGTGGAGTCTGGGGGAGG iGAGTGGGTTTCATTTATACGCAAGGATGGAGCTAGTAAATACTATGGAGACTCCGTGAAGGG CCTGGGTTACTTTGACTACTGGGGCCAGGGAACCCTGGTCACCGTCTCCTCAGGAAATTGTGT 年

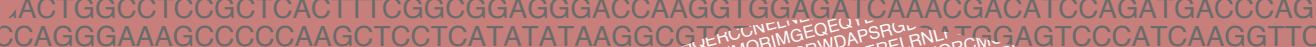
ACTCACTITCGGCGGAGGGACCAAGGTATAAGG ACAGGACTTTCGGCGGAGGGACCAAGGTGGA

AAAGATAAGGTTCCAGACTATGACGAGGTC

ITCAGTCGTTATGGCATGCAGTGGGTCCGPUNNQRCICO

¿TCCTGCAGGGCGCGLAPQRCD

tGCCTAGAGCCT

GCCTGATGAT

ATGGCCAGTC

;CTGGGGGGTCC

CCATCTCCAGAG

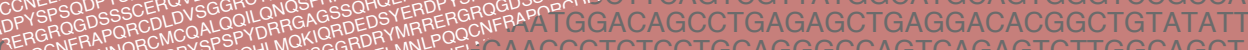

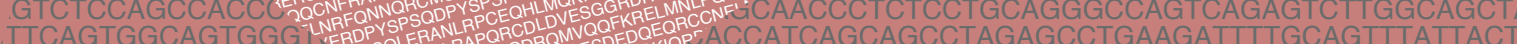

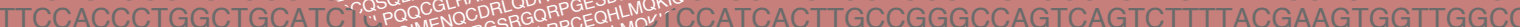

CAGTGGGTCTGGGACAGAGTTU

ACAGCCGGGGGGGTCCCTGAGACTGTCCTGTGCAGCCTCTGGATTCACGTTTAGGAGATATGCTTTGAGTTGGGTCCGC ICTTCACCATCTCCAGAGACAATGCCAAAAACATCCTGTTTCTGCAAATGGCCAGTCCGAGAGTCGAAGACGCGGCCGTT TCAGCAGGTGCAGCTGGTGGAGTCTGGGGGAGGCGTGGTCCAGCCTGGGGG AATGGAGCTAGTAAATACTATGGAGACTCCGTGAAGGGCCGATTCACCATCTCC 'TCCTCATCTATGATGCATCCAACAGGGCCACTGGCATCCCAGCCAGGTTCAGTG AGAGGGACCAAGGTGGAGATCAAACGACATCCAGATGACCCAGTCTCCTTCCACC , CATATATAAGGCGTCTAATTTACAAGATGGAGTCCCATCAAGGTTCAGCGGCAGTG तAGGTGGAAATCAAACGAGGTGCAGTTGGTGGAGTCTGGGGGAGGCTTGGTACA NGCATCAGTGGCAGTGGAAACAAAACATATTATCGAGACTCTGTGAAGGGCC GGGTCCGCCAGGCTCCAGGCAAGGGGCTGGAGTGGGTTTCATTTATACGCAAGG GGCTGTATATTACTGTGCGAAAGATAGGTCCCTGGGTTACTTTGACTACTGGGGCC :TTGGCAGCTACTTAGCCTGGTACCAACATAAACCTGGCCAGGCTCCCAGGCTCC 'CAGGGAAAGCCCCCAAGCTCCTCATATATAA ACTTTCGGCGGAGGGACCAAGGTGGAAATCAAACGAGGTGC

IGCTGGAGTGGGTCTCAGGCATCAGTGGCAGTGGAAACAAAA GGTTCCAGACTATGACGAGGTCGAGATTATGGACGTCTGG TGGCATGCAGTGGGTCCGCCAGGCTCCAGGCAAGGGGC CTGAGGACACGGCTGTATATTACTGTGCGAAAGATAGGT AGTCAGAGTCTTGGCAGCTACTTAGCCTGGTACCAACAT AGATTTTGCAGTTTATTACTGTCAGCAGCGTAGCGACTG TTACGAAGTGGTTGGCCTGGTATCAGCAGAAACCAGG ACTTACTACTGTCAACAATATGATAGTGCCCTACTCACT CTTTGAGTTGGGTCCGCCAGGCTCCAGGGCAGGGG GAAGACGCGGCCGTTTATTACTGTGCGAAAGATAAGC 'TCTCCTGTGTAGCGTCTGGATTCACCTTCAGTCGTTA AAAGACACTCTCTTTCTGCAAATGGACAGCCTGAGAG iTCTCCAGGGGAAAGAGCAACCCTCTCCTGCAGGGC
CAGACTTCACTCTCACCATCAGCAGCCTAGAGCCTG GAGACAGAGTCTCCATCACTTGCCGGGCCAGTCAG 4CCCTCACCATCAGCAGTCTGCAGCCTGATGATTTT TGTCCTGTGCAGCCTCTGGATTCACGTTTAGGAGAT AAAAACATCCTGTTTCTGCAAATGGCCAGTCCGAGA ̇̈GGGAGGCGTGGTCCAGCCTGGGGGGTCCCTGA
TCCGTGAAGGGCCGATTCACCATCTCCAGAGACAAT Aunum TCCGTGAAGGGCCGATTCACCATCTCCAGAGACAAT

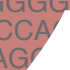
TAGAGACTCTCCTGTGTAGCGTCT
CAATTCCAAAGACACTCTCTTTCTI TGTCTTTGTCTCCAGGGGAAAGA GTCTGGGACAGACTTCACTCTCA GTCTGGGACAGACTTCACTCTCA TCTGTAGGAGACAGAGTCTCCAT,
AGAGTTCACCCTCACCATCAGCA AGAGTTCACCCTCACCATCAGCA
CTGAGACTGTCCTGTGCAGCCT ACAATGCCAAAAACATCCTGTTTC GAGTCTGGGGGAGGCGTGGT GGAGACTCCGTGAAGGGCCGA GGAGACTCCGTGAAGGGCCGA CTCCTCAGGAAATTGTGTTGACA
GGGCCACTGGCATCCCAGCCAC ACATCCAGATGACCCAGTCTCC AAGTCCCATCAAGGTTCAGCGG GAGTCTGGGGGAGGCTTGGTA (A) CGAGACTCTGTGAAGGGCCGC GGACCACGGTCATCGTCTCCTC,
GGTTTCATTTATACGCAAGGATG TACTTTGACTACTGGGGCCAGG GCCAGGCTCCCAGGCTCCTCAT CTCACTTTCGGCGGAGGGACC CCAAGCTCCTCATATATAAGGD CCAAGCTCCTCATATATAAGGC GGTCTCAGGCATCAGTGGCAG CTATGACGAGGTCGAGATTATG CAGTGGGTCCGCCAGGCTCCAC CACGGCTGTATATTACTGTGCG CACGGCTGIATATTACTGTGCG AGTCTTGGCAGCTACTTAGCCTC TGCAGTITATTACTGTCAGCAGC GAAGTGGTTGGCCTGGTATCAG ACTACTGTCAACAATATGATAGTC GAGTTGGGTCCGCCAGGCTCCA ACGCGGCCGTITATTACTGTGC TGTGTAGCGTCTGGATTCACCT CACTCTCTTTCTGCAAATGGAC CAGGGGAAAGAGCAACCCTCT CAGGGCCACTGGCATCCCAGCCAGGTTCAGTGGCAGTGGGTCTGGGACAGACTTCACTCTCACCATCAGCAG ACGACATCCAGATGACCCAGTCTCCTTCCACCCTGGCTGCATCTGTAGGAGACAGAGTCTCCATCACTTGCCGG GATGGAGTCCCATCAAGGTTCAGCGGCAGTGGGTCTGGGACAGAGTTCACCCTCACCATCAGCAGTCTGCAGCC TGGTGGAGTCTGGGGGAGGCTTGGTACAGCCGGGGGGGTCCCTGAGACTGTCCTGTGCAGCCTCTGGATTCAC ¿ATATTATCGAGACTCTGTGAAGGGCCGCTTCACCATCTCCAGAGACAATGCCAAAAACATCCTGTTTCTGCAAATGC GCCAGGGGACCACGGTCATCGTCTCCTCAGCAGGTGCAGCTGGTGGAGTCTGGGGGAGGCGTGGTCCAGCCTGG GGAGTGGGTTTCATTTATACGCAAGGATGGAGCTAGTAAATACTATGGAGACTCCGTGAAGGG

¿CCTGGGTTACTTTGACTACTGGGGCCAGGGAACCCTGGTCACCGTCTCCTCAGGAAATTGTC

ITAAACCTGGCCAGGCTCCCAGGCTCCTCATCTATGATGCATCCAACAGGGCCACTGGCATCr

IGGCCTCCGCTCACTTTCGGCGGAGGGACCAAGGTGGAGATCAAACGACATCCAGATGACCC

AGGGAAAGCCCCCAAGCTCCTCATATATAAGGCGTCTAATTTACAAGATGGAGTCCCATCAAGC

CACTTTCGGCGGAGGGACCAAGGTGGAAATCAAACGAGGTGCAGTTGGTGGAGTCTGGGGG/

GGCTGGAGTGGGTCTCAGGCATCAGTGGCAGTGGAAACAAAACATATTATCGAGACTCTGTG,

\AGGTTCCAGACTATGACGAGGTCGAGATTATGGACGTCTGGGGCCAGGGGACCACGGTCATCG I

1TATGGCATGCAGTGGGTCCGCCAGGCTCCAGGCAAGGGGCTGGAGTGGGTTTCATTTATACGCAAGGATGGAGi I AG I ¿AGAGCTGAGGACACGGCTGTATATTACTGTGCGAAAGATAGGTCCCTGGGTTACTTTGACTACTGGGGCCAGGGAACCCTG AGGGCCAGTCAGAGTCTTGGCAGCTACTTAGCCTGGTACCAACATAAACCTGGCCAGGCTCCCAGGCTCCTCATCTATGATG GAGCCTGAAGATTTTGCAGTTTATTACTGTCAGCAGCGTAGCGACTGGCCTCCGCTCACTTTCGGCGGAGGGACCAAGGTGGA 


\section{General introduction}

GGC,

TCTCL

AGCGG

TTGGTAC

CTCCTCAG

AAGGATGGA

GGCCAGGGA

TCCTCATCTAT

GGGACCAAGG 7 ,
CATAAGGCGTCT/

GAAATCAAACGAC

'TGGCAGTGGAAA!

AGATTATGGACGT

GGCTCCAGGCAAG

ACTGTGCGAAAGATAL

ACTTAGCCTGGTACCAAC,

GTCAGCAGCGTAGCGACTGG

TTGGTATCAGCAGAAACCAGGGAA,

TATGATAGTGCCCTACTCACTTTCGGL

CCAGGCTCCAGGGCAGGGGCTGGAGTGG

TATTACTGTGCGAAAGATAAGGTTCCAGACTA
CAIL.
CACTTGL

AGTCTGCAG

¿ATCTGGATTC

GTTTCTGCAAAT

ITGGTCCAGCCTGGL, CCTL

ITGTTGACACAGTCTCCAGCCACCCTC

CCCAGCCAGGTTCAGTGGCAGTGGGT

:CCAGTCTCCTTCCACCCTGGCTGCATC; ITGTTCAGCGGCAGTGGGTCTGGGACAGA'

TGCTTGGTACAGCCGGGGGGGTCCCTC

4GGGCCGCTTCACCATCTCCAGAGAC

TGTCTCCTCAGCAGGTGCAGCTGGTG

ICAAGGATGGAGCTAGTAAATACTATG

İGGGCCAGGGAACCCTGGTCACCGTC

ìGCTCCTCATCTATGATGCATCCAACAGi

i्GAGGGACCAAGGTGGAGATCAAACvvC

NATATATAAGGCGTCTAATTTACAAGATG

AGGTGGAAATCAAACGAGGTGCAGTTGGTGG IGGCATCAGTGGCAGTGGAAACAAAACATATTATC ¿GAGGSUAGATTATGGACGTCTGGGGCCAGGGI GT:

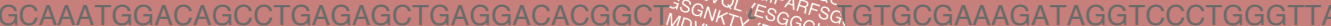

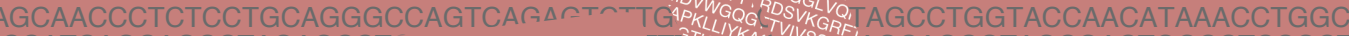
CCATCAGCAGCCTA'

CACTTGCCGGGO

GTCTGCAGCCTr

STGGATTCACGT

TTGCAAATGGCC.

CCAGCCTGGGGG

TCACCATCTCCAG

CAGTCTCCAGCCAC
GGTTCAGTGGCAGTG

TTCCACCCTGGCTGCA

CAGTGGGTCTGGGACA

CAGCCGGGGGGGTCCC

TTCACCATCTCCAGAGAC

AGCAGGTGCAGCTGGTGC

GAGCTAGTAAATACTATG

GAACCCTGGTCACCGTC

CTATGATGCATCCAAC

AAGGTGGAGATr"

GTCTAATTTA

AACGAGGTGC

TGGAAACAAl

GGCAAGGG'

AAAGATAC

AGTACCA

GTAGCG

CAGAAACL.

GCCCTACTCAL

GGGCAG!

GAAAGA

TCAGTC

AGCCT

CCTG

CCTA

GCCA

TGA

GTT

ac

ar

ITT

AAL

IAT,

ITACL.

ICTTAC

CTTTGA

?GAAGAC

TCTCCTG

AAGACAC

TCTCCAG

:AGACTTC,

CTCACCATL

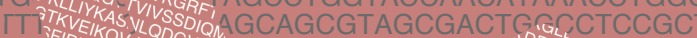
TH

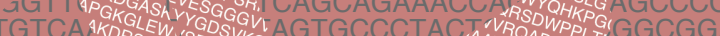

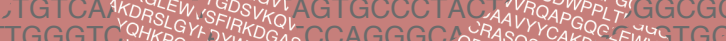

?GGCCG

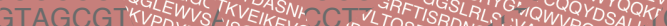

TCTCTTTCW WYDE

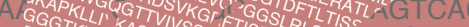

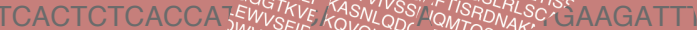

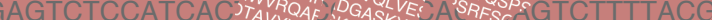

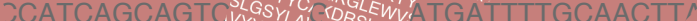

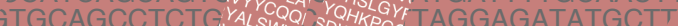

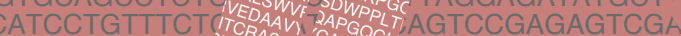

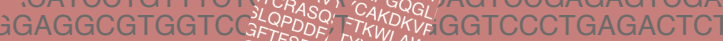

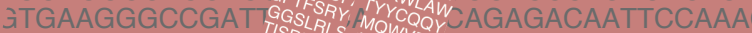
AAATTGTGTTGACA SPATNSY,

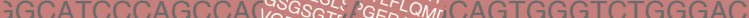

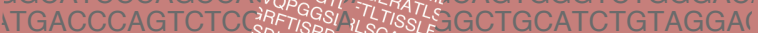

TCAAGGTTCAGCGDOMMTO TAKAASECTGGGACAGAGTTCACr

ICAAGGTTCAGCGDG MVIO TAKN/ALCCTGGGACAGAGTTCAC

CTGTGAAGGGCCGCTTCRGGS:TCTCCAGAGACAATGCC

ITCATCGTCTCCTCAGCAGGTGCAGCTGGTGGAGTCTC

ATACGCAAGGATGGAGCTAGTAAATACTATGGAGAC

OTGGGGCCAGGGAACCCTGGTCACCGTCTCCTC

TTCCTCATCTATGATGCATCCAACAGGGCC TGGACCAAGGTGGAGATCAAACVVGA ^

TATAAGGCGTCTAATTTACAAGAT

TAAATCAAACGAGGTGCAGTTr

GGCAGTGGAAACAAAACAT

TTATGGACGTCTGGGGCC

CCAGGCAAGGGGCTGGA

TGCGAAAGA ${ }^{-1}$ TGTCCCTr

AGCAGCG

ATCAGCA

IATAGTG

GGCTCCA

ITACTGTG

GATTCACr

CAAATGGA

İCAACCCTC

CATCAGCAC

CACTTGCCG

AGTCTGCAGCC

CCTGTGCAGCCTCTGGATTCACG I

AACATCCTGTTTCTGCAAATGGCCA

iGAGGCGTGGTCCAGCCTGGGGG

TAAGGGCCGATTCACCATCTCCA'

ITTGTGTTGACACAGTCTCCAGC

ITCCCAGCCAGGTTCAGTGGC

CCAGTCTCCTTCCACCCTG 


\section{Food allergy}

Food allergy is generally defined as an IgE-mediated immunological disorder leading to reactions against normally harmless proteins. Although allergenic proteins can be present in virtually any food, the major foods responsible for most food allergic reactions are peanut, tree nuts, cow's milk, hen's egg and (shell)fish ${ }^{1}$. The major causative food, however, varies regarding geographical location and age ${ }^{2,3}$. While cow's milk and hen's egg are responsible for most food allergic reactions in children, peanut and tree nut allergies are more common in adults ${ }^{4}$. Regardless of the causative food, subjects can experience symptoms ranging from mild oral itching to even, in rare cases, lethal anaphylaxis. Despite the risk of unexpected severe allergic reactions, allergen immunotherapy is still largely under investigation. The only treatment options are elimination diets and prescription of epinephrine auto-injectors ${ }^{5,6}$. To prevent unnecessary food restrictions, highly accurate diagnostic strategies, preferably minimally invasive, are required.

\section{Food allergy diagnostics}

In daily practice, food allergy diagnostics is comprised of careful evaluation of patient's medical history, complementary in vitro (measuring specific $\lg \mathrm{E}(\mathrm{s} \lg \mathrm{E})$ ) and/or in vivo (skin prick test) measurements, and double-blind placebo-controlled food challenges (DBPCFC) as the gold standard. DBPCFCs are characterised by gradual administration of increasing food doses to patients with suspected food allergy in comparison to placebo administration ${ }^{7}$. Those DBPCFCs, however, are burdensome for patients, costly and require dedicated hospital facilities accompanied by trained personnel. Hence, it is very desirable to reduce the number of required food challenges in the future. Since slgE measurements are partly hampered by a lack of sensitivity (nondetectable clinically relevant sensitisation $\rightarrow$ „false-negative“) or specificity (detection of clinically irrelevant sensitisation $\rightarrow$ „false-positive“), it is currently not advised to replace food challenges by slgE measurements in cases of uncertainty about the severity and/or the causative culprit food ${ }^{8-10}$. These obstacles indicate the strong need to develop improved platforms for slgE measurements or novel minimally invasive in vitro diagnostic strategies. 


\section{Specific IgE measurements in food allergy diagnostics}

Most commonly, slgE levels are measured against a crude extract of the suspected food. Such extracts consist of allergenic and non-allergenic proteins solubilised in an appropriate buffer, which could lead to the potential lack of the responsible allergen in such extracts ${ }^{11}$. Moreover, clinically irrelevant sensitisation might be measured due to the presence of less relevant allergens next to more relevant ones. A promising approach to overcome these limitations was the introduction of component-resolved diagnostics (CRD). CRD is characterised by slgE measurement against a single fulllength allergenic component instead of measuring slgE against whole food extracts ${ }^{12}$. In peanut allergy, the replacement of crude peanut extract by the major peanut allergen Ara $\mathrm{h} 2$, belonging to the $2 \mathrm{~S}$ albumin family, increased the specificity of slgE measurements from $59 \%$ to approximately $84 \%$ using $\geq 0.35 \mathrm{kU} / \mathrm{l}$ as a cut-off level ${ }^{13-17}$. Moreover, CRD allows not only complexity reduction but also enables slgE measurements against allergens absent in commercially available food extracts such as hydrophobic proteins. Hydrophobic proteins such as the oil-body associated oleosins have been described for peanut as a potential marker for (severe) peanut allergy ${ }^{18}$. Such proteins have also been described as potential allergens in hazelnut and sesame seed ${ }^{19,20}$. Despite such improvements by CRD, slgE measurements are still partly hampered by false-negative and false-positive test outcomes that contradict clinical diagnosis ${ }^{21}$.

We hypothesise that

- false-negative test outcomes might be prevented by identifying the missing single allergenic component for CRD

- false-positive test outcomes might be prevented by exploring differences in specific antibody repertoires between allergic and sensitised but tolerant patients regarding epitope (antibody's binding sites) recognition pattern, affinity or blocking ability of non-IgE antibodies to prevent slgE binding. 


\section{Antibody repertoire in allergy}

Generally, antibodies are comprised of two heavy and two corresponding light chains, which form the characteristic $Y$-shape and they are stabilised by disulphide bridges. The heavy and the light chain are divided into a variable and a constant region ${ }^{22}$. While the constant region defines the isotype (e.g. $\lg \mathrm{E}$ ) and therefore the effector function, the variable region is responsible for antigen binding ${ }^{23}$ (Figure 1a). The variable region of the heavy chain is formed by recombination of variable (V), diversity (D) and joining (J) gene segments during maturation of naïve B cells (Figure $1 \mathrm{~b}$ ) The variable region of the light chain, on the other hand, is formed by the same mechanism but lacks the $\mathrm{D}$ gene segment ${ }^{24}$. Recombination of those genes results in variable regions characterised by four frame work regions (FR) and three complementarity-determining regions (CDR). Correct folding of the antibody allows the formation of an antigen-binding site (paratope) consisting of all three CDRs of which CDR3 is largely determining antibody's specificity ${ }^{25}$. V(D)J gene recombination forms the basis for the high diversity of antibodies binding virtually any antigen. This diversity is even enlarged by somatic hypermutations preferably introduced within CDRs during $B$ cell maturation in the germinal centre accompanied by antigen presentation ${ }^{26}$. Potential differences in HCDR3 sequences or $\mathrm{VH}$ gene usage by allergic versus sensitised but tolerant individuals can be defined by analysing the repertoire of $V(D) J$ sequences encoding allergen specific antibodies.

A

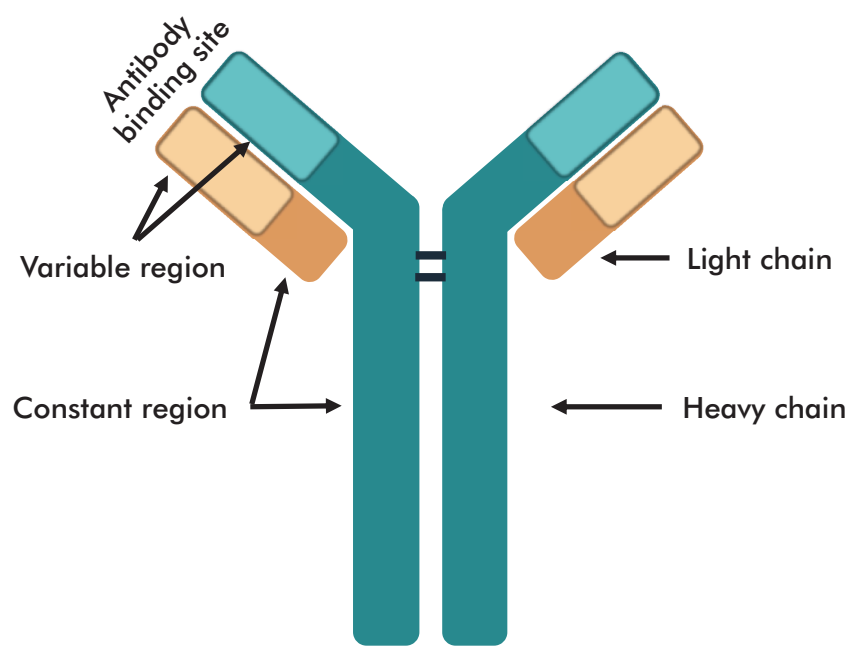

B

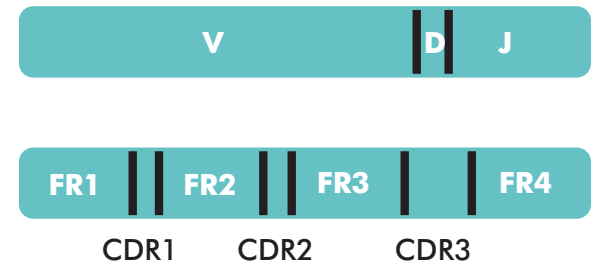

Figure 1: General antibody structure $\boldsymbol{A}$ Antibodies are comprised of two heavy and light chain consisting of a constant and a variable part; $\boldsymbol{B}$ variable regions are recombined by $V, D$ and $J$ gene segments resulting in four frame work regions (FR) and 3 complementarity-determining regions 


\section{Thesis outline}

The overall aimed of the present thesis is to define new and accurate testing strategies to prevent future discrepancies between in vitro and clinical diagnoses. All approaches used within this thesis are summarised in Figure 2.

Part 1 focusses on the prevention of "false-negative" test outcomes resulting from nondetectable clinically relevant sensitisation in food allergic patients. We aimed to overcome this limitation by

- the identification of novel allergenic components from macadamia nut for stratifying macadamia nut allergic patients into patients experiencing rather mild versus severe symptoms (chapter 2)

- examining the diagnostic value of sesame oleosins in order to diagnose sesame allergic patients without detectable specific slgE using conventional slgE testing (chapter 3)

Part 2 focusses on the prevention of "false-positive“ test outcomes resulting from detectable clinically irrelevant sensitisation in sensitised but tolerant subjects. We aimed to overcome this limitation by defining differences in allergen specific antibody repertoires in allergic and sensitised but tolerant patients. To gain insights on these antibody repertoires, we performed

- linear epitope mapping:

- of the hen's egg components Gal d 1 and 3 in order to evaluate the potential of slgE binding to these epitopes in discriminating between hen's egg allergic and tolerant adults both sensitised to the full-length proteins (chapter 4)

- of Ara h 7 isoforms, upon comparing them with Ara h 2 and 6 regarding their ability to induce degranulation, in order to elucidate which epitopes may explain observed divergent potencies in inducing degranulation (chapter 5 and chapter 6).

and

- analysis of peanut $2 S$ albumin (Ara h 2 and 6) specific B cells and their corresponding antibodies on single cell level:

- In chapter 7, we evaluated the advantages of specific monoclonal antibodies (mAbs) compared to patient sera in order to define epitopes associated with allergy or tolerance and how to implement this knowledge into state-of-theart CRD. 
- In chapter 8, we compared two different methods to generate human mAbs from peripheral blood including single cell sequencing combined with heterologous expression and B cell immortalisation by Epstein-Barr Virus followed by limiting dilution.

- In chapter 9, we evaluated peanut 2S albumin-binding B cells from peanut allergic and peanut sensitised but tolerant subjects regarding their genetic features as VH family gene usage and HCDR3 sequence motifs. These insights may provide the basis for the development of novel in vitro diagnostic strategies.

Chapter 10 provides an overall discussion of the main findings in the context of the current literature.

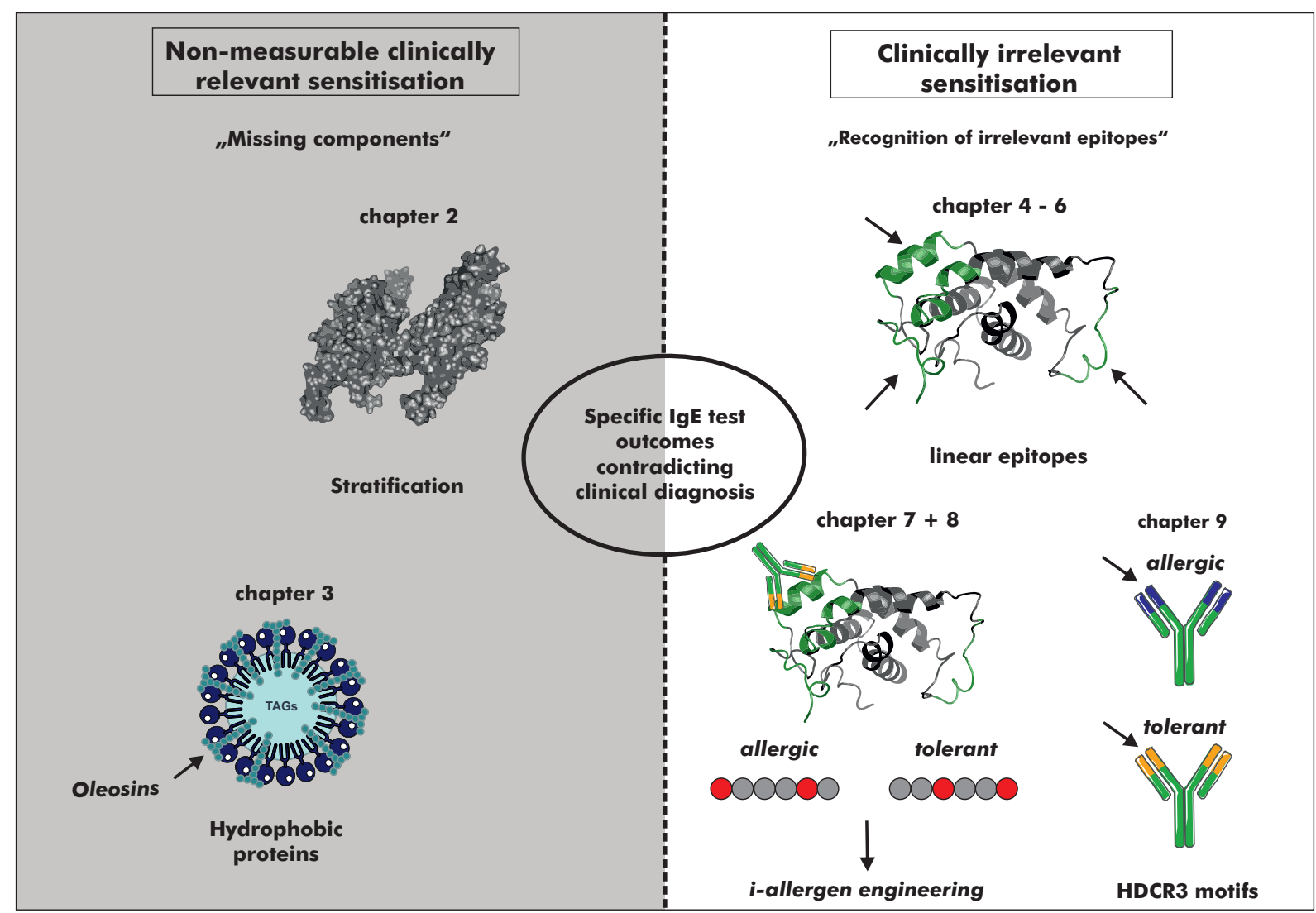

Figure 2: Overview on approaches to overcome „false-negative“ and „false-positive" test outcomes contradicting clinical diagnosis 


\section{References}

1. Ho, M. H.-K., Wong, W. H.-S. \& Chang, C. Clinical Spectrum of Food Allergies: a Comprehensive Review. Clin Rev Allergy Immunol 46, 225-40 (2012).

2. Lyons, S. A. et al. Food Allergy in Adults: Substantial Variation in Prevalence and Causative Foods Across Europe. J Allergy Clin Immunol Pract 7, 19208.e11 (2019).

3. Lyons, S. A. et al. Prevalence of Food Sensitization and Food Allergy in Children Across Europe. J Allergy Clin Immunol Pract 8, 2736-46.e9 (2020).

4. Sicherer, S. H. \& Sampson, H. A. Food allergy: Epidemiology, pathogenesis, diagnosis, and treatment. J Allergy Clin Immunol 133, 291-307.e5 (2014).

5. Pajno, G. B. et al. EAACI Guidelines on allergen immunotherapy: IgE-mediated food allergy. Allergy 73, 799-815 (2017).

6. Lanser, B. J. et al. Current Options for the Treatment of Food Allergy. Ped Clin North Am 62, 1531-49 (2015).

7. Van Erp, F. C. et al. Standardized food challenges are subject to variability in interpretation of clinical symptoms. Clin Transl Allergy 4, 43 (2014).

8. Van Maaren, M. S. \& Dubois, A. E. J. Dutch guideline on food allergy. Neth $J$ Med 74, 376-82 (2016).

9. Muraro, A. et al. EAACI Food Allergy and Anaphylaxis Guidelines: diagnosis and management of food allergy. Allergy 69, 1008-25 (2014).

10. Ansotegui, I. J. et al. IgE allergy diagnostics and other relevant tests in allergy, a World Allergy Organization position paper. World Allergy Organ J 13, 100080 (2020).

11. David, N. A., Penumarti, A., Burks, A. W. \& Slater, J. E. Food allergen extracts to diagnose food-induced allergic diseases. Ann Allergy Asthma Immunol 119, 101-7 (2017).

12. Sato, S., Yanagida, N. \& Ebisawa, M. How to diagnose food allergy. Curr Opinion Allergy Clin Immunol 18, 214-21 (2018).

13. Soares-Weiser, K. et al. The diagnosis of food allergy: a systematic review and meta-analysis. Allergy 69, 76-86 (2013).

14. Nilsson, C. et al. Accuracy of component-resolved diagnostics in peanut allergy: Systematic literature review and meta-analysis. Ped Allergy Immunol 31, 30314 (2020).

15. Hemmings, O. et al. Ara $\mathrm{h} 2$ is the dominant peanut allergen despite similarities with Ara h 6. J Allergy Clin Immunol 146, 621-30.e5 (2020). 
16. Klemans, R. J. B. et al. The diagnostic value of specific $\lg \mathrm{E}$ to Ara $\mathrm{h} 2$ to predict peanut allergy in children is comparable to a validated and updated diagnostic prediction model. J Allergy Clin Immunol 131, 157-63 (2013).

17. Klemans, R. J. B. et al. Ara h 2 Is the Best Predictor for Peanut Allergy in Adults. J Allergy Clin Immunol Pract 1, 632-8.e1 (2013).

18. Schwager, C. et al. Peanut oleosins associated with severe peanut allergy - importance of lipophilic allergens for comprehensive allergy diagnostics. J Allergy Clin Immunol 140, 1331-8.e8 (2017).

19. Leduc, V. et al. Identification of oleosins as major allergens in sesame seed allergic patients. Allergy 61, 349-56 (2006).

20. Zuidmeer-Jongejan, L. et al. Oil body-associated hazelnut allergens including oleosins are underrepresented in diagnostic extracts but associated with severe symptoms. Clin Transl Allergy 4, 4 (2014).

21. Hamilton, R. G. \& Kleine-Tebbe, J. Molecular Allergy Diagnostics: Analytical Features That Support Clinical Decisions. Curr Allergy Asthma Rep 15, 57 (2015).

22. Ramsland, P. A. \& Farrugia, W. Crystal structures of human antibodies: a detailed and unfinished tapestry of immunoglobulin gene products. $J \mathrm{Mol} R e c o g$ 15, 248-59 (2002).

23. Davies, D. R. \& Metzger, H. Structural Basis of Antibody Function. Ann Rev Immunol 1, 87-115 (1983).

24. Gough, N. M. \& Bernard, O. Sequences of the joining region genes for immunoglobulin heavy chains and their role in generation of antibody diversity. Proc Natl Acad Sci U S A 78, 509-13 (1981).

25. Xu, J. L. \& Davis, M. M. Diversity in the CDR3 Region of VH Is Sufficient for Most Antibody Specificities. Immunity 13, 37-45 (2000).

26. Di Noia, J. M. \& Neuberger, M. S. Molecular Mechanisms of Antibody Somatic Hypermutation. Ann Rev Biochem 76, 1-22 (2007). 

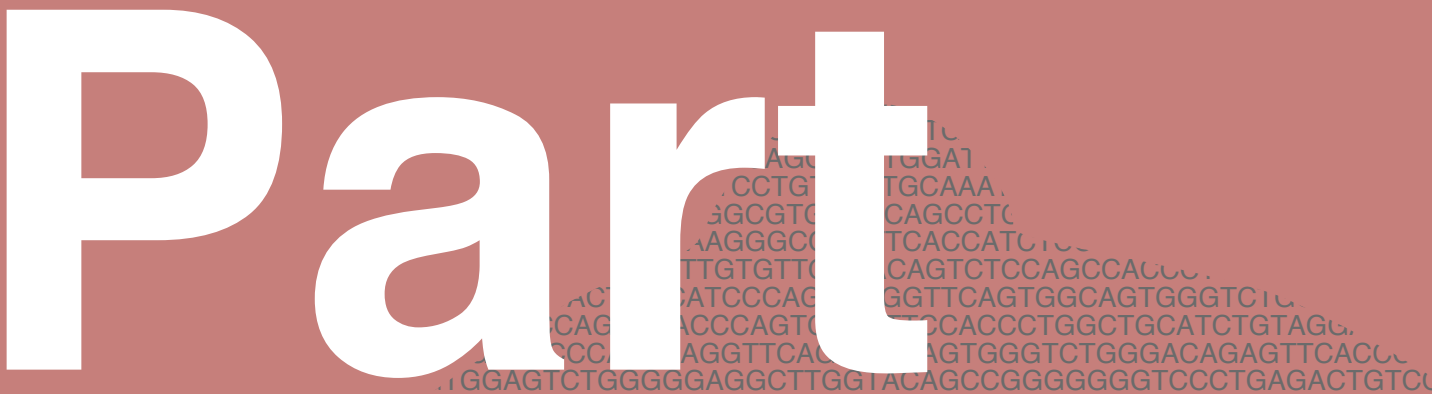

ITATCGAGACTCTGTGAAGGGCCGCTTCACCATCTCCAGAGACAATGCCAAAAACh

ऽAGGGGACCACGGTCATCGTCTCCTCAGCAGGTGCAGCTGGTGGAGTCTGGGGGAGGL

¿GAGTGGGTTTCATTTATACGCAAGGATGGAGCTAGTAAATACTATGGAGACTCCGTGAAGGGy

ICCCTGGGTTACTTTGACTACTGGGGCCAGGGAACCCTGGTCACCGTCTCCTCAGGAAATTGTGT

AAACCTGGCCAGGCTCCCAGGCTCCTCATCTATGATGCATCCAACAGGGCCACTGGCATCCC

ACTGGCCTCCGCTCACTTTCGGCGGAGGGACCAAGGTGGAGATCAAACGACATCCAGATGACCCAG

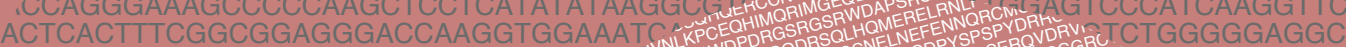

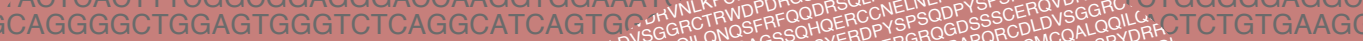

AAAGATAAGGTTCCAGACTATGACGAGGTCGAOLDVSLOQILONRGAGSEDSY ERRRERGR FPAPONORCMCYSPPYHLMOK GGTCATCGT

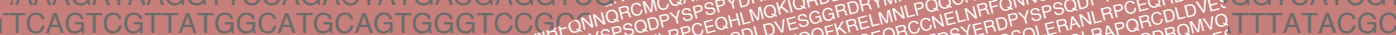

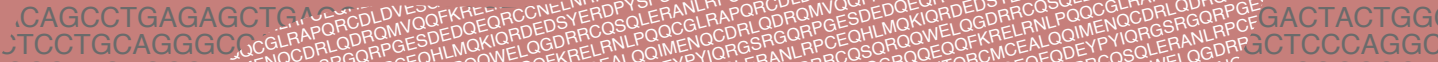

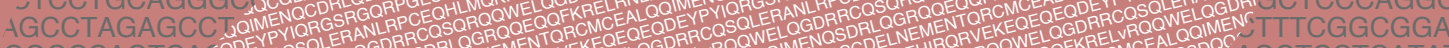
;GGGCCAGTCA

GCCTGATGATT

ATGGCCAGTC

CCATGGGGGTCCOCCAGAG

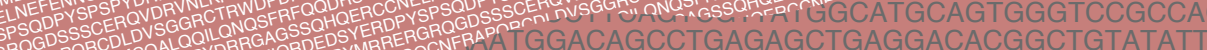

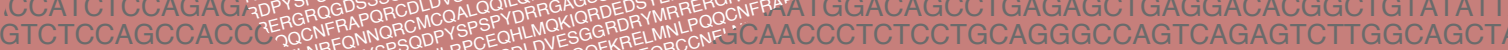

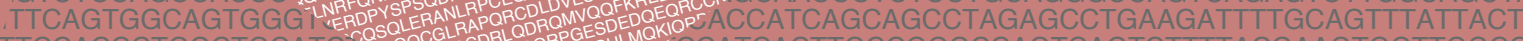

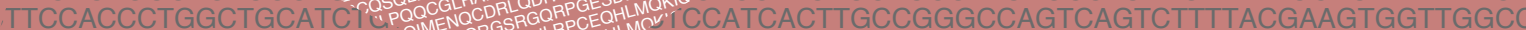
iCAGTGGGTCTGGGACAGAGTTCPYORLEANLRPEQHLMTCAGCAGTCTGCAGCCTGATGATITTGCAACTTACTACTGTCAACAA ACAGCCGGGGGGGTCCCTGAGACTGTCCTGTGCAGCCTCTGGATTCACGTTTAGGAGATATGCTTTGAGTTGGGTCCGC GCTTCACCATCTCCAGAGACAATGCCAAAAACATCCTGTTTCTGCAAATGGCCAGTCCGAGAGTCGAAGACGCGGCCGTI TCAGCAGGTGCA

AATGGAGCTAGTAA

ICCAGGGAACCCTGGTCACC

„GAGGGACCAAGGTGGAGATCAAAC

. CATATATAAGGCGTCTAATTTACAAGAT

AAGGTGGAAATCAAACGAGGTGCAGTTGG

ACGAGGTCGAGATTATGGACGTCTGGGGCCAG

GGGTCCGCCAGGCTCCAGGCAAGGGGCTGGAGT

GGCTGTATATTACTGTGCGAAAGATAGGTCCCTGGG

TTGGCAGCTACTTAGCCTGGTACCAACATAAACC

TTITTACTGTCAGCAGCGTAGCGACTGGCCTC

IACTTTCGG

IGCTGGAGT

GGTTCCAG

TGGCATG

CTGAGGA

AGTCAG

AGATT

TCATA

CTTTC

:GAAG

'TCTC

AAAG

ITCT

GAG

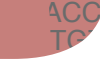

GGTCCAGCCTGGGGGGTCCCTGAGACTCTCCTGTGTAGCGTCT

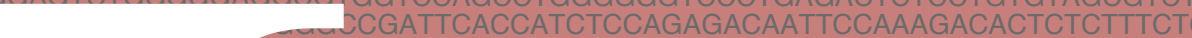
TGTGTTGACACAGTCTCCAGCCACCCTGTCTITGTCTCCAGGGGAAAG/ ATCCCAGCCAGGTTCAGTGGCAGTGGGTCTGGGACAGACTTCACTCTCA ACCCAGTCTCCTTCCACCCTGGCTGCATCTGTAGGAGACAGAGTCTCCAT AGGTTCAGCGGCAGTGGGTCTGGGACAGAGTTCACCCTCACCATCAGCA GAGGCTTGGTACAGCCGGGGGGGTCCCTGAGACTGTCCTGTGCAGCCTC TAAGGGCCGCTTCACCATCTCCAGAGACAATGCCAAAAACATCCTGTTTC ATCGTCTCCTCAGCAGGTGCAGCTGGTGGAGTCTGGGGGAGGCGTGGT ACGCAAGGATGGAGCTAGTAAATACTATGGAGACTCCGTGAAGGGCCGA ACGCAAGGATGGAGCTAGTAAATACTATGGAGACTCCGTGAAGGGCCGA TTGGGGCCAGGGAACCCTGGTCACCGTCTCCTCAGGAAATTGTGTTGACA AGGCTCCTCATCTATGATGCATCCAACAGGGCCACTGGCATCCCAGCCA ATATATAAGGCGTCTAATTTACAAGATGGAGTCCCATCAAGGTTCAGCGG GTGGAAATCAAACGAGGTGCAGTTGGTGGAGTCTGGGGGAGGCTTGGTA (1) TCAGTGGCAGTGGAAACAAAACATATTATCGAGACTCTGTGAAGGGCCGC
TCGAGATTATGGACGTCTGGGGCCAGGGGACCACGGTCATCGTCTCCTC, CAGGCTCCAGGCAAGGGGCTGGAGTGGGTITCATTTATACGCAAGGATG TTACTGTGCGAAAGATAGGTCCCTGGGTTACTTTGACTACTGGGGCCAGG TACTTAGCCTGGTACCAACATAAACCTGGCCAGGCTCCCAGGCTCCTCAT TGTCAGCAGCGTAGCGACTGGCCTCCGCTCACTITCGGCGGAGGGACC TGGTATCAGCAGAAACCAGGGAAAGCCCCCAAGCTCCTCATATATAAGGC TGGTATCAGCAGAAACCAGGGAAAGCCCCCAAGCTCCTCATATATAAGGC TATGATAGTGCCCTACTCACTTTCGGCGGAGGGACCAAGGTGGAAATCA CCAGGCTCCAGGGCAGGGGCTGGAGTGGGTCTCAGGCATCAGTGGCAG TGGATTCACCTTCAGTCGTTATGGCATGCAGTGGGTCCGCCAGGCTCCAC GCAAATGGACAGCCTGAGAGCTGAGGACACGGCTGTATATTACTGTGCG GCAACCCTCTCCTGCAGGGCCAGTCAGAGTCTTGGCAGCTACTTAGCCTC CATCAGCAGCCTAGAGCCTGAAGATTTTGCAGTTTATTACTGTCAGCAGC TCACTTGCCGGGCCAGTCAGTCTTITACGAAGTGGTTGGCCTGGTATCAC GTCTGCAGCCTGATGATTTTGCAACTTACTACTGTCAACAATATGATAGT

TGGATTCACGTTTAGGAGATATGCTTTGAGTTGGGTCCGCCAGGCTCCA

ATGGCCAGTCCGAGAGTCGAAGACGCGGCCGTITATTACTGTGC TCCGTGAAGGGCCGATTCACCATCTCCAGAGACAATTCCAAAGACACTCTCTTTCTGCAAATGGAC CTCCTCAGGAAATTGTGTTGACACAGTCTCCAGCCACCCTGTCTTTGTCTCCAGGGGAAAGAGCAACCCTCT CAGGGCCACTGGCATCCCAGCCAGGTTCAGTGGCAGTGGGTCTGGGACAGACTTCACTCTCACCATCAGCAG ACGACATCCAGATGACCCAGTCTCCTTCCACCCTGGCTGCATCTGTAGGAGACAGAGTCTCCATCACTTGCCGG GATGGAGTCCCATCAAGGTTCAGCGGCAGTGGGTCTGGGACAGAGTTCACCCTCACCATCAGCAGTCTGCAGCD ITGGTGGAGTCTGGGGGAGGCTTGGTACAGCCGGGGGGGTCCCTGAGACTGTCCTGTGCAGCCTCTGGATTCAC ATATTATCGAGACTCTGTGAAGGGCCGCTTCACCATCTCCAGAGACAATGCCAAAAACATCCTGTITCTGCAAATGC GCCAGGGGACCACGGTCATCGTCTCCTCAGCAGGTGCAGCTGGTGGAGTCTGGGGGAGGCGTGGTCCAGCCTGG GAGTGGGTTTCATTTATACGCAAGGATGGAGCTAGTAAATACTATGGAGACTCCGTGAAGGG

CCTGGGTTACTTTGACTACTGGGGCCAGGGAACCCTGGTCACCGTCTCCTCAGGAAATTGTC

ITAAACCTGGCCAGGCTCCCAGGCTCCTCATCTATGATGCATCCAACAGGGCCACTGGCATCr

IGGCCTCCGCTCACTTTCGGCGGAGGGACCAAGGTGGAGATCAAACGACATCCAGATGACCC AGGGAAAGCCCCCAAGCTCCTCATATATAAGGCGTCTAATTTACAAGATGGAGTCCCATCAAGC CACTTTCGGCGGAGGGACCAAGGTGGAAATCAAACGAGGTGCAGTTGGTGGAGTCTGGGGG/

GGCTGGAGTGGGTCTCAGGCATCAGTGGCAGTGGAAACAAAACATATTATCGAGACTCTGTG,

\AGGTTCCAGACTATGACGAGGTCGAGATTATGGACGTCTGGGGCCAGGGGACCACGGTCATCGI

ITATGGCATGCAGTGGGTCCGCCAGGCTCCAGGCAAGGGGCTGGAGTGGGTTTCATTTATACGCAAGGATGGAGiL I AG I ¿AGAGCTGAGGACACGGCTGTATATTACTGTGCGAAAGATAGGTCCCTGGGTTACTTTGACTACTGGGGCCAGGGAACCCTG ,AGGGCCAGTCAGAGTCTTGGCAGCTACTTAGCCTGGTACCAACATAAACCTGGCCAGGCTCCCAGGCTCCTCATCTATGATGC GAGCCTGAAGATTTTGCAGTTTATTACTGTCAGCAGCGTAGCGACTGGCCTCCGCTCACTTTCGGCGGAGGGACCAAGGTGGA 


\section{Preventing „false-negative“" slgE testing: non-detectable clinically relevant sensitisation}

CCAGGCTCCAGGGCAGGGGCTGGAGTGG

ГATTACTGTGCGAAAGATAAGGTTCCAGACTA

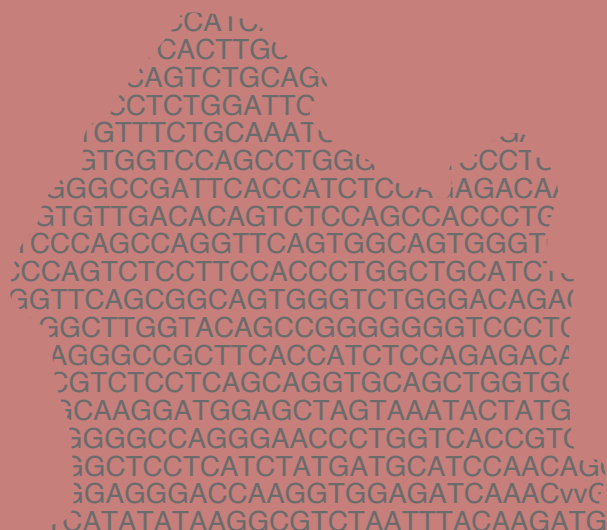
IGGCATCAGTGGCAGTGGAAACAAAACATATTATC
¿GAGGUS:

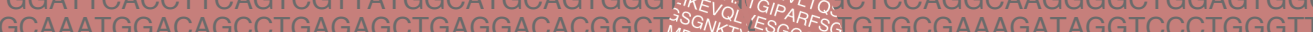

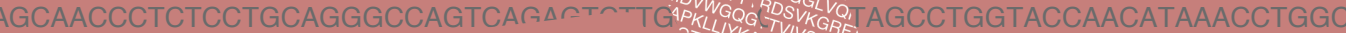
CCATCAGCAGCCTA' CACTTGCCGGGCr GTCTGCAGCCTr STGGATTCACG

STGCAAATGGCC.

CCAGCCTGGGGG

TCACCATCTCCAG

CAGTCTCCAGCCAC
GGTTCAGTGGCAGTG

TTCCACCCTGGCTGCA

CAGTGGGTCTGGGACA

CAGCCGGGGGGGTCCC

TTCACCATCTCCAGAGAC,

AGCAGGTGCAGCTGGTGC

GAGCTAGTAAATACTATGS

GAACCCTGGTCACCGTC

CTATGATGCATCCAAC

AAGGTGGAGATC

GTCTAATTTA

AACGAGGTGC

TGGAAACAA

AGCAAGGG'

AAAGATAC

AGTACCA

GTAGCC

CAGAAACL

GCCCTACTCAL GGGCAG! $\rightarrow$ Ci

GAAAGA

TCAGTC

AGCCT

TGTCA

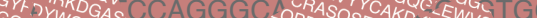

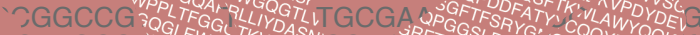

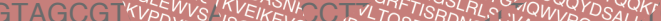

TCTCTTTC'WYDE

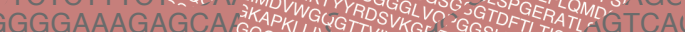

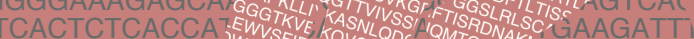

AAGTCTCCATCACTTARAE UGA

OCATCAGCAGTCLGSY

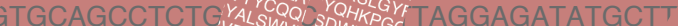

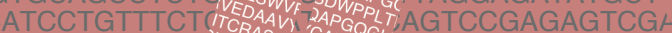

iGAGGCGTGGTCG

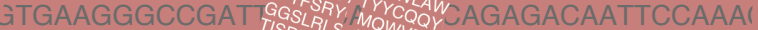

AAATTGTGTTGACA

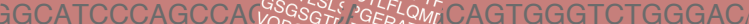

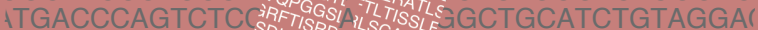

CAAGGTTCAGCG DDOMTD TAK NA ASCCTGGGACAGAGTTCACr

GGGGAGGCTTGG

CTGTGAAGGGCCGCTTC

TTCATCGTCTCCTCAGCAGGTGCAGCTGGTGGAGTCTC

ATACGCAAGGATGGAGCTAGTAAATACTATGGAGAC

$\because$ TGGGGCCAGGGAACCCTGGTCACCGTCTCCTC

OTCCTCATCTATGATGCATCCAACAGGGCC

IGGACCAAGGTGGAGATCAAACvVGAГ

TATAAGGCGTCTAATTTACAAGATr

GAAATCAAACGAGGTGCAGTTr

GGCAGTGGAAACAAAACAT

ITTATGGACGTCTGGGGCC

ICCAGGCAAGGGGCTGGA

TGCGAAAGA ${ }^{-1}$ TGTCCT

AGCAGCG

ATCAGCA

IATAGTGS

TACTGTG

GATTCACr

CAAATGGA

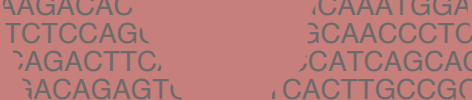

$\begin{array}{lr}\text { AAGACAC } & \text {.CAAATGGA } \\ \text { TCTCCAGL } & \text { iCAACCCTC } \\ \text { :ACACTTC, } & \text {,CATCAGCAC } \\ \text { IACAGAGTL } & \text { CACTTGCCGC }\end{array}$

CACAGAGTL JAGTCTGCAGCC

CCTGTGCAGCCTCTGGATTCACGi

AACATCCTGTTTCTGCAAATGGCCA

iGAGGCGTGGTCCAGCCTGGGGG

3AAGGGCCGATTCACCATCTCCA 
MIUT:

AGCCTCTGGA7

CCTGTTTCTGCAAA

iGCGTGGTCCAGCCTC

AGGGCCGATTCACCATL ,

AATTGTGTTGACACAGTCTCCAGCCALL

UTGGCATCCCAGCCAGGTTCAGTGGCAGTGGG'TC I

$\because$ CAGATGACCCAGTCTCCTTCCACCCTGGCTGCATCTGTAGL

AGTCCCATCAAGGTTCAGCGGCAGTGGGTCTGGGACAGAGTTCACL

FAGTCTGGGGGAGGCTTGGTACAGCCGGGGGGGTCCCTGAGACTGTCL

ITATCGAGACTCTGTGAAGGGCCGCTTCACCATCTCCAGAGACAATGCCAAAAACh ¿AGGGGACCACGGTCATCGTCTCCTCAGCAGGTGCAGCTGGTGGAGTCTGGGGGAGG iGAGTGGGTTTCATTTATACGCAAGGATGGAGCTAGTAAATACTATGGAGACTCCGTGAAGGG ATAAACCTGGCCAGGCTCCCAGGCTCCTCATCTATGATGCATCCAACAGGGCCACTGGCATCCC ACTGGCCTCCGCTCACTTTCGGCGGAGGGACCAAGGTGGAGATCAAACGACATCCAGATGACCCAG

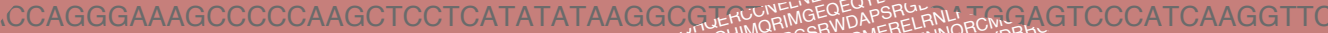

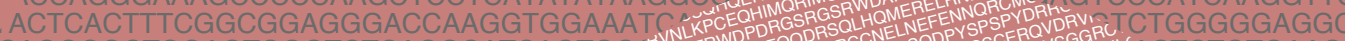

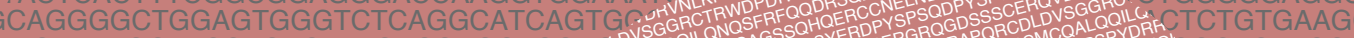

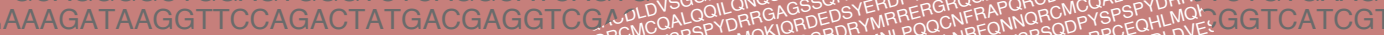

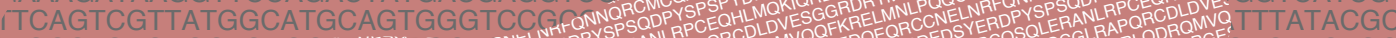

TCCTGCAGGGCCTGAAPORCDLVM

STCCTGCAGGGCSGGLPAP

tGCCTAGAGCCT

GCCTGATGAT

CACGTTTAG

ATGGCCAGTC

CCATCTCCAGAG

GTCTCCAGCCACC

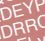

A

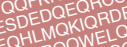

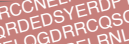

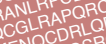

\section{RQMVQQKEDQEQRC}

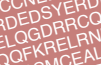

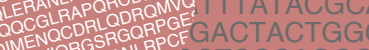

LGG

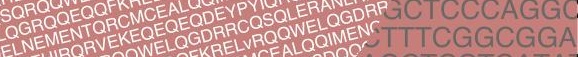
KQELQGDRRC

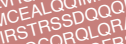
DOIMENOSDRLCCNENEFENYDSYDIRDOCOR GGGACCAAGGTG HQN

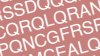
PISSPTPYQGRQO GEQEQYDSRGD PSPYDRRGAGKPCEQHIMQRIMRGSRWDAMEREL KREG GTTCTCAGGCATCAG

\section{ARCDL}

年

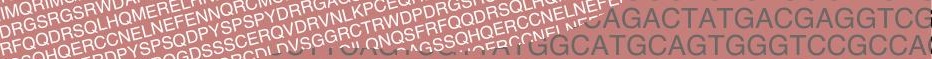

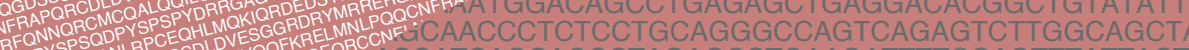

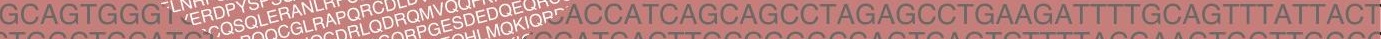

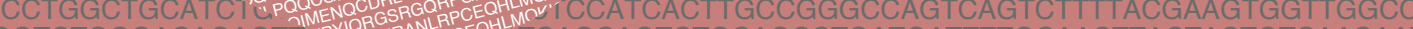

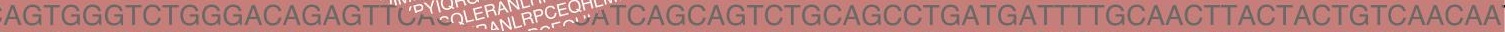
ACAGCCGGGGGGGTCCCTGAGACTGTCCTGTGCAGCCTCTGGATTCACGTTTAGGAGATATGCTTTGAGTTGGGTCCGC GCTTCACCATCTCCAGAGACAATGCCAAAAACATCCTGTTTCTGCAAATGGCCAGTCCGAGAGTCGAAGACGCGGCCGTTI TCAGCAGGTGCAGCTGGTGGAGTCTGGGGGAGGCGTGGTCCAGCCTGGGGGGTCCCTGAGACTCTCCTGTGTAGCGTC) AATGGAGCTAGTAAATACTATGGAGACTCCGTGAAGQTTCCAGAGACAATTCCAAAGACACTCTCTTTCT ¿CCAGGGAACCCTGGTCACCGTCTCCTCAGGAAAT SCCACCCTGTCTTTGTCTCCAGGGGAAAGA ¿TCCTCATCTATGATGCATCCAACAGGGCCACTG CATATATAAGGCGTCTAATTTACAAGATGGAGTCCCA AAGGTGGAAATCAAACGAGGTGCAGTTGGTGGAGTCT जGCATCAGTGGCAGTGGAAACAAAACATATTATCGAGAC AACGAGGTCGAGATTATGGACGTCTGGGGCCAGGGGACCA GGGTCCGCCAGGCTCCAGGCAAGGGGCTGGAGTGGGTTTC
GGCTGTATATTACTGTGCGAAAGATAGGTCCCTGGGTTACTTT ITTGGCAGCTACTTAGCCTGGTACCAACATAAACCTGGCCAGG
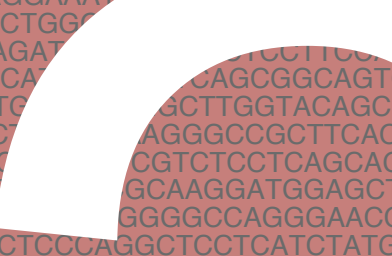

AGGCCGCTTCACCATC CGTCTCCTCAGCAGGTG GAAGGATGGAGCTAGTA GGGCCAGGGAACCCTGG GGTCCTCATCTATGATGCI

TTTATTACTGTCAGCAGCGTAGCGACTGGCCTCCGCTCACTTTCGGCGGAGGGACCAAGGTGGAC AACTTTCGGCGGAGGGACCAAGGTGGAAATCAAACGAGGT IGCTGGAGTGGGTCTCAGGCATCAGTGGCAGTGGAAAC GGTTCCAGACTATGACGAGGTCGAGATTATGGACGT TGGCATGCAGTGGGTCCGCCAGGCTCCAGGCAA CTGAGGACACGGCTGTATATTACTGTGCGAAA 'AGTCAGAGTCTTGGCAGCTACTTAGCCTG AGATTTTGCAGTTTATTACTGTCAGCAG TTACGAAGTGGTTGGCCTGGTATCAG TTACGAAGTGGTTGGCCTGGTATCA
ACTTACTACTGTCAACAATATGATA ACTTACTACTGTCAACAATATG
¿TTTGAGTTGGGTCCGCCAG :GAAGACGCGGCCGTTTATI 'TCTCCTGTGTAGCC AAAGACACTCTCT iTCTCCAGGGGAAA CAGACTTCACT GAGACAGAGTC ACCCTCACCA ;AAAAACATCO ìGGGGAGGC AUTumuTCCGTGAAG GCATCTGTAGGAGACAGAGTCTCCAT ACAGAGTTCACCCTCACCATCAGCA CCCTGAGACTGTCCTGTGCAGCCTC GACAATGCCAAAAACATCCTGTTTC TGGAGTCTGGGGGAGGCGTGGTC TGGAGACTCCGTGAAGGGCCGA CTCCTCAGGAAATTGTGTTGACA AGGGCCACTGGCATCCCAGCCAC GACATCCAGATGACCCAGTCTCC GGAGTCCCATCAAGGTTCAGCGG GGAGTCTGGGGGAGGCTTGGTA ATCGAGACTCTGTGAAGGGCCGO TATCGAGACTCTGTGAAGGGCCGO
GGGGACCACGGTCATCGTCTCCTC,
TGGGTTTCATTTATACGCAAGGATG GTGGGTTTCATTTATACGCAAGGATG ACCTGGCCAGGCTCCCAGGCTCCTCAT AAACCTGGCCAGGCTCCCAGGCTCCTCAT GGCCTCCGCTCACTTTCGGCGGAGGGACCA
GAGACCCAAGCTCCTCATATATAAGGC TTTCGGCGGAGGGACCAAGGTGGAAATCA GCTGGAGTGGGTCTCAGGCATCAGTGGCAG TAAGGTTCCAGACTATGACGAGGTCGAGATTATG A A GCCTGAGAGCTGAGGACACGGCTGTATATTACTGTGCG LAGCCTGAGAGCTGAGGACACGGCTGTATATTACTGTGCG
CCTGCAGGGCCAGTCAGAGTCTTGGCAGCTACTTAGCCTC CTCCTGCAGGGCCAGTCAGAGTCTTGGCAGCTACTTAGCCTC
GCCTAGAGCCTGAAGATTTTGCAGTTTATTACTGTCAGCAGC GCCGGGCCAGTCAGTCTTTTACGAAGTGGTTGGCCTGGTATCAC TGCAGCCTGATGATTTTGCAACTTACTACTGTCAACAATATGATAGT GAGTTGGGTCCGCCAGGCTCCA GACGCGGCCGTITATTACTGTGD CTGTGTAGCGTCTGGATTCACCT ACACTCTCTTTCTGCAAATGGAC CCAGGGGAAAGAGCAACCCTCTI

CAGGGCCACTGGCATCCCAGCCAGGTTCAGTCGCAGTCGGTCTGGGACAGACTTCACTCTCACCATCAGCAG ACGACATCCAGATGACCCAGTCTCCTTCCACCCTGGCTGCATCTGTAGGAGACAGAGTCTCCATCACTTGCCGG GATGGAGTCCCATCAAGGTTCAGCGGCAGTGGGTCTGGGACAGAGTTCACCCTCACCATCAGCAGTCTGCAGCO ITGGTGGAGTCTGGGGGAGGCTTGGTACAGCCGGGGGGGTCCCTGAGACTGTCCTGTGCAGCCTCTGGATTCAD ATATTATCGAGACTCTGTGAAGGGCCGCTTCACCATCTCCAGAGACAATGCCAAAAACATCCTGTTICTGCAAATG GCCAGGGGACCACGGTCATCGTCTCCTCAGCAGGTGCAGCTGGTGGAGTCTGGGGGAGGCGTGGTCCAGCCTGG GGAGTGGGTTTCATTTATACGCAAGGATGGAGCTAGTAAATACTATGGAGACTCCGTGAAGGG

ICCTGGGTTACTTTGACTACTGGGGCCAGGGAACCCTGGTCACCGTCTCCTCAGGAAATTGT
ITAACCTGGCCAGGCTCCCAGGCTCCTCATCTATGATGCATCCAACAGGGCCACTGGCATCr

IGGCCTCCGCTCACTTTCGGCGGAGGGACCAAGGTGGAGATCAAACGACATCCAGATGACC AGGGAAAGCCCCCAAGCTCCTCATATATAAGGCGTCTAATTTACAAGATGGAGTCCCATCAAGE CACTTTCGGCGGAGGGACCAAGGTGGAAATCAAACGAGGTGCAGTTGGTGGAGTCTGGGGG/ GGCTGGAGTGGGTCTCAGGCATCAGTGGCAGTGGAAACAAAACATATTATCGAGACTCTGTG

IAGGTTCCAGACTATGACGAGGTCGAGATTATGGACGTCTGGGGCCAGGGGACCACGGTCATCGI

ITATGGCATGCAGTGGGTCCGCCAGGCTCCAGGCAAGGGGCTGGAGTGGGTTTCATTTATACGCAAGGATGGAGi I AG I iAGAGCTGAGGACACGGCTGTATATTACTGTGCGAAAGATAGGTCCCTGGGTTACTTTGACTACTGGGGCCAGGGAACCCTG AGGGCCAGTCAGAGTCTTGGCAGCTACTTAGCCTGGTACCAACATAAACCTGGCCAGGCTCCCAGGCTCCTCATCTATGATG GAGCCTGAAGATTTTGCAGTTTATTACTGTCAGCAGCGTAGCGACTGGCCTCCGCTCACTTTCGGCGGAGGGACCAAGGTGGA 


\section{IgE-binding to vicilin-like antimicrobial peptides is associated with systemic reactions to macadamia nut}
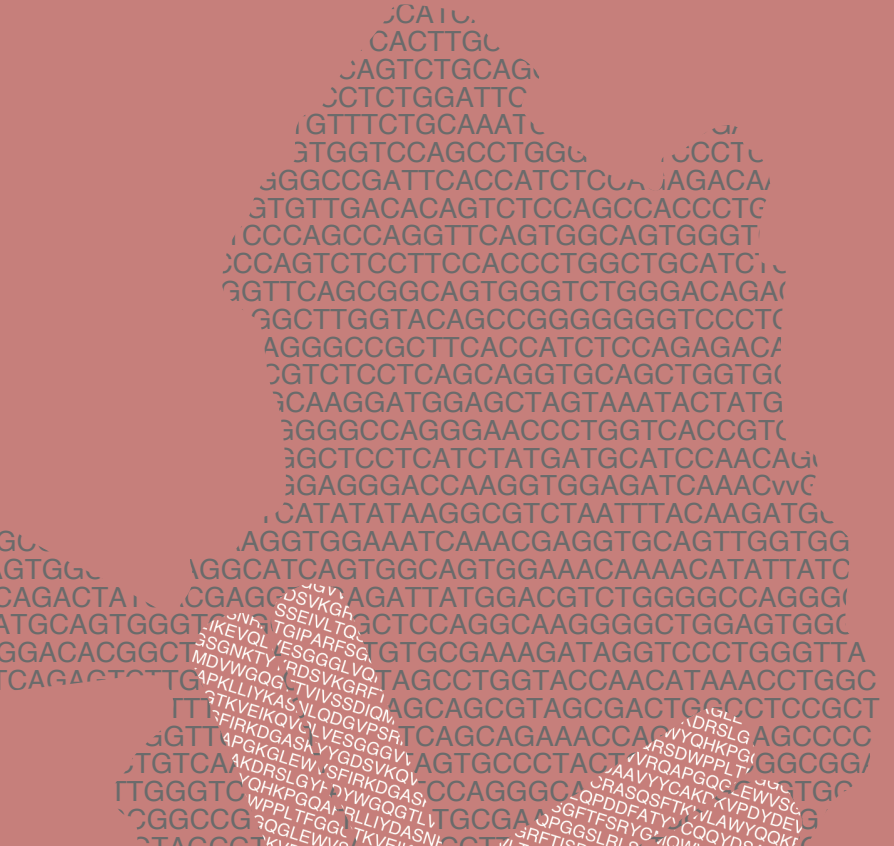

published as letter in Clin Transl Allergy 2020;10:55.

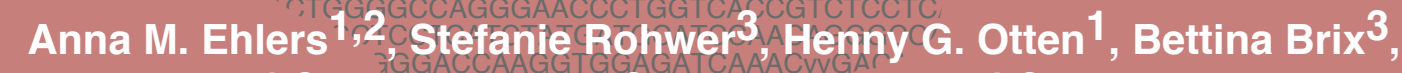

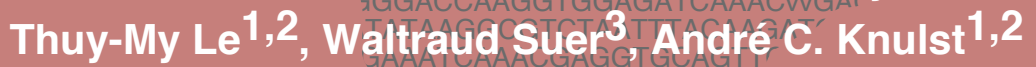

1 Center for TranslationalimmuGG A Utrecht University, Netherlands

CTRepartment of Dêrtûatólólogy/Allergology, University Medical Center Utrecht, Utiech University, Netherlands

3GACAC Unimmun AGAAATGGA Gubeck, Germany

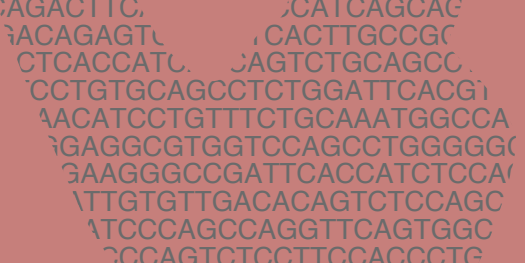




\section{Abstract}

\section{Background}

Macadamia nut can induce fatal allergic reactions and changes in dietary habits will raise their consumption in industrialised countries. So far, diagnosis of macadamia nut allergy by slgE solely relies on the macadamia nut extract, but single components are lacking.

\section{Methods}

Macadamia nut proteins recognised by $\operatorname{lgE}$ from 2 macadamia nut extract positive sera were identified by mass spectrometry (vicilin-like antimicrobial peptides: VLAP). Sensitisation to macadamia nut extract and heterologously expressed isoform VLAP-2-3 was evaluated in 82 nut allergic (NA) and 27 nut tolerant (NT) patients (no tree nut allergy reported) comprehending 10 macadamia nut allergic (MA) and 18 explicitly reported macadamia nut tolerant patients (MT), using line blots. Co-sensitisation to additional VLAP isoforms and other vicilins was evaluated in $8 \mathrm{MA}, 12 \mathrm{MT}$ and 14 NA patients sensitised to VLAP-2-3. Functional properties were determined by indirect basophil activation.

\section{Results}

Even though proteins recognised by IgE were identified as VLAP-2-1, 2-2 and 2-3, only peptides specifically belonging to VLAP-2-3 were detected by mass spectrometry. The macadamia nut extract was recognised by $33 \%$ of NA patients (27/82) including 3 MA patients and $26 \%$ of NT patients (7/27, 3 MT). Similarly, $29 \%$ of NA (24/82) patients showed partly strong slgE-binding to VLAP-2-3 including 3 MA patients with systemic reactions to macadamia nut. Contrary, VLAP-2-3 was recognised by only 2 NT (1 MT) patients $(7 \%)$ with very low slgE titres. Simultaneous recognition of the isoforms VLAP2-1 and 2-2 was observed in all patients positive for VLAP-2-3 with partly reduced slgE titres in $59 \%$ of these patients. Additionally, all three VLAP isoforms were able to repeatedly induce basophil activation upon sensitisation with one MA serum.

\section{Conclusion}

VLAP proteins are the first described macadamia nut components with serological and functional allergenic properties and they are associated with systemic reactions to macadamia nut. 


\section{Introduction}

Tree nut allergies are classified as allergy against edible fruits or seeds growing on woody plants including almonds, Brazil nut, cashew nut, hazelnut, macadamia nut, pecan, pine nuts, pistachio nuts and walnuts. Probable tree nut allergy, defined as selfreported allergy accompanied by doctor's diagnosis and/or sensitisation to the specific tree nut, was evaluated in a systematic review to range from 0.05 to $4.9 \%$ in children from different regions and was mostly caused by almonds (UK), cashew nut (US), hazelnut (Europe) or walnut (UK, US) ${ }^{1}$. The relative contribution of macadamia nut allergy ranged from 0.8 to $9 \%$ in clinical studies ${ }^{2,3}$. In the Western World, a raise in the number of macadamia nut allergies is expected due to steadily growing demand on healthy food and their increasing use in pastry and confectionery 4 .

In several case reports, ingestion of macadamia nut was able to trigger fatal allergic reactions including anaphylaxis ${ }^{5-8}$. Although part of macadamia allergic (MA) patients showed false-negative slgE against macadamia nut extract ${ }^{9-11}$, proteins with molecular masses of 12, 17, 40, 45 and $50 \mathrm{kDa}$ were recognised by IgE in western blot analy$\operatorname{ses}^{7,8}$. Successful inhibition experiments with hazelnut extract or the walnut components Jug $r 1$ and Jug $r 4$ pinpointed towards $2 S$ albumins or $11 S$ globulins as potential allergens in macadamia nut ${ }^{6,12}$. Another seed storage protein family with known allergenic properties are vicilins ${ }^{13,14}$ and a 666 amino acid large vicilin precursor has been described for macadamia nut ${ }^{15}$. Post-translational processing of this precursor results in highly abundant antimicrobial peptides with a molecular mass of $8.1 \mathrm{kDa}$ and a isoelectric point $(\mathrm{pl})$ of $10.1^{16,17}$. Generally, vicilins have been described as marker for severe, non-pollen related walnut and hazelnut allergy ${ }^{18-20}$.

Characterisation of single components from macadamia nut may improve the diagnosis and stratification of macadamia nut allergic patients in the future. Hence, we screened macadamia nut extract for proteins being recognised by $\lg \mathrm{E}$ and characterised vicilinlike antimicrobial peptides (VLAP) 2-1, 2-2 and 2-3 as first single components in nut allergic patients including macadamia nut allergic ones. We further addressed cosensitisation to vicilins from tree nuts, seeds and legumes and the ability of VLAP isoforms to induce degranulation. 


\section{Methods}

\section{Patient selection}

For the serological characterisation of VLAP from macadamia integrifolia, we aimed for sera from MA patients. However, macadamia nut is not a very common cause of food allergy yet, resulting in a small group of MA patients $(n=10)$. A small group involves the risk that minor allergens, potentially recognised in only 10 to $20 \%$ of the patients, may stay undetected ${ }^{21}$. Hence, our population was enlarged with 72 nut allergic (NA) patients (in total $n=82$ ), potentially exhibiting slgE against macadamia nut allergens and theoretically resulting in at least 8 patients recognising VLAP (82 patients $\cdot 0.1=$ 8 patients). Patients $(n=27)$ without known history of a tree nut allergy served as a nut tolerant (NT) reference group. It was not required that tolerance was explicitly reported for all tree nuts, resulting in 18 patients with explicitly reported macadamia nut tolerance.

For the recruitment, we retrospectively screened patients who visited the outpatient clinic of the University Medical Center (UMC) Utrecht between 2008 and 2018 and were diagnosed with a nut allergy (almonds, Brazil nut, cashew nut, hazelnut, macadamia nut, pistachio and/or walnut) or with tolerance to tree nuts. Diagnoses were confirmed by either double-blind placebo-controlled food challenge (DBPCFC) or suggestive history by a trained physician. Suggestive history was defined as typical IgE-mediated symptoms like oral allergy syndrome (OAS), skin reactions, gastrointestinal, respiratory or cardiovascular symptoms and an onset within 2 hours upon ingestion. Severity of the reported symptoms in MA patients was graded by Sampsons' classification ${ }^{22}$. A subpopulation with sufficient material for further analyses was comprised of $8 \mathrm{MA}, 12$ macadamia nut tolerant (MT) and 14 NA patients sensitised to VLAP-2-3. Ethical approval (number 18-428) was acquired from the biobank committee of the UMC Utrecht and detailed patient characteristics are given in Table 1. 
Table 1: Patient characteristics

\begin{tabular}{lcc}
\hline & Nut allergic & Nut tolerant \\
\hline Number [n] & 82 & 27 \\
Macadamia nut allergy & 10 & NA \\
Macadamia nut tolerance & 3 & 15 \\
Not explicitly reported & 69 & 12 \\
Age (median [IQR]) & $28[18-62]$ & $33[20-55]$ \\
Sex female [n, \%] & $57[70 \%]$ & $20[74 \%]$ \\
Food challenge ${ }^{\mathbf{A}}[\mathrm{n}, \%]$ & $13[16 \%]$ & $13[48 \%]$ \\
& &
\end{tabular}

\begin{tabular}{lcc}
\hline Patient & Reported symptoms & Severity $^{\mathbf{A}}$ \\
\hline MA-1 & swelling lips, OAS ${ }^{\mathbf{B}}$, vomiting & Grade 3 \\
MA-2 & itching palate, swelling throat & Grade 3 \\
MA-3 & OAS & Grade 1 \\
MA-4 & OAS & Grade 1 \\
MA-5 & OAS & Grade 1 \\
MA-6 & OAS & Grade 1 \\
MA-7 & OAS, swelling throat, hoarseness, & Grade 4 \\
& dyspnoea & \\
MA-8 & OAS, vomiting, dyspnoea & Grade 3 \\
MA-9 & OAS, angio-oedema, dyspnoea & Grade 4 \\
MA-10 & OAS, dyspnoea & Grade 4 \\
A Severity score based on Sampson score & \\
B OAS $=$ oral allergy syndrome &
\end{tabular}

\section{Sera for the identification of proteins recognised by $\lg E$}

Proteins of the macadamia nut extract recognised by IgE were identified with 2 macadamia nut sensitised samples from AbBaltis Reagents Ltd, Dublin, Ireland and Aalto Bio Reagents Ltd, Dublin, Ireland.

\section{Protein extraction from macadamia nut}

Roasted and salted commercially purchased macadamia nut kernels were ground and subsequently threefold defatted with acetone. Defatted and dried macadamia nut debris was resuspended in phosphate buffer saline (PBS, $50 \mathrm{mM}$ sodium phosphate, $150 \mathrm{mM} \mathrm{NaCl}, \mathrm{pH}$ 7.4) and incubated at $4{ }^{\circ} \mathrm{C}$ for 3 hours in an incubation shaker, followed by filtration and centrifugation ( $3.300 \times \mathrm{g}$ at $\left.4{ }^{\circ} \mathrm{C}, 30 \mathrm{~min}\right)$. The pellet, achieved by $100 \%$ ammonium sulfate precipitation, was resuspended in PBS and subsequently dialysed against PBS. 


\section{Preparative gel electrophoresis}

Crude macadamia extract was precipitated with cooled acetone and incubated at $-20^{\circ} \mathrm{C}$ for 2 hours. For isoelectric focusing (IEF), the resulted pellet $\left(20.000 \times \mathrm{g}\right.$ at $4^{\circ} \mathrm{C}$ for $30 \mathrm{~min}$ ) was resuspended in IEF buffer (2.5\% sulfobetaine 3-10, $4 \mathrm{M}$ urea, $2 \mathrm{M}$ thiourea, $0.5 \%$ (v/v) Carrier Ampholyte pH 3-10, $40 \mathrm{mM} \mathrm{DTT}$ and 0.003\% bromophenol blue) and an IPG-strip (ReadyStrip IPG Strips $7 \mathrm{~cm} \mathrm{pH} 3$ to 10, linear pH gradient, Bio-Rad) was rehydrated with this sample overnight at room temperature. Proteins were separated in the first dimension by isoelectric focusing using a PROTEAN i12 IEF System (final voltage: $4000 \mathrm{~V}$ for $15.000 \mathrm{Vh}$, Bio-Rad) and in the second dimension on the basis of their apparent molecular mass by polyacrylamide gel electrophoresis upon reduction and alkylation with $15 \mathrm{mM}$ DTT and $135 \mathrm{mM}$ IAA. Proteins were either stained with colloidal blue-silver staining ${ }^{23}$ or transferred to a nitrocellulose membrane. Successful transfer was confirmed by reversible Ponceau $\mathrm{S}$ staining.

\section{Mass spectrometry}

Proteins recognised by IgE and separated by 2D gel electrophoresis were identified by mass spectrometry as described previously ${ }^{24}$. Briefly, destained proteins were digested in-gel by trypsin for 3 hours at $37{ }^{\circ} \mathrm{C}$ and generated peptides, mixed with a-cyano-4hydroxycinnamic acid, were spotted onto a MTP Anchor Chip 384 TF target (Bruker, Daltonics, Bremen, Germany). Spotted peptides were measured by matrix-assisted laser desorption/ionisation-time of flight/TOF mass spectrometry (MALDI-TOF/TOF) using an Autoflex III smartbeam TOF/TOF200 System (Bruker,Daltonics, Bremen, Germany) together with the flexControl 3.04 software. For peptide mass fingerprinting (PMF), MS spectra were acquired in a positive ion reflector mode with 6000 shots ranging from 600 to $4.000 \mathrm{Da}$. Spectra were calibrated with external commercially available Peptide Calibration Standard II processed with the flexAnalysis 3.04 software and BioTools 3.2 was used to evaluate the resulting peak lists. Database search was performed with the MASCOT search engine MASCOT Server 2.3 (Matrix Science, London, U.K.) using the NCBI database (2013/12/09) including 111 proteins from macadamia integrifolia and the search parameters were set to mass tolerance of $80 \mathrm{ppm}$, acceptance of one missed trypsin cleavage site, carbamidomethylation of cysteine residues as fixed modification, and oxidation of methionine residues as variable modification. Protein hits with $p<0.05$ were assigned as significant and the hits were confirmed by MS/MS measurements. For these measurements, two to five peptides of each identified protein were selected with the WARP feedback mechanism of BioTools for MS/MS and parent and fragment masses were recorded with 400 and 1,000 shots, respectively. MS/MS spectra were processed and analysed in the same way as the PMF spectra with a fragment mass tolerance of $0.7 \mathrm{Da}$. All measurements were performed in duplicates. 


\section{Heterologous expression and purification of recombinant allergens}

VLAP-2-1, 2-2, 2-3, the C-terminal VLAP-2-3 fragment (aa 178-625) and vicilins from seeds, tree nuts and legumes (Ses i 3: Q9AUD0, Ana o 1: Q8L5L5, Pis v 3: B4X640, Jug r 2: Q9SEW4, Cor a 11: Q8S4P9, Ara h 1: P43238, Gly m 5.01: O22120, Gly m 5.02: Q9FZP9, Gly m 5.03: P25974, Pin k 2: V9VGU0) were heterologously expressed as fusion proteins containing a N-terminal-His (6x)-tag in E. coli as described previously ${ }^{25}$. Purification of heterologously expressed proteins was performed under denaturing conditions using an immobilized metal ion chromatography.

\section{IgE binding capacity}

Specific IgE binding to macadamia nut proteins from the crude extract was evaluated by western blot analyses. Shortly, nitrocellulose membrane was blocked with working strength universal buffer (WSUB, Article number ZD1100, EUROIMMUN AG, Germany) supplemented with $5 \%$ milk powder and upon extensive washing, sera diluted 1:10 in WSUB were applied for 1 hour at room temperature. Specific IgE binding was detected with a-human IgE antibodies coupled with alkaline phosphatase and visualisation was provided by applying nitro-blue tetrazolium/5-bromo-4-chloro-3'-indolyphosphate substrate for $10 \mathrm{~min}$.

Crude macadamia extract, heterologously expressed VLAP isoforms and vicilins from seeds, tree nuts and legumes were coated on a line blot by EUROIMMUN AG, Germany. Evaluation of slgE binding was performed as described above with the following modifications. Sera were diluted 1:11 in WSUB and applied overnight at room temperature. Specific IgE levels were evaluated as EUROLINE (EL)-intensities (RU) using the EUROLineScan 3.1 software.

\section{Indirect basophil activation test}

The indirect basophil activation test (BAT) was performed as previously described with minor modifications ${ }^{26}$. Briefly, isolated PBMCs were treated with lactic acid buffer (13.4 mM lactate, $140 \mathrm{mM} \mathrm{NaCl}$, and $5 \mathrm{mM} \mathrm{KCl}, \mathrm{pH}$ 3.9) for $2 \mathrm{~min}$ at room temperature to strip receptor-bound $\mathrm{lgE}$. The reaction was stopped by applying neutralisation buffer to the cells (20 mM HEPES buffer, $\mathrm{pH} 7.4,132 \mathrm{mM} \mathrm{NaCl}, 6 \mathrm{mM} \mathrm{KCl}, 1 \mathrm{mM}$ MgSO4, 1.2 mM KH2PO4, $5.5 \mathrm{mM}$ glucose, 0.5\% HSA) and washing them twice with the same buffer. For re-loading $\mathrm{IgE}$, individual serum samples, diluted in neutralisation buffer (1:2) supplemented with $10 \mu \mathrm{g} / \mathrm{ml}$ heparin and $8 \mathrm{mM}$ EDTA, were applied to the cells and incubated for $90 \mathrm{~min}$ at $37^{\circ} \mathrm{C}$. Re-loaded cells were allowed to rest overnight in RPMI supplemented with $10 \%$ fetal calf serum, $1 \%$ penicillin-streptomycin and $1 \mathrm{ng} / \mathrm{ml}$ 
IL-3 at $37{ }^{\circ} \mathrm{C}$ and $5 \% \mathrm{CO} 2$. Rested cells were stimulated with the macadamia nut extract, VLAP-2-1, 2-2, 2-3 and the C-terminal fragment of VLAP 2-3 diluted in RPMI + $1 \mathrm{ng} / \mathrm{ml} \mathrm{IL}-3$ for $30 \mathrm{~min}$ at $37{ }^{\circ} \mathrm{C}$ (range from $1 \mu \mathrm{g} / \mathrm{ml}$ to $1 \mathrm{ng} / \mathrm{ml}$ ). The following samples were used as controls: RPMI $+1 \mathrm{ng} / \mathrm{ml} \mathrm{IL-3,} 100 \mathrm{ng} / \mathrm{ml} \mathrm{C5a} \mathrm{(R} \mathrm{and} \mathrm{D} \mathrm{Systems),}$ $1 \mu \mathrm{g} / \mathrm{ml}$ a-human IgE (Vector Laboratories), $10 \mu \mathrm{M}$ fMLP and a serum from peanut allergic patients showing CD63 upregulation upon stimulation with Ara $\mathrm{h} 2$. Stimulated cells were stained with CD123-FITC (Biolegend), CD63-PE (Monosan), CD203c-APC (Sony) and CD193-PerCP-Cy5.5 (Biolegend). Basophils were defined as CD203c ${ }^{+}$, $\mathrm{CD} 123^{+} \mathrm{CD} 193^{+}$and basophil activation was calculated as the percentage of $\mathrm{CD}^{+} 3^{+}$ basophils. Threshold for basophil degranulation was set to $5 \% \mathrm{CD}^{2} 3^{+}$cells. All measurements were performed in duplicates.

\section{Results}

\section{Vicilin-like antimicrobial peptides from macadamia integrifolia are recognized by $\lg E$}

Particularly proteins with a molecular mass between 53 and $67 \mathrm{kDa}$ and a pl between 6.3 and 8.7 (Figure 1a) were strongly bound by slgE from 2 macadamia nut sensitised sera (Serum 1: 18 RU VLAP-2-3 [A]; Serum 2: 114 RU VLAP-2-3 [B]). As shown in Table 2, these proteins (spots 20-28, Figure $1 \mathrm{~b}$ and $\mathrm{c}$ ) were identified as vicilin-like antimicrobial peptides (VLAP) 2-1 (Q9SPL5), 2-2 (Q9SPL4) and 2-3 (Q9SPL3) whereof VLAP-2-3 showed the highest probability scores in MS analyses (Suppl. Table S1) due to the detection of peptides solely specific for VLAP-2-3. Although full-length VLAPS possess molecular masses of $74.6 \mathrm{kDa}(2-3)$ and $79.4 \mathrm{kDa}(2-1$ and 2-2), their dominant spots were 7 to $15 \mathrm{kDa}$ smaller and no peptide belonging to the $\mathrm{N}$-terminus was detected in MS/MS experiments. Due to this observation, we chose to express VLAP 2-1, 2-2 and 2-3 as full-length variants including a modified VLAP 2-3 variant lacking the N-terminus (aa 178-625).

Moreover, proteins with a molecular mass between 20 and $25 \mathrm{kDa}$ and a pl between 6.5 and 7.9 (spots 42-44, 47, 48) were also strongly bound by slgE in western blot analyses (Figure 1a). Despite high quality MS spectra with a great range of detected masses and high peak intensities, these proteins were not identified by aligning the spectra to the NCBI database, probably due to the limited number of proteins $(n=111)$ described and listed for macadamia integrifolia. 
A

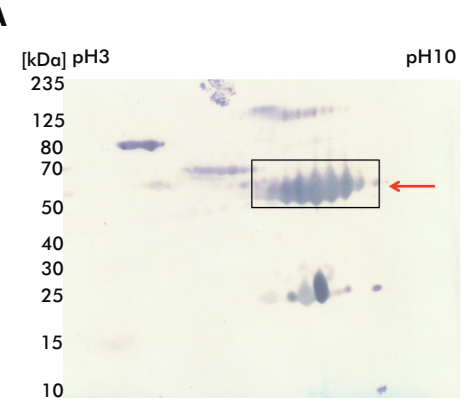

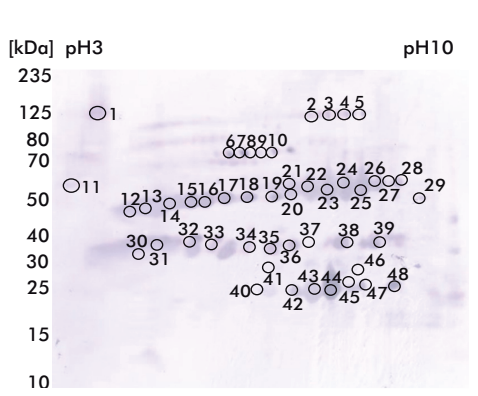

C

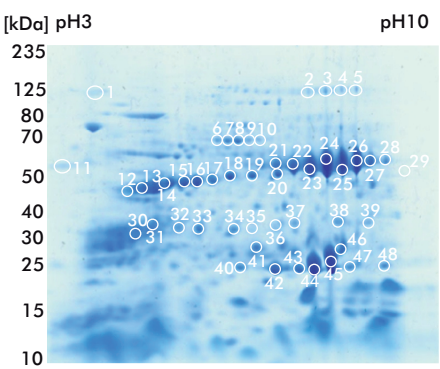

Figure 1: Identification of macadamia nut proteins recognised by slgE

Macadamia nut proteins recognised by lgE were identified by 2D gel electrophoresis (4-20\% SDS$P A G E)$ under reducing conditions, western blot analysis and subsequent mass spectrometry; $\boldsymbol{A}$ and B 2D western blot incubated with two different sera with slgE against macadamia nut; $\boldsymbol{C} 2 D$ SDS-PAGE corresponding to $\boldsymbol{B}$ stained with colloidal Commassie stain. Analysed proteins are indicated with black circles and consecutive numbering

Table 2: Identified proteins by MS analysis

\begin{tabular}{lc}
\hline Protein (accession number) & Spots \\
\hline Vicilin-like antimicrobial peptides 2-3 (gi/75207035) & $1,2,3,4,5,11,20-28,41,46$ \\
Vicilin-like antimicrobial peptides 2-2 (gi/75266171) & $1,3,4,11,21-28,41,46$ \\
Vicilin-like antimicrobial peptides 2-1 (gi/75207036) & $1,3,4,11,21-28,41,46$ \\
& \\
VLAP-2-3 may have the potential to distinguish between clinically \\
relevant and irrelevant sensitisation to macadamia nut extract
\end{tabular}

As VLAP-2-3 was the dominant isoform detected in MS analyses, its serological recognition was evaluated in $82 \mathrm{NA}$ and 27 NT patients comprehending $10 \mathrm{MA}$ and $18 \mathrm{MT}$ patients, in comparison with the macadamia nut extract (Figure 2a). The macadamia nut extract was recognised by $33 \%(27 / 82)$ NA and $26 \%$ (7/27) NT patients including $3 \mathrm{MA}(30 \%, 3 / 10)$ and $3 \mathrm{MT}(17 \%, 3 / 18)$ patients. A comparable number of NA patients (24/82, 29\%), including the same 3 MA patients, showed slgE-binding to VLAP-2-3. The percentage of NT patients, however, was lowered to only $7 \%$ (2/27 NT, 1/18 MT) with almost negligible slgE titres. Whilst 8 NA patients with increased slgE titres to the extract did not recognise VLAP-2-3 (range: 4-31 RU), 5 NA patients without detectable slgE against the extract recognised VLAP-2-3 with mostly slightly increased slgE titres (range: 3-25 RU). Taken together, VLAP-2-3 may have the potential to discriminate between macadamia nut allergic and tolerant patients as clinically irrelevant sensitisation to macadamia nut extract was not explained by slgE binding to the component VLAP-2-3 in nut allergic patients. 


\section{Specific IgE against VLAP-2-3 was mainly present in macadamia nut allergic patients with severe symptoms}

MA patients (3/10), recognising both the macadamia nut extract and VLAP-2-3, showed increased slgE titres against VLAP-2-3 (23 to $105 \mathrm{RU}$ ) compared with the extract (17 to $70 \mathrm{RU})$. Reversed sensitisation patterns were observed for the 3 MT patients positive for macadamia nut extract (3 to $18 \mathrm{RU}$ ) whose slgE titres to VLAP-2-3 were lower compared with the extract. Their slgE titres against VLAP-2-3 were either very low (5 RU) or even undetectable. While all 3 MA patients positive for VLAP-2-3 experienced moderate to severe symptoms upon ingestion, the other 7 MA patients without slgE against the extract or VLAP-2-3 suffered from rather mild to moderate symptoms except MA-7 (Table 1). Most of these patients (6/7) showed sensitisation to Bet $v 1$ and had a history of a pollen-related oral food allergy. The remaining MA patient showed sensitisation to Bet $v 2$, the profilin from birch pollen, which has also been associated with food sensitisation and oral allergy ${ }^{27}$.

A

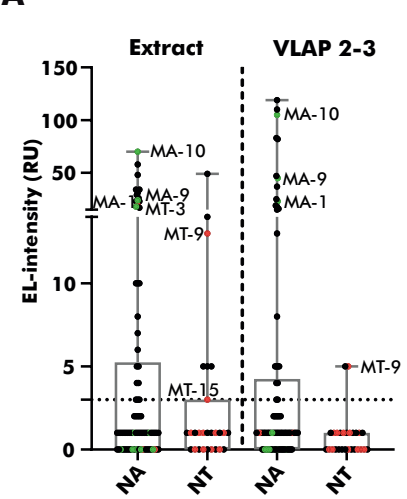

B

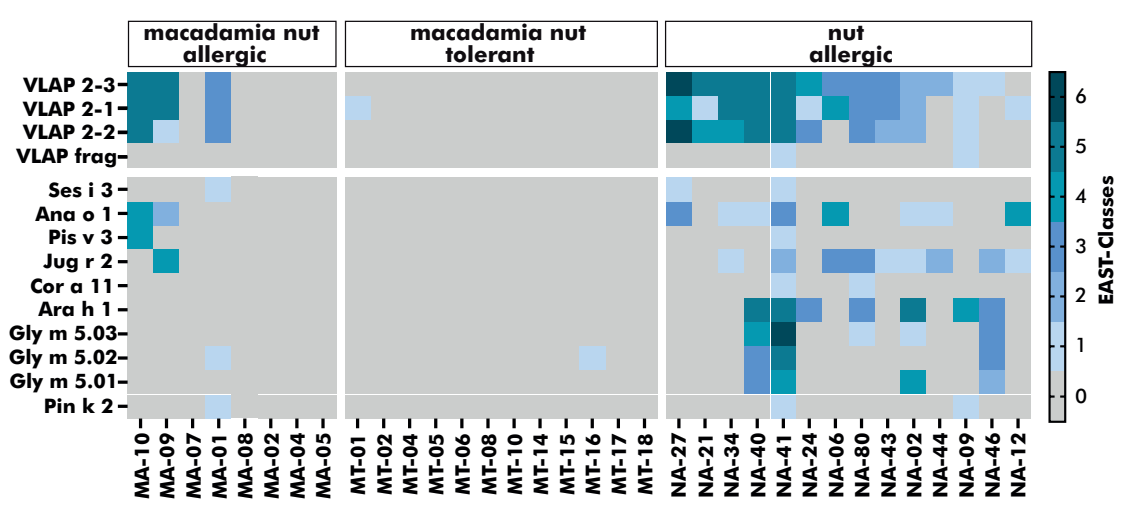

Figure 2: Sensitisation to macadamia nut, VLAP and vicilins from different sources $\boldsymbol{A}$ Sensitisation to macadamia nut extract and VLAP-2-3 was evaluated in 82 nut allergic (NA) and 27 nut tolerant (NT) patients including 10 macadamia nut allergic (MA) and 18 macadamia nut tolerant (MT) patients. Specific IgE titres from MA patients are indicated in green and slgE titres from MT patients are shown in red; $\boldsymbol{B}$ Sensitisation to VLAP isoforms, a C-terminal VLAP-2-3 fragment (aa 178-625) and vicilins from seeds, tree nuts and legumes was evaluated in 8 MA, 12 MT and 14 NA patients sensitised to VLAP 2-3. MA patients are sorted by severity of their reaction to macadamia nut. Specific IgE levels are expressed as EAST classes; $\mathrm{Cl}$. $0=0$ to $2 \mathrm{RU}, \mathrm{Cl} .1=3$ to $6 \mathrm{RU}, \mathrm{Cl} .2=7$ to $15 \mathrm{RU}, \mathrm{Cl} .3=16$ to $30 \mathrm{RU}, \mathrm{Cl} .4=31$ to $50 \mathrm{RU}, \mathrm{Cl} .5=51$ to $100 \mathrm{RU}, \mathrm{Cl} .6=>100 \mathrm{RU}$

\section{$\mathrm{N}$-terminal substitutions may explain divergent recognition of VLAP isoforms}

Recognition of the different VLAP isoforms and the C-terminal fragment (aa 178-625) of VLAP-2-3, generated based on the lack of detected N-terminal peptides in MS analysis, was studied in a subgroup of 8 MA, 12 MT and 14 additional NA patients (Figure 2b). This subgroup was formed on both suggestive history to macadamia nut and sensiti- 
sation to VLAP-2-3. Overall, patients recognising VLAP-2-3 were mostly also positive for the isoforms VLAP-2-1 and 2-2 but their slgE binding to VLAP-2-1 and 2-2 was reduced in $59 \%$ of the patients. Lower or almost negligible slgE binding was observed for the C-terminal fragment of VLAP-2-3, pointing towards relevant IgE binding sites and amino acid substitutions on the $\mathrm{N}$-terminus not included in the sequence of the C-terminal VLAP-2-3 fragment. An amino acid sequence alignment in Figure 3 showed 13 amino acid substitutions on the N-terminus (aa 1-177 of VLAP-2-3) which were always consistent between two of the isoforms. In total, we observed 3 substitutions for VLAP-2-1, 4 substitutions for VLAP-2-2 and 6 substitutions for VLAP-2-3.

\footnotetext{
VLAP 2- 1 ESEFDROEYEECKROCMQLETSGQMRRCVSOCDKRFEEDIDWSKYDNOEDPOTECQOCQRRCRQQESGPRQQQYCQRRCKEICEEEEEYNRQRDPQQQYE 100 VLAP 2- 2 ESEFDROEYEECKROCMOLETSGOMRRCVSOCDKRFEEDIDWSKYDNODDPOTDCOOCORRCROOESGPROOOYCORRCKEICEEEEEYNRORDPOOOYE 100 VLAP 2 - 3 --------QCMQLETSGQMRRCVSQCDKRFEEDIDWSKYDNQEDPQTECQQCQRRCRQQESDPRQQQYCQRRCKEICEEEEEYNRQRDPQQQYE 86

VLAP 2- 1 QCQKHCQRRETEPRHMQTCQQRCERRYEKEKRKQQKRYEEQQREDEEKYEERMKEEDNKRDPQQREYEDCRRRCEQQEPRQQHQCQLRCREQQRQHGRGG 200 VLAP 2- 2 QCQERCQRHETEPRHMQTCQQRCERRYEKEKRKQQKRYEEQQREDEEKYEERMKEEDNKRDPQQREYEDCRRRCEQQEPRQQYQCQRRCREQQRQHGRGG 200 VLAP 2 - 3 QCQKRCQRRETEPRHMQICQQRCERRYEKEKRKQQKRYEEQQREDEEKYEERMKEGDNKRDPQQREYEDCRRHCEQQEPRLQYQCQRRCQEQQRQHGRGG 186

VLAP 2-1 DMMNPQRGGSGRYEEGEEEQSDNPYYFDERSLSTRFRTEEGHISVLENFYGRSKLLRALKNYRLVLLEANPNAFVLPTHLDADAILLVIGGRGALKMIHH 300 VLAP 2-2 DLINPQRGGSGRYEEGEEKOSDNPYYFDERSLSTRFRTEEGHISVLENFYGRSKLLRALKNYRLVLLEANPNAFVLPTHLDADAILLVTGGRGALKMIHR 300 VLAP 2 - 3 DIMNPQRGGSGRYEEGEEKQSDNPYYFDERSLSTRFRTEEGHISVLENFYGRSKLLRALKNYRLVLLEANPNAFVLPTHLDADAILLVIGGRGALKMIHR 286

VLAP 2- 1 DNRESYNLECGDVIRIPAGTTFYLINRDNNERLHIAKFLQTISTPGQYKEFFPAGGQNPEPYLSTFSKEILEAALNTQTEKLRGVFGQQREGVIIRASQE 400 VLAP 2- 2 DNRESYNLECGDVIRIPAGTTFYLINRDNNERLHIAKFLQTISTPGQYKEFFPAGGQNPEPYLSTFSKEILEAALNTQAERLRGVLGQQREGVIISASQE 400 VLAP 2 - 3 DNRESYNLECGDVIRIPAGTTFYLINRDNNERLHIAKFLQTISTPGQYKEFFPAGGQNPEPYLSTFSKE ILEAALNTQTERLRGVLGQQREGVIIRASQE 386

VLAP 2- 1 QIRELTRDDSESRHWHIRRGGESSRGPYNLFNKRPLYSNKYGQAYEVKPEDYRQLQDMDLSVFIANVTQGSMMGPFFNTRSTKVVVVASGEADVEMACPH 500 VLAP 2- 2 QIRELTRDDSESRRWHIRRGGESSRGPYNLFNKRPLYSNKYGQAYEVKPEDYRQLQDMDVSVFIANITQGSMMGPFFNTRSTKVVVVASGEADVEMACPH 500

VLAP 2 - 3 QIRELTRDDSESRRWHIRRGGESSR GPYNLFNKRPLYSNKYGQAYEVKPEDYRQLQDMDVSVFI ANITQGSMMGPFFNTRSTKVVVVASGEADVEMACPH 486

VLAP 2- 1 LSGRHGGRGGGKRHEEEEDVHYEOVRARLSKREAIVVLAGHPVVFVSSGNENLLLFAFGINAONNHENFLAGRERNVLOOIEPOAMELAFAAPRKEVEES 600 VLAP 2- 2 LSGRHGGRRGGKRHEEEEDVHYEQVKARLSKREAIVVPVGHPVVFVSSGNENLLLFAFGINAQNNHENFLAGRERNVLQQIEPQAMELAFAAPRKEVEEL 600 VLAP 2 - 3 LSGRHGGRGGGKRHEEEEEVHYEQVRARLSKREAIVVLAGHPVVFVSSGNENLLLFAFGINAQNNHENELAGRERNVLQQIEPQAMELAFAASRKEVEEEL 586

VLAP 2- 1 FNSQDQSIFFPGPRQHQQQSPRSTKQQQPLVSILDFVGF 639

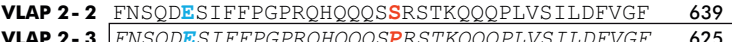

Figure 3: Amino acid sequence alignment of macadamia nut VLAP isoforms Amino acid sequence alignment of the different VLAP isoforms with highlighted amino acid substitutions; Cyan: VLAP-2-1 $\neq$ VLAP-2-2 and VLAP-2-3; Red: VLAP-2-2 $\neq$ VLAP-2-1 and VLAP-2-3 Green: VLAP2-3 $\neq$ VLAP-2-1 and VLAP-2-2; the C-terminal VLAP-2-3 fragment is indicated with a black box

\section{Co-recognised vicilins were often associated with a respective nut allergy}

Co-sensitisation to vicilins from seeds, tree nuts and legumes was determined in the same subpopulation. Recognition of VLAP isoforms was accompanied by slgE binding to Ana o 1 (cashew nut) and Jug $r 2$ (walnut) in $59 \%$ of the patients sensitised to VLAP (Figure 2b). Regarding MA patients, Ana o 1 was co-recognised in 2 and Jug $r 2$ in 1 out of 3 MA patients. The recognition of additional vicilins was mostly but not exclusively associated with a corresponding food allergy (Supplementary, Online Repository). In contrast, hazelnut allergy was not at all associated with recognising Cor a 11 and peanut allergy was only partly associated with Ara $\mathrm{h} 1$ recognition. 


\section{Vicilin-like antimicrobial peptides can induce degranulation}

Besides serological characterisation of VLAP-2-3 and its isoforms, their ability to induce degranulation was evaluated by indirect BAT (Figure 4). Patient MA-10 (NA-29) showed dose-dependent BAT reactivity upon stimulation with all three VLAP isoforms starting from $10 \mathrm{ng} / \mathrm{ml}$ and reaching a plateau at around $100 \mathrm{ng} / \mathrm{ml}$ for VLAP-2-2 and 2-3 and at $1000 \mathrm{ng} / \mathrm{ml}$ for VLAP-2-1. Consistent with sensitisation data, stimulation with the C-terminal VLAP-2-3 fragment did not result in any upregulation of CD63 surface expression at all considered concentrations. This dose-dependent BAT reactivity was confirmed by repeating the experiment under the same conditions on a second day with freshly obtained basophils (Figure 4a). These basophils, however, showed a more flatangel reactivity curve and the plateau was reached at tenfold higher concentrations. In comparison, stimulation with native macadamia nut extract resulted in a stronger CD63 upregulation than stimulation with recombinant VLAP components. To ensure that no activation was caused by remaining donor IgE, basophils were stimulated upon stripping with the different VLAP isoforms and macadamia nut extract leading to no CD63 upregulation (Figure $4 b$ ). As internal positive control, stripped basophils were loaded with serum from a peanut allergic patients sensitised to Ara $\mathrm{h} 2$ (Figure 4c) and stimulation with Ara $\mathrm{h} 2$ resulted in a dose-dependent upregulation of CD63. Overall, VLAP 2-3 and its isoforms are generally capable of inducing degranulation. 
A

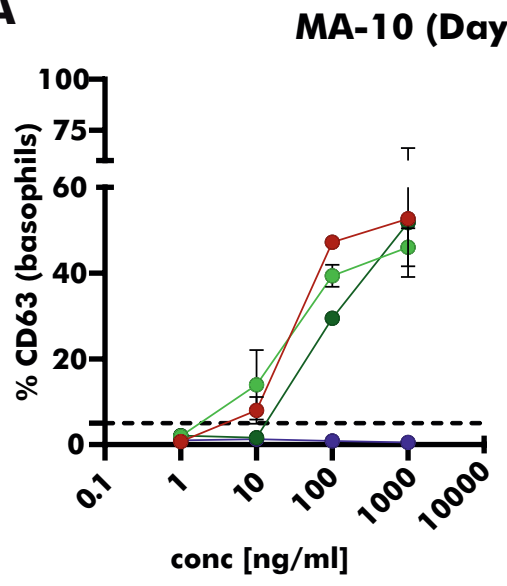

B

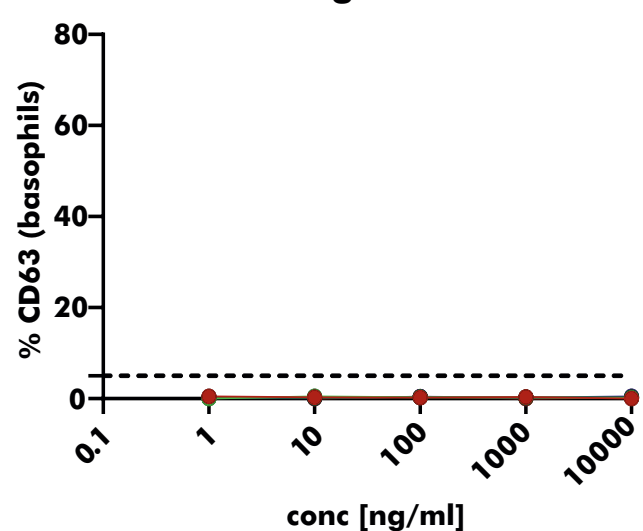

VLAP 2-1

VLAP 2-3
C

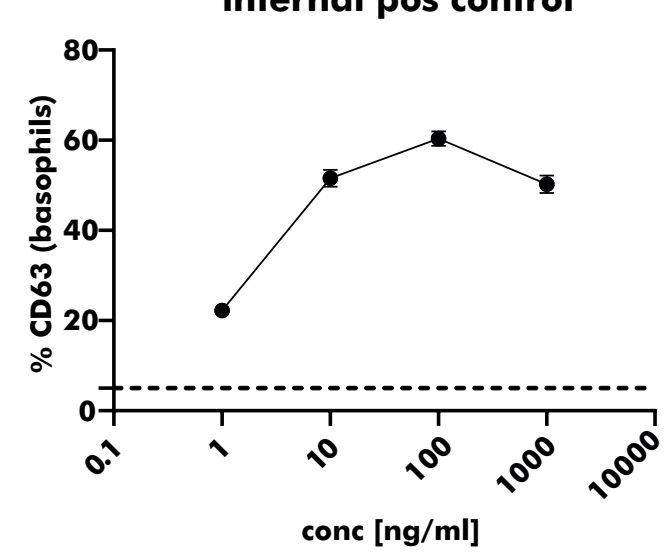

$\rightarrow$ Ara h 2

MA-10 (Day 2)

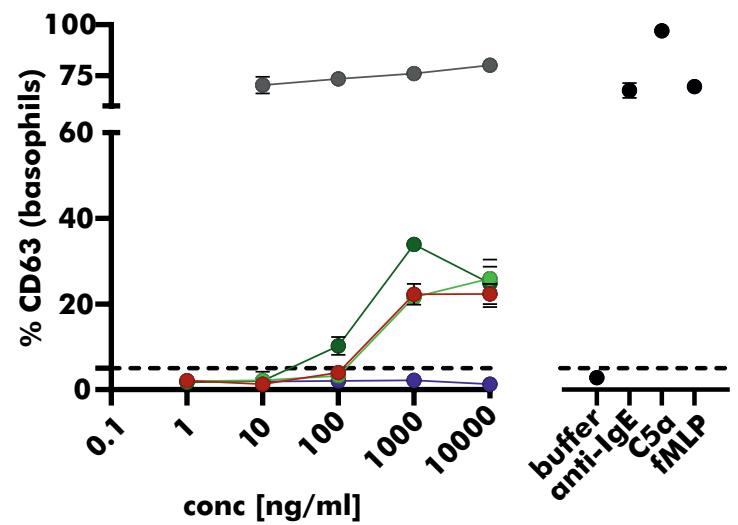

internal pos control

Figure 4: Indirect basophil activation by macadamia nut VLAP isoforms

Indirect basophil activation test to evaluate the potential of VLAP isoforms and the C-terminal VLAP-2-3 to induce degranulation

A Dose-response curves upon stimulation with VLAP isoforms, C-terminal VLAP 2-3 fragment and macadamia nut extract on two different days with freshly obtained basophils; [B] Dose-response curves of stripped basophils with remaining donor IgE stimulated under the same conditions as $\boldsymbol{A}$ (negative control); $C$ Dose-response curve of basophils loaded with sera from a peanut allergic patient sensitised to Ara $h 2$ upon stimulation with Ara $h 2$ 


\section{Discussion}

To date, the diagnosis of macadamia nut allergy by slgE solely relies on the macadamia nut extract and single components are lacking. In the present study, macadamia nut proteins recognised by slgE were identified as VLAPs with serological recognition in $33 \%$ of NA and $30 \%$ of MA patients and the ability to induce basophil activation. While MA patients recognised VLAPs with highly increased slgE titres (23 to $105 \mathrm{RU}$ ), MT patients recognised VLAPs with almost negligible slgE titres, indicating the potential of VLAPs to discriminate between MA and MT patients positive for the macadamia nut extract. Moreover, MA patients sensitised to VLAPs experienced moderate to severe symptoms upon ingestion, highlighting the potential of VLAPs as potential markers for systemic reactions to macadamia nut.

VLAP-2-3 and its isoforms, belonging to the vicilin family, were identified as macadamia nut proteins predominately recognised by slgE from MA patients with moderate to severe symptoms. Accordingly, the vicilin from walnut, Jug $r 2$, has been described as a potential marker for severe allergic reactions in patients without pollen-related sensitisation ${ }^{18,19}$. Moreover, sensitisation to the vicilin from hazelnut, Cor a 11, was observed in children with severe hazelnut allergies, while Cor a 11 was scarcely recognised by adults with OAS upon hazelnut ingestion ${ }^{20}$. Specific IgE binding to cross-reactive carbohydrate determinants (CCDs) has been excluded by the use of recombinant allergens in those studies. Overall, vicilins, including the one from macadamia nut, appear to be markers for severe, potentially non-pollen related, allergies to tree nuts.

In contrast, MA patients, primarily suffering from mild symptoms upon ingestion, recognised neither macadamia nut extract nor VLAP proteins and most of these patients (6/7) were known for their Bet v 1-related oral food allergies. Since PR-10 proteins are underrepresented in aqueous extracts of several foods like hazelnut, a Bet v 1-homologue might also be underrepresented in the macadamia nut extract, theoretically explaining the lack of detected slgE in those patients ${ }^{28,29}$. Moreover, no slgE binding to VLAP-2-3 by patients with rather mild to moderate symptoms supports the potential of VLAP proteins to stratify MA patients into patients with rather mild and patients with rather severe symptoms upon macadamia nut ingestion.

While NA patients sensitised to VLAP 2-3 showed also slgE-binding to the isoforms VLAP-2-1 and 2-2, nearly no recognition was observed for the C-terminal VLAP-2-3 fragment (aa 178-625). Accordingly, basophil activation was repeatedly achieved by stimulation with VLAP full-length proteins but not with the C-terminal VLAP-2-3 fragment using one serum, suggesting the location of critical epitopes on the $\mathrm{N}$-terminus of VLAP. Contrary, no peptides belonging to the N-terminus were detected by mass spectrometry, potentially due to a mixture of degradation products present at the same molecular mass. Compared with literature, shared linear epitopes between VLAP and 
Jug $r 2$ as well as structural epitopes of different vicilins (Ara h 1, Jug r 2, Cor a 11, Ana 0 1) were predicted to be mainly located on the C-terminus ${ }^{14,17}$, suggesting only co-sensitisation but not cross-reactivity between VLAP and vicilins from other tree nuts. This hypothesis is supported by negligible inhibition of slgE-binding to macadamia nut extract with Jug $r 2$ in a study by Teuber and colleagues ${ }^{12}$.

Additional macadamia nut proteins recognised by slgE but not identified by MS analysis were detected at molecular masses of around 25, 35 and $45 \mathrm{kDa}$ and a corresponding pl of 7.3 to $9.1,4.1$ to 8.9 and 4.2 to 6.1 , respectively. These characteristics might correspond to either $2 \mathrm{~S}$ albumins $(25 \mathrm{kDa})$ or $11 \mathrm{~S}$ globulins ( $25 \mathrm{kDa}$ : basic subunit and $35 \mathrm{kDa}$ : acidic subunit) described as allergens for several other tree nut and legume allergies $^{30-34}$. Accordingly, previous inhibition experiments showed reduction in slgE binding to the macadamia nut extract upon pre-incubation with Jug $r 1$ (2S albumin) and Jug $r 4$ (11S globulin), supporting the hypothetical presence of cross-reactive 2S albumins and $11 \mathrm{~S}$ globulins in the macadamia nut extract ${ }^{12}$. The presence would explain IgE binding to the macadamia nut extract without recognition of VLAP-2-3 in 8 NA patients. The identification of the remaining proteins recognised by slgE might be additionally beneficial for component-resolved diagnostics in macadamia nut allergy. Overall, VLAPs are the first described single components from macadamia nut with serological and functional allergenic properties. These components appear to be supportive in identifying patients with systemic reactions to macadamia nut.

\section{Acknowledgements}

We would like to thank Y. Denno (EUROIMMUN AG, Lübeck, Germany) for technical assistance and fruitful discussions regarding mass spectrometry analyses. Line blots and reagents were kindly provided by EUROIMMUN AG, Lübeck, Germany.

This study was funded by a grant from the European Regional Development Fund of the European Union (V-630-1-301-2013/185).

\section{Informed consent statement}

This study was carried out in accordance with the University Medical Centre Utrecht, Biobank Regulations, which are in compliance with the applicable national and international laws and regulations. These regulations permit the use of 'residual material from diagnostic testing' for research, unless the patient objects (Article 8, 'no objection' procedure). None of the included patients objected the use of their serum. The protocol was approved by the Biobank Research Ethics Committee of the University Medical Centre Utrecht under the protocol number 18-428. 


\section{References}

1. McWilliam, V. et al. The Prevalence of Tree Nut Allergy: A Systematic Review. Curr Allergy Asthma Rep 15, 54 (2015).

2. Sasaki, M. et al. Prevalence of clinic-defined food allergy in early adolescence: The SchoolNuts study. J Allergy Clin Immunol 141, 391-8.e4 (2018).

3. Jeong, K. et al. A multicenter study on anaphylaxis caused by peanut, tree nuts, and seeds in children and adolescents. Allergy 72, 507-10 (2017).

4. Global, A. The European market potential for macadamia nuts Dutch Ministry of Foreign Affairs. Government Document. 2020. https : //www . cbi . eu/marketinformation/processed-fruit-vegetables-edible-nuts/macadamia-nuts/ market-potential\%20(2020-05-18).

5. Pallares, D. E. Allergy to macadamia nut. Ann Allergy Asthma Immunol 85, 3856 (2000).

6. Sutherland, M. F., O'Hehir, R. E., Czarny, D. \& Suphioglu, C. Macadamia nut anaphylaxis: Demonstration of specific lgE reactivity and partial cross-reactivity with hazelnut. J Allergy Clin Immunol 104, 889-90 (1999).

7. Yoshida, K. et al. Clinical features and allergen analysis in five children with macadamia nut allergy. J Allergy Clin Immunol 141 (2018).

8. Herbst, R. A., Wahl, R. \& Frosch, P. J. Specific IgE reactivity and identification of potential allergens in macadamia allergy. J Eur Acad Dermatol Venereol 24, 1361-3 (2010).

9. de Knop, K. J. et al. Macadamia nut allergy: 2 case reports and a review of the literature. Acta Clin Belg 65, 129-32 (2010).

10. Knott, E. et al. Macadamia nut allergy. J Europ Acad Dermatol Venereol 22, 1394-5 (2008).

11. Lerch, M., Egger, C. \& Bircher, A. J. Allergic reactions to macadamia nut. Allergy 60, 130-1 (2005).

12. Teuber, S. S. \& Peterson, W. R. IgE-binding Proteins in Macadamia Nut Crossreact with Walnut. J Allergy Clin Immunology 125, AB222 (2010).

13. Dubiela, P. et al. Jug $r 6$ is the allergenic vicilin present in walnut responsible for $\lg$ E cross-reactivities to other tree nuts and seeds. Sci Rep 8, 11366 (2018).

14. Barre, A. et al. Vicilin allergens of peanut and tree nuts (walnut, hazelnut and cashew nut) share structurally related IgE-binding epitopes. Mol Immunol 45, 1231-40 (2008). 
15. Marcus, J. P., Green, J. L., Goulter, K. C. \& Manners, J. M. A family of antimicrobial peptides is produced by processing of a $7 \mathrm{~S}$ globulin protein in Macadamia integrifolia kernels. Plant Journal 19, 699-710 (1999).

16. Marcus, J. P. et al. Purification, Characterisation and cDNA Cloning of an Antimicrobial Peptide from Macadamia Integrifolia. Eur J Biochem 244, 743-9 (1997).

17. Rost, J., Muralidharan, S. \& Lee, N. A. A label-free shotgun proteomics analysis of macadamia nut. Food Res Int 129, 108838 (2020).

18. Teuber, S. S. et al. Identification and cloning of a complementary DNA encoding a vicilin-like proprotein, Jug $r$ 2, from English walnut kernel (Juglans regia), a major food allergen. J Allergy Clin Immunol 104, 1311-20 (1999).

19. Pastorello, E. A. et al. Lipid transfer protein and vicilin are important walnut allergens in patients not allergic to pollen. J Allergy Clin Immunol 114, 908-14 (2004).

20. Verweij, M. et al. Age-Dependent Sensitization to the 7S-Vicilin-Like Protein Cor a 11 From Hazelnut (Corylus avellana) in a Birch-Endemic Region. J Invest A/lergol Clin immunol 22, 245-51 (2012).

21. Marsh, D. G. et al. Allergen nomenclature. Allergy 43, 161-8 (1986).

22. Sampson, H. A. Anaphylaxis and Emergency Treatment. Pediatrics 111, 16018 (2003).

23. Candiano, G. et al. Blue silver: A very sensitive colloidal Coomassie G-250 staining for proteome analysis. ELECTROPHORESIS 25, 1327-33 (2004).

24. Scharf, M. et al. A Spectrum of Neural Autoantigens, Newly Identified by HistoImmunoprecipitation, Mass Spectrometry, and Recombinant Cell-Based Indirect Immunofluorescence. Front Immunol 9, 1447 (2018).

25. Sitaru, C. et al. Enzyme-linked immunosorbent assay using multimers of the 16th non-collagenous domain of the BP180 antigen for sensitive and specific detection of pemphigoid autoantibodies. Exp Dermatol 16, 770-7 (2007).

26. Santos, A. F. et al. IgG4 inhibits peanut-induced basophil and mast cell activation in peanut-tolerant children sensitized to peanut major allergens. J Allergy Clin Immunol 135, 1249-56 (2015).

27. Valenta, R. et al. Profilins constitute a novel family of functional plant pan-allergens. The Journal of Experimental Medicine 175, 377-85 (1992).

28. Huss-Marp, J., Raulf, M. \& Jakob, T. Spiking with recombinant allergens to improve allergen extracts: benefits and limitations for the use in routine diagnostics. Allergo J Int 24, 236-43 (2015). 
29. Andersson, K. et al. Enhancement of hazelnut extract for IgE testing by recombinant allergen spiking. Allergy 62, 897-904 (2007).

30. Pastorello, E. A. et al. Sensitization to the major allergen of Brazil nut is correlated with the clinical expression of allergy. J Allergy Clin Immunol 102, 1021-7 (1998).

31. Masthoff, L. J. N. et al. Sensitization to Cor a 9 and Cor a 14 is highly specific for a hazelnut allergy with objective symptoms in Dutch children and adults. $J$ Allergy Clin Immunol 132, 393-9 (2013).

32. Klemans, R. J. B. et al. Ara h 2 Is the Best Predictor for Peanut Allergy in Adults. J Allergy Clin Immunol Pract 1, 632-8.e1 (2013).

33. Wolff, N. et al. Allergy to sesame in humans is associated primarily with IgE antibody to a $14 \mathrm{kDa} 2 \mathrm{~S}$ albumin precursor. Food Chem Toxicol 41, 1165-74 (2003).

34. Ebisawa, M. et al. Gly $\mathrm{m} 2 \mathrm{~S}$ albumin is a major allergen with a high diagnostic value in soybean-allergic children. J Allergy Clin Immunol 132, 976-8.e5 (2013). 


\section{Supplementary}

Detailed description of individual patients characteristics are available at the Online Repository of the Journal Clinical and Translational Allergy (doi: 10.1186/s13601-02000364-5).

Table S1: Enclosed proteins detected by $2 D$ gel electrophoresis and mass spectrometry analysis The macadamia nut extract was separated by IEF and SDS-PAGE. Spots corresponding to slgE binding were analysed by mass spectrometry using peptide mass fingerprinting. The table shows the identified proteins in this fraction and MS/MS results are displayed in italic

\begin{tabular}{|c|c|c|c|c|c|}
\hline Spot & Protein (Acession number) & Cut-Off & $\begin{array}{l}\text { Protein } \\
\text { score }\end{array}$ & $\begin{array}{l}\text { Seq cover- } \\
\text { age }[\%]\end{array}$ & $\begin{array}{l}\text { Molecular } \\
\text { mass [kDa] }\end{array}$ \\
\hline \multirow{3}{*}{1} & $\begin{array}{l}\text { Vicilin-like antimicrobial peptides 2-3 } \\
\text { (gil75207035) }\end{array}$ & $74 / 42$ & $149 / 55$ & $31 / 1$ & 74.6 \\
\hline & $\begin{array}{c}\text { Vicilin-like antimicrobial peptides 2-2 } \\
\text { (gil75266171) }\end{array}$ & $74 / 42$ & $77 / 55$ & $16 / 1$ & 79.4 \\
\hline & $\begin{array}{l}\text { Vicilin-like antimicrobial peptides 2-1 } \\
\text { (gil75207036) }\end{array}$ & 142 & 155 & 1 & 79.4 \\
\hline 2 & $\begin{array}{l}\text { Vicilin-like antimicrobial peptides 2-3 } \\
\text { (gil75207035) }\end{array}$ & 74 & 113 & 24 & 74.6 \\
\hline \multirow{3}{*}{3} & $\begin{array}{l}\text { Vicilin-like antimicrobial peptides 2-3 } \\
\text { (gil75207035) }\end{array}$ & $74 / 40$ & $98 / 18$ & $22 / 1$ & 74.6 \\
\hline & $\begin{array}{c}\text { Vicilin-like antimicrobial peptides 2-2 } \\
\text { (gil75266171) }\end{array}$ & 40 & 18 & 1 & 79.4 \\
\hline & $\begin{array}{l}\text { Vicilin-like antimicrobial peptides 2-1 } \\
\text { (gil75207036) }\end{array}$ & 40 & 18 & 1 & 79.4 \\
\hline \multirow{3}{*}{4} & $\begin{array}{l}\text { Vicilin-like antimicrobial peptides 2-3 } \\
\text { (gil75207035) }\end{array}$ & $74 / 42$ & $191 / 113$ & $35 / 4$ & 74.6 \\
\hline & $\begin{array}{c}\text { Vicilin-like antimicrobial peptides 2-2 } \\
\text { (gil75266171) }\end{array}$ & $74 / 42$ & $110 / 113$ & $24 / 3$ & 79.4 \\
\hline & $\begin{array}{l}\text { Vicilin-like antimicrobial peptides 2-1 } \\
\text { (gil75207036) }\end{array}$ & $74 / 42$ & $80 / 113$ & $21 / 3$ & 79.4 \\
\hline 5 & $\begin{array}{l}\text { Vicilin-like antimicrobial peptides 2-3 } \\
\text { (gil75207035) }\end{array}$ & 74 & 146 & 32 & 74.6 \\
\hline 6 & - & - & - & - & - \\
\hline 7 & - & - & - & - & - \\
\hline 8 & - & - & - & - & - \\
\hline 9 & - & - & - & - & - \\
\hline 10 & - & - & - & - & - \\
\hline \multirow{3}{*}{11} & $\begin{array}{l}\text { Vicilin-like antimicrobial peptides 2-3 } \\
\text { (gil75207035) }\end{array}$ & $74 / 42$ & $176 / 57$ & $37 / 1$ & 74.6 \\
\hline & $\begin{array}{c}\text { Vicilin-like antimicrobial peptides 2-2 } \\
\text { (gil75266171) }\end{array}$ & $74 / 42$ & $99 / 57$ & $24 / 1$ & 79.4 \\
\hline & $\begin{array}{l}\text { Vicilin-like antimicrobial peptides 2-1 } \\
\text { (gil75207036) }\end{array}$ & $74 / 42$ & $99 / 57$ & $24 / 1$ & 79.4 \\
\hline 12 & - & - & - & - & - \\
\hline 13 & - & - & - & - & - \\
\hline
\end{tabular}




\begin{tabular}{|c|c|c|c|c|c|}
\hline Spot & Protein (Acession number) & Cut-Off & $\begin{array}{l}\text { Protein } \\
\text { score }\end{array}$ & $\begin{array}{l}\text { Seq cover- } \\
\text { age }[\%]\end{array}$ & $\begin{array}{l}\text { Molecular } \\
\text { mass [kDa] }\end{array}$ \\
\hline 14 & - & - & - & - & - \\
\hline 15 & - & - & - & - & - \\
\hline 16 & - & - & - & - & - \\
\hline 17 & - & - & - & - & - \\
\hline 18 & - & - & - & - & - \\
\hline 19 & - & - & - & - & - \\
\hline 20 & $\begin{array}{l}\text { Vicilin-like antimicrobial peptides 2-3 } \\
\text { (gil75207035) }\end{array}$ & 74 & 91 & 29 & 74.6 \\
\hline \multirow{3}{*}{21} & $\begin{array}{l}\text { Vicilin-like antimicrobial peptides 2-3 } \\
\text { (gil75207035) }\end{array}$ & $74 / 44$ & $236 / 148$ & $41 / 4$ & 74.6 \\
\hline & $\begin{array}{c}\text { Vicilin-like antimicrobial peptides 2-2 } \\
\text { (gil75266171) }\end{array}$ & $74 / 44$ & $164 / 148$ & $33 / 3$ & 79.4 \\
\hline & $\begin{array}{l}\text { Vicilin-like antimicrobial peptides 2-1 } \\
\text { (gil75207036) }\end{array}$ & $74 / 44$ & $94 / 148$ & $27 / 3$ & 79.4 \\
\hline \multirow{3}{*}{22} & $\begin{array}{l}\text { Vicilin-like antimicrobial peptides 2-3 } \\
\text { (gil75207035) }\end{array}$ & $74 / 44$ & $348 / 156$ & $43 / 3$ & 74.6 \\
\hline & $\begin{array}{c}\text { Vicilin-like antimicrobial peptides 2-2 } \\
\text { (gil75266171) }\end{array}$ & $74 / 44$ & $233 / 156$ & $34 / 3$ & 79.4 \\
\hline & $\begin{array}{l}\text { Vicilin-like antimicrobial peptides 2-1 } \\
\text { (gil75207036) }\end{array}$ & 74 & 131 & 30 & 79.4 \\
\hline \multirow{3}{*}{23} & $\begin{array}{l}\text { Vicilin-like antimicrobial peptides 2-3 } \\
\text { (gil75207035) }\end{array}$ & $74 / 45$ & $366 / 20$ & $44 / 1$ & 74.6 \\
\hline & $\begin{array}{c}\text { Vicilin-like antimicrobial peptides 2-2 } \\
\text { (gil75266171) }\end{array}$ & $74 / 45$ & $245 / 20$ & $37 / 1$ & 79.4 \\
\hline & $\begin{array}{l}\text { Vicilin-like antimicrobial peptides 2-1 } \\
\text { (gil75207036) }\end{array}$ & 74 & 166 & 31 & 79.4 \\
\hline \multirow{3}{*}{24} & $\begin{array}{l}\text { Vicilin-like antimicrobial peptides 2-3 } \\
\text { (gil75207035) }\end{array}$ & $74 / 44$ & $414 / 145$ & $48 / 3$ & 74.6 \\
\hline & $\begin{array}{c}\text { Vicilin-like antimicrobial peptides 2-2 } \\
\text { (gil75266171) }\end{array}$ & $74 / 44$ & $265 / 145$ & $37 / 3$ & 79.4 \\
\hline & $\begin{array}{l}\text { Vicilin-like antimicrobial peptides 2-1 } \\
\text { (gil75207036) }\end{array}$ & 74 & 170 & 32 & 79.4 \\
\hline \multirow{3}{*}{25} & $\begin{array}{l}\text { Vicilin-like antimicrobial peptides 2-3 } \\
\text { (gil75207035) }\end{array}$ & $74 / 44$ & $409 / 93$ & $45 / 2$ & 74.6 \\
\hline & $\begin{array}{c}\text { Vicilin-like antimicrobial peptides 2-2 } \\
\text { (gil75266171) }\end{array}$ & 74 & 259 & 32 & 79.4 \\
\hline & $\begin{array}{l}\text { Vicilin-like antimicrobial peptides 2-1 } \\
\text { (gil75207036) }\end{array}$ & 74 & 170 & 30 & 79.4 \\
\hline \multirow{3}{*}{26} & $\begin{array}{l}\text { Vicilin-like antimicrobial peptides 2-3 } \\
\text { (gil75207035) }\end{array}$ & $74 / 44$ & $382 / 83$ & $43 / 2$ & 74.6 \\
\hline & $\begin{array}{c}\text { Vicilin-like antimicrobial peptides 2-2 } \\
\text { (gil75266171) }\end{array}$ & 74 & 276 & 34 & 79.4 \\
\hline & $\begin{array}{l}\text { Vicilin-like antimicrobial peptides 2-1 } \\
\text { (gil75207036) }\end{array}$ & $74 / 44$ & $186 / 83$ & $30 / 2$ & 79.4 \\
\hline
\end{tabular}




\begin{tabular}{|c|c|c|c|c|c|}
\hline Spot & Protein (Acession number) & Cut-Off & $\begin{array}{l}\text { Protein } \\
\text { score }\end{array}$ & $\begin{array}{l}\text { Seq cover- } \\
\text { age }[\%]\end{array}$ & $\begin{array}{l}\text { Molecular } \\
\text { mass [kDa] }\end{array}$ \\
\hline \multirow{3}{*}{27} & $\begin{array}{l}\text { Vicilin-like antimicrobial peptides 2-3 } \\
\text { (gil75207035) }\end{array}$ & 74 & 289 & 41 & 74.6 \\
\hline & $\begin{array}{l}\text { Vicilin-like antimicrobial peptides 2-2 } \\
\text { (gil75266171) }\end{array}$ & 74 & 201 & 31 & 79.4 \\
\hline & $\begin{array}{l}\text { Vicilin-like antimicrobial peptides 2-1 } \\
\text { (gil75207036) }\end{array}$ & $74 / 45$ & $148 / 151$ & $31 / 5$ & 79.4 \\
\hline \multirow{3}{*}{28} & $\begin{array}{l}\text { Vicilin-like antimicrobial peptides 2-3 } \\
\text { (gil75207035) }\end{array}$ & 74 & 165 & 37 & 74.6 \\
\hline & $\begin{array}{l}\text { Vicilin-like antimicrobial peptides 2-2 } \\
\text { (gil75266171) }\end{array}$ & 74 & 119 & 28 & 79.4 \\
\hline & $\begin{array}{l}\text { Vicilin-like antimicrobial peptides 2-1 } \\
\text { (gil75207036) }\end{array}$ & $74 / 44$ & $131 / 192$ & $33 / 8$ & 79.4 \\
\hline 29 & - & - & - & - & - \\
\hline 30 & - & - & - & - & - \\
\hline 31 & - & - & - & - & - \\
\hline 32 & - & - & - & - & - \\
\hline 33 & - & - & - & - & - \\
\hline 34 & - & - & - & - & - \\
\hline 35 & - & - & - & - & - \\
\hline 36 & - & - & - & - & - \\
\hline 37 & - & - & - & - & - \\
\hline 38 & - & - & - & - & - \\
\hline 39 & - & - & - & - & - \\
\hline 40 & - & - & - & - & - \\
\hline \multirow{3}{*}{41} & $\begin{array}{l}\text { Vicilin-like antimicrobial peptides 2-3 } \\
\text { (gil75207035) }\end{array}$ & $74 / 45$ & $190 / 139$ & $34 / 3$ & 74.6 \\
\hline & $\begin{array}{l}\text { Vicilin-like antimicrobial peptides 2-2 } \\
\text { (gil75266171) }\end{array}$ & $74 / 45$ & $116 / 139$ & $27 / 3$ & 79.4 \\
\hline & $\begin{array}{l}\text { Vicilin-like antimicrobial peptides 2-1 } \\
\text { (gil75207036) }\end{array}$ & $74 / 45$ & $117 / 139$ & $29 / 3$ & 79.4 \\
\hline 42 & - & - & - & - & - \\
\hline 43 & - & - & - & - & - \\
\hline \multirow{3}{*}{46} & $\begin{array}{l}\text { Vicilin-like antimicrobial peptides 2-3 } \\
\text { (gil75207035) }\end{array}$ & $74 / 44$ & $180 / 30$ & $32 / 1$ & 74.6 \\
\hline & $\begin{array}{c}\text { Vicilin-like antimicrobial peptides 2-2 } \\
\text { (gil75266171) }\end{array}$ & $74 / 44$ & $113 / 30$ & $22 / 1$ & 79.4 \\
\hline & $\begin{array}{l}\text { Vicilin-like antimicrobial peptides 2-1 } \\
\text { (gil75207036) }\end{array}$ & 74 & 95 & 22 & 79.4 \\
\hline 47 & - & - & - & - & - \\
\hline 48 & - & - & - & - & - \\
\hline
\end{tabular}


MIUT:

AGCCTCTGGA7

CCTGTTTCTGCAAA

¿GCGTGGTCCAGCCTC

AGGGCCGATTCACCATL ,

AATTGTGTTGACACAGTCTCCAGCCALL

UTGGCATCCCAGCCAGGTTCAGTGGCAGTGGG'TC I

$\because$ CAGATGACCCAGTCTCCTTCCACCCTGGCTGCATCTGTAG

AGTCCCATCAAGGTTCAGCGGCAGTGGGTCTGGGACAGAGTTCACL

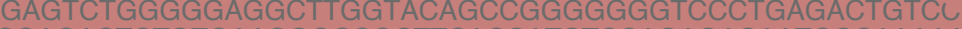

ITATCGAGACTCTGTGAAGGGCCGCTTCACCATCTCCAGAGACAATGCCAAAAAC ¿AGGGGACCACGGTCATCGTCTCCTCAGCAGGTGCAGCTGGTGGAGTCTGGGGGAGG iGAGTGGGTTTCATTTATACGCAAGGATGGAGCTAGTAAATACTATGGAGACTCCGTGAAGGG , CCTGGGTTACTTTGACTACTGGGGCCAGGGAACCCTGGTCACCGTCTCCTCAGGAAATTGTGT ACTGGCCTCCGCTCACTTTCGGCGGAGGGACCAAGGTGGAGATCAAACGACATCCAGATGACCCAG

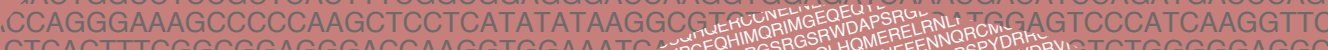
ACTCACTTTCGGCGGAGGGACCAAGGTGGAAAT

¿CAGGGGCTGGAGTGGGTCTCAGGCATCAGTGS

AAAGATAAGGTTCCAGACTATGACGAGGTCE

ITCAGTCGTTATGGCATGCAGTGGGTCCGPUNNORCMCOR

:CAGCCTGAGAGCTG.APPRCDLDVE

tGCCTAGAGCCT

GCCTGATGAT

CACGTTTAGG

ATGGCCAGTCE

CCATCTCCAGAG

GTCTCCAGCCACC

\author{
N
}

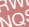

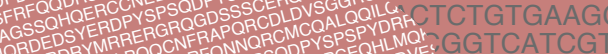

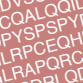

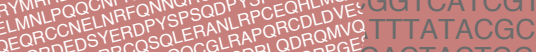
SDEDQEQRODE GKRELPNLP FEYYQSQLEF

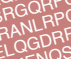

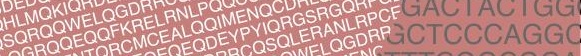
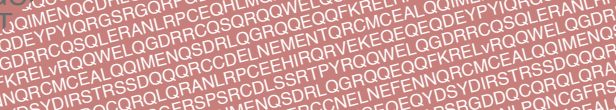

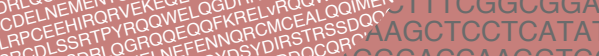
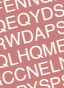

AERE LNLPOAMCEALA
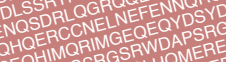

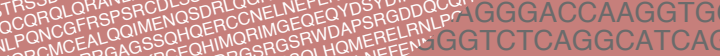

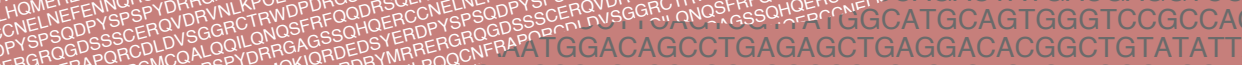

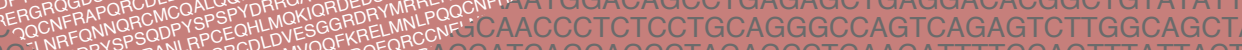

GGCAGTGGG UERD SOLERANRAPQRCDROMVESDEDGEORSACCATCAGCAGCCTAGAGCCTGAAGATTTTGCAGTTTATTACT

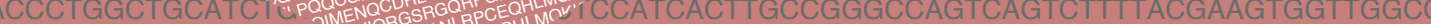
CAGTGGGTCTGGGACAGAGTT UYM CRLERANLPCEOHL ATCAGCAGTCTGCAGCCTGATGATTITGCAACTTACTACTGTCAACAA ACAGCCGGGGGGGTCCCTGAGACTGTCCTGTGCAGCCTCTGGATTCACGTTTAGGAGATATGCTTTGAGTTGGGTCCGC जCTTCACCATCTCCAGAGACAATGCCAAAAACATCCTGTTTCTGCAAATGGCCAGTCCGAGAGTCGAAGACGCGGCCGTT TCAGCAGGTGCAGCTGGTGGAGTCTGGGGGAGGCGTGGTCCAGCCTGGGGGGTCCCTGAGACTCTCCTGTGTAGCGTC aATGGAGCTAGTAAATACTATGGAGACTCCGTGAAG $\triangle$ TCTCCAGAGACAATTCCAAAGACACTCTCTTTCT iCCAGGGAACCCTGGTCACCGTCTCCTCAGGAAAD TTCTCATCTATGATGCATCCAACAGGGCCACTG aGAGGGACCAAGGTGGAGATCAAACGACATCCAGA . CATATATAAGGCGTCTAATTTACAAGATGGAGTCCC AAGGTGGAAATCAAACGAGGTGCAGTTGGTGGAGTCT ACGAGGTCGAGATTATGGACGTCTGGGGCCAGGGGACC GGGTCCGCCAGGCTCCAGGCAAGGGGCTGGAGTGGGTTTC
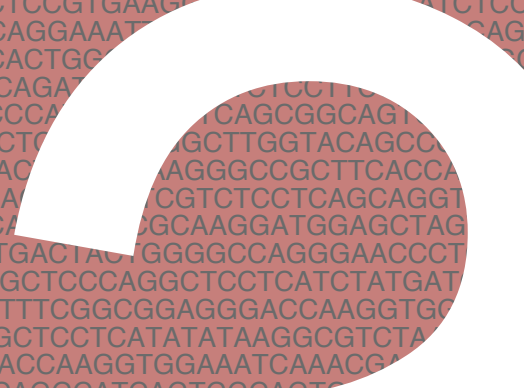
CCACCCTGTCTTTGTCTCCAGGGGAAAG/ AGTGGGTCTGGGACAGACTTCACTCTCA TTGATCTGTAGGAGACAGAGTCTCCAT GGACAGAGTTCACCCTCACCATCAGCA GTCCCTGAGACTGTCCTGTGCAGCCTC IAGACAATGCCAAAAACATCCTGTTTC GGTGGAGTCTGGGGGAGGCGTGGTC TATGGAGACTCCGTGAAGGGCCGA SGTCTCCTCAGGAAATTGTGTTGACA ACAGGGCCACTGGCATCCCAGCCAC ACGACATCCAGATGACCCAGTCTCC ATGGAGTCCCATCAAGGTTCAGCGG TGGTGGAGTCTGGGGGAGGCTTGGTA ATATTATCGAGACTCTGTGAAGGGCCGO LGGCCAGGGGACCACGGTCATCGTCTCCTC. GGTTCCAGACTATGACGAGGTCGAGAT TGGCATGCAGTGGGTCCGCCAGGCT CTGAGGACACGGCTGTATATTACTG

AGTCAGAGTCTTGGCAGCTACTTAG

ATTTTTGCAGTTTATTACTGTCAG

TTACGAAGTGGTTGGCCTGGTATCAGCAGAAACC  CTITGAGTTGGGTCCGCCAGGCTCCAGGGCAGGG :GAAGACGCGGCCGTTTATTACTGTGCGAAAGATAAG ATGTAGCGTGGATTCACCTTCAGTCGTTA AAAGACACTC iTCTCCAGGG CAGACTTCA GAGACAGAG 4CCCTCACCAT TGTCC ;AAAAACATCCTG IGGGGAGGCGTGG

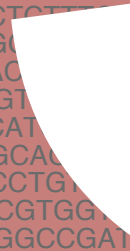
CAAATGGACAGCCTGAGA CAACCCTCTCCTGCAGGG ATCAGCAGCCTAGAGCC ACTTGCCGGGCCAGT TGGAGTGGGTTTCATTTATACGCAAGGATG CTGGCCAGGCTCCCAGGCTCCTCAT CGCTCACTTTCGGCGGAGGGACC CCCCAAGCTCCTCATATATAAGGC GGAGGGACCAAGGTGGAAATCA GGGTCTCAGGCATCAGTGGCAG ACTATGACGAGGTCGAGATTATG ICAGTGGGTCCGCCAGGCTCCAC ACACGGCTGTATATTACTGTGCG AAGTCTTGGCAGCTACTTAGCCTC GAGTCTTGGCAGCTACTTAGCCTC
TTGCAGTTTATTACTGTCAGCAGC CGAAGTGGTTGGCCTGGTATCAC ACTACTGTCAACAATATGATAGT TGAGTTGGGTCCGCCAGGCTCCA AAGACGCGGCCGTITATTACTGTGC GAAGACGCGGCCGTITATTACTGTGC
CTCCTGTGTAGCGTCTGGATTCACC Aunum TCCGTGAAGGGCCGA

ATTGTGTTGACACA CAAAGACACTCTCTTTCTGCAAATGGAC

ACGACATCCAGATGACCCAGTCTCCTTCCACCCTGGCTGCATCTGTAGGAGACAGAGTCTCCATCACTTGCCGG GATGGAGTCCCATCAAGGTTCAGCGGCAGTGGGTCTGGGACAGAGTTCACCCTCACCATCAGCAGTCTGCAGCC ITGGTGGAGTCTGGGGGAGGCTTGGTACAGCCGGGGGGGTCCCTGAGACTGTCCTGTGCAGCCTCTGGATTCAC ATATTATCGAGACTCTGTGAAGGGCCGCTTCACCATCTCCAGAGACAATGCCAAAAACATCCTGTITCTGCAAATGC GCCAGGGGACCACGGTCATCGTCTCCTCAGCAGGTGCAGCTGGTGGAGTCTGGGGGAGGCGTGGTCCAGCCTGG GGAGTGGGTTTCATTTATACGCAAGGATGGAGCTAGTAAATACTATGGAGACTCCGTGAAGGG

¿CCTGGGTTACTTTGACTACTGGGGCCAGGGAACCCTGGTCACCGTCTCCTCAGGAAATTGTऽ

ITAAACCTGGCCAGGCTCCCAGGCTCCTCATCTATGATGCATCCAACAGGGCCACTGGCATCr

rGGCCTCCGCTCACTTTCGGCGGAGGGACCAAGGTGGAGATCAAACGACATCCAGATGACCC AGGGAAAGCCCCCAAGCTCCTCATATATAAGGCGTCTAATTTACAAGATGGAGTCCCATCAAGC CACTTTCGGCGGAGGGACCAAGGTGGAAATCAAACGAGGTGCAGTTGGTGGAGTCTGGGGG GGCTGGAGTGGGTCTCAGGCATCAGTGGCAGTGGAAACAAAACATATTATCGAGACTCTGTG

\AGGTTCCAGACTATGACGAGGTCGAGATTATGGACGTCTGGGGCCAGGGGACCACGGTCATCGI

ITATGGCATGCAGTGGGTCCGCCAGGCTCCAGGCAAGGGGCTGGAGTGGGTTTCATTTATACGCAAGGATGGAGiL I AG I iAGAGCTGAGGACACGGCTGTATATTACTGTGCGAAAGATAGGTCCCTGGGTTACTTTGACTACTGGGGCCAGGGAACCCTG AGGGCCAGTCAGAGTCTTGGCAGCTACTTAGCCTGGTACCAACATAAACCTGGCCAGGCTCCCAGGCTCCTCATCTATGATG GAGCCTGAAGATTTTGCAGTTTATTACTGTCAGCAGCGTAGCGACTGGCCTCCGCTCACTTTCGGCGGAGGGACCAAGGTGGA 


\section{Sesame oleosins are minor allergens}

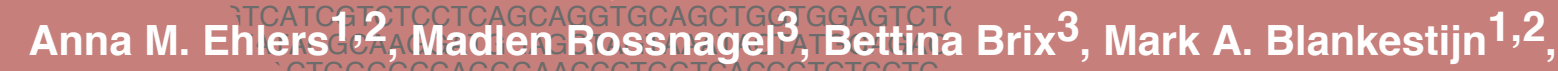

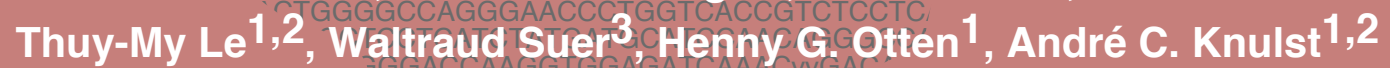

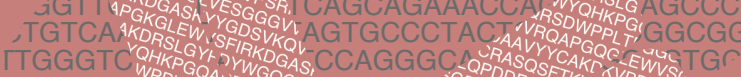

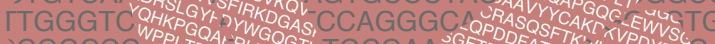

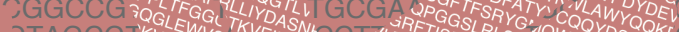

ГCTCTTTC'WYODE

iGGGAAAGAGCA/

ITCACTCTCACCA

XCATCAGCAGTC

iTGCAGCCTCTG

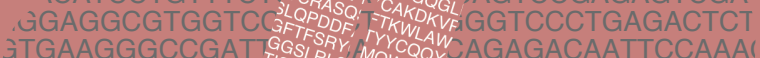

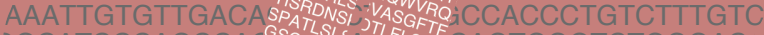

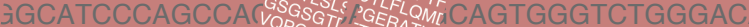

ITGACCCAGTCTCGRTG

ICAAGGTTCAGCGDGWTO TAKN A ASECTGGGACAGAGTTCACr

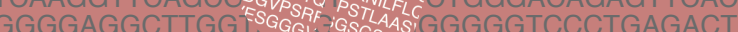

Clin TransI Allergy 2019;9:32.

AATCTGCAG

GTTTCTGCAAAT

AGCCGATTCACCATCTC

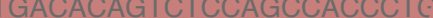

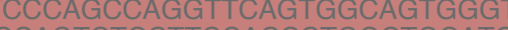

GTTCAGCGGCAGTGGGTCTGGGACAGAr

GGCCGCTTCACCATCTCCAGAGAC

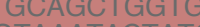
IGAGGGACCAAGGTGGAGATCAAACVVG

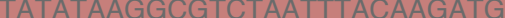
GGCATCAGTGGCAGTGGAAACAAAACATATTATC GAGGJVAGATTATGGACGTCTGGGGCCAGGG

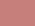

${ }^{1}$ Laboratory of Trañsfâtionalifinmuthology, Utrecht University, University Medical Center Utrecht, Utrecht, 1 The Netherlands A2Department of Dermatology/Aliergology, University Medical Center Utrecht, Uffecht University A Utrecht, The Netherlands

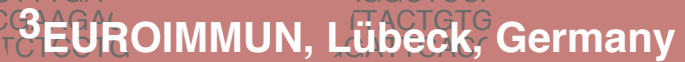

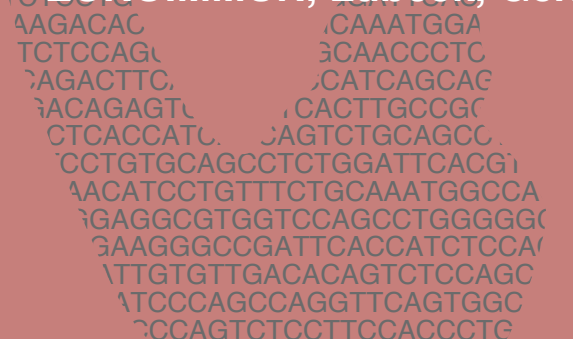




\section{Abstract}

\section{Background}

In daily practice, one-third of sesame allergic patients, confirmed by clinical history or food challenge, do not show any detectable specific lgE using current diagnostics. Currently used sesame extracts are water-based and therefore lacking hydrophobic proteins like oleosins. Oleosins, the stabilizer of lipid droplets in plants, are described as allergens in sesame, peanut, and hazelnut. In this study, we examined the role of oleosins in sesame allergy and their potential cross-reactivity between sesame and (pea)nuts.

\section{Methods}

Specific IgE and IgG sensitisation to native and heterologously expressed sesame components and oleosins from other nuts, free of seed storage proteins, was assessed by line blot and sera from 17 sesame allergic patients without detectable specific IgE sensitisation to sesame extract, and compared to 18 sesame allergic and 13 tolerant patients with specific $\lg \mathrm{E}$ sensitisation to sesame extract.

\section{Results}

Sesame allergic patients without sensitisation showed no specific $\lg E$ to the tested sesame oleosins or components. Low levels of specific lgE to sesame oleosins were detected in $17 \%$ of sesame allergic and $15 \%$ of tolerant patients with slgE sensitisation. Oleosins were recognised by serum IgG from multiple patients confirming immune reactivity and excluding technical issues leading to lack of specific lgE-binding to oleosins.

\section{Conclusion}

Sesame oleosins are minor allergens and appear to have no additonal value in diagnosing sesame allergy in adults based on slgE and slgG detection. There is a high need for additional diagnostic tools in those patients to minimise the number of required food challenges. 


\section{To the editor:}

Diagnosis of sesame allergy by measuring specific $\lg E$ (slgE) is based on extracts or the major allergen: $2 S$ albumin Ses i 1 . However, this leads to false negative results in around $30 \%$ of sesame allergic patients, a high frequency compared to other food allergies $^{1}$. To overcome this obstacle, we evaluated the role of oleosins in sesame allergy. Oleosins are oil-body stabilising proteins and might be lacking in water-based extracts due to its hydrophobicity².

Adult sesame allergic and tolerant but sensitised patients who visited the outpatient clinic of the University Medical Centre Utrecht, The Netherlands, were retrospectively selected and allergy $(n=35)$ or tolerance $(n=13)$ was confirmed by food challenge or an experienced physician diagnosis. Sesame allergic patients were subdivided into patients without and with detectable slgE (ImmunoCAP sesame extract $\geq 0.35 \mathrm{kU} / \mathrm{L}$; without $n=17$; with $n=18$ ). Patient characteristics are described in Table 1. Sera with slgE against native and heterologously expressed oleosins from different nuts and seeds acted as positive controls. Ethical approval was acquired from the biobank committee of the University Medical Centre Utrecht, number 18-428.

Known sesame components and oleosins (from sesame, walnut, hazelnut, peanut and soy) with and without the hydrophobic domain based on the TMHMM model (prediction of transmembrane helices being not available for antibody binding) were heterologously expressed $^{3}$. For comparisons to the native form, oil-body associated proteins (OAPs) were isolated from sesame, walnut and pecan nut using a modified previously described method with an additional hydrophobic interaction chromatography (HIC) instead of a preparative gel electrophoresis to separate traces of seed storage proteins with similar molecular masses ${ }^{4}$. To examine the absence of seed storage proteins, enclosed proteins were identified by mass spectrometry and detected by western blot with a-human IgE-alkaline phosphatase (AP). Sensitisation to these components was deeply investigated by measuring slgE and sIgG levels using line blots (EUROLINE, EUROIMMUN, Luebeck, Germany) according to manufacturer's instructions. IgG subtypes were examined using an ELISA coated with different heterologously expressed oleosins (Ses i 4, Ses i Oleosin, Jug r Oleosin-1, Jug r Oleosin-2) and detected with a-human IgG1-4 AP-conjugates. Detailed description of the methods is listed in the Method section.

Overall, the median age was 37 and allergic subjects suffered from typical symptoms being in line with an IgE-mediated hypersensitivity (characteristics are shown in Table 1). Gastro-intestinal symptoms were always accompanied by OAS or skin reactions (Suppl. Figure S1) and the allergic reactions took place within several minutes to half an hour after ingestion (data from 8/35), supporting the diagnosis 'food allergy'. 
Table 1: Patient demography of included sesame allergic and tolerant patients; Allergy was defined by food challenge or an experienced physician

\begin{tabular}{lcccc}
\hline & Group 1a $^{\mathbf{A}}$ & Group 1b $^{\mathbf{A}}$ & Group 2 $^{\mathbf{A}}$ & $\mathbf{p}$-value \\
\hline Age [median (range)] & $53(29-85)$ & $35(27-51)$ & $32(27-58)$ & 0.001 \\
Sex female & $14(82 \%)$ & $10(56 \%)$ & $10(77 \%)$ & 0.1865 \\
Food challenge & $8(47 \%)$ & $1(6 \%)$ & $1(8 \%)$ & 0.0034 \\
Total IgE (kU/l) & 295 & 4470 & $>5000$ & $<0.0001$
\end{tabular}

\begin{tabular}{lllll}
\hline Symptoms $^{\text {B }}$ & & & & \\
\hline Mild (Müller 0) & $6(35 \%)$ & $6(33 \%)$ & N/A & \\
Moderate (Müller 1+2) & $6(35 \%)$ & $6(33 \%)$ & N/A & 0.9693 \\
Severe (Müller 3+4) & $5(29 \%)$ & $6(33 \%)$ & N/A &
\end{tabular}

\begin{tabular}{lcccc}
\multicolumn{5}{l}{ Sensitisation ${ }^{\mathrm{C}}$ (n, median, range) } \\
\hline Sesame extract & $17(0,0-0.32 \mathrm{kU} / \mathrm{l})$ & $18(4.5,0.5-75 \mathrm{kU} / \mathrm{l})$ & $13(3.9,0.4-48 \mathrm{kU} / \mathrm{l})$ & 0.5338 \\
ImmunoCAP & $6(0 \mathrm{ISU})$ & $10(2.9,0-22.5 \mathrm{ISU})$ & $3(0,0-6 \mathrm{ISU})$ & 0.4336 \\
Ses i 1 ISAC & &
\end{tabular}

${ }^{A} \mathrm{G} 1 \mathrm{a}$ : sesame allergic patients without detectable slgE sensitisation; G1b: sesame allergic patients with slgE sensitisation; G2: sesame tolerant patients with slgE sensitisation

B Symptom distribution of each group is shown in the Supplementary

$\mathrm{C}^{\mathrm{C}}$ ImmunoCAP and ISAC data were compared between group $1 \mathrm{~b}$ and 2 since group $1 \mathrm{a}$ was selected by lacking slgE sensitisation; CAP $>0.35 \mathrm{kU} / \mathrm{L}$ was considered as positive

The sesame OAPs fraction (pool of HIC-fractions 4 and 5) used for slgE measurements was free of seed storage protein traces as confirmed by western blot analysis with sera positive for these proteins from the study cohort (Figure 1a). Before performing a HIC, these sera reacted with proteins around $15 \mathrm{kDa}$, similar molecular masses as oleosins. After the additional purification step, only the positive control (PC) still recognised proteins at this height. Additionally, no seed storage proteins but sesame oleosins were detected by mass spectrometry (Suppl. Figure S2 and Suppl. Table S1 to S3).

Specific IgE-binding to sesame oleosins with levels above the detection limit (EASTclass $1-2$ ) was detected in $17 \%$ of sensitised allergic and $15 \%$ of tolerant patients. However, none of the non-sensitised sesame allergic patients showed slgE-binding neither to sesame oleosins nor to any other sesame component, except one serum showing low slgE level to the $11 \mathrm{~S}$ globulin Ses i 7 (G1a_6). Control sera, selected for slgE binding to sesame $(\mathrm{PC})$ or peanut oleosins (C1-3), showed slgE binding to oleosins from different sources, confirming the binding capacity of heterologously expressed and native oleosins. Specific lgE-binding to oleosins in sensitised allergic patients was accompanied by recognising other sesame components, especially Ses $i 1$ and 2 whilst in tolerant patients was not (Figure $1 \mathrm{~b}$ and $\mathrm{c}$ ). Patients showing IgE reactivity 
to heterologously expressed sesame oleosins were co-sensitised to heterologously expressed oleosins from walnut, hazelnut, and peanut, indicating potential cross-reactivity (G1b_15, G1b_17). All oleosins expressed as full-length variants were recognised at least by one serum, except Cor a 12. In case of Cor a 13, the full-length and the hpvariant of Cor a 13 were recognised in parallel (G1b_17, PC).

Although oleosins were infrequently recognised by slgE, they were bound by serum IgG from multiple patients across all groups confirming immune reactivity and excluding technical issues leading to lack of specific $\lg E$ binding. Depletion of IgG by protein $\mathrm{G}$ columns in these sera did not result in slgE binding (Suppl. Figure S3), excluding competition between IgG and IgE. Specific IgG was mainly detected for Ses i 4, Ses i OI, Jug r Ol-1 and Jug r Ol-2 and significantly increased in sesame allergic patients without detectable slgE sensitisation compared with the other groups (Ses i 4: G1a vs G1b, p=0.0014; Ses i Ol: G1a vs G2, p=0.03; Jug r Ol-2: G1a vs $G 1 b, p=0.0049)$. The most prevalent subtype recognising oleosins was IgG1, while IgG2, IgG3 and IgG4 levels were dependent on the oleosin of interest (Suppl. Figure S4 and S5).

Up to $30 \%$ of sesame allergic patients, including those suffering from severe reactions, cannot be diagnosed by commercially available diagnostic tests ${ }^{1,5}$. Launched aqueous sesame extracts do not contain hydrophobic proteins like oleosins. We demonstrated that oleosins were clearly recognised in $17 \%$ of sensitised sesame allergic patients but did not have any additional diagnostic value compared to sesame extract and Ses i 1, especially in patients without detectable slgE sensitisation. Contrary to our findings, $90 \%$ recognition in sesame allergic patients (31\% without slgE to sesame extract) was reported previously ${ }^{6}$. Moreover, OAPs reactivity was detected in $36 \%$ of hazelnut allergic patients and therefore considered as relevant diagnostic markers, particularly in patients without detectable $\operatorname{sig}^{7}$. This discrepancy might be explained by the absence of seed storage proteins or fragments thereof ${ }^{8}$ in our native preparation accomplished by HIC, which was confirmed by western blot analysis and mass spectrometry.

Oleosins were recognised by slgE levels slightly above the detection limit (EAST-class 1-2). This is in line with a hazelnut study across Europe showing a prevalence of $20 \%$ for the hazelnut oleosin Cor a 12, but low slgE titre up to $1 \mathrm{kU} / \mathrm{l}(=\mathrm{EAST} \text {-class } 2)^{9}$. In our test system, even lower slgE titres might be explained by competition between different oleosins coated on the same line blot.

In conclusion, sesame oleosins are minor allergens and appear to have no additional value in diagnosing sesame allergy in adults based on slgE and slgG detection. We propose a prospective study to evaluate the diagnostic value of direct basophil activation tests due to the high need for additional diagnostic tools in those patients. 


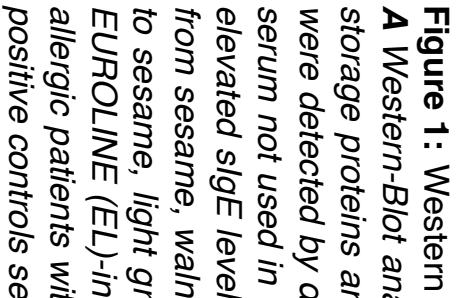

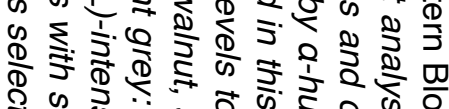

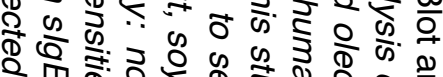
o

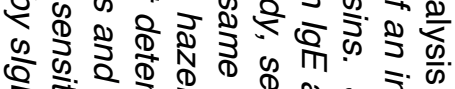

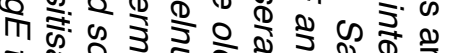
ธ。

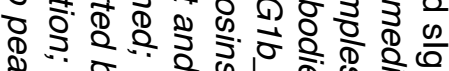
วั

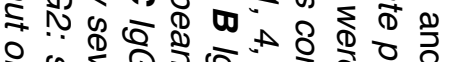
ㅇํㅇำ D की क क्ञ

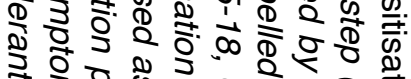

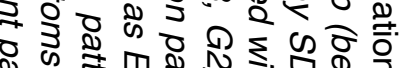
क क आ 0 N

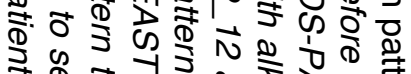

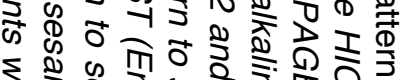

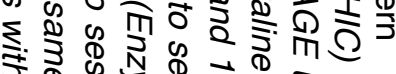

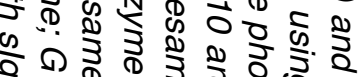
而 0 व

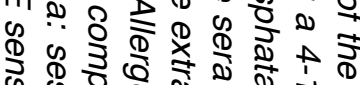
की की

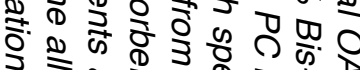
(1) ग0.

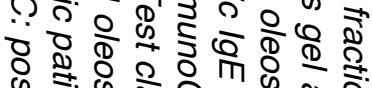

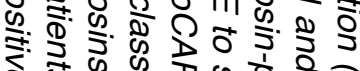
का के की की §

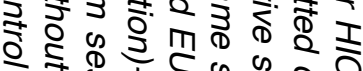

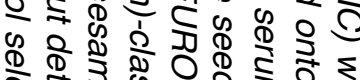

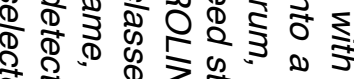

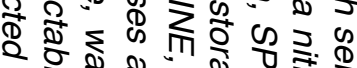

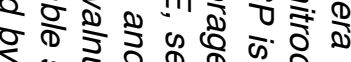

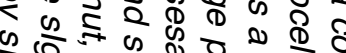
क⿺辶

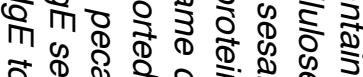

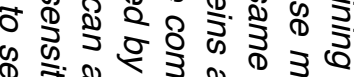
की कू

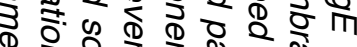
눈

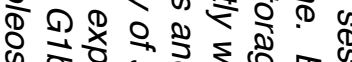

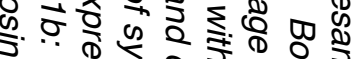
की

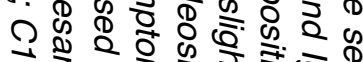

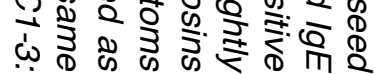
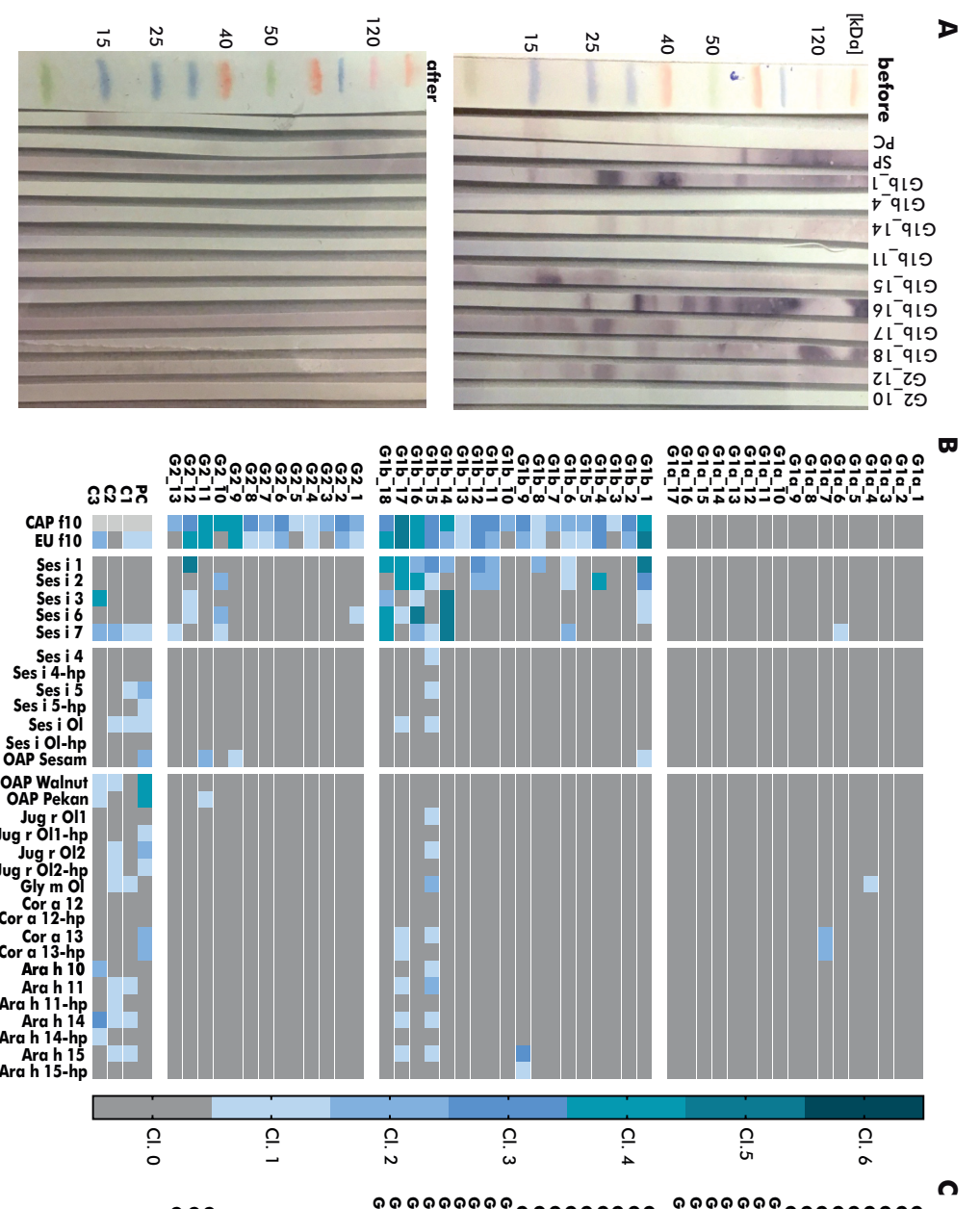
ค

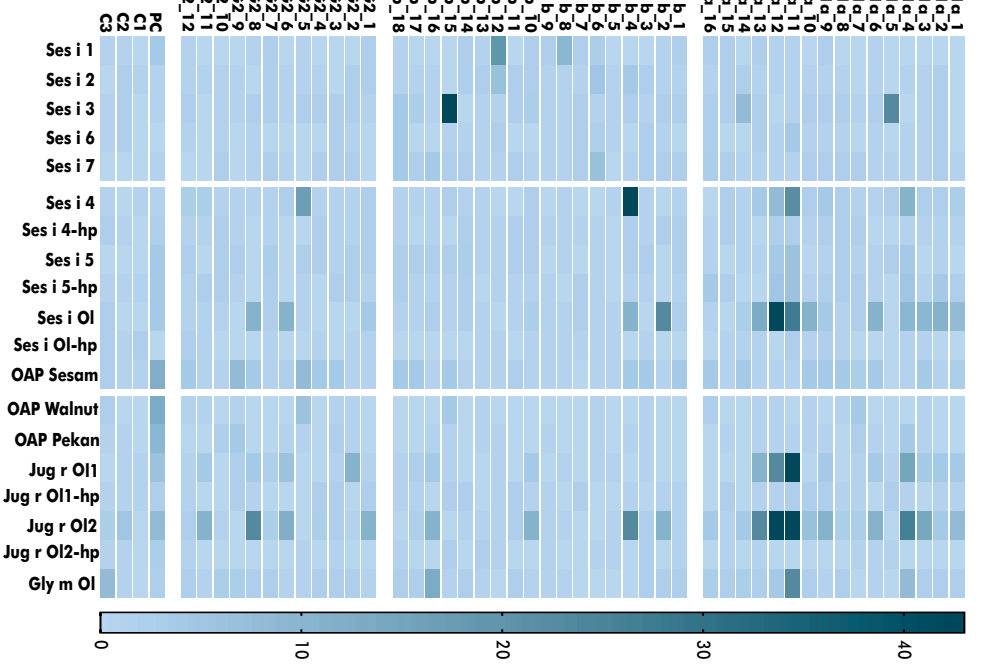




\section{Methods}

\section{Patient selection}

Sesame allergic and tolerant patients who visited the outpatient clinic of the University Medical Centre Utrecht, The Netherlands, between 2012 and 2017 were retrospectively selected. The selected patients were first divided into one of two groups regarding allergy $(n=35)$ or tolerance $(n=13)$ confirmed by food challenge or an experienced physician. Allergic patients were subdivided into patients without $(n=17)$ and with $(n=18)$ detectable slgE sensitisation (ImmunoCAP sesame extract $\geq 0.35 \mathrm{kU} / \mathrm{L}$ ). Tolerant patients without detectable slgE sensitisation were excluded. Positive controls were sera with slgE against native and heterologously expressed oleosins from different nuts and seeds. Ethical approval was acquired from the biobank committee of the University Medical Centre Utrecht under the number 18-428.

\section{Purification of oil-body associated proteins}

Oil-body associated proteins (OAPs) were isolated from sesame, walnut and pecan nut as described previously with the following modifications ${ }^{2,4,7}$. Sesame seeds were ground 1:2 w/v in $50 \mathrm{mM} \mathrm{NaH} \mathrm{PO}_{4}$ buffer $\mathrm{pH} 7.4$ containing $1 \mathrm{mM}$ EDTA, $10 \mathrm{mM}$ $\mathrm{KCl}, 2 \mathrm{mM}$ DTT and $20 \% \mathrm{w} / \mathrm{v}$ sucrose (grinding buffer) using a blender. The ground sesame seeds were filtered over a gauze and layered 1:1 v/v with flotation buffer $(50 \mathrm{mM}$ $\mathrm{NaH}_{2} \mathrm{PO}_{4}, 1 \mathrm{mM}$ EDTA, $10 \mathrm{mM} \mathrm{KCl}, 2 \mathrm{mM}$ DTT, pH 7.4). The gradient was centrifuged for $30 \mathrm{~min}$ at $4{ }^{\circ} \mathrm{C}$ using a swing-bucket rotor at $16.000 \times \mathrm{g}$. After centrifugation, a white fat pad was formed on top of the gradient. After resuspension of the fat pad in urea buffer (50 $\mathrm{mM} \mathrm{NaH}_{2} \mathrm{PO}_{4}, 9 \mathrm{M}$ urea, $\mathrm{pH}$ 7.4), the suspension was incubated for $15 \mathrm{~min}$ at room temperature, layered $1: 1 \mathrm{v} / \mathrm{v}$ with $50 \mathrm{mM} \mathrm{NaH} \mathrm{PO}_{4}$ buffer $\mathrm{pH} 7.4$ and centrifuged under the same conditions. This step was repeated twice, and the resulting fat pad was resuspended in grinding buffer containing $0.1 \%$ Tween-20 and layered $1: 1 \mathrm{v} / \mathrm{v}$ with $50 \mathrm{mM} \mathrm{NaH}_{2} \mathrm{PO}_{4}$ buffer $\mathrm{pH}$ 7.4. After centrifugation, the fat pad was resuspended in grinding buffer containing $2 \mathrm{M} \mathrm{NaCl}$ and layered with flotation buffer containing $2 \mathrm{M}$ $\mathrm{NaCl}$. After resuspension of the fat pad in grinding buffer, proteins were precipitated with a fourfold volume of ice-cold acetone and washed twice. After air-drying, the pellet was solubilised in a detergent-containing buffer and dialyzed against a $50 \mathrm{mM}$ Tris- $\mathrm{HCl}$ buffer, $\mathrm{pH}$ 7.5. To separate traces of seed storage proteins, the protein solution was applied on a hydrophobic interaction chromatography column according to manufacturer's instructions. 


\section{Heterologous expression and purification of recombinant allergens}

Sesame components (Ses i 1, 2, 3, 6 and 7) and oleosins (sesame, walnut, hazelnut and peanut) were expressed as fusion proteins with N-terminal His(6x) in E. coli as previously described ${ }^{10,11}$. The hp-variants of oleosins were expressed without the hydrophobic part determined by using the TMHMM model ${ }^{3}$. All heterologous expressed proteins were purified by immobilised metal ion chromatography under denaturing conditions. Full-length oleosins were dialyzed against citrate buffer $\mathrm{pH} 5.5$ for applying on the line blot.

\section{Mass spectrometry}

Enclosed proteins of the OAPs fractions from sesame, walnut and pecan nut were identified by mass spectrometry. Reduced (0.02 M DTT) and alkylated ( $0.06 \mathrm{M}$ iodoacetamide) proteins were separated in a $4-12 \%$ polyacrylamide gel stained with colloidal Coomassie. After excising and achromatising, these proteins were digested in-gel by trypsin for 3 hours at $37{ }^{\circ} \mathrm{C}$. The resulting peptides were subsequently extracted and spotted with a-cyano-4-hydroxycinnamic acid onto a MTP Anchor Chip 384 TF target (Bruker, Billerica, Massachusetts, US). Spotted peptides were measured by matrixassisted laser desorption/ionisation-time of flight/TOF mass spectrometry (MALDI-TOF ITOF) using an Autoflex III smartbeam TOF/TOF200 System (Bruker, Billerica, Massachusetts, US) combined with the flexControl 3.4 software. MS spectra for peptide mass fingerprinting (PMF) were acquired in a positive ion reflector mode with 6000 shots ranging from 600 to $4.000 \mathrm{Da}$. For spectra calibration, external commercially available Peptide Calibration Standard II was processed with flexAnalysis 3.4 and the resulting peak lists were examined with BioTools 3.2. MS spectra for protein identification were analysed by submitting them to the MASCOT search engine MASCOT Server 2.3 (Matrix Science, London, U.K.) searching against the NCBI database (2016/12/19) including 33.467 proteins from Sesamum indicum, 56.036 proteins from Juglans regia and 278 proteins from Carya illinoinensis. Search parameters were set as follows: mass tolerance of $80 \mathrm{ppm}$, acceptance of one missed trypsin cleavage site, carbamidomethylation of cysteine residues as fixed modification, and oxidation of methionine residues as variable modification. For evaluation of protein hits, significance was defined as $p$ $<0.05$. PMF hits were confirmed by selecting two to five peptides of each identified protein with the WARP feedback mechanism of BioTools for MS/MS measurements. Recording of parent and fragment masses were performed with 400 and 1,000 shots, respectively. Resulted spectra were processed and analysed as described above with a fragment mass tolerance of $0.7 \mathrm{Da}$. These measurements were performed twice with different preparations in duplicates. 


\section{Line Blot}

Sensitisation was defined by measuring slgE and slgG levels using a line blot (EUROLINE, EUROIMMUN, Luebeck, Germany) according manufacturer's instructions. For IgE detection, line blots were incubated overnight with $1 \mathrm{ml}$ diluted serum (1:11 in universal buffer), some of them IgG-depleted (Protein G columns, GE Healthcare, Buckinghamshire, Great Britain), at room temperature on a rocket shaker. For detection of slgG levels, $0.51 \mathrm{ml}$ diluted serum (1:51 in universal buffer) was incubated for one hour at room temperature. Bound serum antibodies were detected with a-human IgE or IgG alkaline phosphatase-coupled conjugate and visualised by adding nitro-blue tetrazolium/5-bromo-4-chloro-3'-indolyphosphate substrate. The binding of antibodies was evaluated using the EUROLINEScan software. For IgE measurement, band intensities were reported as intensity levels or classes, leaned on the Enzyme-Allergo-Sorbent Test (EAST) classification ${ }^{12}$. EUROLINE intensities (EL-intensities) of 3 or greater were considered as positive, corresponding to classes from 1 to 6 . For lgG measurement, band intensities smaller than 8 were considered as negative. These measurements were performed once.

\section{Total IgE levels}

Total IgE levels were determined using the ELISA technique (EUROIMMUN, Lübeck, Germany). In brief, $100 \mu \mathrm{l}$ of diluted serum (1:10 in universal buffer) were applied per well and incubated for $30 \mathrm{~min}$ at room temperature. Bound IgE was detected by applying a-lgE horse radish peroxidase-coupled conjugate and visualised by adding tetramethylbenzidine. Optical intensities (OD at $450 \mathrm{~nm}$ ) were evaluated with a calibration curve and converted into $\mathrm{IU} / \mathrm{ml}$. These measurements were performed once in duplicates.

\section{IgG subtype determination}

Microtiter plates were coated with heterologously expressed full-length oleosins $(9 \mu \mathrm{g} / \mathrm{mL}$ in PBS, pH 7.5) over night at $4{ }^{\circ} \mathrm{C}$. After blocking, $100 \mu$ l of diluted serum (1:25 in blocking buffer) was applied in duplicates and allowed to react for 1 hour at room temperature on an orbital shaker. Bound antibodies were detected using a-human IgG1-4 AP-conjugates and stained with p-Nitrophenyl Phosphate Disodium salt (Thermofisher Scientific, Waltham, MA, USA). The OD was read at $405 \mathrm{~nm}$ using an automated spectrophotometer (iMark, Bio-Rad, Hercules, CA, USA). OD values of each IgG subtype measured for sera previously considered as positive (line blot) were divided by the OD value of the negative control. These measurements were performed twice in duplicates. 


\section{Data analysis}

The baseline data were statistically analysed using one-way ANOVA or Mann-WhitneyU-test for continuous data and Fisher's exact test for categorical data. For comparison of slgG levels between the patient groups, Kruskal-Wallis and Dunn's post-hoc tests were used. Statistical evaluation was performed with GraphPad Prism 7 (GraphPad Software, La Jolla, CA, USA) and SPSS Statistics 21 (IBM Corporation, Armonk, NY, USA). $P$ values $\leq 0.05$ were considered as statistically significant.

\section{Informed consent statement}

This study was carried out in accordance with the University Medical Centre Utrecht, Biobank Regulations, which are in compliance with the applicable national and international laws and regulations. These regulations permit the use of 'residual material from diagnostic testing' for research, unless the patient objects (Article 8, 'no objection' procedure). None of the included patients objected the use of their serum. The protocol was approved by the Biobank Research Ethics Committee of the University Medical Centre Utrecht under the protocol number 18-428.

\section{Acknowledgements}

We would like to thank M. Klug and S. Brandhoff for technical assistance, Y. Weimann for fruitful discussions and $Y$. Denno for performing the mass spectrometry analyses. Line blots and corresponding reagents were kindly provided by EUROIMMUN AG, Lübeck, Germany. 


\section{References}

1. Permaul, P. et al. Sesame allergy: Role of specific lgE and skin-prick testing in predicting food challenge results. Allergy Asthma Proc 30, 643-8 (2009).

2. Tzen, J. T. C. et al. A New Method for Seed Oil Body Purification and Examination of Oil Body Integrity Following Germination. J Biochem 121, 762-8 (1997).

3. Sonnhammer, E. L. L., van Heijne, G. \& Krogh, A. A Hidden Markov Model for Predicting Transmembrane Helices in Protein Sequences. Int Conf Intel Sys Mol Biol 6, 175-82 (1998).

4. Schwager, C. et al. Development of a Novel Strategy to Isolate Lipophilic Allergens (Oleosins) from Peanuts. PLOS ONE 10 (ed Chardot, T.) e0123419 (2015).

5. Derby, C. J., Gowland, M. H. \& Hourihane, J. O. Sesame allergy in Britain: A questionnaire survey of members of the Anaphylaxis Campaign. Pediatric Allergy and Immunology 16, 171-5 (2005).

6. Leduc, V. et al. Identification of oleosins as major allergens in sesame seed allergic patients. Allergy 61, 349-56 (2006).

7. Zuidmeer-Jongejan, L. et al. Oil body-associated hazelnut allergens including oleosins are underrepresented in diagnostic extracts but associated with severe symptoms. Clin Transl Allergy 4, 4 (2014).

8. Koppelman, S. J. et al. Peanut allergen Ara $\mathrm{h}$ 3: Isolation from peanuts and biochemical characterization. Allergy 58, 1144-51 (2003).

9. Datema, M. R. et al. Hazelnut allergy across Europe dissected molecularly: A EuroPrevall outpatient clinic survey. J Allergy Clin Immunol 136, 382-91 (2015).

10. Hochuli, E. et al. Genetic Approach to Facilitate Purification of Recombinant Proteins with a Novel Metal Chelate Adsorbent. Nat Biotechnol 6, 1321-5 (1988).

11. Trigoso, Y. D., Evans, R. C., Karsten, W. E. \& Chooback, L. Cloning, Expression, and Purification of Histidine-Tagged Escherichia coli Dihydrodipicolinate Reductase. PLOS ONE 11, e0146525 (2016).

12. Williams, P. B., Barnes, J. H., Szeinbach, S. L. \& Sullivan, T. J. Analytic precision and accuracy of commercial immunoassays for specific IgE: Establishing a standard. Journal of Allergy and Clinical Immunology 105, 1221-1230 (June 2000). 


\section{Supplementary}
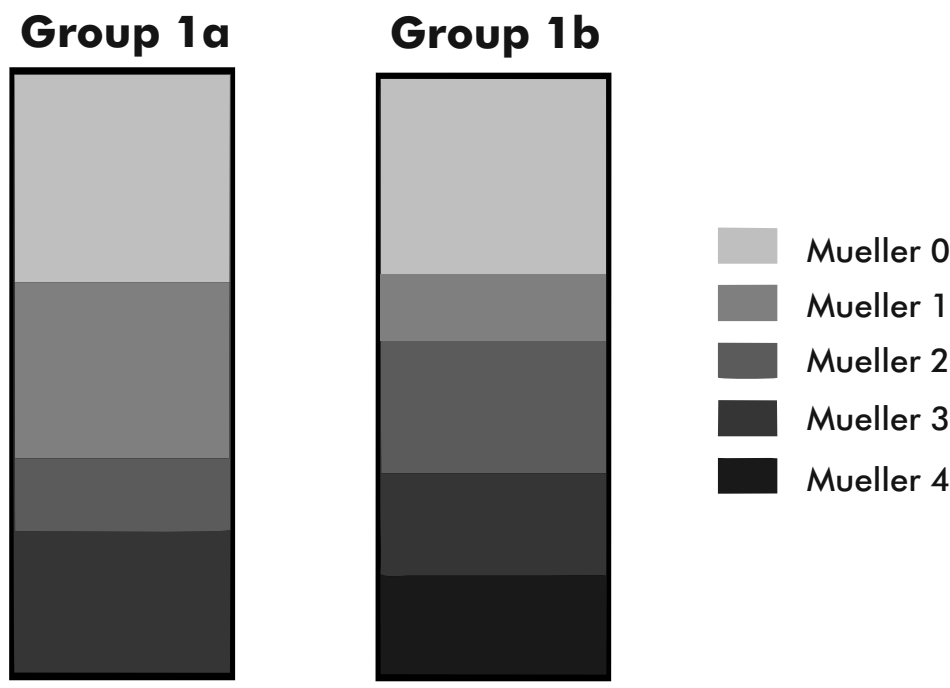

Figure S1: Distinct symptom distribution between sesame allergic patients with and without detectable slgE sensitisation

Sesame allergic patients without detectable slgE sensitisation (G1a) showed more often skin related reactions (Mueller 1) compared to patients with sensitisation (G1b). The other way around, patients of G1b showed more often gastro-intestinal symptoms (Mueller 2) and cardiovascular reactions (Mueller 4)
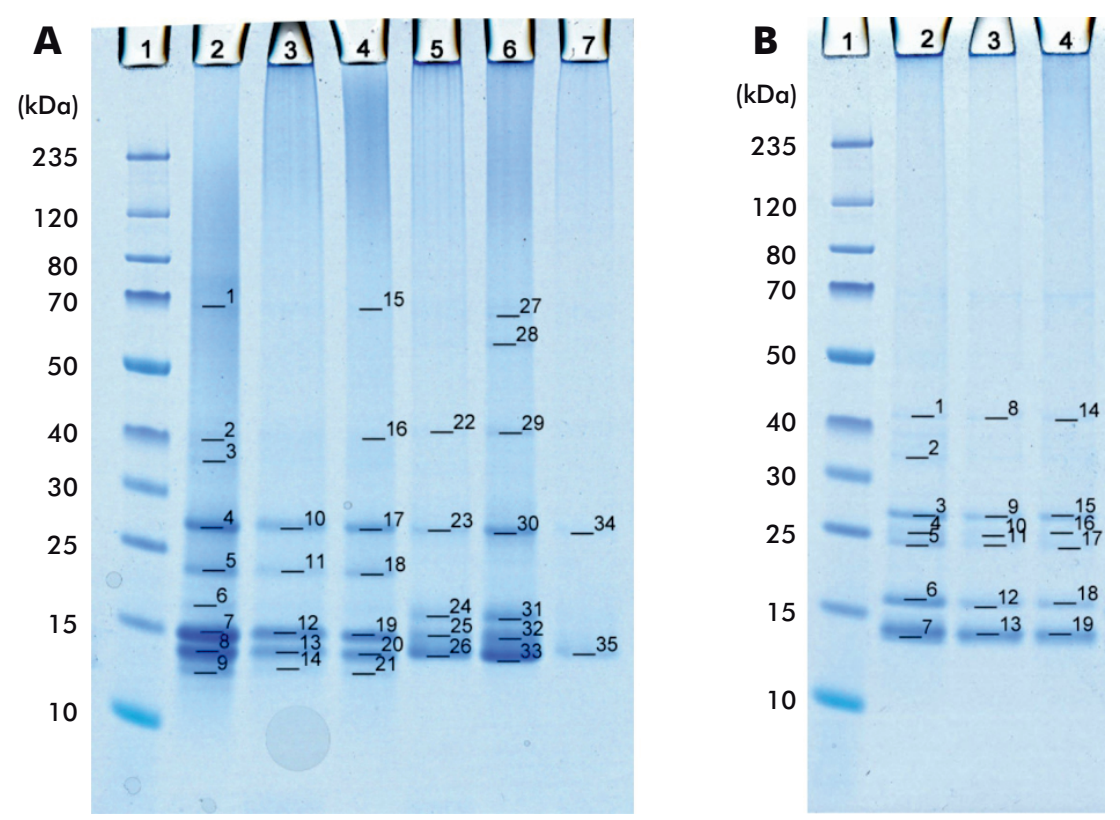
1 - Marker
2 - OAPs sesame HIC-fraction 3
3 - OAPs sesame HIC-fraction 4
4 - OAPs sesame HIC-fraction 5
5 - OAPs walnut HIC-fraction 3
6 - OAPs walnut HIC-fraction 4
7 - OAPs walnut HIC-fraction 5
1 - Marker
2 - OAPs pecan nut HIC-fraction 3
3 - OAPs pecan nut HIC-fraction 4
4 - OAPs pecan nut HIC-fraction 5

B

Figure S2: Gel images for mass spectrometry analyses of OAPs fractions Gel images of elution fraction of OAPs from sesame, walnut and pecan nut after hydrophobic interaction chromatography used for mass spectrometry analyses 
Table S1: The native sesame OAPs fractions were analysed by mass spectrometry using peptide mass fingerprint. The table shows the identified proteins in these fractions, italic: MS/MS analysis

\begin{tabular}{llllcc}
\hline Band & $\begin{array}{c}\text { Protein } \\
\text { (Accession Number) }\end{array}$ & Cut-Off & Protein Score & $\begin{array}{c}\text { Sequence } \\
\text { Coverage [\%] }\end{array}$ & $\begin{array}{c}\text { Molecular } \\
\text { Mass [kDa] }\end{array}$ \\
\hline
\end{tabular}

Sesame OAPs: HIC-Elution-Fraction 3

\begin{tabular}{|c|c|c|c|c|c|}
\hline \multirow{5}{*}{1} & uncharacterised protein & & & & \\
\hline & LOC105177100 & 49 & 45 & 10 & 17.4 \\
\hline & (NP_001292915.1) & & & & \\
\hline & $\begin{array}{l}15 \mathrm{kDa} \text { oleosin } \\
\text { (AAD42942.1) }\end{array}$ & 49 & 23 & 6 & 15.2 \\
\hline & $\begin{array}{l}\text { Predicted: oleosin 1-like } \\
\text { (XP_011076526.1) }\end{array}$ & 49 & 23 & 4 & 24.0 \\
\hline 2 & $\begin{array}{l}\text { 11-beta-hydroxysteroid } \\
\text { dehydrogenase-like } 5 \\
\text { (NP_001291330.1) }\end{array}$ & $79 / 50$ & $165 / 55$ & $54 / 4$ & 41.3 \\
\hline \multirow[t]{2}{*}{3} & $\begin{array}{l}\text { 11-beta-hydroxysteroid } \\
\text { dehydrogenase-1B-like } \\
\text { (NP_001291322.1) }\end{array}$ & $79 / 50$ & $123 / 31$ & $50 / 2$ & 39.7 \\
\hline & $\begin{array}{c}\text { 11-beta-hydroxysteroid } \\
\text { dehydrogenase-like } 5 \\
(\text { NP_001291330.1) }\end{array}$ & $79 / 50$ & $87 / 20$ & $41 / 4$ & 41.3 \\
\hline 4 & $\begin{array}{c}\text { peroxygenase } \\
\left(\mathrm{NP} \_001291323.1\right)\end{array}$ & $79 / 50$ & $76 / 115$ & $40 / 23$ & 27.8 \\
\hline \multirow{3}{*}{5} & $\begin{array}{l}\text { PREDICTED: legumin-B-like } \\
\text { (XP_011083024) }\end{array}$ & $79 / 50$ & $93 / 59$ & $35 / 8$ & 52.1 \\
\hline & $\begin{array}{c}\text { peroxygenase } \\
\text { (NP_001291323.1) }\end{array}$ & $79 / 50$ & $80 / 81$ & $32 / 10$ & 27.8 \\
\hline & $\begin{array}{l}\text { 11S globulin precursor } \\
\text { isoform } 4 \\
(\mathrm{ABB} 60055.1)\end{array}$ & $79 / 50$ & $80 / 59$ & $32 / 8$ & 53.0 \\
\hline \multirow{4}{*}{6} & $\begin{array}{l}\text { PREDICTED: legumin-B-like } \\
\text { (XP_011083024) }\end{array}$ & 79 & 72 & 33 & 52.1 \\
\hline & $\begin{array}{c}\text { 11S globulin precursor } \\
\text { isoform } 4 \\
\text { (ABB60055.1) }\end{array}$ & 79 & 60 & 28 & 53.0 \\
\hline & $\begin{array}{l}15 \mathrm{kDa} \text { oleosin } \\
\text { (AAD42942.1) }\end{array}$ & 50 & 22 & 6 & 15.2 \\
\hline & $\begin{array}{l}\text { PREDICTED: oleosin 1-like } \\
\text { (XP_011076526.1) }\end{array}$ & 50 & 22 & 4 & 24.0 \\
\hline \multirow[t]{2}{*}{7} & $\begin{array}{l}15 \mathrm{kDa} \text { oleosin } \\
\text { (AAD42942.1) }\end{array}$ & 50 & 61 & 6 & 15.2 \\
\hline & $\begin{array}{l}\text { PREDICTED: oleosin 1-like } \\
\text { (XP_011076526.1) }\end{array}$ & 50 & 61 & 4 & 24.0 \\
\hline
\end{tabular}




\begin{tabular}{|c|c|c|c|c|c|}
\hline Band & $\begin{array}{c}\text { Protein } \\
\text { (Accession Number) }\end{array}$ & Cut-Off & $\begin{array}{l}\text { Protein } \\
\text { Score }\end{array}$ & $\begin{array}{c}\text { Sequence } \\
\text { Coverage [\%] }\end{array}$ & $\begin{array}{l}\text { Molecular } \\
\text { Mass [kDa] }\end{array}$ \\
\hline \multirow{3}{*}{8} & uncharacterised protein & & & & \\
\hline & LOC105177100 & 51 & 43 & 10 & 17.4 \\
\hline & (NP_001292915.1) & & & & \\
\hline \multirow{7}{*}{9} & uncharacterised protein & & & & \\
\hline & LOC105177100 & 49 & 40 & 10 & 17.4 \\
\hline & (NP_001292915.1) & & & & \\
\hline & 15 kDa oleosin & & & & \\
\hline & (AAD42942.1) & 49 & 35 & 6 & 15.2 \\
\hline & $\begin{array}{l}\text { PREDICTED: oleosin 1-like } \\
\text { (XP_011076526.1) }\end{array}$ & 49 & 35 & 4 & 24.0 \\
\hline & $\begin{array}{l}\text { PREDICTED: oleosin 16.4 kDa } \\
\text { (XP_011097414.1) }\end{array}$ & 49 & 20 & 10 & 17.4 \\
\hline
\end{tabular}

Sesame OAPs: HIC-Elution-Fraction 4

\begin{tabular}{|c|c|c|c|c|c|}
\hline 10 & $\begin{array}{c}\text { Peroxygenase } \\
(\text { NP_001291323.1) }\end{array}$ & $79 / 49$ & $108 / 27$ & $41 / 8$ & 27.8 \\
\hline 11 & $\begin{array}{c}\text { Peroxygenase } \\
(\text { NP_001291323.1) }\end{array}$ & 51 & 81 & 10 & 27.8 \\
\hline \multirow{5}{*}{12} & uncharacterised protein & & & & \\
\hline & LOC105177100 & 79 & 57 & 39 & 17.4 \\
\hline & (NP_001292915.1) & & & & \\
\hline & $\begin{array}{l}15 \text { kDa oleosin } \\
(\text { AAD42942.1) }\end{array}$ & 50 & 47 & 6 & 15.2 \\
\hline & $\begin{array}{l}\text { PREDICTED: oleosin 1-like } \\
\text { (XP_011076526.1) }\end{array}$ & 50 & 47 & 4 & 24.0 \\
\hline \multirow{5}{*}{13} & uncharacterised protein & & & & \\
\hline & $\begin{array}{c}\text { LOC105177100 } \\
(\text { NP_001292915.1) }\end{array}$ & 51 & 24 & 10 & 17.4 \\
\hline & $\begin{array}{l}\text { PREDICTED: oleosin 1-like } \\
\text { (XP_011081346.1) }\end{array}$ & 51 & 22 & 7 & 14.8 \\
\hline & $\begin{array}{l}15 \text { kDa oleosin } \\
\text { (AAD42942.1) }\end{array}$ & 51 & 21 & 6 & 15.2 \\
\hline & $\begin{array}{l}\text { PREDICTED: oleosin 1-like } \\
\text { (XP_011076526.1) }\end{array}$ & 51 & 21 & 4 & 24.0 \\
\hline \multirow{3}{*}{14} & PREDICTED: DDB1- and & & & & \\
\hline & $\begin{array}{c}\text { CUL4-associated factor } 8 \\
\qquad\left(X P \_011089371.1\right) \\
\text { uncharacterised protein }\end{array}$ & 79 & 58 & 22 & 54.1 \\
\hline & $\begin{array}{c}\text { LOC105177100 } \\
(\text { NP_001292915.1) }\end{array}$ & 49 & 15 & 10 & 17.4 \\
\hline
\end{tabular}




\begin{tabular}{|c|c|c|c|c|c|}
\hline Band & $\begin{array}{c}\text { Protein } \\
\text { (Accession Number) }\end{array}$ & Cut-Off & $\begin{array}{l}\text { Protein } \\
\text { Score }\end{array}$ & $\begin{array}{c}\text { Sequence } \\
\text { Coverage [\%] }\end{array}$ & $\begin{array}{l}\text { Molecular } \\
\text { Mass [kDa] }\end{array}$ \\
\hline \multicolumn{6}{|c|}{ Sesame OAPs: HIC-Elution-Fraction 5} \\
\hline 15 & - & - & - & - & - \\
\hline 16 & $\begin{array}{c}\text { 11-beta-hydroxysteroid } \\
\text { dehydrogenase-like-5 } \\
\text { (NP_001291330.1) }\end{array}$ & $79 / 50$ & $190 / 70$ & $53 / 7$ & 41.3 \\
\hline 17 & $\begin{array}{c}\text { Peroxygenase } \\
(\text { NP_001291323.1) }\end{array}$ & $79 / 50$ & $119 / 73$ & $53 / 13$ & 27.8 \\
\hline 18 & $\begin{array}{c}\text { Peroxygenase } \\
(\text { NP_001291323.1) }\end{array}$ & $79 / 50$ & $80 / 63$ & $27 / 10$ & 27.8 \\
\hline \multirow[t]{3}{*}{19} & $\begin{array}{l}\text { uncharacterised protein } \\
\text { LOC105177100 } \\
\text { (NP_001292915.1) }\end{array}$ & 50 & 67 & 10 & 17.4 \\
\hline & $\begin{array}{l}15 \text { kDa oleosin } \\
(\text { AAD42942.1) }\end{array}$ & 50 & 52 & 6 & 15.2 \\
\hline & $\begin{array}{l}\text { PREDICTED: oleosin 1-like } \\
\text { (XP_011076526.1) }\end{array}$ & 50 & 52 & 4 & 24.0 \\
\hline \multirow[t]{2}{*}{20} & $\begin{array}{l}\text { PREDICTED: oleosin 1-like } \\
\text { (XP_011081346.1) } \\
\text { uncharacterised protein }\end{array}$ & 51 & 46 & 7 & 14.8 \\
\hline & $\begin{array}{c}\text { LOC105177100 } \\
(\text { NP_001292915.1) }\end{array}$ & 51 & 43 & 10 & 17.4 \\
\hline \multirow[t]{3}{*}{21} & $\begin{array}{l}\text { PREDICTED: DDB1- and } \\
\text { CUL4-associated factor } 8 \\
\quad\left(X P \_011089371.1\right)\end{array}$ & 79 & 91 & 36 & 54.1 \\
\hline & uncharacterised protein & & & & \\
\hline & $\begin{array}{c}\text { LOC105177100 } \\
(\text { NP_001292915.1) }\end{array}$ & 49 & 17 & 10 & 17.4 \\
\hline
\end{tabular}


Table S2: The native walnut OAPs fractions were analysed by mass spectrometry using peptide mass fingerprint. The table shows the identified proteins in these fractions.

\begin{tabular}{lccccc}
\hline Band & $\begin{array}{c}\text { Protein } \\
\text { (Accession Number) }\end{array}$ & Cut-Off & Protein Score & $\begin{array}{c}\text { Sequence } \\
\text { Coverage [\%] }\end{array}$ & $\begin{array}{c}\text { Molecular Mass } \\
{[\mathrm{kDa}]}\end{array}$ \\
\hline
\end{tabular}

Walnut OAPs: HIC-Elution-Fraction 3

$\begin{array}{lccccc}22 & - & - & - & - & - \\ 23 & - & - & - & - & - \\ 24 & - & - & - & - & - \\ 25 & - & - & - & - & 14.7 \\ 26 & \text { Oleosin } & 79 & 40 & 38 & \end{array}$

Walnut OAPs: HIC-Elution-Fraction 4

\begin{tabular}{lccccc}
\hline 27 & - & - & - & - & - \\
28 & - & - & - & - & - \\
29 & - & - & - & - & - \\
30 & - & - & - & - & - \\
31 & - & - & - & - & - \\
32 & - & - & - & 38 & 14.7 \\
33 & Oleosin & 79 & 48 & & - \\
Walnut OAPs: HIC-Elution-Fraction 5 & - & & 14.7 \\
\hline 34 & - & - & - & 35
\end{tabular}


Table S3: The native pecan nut OAPs fractions were analysed by mass spectrometry using peptide mass fingerprint. The table shows the identified proteins in these fractions.

\begin{tabular}{lccccc}
\hline Band & $\begin{array}{c}\text { Protein } \\
\text { (Accession Number) }\end{array}$ & Cut-Off & Protein Score & $\begin{array}{c}\text { Sequence } \\
\text { Coverage [\%] }\end{array}$ & $\begin{array}{c}\text { Molecular Mass } \\
{[\mathrm{kDa}]}\end{array}$ \\
\hline
\end{tabular}

\section{Pecan nut OAPs: HIC-Elution-Fraction 3}

\begin{tabular}{llllll}
\hline 1 & - & - & - & - & - \\
2 & - & - & - & - & - \\
3 & - & - & - & - & - \\
4 & - & - & - & - & - \\
5 & - & - & - & - & - \\
6 & - & - & - & - & -
\end{tabular}

Pecan OAPs: HIC-Elution-Fraction 4

$\begin{array}{llllll}8 & - & - & - & - & - \\ 9 & - & - & - & - & - \\ 10 & - & - & - & - & - \\ 11 & - & - & - & - & - \\ 12 & - & - & - & - & - \\ 13 & - & - & - & - & -\end{array}$

Pecan nut OAPs: HIC-Elution-Fraction 5

$\begin{array}{llllll}14 & - & - & - & - & - \\ 15 & - & - & - & - & - \\ 16 & - & - & - & - & - \\ 17 & - & - & - & - & - \\ 18 & - & - & - & - & - \\ 19 & - & - & - & - & -\end{array}$



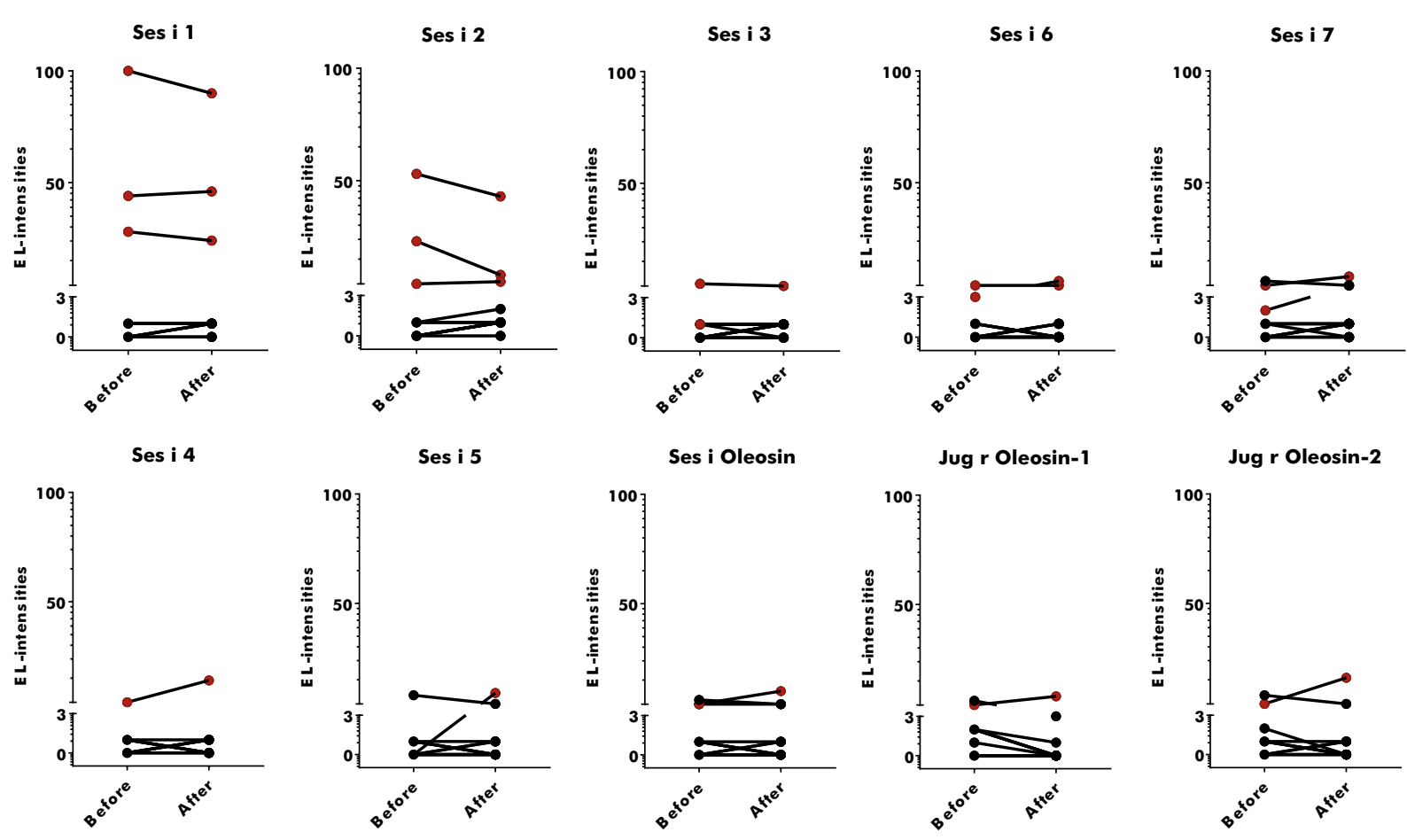

Figure S3: IgG depletion did not influence the recognition of sesame oleosins by slgE

Sera with high slgG levels to oleosins (G1a_4, G1a_12, G1a_13 and G2_8) showed no increase in slgE levels to sesame components or oleosins while sera with low slgG levels but IgE sensitisation to sesame (G1b_1, G1b_15, G1b_17, G2_9 and G2_11) showed a scarce decrease in slgE levels after lgG depletion, confirming no depletion of IgE during IgG depletion. One serum positive for sesame oleosins, G1b_15, displayed an increase in slgE levels to oleosins although no slgG to these components were detected. Sera with slgE levels greater than an EUROLINE (EL-) intensity of 3 were marked accordingly to their study group (green: group $1 a$ - sesame allergic without sensitisation, red: groub $1 b-$ sesame allergic with sensitisation, black: group 2 - sesame tolerant with sensitisation) 


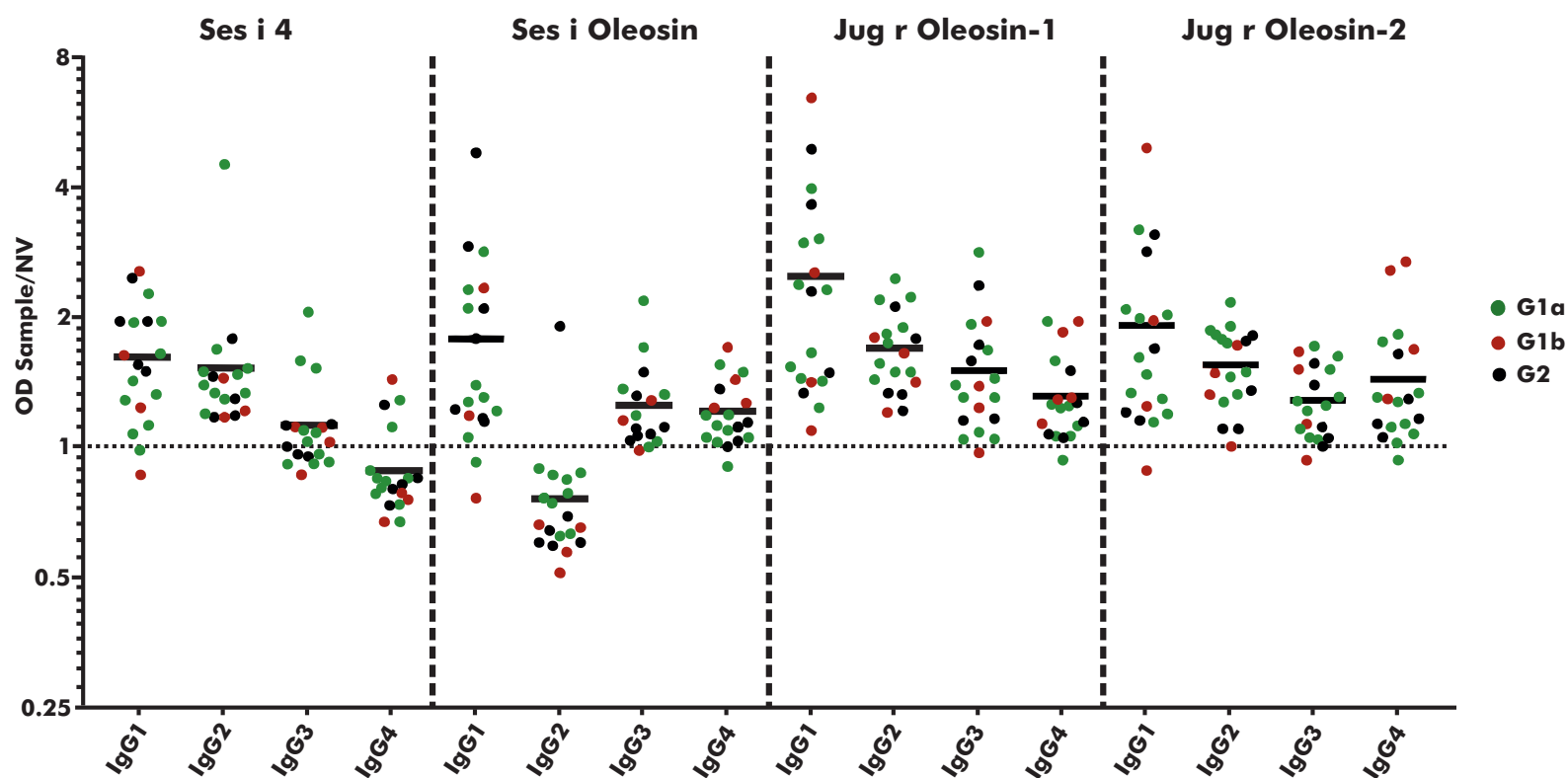

Figure S4: Subtype analysis of sera with elevated slgG levels for Ses i 4, Ses i Ol, Jug r Ol-1 and Jug $\mathrm{r} \mathrm{Ol}-2$

Detection of the IgG subtype bound to Ses i 4, Ses i Oleosin, Jug r Oleosin-1 and Jug r Oleosin-2 from serum with specific IgG to oleosins (EUROLINE-intensities $>8$ ); Scatter-blot with the measured OD value ratios (sample/negative value [NV]) divided by subtype for each oleosin. Horizontal lines mark the mean value. Green: Group $1 a$ - sesame allergic patients without detectable slgE sensitisation; Red: Group $1 b$ - sesame allergic patients with slgE sensitisation; Black: Group 2 - sesame tolerant patients with slgE sensitisation 
Ses i 4

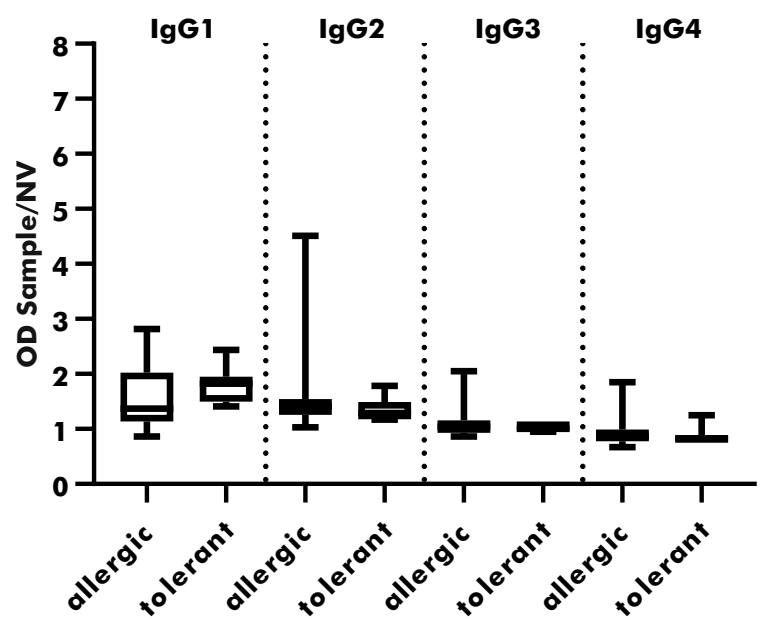

Jug r Oleosin-1

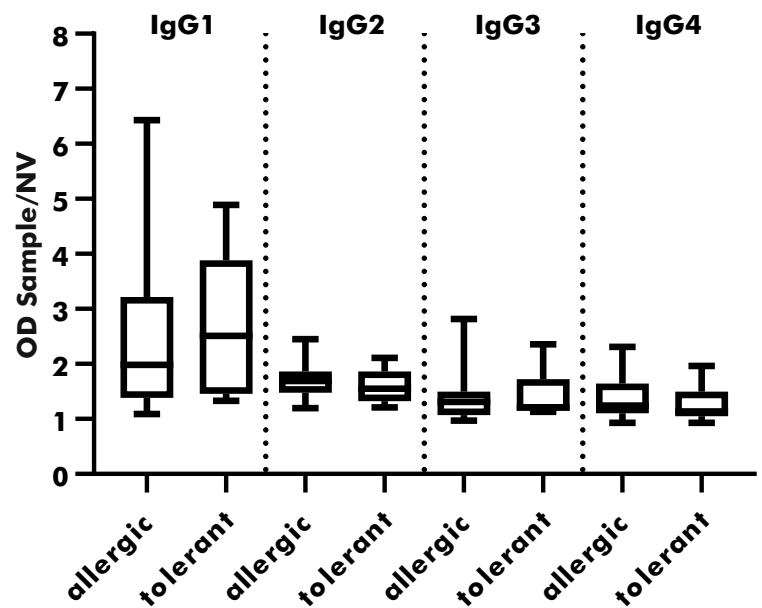

Ses i Oleosin

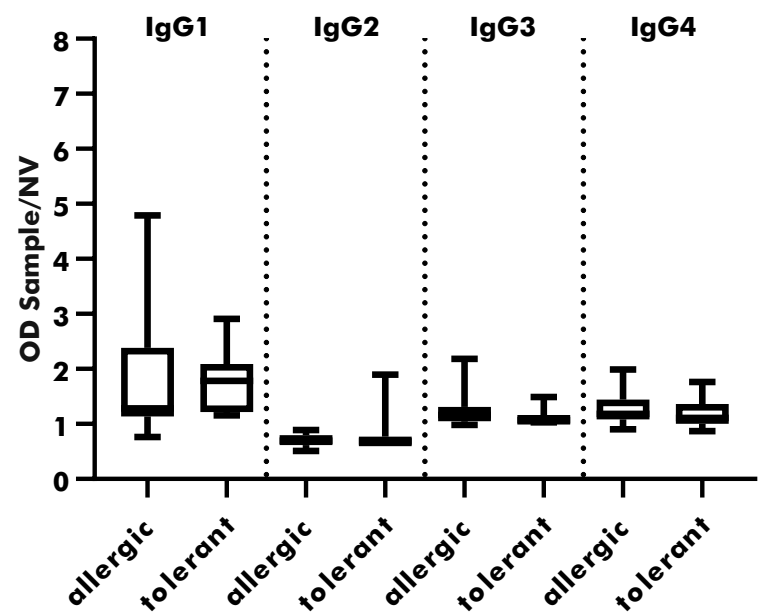

Jug r Oleosin-2

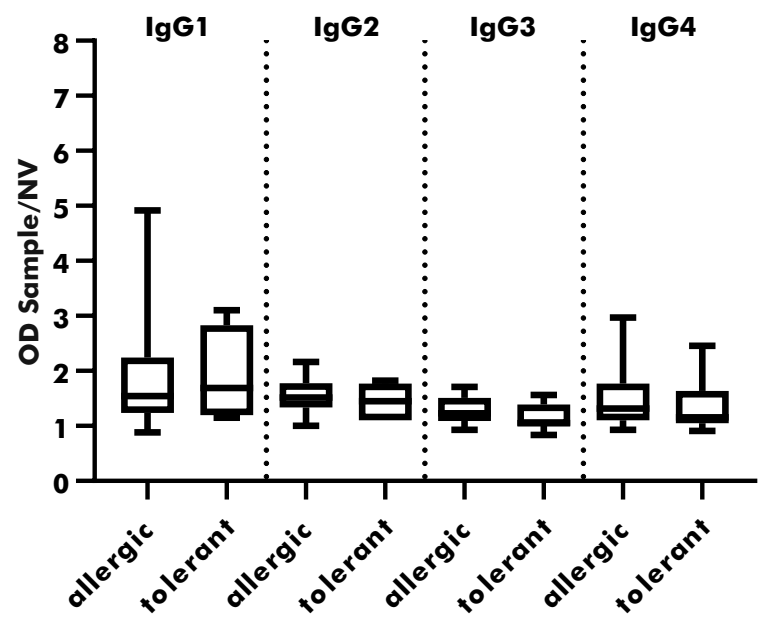

Figure S5: IgG subtype comparison between allergic and tolerant patients

Detection of the IgG subtype bound to Ses i 4, Ses i Oleosin, Jug r Oleosin-1 and Jug r Oleosin-2 from serum with specific IgG to oleosins (EUROLINE-intensities $>8$ ); the data were separated by allergic and tolerant patients, IgG subtype and protein (oleosin) 

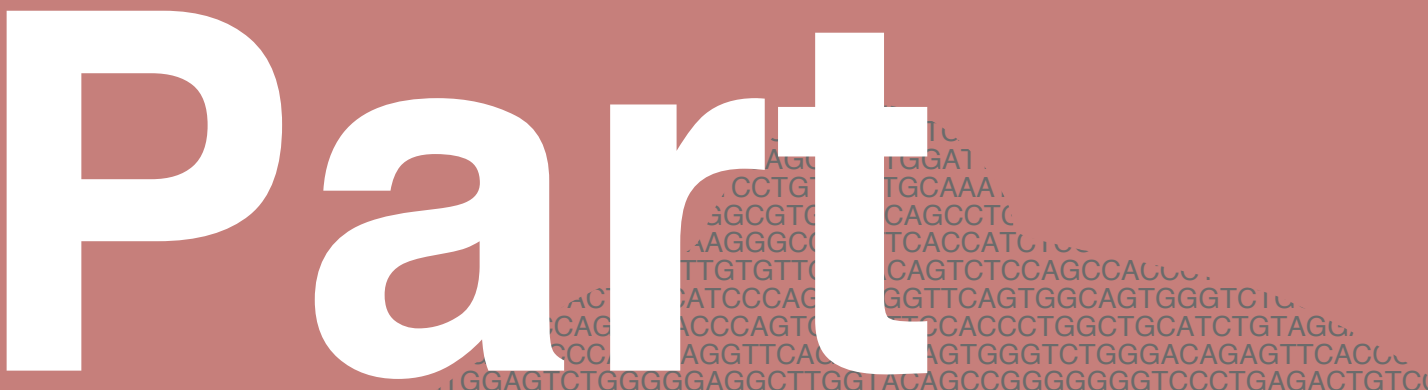

TATCGAGACTCTGTGAAGGGCCGCTTCACCATCTCCAGAGACAATGCCAAAAAC

¿AGGGGACCACGGTCATCGTCTCCTCAGCAGGTGCAGCTGGTGGAGTCTGGGGGAGG

iGAGTGGGTTTCATITATACGCAAGGATGGAGCTAGTAAATACTATGGAGACTCCGTGAAGGG

ICCCTGGGTTACTTTGACTACTGGGGCCAGGGAACCCTGGTCACCGTCTCCTCAGGAAATTGTGT

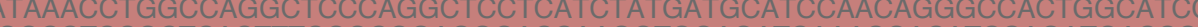

ACTGGCCTCCGCTCACTTTCGGCGGAGGGACCAAGGTGGAGATCAAACGACATCCAGATGACCCAG

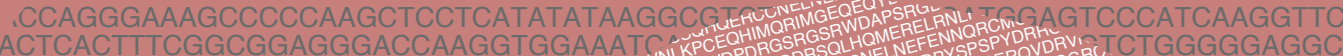

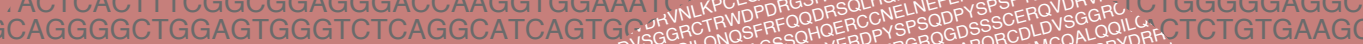

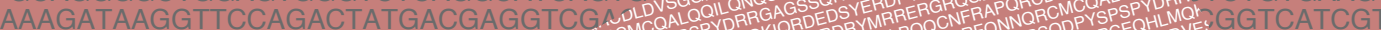

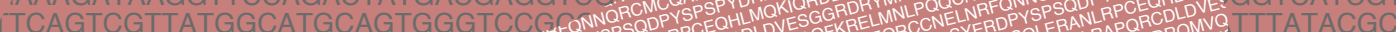

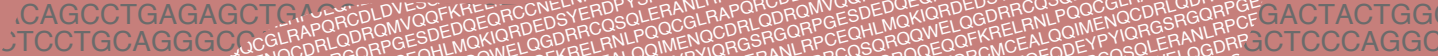

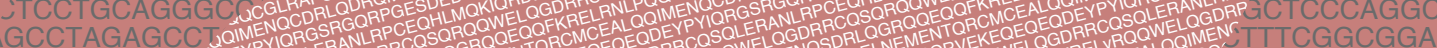

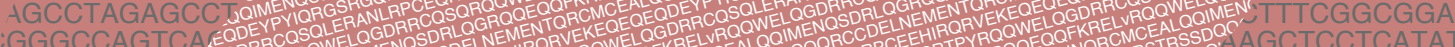

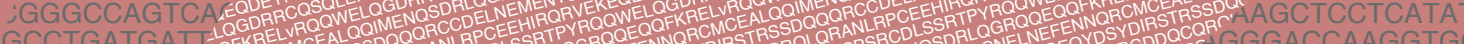

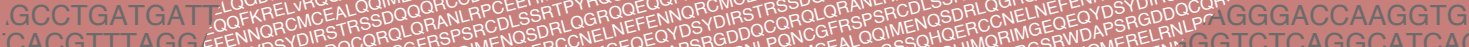

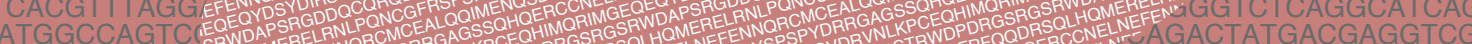

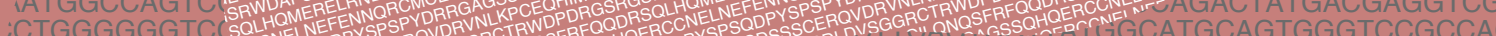

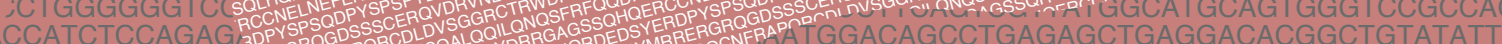

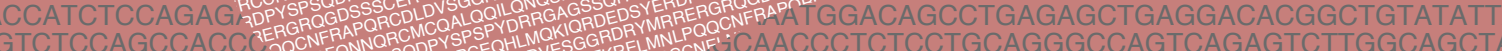

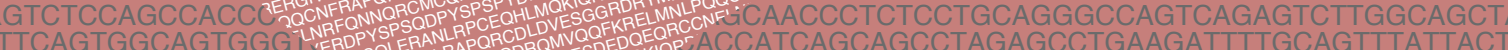

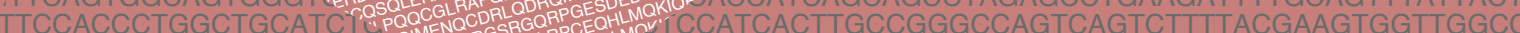

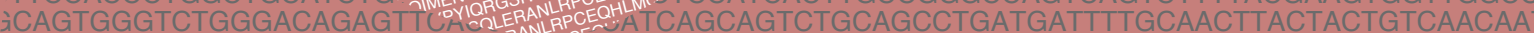
ACAGCCGGGGGGGTCCCTGAGACTGTCCTGTGCAGCCTCTGGATTCACGTTTAGGAGATATGCTTTGAGTTGGGTCCGC ACAGCCGGGGGGGTCCCTGAGACT GTCCTGTGCAGCCTCTGGATTCACGTITAGGAGATATGCTAGAGGGGCCGT

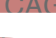

UTCCTCATC GGAGGGACCAAG . CATATATAAGGCG AAGGTGGAAATCAAAC जGCATCAGTGGCAGTGG ^ACGAGGTCGAGATTATGGA GGGTCCGCCAGGCTCCAGGC GGCTGTATATTACTGTGCGAAA TTGGCAGCTACTTAGCCTGGT

TTTATTACTGTCAGCAGCGTA

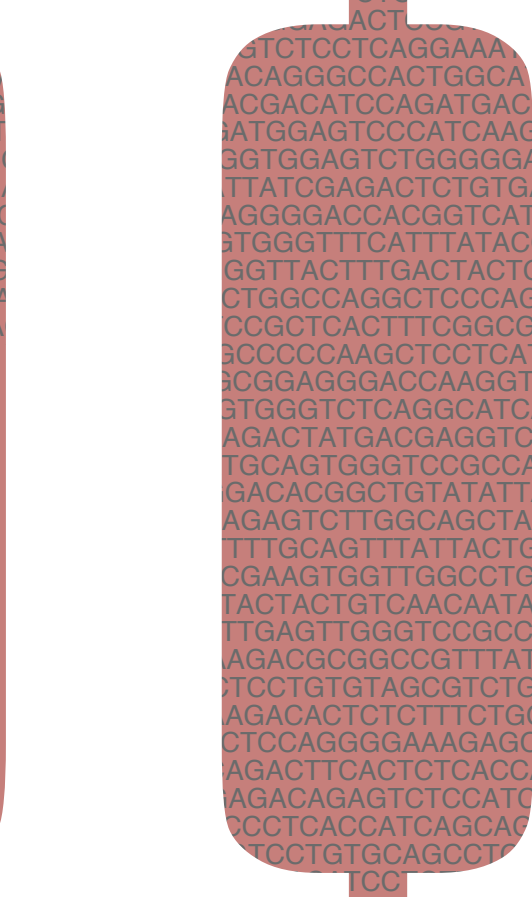
GGGTCCCTGAGACTCTCCTGTGTAGCGTC
CCAGAGACAATTCCAAAGACACTCTCTTTCT TCCAGCCACCCTGTCTTTGTCTCCAGGGGAAAG/ CAGTGGCAGTGGGTCTGGGACAGACTTCACTCTCA CACCCTGGCTGCATCTGTAGGAGACAGAGTCTCCAT GTGGGTCTGGGACAGAGTTCACCCTCACCATCAGCA GCCGGGGGGGTCCCTGAGACTGTCCTGTGCAGCCT ACCATCTCCAGAGACAATGCCAAAAACATCCTGTTTC AGGTGCAGCTGGTGGAGTCTGGGGGAGGCGTGGT CTAGTAAATACTATGGAGACTCCGTGAAGGGCCGA CCCTGGTCACCGTCTCCTCAGGAAATTGTGTTGACA TGATGCATCCAACAGGGCCACTGGCATCCCAGCCA GTGGAGATCAAACGACATCCAGATGACCCAGTCTCC CTAATTTACAAGATGGAGTCCCATCAAGGTTCAGCGG AGGTGCAGTTGGTGGAGTCTGGGGGAGGCTTGGTA AACAAAACATATTATCGAGACTCTGTGAAGGGCCGC AAACAAAACATATTATCGAGACTCTGTGAAGGGCCGC GTCTGGGGCCAGGGGACCACGGTCATCGTCTCCTC, ATAGGTCCCTGGGTTACTTTGACTACTGGGGCCAGG CCAACATAAACCTGGCCAGGCTCCCAGGCTCCTCAT GCGACTGGCCTCCGCTCACTTTCGGCGGAGGGACC GCGACT GGCCTCCGCTCACT ITCGCGGAGGGACC AACCAGGGAAAGCCCCCAAGCTCCTCATATATAAGGQ TACTCACT TCGGCGGAGGGACCAAGGTGGAAATCA CAGGGGCTGGAGTGGGTCTCAGGCATCAGT GGCAG GATAAGGTTCCAGACTATGACGAGGTCGAGATTATG GTCGTTATGGCATGCAGTGGGTCCGCCAGGCTCCAC CTGAGAGCTGAGGACACGGCTGTATATTACTGTGCG CAGGGCCAGTCAGAGTCTTGGCAGCTACTTAGCCTC GAGCCTGAAGATTTTGCAGTTTATTACTGTCAGCAGC CAGTCAGTCTTTTACGAAGTGGTTGGCCTGGTATCAC TGATTITGCAACTTACTACTGTCAACAATATGATAGT GGAGATATGCTTTGAGTTGGGTCCGCCAGGCTCCA AAGTCGAAGACGCGGCCGTITATTACTGTGO TCCGTGAAGGGCCGATTCACCATCTCCAGAGACAATTCCAAAGACACTCTCTTCTGGATTCACCT CTCCTCAGGAATTGTGTTGACACAGTCTCCAGCCACCCTGTCTITGTCTCCAGGGGAAAGAGCAACCCTCTI CAGGGCACTGGCATCCCAGCCAGGTTCAGTGGCAGTGGGTCTGGGACAGACTTCACTCTCACCATCAGCAG ACGACATCCAGATGACCCAGTCTCCTTCCACCCTGGCTGCATCTGTAGGAGACAGAGTCTCCATCACTTGCCGG GATGGAGTCCCATCAAGGTTCAGCGGCAGTGGGTCTGGGACAGAGTTCACCCTCACCATCAGCAGTCTGCAGCO TGGTGGAGTCTGGGGGAGGCTTGGTACAGCCGGGGGGGTCCCTGAGACTGTCCTGTGCAGCCTCTGGATTCAC ATATTATCGAGACTCTGTGAAGGGCCGCTTCACCATCTCCAGAGACAATGCCAAAAACATCCTGTTTCTGCAAATGC GCCAGGGGACCACGGTCATCGTCTCCTCAGCAGGTGCAGCTGGTGGAGTCTGGGGGAGGCGTGGTCCAGCCTGG GGAGTGGGTTTCATTTATACGCAAGGATGGAGCTAGTAAATACTATGGAGACTCCGTGAAGGG

ICCTGGGTTACTITGACTACTGGGGCCAGGGAACCCTGGTCACCGTCTCCTCAGGAAATTGTC

ITAAACCTGGCCAGGCTCCCAGGCTCCTCATCTATGATGCATCCAACAGGGCCACTGGCATCr

IGGCCTCCGCTCACTTTCGGCGGAGGGACCAAGGTGGAGATCAAACGACATCCAGATGACC AGGGAAAGCCCCCAAGCTCCTCATATATAAGGCGTCTAATTTACAAGATGGAGTCCCATCAAGC CACTTTCGGCGGAGGGACCAAGGTGGAAATCAAACGAGGTGCAGTTGGTGGAGTCTGGGGG GGCTGGAGTGGGTCTCAGGCATCAGTGGCAGTGGAAACAAAACATATTATCGAGACTCTGTG

\AGGTTCCAGACTATGACGAGGTCGAGATTATGGACGTCTGGGGCCAGGGGACCACGGTCATCGI

1TATGGCATGCAGTGGGTCCGCCAGGCTCCAGGCAAGGGGCTGGAGTGGGTTTCATTTATACGCAAGGATGGAGi I AG I ¿AGAGCTGAGGACACGGCTGTATATTACTGTGCGAAAGATAGGTCCCTGGGTTACTTTGACTACTGGGGCCAGGGAACCCTG ,AGGGCCAGTCAGAGTCTTGGCAGCTACTTAGCCTGGTACCAACATAAACCTGGCCAGGCTCCCAGGCTCCTCATCTATGATGC GAGCCTGAAGATTTTCAGTTTATTACTGTCAGCAGCGTAGCGACTGGCCTCCGCTCACTTTCGGCGGAGGGACCAAGGTGGA 


\section{Preventing ,false-positive“ slgE testing: clinically irrelevant sensitisation}

CAGGCTCCAGGGCAGGGGCTGGAGTGG

ГATTACTGTGCGAAAGATAAGGTTCCAGACTA

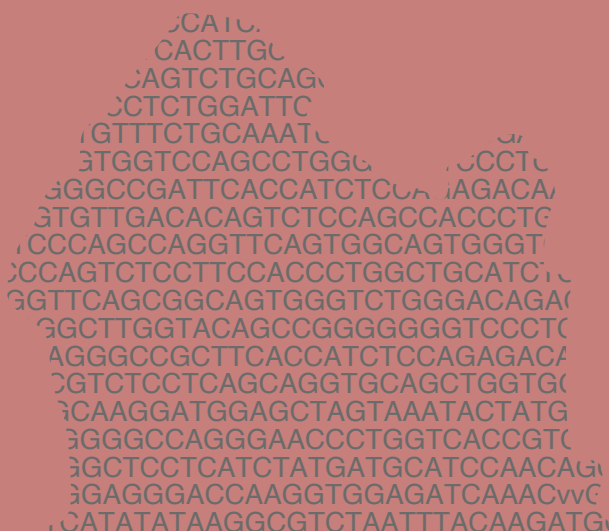


TIUTCTL

AGCCTCTGGA7

CCTGTTTCTGCAAA

¿GCGTGGTCCAGCCTC

AGGGCCGATTCACCATL ,

$\checkmark A A T T G T G T T G A C A C A G T C T C C A G C C A L L$

UTGGCATCCCAGCCAGGTTCAGTGGCAGTGGGTC I

$\checkmark C A G A T G A C C C A G T C T C C T T C C A C C C T G G C T G C A T C T G T A G L$

AGTCCCATCAAGGTTCAGCGGCAGTGGGTCTGGGACAGAGTTCACL

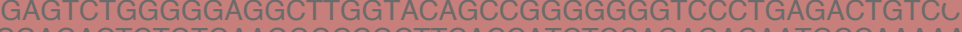

ITATCGAGACTCTGTGAAGGGCCGCTTCACCATCTCCAGAGACAATGCCAAAAACh ¿AGGGGACCACGGTCATCGTCTCCTCAGCAGGTGCAGCTGGTGGAGTCTGGGGGAGGL ¿GAGTGGGTTTCATITATACGCAAGGATGGAGCTAGTAAATACTATGGAGACTCCGTGAAGGGy

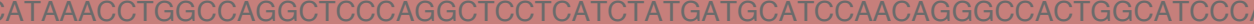
ACTGGCCTCCGCTCACTTTCGGCGGAGGGACCAAGGTGGAGATCAAACGACATCCAGATGACCCAG

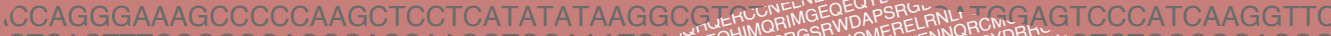
ACTCACTTTCGGCGGAGGGACCAAGGTGGAAATG iCAGGGGCTGGAGTGGGTCTCAGGCATCAGTG

AAAGATAAGGTTCCAGACTATGACGAGGTC

TCAGTCGTTATC

¿TAGCCTGAGAGCTG.NO

tGCCTAGAGCC

GCCTGATGAT

CACGTTTAGG

:ATGGCCAGTC

CCATCTCCAGAG

GTCTCCAGCCACCO

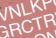

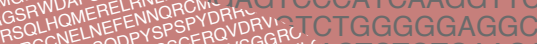

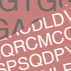

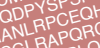

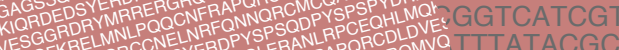

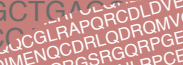

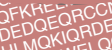
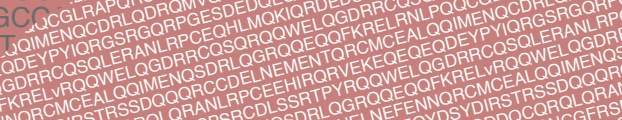

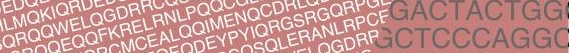
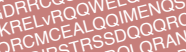

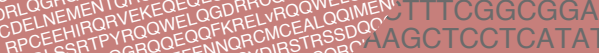
SDQQQRANLRPCEL
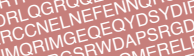

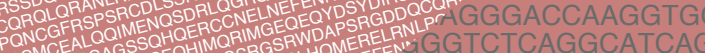

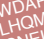

MRELRNPRCMCEALQ

SEDPYSPSPYDRRVNL KTRWDPDRGODRSRCNELNEAGACTATGACGAGGTCG

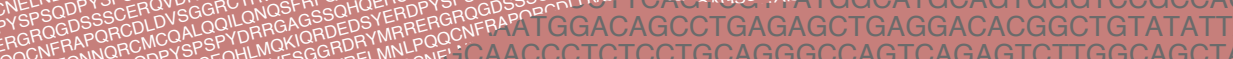
作 CAGTGGGTCTGGGACAGAGTT"DVMLLERANLPCEOHLMTCAGCAGTCTGCAGCCTGATGATIITGCAACTTACTACTGTCAACAA ACAGCCGGGGGGGTCCCTGAGACTGTCCTGTGCAGCCTCTGGATTCACGTTTAGGAGATATGCTTTGAGTTGGGTCCGC GCTTCACCATCTCCAGAGACAATGCCAAAAACATCCTGTTICTGCAAATGGCCAGTCCGAGAGTCGAAGACGCGGCCGTI TCAGCAGGTGCAGCTGGTGGAGTCTGGGGGAGGCGTGGTCCAGC AATGGAGCTAGTAAATACTATGGAGACTCCGTGAAGGGCCGATTCA UTCCTCATCTATGATGCATCCAACAGGGCCACTGGCATCCCAGCCAG aGAGGGACCAAGGTGGAGATCAAACGACATCCAGATGACCCAGTCTC . CATATATAAGGCGTCTAATTTACAAGATGGAGTCCCATCAAGGTTCAGC तAGGTGGAAATCAAACGAGGTGCAGTTGGTGGAGTCTGGGGGAGGCTTC ACGAGGTCGAGATTATGGACGTCTGGGGCCAGGGGACCACGGTCATCGTC जGGTCCGCCAGGCTCCAGGCAAGGGGCTGGAGTGGGTTTCATTTATACGC GGCTGTATATTACTGTGCGAAAGATAGGTCCCTGGGTTACTTTGACTACTGG

TTTATTACTGTCAGCAGCGTAGCGACTGGCCTCCGCTCACTTTCGGCG

-n^n.TGGTATCAfn n 'CAGGGAAAGCCCCCAAGCTCCTC

AATTTCGGCGGAGGGACCAAG

GCTGGAGTGGGTCTCAGG

GGTTCCAGACTATGACGAC TGGCATGCAGT

AGTCAGAGTCTTC

AGATTTTGCAGT

TTACGAAGTG

ACTTACTACTG

CTTTGAGTT

GAAGACGC

'TCTCCTGT

AAAGACA

TTCTCCAGC

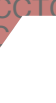

GGTCCCTGAGACTCTCCTGTGTAGCGTC CCACCCTGTCTTTGTCTCCAGGGGAAAGR CAGTGGGTCTGGGACAGACTTCACTCTCA GCTGCATCTGTAGGAGACAGAGTCTCCAT TGGGACAGAGTTCACCCTCACCATCAGCA IGGACAGAGTICACCCTCACCATCAGCA CCAGAGACAATGCCAAAAACATCCTGTTTC GCTGGTGGAGTCTGGGGGAGGCGTGGT ATACTATGGAGACTCCGTGAAGGGCCGA CACCGTCTCCTCAGGAAATTGTGTTGACA TCCAACAGGGCCACTGGCATCCCAGCCAC TAAACGACATCCAGATGACCCAGTCTCC CAAGATGGAGTCCCATCAAGGTTCAGCGG GTTGGTGGAGTCTGGGGGAGGCTTGGTA CATATTATCGAGACTCTGTGAAGGGCCGO GCCAGGGGACCACGGTCATCGTCTCCTC. GCCAGGGGACCACGGTCATCGTCTCCTC, CCTGGGTTACTTTGACTACTGGGGCCAGG AAACCTGGCCAGGCTCCCAGGCTCCTCAT ACCTCCGCTCACTTTCGGCGGAGGGACC GCCTCCGCTCACTICGGCGGAGGGACC IAAAGCCCCCAAGCTCCTCATATATAAGGC
TCGGCGGAGGGACCAAGGTGGAAATCA GGTCTCAGGCATCAGTGGCAG TATGACGAGGTCGAGATTATG AAGTGGGTCCGCCAGGCTCCAC CACGGCTGTATATTACTGTGCG

CAGACTTCACTCTCACCATCAGCAGCCTA GAGACAGAGTCTCCATCACTTGCCGGGC ACCCTCACCATCAGCAGTCTGCAGCCTGA TGTCCTGTGCAGCCTCTGGATTCACGTTT AAAAACATCCTGTTTCTGCAAATGGCCAG İGGGGAGGCGTGGTCCAGCCTGGGGGG
TCCGTGAAGGGCCGATTCACCATCTCCAG Aunum TCCGTGAAGGGCCGATTCACCATCTCCAGA TITGTCTCCAGGGGAAAGAGCAACCCTCT ACGACATCCAGATGACCCAGTCTCCTTCCACCCTGGCTGCATCTGTAGGAGACAGAGTCTCCATCACTTGCCGG GATGGAGTCCCATCAAGGTTCAGCGGCAGTGGGTCTGGGACAGAGTTCACCCTCACCATCAGCAGTCTGCAGCD TGGTGGAGTCTGGGGGAGGCTTGGTACAGCCGGGGGGGTCCCTGAGACTGTCCTGTGCAGCCTCTGGATTCAC ¿ATATTATCGAGACTCTGTGAAGGGCCGCTTCACCATCTCCAGAGACAATGCCAAAAACATCCTGTTTCTGCAAATGC GCCAGGGGACCACGGTCATCGTCTCCTCAGCAGGTGCAGCTGGTGGAGTCTGGGGGAGGCGTGGTCCAGCCTGG GGAGTGGGTTTCATITATACGCAAGGATGGAGCTAGTAAATACTATGGAGACTCCGTGAAGGG

¿CCTGGGTTACTTTGACTACTGGGGCCAGGGAACCCTGGTCACCGTCTCCTCAGGAAATTGTC

ITAAACCTGGCCAGGCTCCCAGGCTCCTCATCTATGATGCATCCAACAGGGCCACTGGCATCr

IGGCCTCCGCTCACTTTCGGCGGAGGGACCAAGGTGGAGATCAAACGACATCCAGATGACCC AGGGAAAGCCCCCAAGCTCCTCATATATAAGGCGTCTAATTTACAAGATGGAGTCCCATCAAGC CACTTTCGGCGGAGGGACCAAGGTGGAAATCAAACGAGGTGCAGTTGGTGGAGTCTGGGGG^

GGCTGGAGTGGGTCTCAGGCATCAGTGGCAGTGGAAACAAAACATATTATCGAGACTCTGTG

AAGGTTCCAGACTATGACGAGGTCGAGATTATGGACGTCTGGGGCCAGGGGACCACGGTCATCG I

1TATGGCATGCAGTGGGTCCGCCAGGCTCCAGGCAAGGGGCTGGAGTGGGTTTCATTTATACGCAAGGATGGAGi I AG I

¿AGAGCTGAGGACACGGCTGTATATTACTGTGCGAAAGATAGGTCCCTGGGTTACTTTGACTACTGGGGCCAGGGAACCCTG AGGGCCAGTCAGAGTCTTGGCAGCTACTTAGCCTGGTACCAACATAAACCTGGCCAGGCTCCCAGGCTCCTCATCTATGATG GAGCCTGAAGATTTTGCAGTTTATTACTGTCAGCAGCGTAGCGACTGGCCTCCGCTCACTTTCGGCGGAGGGACCAAGGTGGA 


\section{Detection of specific lgE against linear epitopes from Gal d 1 has additional value in diagnosing hen's egg allergy in adults}

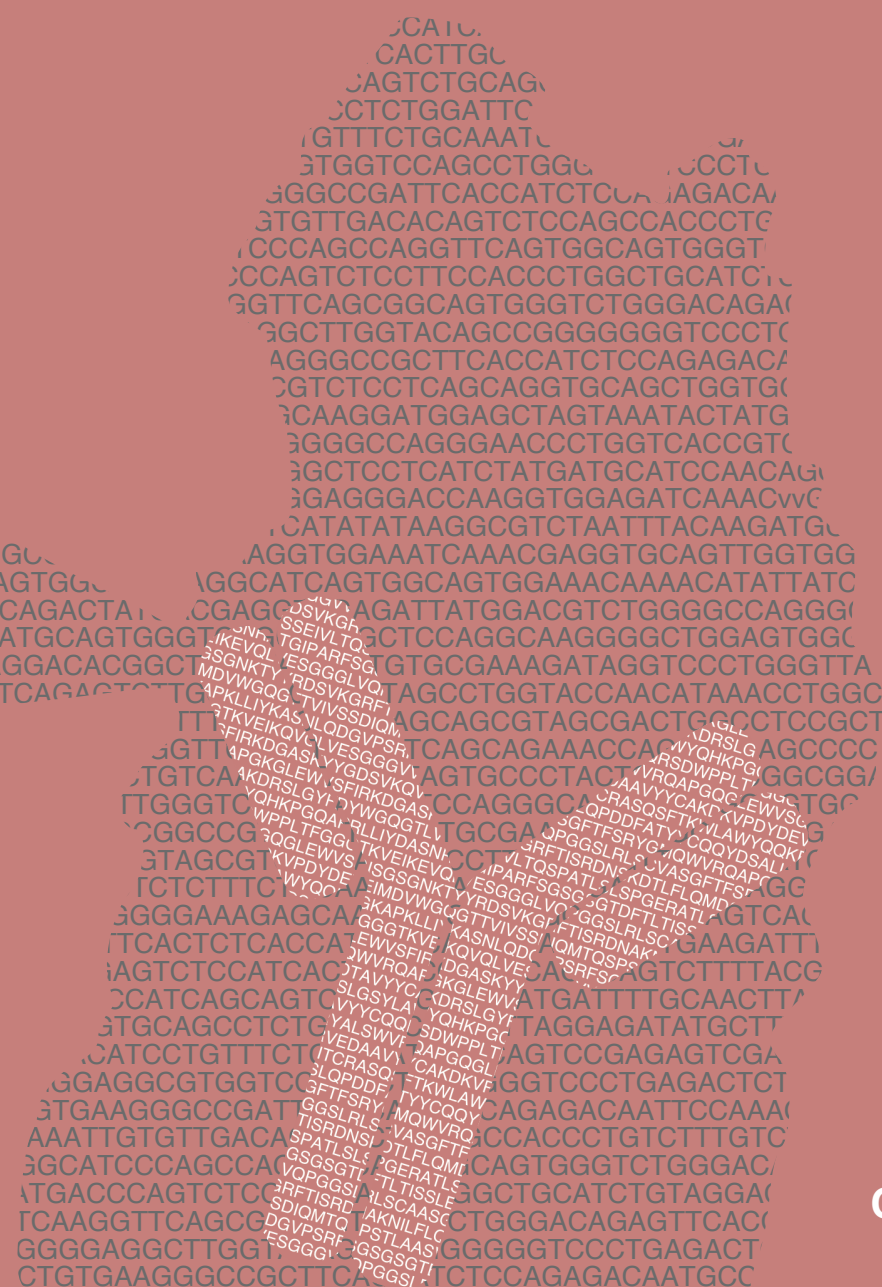

Clin Exp Allergy 2020;00:1-9

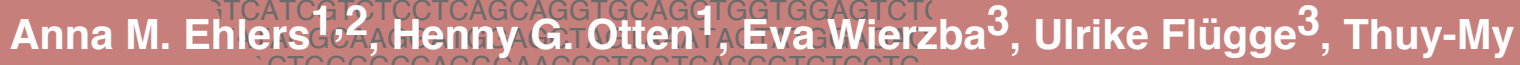

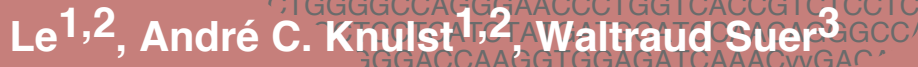

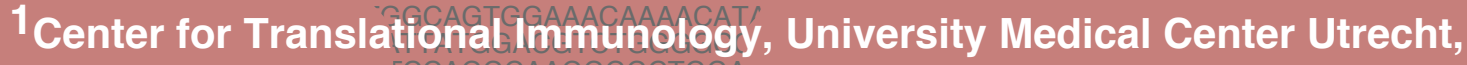
Utrecht University, Netherlands

A 2 Department of Dermatology/Ailergology, University Medical Center Utrecht, ICUtrecht Universityia Netherlands

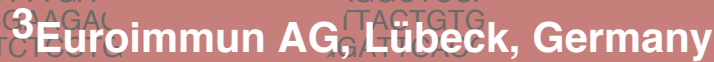

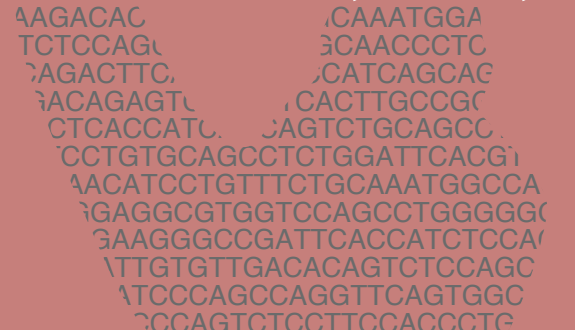




\section{Abstract}

\section{Background}

Although hen's egg allergy is more prevalent in children, up to $0.6 \%$ of adults from different European countries suffers from a persistent or newly-onset hen's egg allergy, making accurate diagnosis in adults necessary. However, sensitisation to hen's egg extracts, components and linear epitopes are solely studied in children.

\section{Methods}

Hen's egg allergic $(n=16)$ and tolerant $(n=20)$ adults were selected by sensitisation towards recombinant components $\mathrm{rGal} d \mathrm{~d}$ and/or 3 . Sensitisation profiles towards egg white and yolk extract and the native components Gal d 1, 2, 3 and 4 were respectively evaluated with the ImmunoCAP or the EUROLINE system. Characterisation of linear epitopes was performed with a peptide microarray containing $15 \mathrm{mer}$ peptides representing the entire sequence of mature Gal $d 1$ and 3.

\section{Results}

Overall, slgE titres against hen's egg extracts and single components overlapped largely between allergic and tolerant adults. Although the median slgE/slgG4 ratio to Gal d 1 was increased in allergic adults, the range was comparable between both groups. Clinically relevant sensitisation to Gal $d 1$ was confirmed by slgE binding to the linear epitopes aa 30-41, aa 39-50 or aa 84-95 in 6/13 allergic adults, mainly suffering from objective symptoms. In comparison, these epitopes were recognized by $1 / 15$ tolerant patient. Only a few linear epitopes were detected for Gal d 3, suggesting a greater importance of conformational epitopes for the recognition of Gal d 3.

\section{Conclusion and Clinical Relevance}

Specific IgE binding to linear epitopes of Gal d 1 is highly specific in identifying hen's egg allergic adults with objective symptoms. 


\section{Introduction}

Hen's egg is known as a major cause of food allergic reactions in children and this allergy is often outgrown by the age of five. Nevertheless, the prevalence of hen's egg allergy in adults average 0.02 to $0.6 \%$ across European countries ${ }^{1,2}$. Although hen's egg allergy in adulthood is predominantly a persistent allergy developed in childhood, it can also be newly developed later in life ${ }^{3}$. In a study conducted in the US, $29 \%$ of all hen's egg allergic adults suffered from an adult-onset allergy ${ }^{4}$.

Diagnosis of hen's egg allergy is comprised of anamnesis, skin prick test, measuring slgE and food challenges. Food challenges are the gold standard, but they are burdensome, expensive and require dedicated hospital facilities and personnel. To avoid or replace food challenges, intensive research has been performed to improve the diagnostic value of slgE measurements in hen's egg allergic children. In a systematic review, an evaluation of slgE measurements towards egg white extract in children ranging from infants to adolescents showed an overall sensitivity of 0.93 , but only a specificity of $0.49^{5}$.

Component-resolved diagnostics improved the accuracy of slgE measurement in several food allergies ${ }^{6}$. Major allergenic components of hen's egg white, which are responsible for most of its allergenicity, are ovomucoid (Gal d 1), a thermo-stable allergen, ovalbumin (Gal d 2), ovotransferrin (Gal d 3) and lysozyme (Gal d 4). Ovomucoid, the major allergen of egg white, is by far the most studied component in relation to hen's egg allergy in children and although ovomucoid is classified as a prognostic marker for persistent hen's egg allergy, its superior role compared to egg white extract has been debated $^{7}$.

Patients' sera contain polyclonal IgE antibodies recognising a broad range of epitopes comprised of either sequential residues of the amino acid sequence (linear) or amino acids closely located upon folding (conformational). Epitope mapping approaches aim to identify clinically relevant epitopes which are undetectable by measuring slgE against extracts or full-length single components. In hen's egg allergy, linear epitope mapping of Gal d 1 identified epitopes (aa 1-10, aa 11-20, aa 47-56 and aa 113-122) exclusively recognised by children with persistent hen's egg allergy ${ }^{8}$. Comparable allergenic parts (aa 1-10, aa 11-20 and aa 47-56) were described as immunodominant linear epitopes by several other studies ${ }^{9-11}$.

So far, the impact of slgE titres to hen's egg components and slgE binding to their linear epitopes on discriminating between clinically relevant and irrelevant sensitisation is poorly studied in hen's egg allergic and tolerant adults. To this end, we evaluated sensitisation patterns and slgE titres to hen's egg components (Gal d 1, 2, 3 and 4) in allergic and tolerant, but sensitised adults. Since Gal d 1 is known as the most important single component for diagnosing hen's egg allergy in children, recognition of linear 
epitopes derived from Gal d 1 was evaluated by peptide chip analysis. Since the role of Gal $\mathrm{d} 2$ is controversially discussed ${ }^{7}$, we decided to additionally map the linear epitopes of Gal d 3, another major egg white allergen of which little information is known so far.

\section{Methods}

\section{Patient selection}

Patients $(n=35)$ sensitised to at least one of the recombinant hen's egg components rGal d 1 and rGal d 3, examined in 121 patients with hen's egg related sensitisation (SPT, ImmunoCAP, ISAC) by Western Blot, were retrospectively selected from patients who visited the Dermatology/Allergology outpatient clinic of the University Medical Center (UMC) Utrecht between 2008 and 2018. These patients were divided into (a) hen's egg allergic $(n=16)$ and (b) hen's egg tolerant $(n=20)$ patients based on either doubleblind placebo-controlled food challenge (DBPCFC) with heated egg or convincing history confirmed by a trained physician (challenged: 44\% allergic group, $29 \%$ tolerant group). Convincing history was defined as immediate symptoms including oral allergy syndrome, skin reactions, gastrointestinal, respiratory, or cardiovascular symptoms and an onset within 2 hours after ingestion. Gastrointestinal symptoms had to be combined with at least one additional immediate type symptom.

For the epitope discovery, sera from 13 allergic (8 suffering from objective symptoms) and 15 tolerant patients were applied on the peptide chip. Ethical approval (number 18-428) was acquired from the biobank committee of the UMC Utrecht.

\section{Heterologous expression of hen's egg components}

The mature hen's egg components Gal d 1 (accession number: P01005) and Gal d 3 (accession number: P02789) were heterologously expressed as fusion proteins with $\mathrm{N}$-terminal-His (6x)-tag in E. coli and purified as previously described ${ }^{12,13}$. All heterologously expressed proteins were purified by immobilised metal ion chromatography under denaturing conditions. Purified $\mathrm{rGal} \mathrm{d} 1$ and 3 were separated by gel electrophoresis and blotted onto a nitrocellulose membrane.

\section{Determination of slgE and slgG4 sensitisation}

Sensitisation to egg white and yolk extract was determined using the commercially available ImmunoCAP system and slgE and slgG4 sensitisation to the native components Gal d 1, 2, 3 and 4 was measured using the EUROLINE immunoblot strip 
"Paediatrics' 1" (DP 3812-1601-1 E, EUROIMMUN AG, Germany) according to manufacturer's instructions. Briefly, the immunoblots were manually incubated overnight at room temperature with serum diluted 1:11 (IgE) or 1:51 (IgG4) in working strength universal buffer (WSUB). After extensive washing with WSUB, bound IgE and IgG4 antibodies were detected with a-human IgE or IgG4-conjugate coupled with alkaline phosphatase. Upon another extensive washing step, visualisation was provided by applying nitro-blue tetrazolium/5-bromo-4-chloro-3'-indolyphosphate substrate for ten minutes and specific IgE levels were evaluated as EUROLINE (EL)-intensities and expressed as response units. Specific IgE binding to heterologously expressed hen's egg components were determined under the same conditions.

\section{Microarray design}

A microarray with synthetic 15 mer peptides, comprising the sequence of the mature Gal d 1 (accession number: P01005) and Gal d 3 (accession number: P02789) (offset=3 due to limited space), was commercially obtained (PEPperPRINT). The peptide length of 15 amino acids was in accordance with the experience of PEPperPRINT to provide sufficient sensitivity without significant formation of secondary structures. All peptides were printed in triplicates with a linker consisting of $2 \mathrm{~B}$-alanine and one aspartic acid. This linker was chosen to circumvent the binding of negatively charged fluorescent dyes to positively charged amino acids which are close to the array surface.

\section{Microarray design}

The microarray incubation was performed as previously described ${ }^{14}$. Briefly, patient sera were diluted 1:4 in WSUB and incubated overnight. For detecting bound specific $\lg$ E and IgG4, a biotinylated a-lgE antibody (clone MHE-18 1:5000, BioLegend) and simultaneously a biotinylated a-human IgG4 coupled with Neutravidin DyLight 680 (clone HP6025, 1:5000, Southern Biotech) were applied on the microarray and incubated for $60 \mathrm{~min}$ at room temperature. Bound biotinylated human $\mathrm{a}-\lg \mathrm{E}$ antibodies were visualised by adding Neutravidin DyLight 800 (1:5000, Thermo Fisher) for 60 min at room temperature. After extensive washing and drying, the microarray slides were scanned at a wavelength of $700 \mathrm{~nm}$ for IgG4 and $800 \mathrm{~nm}$ for IgE (intensity: 8.5) and the focus was set to $0.8 \mathrm{~mm}$ and the resolution to $21 \mu \mathrm{m}$. 


\section{Microarray evaluation}

For data evaluation, the fluorescent signals for each peptide were obtained using the Pepslide Analyzer Software (SICASYS) with the fixed-spot adjustment and the logarithmic signal-to-noise ratios $(S)$ were computed according to the following quotation:

$$
\mathrm{S}_{i}=\log _{2} \cdot \frac{\text { Total fluorescene (Peptide) }}{\text { Background Flourescence (Peptide) }}
$$

For normalisation, the S-values were compared to the S-values of blank spots, resulting in z-scores defined as:

$$
\mathbf{Z}_{i}=\frac{S_{i}-\text { Median }(\text { SBlank })}{\text { Median Deviation (SBlank) }}
$$

Epitopes were defined as recognition of 2-4 contiguous peptides with a median z-score $\geq 3.0$ and the amino acid residues were counted based on the amino acid sequence without signal peptide.

\section{Determination of surfaced exposed epitopes}

Surface exposed residues of an epitope were determined by submitting the 3D structure (Gal d 3, PDB ID: 1OVT) to the http://curie.utmb.edu/getarea.html interface ${ }^{15}$. Under the conditions (default settings) as radius of the water probe set to 1.4 and no gradient in calculations, the algorithm calculates the probability of each residue to be solvent accessible. For an epitope, at least $25 \%$ of its residues must have a greater probability than $50 \%$ to be solvent accessible for calling this epitope „surface-exposed“. The definition was confirmed by mapping the linear epitopes onto the 3D structure of Gal d 3 (pdb: 1OVT) using PyMol 1.3 (Schrödinger, Inc, US). The corresponding images are shown in Suppl. Figure $\mathrm{S} 1$.

\section{Statistical analyses}

Statistical differences between hen's egg allergic and tolerant adults regarding their sensitisation profiles were evaluated with the non-parametric Mann-Whitney-U-test and visualised by GraphPad Prism 8.3. For peptides and epitopes derived from Gal d 1, their recognition by $\operatorname{lgE}$ was evaluated by principle component analyses in R. Heat maps were generated in $\mathrm{R}$ using the 'ComplexHeatmap' package ${ }^{16}$. 


\section{Results}

\section{Patient characteristics}

Patients sensitised to the recombinant components rGal d 1 and/ or rGal d 3 were divided into (a) allergic (75\% female) and (b) tolerant patients (52\% female) based on food challenge outcome or convincing history. Patients with subjective symptoms were more often diagnosed by a food challenge (4/6: 67\%) compared with patients with objective symptoms (3/7: $43 \%$ ), reducing at least the risk of misclassification. Allergic patients showed a median age of 25 and were overall younger than the tolerant patients with a median age of 28 , although the age range was comparable $(p=0.63)$. Even though the majority of patients were co-sensitised to nGal d 1 and nGal d 3, one allergic and one tolerant patient were mono-sensitised to nGal $\mathrm{d} 1$ and one tolerant patient was mono-sensitised to nGal d 3. Interestingly, up to $94 \%$ of all included patients, irrespective of allergy or tolerance, suffered from atopic dermatitis. Besides, more than $60 \%$ of all included patients experienced symptoms related to allergic asthma (allergic $63 \%$, tolerant $75 \%$ ) and allergic rhinitis (allergic $81 \%$, tolerant $60 \%$ ). All characteristics are shown in Table 1. 
Table 1: Patient characteristics and sensitisation data

\begin{tabular}{lcc}
\hline & Allergic & Tolerant \\
\hline Number [n] & 16 & 19 \\
Age (median [IQR]) & $25[19-65]$ & $28[19-70]$ \\
Sex female [n, \%] & $12[75 \%]$ & $11[58 \%]$ \\
Food challenge $^{\mathrm{A}}[\mathrm{n}, \%]$ & $7[44 \%]$ & $5[26 \%\}$ \\
& & \\
\hline Symptoms [n, \%] & & $\mathrm{N} / \mathrm{A}$ \\
\hline Subjective & $6[37 \%]$ & $\mathrm{N} / \mathrm{A}$ \\
Objective & $10[63 \%]$ & $19[100 \%]$ \\
No symptoms & $\mathrm{N} / \mathrm{A}$ &
\end{tabular}

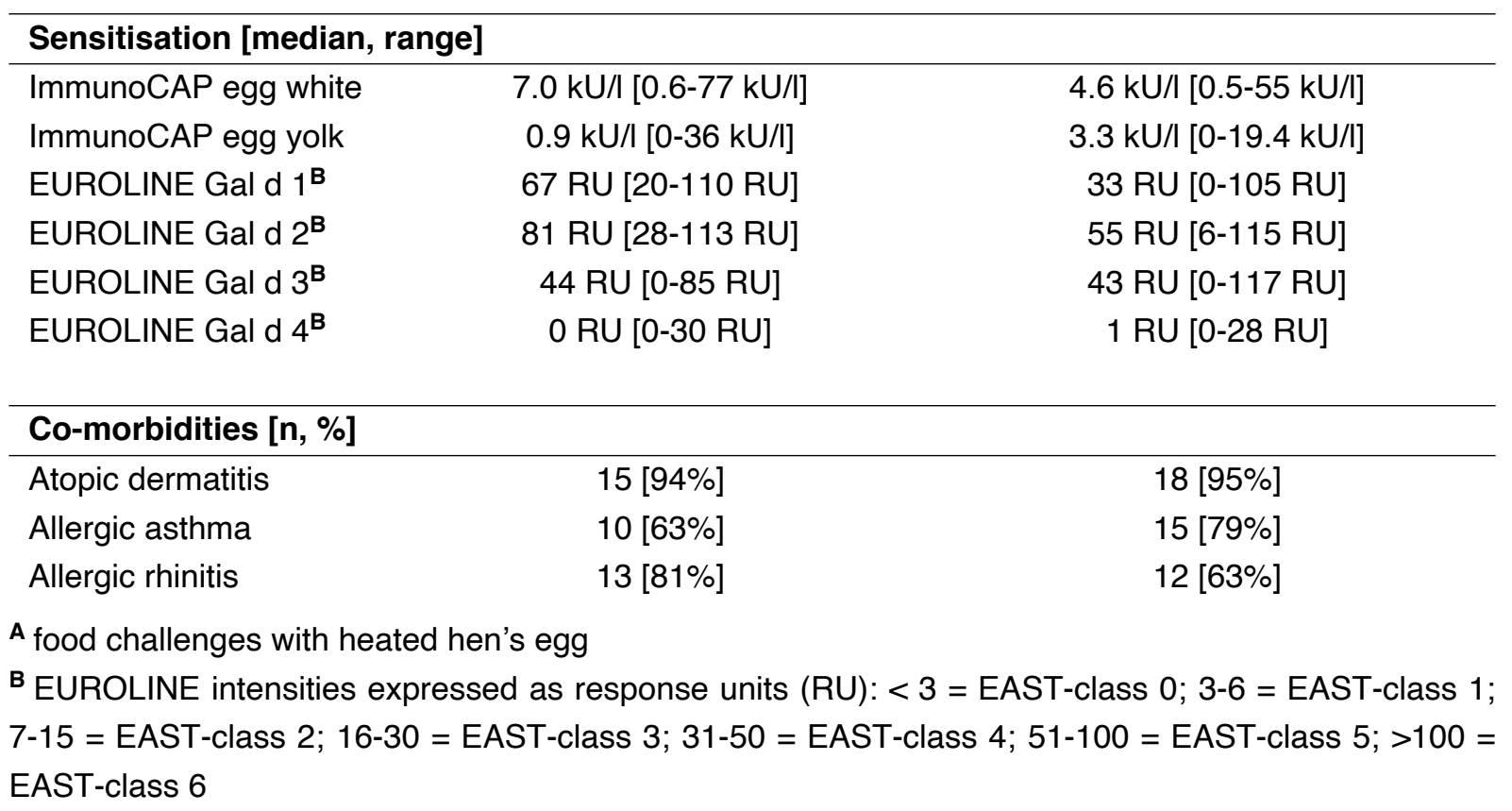

\section{slgE levels towards hen's egg extracts overlapped largely between allergic and tolerant but sensitised patients}

All patients of this study were included based on their sensitisation to at least one heterologously expressed hen's egg component ( $\mathrm{Gal}$ d 1 and/or 3) and hence, detectable sensitisation to hen's egg extracts was detected in all tested patients. Specific lgE titres towards egg white and yolk extract overlapped greatly between allergic and tolerant adults, resulting in low specificity even at increased cut-off levels ( 0.53 at $5 \mathrm{kU} / \mathrm{l})$. Overall, tolerant patients tend to have lower slgE titres to egg white extract than allergic patients (median $4.6 \mathrm{kU} / \mathrm{l}$ vs $7.0 \mathrm{kU} / \mathrm{l}$ ). On the other hand, slgE levels to yolk extract were even higher in tolerant patients (median $3.3 \mathrm{kU} / \mathrm{l}$ ) compared with allergic patients (median $0.9 \mathrm{kU} / \mathrm{l}$ ), suggesting greater relevance of hen's egg white proteins compared to yolk-derived ones (Figure 1a). No statistically significant differences were observed for egg white and egg yolk extract. 
A
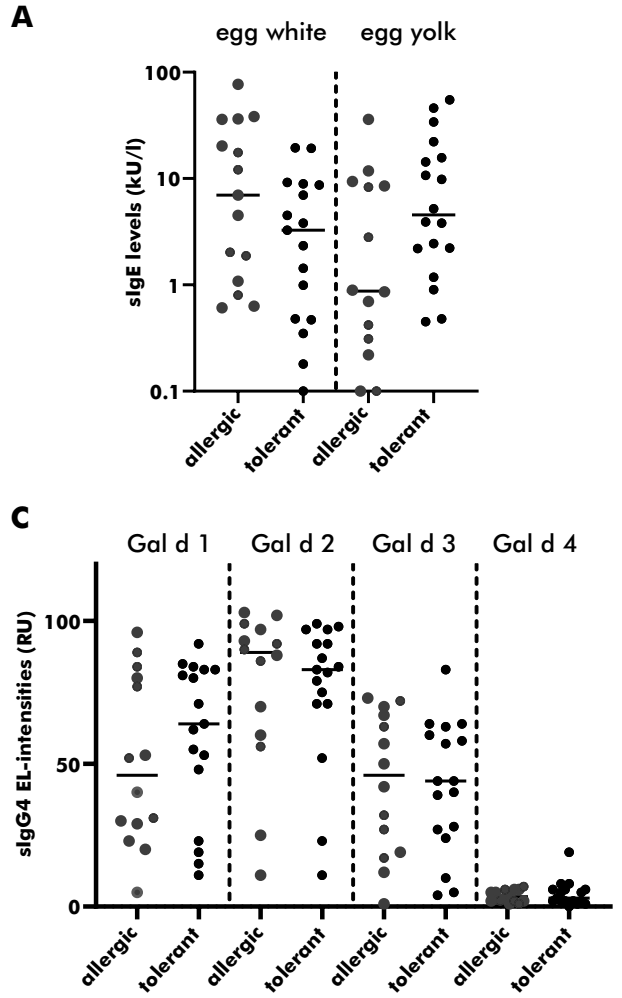

B

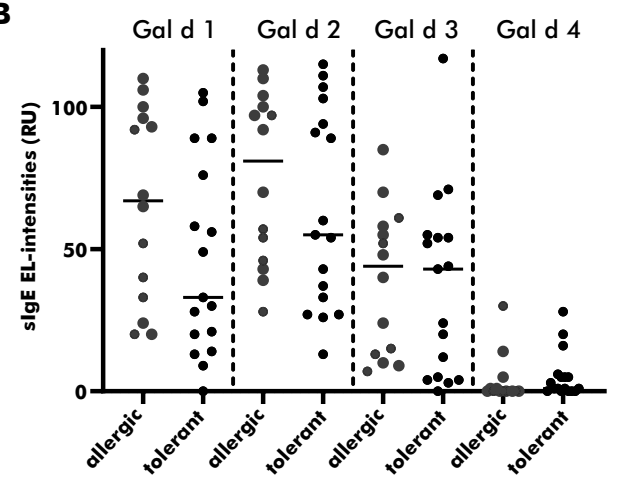

D

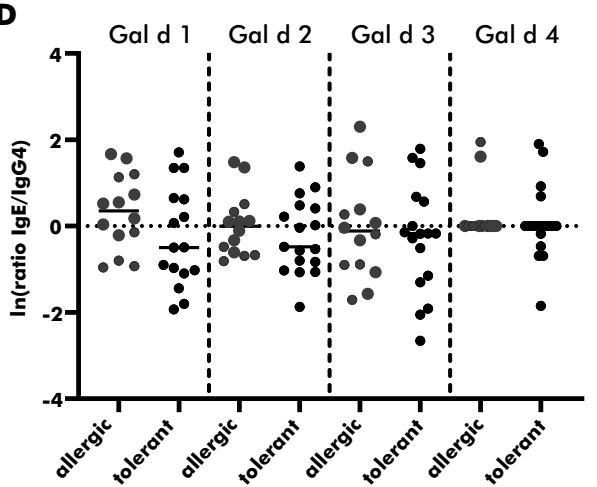

Figure 1: Sensitisation profiles of tolerant and allergic patients to hen's egg extracts and components $\boldsymbol{A}$ slgE levels to egg white and egg yolk extract measured with the ImmunoCAP system (kU/l) split by allergic and tolerant patients; represented with the median; $\boldsymbol{B}$ and $\boldsymbol{C}$ slgE and slgG4 levels to native hen's egg components Gal d 1, 2, 3 and 4 measured with the EUROLINE-immunoblot (EL-intensities, $R U)$ split by allergic and tolerant patients; represented with the median; $\boldsymbol{D}$ Log-transformed slgE/IgG4 ratios to native hen's egg components Gal d 1,2, 3 and 4 resulting from the measures shown in $B$ and $\mathrm{C}$; represented with the median

\section{Gal d 1 slgE/sigG4 ratios were higher in allergic patients}

As slgE levels towards hen's egg white extract were on average higher in allergic than in tolerant patients, we next analysed the relevance of antibodies against native components present in egg white. Although allergic and tolerant patients showed similar sensitisation patterns towards nGal d 1, 2, 3 and 4 (Suppl. Figure S2), the tolerant group showed a decreased median of 33 EL-intensities towards nGal $d 1$ whilst allergic patients showed a median of 67 EL-intensities (Figure 1b). On the other hand, the tolerant group showed an increased median of slgG4 levels against nGal d 1 (tolerant: 71 EL-intensities, allergic: 46 EL-intensities), resulting in a decreased median of slgE/slgG4 ratios for nGal d 1 (median:0.5 vs 0.35 , Figure $1 \mathrm{~b}$ to $\mathrm{d}$ ) in tolerant compared to allergic adults. The range, however, was comparable between both groups. Regarding nGal d 2, 3 and 4, also no statistically significant differences were observed. 
A
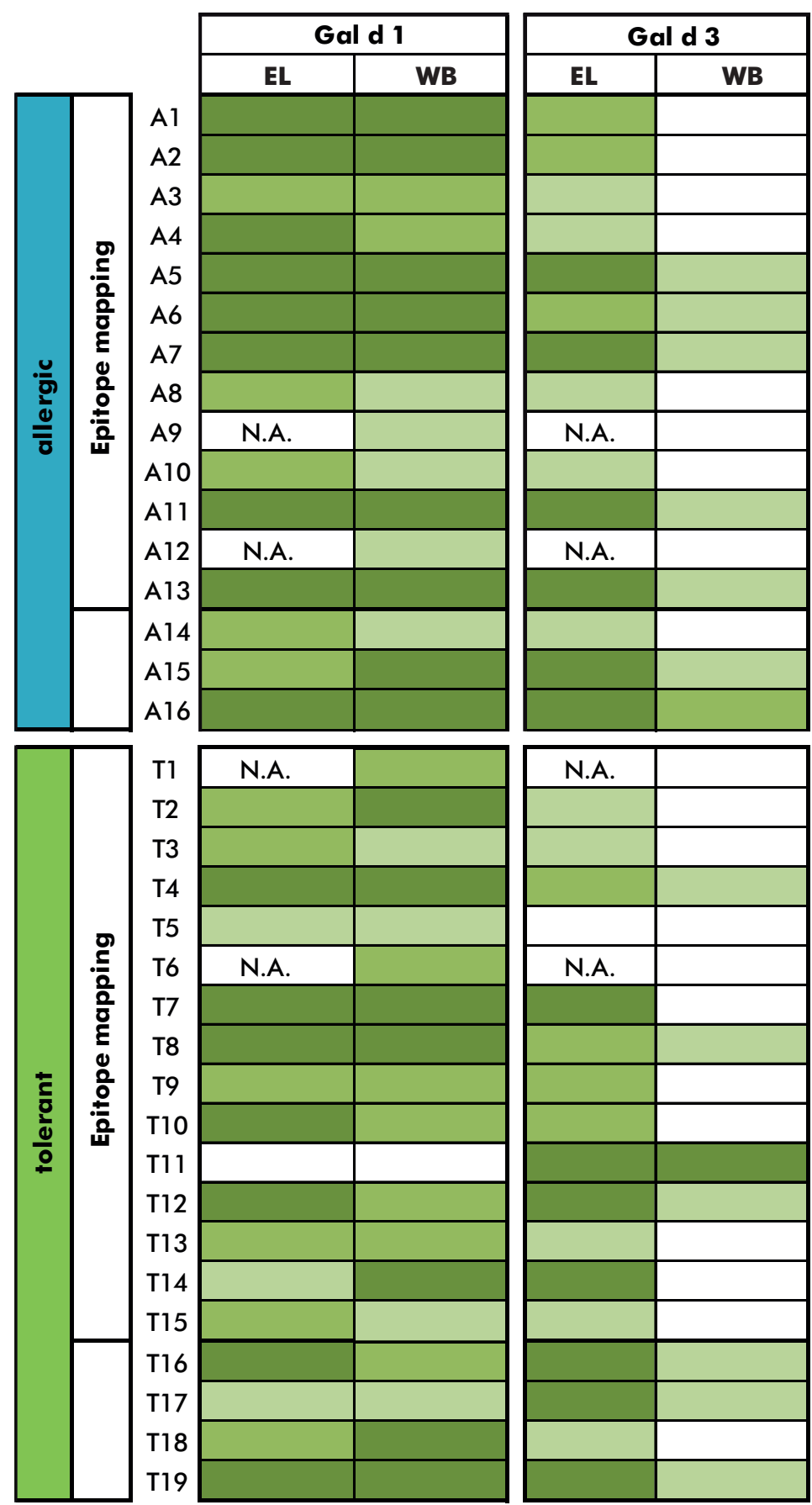

$\begin{array}{ccc}\text { category } & \text { RU (EL) } & \text { WB } \\ 0 & 0 \text { to } 2 & - \\ 1 & 3 \text { to } 15 & + \\ 2 & 16 \text { to } 50 & ++ \\ 3 & 51 \text { to } 120 & +++\end{array}$

B

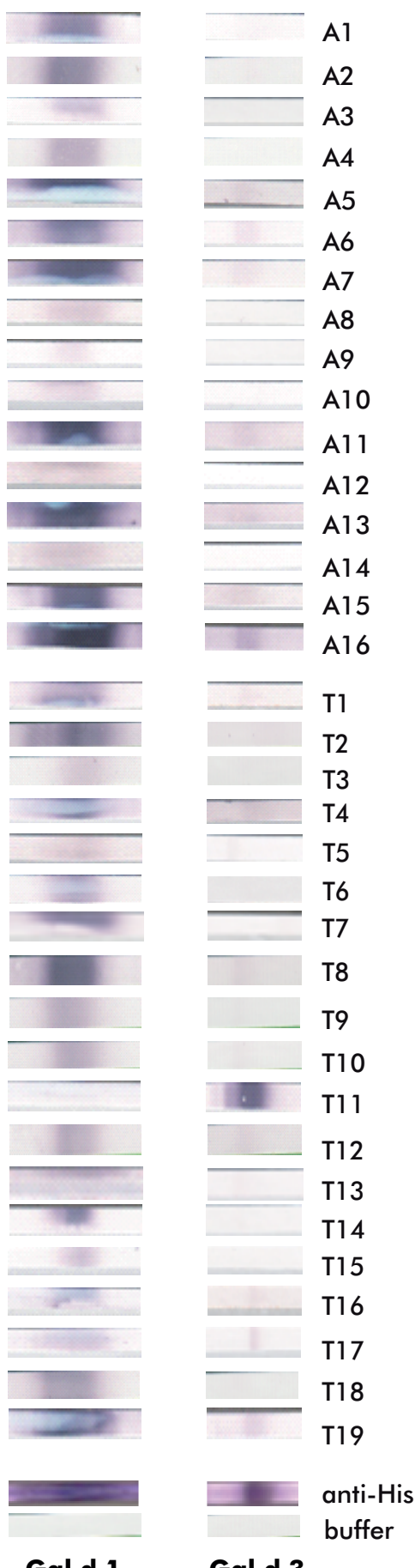

Gal d $1 \quad$ Gal d 3

Figure 2: Linearization effects slgE binding to Gal d 3

A Comparison of IgE-binding capacity between native (EL) and linearised Gal $d 1$ and Gal $d 3$ (WB); the number of bound IgE is divided into categories defined as low (light green), moderate (green) and high (dark green); $\boldsymbol{B}$ original WB showing slgE-binding to the recombinant components rGal $d 1$ and $r G a l d 3$ 


\section{Binding of slgE to Gal d 3 was greatly reduced upon linearisation}

Although nGal d 3 was recognised by lower slgE titres compared with nGal $d 1$ and nGal d 2, nGal d 3 was recognised by all allergic patients and $93 \%$ of tolerant patients. As shown in Figure 2, the binding capacity of nGal d 3 was greatly reduced upon linearisation (western blot (WB)) of the heterologously expressed rGal d 3 compared to the native component. For instance, patient $A 6$ reacted strongly to the native form of nGal $\mathrm{d} 3$ while no binding to the linearized form was observed, indicating the importance of conformational epitopes for the recognition of Gal d 3 by IgE. In contrast, the binding capacity of rGal d 1 was hardly affected by linearisation, pointing to the relevance of linear epitopes in recognising Gal d 1.

\section{Linear epitope recognition of Gal d 1 confirms clinically relevant sensitisation in allergic patients with objective symptoms}

As linearisation of rGal d 1 only marginally affected IgE binding, we next analysed linear epitope recognition with a peptide microarray. By means of principle component analysis with normalized values (z-scores) for each peptide, one allergic patient was separated from the remaining patients within the first dimension and four allergic patients were separated within the second dimension. This effect was even more dominant by repeating the principle component analysis with the mean z-scores of consensus sequences (epitopes) (Suppl. Figure S3). Principle component 1 (dimension 1) was dominantly driven by epitope aa 45-56 (97\%) while principle component 2 was mainly driven by aa 30-41 (49\%) and aa 84-95 (47\%), having the greatest impact on discrimination. Accordingly to the principle component analysis, 6/13 allergic (46\%) - 4 suffered from objective symptoms - and $1 / 15(7 \%)$ tolerant patient recognised at least one of the epitopes aa 30-41, aa 39-50 and aa 84-95 as shown in Figure 3a, indicating the potential of these epitopes to confirm clinically relevant sensitisation to Gal d 1. Even though these findings have to be validated in a larger cohort, the recognition of these epitopes were highly specific (0.93) compared to slgE titres to egg white extract $(0.53$ at $5 \mathrm{kU} / \mathrm{l})$ or slgE/slgG4 ratios for Gal d 1 (0.63 at a ratio of 1$)$. 
Allergic patients who recognised aa 30-41 combined with aa 84-95, experienced respiratory symptoms $(n=2)$ or severe OAS $(n=1)$. The epitope aa $45-56$, known to be exclusively recognised by children with persistent hen's egg allergy ${ }^{8}$, was recognised by 5 allergic (38\%) but also by 4 tolerant patients (27\%). Moreover, most of the epitopes recognised by $\lg E$ were simultaneously recognised by IgG4 derived from the same individual. However, 3 allergic patients (23\%), all suffering from mild subjective symptoms as manifested by food challenges with heated hen's egg, did not recognise any epitope by either $\lg \mathrm{E}$ or $\lg \mathrm{G} 4$, suggesting a higher relevance of conformational epitopes in recognising Gal $d 1$ by patients with mild symptoms.

\section{Most patients with objective symptoms also recognised linear epi- topes of Gal d 3}

Additionally, a linear epitope mapping was also performed for Gal d 3. Regarding Gal d 3, only a small number of different epitopes $(n=11)$ were recognised by $\lg E$ in relation to its molecular mass $(78 \mathrm{kDa})$ as already indicated by the reduction in slgE binding upon linearisation. In total, only nine different epitopes were recognised by six allergic patients while two epitopes were recognised by one tolerant patient (Figure 3b). These epitopes bound by lgE were mostly located on the surface of Gal d 3 and therefore easily accessible for antibody binding. Surface-exposed epitopes were defined as possessing $\geq 3$ residues ( $\geq 25 \%$ ) facing the outside of the three-dimensional structure (indicated with red stars in Figure $3 b$ ). Three of the allergic patients and one tolerant recognised at least one surface-exposed epitope by slgE. These three patients, all suffering from objective symptoms, were the same patients who recognised at least one of the linear epitopes, which confirmed clinically relevant sensitisation to Gal d 1. In contrast, IgG4 antibodies bound to a larger number $(n=30)$ of epitopes, although most of them were just recognised by IgG4 from one to two individual patients except for the epitopes aa 396-407 and aa 483-494 not located on the protein surface. Taken together, slgE binding to linear epitopes of Gal d 3 has no additional value compared to linear epitope recognition of Gal d 1. 

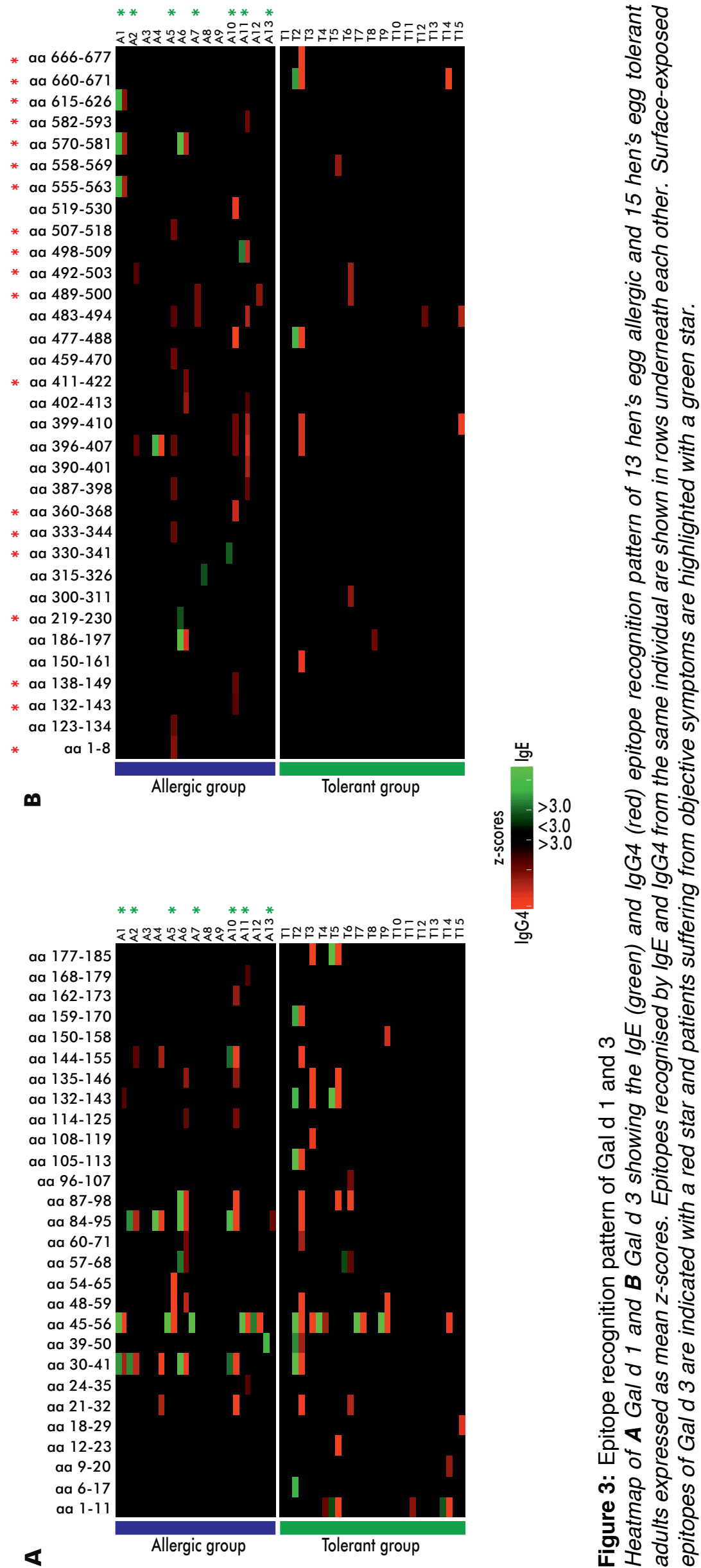


\section{Discussion}

So far, sensitisation to hen's egg extracts, components and linear epitopes is solely studied in children, although persistent and newly-onset hen's egg allergy do appear in adults with a prevalence of 0.02 to $0.6 \%$ across European countries ${ }^{1,2}$. In the present study, we showed great overlap in slgE levels to hen's egg extracts or single components between allergic and tolerant, but sensitised adults. Clinically relevant sensitisation to Gal d 1 was confirmed by slgE binding to the linear epitopes aa 30-41, aa 39-50 or aa 84-95 in 6 out of 13 hen's egg allergic adults, mainly suffering from objective symptoms. In contrast, patients with mild subjective symptoms showed no binding to linear epitopes of Gal d 1.

This is, to our knowledge, the first study focussing on sensitisation patterns in hen's egg allergic and tolerant adults. While largely overlapping slgE titres to egg white extract were not able to clearly discriminate between allergy and tolerance in adults, the definition of clinically relevant cut-off levels appeared to be supportive in diagnosing raw or heated hen's egg allergy in children ${ }^{5,17-19}$. Despite lacking the complete information about heated egg tolerance in our study population, a similar tendency of higher sigE titres to egg white extract was observed in allergic (median: $7.0 \mathrm{kU} / \mathrm{l}$ ) compared with tolerant adults (median: $4.6 \mathrm{kU} / \mathrm{l}$ ). Although our cohort selection based on sensitisation towards at least one heterologously expressed hen's egg component (rGal d 1 and/or rGal d 3) resulted in a certain selection bias, overlapping slgE titres to Gal d 1 (up to $10 \mathrm{kU} / \mathrm{l}$ ) and therefore to egg white extract, containing Gal d 1 as major allergen, were also described in hen's egg allergic and tolerant children ${ }^{20}$.

While slgE binding to Gal d 3 strongly decreased upon linearisation (SDS-PAGE under reducing conditions and western blotting), the slgE binding to Gal d 1 was only slightly altered, pointing to the importance of linear epitopes for the recognition of Gal $d 1$. Nevertheless, $23 \%$ of the allergic (subjective symptoms) and $53 \%$ of the tolerant patients who showed slgE binding to the linearised form of Gal d 1 did not show any $\lg E$ binding towards linear epitopes on the microarray, suggesting incomplete linearisation of Gal d 1 potentially due to reduced accessibility of disulphide bridges by reducing agents $^{21}$. Incomplete linearisation and the lack of linear epitope recognition in a part of the patients pinpoint to the importance of conformational epitopes for the recognition of Gal d 1 in those patients. A similar observation was made by Martínez-Botas and colleagues where $34 \%$ of hen's egg allergic children strongly positive to Gal d 1 did not recognise any linear epitope by $\lg ^{10}$, suggesting exclusive recognition of conformational epitopes in a subpopulation of hen's egg allergic patients.

The epitopes aa 30-41, aa 39-50 and aa 84-95 from Gal d 1 were mostly recognised by allergic patients (6/13 allergic vs $1 / 15$ tolerant patients) who suffered from objective symptoms (4/7) upon hen's egg ingestion, confirming clinically relevant sensitisation to 
Gal d 1 despite overlapping slgE titres between allergic and tolerant adults. Although these epitopes were described independently in different studies with hen's egg allergic children ${ }^{9,11,22}$, they did not belong to the so-called "informative" epitopes (aa 1-10, aa 11-20, aa 47-56 and aa 113-122) which showed great potential to predict persistent hen's egg allergy in children ${ }^{8}$. The epitope aa $47-56$, however, was recognised by $38 \%$ of allergic vs $27 \%$ of tolerant adults (overall $32 \%$ of all patients) in the present study, suggesting divergent IgE specificities in adulthood compared to childhood. These differences may also be related to study design and inclusion criteria.

Notably, epitopes recognised by $\lg E$ were often simultaneously bound by $\lg \mathrm{G} 4$ from the same individual irrespectively of their status, suggesting a clonal relationship between the $\lg E$ and $\lg G 4$ antibodies. A clonal relationship would imply that $\lg E$ producing $B$ cells partially originate from $\operatorname{lgG} 4^{+} \mathrm{B}$ cells or that some $\lg \mathrm{E}^{+}$and $\operatorname{lgG} 4^{+} \mathrm{B}$ cells share the same origin. Clonal analysis of allergic patients showed a predominately clonal relationship between $\lg \mathrm{G}_{1}{ }^{+}$and $\lg \mathrm{E}^{+} \mathrm{B}$ cells, suggesting that $\lg \mathrm{G} 1{ }^{+} \mathrm{B}$ cells might be the shared origin ${ }^{23}$. Compared to children, this potential clonal relationship between $\operatorname{lgE}$ and IgG4 appeared to be less dominant, since lgE-binding overlapped only partially with the binding characteristics of $\lg G 4^{10}$. This discrepancy between children and adults regarding potential clonal relationship between $\lg E$ and $\lg \mathrm{G}_{4}{ }^{+} \mathrm{B}$ cells might indicate the alteration of the origin for $\lg \mathrm{E}^{+}$and $\lg 4^{+} \mathrm{B}$ cells over time. However, more research is needed to confirm this hypothesis.

As a step forward, the promising results of the present study should be validated in a prospective cohort exclusively diagnosed by food challenge, minimising the risk of misclassification. Moreover, inclusion based on suspicion of egg allergy provides a broader population with more distinct sensitisation patterns such as mono-sensitisation to Gal d 2.

In conclusion, slgE-binding to linear epitope of Gal d 1 (aa 30-41, aa 39-50 or aa 8495 ) is highly specific to identify hen's egg allergic adults, mainly suffering from objective symptoms and may improve slgE diagnostics as an additional tool to conventional testing using egg extracts and single allergen components. 


\section{Acknowledgments}

We would like to thank B. Brix and M. Klinge for critical and fruitful discussions. Line blots and corresponding reagents were kindly provided by EUROIMMUN AG, Lübeck, Germany. This study was funded by a grant from the European Regional Development Fund of the European Union (TBI-V-1-098-E).

\section{Informed consent statement}

This study was carried out in accordance with the University Medical Center Utrecht, Biobank Regulations, which are in compliance with the applicable national and international laws and regulations. These regulations permit the use of 'residual material from diagnostic testing' for research, unless the patient objects (Article 8, 'no objection' procedure). None of the included patients objected the use of their serum. The protocol was approved by the Biobank Research Ethics Committee of the University Medical Center Utrecht under the protocol number 18-428. 


\section{References}

1. Lyons, S. A. et al. Food Allergy in Adults: Substantial Variation in Prevalence and Causative Foods Across Europe. J Allergy Clin Immunol Pract 7, 19208.e11 (2019).

2. Zuberbier, T. et al. Prevalence of adverse reactions to food in Germany - a population study. Allergy 59, 338-45 (2004).

3. Kamdar, T. A. et al. Prevalence and characteristics of adult-onset food allergy. J Allergy Clin Immunol Pract 3, 114-5.e1 (2015).

4. Gupta, R. S. et al. Prevalence and Severity of Food Allergies Among US Adults. JAMA Network Open 2, e185630 (2019).

5. Soares-Weiser, K. et al. The diagnosis of food allergy: a systematic review and meta-analysis. Allergy 69, 76-86 (2013).

6. Luengo, O. \& Cardona, V. Component resolved diagnosis: when should it be used? ClinTransl Allergy 4 (2014).

7. Chokshi, N. Y. \& Sicherer, S. H. Molecular diagnosis of egg allergy: an update. Expert Rev Mol Diagn 15, 895-906 (2015).

8. Järvinen, K.-M. et al. Specificity of $\lg \mathrm{E}$ antibodies to sequential epitopes of hen's egg ovomucoid as a marker for persistence of egg allergy. Allergy 62, 758-65 (2007).

9. Cooke, S. K. \& Sampson, H. A. Allergenic properties of ovomucoid in man. $J$ Immunol 159, 2026-32 (1997).

10. Martínez-Botas, J. et al. Mapping of the IgE and IgG4 Sequential Epitopes of Ovomucoid with a Peptide Microarray Immunoassay. Int Arch Allergy Immunol 161, 11-20 (2013).

11. Holen, E., Bolann, B. \& Elsayed, S. Novel B and T cell epitopes of chicken ovomucoid (Gal d 1) induce T cell secretion of IL-6, IL-13, and IFN-gamma. Clin Exp Allergy 31, 952-64 (2001).

12. Hochuli, E. et al. Genetic Approach to Facilitate Purification of Recombinant Proteins with a Novel Metal Chelate Adsorbent. Nat Biotechnol 6, 1321-5 (1988).

13. Trigoso, Y. D., Evans, R. C., Karsten, W. E. \& Chooback, L. Cloning, Expression, and Purification of Histidine-Tagged Escherichia coli Dihydrodipicolinate Reductase. PLOS ONE 11, e0146525 (2016).

14. Ehlers, A. M. et al. Ara h 7 isoforms share many linear epitopes: Are 3D epitopes crucial to elucidate divergent abilities? Clin Exp Allergy 49, 1512-9 (2019). 
15. Fraczkiewicz, R. \& Brau, W. Exact and Efficient Analytical Calculation of the Accessible Surface Areas and Their Gradients for Macromolecules. J Comp Chem 19, 319-33 (1988).

16. Gu, Z., Eils, R. \& Schlesner, M. Complex heatmaps reveal patterns and correlations in multidimensional genomic data. Bioinformatics 32, 2847-9 (2016).

17. Peters, R. L. et al. Skin prick test responses and allergen-specific lgE levels as predictors of peanut, egg, and sesame allergy in infants. J Allergy Clin Immunol 132, 874-80 (2013).

18. Sicherer, S. H. \& Sampson, H. A. Food allergy: Epidemiology, pathogenesis, diagnosis, and treatment. J Allergy Clin Immunol 133, 291-307.e5 (2014).

19. Benhamou-Senouf, A. H., Borres, M. P. \& Eigenmann, P. A. Native and denatured egg white protein IgE tests discriminate hen's egg allergic from egg-tolerant children. Ped Allergy Immunol 26, 12-7 (2015).

20. Ando, H. et al. Utility of ovomucoid-specific lgE concentrations in predicting symptomatic egg allergy. J Allergy Clin Immunol 122, 583-8 (2008).

21. Jocelyn, P. C. Chemical reduction of disulfides. Methods Enzymol 143, 246-56 (1987).

22. Mine, Y. \& Zhang, J. W. Identification and Fine Mapping of IgG and IgE Epitopes in Ovomucoid. Biochem Biophys Res Commun 292, 1070-4 (2002).

23. Looney, T. J. et al. Human B-cell isotype switching origins of IgE. J Allergy Clinical Immunol 137, 579-86.e7 (2016). 


\section{Supplementary}

Detailed description of individual patients characteristics are available at the Online Repository of the Journal Clinical and Experimental Allergy (doi: 10.1111/cea.13730).

Table S1: Identified epitopes of Gal $d 1$ in comparison to the literature

\begin{tabular}{|c|c|c|c|c|}
\hline Peptide & Residues $^{A}$ & $\lg E$ & IgG4 & $\begin{array}{c}\text { Publication } \\
\lg ^{B}\end{array}$ \\
\hline AEVDCSRFPNA & $1-11$ & $x$ & $\mathrm{X}$ & $1,2,4$ \\
\hline SRFPNATDKEGK & $6-17$ & $x$ & & 1,5 \\
\hline PNATDKEGKDVL & $9-20$ & $x$ & & $1,4,5$ \\
\hline TDKEGKDVLVCN & $12-23$ & $x$ & & 2 \\
\hline DVLVCNKDLRPI & $18-29$ & $x$ & & \\
\hline VCNKDLRPICGT & $21-32$ & $x$ & & \\
\hline KDLRPICGTDGV & $24-35$ & $x$ & & \\
\hline CGTDGVTYTNDC & $30-41$ & $x$ & $x$ & $2,3,4$ \\
\hline NDCLLCAYSIEF & $39-50$ & $x$ & $\mathrm{x}$ & 3,4 \\
\hline AYSIEFGTNISK & $45-56$ & $x$ & $x$ & $1,4,5$ \\
\hline IEFGTNISKEHD & $48-59$ & $x$ & & \\
\hline ISKEHDGECKET & $54-65$ & $x$ & & 2,3 \\
\hline EHDGECKETVPM & $57-68$ & $x$ & $x$ & 2,4 \\
\hline GECKETVPMNCS & $60-71$ & $x$ & & \\
\hline CSSYAN & $71-75$ & & & 3 \\
\hline DGKVMVLCNRA & $80-90$ & & & 3 \\
\hline MVLCNRAFNPVC & $84-95$ & $x$ & $x$ & 1,4 \\
\hline CNRAFNPVCGTD & $87-98$ & $x$ & & \\
\hline GTDGVTYDNECL & $96-107$ & $x$ & & 3,5 \\
\hline ECLLCAHKV & $105-113$ & $x$ & $x$ & 2,4 \\
\hline LCAHKVEQGASV & $108-119$ & $x$ & & \\
\hline EQGASVDKRHDG & $114-125$ & $x$ & & 4 \\
\hline KRHDGGCRKELAAV & $121-134$ & & & 2,3 \\
\hline AAVSVDCSEYPK & $132-143$ & $x$ & $x$ & 4 \\
\hline SVDCSEYPKPDC & $135-146$ & $x$ & & \\
\hline PDCTAEDRPLCG & 144-155 & $x$ & $x$ & \\
\hline DRPLCGSDN & $150-158$ & $x$ & & 4 \\
\hline KTYGNKCNFCNA & $159-170$ & $x$ & $x$ & 3 \\
\hline GNKCNFCNAVVE & $162-173$ & $x$ & & 3 \\
\hline CNAVVESNGTLT & $168-179$ & $x$ & & \\
\hline TLTLSHFGK & $177-185$ & $x$ & $\mathrm{X}$ & 1,3 \\
\hline
\end{tabular}

A without signal sequence

B 1: Cooke et al. 1997; 2: Holen et al. 2001; 3: Mine and Zhang 2002; 4: Järvinen et al. 2007, italic = informative; 5: Martínez-Botas et al. 2013 
A

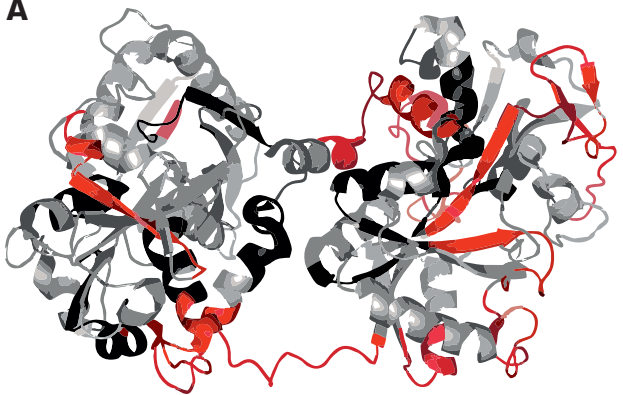

B

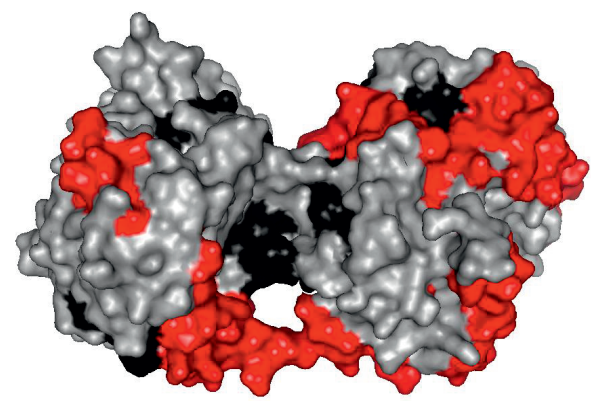

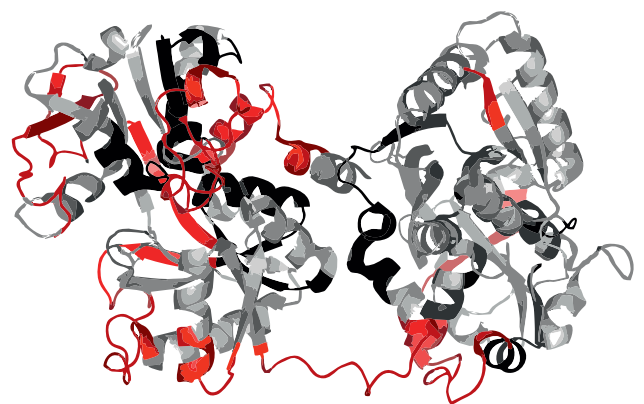

D

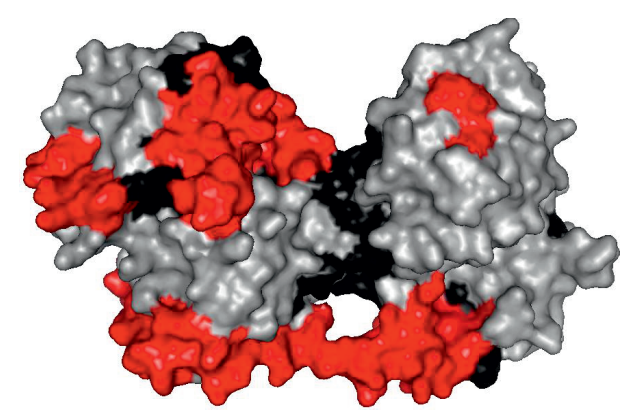

Figure S1: Mapping of linear epitopes onto the 3D structure of Gal d 3

The as surface-exposed (red) and non-surface exposed (black) defined epitopes were mapped onto the $3 D$ structure of Gal d 3 (pdb: 1OVT); A Cartoon view highlighting the dominant location of as surfaceexposed defined epitopes in the loops of Gal d 3; $\boldsymbol{B}$ View on the solvent-accessible surface of Gal $d$ 3; C Cartoon view as in $A$ but turned for $180^{\circ} ; \boldsymbol{D}$ View on the solvent-accessible surface of Gal $d 3$ as in $B$ but turned for $180^{\circ}$
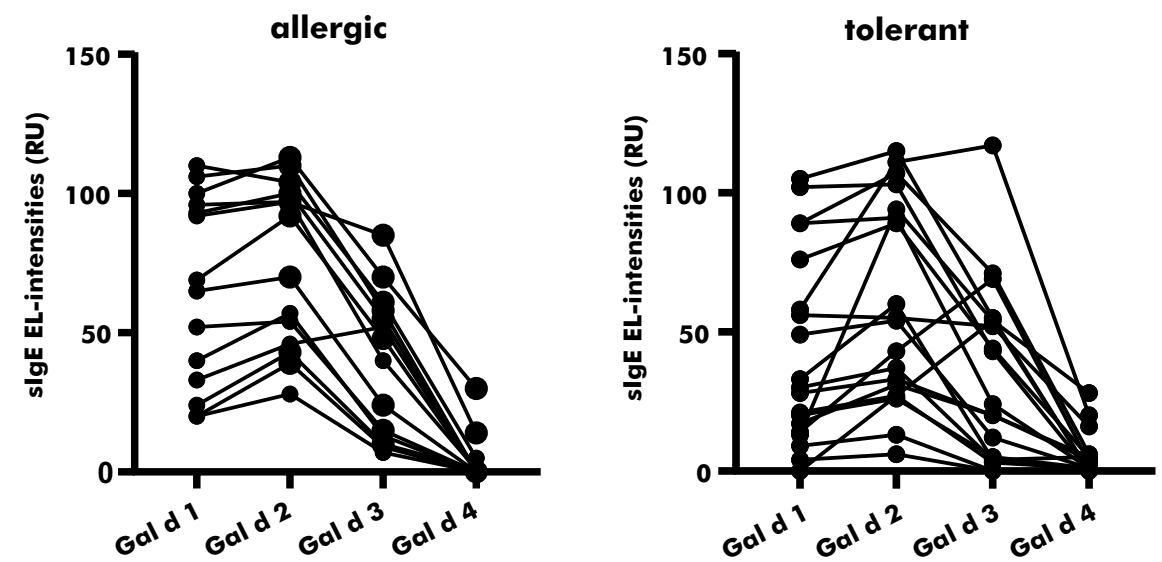

Figure S2: Patients' sensitisation profiles

Sensitisation profiles of hen's egg allergic and tolerant patients to the native hen's egg components Gal d 1, Gal d 2, Gal d 3 and Gal d 4 are separately shown. 
A

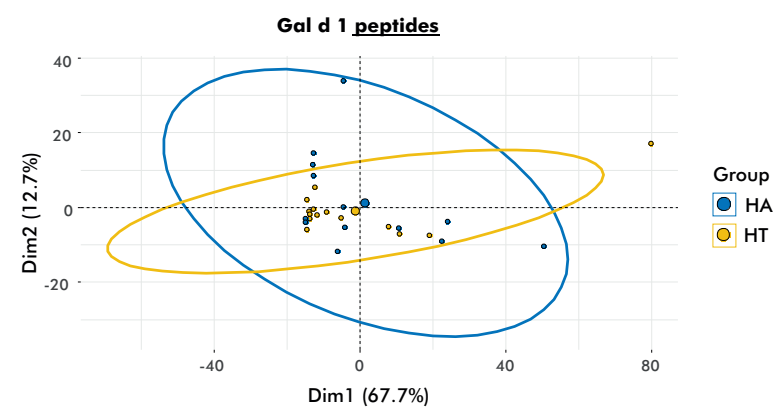

C

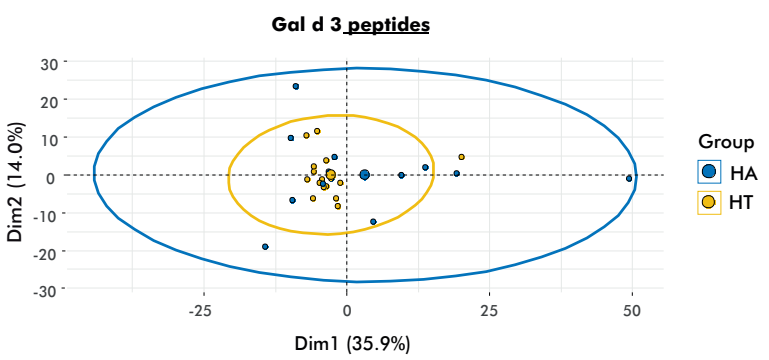

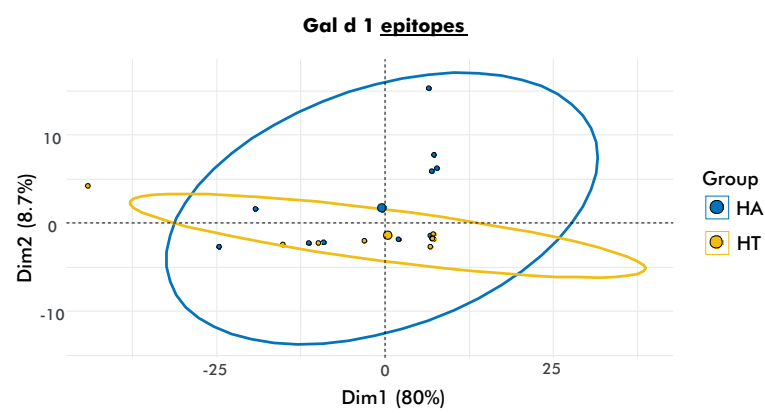

D

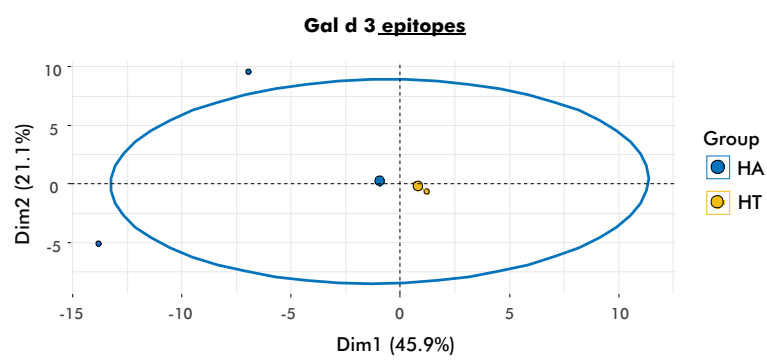

Figure S3: Principle component analysis

$\boldsymbol{A}$ Principle component analysis of peptides derived from Gal $d 1$ recognised by $\lg E$ and $\boldsymbol{B}$ principle component analysis of epitopes derived from Gald 1 recognised by $\mathrm{lgE}$; $\boldsymbol{C}$ Principle component analysis of peptides derived from $\mathrm{Gal} d 3$ recognised by $\lg E$ and $\boldsymbol{D}$ principle component analysis of epitopes derived from $\mathrm{Gal} d \mathrm{~d}$ recognised by $\lg E$ 
CIUTCT

AGCCTCTGGA7

CCTGTTTCTGCAAA

¿GCGTGGTCCAGCCTC

AGGGCCGATTCACCATL,

AATTGTGTTGACACAGTCTCCAGCCALU

UTGGCATCCCAGCCAGGTTCAGTGGCAGTGGG'TC I

$\because$ CAGATGACCCAGTCTCCTTCCACCCTGGCTGCATCTGTAG

AGTCCCATCAAGGTTCAGCGGCAGTGGGTCTGGGACAGAGTTCACL

IGAGTCTGGGGGAGGCTTGGTACAGCCGGGGGGGTCCCTGAGACTGTCL

TATCGAGACTCTGTGAAGGGCCGCTTCACCATCTCCAGAGACAATGCCAAAAAC ¿AGGGGACCACGGTCATCGTCTCCTCAGCAGGTGCAGCTGGTGGAGTCTGGGGGAGG iGAGTGGGTTTCATTTATACGCAAGGATGGAGCTAGTAAATACTATGGAGACTCCGTGAAGGG ATAAACCTGGCCAGGCTCCCAGGCTCCTCATCTATGATGCATCCAACAGGGCCACTGGCATCCC ACTGGCCTCCGCTCACTITCGGCGGAGGGACCAAGGTGGAGATCAAACGACATCCAGATGACCCAG

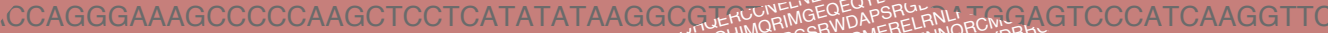

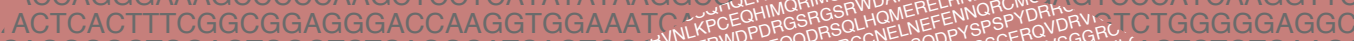

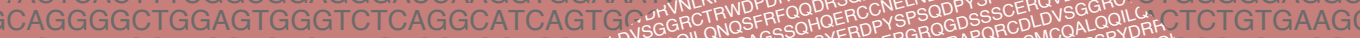

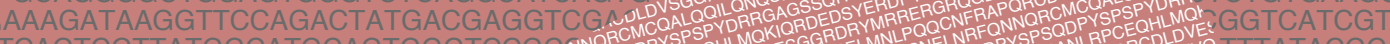

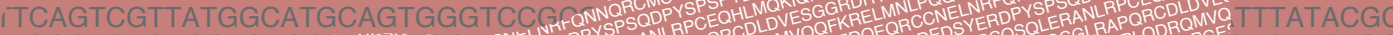

TCCTGCAGGGCGGLRAORO tGCCTAGAGC

\section{GCCTGATGAT}

\section{CACGTTTAGG}

ATGGCCAGTC

CCATCTCCAGAG

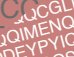

\section{GLRAPQRDRQN}

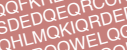

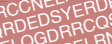

MENQCDRLS

\section{Q}

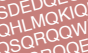

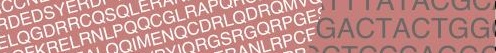

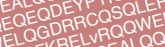

OMMENQSDRDELNEMENTRVEKE

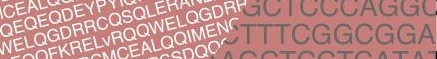

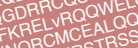

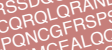

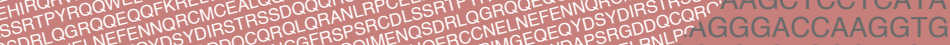
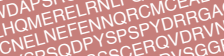

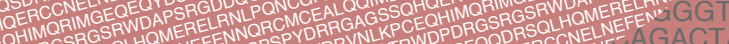

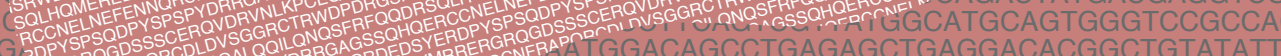

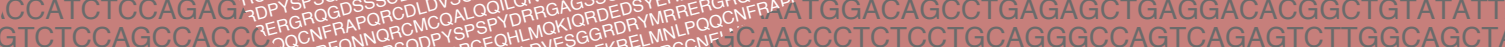

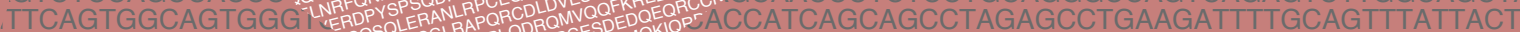
TTCCACCCTGGCTGCATC' 'PQQCNOCDRLGQRPGEOHLMW' CCATCACTTGCCGGGCCAGTCAGTCTTTTACGAAGTGGTTGGCC CAGTGGGTCTGGGACAGAGTTCrOALERANLPCEOHLTCAGCAGTCTGCAGCCTGATGATITTGCAACTTACTACTGTCAACAA ACAGCCGGGGGGGTCCCTGAGACTGTCCTGTGCAGCCTCTGGATTCACGTTTAGGAGATATGCTTTGAGTTGGGTCCGC GCTTCACCATCTCCAGAGACAATGCCAAAAACATCCTGTTTCTGCAAATGGCCAGTCCGAGAGTCGAAGACGCGGCCGTT TCAGCAGGTGCAGCTGGTGGAGTCTGGGGG AATGGAGCTAGTAAATACTATGGAGACTCCGT ¿CCAGGGAACCCTGGTCACCGTCTCCTCAGGA aGAGGGACCAAGGTGGAGATCAAACGACATCCAGAT CATATATAAGGCGTCTAATTTACAAGATGGAGTCCCAT AAGGTGGAAATCAAACGAGGTGCAGTTGGTGGAGTCTGG जGCATCAGTGGCAGTGGAAACAAAACATATTATCGAGACTC ACGAGGTCGAGATTATGGACGTCTGGGGCCAGGGGACCACG जेGTCCGCCAGGCTCCAGGCAAGGGGCTGGAGTGGGTTTCATT GGCTGTATATTACTGTGCGAAAGATAGGTCCCTGGGTTACTTTGA TTGGCAGCTACTTAGCCTGGTACCAACATAAACCTGGCCAGGC

TTTATTACTGTCAGCAGCGTAGCGACTGGCCTCCGCTCACT

CAGGGAAAGCCCCCAAGC

ACTTTCGGCGGAGGGAC

GCTGGAGTGGGTCTCA

GGTTCCAGACTATGAD TGGCATGCAGTGGG CTGAGGACACGGC AGTCAGAGTCT AGATTTTGCAGTTTATTACTGTCAGCAGCGTAGCGAC

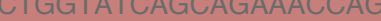
ACTTACTACTGTCAACAATATGATAGTGCCCTACTCAC CTTTGAGTTGGGTCCGCCAGGCTCCAGGGCAGGG :GAAGACGCGGCCGTTTATTACTGTGCGAAAGATAAG 'TCTCCTG AAAGACACT iTCTCCAGGG CAGACTTC GAGACAGAG 4CCCTCACCAT ;AAAAACATCCTC ;AAAAACATCCTG

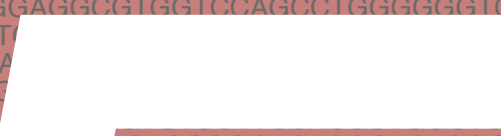
CTGAGACTCTCCTGTGTAGCGTC ACAATTCCAAAGACACTCTCTTTCT CCTGTCTITGTCTCCAGGGGAAAG GGTCTGGGACAGACTTCACTCTCA ATCTGTAGGAGACAGAGTCTCCAT ATCTGTAGGAGACAGAGTCTCCAT,
ACAGAGTTCACCCTCACCATCAGCA CAGCGGCAGTGGGTCTGGGACAGAGTTCACCCTCACCATCAGCA GGCCGCTTCACCATCTCCAGAGACAATGCCAAAAACATCCTGTTT CTCCTCAGCAGGTGCAGCTGGTGGAGTCTGGGGGAGGCGTGGT AGGATGGAGCTAGTAAATACTATGGAGACTCCGTGAAGGGCCGA GGCCAGGGAACCCTGGTCACCGTCTCCTCAGGAAATTGTGTTGACA GGCCAGGGAACCCTGGTCACCGTCTCCTCAGGAAATT GTGTTGACA
CTCCTCATCTATGATGCATCCAACAGGGCCACTGGCATCCCAGCCAC TCCGTGAAGGGCCGAT

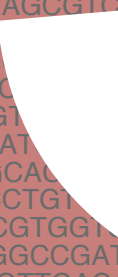

GGATTCACCTTCAGTCGT
CAAATGGACAGCCTGAGA

AACCCTCTCCTGCAGG

ACTTGCCGGGCCAGT GAGATCAAACGACATCCAGATGACCCAGTCTCC CAAGATGGAGTCCCATCAAGGTTCAGCGC ATTATCGAGACTCTGTGAAGGGCCGC AGGGGACCACGGTCATCGTCTCCTC TTGGGTTTCATTTATACGCAAGGATG GTTACTTTGACTACTGGGGCCAGG GGCCAGGCTCCCAGGCTCCTCAT GCTCACTTTCGGCGGAGGGACC CCCAAGCTCCTCATATATAAGGC GGAGGGACCAAGGTGGAAATCA GGGTCTCAGGCATCAGTGGCAG ACTATGACGAGGTCGAGATTATG GCAGTGGGTCCGCCAGGCTCCAC ACACGGCTGTATATTACTGTGCG ACACGGCTGTATATACTGTCG AAGTCTTGGCAGCTACTTAGCCTC TGCAGTTIATTACTGTCAGCAGQ CACTACTGTCAACAATATGATAGT ITGAGTTGGGTCCGCCAGGCTCCA 年 CGAAGACGCGGCCGTITATTACTGTGC
CTCTCCTGTGTAGCGTCTGGATTCACCT
AAAGACACTCTCTTTCTGCAAATGGAC, TTCCAAAGACACTCTCTTTCTGCAAATGGAC COCTCCTCAGGAAATTGTGTTGACACAG

作 ACGACATCCAGATGACCCAGTCTCCTTCCACCCTGGCTGCATCTGTAGGAGACAGAGTCTCCATCACTTGCCGG GATGGAGTCCCATCAAGGTTCAGCGGCAGTGGGTCTGGGACAGAGTTCACCCTCACCATCAGCAGTCTGCAGCO ITGGTGGAGTCTGGGGGAGGCTTGGTACAGCCGGGGGGGTCCCTGAGACTGTCCTGTGCAGCCTCTGGATTCAC ¿ATATTATCGAGACTCTGTGAAGGGCCGCTTCACCATCTCCAGAGACAATGCCAAAAACATCCTGTTTCTGCAAATGC GCCAGGGGACCACGGTCATCGTCTCCTCAGCAGGTGCAGCTGGTGGAGTCTGGGGGAGGCGTGGTCCAGCCTGG GGAGTGGGTTTCATTTATACGCAAGGATGGAGCTAGTAAATACTATGGAGACTCCGTGAAGGG

¿CCTGGGTTACTTTGACTACTGGGGCCAGGGAACCCTGGTCACCGTCTCCTCAGGAAATTGTC

ITAAACCTGGCCAGGCTCCCAGGCTCCTCATCTATGATGCATCCAACAGGGCCACTGGCATC

IGGCCTCCGCTCACTTTCGGCGGAGGGACCAAGGTGGAGATCAAACGACATCCAGATGACC

AGGGAAAGCCCCCAAGCTCCTCATATATAAGGCGTCTAATTTACAAGATGGAGTCCCATCAAGC

CACTTTCGGCGGAGGGACCAAGGTGGAAATCAAACGAGGTGCAGTTGGTGGAGTCTGGGGG

GGCTGGAGTGGGTCTCAGGCATCAGTGGCAGTGGAAACAAAACATATTATCGAGACTCTGTG

IAGGTTCCAGACTATGACGAGGTCGAGATTATGGACGTCTGGGGCCAGGGGACCACGGTCATCGI

ITATGGCATGCAGTGGGTCCGCCAGGCTCCAGGCAAGGGGCTGGAGTGGGTTTCATTTATACGCAAGGATGGAGi I AG I iAGAGCTGAGGACACGGCTGTATATTACTGTGCGAAAGATAGGTCCCTGGGTTACTTTGACTACTGGGGCCAGGGAACCCTG ,AGGGCCAGTCAGAGTCTTGGCAGCTACTTAGCCTGGTACCAACATAAACCTGGCCAGGCTCCCAGGCTCCTCATCTATGATGC GAGCCTGAAGATTTTCAGTTTATTACTGTCAGCAGCGTAGCGACTGGCCTCCGCTCACTTTCGGCGGAGGGACCAAGGTGGA 


\section{$2 S$ protein Ara $\mathrm{h} 7.0201$ has unique epitopes compared to other Ara $h 7$ isoforms and is comparable to $2 S$ proteins Ara $h 2$ and 6 in basophil degranulation capacity}
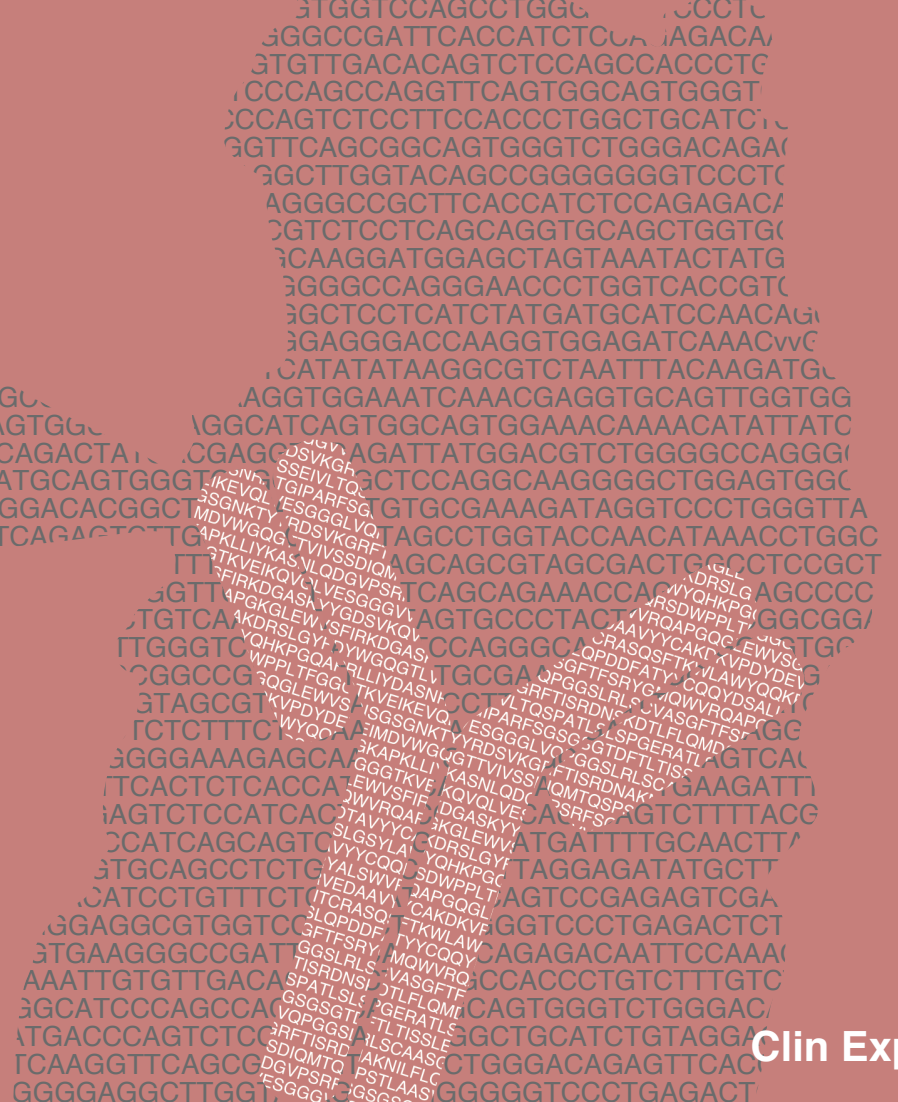

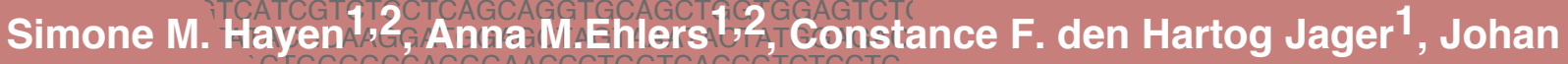

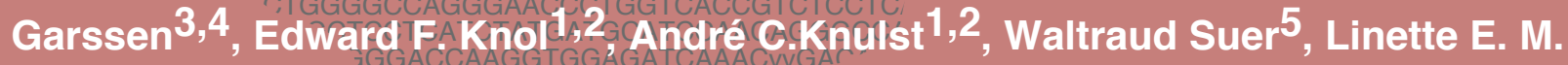

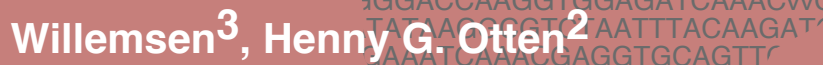

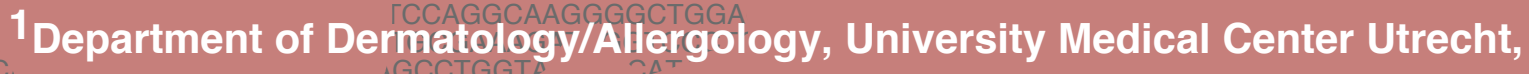
Utrecht University, Altrecht, The Netherlands

2 Laboratory of TrâñSIatitional Immunology, Utrecht University, University Medical Center Utrecht, Utrechits The Netherlands

3GACA C

Facalty of Sciencegutrecht University, Utrecht, The Netherlands

${ }^{4}$ NutriciaAResearch Timmunology, Utrecht, The Netherlands

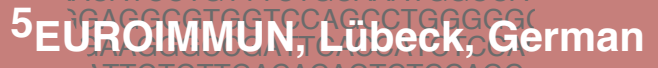




\section{Abstract}

\section{Background}

Screening for specific IgE against $2 S$ albumin proteins Ara $\mathrm{h} 2$ and 6 has a good positive predictive value in diagnosing peanut allergy. From the third $2 S$ albumin member Ara $\mathrm{h} 7$, three isoforms have been identified. Their allergenicity has not been elucidated.

\section{Objective}

This study investigated the allergenicity of Ara h 7 isoforms compared to Ara $\mathrm{h} 2$ and 6 .

\section{Methods}

Sensitization of 15 DBPCFC-confirmed peanut allergic patients to recombinant Ara $\mathrm{h}$ $2.0201,6.01$ and isoforms of recombinant Ara h 7 was determined by lgE immunoblotting strips. A basophil activation test (BAT) was performed in nine patients to determine $\lg$ E-crosslinking capacities of the allergens. Sensitivity to the allergens was tested in five patients that were sensitized to at least one Ara $\mathrm{h} 7$ isoform, by a concentration range in the BAT. 3D-prediction models and sequence alignments were used to visualize differences between isoforms and to predict allergenic epitope regions.

\section{Results}

Sensitization to Ara h 7.0201 was most frequent (80\%) and showed to be equally potent as Ara h 2.0201 and 6.01 in inducing basophil degranulation. Sensitization to Ara h 7.0201 together with Ara h 2.0201 and/or 6.01 was observed, indicating the presence of unique epitopes compared to the other two isoforms. Differences between the three Ara $\mathrm{h} 7$ isoforms were observed in C-terminal cysteine residues, pepsin and trypsin cleavage sites and three single amino acid substitutions.

\section{Conclusion and Clinical relevance}

The majority of peanut-allergic patients are sensitized to isoform Ara $\mathrm{h} 7.0201$, which is functionally as active as Ara h 2.0201 and 6.01. Unique epitopes are most likely located in the $\mathrm{C}$-terminus or an allergenic loop region which is a known allergenic epitope region for Ara h 2.0201 and 6.01. Due to its unique epitopes and allergenicity, it is an interesting candidate to improve the diagnostic accuracy for peanut allergy. 


\section{Introduction}

It is estimated that approximately $11.4-13.1 \%$ of children (0-17 years) and $3.2-5.1 \%$ of adults (>18 years) in European countries are sensitized against at least one food allergen, based on detection of specific $\lg E$ in serum ${ }^{1}$. Most food allergies are $\lg \mathrm{E}$ mediated, and symptoms develop within minutes to a few hours after ingestion of the specific allergen. Among food allergies, peanut allergens are most frequent in eliciting a fatal food reaction, and it is estimated that $0.6 \%$ of the total population is affected by peanut allergy ${ }^{2}$. By determining specific IgE to recombinant or purified peanut proteins rather than crude peanut extract, component-resolved diagnostics (CRD) has proven to be a useful tool to improve diagnostic accuracy for peanut allergy ${ }^{3}$. However, the functionality of several potentially relevant allergens remains unknown, since their capacity to induce effector cell degranulation has never been tested in patient samples before.

To date, seventeen peanut allergens (Ara h 1-17) are known and most of them have been sequenced and cloned ${ }^{3-6}$. Previous research indicated that screening for specific $\lg \mathrm{E}$ against $2 \mathrm{~S}$ albumins Ara $\mathrm{h} 2$ and 6 is to date most effective in diagnosing peanut allergy, since the majority of peanut-allergic patients have specific $\lg E$ against these allergens $^{6-9}$. Ara $\mathrm{h} 2$ and 6 are both proteins belonging to the conglutin family, and Ara h 6 has approximately $53 \%$ sequence identity with Ara $h 2^{5,6}$.

Besides determining specific IgE against Ara $\mathrm{h} 2$ and 6 for diagnosing peanut allergy, a third $2 S$ protein Ara $\mathrm{h} 7$ is currently gaining attention as predictor for peanut allergy ${ }^{10}$. Although Ara $\mathrm{h} 7$ makes up only $0.5 \%$ of total peanut protein ${ }^{3}$, it is a storage protein and therefore considered an allergen with a potential strong diagnostic value ${ }^{11}$. In comparison, the abundance of Ara $\mathrm{h} 2$ ranges between 5.9-9.3\% of total peanut protein and for Ara $\mathrm{h} 6$, this is 4-14\% $\%^{3,12}$. By phage display technology, isoforms Ara $\mathrm{h} 7.0101$ and Ara h 7.0201 were previously identified as allergens, but only Ara h 7.0201 was identified in peanut-extract ${ }^{5}$. Ara $\mathrm{h} 7.0101$ shares $42 \%$ sequence identity with Ara $\mathrm{h} 2$ and 45\% with Ara h 6, whereas Ara h 7.0201 shares 44\% sequence identity with Ara h 2 and $52 \%$ with Ara h 6,13. Besides these two isoforms of Ara $h 7$, a third isoform labelled Ara $\mathrm{h} 7.0301$ shares $70 \%$ sequence identity with Ara h 7, and was also identified in peanut-extract ${ }^{5,14}$. Previous research indicated the presence of unique epitopes on these Ara $\mathrm{h} 7$ isoforms, and therefore they may be relevant in peanut allergy diagnosis $^{10}$. 
Since the functionality of Ara $\mathrm{h} 7$ has not yet been studied extensively, the aim of this study was to identify whether the capacity of Ara $\mathrm{h} 7$ to induce basophil degranulation using whole blood from peanut-allergic patients is similar to Ara h 2 and 6 . In addition, the allergen recognition pattern of the three isoforms of Ara $h 7$ by peanut allergic patients was studied. Furthermore, in relation to their functionality, the amino acid sequence and a 3D-prediction protein model were used to predict epitopes or regions of Ara $\mathrm{h} 7$ that are important in inducing basophil degranulation and allergenicity.

\section{Materials and Methods}

\section{Study design and study population}

Assessment of sensitization to $2 \mathrm{~S}$ peanut allergens was performed with residual plasma of fifteen peanut-allergic patients that visited the outpatient clinic of Dermatology/Allergology at the University Medical Center Utrecht in 2015-2017 for clinical research. Table 1 shows data on gender, age and historical data on SPT, subjective and objective doses determined by DBPCFC and Müller score. Of these fifteen patients, nine random patients that were scheduled for visiting the UMC for clinical research were able to donate blood for the functional basophil activation test. Five random patients from the complete cohort that were sensitized to at least one Ara $\mathrm{h} 7$ isoform were recruited for the concentration range BAT. Inclusion criteria consisted of a type I allergic reaction to peanut, confirmed by a positive double-blind placebo-controlled food challenge (DBPCFC). Use of prednisone, other immunosuppressants or pregnancy were exclusion criteria. Informed consent was obtained of all patients prior to the study. The study was reviewed and approved by the Ethics Committee of the University Medical Center Utrecht (NL51606.041.15).

\section{Expression and purification of crude peanut extract and recombi- nant allergens}

Crude peanut extract (CPE) was obtained by blending raw peanuts, followed by extraction at room temperature with Tris/NaCL buffer (20 mM Tris, $150 \mathrm{mM} \mathrm{NaCL}, \mathrm{pH}$ 7.2). Supernatant was filtered twice and diluted in 1x PBS to the appropriate concentration. Recombinant peanut allergens Ara h 2.0201 (Acc.no. Q6PSU2), Ara h 6.01 (Acc.no Q647G9), Ara h 7.0101 (Acc.no. Q9SQH1), Ara h 7.0201 (Acc.no. B4XID4) and Ara h 7.0301 (Acc. No. Q647G8) were provided by Euroimmun and produced as described previously ${ }^{10,15}$. 


\section{Immunoblot}

Immunoblots (Euroline, Euroimmun, Lübeck, Germany) were used to determine sensitization of fifteen patients to isoforms of recombinant peanut proteins of Ara $\mathrm{h}$ 2.0201, Ara h 6.01, Ara h 7.0101, Ara h 7.0201 and Ara h 7.0301. Immunoblots and reagents were kindly provided by Euroimmun and experiments were performed according to the manufacturer's instructions. In short, the Euroline strips were incubated on a rocking shaker overnight at RT with $100 \mu \mathrm{L}$ of $1: 11$ diluted patient plasma in universal buffer. Binding of antibodies was visualized with an enzyme-labelled a-human IgE antibody in combination with substrate nitro-blue tetrazolium/5-bromo-4-chloro-3'-indolyphosphate. The results were evaluated with EuroLineScan software. The intensity of the bands was measured and an intensity level of 3 or higher was considered positive (arbitrary units). Line blot intensity values of 2 and lower were considered negative.

\section{Basophil activation test}

Whole heparinized blood was obtained from 9 out of 15 peanut-allergic patients and a BAT was performed. Blood samples were stimulated for 30 minutes with increasing concentrations or $1000 \mathrm{ng} / \mathrm{mL}$ of crude peanut extract (CPE), or separate recombinant peanut allergens in RPMI 1640 medium (Gibco, Life Technologies) supplemented with $1 \mathrm{ng} / \mathrm{mL} \mathrm{IL}-3$ (R and D Systems). Control samples for the basophil activation test were rVP40 (recombinant VP40, control protein) and buffer. Leukocytes were stained with an antibody cocktail of CD45-PO (Life Technologies), CD123-FITC (Biolegend), HLA-DR-PB (Biolegend), CD63-PE (Monosan), CD41-PE-Cy7 (Beckman coul-

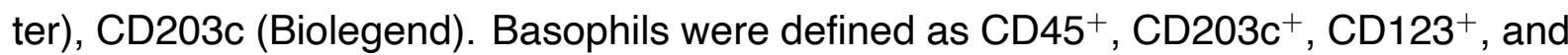
$\mathrm{HLA}^{-D R}{ }^{-}, \mathrm{CD}_{41}^{-}$, and degranulation was quantified by determining the surface expression of CD63. Results were expressed as percentage of CD63 bright basophils. A threshold above $5 \%$ degranulation was considered positive.

\section{D protein models and distance mapping}

3D protein models were created with Protein Homology/analogY Recognition Engine $(\mathrm{PHYRE})^{16}$. Further graphics and analyses such as distance mapping were performed with UCSF Chimera, developed by the Resource for Biocomputing, Visualization, and Informatics at the University of California, San Francisco ${ }^{17}$. 


\section{Data analysis and statistics}

Correlation between percentage degranulation of basophils and intensity of the Euroline strips was determined with Spearman's correlation coefficient, since the data were not normally distributed. GraphPad Prism 7 (GraphPad Software, USA) was used for statistical testing and visualizing data.

Table 1: Patient characteristics.

Sex, age, Skin Prick Test (SPT), results of DBPCFC and Müller score per peanut-allergic subject

\begin{tabular}{ccccccc}
\hline Patient & Sex (M/F) & Age (y) & $\begin{array}{c}\text { SPT peanut } \\
\left(\mathbf{m m}^{\mathbf{A}}\right)\end{array}$ & $\begin{array}{c}\text { Subjective ED } \\
\left.\mathbf{( m g}^{\mathbf{B}}\right)\end{array}$ & $\begin{array}{c}\text { Objective ED } \\
(\mathbf{m g})^{\mathbf{B}}\end{array}$ & Müller score $^{\mathbf{C}}$ \\
\hline N01 & $\mathrm{F}$ & 41 & $3+$ & 10 & - & 2 \\
N02 & M & 37 & $3+$ & 0.1 & 300 & 4 \\
N03 & M & 45 & $4+$ & 100 & - & 2 \\
N04 & F & 50 & $4+$ & 10 & 10 & 3 \\
N05 & F & 35 & $4+$ & 0.1 & - & 4 \\
N06 & F & 27 & $4+$ & 4 & 40 & 2 \\
N07 & M & 42 & $5+$ & not known & 300 & 3 \\
N08 & M & 24 & $4+$ & 100 & $>30000$ & 1 \\
N09 & F & 24 & $3+$ & not known & $>30000$ & 3 \\
N10 & F & 18 & $4+$ & 300 & 1000 & 3 \\
N11 & F & 32 & $4+$ & 10 & 3000 & 2 \\
N12 & M & 27 & $5+$ & 0.1 & 1000 & 3 \\
N13 & M & 25 & $3+$ & 10 & - & 2 \\
N14 & F & 26 & $4+$ & 0.1 & 100 & 3 \\
N15 & F & 34 & $4+$ & 40 & 12000 & 2 \\
A Skin prick test (mm), a diameter of 3 mm (3+) was considered positive & \\
B Subjective and objective effective dose (ED) during DBPCFC indicated in mg. \\
C Müller score 0: Symptoms oral cavity, 1: Symptoms of the skin and mucous membranes, 2: Gastro- \\
intestinal symptoms, 3: Respiratory symptoms, 4: Cardiovascular symptoms.
\end{tabular}

\section{Results}

\section{Peanut-allergic patients can be sensitized to Ara h 2.0201, Ara h 6.01 and all isoforms of Ara $\mathrm{h} 7$}

In fifteen peanut-allergic patients, sensitization to Ara $\mathrm{h} 2.0201,6.01$ and the three isoforms of Ara $\mathrm{h} 7$ was established by means of the immunoblot strips (Figure 1a). Sensitization to Ara h 2.0201 was most abundant; 14 out of 15 patients were sensitized to this allergen, followed by sensitization to Ara h 6.01 and 7.0201, which were both recognized by 12 patients (80\%). Two patients were mono-sensitized to Ara h 2.0201 (N01, N09), while also co-sensitization for multiple allergens occurred. $40 \%$ of the patients were sensitized to all allergens, while one patient (N15) recognized all allergens except 
Ara h 2.0201 (Figure 1b). Ara h 7.0201 sensitization in combination with Ara h 2.0201 and/or Ara h 6.01 was found for three patients (N03, N04, N11), while sensitization to Ara h 7.0101 or Ara h 7.0301 was never observed in the absence of Ara h 7.0201.

A

\begin{tabular}{|l|c|c|c|c|c|c|}
\cline { 3 - 7 } \multicolumn{1}{l|}{} & Line blot intensity (Arbitrary Units) \\
\hline Patient & $\begin{array}{c}\text { CAP peanut } \\
(\mathrm{kU} / \mathrm{L})\end{array}$ & Ara h 2.0201 & Ara h 6.01 & Ara h 7.0101 & Ara h 7.0201 & Ara h 7.0301 \\
\hline N01 & 1.7 & 4 & 2 & 2 & 2 & 2 \\
\hline N02 & 44 & 67 & 71 & 5 & 3 & 0 \\
\hline N03 & 1.8 & 3 & 11 & 0 & 7 & 0 \\
\hline N04 & 12 & 4 & 0 & 2 & 5 & 1 \\
\hline N05 & 85 & 18 & 31 & 10 & 34 & 5 \\
\hline N06 & 12.8 & 3 & 62 & 8 & 3 & 1 \\
\hline N07 & 42.7 & 57 & 58 & 26 & 74 & 25 \\
\hline N08 & 1.9 & 4 & 5 & 1 & 2 & 0 \\
\hline N09 & 1.0 & 3 & 2 & 2 & 2 & 0 \\
\hline N10 & $>100$ & 167 & 81 & 56 & 115 & 106 \\
\hline N11 & n.d. & 12 & 26 & 1 & 5 & 1 \\
\hline N12 & 66 & 74 & 96 & 6 & 106 & 12 \\
\hline N13 & 11.2 & 29 & 4 & 3 & 40 & 4 \\
\hline N14 & 9.7 & 50 & 61 & 8 & 54 & 4 \\
\hline N15 & 1.6 & 2 & 17 & 8 & 12 & 3 \\
\hline
\end{tabular}

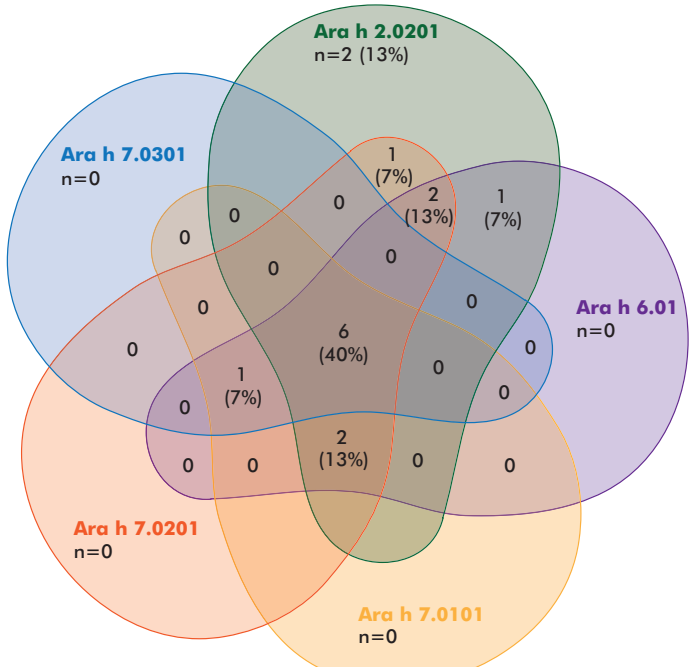

Figure 1: Co-sensitization to peanut allergens of fifteen peanut-allergic patients $\boldsymbol{A}$ Specific IgE $(\mathrm{kU} / \mathrm{L})$ against peanut determined by ImmunoCAP are indicated per patient in the left column. Sensitization to allergens Ara $h$ 2.0201, 6.0101, 7.0101, 7.0201 and 7.0301 by lineblot are shown as intensity values (arbitrary units) with intensity values $>3$ considered positive.; $\boldsymbol{B}$ Venn diagram illustrating co-sensitization to peanut allergens Ara $h$ 2.0201, 6.0101, 7.0101, 7.0201 and 7.0301 based on intensity level Euroline strips $(n=15)$.

\section{All Ara $\mathbf{h} \mathbf{7}$ isoforms can induce basophil degranulation, and their line blot intensity levels correlate with basophil degranulation}

Basophil degranulation experiments were performed for nine peanut-allergic patients, with the optimal allergen concentration of $1000 \mathrm{ng} / \mathrm{mL}$ (Figure 2a). All allergens were able to induce basophil degranulation. Of the three Ara $h 7$ isoforms, Ara $h 7.0201$ was most effective, since it induced basophil degranulation in six patients, followed by Ara h 7.0301 which induced basophil degranulation in two patients. Ara h 7.0101 was able to induce degranulation in only one patient. Besides basophil degranulation, the correlation between the intensity levels of the Euroline strips, which were directly related to levels of specific $\lg \mathrm{E}$, and the percentage of basophil degranulation was determined (Figure 2a). A significant correlation ( $p<0.05$ ), $r=0.8-0.9$ was observed between the lineblot intensity and basophil degranulation upon Ara h 2.0201, 6.01 or 7.0201 exposure (Figure 2b). 

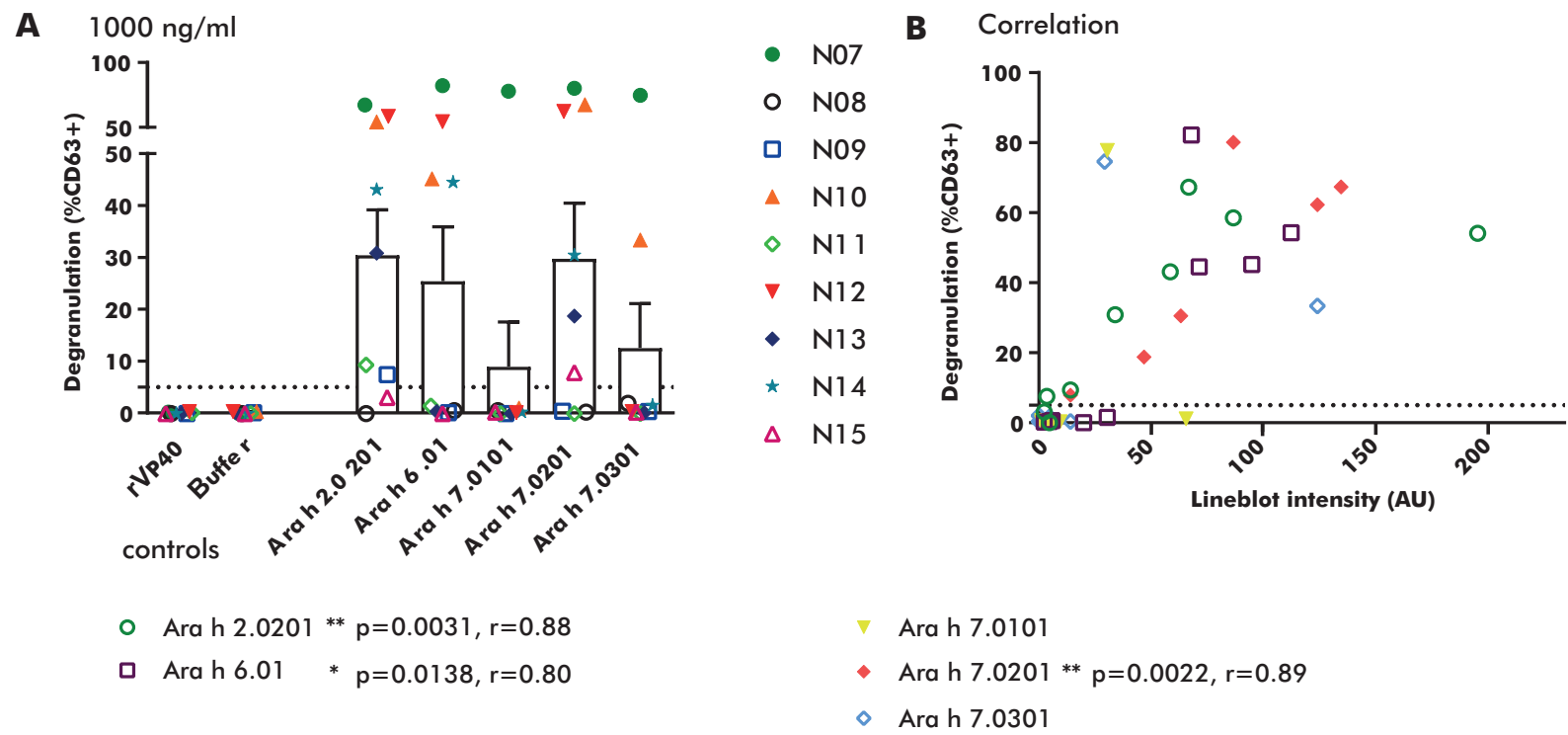

Figure 2: Functionality Ara $\mathrm{h} 7$ isoforms in basophil activation test

A BAT assay in peanut-allergic patients at an allergen concentration of $1000 \mathrm{ng} / \mathrm{mL}$. Degranulation is indicated as percentage of $\mathrm{CD} 3^{+}$cells $(n=9)$.; B Spearman correlation between degranulation in the $B A T$ assay versus the intensity levels of the lineblot strips $(n=9),{ }^{*} p<0.05,{ }^{* *} p<0.01$.

\section{Isoforms Ara h 7.0201 and Ara h 7.0301 can induce basophil degran- ulation at least as well as Ara h 2.0201 and 6.01}

To compare the ability to induce basophil degranulation at low allergen concentrations, a concentration range of allergens was used in the BAT in whole blood of five patients that were sensitized against at least one isoform of Ara $\mathrm{h} 7$. Althouh patient N06 was sensitized to Ara h 2.0201, 6.01, 7.0101 and 7.0201, basophil degranulation was only detected upon exposure to CPE and Ara h 6.01 (Figure 3a), which was probably related to relatively low intensity levels of sensitization for Ara h 2.0201 and 7. In comparison, $\mathrm{N} 10$ and N14 showed already high basophil degranulation upon low concentrations (0.3 ng/mL) of isoforms Ara h 7.0201 and Ara h 7.0301 (Figure 3c and e), while basophils of other patients degranulated around $10 \mathrm{ng} / \mathrm{mL}$ of Ara h 2, 6 and 7 . In the other two patients (N07, N12) the maximal degranulation upon exposure to the Ara $\mathrm{h} 7$ isoforms was comparable to Ara h 2.0201 and 6.01. These data indicate that isoform Ara h 7.0201 is at least as effective as recombinant Ara h 2.0201 and Ara h 6.01 in terms of inducing basophil degranulation. 

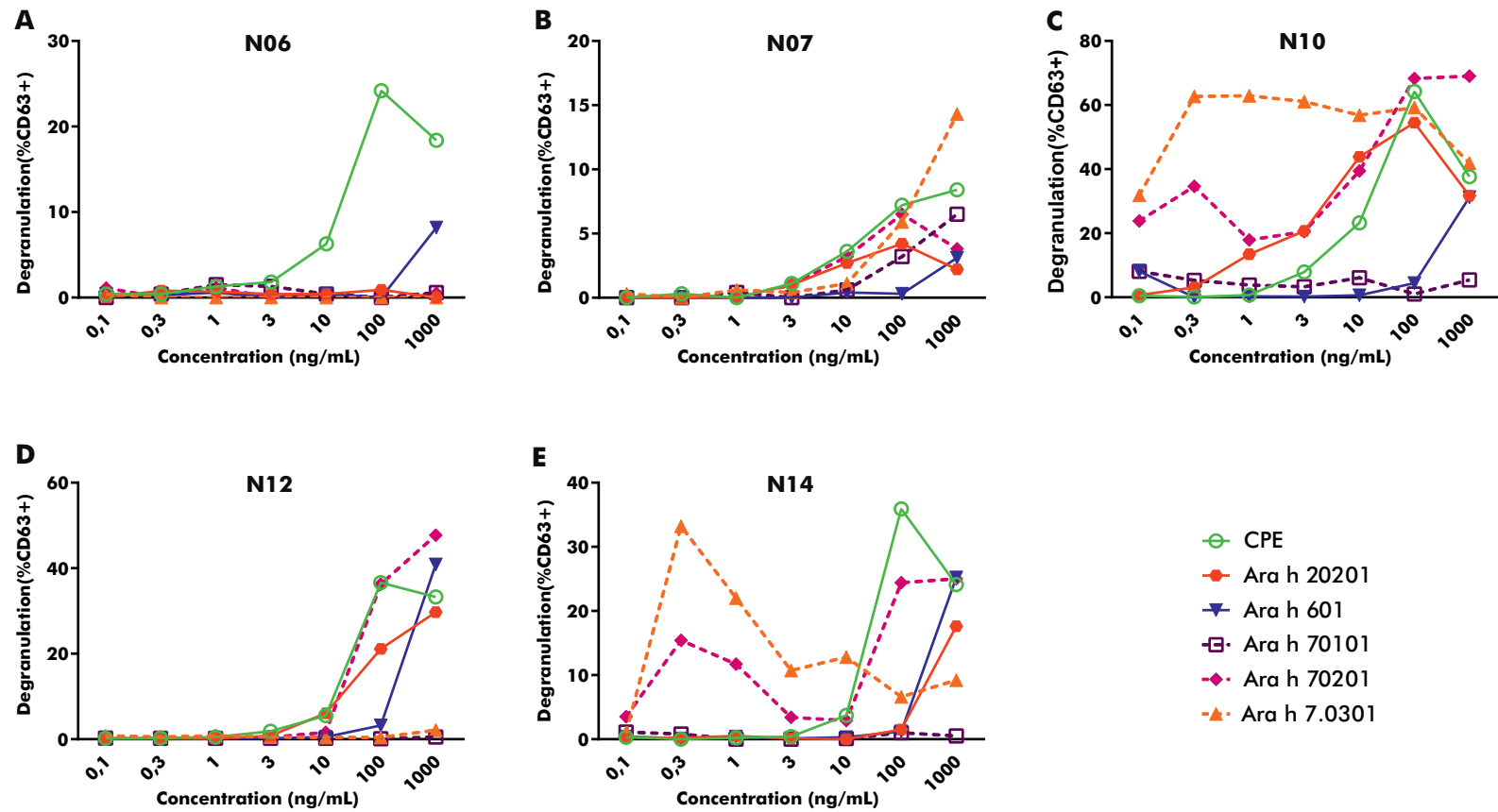

$$
\begin{aligned}
& \because \text { CPE } \\
& - \text { Ara h } 20201 \\
& - \text { Ara h } 601 \\
& -\because \cdot \text { Ara h } 70101 \\
& -\bullet \text { Ara h } 70201 \\
& -\star \text { Ara h } 7.0301
\end{aligned}
$$

Figure 3: Degranulation of basophils after exposure to different allergen concentration A-E Basophil activation of 5 peanut allergic patients in a BAT assay with an allergen concentration range (CPE, Ara h 2.0201, 6.0101, 7.0101, 7.0201 and 7.0301).

\section{Sequential differences in sequence alignment of Ara $\mathrm{h} 7$ isoforms compared to Ara h 2.0201 and 6.01}

A sequence alignment between Ara h 2.0201, 6.01 and the three Ara $\mathrm{h} 7$ isoforms was performed to explain the difference in efficacy of Ara $\mathrm{h} 7.0201$ to induce basophil degranulation in more patients compared to the other two Ara $\mathrm{h} 7$ isoforms (Figure 4a). Known linear epitopes of Ara $\mathrm{h} 2$ and 6 recognized by allergic patients are highlighted in color $^{18}$. Of the three isoforms, Ara h 7.0201 showed most sequence similarity with Ara h 2.0201 and 6.01 in the C-terminal regions that are known to carry allergenic linear epitopes of Ara h 2 and 6 (orange underlined sequence) ${ }^{18}$. Moreover, Ara h 7.0201 is the only isoform containing 8 cysteine residues (underlined C-residues), similar to the conserved cysteine pattern of Ara h 2.0201 and 6.01, whereas Ara h 7.0101 and 7.0301 only contain 6 cysteine residues. Cysteine residues play an important role in the folding and stability of proteins ${ }^{11}$. Furthermore, Ara $\mathrm{h} 7.0201$ differed in three amino acid positions from both other isoforms (Figure 4b, arrows). These differences influence polarity, hydrophobicity, charge, and trypsin cleavage sites (blue residues). In addition, more differences in trypsin (blue) and pepsin (red) cleavage sites were observed in the C-terminus (highlighted end) between the three different isoforms, which play an important role in the enzymatic digestion and thus can influence stability and allergenicity of proteins (Figure $4 b$ ). 


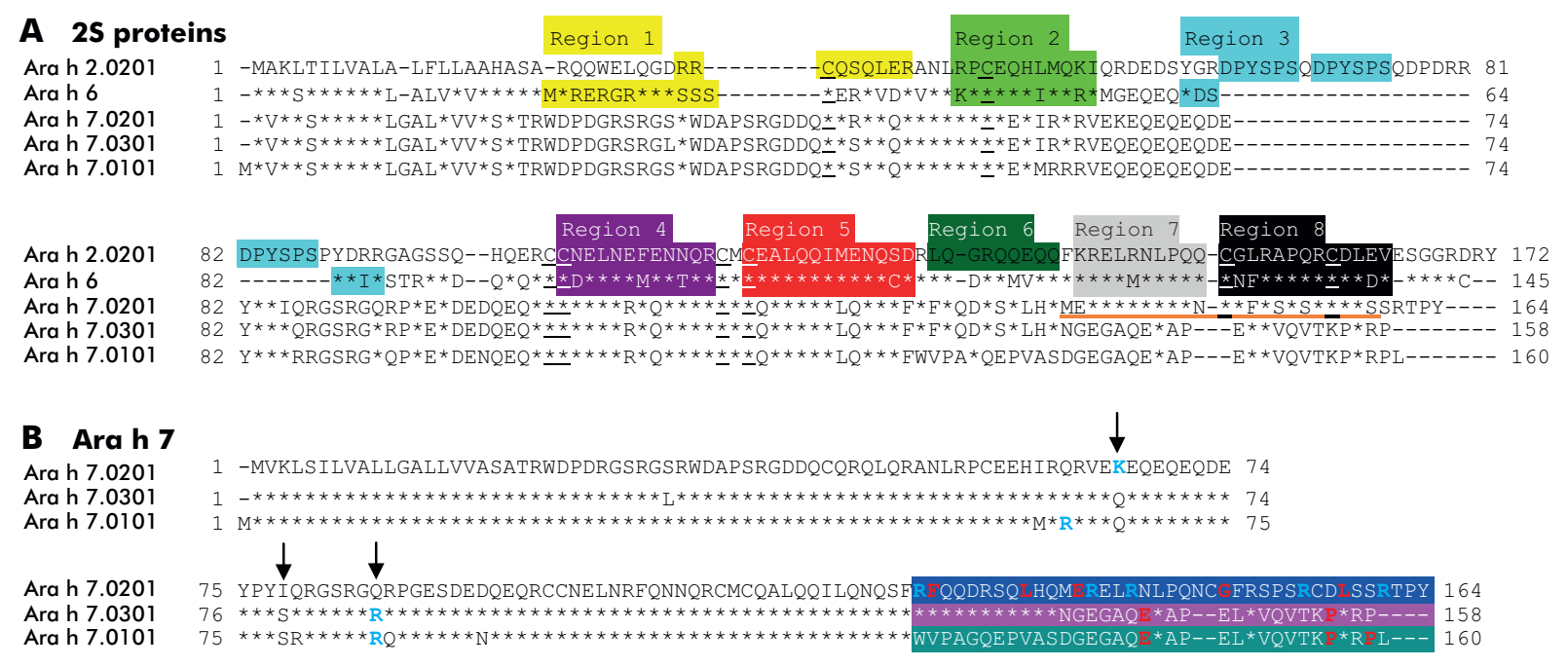

Figure 4: Sequence alignment of Ara h 2.0201, 6.0101, 7.0201, 7.0101 and 7.0301

A Known allergenic epitopes of Ara h 2.0201 and 6 are color highlighted and explained below. Cysteine residues are underlined. Stars indicate similarities compared to Ara $h$ 2.0201. C-terminal similarity of Ara $h 7.0201$ compared to Ara $h 2.0201$ and 6 is underlined in orange.; $\boldsymbol{B}$ Sequence alignment of Ara $h$ 7.0201, 7.0101 and 7.0301. Similarities compared to Ara $h 7.0201$ are indicated with stars. Arrows indicate differences in amino acid sequence of Ara $h 7.0201$ to both other isoforms. Blue residues indicate trypsin cleavage sites and red residues pepsin cleavage sites. The C-terminus is highlighted at the end.

\section{D-structural differences between Ara $\mathrm{h} 7$ isoforms related to known allergenic epitope sites of Ara h 2.0201 and 6.01}

In contrast to Ara h 2 and 6, of which crystal structures have been described ${ }^{2,19}$, no crystal structure is available for the isoforms of Ara $\mathrm{h} 7$ and isoforms Ara h 2.0201 and 6.01. In addition to the sequence alignment, predictive protein 3D-models of all isoforms were therefore created with PHYRE and UCSF Chimera (Figure 5). Known allergenic linear epitopes of Ara h 2.0201 and 6.01 are highlighted in the same colors as displayed in Figure 4 (Figure $5 a$ and $b)^{18}$. Figure $5 \mathrm{c}$-e shows the predicted 3D models of the Ara $\mathrm{h} 7$ isoforms. The sequence alignment indicated that most differences were located in the C-terminus. In the 3D-models, a main structural difference with Ara h 7.0101 was observed in this C-terminus (turqoise), and some smaller differences were observed between Ara h 7.0201 and 7.0301 in this region (pink vs blue). The three amino acids that differ between these three isoforms (light blue) were all located in a loop which is a known epitope region for Ara h 2.0201 and 6.01 (region 3). Since these changes in amino acids can influence hydrophobicity, polarity and charge, an amino acid distance analysis was performed (UCSF Chimera, Suppl. Figure S1). Indeed, mainly in the loop region (green circle), differences in distance between amino acid residues were observed (Suppl. Figure S1). 
A Ara h 2.0201
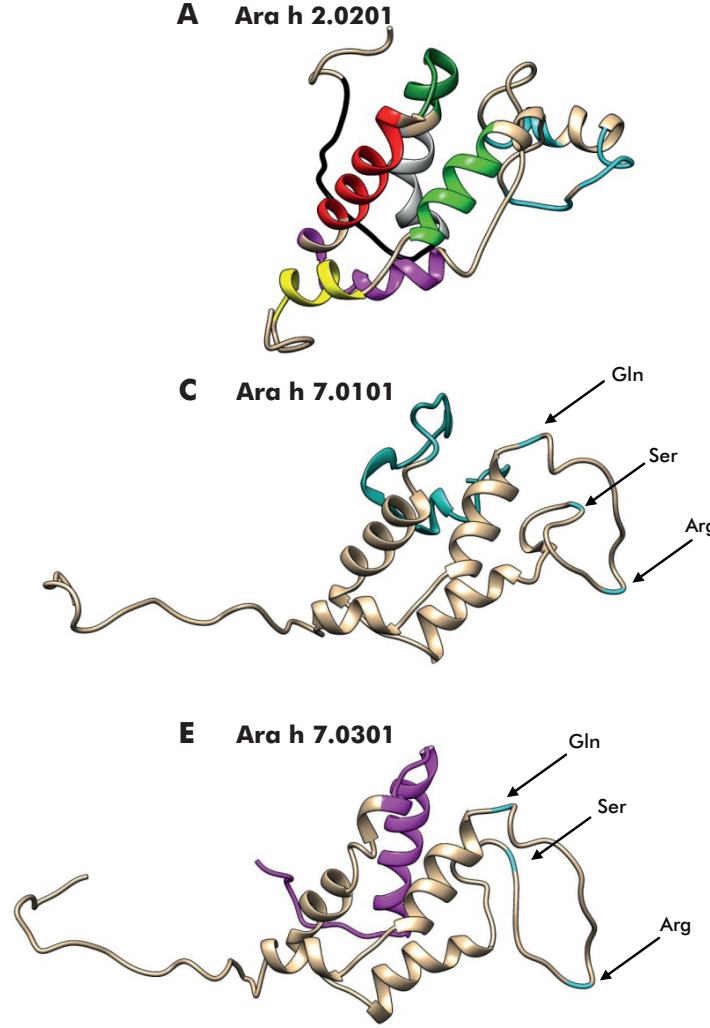

B Ara h 6.01

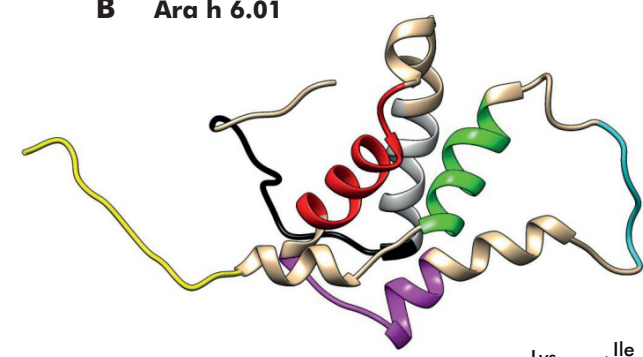

D Ara h 7.0201

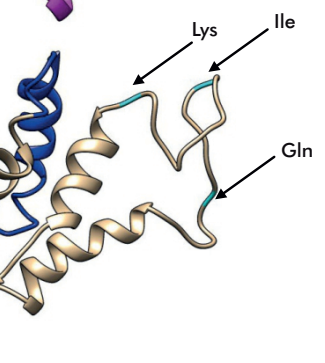

Ara h 6.01

MRRERGRQGDSSS

KPCEQHIMQR

YDSYDIR

CDELNEMMENTQR

CEALQQIMENQCD

KRELMNLPQQ

CNFRAPQRCDLDV

Figure 5: 3D protein prediction models of Ara $\mathrm{h} 2.0201,6.0101$ and 7

A-B 3D-model of Ara $h 2.0201$ and 6, known allergenic epitopes are indicated in color according to the color-scheme of Figure 4.; C-E 3D prediction models of the three Ara $h 7$ isoforms. Turquoise (Ara $h$ 7.0101), blue (Ara $h$ 7.0201) or pink (Ara $h$ 7.0301) indicates the $C$-terminus, light blue residues indicated with arrows indicate the main differences between Ara $h 7.0201$ and the other two isoforms in loop region 3.

\section{Discussion}

Ara $\mathrm{h} 2$ and 6 have proven to be two of the most informative peanut-allergens in the diagnosis of peanut allergy, since most patients have specific lgE against one or both allergens $^{7}$. In addition, the current study showed that $80 \%$ of the 15 peanut-allergic patients studied, were sensitized to one or multiple isoforms of a third recombinant $2 S$ albumin member Ara h 7, mostly in combination with recombinant Ara h 2.0201 or 6.01. This is probably explained by the sequence identity between these three isoforms and Ara h 2.0201 and 6.012,5,6,8,9. Ara h 7.0201 showed the highest sensitization frequency amongst peanut-allergic patients $(80 \%)$, which was comparable to sensitization to Ara h 2.0201 and 6.01 (93 and $80 \%$, respectively).

To the best of our knowledge, this is the first time that the functionality of recombinant Ara $\mathrm{h} 7$ isoforms was tested, rather than only determining specific lgE binding in patient samples. Although the BAT assay can be a variable assay, grouped results indicate that Ara h 7.0201 was overall able to induce basophil degranulation comparable to Ara h 2.0201 and 6.01. In two independent patients, Ara h 7.0201 and 7.0301 were able 
to induce basophil degranulation at relatively low concentrations of allergen comparable to CPE and Ara h 2.0201 and 6.01, suggesting that these specific Ara h 7 isoforms can be recognized by sensitized individuals and increase efficacy in stimulating basophil degranulation. While this could not be directly related to sensitization levels of the line blot strips, it indicates that some patients can react to low concentrations of Ara $\mathrm{h} 7$. Although Ara h 7 represents only $0.5 \%$ of peanut protein content, in contrast to $4-14 \%$ for Ara $\mathrm{h} 2$ and $6^{3,12}$, this allergen has the potency to induce responses at low concentrations. Sensitization to isoform Ara h 7.0101 was observed in $60 \%$ of peanut allergic patients, although biologic activity was observed in only one patient. Ara h 7.0101 was identified with phage display technology, but could not be retrieved in peanut extract ${ }^{5}$, which is most likely the explanation for this reduced biologic activity. Cross-reactivity between the three isoforms may explain the observed sensitization for this isoform.

A limitation of this study is the lack of native Ara $\mathrm{h} 7$ which is not commercially available and difficult to isolate due to its low abundance. Hence, all experiments were performed with recombinant proteins. Native Ara h 2 and 6 have been shown to induce basophil degranulation at lower concentrations of allergen than those of the recombinant proteins used in this study ${ }^{9}$. Nevertheless, the recombinant Ara $\mathrm{h} 7$ proved to be able to induce basophil degranulation in some patients at already low concentration, indicating that it might even be more reactive in crude peanut extract.

A limitation of the BAT assay is that the response of patients to specific allergens can be significantly variable and does not always follow the typical bell-shaped dose-response curve $^{20}$ as e.g. observed for patient N10 and N14. The BAT tests performed in this study were only performed once for each patient. Nevertheless, Ara h 7.0201 appeared to be the most promising Ara $\mathrm{h} 7$ isoform in optimizing peanut allergy diagnosis, as it possessed a similar sensitization profile and efficacy in basophil degranulation compared to recombinant Ara h 2.0201 and 6.01. Moreover, Ara h 7.0201 contains potentially unique epitopes compared to the other two Ara $\mathrm{h} 7$ isoforms, since patients were more often sensitized to this particular isoform and its recognition was partly exclusively accompanied by Ara h 2.0201 and 6.01 (N03, N04 and N11). Sensitization to one of the other two isoforms in combination with Ara $\mathrm{h} 2.0201$ and/or 6.01 was not observed. Although Ara h 7.0201 contains cross-reactive epitopes with Ara h 2.0201 and 6.01, a previous study indicated that mono-sensitization against Ara $\mathrm{h} 7.0201$ was observed in two out of fifteen patients ${ }^{10}$. This suggests that Ara h 7.0201 indeed contains one or more epitopes not present on the other Ara $h 7$ isoforms or Ara $h 2.0201$ and 6.01. The similarity of Ara $\mathrm{h} 7.0201$ in sensitization and basophil degranulation with Ara h 2.0201 and 6.01 is most likely related to the C-terminus of Ara h 7.0201, as it fits into the conserved cysteine conglutin family pattern of at least eight cysteine residues ${ }^{5}$. These C-terminal cysteine residues are important for protein stability and determine the $\lg E$ binding of allergens ${ }^{21}$. By $3 \mathrm{D}$ protein modeling, the three main differences in 
amino-acid sequence of the Ara $\mathrm{h} 7$ isoforms were visualized in a loop region that is a known allergenic epitope for Ara h 2.0201 and 6.01. Due to these amino acid substitutions, small changes in distances between amino acids occur, which could contribute to enhanced exposure of an epitope. Combining these two findings, it is expected that the unique epitopes of Ara h 7.0201 are located in either the C-terminus or in this loop region 3 . Therefore, differences in enzymatic digestion by pepsin and trypsin may influence the allergenicity of Ara $\mathrm{h} 7$ isoforms. Taken together previous data ${ }^{10}$ and the data presented in this paper, we hypothesize that determining specific IgE to Ara $h 7.0201$ can be of additional value in peanut allergy diagnosis. Ara $\mathrm{h} 7.0201$ contains unique epitopes and is functionally as active as Ara h 2.0201 and 6.01 in inducing basophil degranulation. In addition, in some patients Ara h 7 can already provoke basophil degranulation at low concentrations. Due to cross-reactivity between Ara h 2.0201, 6.01 and 7 , the latter one could have a potential strong diagnostic value.

\section{Acknowledgements}

Line blots and reagents were kindly provided by Euroimmun. Further funding was provided by a Dutch Government STW Funding, project number 12652 NUTRALL (Nutrition-based approach to support antigen-specific immunotherapy for food allergies). 


\section{References}

1. Muraro, A. et al. EAACI Food Allergy and Anaphylaxis Guidelines: diagnosis and management of food allergy. Allergy 69, 1008-25 (2014).

2. Lehmann, K. et al. Structure and stability of $2 \mathrm{~S}$ albumin-type peanut allergens: implications for the severity of peanut allergic reactions. Biochem J 395, 463-72 (2006).

3. Van Erp, F. C. et al. Using Component-Resolved Diagnostics in the Management of Peanut-Allergic Patients. Curr Treat Options Allergy 3, 169-80 (2016).

4. Miller, D. S., Brown, M. P., Howley, P. M. \& Hayball, J. D. Current and emerging immunotherapeutic approaches to treat and prevent peanut allergy. Exp Rev Vaccines 11, 1471-81 (2012).

5. Schmidt, H. et al. Detection and Structural Characterization of Natural Ara h 7, the Third Peanut Allergen of the 2S Albumin Family. J Prot Res 9, 3701-9 (2010).

6. Zhou, Y. et al. Peanut Allergy, Allergen Composition, and Methods of Reducing Allergenicity: A Review. Int J Food Sci 2013, 1-8 (2013).

7. Van Erp, F. C. et al. The IgE and basophil responses to Ara $\mathrm{h} 2$ and Ara $\mathrm{h} 6$ are good predictors of peanut allergy in children. J Allergy Clin Immunol 139, 358-60.e8 (2017).

8. Pedrosa, M. et al. Utility of specific $\lg \mathrm{E}$ to Ara $\mathrm{h} 6$ in peanut allergy diagnosis. Ann Allergy Asthma Immunol 115, 108-12 (2015).

9. Koppelman, S. J. et al. Purification and immunoglobulin E-binding properties of peanut allergen Ara h 6: evidence for cross-reactivity with Ara h 2. Clin Exp Allergy 35, 490-7 (2005).

10. Blankestijn, M. A. et al. Specific lgE to peanut $2 S$ albumin Ara $h 7$ has a discriminative ability comparable to Ara h 2 and 6. Clin Exp Allergy 48, 60-5 (2017).

11. Moreno, F. J. \& Clemente, A. 2S Albumin Storage Proteins: What Makes them Food Allergens? Open Biochem J 2, 16-28 (2008).

12. Koppelman, S. J. et al. Quantification of major peanut allergens Ara $\mathrm{h} 1$ and Ara $\mathrm{h} 2$ in the peanut varieties Runner, Spanish, Virginia, and Valencia, bred in different parts of the world. Allergy 56, 132-7 (2001).

13. Altschul, S. F. et al. Basic local alignment search tool. J Mol Biol 215, 403-10 (1990).

14. Yan, Y.-S. et al. Isolation of peanut genes encoding arachins and conglutins by expressed sequence tags. Plant Science 169, 439-45 (2005). 
15. Sitaru, C. et al. Enzyme-linked immunosorbent assay using multimers of the 16th non-collagenous domain of the BP180 antigen for sensitive and specific detection of pemphigoid autoantibodies. Exp Dermatol 16, 770-7 (2007).

16. Kelley, L. A. \& Sternberg, M. J. E. Protein structure prediction on the Web: a case study using the Phyre server. Nat Prot 4, 363-71 (2009).

17. Pettersen, E. F. et al. UCSF Chimera: A visualization system for exploratory research and analysis. J Comput Chem 25, 1605-12 (2004).

18. Otsu, K., Guo, R. \& Dreskin, S. C. Epitope analysis of Ara h 2 and Ara h 6: characteristic patterns of lgE-binding fingerprints among individuals with similar clinical histories. Clin Exp Allergy 45, 471-84 (2015).

19. Mueller, G. A. et al. Ara h 2: crystal structure and lgE binding distinguish two subpopulations of peanut allergic patients by epitope diversity. Allergy 66, 87885 (2011).

20. Santos, A. F. \& Lack, G. Basophil activation test: food challenge in a test tube or specialist research tool? Clin Transl Allergy 6, 1 (2016).

21. Banerjee, B. et al. C-Terminal Cysteine Residues Determine the IgE Binding of Aspergillus fumigatus Allergen Asp f 2. J Immunol 169, 5137-44 (2002). 


\section{Supplementary}

A

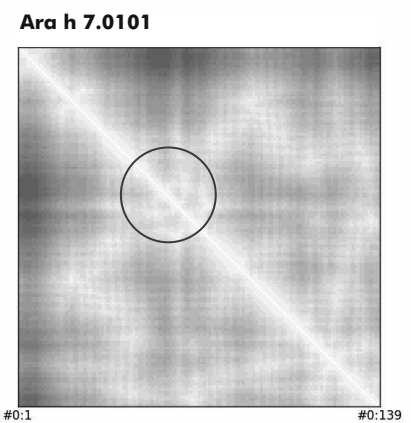

B

Ara h 7.0201

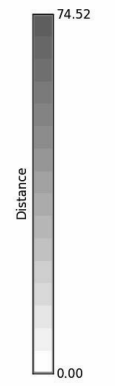

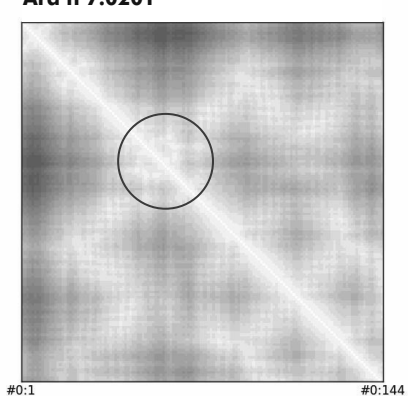

C

Ara h 7.0301

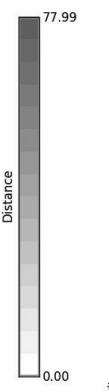

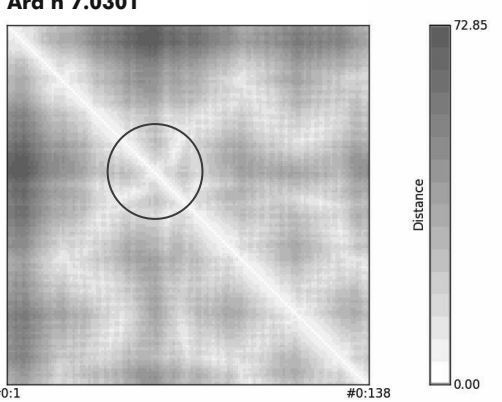

Figure S1: Residue distance map

Distance maps of $\boldsymbol{A}$ Ara $h$ 7.0101, B 0201 and $\boldsymbol{C}$ 0301. The distance of each amino acid is related to every other amino acid. The distances between the residues are indicated according to the color scale $(\AA)$, where white indicates that amino acid residues are in close proximity. 
MIUT:

AGCCTCTGGA7

CCTGTTTCTGCAAA

iGCGTGGTCCAGCCTC

AGGGCCGATTCACCATL,

AATTGTGTTGACACAGTCTCCAGCCALU

'TGGCATCCCAGCCAGGTTCAGTGGCAGTGGG'TC I

CAGATGACCCAGTCTCCTTCCACCCTGGCTGCATCTGTAG

AGTCCCATCAAGGTTCAGCGGCAGTGGGTCTGGGACAGAGTTCACL

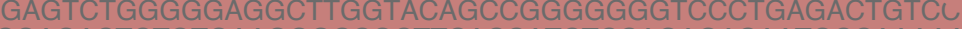

ITATCGAGACTCTGTGAAGGGCCGCTTCACCATCTCCAGAGACAATGCCAAAAAC ¿AGGGGACCACGGTCATCGTCTCCTCAGCAGGTGCAGCTGGTGGAGTCTGGGGGAGG iGAGTGGGTTTCATTTATACGCAAGGATGGAGCTAGTAAATACTATGGAGACTCCGTGAAGGG , CCCTGGGTTACTTTGACTACTGGGGCCAGGGAACCCTGGTCACCGTCTCCTCAGGAAATTGTGT CATAAACCTGGCCAGGCTCCCAGGCTCCTCATCTATGATGCATCCAACAGGGCCACTGGCATCCC ACTGGCCTCCGCTCACTTTCGGCGGAGGGACCAAGGTGGAGATCAAACGACATCCAGATGACCCAG

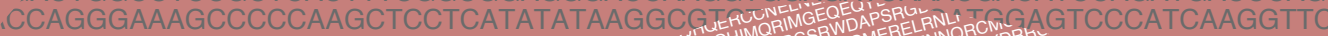

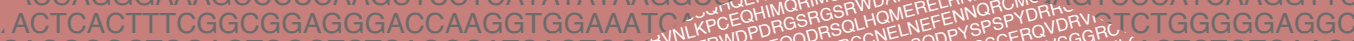

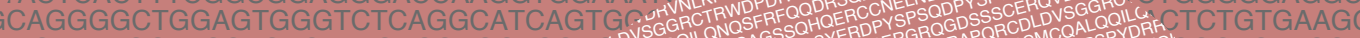

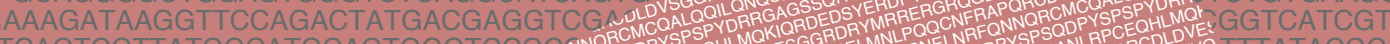

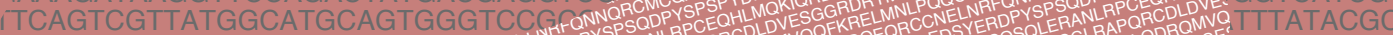

TCCTGCAGGGCGGLRAORO tGCCTAGAGC

\section{GCCTGATGAT}

\section{CACGTTTAGG}

\section{ATGGCCAGTCE}

\section{CCATCTCCAGAG}

GTCTCCAGCCACCE

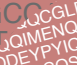

\section{STRGQRPPCER}

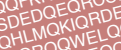

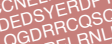

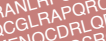

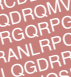

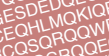

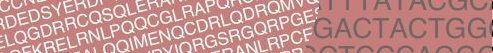

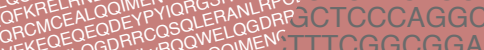

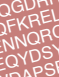
CEALQQ Q

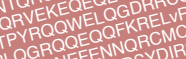
SDQQQRANLPCEEHRTPYRQQUEQOFNARCMCESTSSDO A GCTCCTCATA

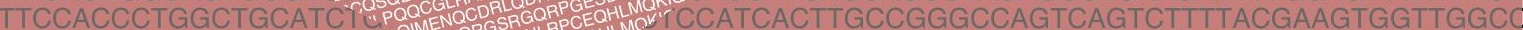

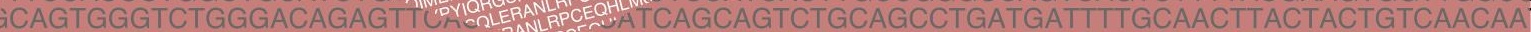
ACAGCCGGGGGGGTCCCTGAGACTGTCCTGTGCAGCCTCTGGATTCACGTTTAGGAGATATGCTTTGAGTTGGGTCCGC GCTTCACCATCTCCAGAGACAATGCCAAAAACATCCTGTTTCTGCAAATGGCCAGTCCGAGAGTCGAAGACGCGGCCGTT TCAGCAGGTGCAGCTGGTGGAGTCTGGGGGAGGCGTGGTCCAGCCTGGGGGGTCCCTGAGACTCTCCTGTGTAGCGTCT aATGGAGCTAGTAAATACTATGGAGACTCCGTGAAGG iCCAGGGAACCCTGGTCACCGTCTCCTCAGGAAATT \&CCACCCTGTCTTTGTCTCCAGGGGAAAG UTCCTCATCTATGATGCATCCAACAGGGCCACTGGC VGTGGGTCTGGGACAGACTTCACTCTCA aGAGGGACCAAGGTGGAGATCAAACGACATCCAGAT . CATATATAAGGCGTCTAATTTACAAGATGGAGTCCCAT जGCATCAGTGGCAGTGGAAACAAAACATATTATCGAGACT ^ACGAGGTCGAGATTATGGACGTCTGGGGCCAGGGGACCAC GGGTGTATATTACTGTGCGAAAGATAGGTCCCTGGGTTACTTTO ?TTGGCAGCTACTTAGCCTGGTACCAACATAAACCTGGCCAGG

TTTATTACTGTCAGCAGCGTAGCGACTGGCCTCCGCTCAC

- $\simeq$ CTTGTATCACPAn.

,ACTTTCGGCGGAGGG ᄀGCTGGAGTGGGTCT GGTTCCAGACTATG TGGCATGCAGTGG CTGAGGACACGG 'AGTCAGAGTCT AGATTTTGCAGT TTACGAAGTGG ACTTACTACTG CTTTGAGTTG :GAAGACGCGG :TCTCCTGTGT AAAGACACTCT iTCTCCAGGGG CAGACTTCAC GAGACAGAGT ACCCTCACCATC TGTCCTGTGCAG ;AAAAACATCCTGT İGGGAGGCGTGG
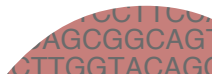
GGGCCGCTTCACCAT GTCTCCTCAGCAGGTG GAGGATGGAGCTAGTA GAAGGATGGAGCTAGTA
GGGAGGGAACCCTGGTCACCGTCTCCTCAGGAAATTGTGTTGACA

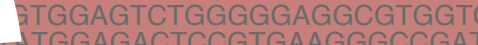
AGCTCCTCATCTATGATGCATCCAACAGGGCCACTGGCATCCCAGCCAC GGAGGGACCAAGGTGGAGATCAAACGACATCCAGATGACCCAGTCTCC ATATATA $\triangle \triangle T T$ TCAAGATGGAGTCCCATCAAGGTTCAGCGG ATATATA
GTGG AGTTGGTGGAGTCTGGGGGAGGCTTGGTA
AATATTATCGAGACTCTGTGAAGGGCCGC CAGGGGACCACGGTCATCGTCTCCTC GTGGGCAAMTITCATTTATACGCAAGGATG GTGCGAAAGATA AGCAGCGTAGCGAC TATCAGCAGAAACCAGG GTATCAGCAGAAACCAG AGGCTCCAGGGCAGGGG TTACTGTGCGAAAGATAAG GGATTCACCTTCAGTCGTTA CAAATGGACAGCCTGAGA AACCCTCTCCTGCAGGG TCAGCAGCCTAGAGCC TTTGCCGGGCCAGTC GGTTACTTTGACTACTGGGGCCAGG GGCCAGGCTCCCAGGCTCCTCAT GCTCACTITCGGCGGAGGGACCA CCCAAGCTCCTCATATATAAGGQ GGAGGGACCAAGGTGGAAATCAA GGGTCTCAGGCATCAGTGGCAG ACTATGACGAGGTCGAGATTATG GCAGTGGGTCCGCCAGGCTCCAC ACACGGCTGTATATTACTGTGCG AGTCTTGGCAGCTACTTAGCCTC TGCAGTITATTACTGTCAGCAGC CGAAGTGGTTGGCCTGGTATCAC TACTACTGTCAACAATATGATAGTC TCCGTGAAGGGCCGA

CCTCCTCAGGAAATTGTGTTGACACAG

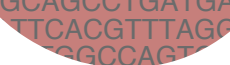
TGAGTTGGGTCCGCCAGGCTCCA AAGACGCGGCCGTITATTACTGTGC CTCCTGTGTAGCGTCTGGATTCACCT IGGTCTGGGACAGACTTCACTCTCACCATCAGCAG ACGACATCCAGATGACCCAGTCTCCTTCCACCCTGGCTGCATCTGTAGGAGACAGAGTCTCCATCACTTGCCGG GATGGAGTCCCATCAAGGTTCAGCGGCAGTGGGTCTGGGACAGAGTTCACCCTCACCATCAGCAGTCTGCAGCG ITGGTGGAGTCTGGGGGAGGCTTGGTACAGCCGGGGGGGTCCCTGAGACTGTCCTGTGCAGCCTCTGGATTCAC ;ATATTATCGAGACTCTGTGAAGGGCCGCTTCACCATCTCCAGAGACAATGCCAAAAACATCCTGTTTCTGCAAATGC GCCAGGGGACCACGGTCATCGTCTCCTCAGCAGGTGCAGCTGGTGGAGTCTGGGGGAGGCGTGGTCCAGCCTGG GGAGTGGGTTTCATITATACGCAAGGATGGAGCTAGTAAATACTATGGAGACTCCGTGAAGGG

¿CCTGGGTTACTTTGACTACTGGGGCCAGGGAACCCTGGTCACCGTCTCCTCAGGAAATTGTS

ITAAACCTGGCCAGGCTCCCAGGCTCCTCATCTATGATGCATCCAACAGGGCCACTGGCATCr

rGGCCTCCGCTCACTTTCGGCGGAGGGACCAAGGTGGAGATCAAACGACATCCAGATGACCC AGGGAAAGCCCCCAAGCTCCTCATATATAAGGCGTCTAATTTACAAGATGGAGTCCCATCAAGE CACTTTCGGCGGAGGGACCAAGGTGGAAATCAAACGAGGTGCAGTTGGTGGAGTCTGGGGG॰ GGCTGGAGTGGGTCTCAGGCATCAGTGGCAGTGGAAACAAAACATATTATCGAGACTCTGTG,

\AGGTTCCAGACTATGACGAGGTCGAGATTATGGACGTCTGGGGCCAGGGGACCACGGTCATCG I .

1TATGGCATGCAGTGGGTCCGCCAGGCTCCAGGCAAGGGGCTGGAGTGGGTTTCATTTATACGCAAGGATGGAGi I AG I iAGAGCTGAGGACACGGCTGTATATTACTGTGCGAAAGATAGGTCCCTGGGTTACTTTGACTACTGGGGCCAGGGAACCCTG AGGGCCAGTCAGAGTCTTGGCAGCTACTTAGCCTGGTACCAACATAAACCTGGCCAGGCTCCCAGGCTCCTCATCTATGATG GAGCCTGAAGATTTTGCAGTTTATTACTGTCAGCAGCGTAGCGACTGGCCTCCGCTCACTTTCGGCGGAGGGACCAAGGTGGA 


\section{Ara $\mathrm{h} 7$ isoforms share many linear epitopes: Are 3D epitopes crucial to elucidate divergent abilities?}

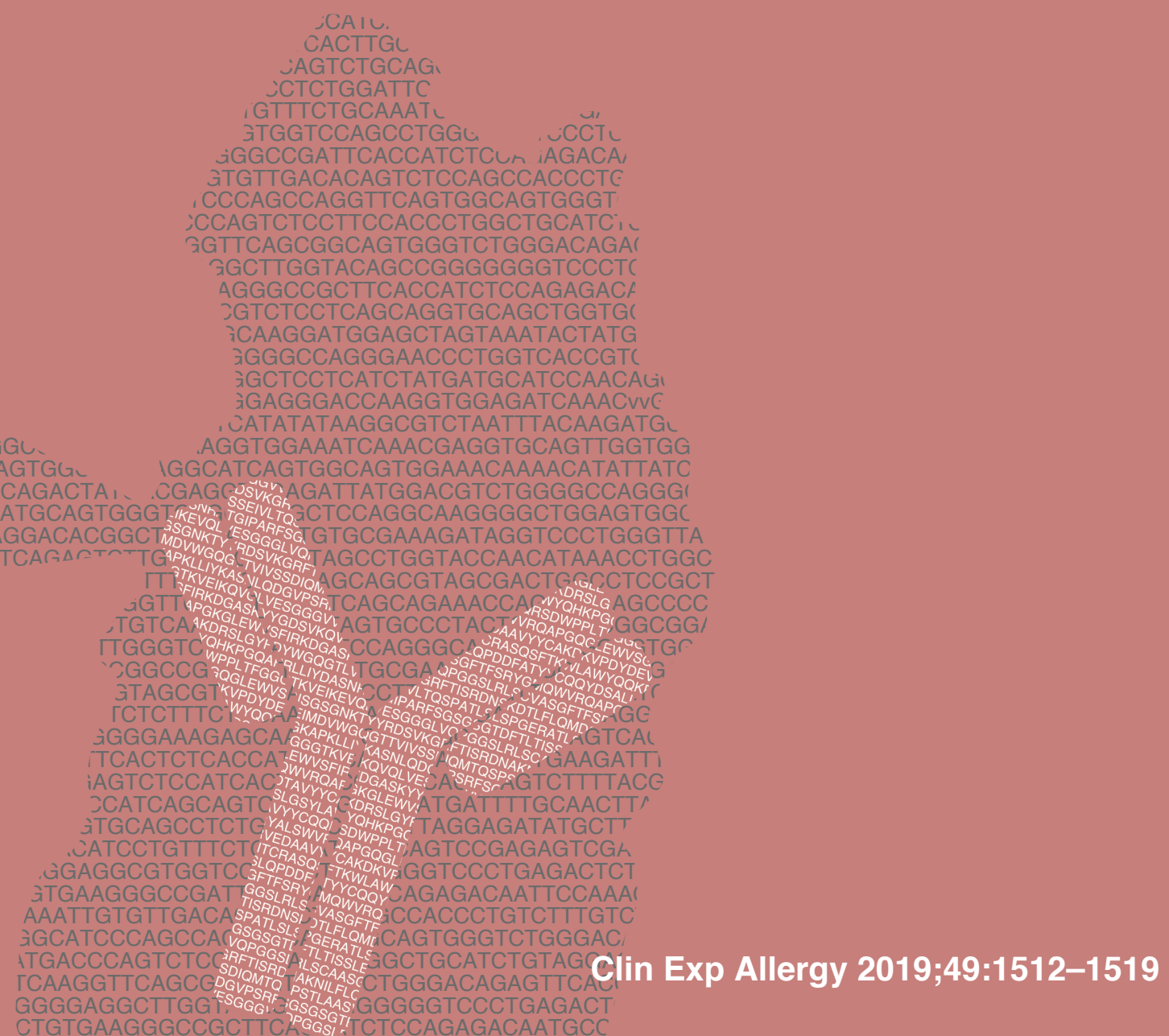

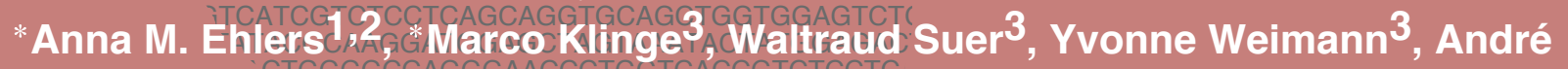

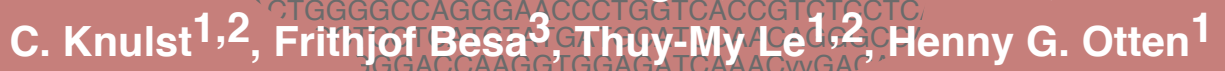

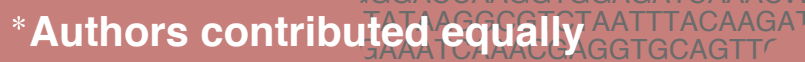

${ }^{1}$ Center for TranslationalimmingGCIOGA Utrecht University, Altrecht, The Netherlands

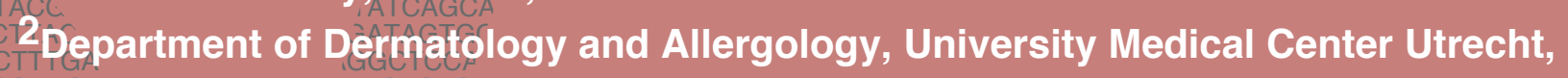
Utrecent University, Utrecht, The Netherlands

BGAGAOIMMUN AGA,CLGGA

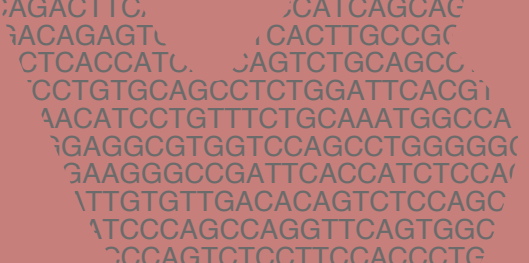




\section{Abstract}

\section{Background}

The peanut allergens Ara h 2, 6 and 7 are potent allergens and can trigger severe reactions. Ara h 7 consists of three isoforms differing in their ability to induce basophil degranulation, whereas the ability of Ara $\mathrm{h} 7.0201$ is comparable to Ara $\mathrm{h} 2$ and 6 as shown in previous literature. Thus, we sought to identify linear epitopes of Ara $h$ 7.0101, 7.0201 and 7.0301 recognised by $\lg E$ and IgG4 from patients sensitised to Ara $\mathrm{h} 7$ and to investigate their potential to elucidate divergent abilities of the Ara $h 7$ isoforms in inducing basophil activation.

\section{Methods}

Linear epitopes recognised by $\lg E$ and $\lg G 4$ were mapped by peptide microarray analysis containing 15mer peptides of Ara h 2.0201, 6, 7.0101, 7.0201 and 7.0301 and 39 peanut allergic patients sensitised to Ara h 7 (discovery). For validation, 20mer peptides containing the minimal epitope and surrounding amino acids were incubated with 25 sensitised patients and 10 controls (validation).

\section{Results}

Three out of 14 linear epitopes were unique for each isoform (Ara h 7.0101: aa 97-109; Ara h 7.0201: aa 122-133; Ara h 7.0301: aa 65-74) but scarcely recognised by lgE. The main linear $\lg E$ epitope (aa 51-57) located in the long flexible loop of all Ara $\mathrm{h} 7$ isoforms was bound by antibodies from $31 \%$ of the patients (discovery and validation cohort). Regarding IgG4, 55\% of the patients recognised an epitope present on all isoforms (aa 55-65) whereas epitope aa 129-137, only present on Ara h 7.0101/.0301, was recognised by $38 \%$ of the patients. Recognition was highly individual, although $20 \%$ of the patients recognised any linear epitope neither by IgE nor by IgG4 despite a low mean $z$-score of $\geq 1.7$. Remarkably, only $50 \%$ of the patients recognised one or more epitopes by lgE.

\section{Conclusion and Clinical Relevance}

Ara $\mathrm{h} 7$ isoforms share many linear epitopes being easily accessible for antibody binding. Unique epitopes, essential to elucidate divergent potencies, were scarcely recognised, suggesting a crucial involvement of conformational epitopes. 


\section{Introduction}

Peanut allergy is one of the most prevalent food allergies worldwide and is often triggered by Ara h 8, a birch pollen-related PR-10 protein, or seed storage proteins such as Ara h 2 and 6 (2S albumins) in Western and Central Europe. While sensitisation to Ara h 8 often results in mild reactions such as oral allergy syndrome, Ara h 2 and 6 can induce severe reactions, including anaphylaxis ${ }^{1,2}$. Ara $\mathrm{h} 7$ is the third member of the $2 S$ albumin family, but it is far less abundant in peanut than Ara $\mathrm{h} 2$ and 6 and shares a sequence homology of $51 \%$ and $61 \%$, respectively ${ }^{3}$.

For diagnosing peanut allergy, IgE antibodies against Ara $\mathrm{h} 2$ and 6 are known to have a very high positive predictive value ${ }^{4,5}$. Recently, IgE binding to Ara $\mathrm{h} 7$ showed a discriminative ability comparable to Ara $\mathrm{h} 2$ and 6 and this $2 \mathrm{~S}$ albumin was as potent as Ara $\mathrm{h} 2$ and 6 in inducing basophil degranulation ${ }^{6}$. For Ara $\mathrm{h} 7$, three different isoforms - Ara h 7.0101, 7.0201, 7.0301 - have been described whilst Ara h 7.0101 has only be detected on cDNA level but not on protein level ${ }^{7}$. Ara $\mathrm{h} 7.0201$ was the most potent isoform to induce degranulation. IgE binding to this allergen were not fully inhibited by Ara $h$ 7.0101, although lgE-binding to Ara $\mathrm{h} 7.0101$ was completely inhibited by Ara $h$ 7.0201. These data suggest the presence of unique epitopes on Ara $h 7.0201^{8}$. By amino acid sequence comparisons, these unique epitopes might be located on the distinctive C-terminus or created by amino acid substitutions within the flexible loops sensitive to pepsin or trypsin digestion ${ }^{6}$.

The aim of the study was to define linear epitopes of Ara $\mathrm{h} 7$ isoforms. To this end, we performed a linear epitope mapping using the peptide microarray technique and applying sera from allergic and tolerant patients sensitised to Ara $h$. 


\section{Methods}

\section{Patient selection}

For identifying linear epitopes, sera with specific IgE to peanut extract (ImmunoCAP) were screened by EUROLINE (EL, Euroimmun AG, Lübeck, Germany) for slgE binding to heterologously expressed Ara $h$ 7.0201. Overall, 39 sera with slgE levels $\geq 16$ intensity units (EL) for Ara h 7.0201 were applied for the peptide microarray analysis, and peanut allergy was confirmed by food challenge according to the international consensus protocol or experienced physician diagnosis (discovery cohort) ${ }^{9}$. The cut-off level was chosen to guarantee the detection of a broad epitope spectrum. In the next phase, the identified epitopes were validated by 25 DBPCFC-confirmed peanut allergic $(n=22)$ or tolerant $(n=3)$ patients with slgE levels $\geq 3$ intensity units for Ara $h 7.0201$ (validation cohort) ${ }^{8,9}$. As control, 10 sera without slgE to Ara h 7.0201 were used. In Table 1 the comparison between CAP-classes, concentrations and Euroline intensity units are shown ${ }^{10}$. Ethical approval was acquired from the biobank committee of the University Medical Center Utrecht, number 18-428.

Table 1: EUROLINE intensities

$\begin{array}{cccccccc}\text { EAST-class } & 0 & 1 & 2 & 3 & 4 & 5 & 6 \\ \begin{array}{c}\text { EL-intensity } \\ \text { level }\end{array} & 0-2 & 3-6 & 7-15 & 16-30 & 31-50 & 51-100 & >100 \\ \begin{array}{c}\text { ImmunoCAP } \\ {[\mathrm{kU} / \mathrm{L}]}\end{array} & <0.35 & 0.35-<0.7 & 0.7-<3.5 & 3.5-<17.5 & 17.5-<50 & 50-<100 & \geq 100\end{array}$

\section{Peptide chip design}

For the discovery phase, a peptide microarray with overlapping 15mer peptides was commercially obtained (PEPperPRINT), comprising the sequences of Ara $h$ 7.0101, 7.0201, 7.0301 (offset: 1), 2.0101, and 6.0101 (offset: 2). According to the experiences of PEPperPRINT, 15 mer peptides have the optimal length to provide sufficient sensitivity without significant induction of secondary structures. All peptides were printed as triplicates with a 3 amino acids linker (2x B-alanine and 1x aspartic acid) to prevent binding of negatively charged fluorescent dyes to positively charged amino acids close to the array surface. For validating the discovered minimal epitopes, a new microarray layout was applied. For each epitope, a 20mer peptide was designed containing the minimal epitope and surrounding amino acids, replenished with glycine and serine amino acids. 


\section{Microarray incubation}

All dilutions and washing steps were performed in working strength universal buffer (EUROIMMUN, purchase order number ZW1100). Patient samples were diluted 1:4 and incubated on the microarrays at $4{ }^{\circ} \mathrm{C}$ overnight. For the detection of bound $\lg \mathrm{E}$ and IgG4 antibodies, the arrays were incubated with biotinylated a-IgE antibody (clone MHE-18 1:5000, BioLegend) and biotin a-human IgG4 coupled with Neutravidin DyLight 680 (clone HP6025, 1:5000, Southern Biotech) for one hour at room temperature. After washing, the arrays were incubated for one hour at room temperature with fluorescent Neutravidin DyLight 800 (Thermo Fisher), diluted 1:5000 for IgE detection. After

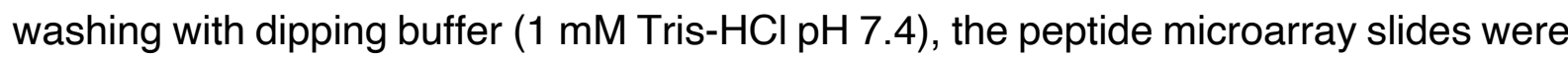
dried and scanned with a Licor Odyssey Imager at a wavelength of $800 \mathrm{~nm}$ (intensity: 10). Image focus was set to $0.8 \mathrm{~mm}$ and an image resolution of $21 \mu \mathrm{m}$ was chosen.

\section{Microarray evaluation}

Fluorescent signals were extracted using Pepslide Analyzer Software (SICASYS) and exported to CSV files. For data evaluation, logarithmic signal-noise-ratios (S) were calculated for each peptide according to:

$$
\mathrm{S}_{i}=\log _{2} \cdot \frac{\text { Total fluorescene (Peptide) }}{\text { Background Flourescence (Peptide) }}
$$

These S-values were normalized against the S-values of blank spots on the array, resulting in a z-score defined as:

$$
\mathbf{Z}_{i}=\frac{S_{i}-\text { Median }(\text { SBlank })}{\text { Mean Deviation (SBlank) }}
$$

Significance levels of positive peptide binding were defined based on z-scores as followed: $z>1.7\left(p<0.05 ;^{*}\right) ; z>2.4\left(p<0.01 ;^{* *}\right) ; z>3.0\left(p<0.001 ;^{* * *}\right) ; z>4.0(p$ $<0.0001$ ). Peptides were only considered if the coefficient of variation for the triplicate of each peptide was lower than $50 \%$. Recognised peptides were defined as epitopes if 3-5 contiguous peptides with a mean z-score $\geq 1.7$ were detected. 


\section{Determination of slgE and slgG4 sensitisation}

Specific IgE and IgG4 sensitisation to the full-length protein Ara $\mathrm{h} 7$ was assessed by line blots (EUROIMMUN, Luebeck, Germany) according to manufacturer's instructions. Briefly, sera were applied (1:11 for lgE, 1:51 for IgG4) overnight and after washing three times with universal buffer, bound IgE were detected by an a-human IgE and ahuman IgG4-antibody labelled with alkaline phosphatase. Visualisation was provided by adding nitro-blue tetrazolium/5-bromo-4-chloro-3'-indolyphosphate substrate for ten minutes after washing three times with universal buffer.

\section{Modelling 3D structure of Ara h 7.0201}

3D structure of Ara h 7.0201 was assessed by Modeller software ${ }^{11}$. Since the amino acid sequences of $2 S$ albumins differ, homology modelling with multiple input sequences and crystal or NMR structures was chosen (Ara $\mathrm{h} 2$ reference 3OB4, Ara $\mathrm{h} 6$ reference $1 \mathrm{~W} 2 \mathrm{Q}$, Ric $\mathrm{c} 3$ reference 1PSY, sunflower $2 \mathrm{~S}$ albumin reference $1 \mathrm{~S} 6 \mathrm{D}$, rapeseed $2 S$ albumin reference 1SM7). Five resulting models were evaluated by DOPE score and the model with the lowest score was selected (DOPE score $=-11083.618$ ).

\section{Model assessment}

To evaluate the 3D model, the DOPE score per residue was assessed. DOPE scores $>-0.3$ indicate levels of relatively high energy, pointing towards structural errors. Additionally, the model was applied to the ModFOLD6 server ${ }^{12,13}$ calculating a residue error plot, a global model quality score (0.5348) and a $p$-value ( $p<0.0001)$ (Figure S1). Overall, the structure was of high quality, apart from the flexible loops which are also experimentally difficult to assess.

\section{Data analysis}

The baseline data were statistically analysed using one-way ANOVA or Mann-WhitneyU-test for continuous data and Fisher's exact test for categorical data. Statistical evaluation was performed with GraphPad Prism 7 (GraphPad Software, La Jolla, CA, USA) and SPSS Statistics 21 (IBM Corporation, Armonk, NY, USA). P values $\geq 0.05$ were considered as statistically significant. 


\section{Results}

\section{Unique linear IgE-epitopes of Ara $\mathrm{h} 7$ isoforms were marginally recog- nised}

To define unique epitopes of Ara $\mathrm{h} 7$ isoforms, we mapped linear epitopes of these proteins by peptide chip analysis using patients' sera with lgE levels for Ara $\mathrm{h} 7 \geq 16$ intensity units (corresponding to ImmunoCAP-class $>2$ ). Patient characteristics are shown in Table 2. Overall, 14 different linear amino acid sequences (A-L) were bound by $\operatorname{lgE}$ (green) or IgG4 (red) as shown in Figure 1a. Epitope codes are listed in Table 3. Epitope $E$ affiliating to all isoforms was recognised by most patients with a frequency of $31 \%$ for $\lg E$ and $5 \%$ for $\lg G 4$ in the discovery cohort. Whilst epitope $E$ was predominately recognised by $\lg E$, epitope $F$ and $L$ were mainly bound by $\lg G 4$ (61.5\% and 54\%). Contrary, the unique epitope $\mathrm{G}$ (Ara $\mathrm{h}$ 7.0301) showed an IgE recognition frequency of only $2.5 \%$ and epitope I (Ara $h$ 7.0101) was not recognised by $\lg E$ at all. These unique epitopes were present in the core of the 3D structure of the proteins and theoretically, they would be only accessible after enzymatic digestion by pepsin or trypsin. In contrast, the unique epitope $\mathrm{K}$ (Ara $\mathrm{h}$ 7.0201) is located on the flexible C-terminus and was recognised by $\lg E$ from $10 \%$ of the included patients. However, this epitope was only recognised by lgG4 (16\%) in the validation cohort (Figure 1b). Overall, epitope E was the main $\lg E$ epitope in the discovery and validation cohort.

\section{Recognition patterns of linear epitopes were highly individual}

All patients showed individual linear epitope recognition patterns as shown in Figure $1 a$ and $b$. Two patients (D-06 and D-35) recognised up to four different epitopes by $\lg E$ in the discovery cohort. Occasionally, epitopes, particularly epitope $E$ and $F$, were bound simultaneously by patients' IgE and IgG4 antibodies (yellow). Moreover, epitopes bound by IgG4 were located in the neighbourhood of an epitope recognised by $\lg E$ in eight patients. For example, epitope $E$ was bound by $\lg E$ and epitopes $D$ and $F$ were bound by lgG4 in patient D-04. Certainly, half of the patients did not show any slgE binding to linear peptides whilst all of them recognised the complete allergens, and ten of them showed at least IgG4-binding. This suggests the importance of conformational epitopes to elucidate strong lgE-binding to the full-length protein. Importance of conformational epitopes was supported by IgE-binding to even fewer linear epitopes in the validation cohort. 


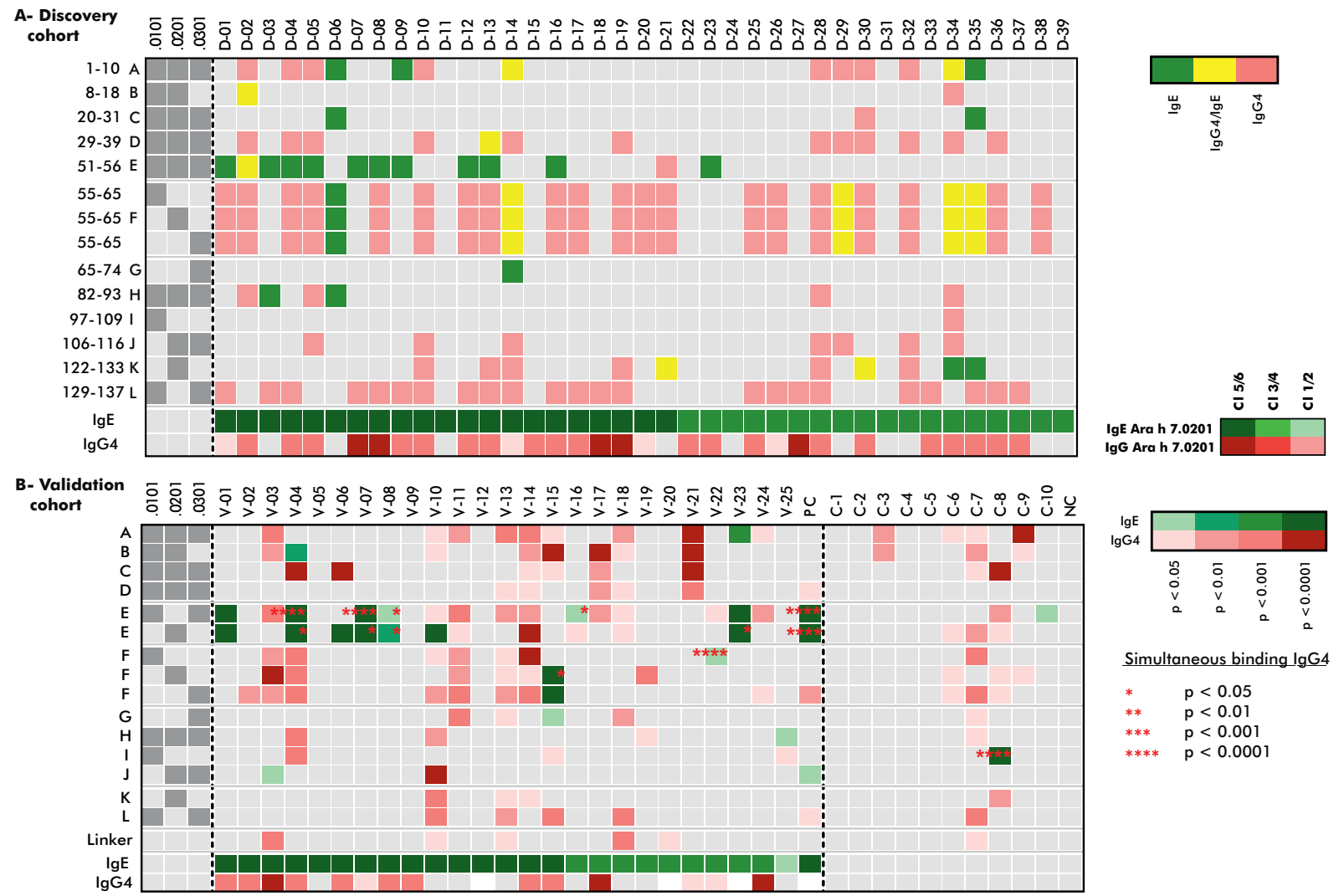

Figure 1: Individual linear epitope recognition

Individual epitope recognition patterns for IgE (green), for IgG4 (red), and for co-recognition by IgE and IgG4 (yellow); on the left-hand side, dark grey dots indicate the respective isoform(s) for each epitope. The relative IgE and IgG4 sensitisation to Ara $h$ 7 is indicated with different gradation of green (IgE) and red (IgG4) in the bottom of each heatmap. A Discovery cohort selected by slgE levels $\geq 16$ intensity units for Ara $h$ 7.0201, the numbers on the left-hand side indicate the different minimal epitopes identified; $B$ Validation cohort selected by slgE levels $\geq 3$ intensity units for Ara $h 7.0201$ and controls without slgE to Ara $h$ 7.0201; for validation purposes, peptides containing the minimal epitope and surrounding amino acids were used resulting occasionally in more than one peptide containing the same minimal epitope (e. g. epitope E). Epitopes recognised by IgE and IgG4 are highlighted based on their significance level $\left({ }^{\star}\right.$ is $p<0.05,{ }^{* *}$ is $p<0.01,{ }^{* * *}$ is $p<0.001,{ }^{\star * * *}$ is $\left.p<0.0001\right)$ 
Table 2: Patient characteristics and sensitisation data

\begin{tabular}{lcccc}
\hline & Discovery cohort & Validation cohort & Control group & p-value \\
\hline Number [n] & 39 & 25 & 10 & \\
Age (median [IQR]) & $25[18-54]$ & $23[18-38]$ & $39[20-66]$ & $0.001^{\mathrm{D}}$ \\
Sex female [n, \%] & $21(54 \%)$ & $8(32 \%)$ & $7(70 \%)$ & 0.084 \\
Food challenge [n, \%] & $6(15 \%)$ & $25(100 \%)$ & $10(100 \%)$ & $<.0001^{\mathrm{E}}$ \\
& & & \\
\hline Symptoms [n, \%] & & & $\mathrm{N} / \mathrm{A}$ & 0.341 \\
\hline Objective & $25(64 \%)$ & $14(56 \%)$ & $\mathrm{N} / \mathrm{A}$ & \\
Subjective & $13(33 \%)$ & $7(28 \%)$ & $8(80 \%)^{\mathbf{A}}$ &
\end{tabular}

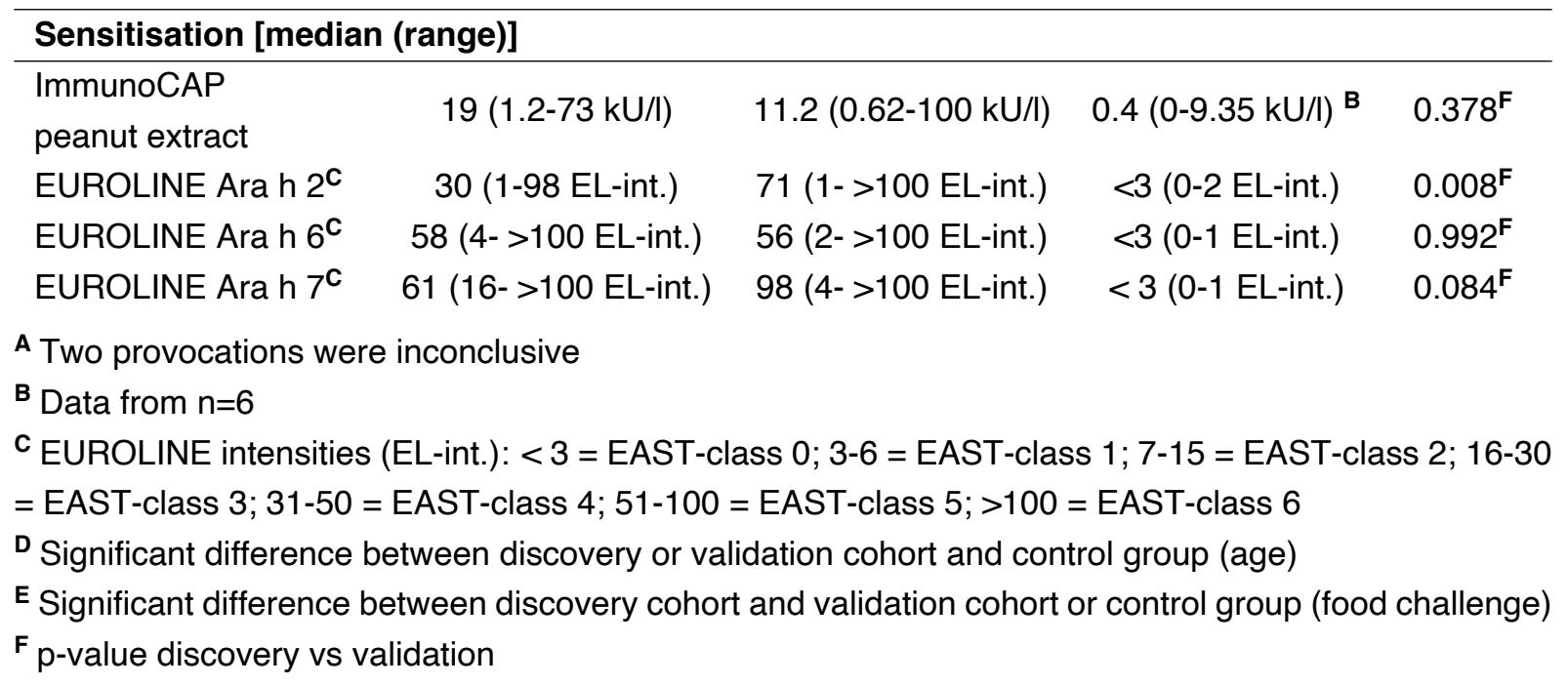

\section{Similar epitope recognition results in divergent ability to induce ba- sophil degranulation}

In a previous study, the ability to induce basophil degranulation was studied for all Ara h 7 isoforms ${ }^{6}$. Patients D-23, V-01 and D-13 correspond to the patients N07, N12 and $\mathrm{N} 14$, respectively, in that study. Even though $\lg E$ antibodies from patients D-23 and $V-01$ recognised only epitope $E$, patient D-23 with high $\lg E$ titres to all full-length proteins showed overall low degranulation, whereas patient V-01, also having high IgE titre, showed strong basophil degranulation after stimulation with Ara h 7.0201. Patient D-13 had a more diverse slgE recognition profile (epitopes $E+D$ which are present on all Ara $\mathrm{h} 7$ isoforms) and degranulation was induced by Ara h 7.0201 and 7.0301, although slgE levels to Ara $\mathrm{h} 7.0301$ were low. These data indicate that IgE recognition patterns of peptides alone cannot elucidate the divergent ability in inducing basophil degranulation. 


\section{Amino acid replacements do not necessarily influence binding and recognition}

A complete overview of identified linear epitopes is shown in Figure 2. Even though epitope $\mathrm{F}$ showed amino acid differences between all isoforms, these differences did not result in divergent minimal epitope recognition within the discovery cohort (Figure 1a). This observation suggested the relevance of residues 55-57 (Tyr-Pro-Tyr) and 6163 (Gly-Ser-Arg) for antibody binding. Surprisingly, antibody binding of epitope F was not influenced by polar amino acids as glutamine and arginine (aa 59, aa 65). However, surrounding amino acids of epitope $F$, as considered in the peptide design for the validation cohort, had a great impact on antibody binding (aa 66; V-02, V-10, V15, V-19, V-22, V-24). Moreover, surrounding residues of epitope $E$ differing between Ara h 7.0101/.0301 and 7.0201 (Lys46GIn and lle58Ser) resulted in divergent antibody binding of the minimal epitope (Figure 1b). IgE antibodies of two patients (V-06 and $\mathrm{V}-10$ ) only recognised the sequence of Ara $\mathrm{h} 7.0201$ and IgG4 antibodies of six patients only the sequence of Ara $h$ 7.0101/.0301. This indicates an oligoclonal antibody repertoire recognising the same minimal epitope.

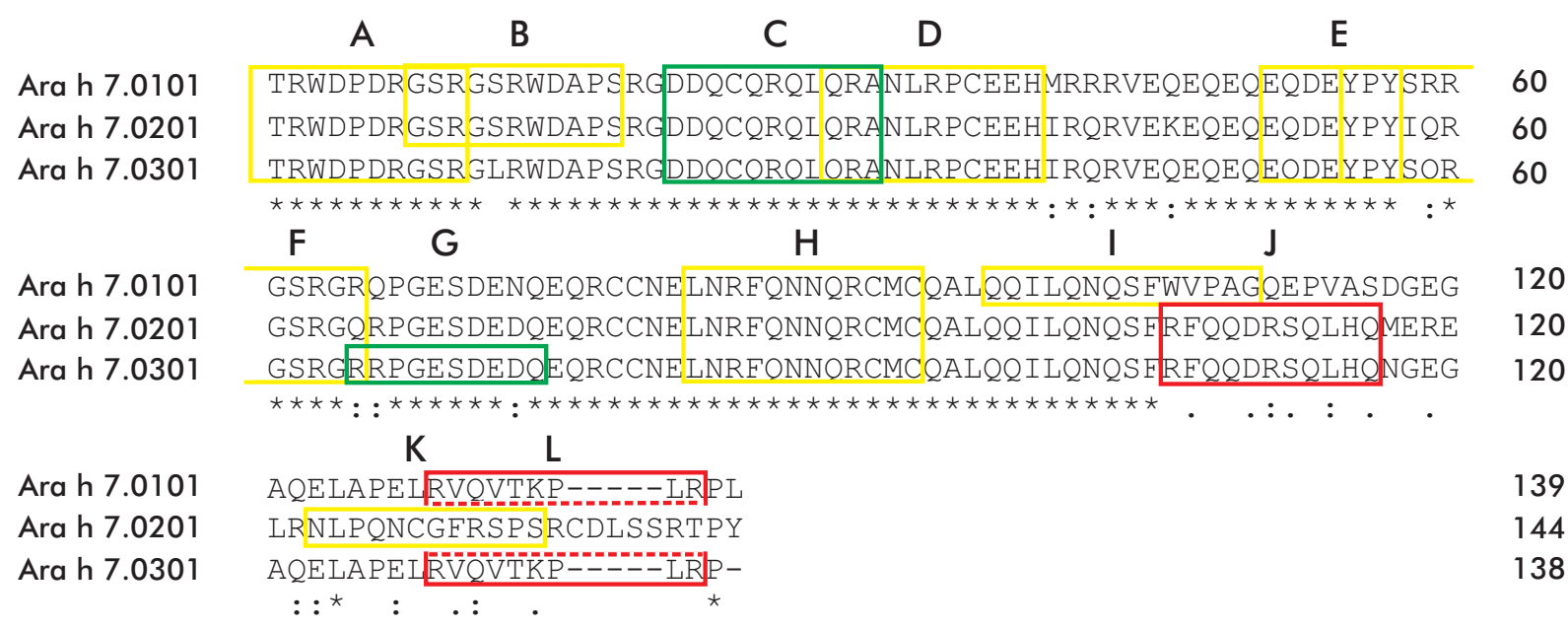

Figure 2: Linear epitopes of Ara $\mathrm{h} 7$ isoforms

Identified linear epitopes highlighted on the amino acid sequences of Ara $h$ 7.0101, .0201 and .0301, green: exclusively recognised by IgE; yellow: recognised by IgE and/or IgG4; red: exclusively recognised by $\lg G 4$ 
Table 3: Linear epitopes of Ara $h 7$ isoforms

\begin{tabular}{|c|c|c|c|c|}
\hline Epitope & Residues $^{A}$ & aa sequence & Isoform & Specificity \\
\hline A & $1-9$ & TRWDPDRGSR & all & $\lg \mathrm{E} / \lg \mathrm{G} 4$ \\
\hline \multirow[t]{2}{*}{ B } & $8-18$ & GSRGSRW- & $.0101, .0201$ & $\lg E / \lg G 4$ \\
\hline & & DAPS & & \\
\hline C & $20-31$ & DDQCQRQIQRA & all & $\lg E / \lg G 4$ \\
\hline D & $29-39$ & QRANLRPCEEH & all & $\lg E / \lg G 4$ \\
\hline \multirow[t]{2}{*}{ E } & $51-57$ & EQDEYPY & all & $\lg E / \lg G 4$ \\
\hline & $55-65$ & YPYSRRGSRGR & .0101 & $\lg E / \lg G 4$ \\
\hline \multirow[t]{2}{*}{$\mathrm{F}$} & $55-65$ & YPYIQRGSRGQ & .0201 & $\lg \mathrm{E} / \lg \mathrm{G} 4$ \\
\hline & $55-65$ & YPYSQRGSRGR & .0301 & $\lg E / \lg G 4$ \\
\hline $\mathrm{G}$ & $65-74$ & RRPGESDEDQ & .0301 & $\lg E$ \\
\hline $\mathrm{H}$ & $82-93$ & $\begin{array}{c}\text { LNRFQNNQR- } \\
\text { CMC }\end{array}$ & all & $\lg E / \lg G 4$ \\
\hline I & $97-109$ & $\begin{array}{l}\text { QQILQNQS- } \\
\text { FWVPA }\end{array}$ & .0101 & $\lg G 4$ \\
\hline J & $106-116$ & RFQQDRSQLHQ & $.0201, .0301$ & $\lg G 4$ \\
\hline K & $122-133$ & $\begin{array}{l}\text { NLPQNCGFR- } \\
\text { SPS }\end{array}$ & .0201 & $\lg E / \lg G 4$ \\
\hline L & $129-137$ & RVQVTKPLR & $.0101, .0301$ & $\lg G 4$ \\
\hline
\end{tabular}

\section{Linear epitopes recognised by IgE are often located on flexible loops}

The location of epitopes was shown by applying epitope recognition patterns of four individual patients on the 3D model of Ara h 7.0201 (Figure 3). Epitopes A, B, E, F, $K$ were situated within the flexible loops of the model and epitopes $D, H, J$ within a combination of a flexible loop and an a-helix. Epitopes A, B, F and $\mathrm{K}$ contained multiple theoretical cutting sites for trypsin and pepsin whilst epitope $E$ contained only cutting sites in the beginning and in the end of its amino acid sequence. Cutting sites of epitope E were altered by substitutions of surrounding amino acids. A cutting site for trypsin was introduced on Ara h 7.0201 (aa 46) and a cutting site for pepsin on Ara h 7.0101/.0301 (aa 56). Contrary, unique linear epitopes of Ara h 7.0101 and .0301 were located in the core, not accessible without enzymatic digestion. Due to less disulphide bridges, these isoforms are probably less resistant against enzymatic digestion, making these amino acid sequences more easily accessible for Ara h 7.0101/.0301 than for Ara $h$ 7.0201. 

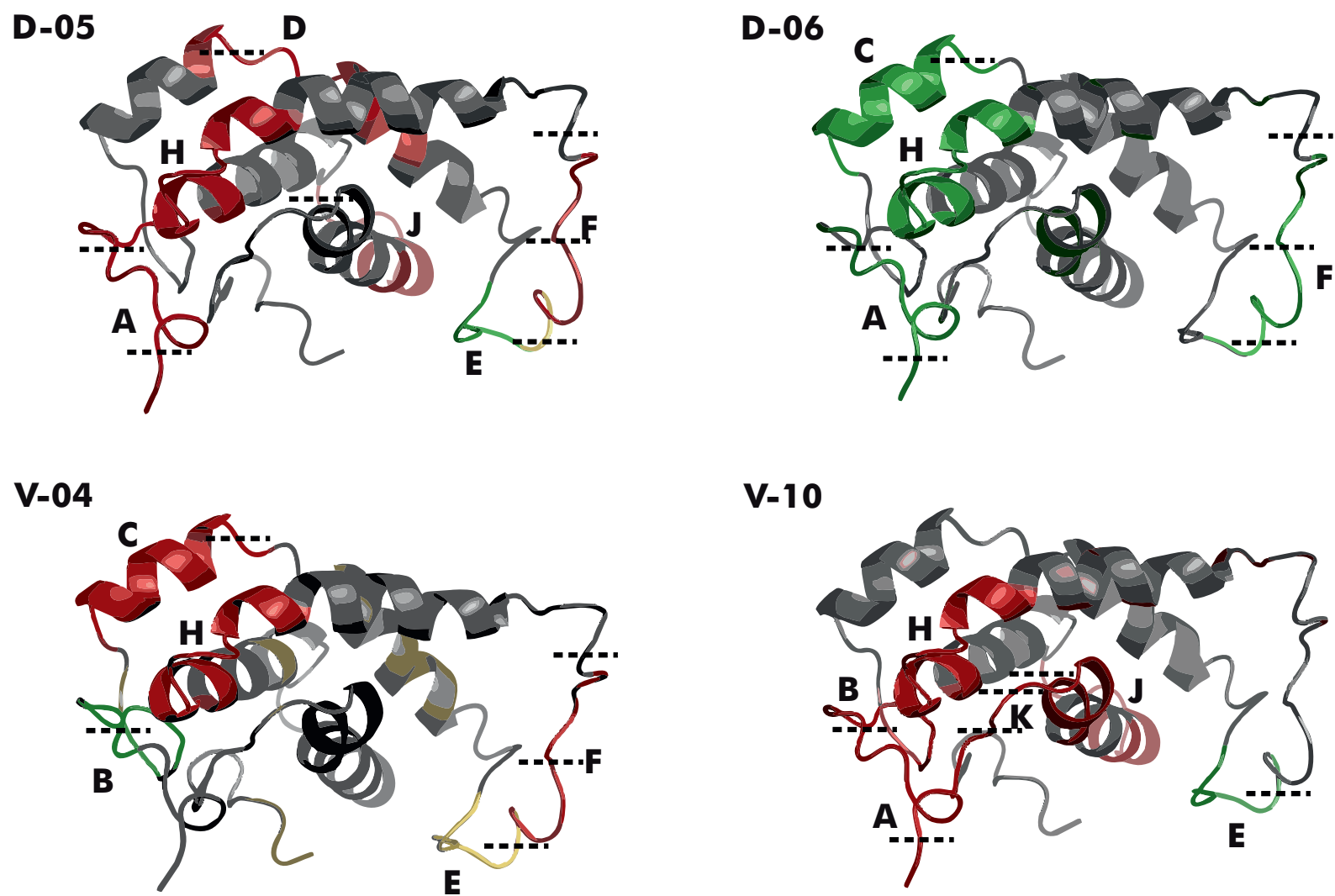

Figure 3: Linear epitopes mapped onto the 3D structure of Ara $\mathrm{h} 7.0201$

Divergent epitope recognition patterns of four selected patients (D-05, D-06, V-04 and V-10) were mapped on the 3D model of Ara $h 7.0201$ and theoretical cutting site were marked with black dash lines. Epitopes recognised by $\mathrm{IgE}$ are highlighted in green and epitopes recognised by IgG4 are highlighted in red.

\section{Discussion}

Divergent abilities of Ara $\mathrm{h} 7$ isoforms in inducing basophil degranulation suggested the presence of unique epitopes for Ara h 7.0201 in comparison with the other isoforms. This hypothesis was supported by inhibition assays showing complete inhibition of lgE binding to Ara h 7.0101 by Ara h 7.0201, although IgE binding to Ara h 7.0201 was not fully inhibited by Ara $\mathrm{h} 7.0101^{6,8}$. These observations can partially be elucidated by the newly mapped linear epitopes in this study. Epitope $E$ was predominately recognised by $\mathrm{IgE}$ in $31 \%$ of the patients whilst epitope $\mathrm{F}$ was immunodominant regarding IgG4 binding (55\%). Nevertheless, unique epitopes of all isoforms were scarcely recognised and linear epitopes in general were infrequently recognised despite the relatively low threshold of a mean $z$-score of $\geq 1.7$. Since the absence of epitope recognition by $\lg E$ was not associated with lower slgE levels to Ara h 7 (Figure 1), lack of sensitivity can be excluded. Thus, our results suggest the importance of conformational epitopes and to lesser extent the importance of $\mathrm{IgE}$ antibodies with deviant affinities to understand divergent abilities of the Ara $\mathrm{h} 7$ isoforms in inducing basophil degranulation. 
The main linear $\lg E$ epitope $(E)$, located in the long flexible loop, was recognised by only $32.5 \%$ of the patients. Other epitopes were detected by even less patients. Limited IgE binding to linear epitopes has also been observed for Ara h 6 and to a fewer extent for Ara h 2. For Ara h 6, seven linear IgE epitopes have been identified whereof the main epitope (aa 97-106 without signal sequence) was recognised by only $30 \%$ of the patients, comparable to our data for Ara h 7 (31\% for epitope E). Regarding Ara $h$ 2 , linear epitopes were recognised by a greater number of patients, varying from $75 \%$ to $100 \%$ of the included patients depending on the study considered (aa 10-18 and aa 42-60 without signal sequence) ${ }^{14,15}$. Contrary to the other two $2 S$ albumins, Ara $\mathrm{h} 2$ is endowed with a very long flexible loop containing the main epitope and five proline residues (cf. 0 for Ara h 6 and 1 for Ara h 7). Proline residues can be modified by Maillard reaction in presence of reducing sugars occurring during the roasting process. This modification has been shown to increase the allergenicity of Ara $\mathrm{h} 2$ and underlined the importance of proline residues ${ }^{16,17}$. However, deletion of flexible loops containing the main linear epitopes diminished the slgE binding only on an individual basis, showing the additive of conformational epitopes to elucidate lgE binding to Ara $\mathrm{h} 2^{16,18}$.

Recognition of linear Ara $\mathrm{h} 7$ epitopes by individual patients was highly distinct, stretching from multiple to none linear epitopes recognised by $\mathrm{lgE}$. Individual recognition patterns were also observed for Ara h 2 in previously conducted studies ${ }^{19,20}$. Occasionally, in the present study epitope recognition by $\lg \mathrm{E}$ was accompanied by $\lg \mathrm{G} 4$ binding for the same epitope. Additionally, IgG4 binding was observed in the neighbourhood of an epitope bound by $\lg \mathrm{E}$. Consistently with our results, overlapping $\lg \mathrm{E}$ and $\lg \mathrm{G} 4$ epitopes for Ara h 1, 2, 3, 6, 8, and 9 were described previously in partly severely reacting peanut allergic patients ${ }^{21}$. Moreover, amino acids important for antibody binding were consistent for $\lg \mathrm{E}$ and $\lg \mathrm{G} 4$ binding which is in line with our observation for epitope $\mathrm{F}^{20}$. Contrary, amino acid replacements in the surrounding area of epitope $E$ influenced the binding of $\lg E$ and IgG4 antibodies. While aa 46 was important for IgG4 binding in six patients, aa 58 was important for IgE binding in two patients, suggesting an oligo- or polyclonal response towards one epitope and limited clonal relation between IgE and IgG4 antibodies, at least in some patients. Overall, IgE and IgG4 can bind the same epitope, also simultaneously, although critical amino acids can differ for IgE and lgG4 antibodies.

Linear epitopes were mostly located in the flexible loops of the 3D protein model. Due to their loci, these epitopes are accessible for antibody binding, but also for enzymatic degradation. Mainly epitope $\mathrm{E}$ was located on the longest flexible loop of the Ara $\mathrm{h} 7$ isoforms. This is in line with the main epitopes of Ara h 2 and 6 showing similar loci. However, unique epitopes of Ara h 7.0101 and 7.0301 were found in the a-helices, making them only accessible after enzymatic digestion.

To validate the applied technique, we additionally mapped linear epitopes of Ara $\mathrm{h} 2$ 
and 6. Compared with previous literature, the same main epitopes were found, although two epitopes of Ara h 2 and two of Ara h 6 were not detected (Suppl. Table S1). However, comparing previous literature among each other, the epitopes described were only partly detected by other studies ${ }^{14,15,21}$.

The identification of conformational epitopes might help elucidating the divergent potencies of the Ara h 7 isoforms. Since detecting and characterising conformational epitopes is more sophisticated than the identification of linear epitopes, especially with polyclonal serum, human monoclonal antibodies might be a suitable tool for the identification of conformational epitopes. Moreover, human monoclonal antibodies directed to one specific epitope can also be characterised for their exact affinity ${ }^{22}$.

In conclusion, recognition of the 14 new mapped linear epitopes belonging to all three Ara $\mathrm{h} 7$ isoforms was highly individual, and epitopes predominately bound by lgG4 varied from epitopes bound by $\mathrm{lgE}$. These recognition patterns scarcely elucidated the divergent potency of Ara $\mathrm{h} 7$ isoforms, indicating the importance of conformational epitopes for the recognition of Ara $\mathrm{h} 7$.

\section{Informed consent statement}

This study was carried out in accordance with the University Medical Center Utrecht, Biobank Regulations, which are in compliance with the applicable national and international laws and regulations. These regulations permit the use of 'residual material from diagnostic testing' for research, unless the patient objects (Article 8, 'no objection' procedure). None of the included patients objected the use of their serum. The protocol was approved by the Biobank Research Ethics Committee of the University Medical Center Utrecht under the protocol number 18-428. 


\section{References}

1. Asarnoj, A. et al. IgE to peanut allergen components: relation to peanut symptoms and pollen sensitization in 8-year-olds. Allergy 65, 1189-95 (2010).

2. Nicolaou, N. et al. Quantification of specific IgE to whole peanut extract and peanut components in prediction of peanut allergy. J Allergy Clin Immunol 127, 684-5 (2011).

3. Kleber-Janke, T. et al. Selective Cloning of Peanut Allergens, Including Profilin and 2S Albumins, by Phage Display Technology. Int Arch Allergy Immunol 119, 265-74 (1999).

4. Klemans, R. J. B. et al. Ara h 2 Is the Best Predictor for Peanut Allergy in Adults. J Allergy Clin Immunol Pract 1, 632-8.e1 (2013).

5. Klemans, R. J. B. et al. The diagnostic value of specific IgE to Ara $\mathrm{h} 2$ to predict peanut allergy in children is comparable to a validated and updated diagnostic prediction model. J Allergy Clin Immunol 131, 157-63 (2013).

6. Hayen, S. M. et al. 2S protein Ara h 7.0201 has unique epitopes compared to other Ara $\mathrm{h} 7$ isoforms and is comparable to $2 \mathrm{~S}$ proteins Ara $\mathrm{h} 2$ and 6 in basophil degranulation capacity. Clin Exp Allergy 48, 890-7 (2018).

7. Schmidt, H. et al. Detection and Structural Characterization of Natural Ara h 7, the Third Peanut Allergen of the 2S Albumin Family. J Prot Res 9, 3701-9 (2010).

8. Blankestijn, M. A. et al. Specific IgE to peanut $2 S$ albumin Ara $\mathrm{h} 7 \mathrm{has}$ a discriminative ability comparable to Ara h 2 and 6. Clin Exp Allergy 48, 60-5 (2017).

9. Taylor, S. L. et al. A consensus protocol for the determination of the threshold doses for allergenic foods: how much is too much? Clin Exp Allergy 34, 689-95 (2004).

10. Popescu, F.-D. \& Vieru, M. Precision medicine allergy immunoassay methods for assessing immunoglobulin $\mathrm{E}$ sensitization to aeroallergen molecules. World $J$ Methodol 8, 17-36 (2018).

11. Šali, A. \& Blundell, T. L. Comparative Protein Modelling by Satisfaction of Spatial Restraints. J Mol Biol 234, 779-815 (1993).

12. Maghrabi, A. H. A. \& McGuffin, L. J. ModFOLD6: an accurate web server for the global and local quality estimation of 3D protein models. Nuc Acids Res 45, W416-21 (2017).

13. McGuffin, L. J. et al. Accurate template-based modeling in CASP12 using the IntFOLD4-TS, ModFOLD6, and ReFOLD methods. Proteins 86, 335-44 (2017). 
14. Otsu, K., Guo, R. \& Dreskin, S. C. Epitope analysis of Ara h 2 and Ara h 6: characteristic patterns of IgE-binding fingerprints among individuals with similar clinical histories. Clin Exp Allergy 45, 471-84 (2015).

15. Stanley, J. S. et al. Identification and Mutational Analysis of the Immunodominant IgE Binding Epitopes of the Major Peanut Allergen Ara h 2. Arch Biochem Biophys 342, 244-53 (1997).

16. Bernard, $\mathrm{H}$. et al. Allergenicity of peanut component Ara $\mathrm{h} 2$ : Contribution of conformational versus linear hydroxyproline-containing epitopes. J Allergy Clin Immunol 135, 1267-74.e8 (2015).

17. Gruber, P., Becker, W.-M. \& Hofmann, T. Influence of the Maillard Reaction on the Allergenicity of rAra h 2, a Recombinant Major Allergen from Peanut (Arachis hypogaea), Its Major Epitopes, and Peanut Agglutinin. J Agricul Food Chem 53, 2289-96 (2005).

18. Tscheppe, A. et al. Influence of Conformational and Linear IgE Epitopes on Ara h 2-Specific IgE-Binding. J Allergy Clin Immunol 139, AB378 (2017).

19. Shreffler, W. G. et al. Microarray immunoassay: Association of clinical history, in vitro IgE function, and heterogeneity of allergenic peanut epitopes. J Allergy Clin Immunol 113, 776-82 (2004).

20. Hansen, C. S. et al. Linear epitope mapping of peanut allergens demonstrates individualized and persistent antibody-binding patterns. J Allergy Clin Immunol 138, 1728-30 (2016).

21. Shreffler, W. G., Lencer, D. A., Bardina, L. \& Sampson, H. A. IgE and IgG4 epitope mapping by microarray immunoassay reveals the diversity of immune response to the peanut allergen, Ara h 2. J Allergy Clin Immunol 116, 893-9 (2005).

22. Ehlers, A. M. et al. Can alternative epitope mapping approaches increase the impact of B-cell epitopes in food allergy diagnostics? Clin Exp Allergy 49, 17-26 (2018). 


\section{Supplementary}

\begin{tabular}{|l|c|c|c|c|}
\hline \multicolumn{5}{|c|}{ Graphical ModFOLD6 results for Ara h 7.0201 } \\
\hline Model name & $\begin{array}{l}\text { Confidence } \\
\text { and } \mathrm{p} \text {-value }\end{array}$ & $\begin{array}{l}\text { Global model } \\
\text { quality score }\end{array}$ & Residue error plot & 3D view of residue error \\
\hline & Ara h 7.0201 & $\begin{array}{c}\text { CERT: } \\
4.792 E-4\end{array}$ & 0.4604 &
\end{tabular}

A

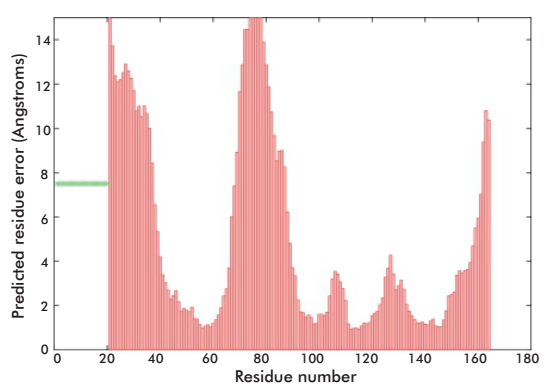

C

\begin{tabular}{|l|l|l|}
\hline $\begin{array}{l}\text { p-value } \\
\text { cut-off }\end{array}$ & Confidence & Description \\
\hline$p<0.001$ & CERT & Less than a $1 / 1000$ chance that the model is incorrect. \\
\hline$p<0.01$ & HIGH & Less than a $1 / 100$ chance that the model is incorrect. \\
\hline$p<0.05$ & MEDIUM & Less than a $1 / 20$ chance that the model is incorrect. \\
\hline$p<0.1$ & LOW & Less than a $1 / 10$ chance that the model is incorrect. \\
\hline$p>0.1$ & POOR & Likely to be a poor model with little or no similarity to the native structure \\
\hline
\end{tabular}

Figure S1: Quality assessment of the 3D structure of Ara $\mathrm{h} 7.0201$

The quality of the Ara $h$ 7.0201 3D model was assessed by ModFOLD6 webserver (https://www.reading.ac.uk/bioinf/ModFOLD/); $\boldsymbol{A}$ Errorresidue plot showing the predicted residue error; the loop regionshave a high predicted residue error whilst a-helices have a lowpredicted residue error. In general, loop regionsare hard to predict and to measure since loops are very flexible; $\boldsymbol{B}$ Predicted $3 D$ model ofArah7.0201 with highlighted residues based on B-factors; $\boldsymbol{C}$ Table overviewof quality scores used bythe ModFOLD6 webserver 
Table S1: Linear epitopes of Ara $h 7$ isoforms italic: residues only present in previous described epitopes

\begin{tabular}{|c|c|c|c|c|c|}
\hline Protein & Peptide & Residues $^{A}$ & Publication ${ }^{B}$ & Identified? & Specificity \\
\hline \multirow{17}{*}{ 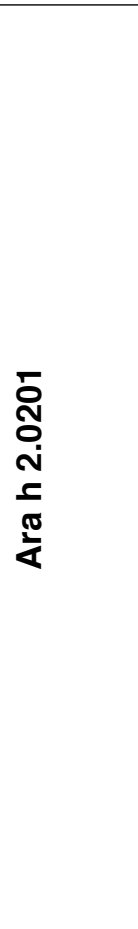 } & HASARQQWEL & $1-5$ & 1 & $\begin{array}{c}\mathrm{x} \\
\text { (overlapped) }\end{array}$ & $\operatorname{lgE} / \operatorname{lgG} 4$ \\
\hline & QWELQGDR & $3-10$ & 1 & & \\
\hline & DRRCQSQLER & $9-18$ & $1,2,3$ & $x$ & $\operatorname{lgG} 4$ \\
\hline & LRPCEG(Q)HLMQ & $21-30$ & 1,3 & $x$ & $\lg G 4$ \\
\hline & KIQRDEDS & $31-38$ & 1 & & \\
\hline & $P E Q H L M Q$ KIQRDEDSY & $25-30 / 31-39$ & 2 & $x$ & $\operatorname{lgG} 4$ \\
\hline & RDPYSP & $41-47 / 60-65$ & $1,2,3$ & $x$ & $\operatorname{lgE} / \operatorname{lgG} 4$ \\
\hline & SQDPYSPS & $47-54$ & 1 & $x$ & $\operatorname{lgE} / \lg G 4$ \\
\hline & DPYSPSPYDRR & $61-71$ & 2,3 & $x$ & $\lg E / \lg G 4$ \\
\hline & RRGAGSSQHQ & $70-79$ & 2 & $x$ & $\lg E$ \\
\hline & CNELNEFENNQR & 83-94 & 2,3 & $x$ & $\lg E$ \\
\hline & CEALQQIMENQSD & $97-109$ & 1,3 & & \\
\hline & QQIMENQ & $101-107$ & 1 & & \\
\hline & LQGRQQEQQ & $111-119$ & 1,3 & $x$ & $\operatorname{lgG} 4$ \\
\hline & KRELRNLPQQ & $121-130$ & $1,2,3$ & $x$ & $\lg E / \operatorname{lgG} 4$ \\
\hline & CGLRAPQRCDLEVE & $137-144$ & 1,3 & $x$ & $\lg E$ \\
\hline & EVESGGRDR & $142-150$ & 2 & & \\
\hline \multirow{11}{*}{$\begin{array}{l}0 \\
\frac{1}{\pi} \\
\frac{\pi}{\alpha}\end{array}$} & MRRERGRGGDSSS & $1-13$ & 3 & $x$ & $\operatorname{lgE} / \lg G 4$ \\
\hline & SSCERQVDRVNLK & $11-24$ & new & $x$ & $\lg G 4$ \\
\hline & KPCEQHIMQRI & $24-34$ & 3 & $x$ & $\operatorname{lgG} 4$ \\
\hline & GEQEQ & $36-40$ & new & $x$ & $\lg E$ \\
\hline & YDSYDIR & $41-47$ & 3 & $x$ & $\lg E / \operatorname{lgG} 4$ \\
\hline & CDELNEMENTQR & $59-70$ & 3 & & \\
\hline & QQQRCCDELNE & $54-64$ & new & $x$ & $\operatorname{lgG} 4$ \\
\hline & CEALQQIMENQCD & $73-85$ & 3 & $x$ & $\lg E$ \\
\hline & KRELRMLPQQ & $97-106$ & 3 & $x$ & $\lg E / \operatorname{lgG} 4$ \\
\hline & CNFRAPQRCDLDV & $107-119$ & 3 & & \\
\hline & RCDLDVSGGRC & $114-124$ & new & $x$ & $\lg E / \lg G 4$ \\
\hline
\end{tabular}

A without signal sequence

B 1: Stanley et al. 1997; 2: Shreffler et al. 2005; 3: Ostu et al. 2015 
MIUI:

AGCCTCTGGA7

CCTGTTTCTGCAAA

iGCGTGGTCCAGCCTC

AGGGCCGATTCACCATL ,

AATTGTGTTGACACAGTCTCCAGCCALU

UTGGCATCCCAGCCAGGTTCAGTGGCAGTGGGTC

$\checkmark$ CAGATGACCCAGTCTCCTTCCACCCTGGCTGCATCTGTAG

AGTCCCATCAAGGTTCAGCGGCAGTGGGTCTGGGACAGAGTTCACL

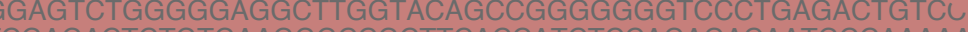

ITATCGAGACTCTGTGAAGGGCCGCTTCACCATCTCCAGAGACAATGCCAAAAAC ¿AGGGGACCACGGTCATCGTCTCCTCAGCAGGTGCAGCTGGTGGAGTCTGGGGGAGG iGAGTGGGTTTCATITATACGCAAGGATGGAGCTAGTAAATACTATGGAGACTCCGTGAAGGG ATAAACCTGGCCAGGCTCCCAGGCTCCTCATCTATGATGCATCCAACAGGGCCACTGGCATCCC ACTGGCCTCCGCTCACTTTCGGCGGAGGGACCAAGGTGGAGATCAAACGACATCCAGATGACCCAG

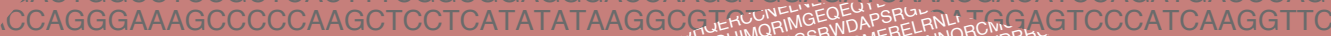
ACTCACTTTCGGCGGAGGGACCAAGGTGGAAAT iCAGGGGCTGGAGTGGGTCTCAGGCATCAGTG

AAAGATAAGGTTCCAGACTATGACGAGGTC

TCAGTCGTTATG

CAGCCTGAGAC

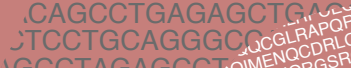

GGGCCAGTCA

GCCTGATGAT

CACGTTTAGG

;CTGGGGGGTCE

CCATCTCCAGAG

GTCTCCAGCCACCE
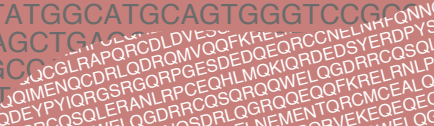

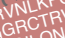

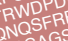

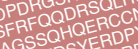

RDPY GRQGDSSSCERDVSGGROULW

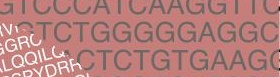

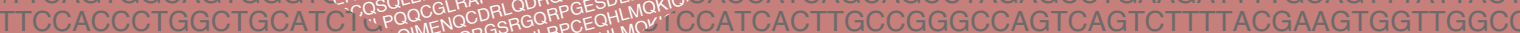

¿CAGTGGGTCTGGGACAGAGTTCNORLERANRPCEHLMTCAGCAGTCTGCAGCCTGATGATITTGCAACTTACTACTGTCAACAA

ACAGCCGGGGGGGTCCCTGAGACTGTCCTGTGCAGCCTCTGGATTCACGTTTAGGAGATATGCTTTGAGTTGGGTCCGC GCTTCACCATCTCCAGAGACAATGCCAAAAACATCCTGTTTCTGCAAATGGCCAGTCCGAGAGTCGAAGACGCGGCCGTI TCAGCAGGTGCAGCTGGTGGAGTCTR aATGGAGCTAGTAAATACTATGGAGAC

¿CCAGGGAACCCTGGTCACCGTCTCCTC LTCCTCATCTATGATGCATCCAACAGGGCC
GAGGGACCAAGGTGGAGATCAAACGACATC CATATATAAGGCGTCTAATTTACAAGATGGAGTC

AGGTGGAAATCAAACGAGGTGCAGTTGGTGGAGTCTGGGGGAGGCTTGGTACAGCC

जGCATCAGTGGCAGTGGAAACAAAACATATTATCGAGACTCTGTGAAGGGCCGCTTCAC

AACGAGGTCGAGATTATGGACGTCTGGGGCCAGGGGACCACGGTCATCGTCTCCTCAGCA

GGGTCCGCCAGGCTCCAGGCAAGGGGCTGGAGTGGGTTTCATTTATACGCAAGGATGG

GGCTGTATATTACTGTGCGAAAGATAGGTCCCTGGGTTACTTTGACTACTGGGGCCAGGG

TTTATTACTGTCAGCAGCGTAGCGACTGGCCTCCGCTCACTTTCGGCGGAGGGACC

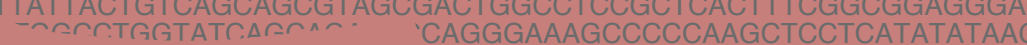

,ACTTTCGGCGGAGGGACCAAGGTGGAAATCA

IGCTGGAGTGGGTCTCAGGCATCAGTGGCA

GGTTCCAGACTATGACGAGGTCGAGAT

TGGCATGCAGTGGGTCCGCCAGGCTC

CTGAGGACACGGCTGTATATTACTG

AGTCAGAGTCTTGGCAGCTACTTAC

TGATTTTGCAGTTTATTACTGTCAG

ACTTACTACTGTCAACAATATGA

CTTTGAGTTGGGTCCGCCAG

:GAAGACGCGGCCGTTTATTA

:TCTCCTGTGTAGCGTCTGG

AAAGACACTCTCTTTCTGCA

iTCTCCAGGGGAAAGAGCA

CAGACTTCACTCTCACC

GAGACAGAGTCTCCATC

ACCCTCACCATCAGCAG

TGTCCTGTGCAGCCTC

:AAAAACATCCTGTTTC

¿̇GGGAGGCGTGGTCC

unum TCCGTGAAGGGCCGATT

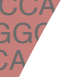

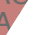

TAAGACTCTCCTGTGTAGCGTC

AATCCAAAGACACTCTCTTCT

GATCCAAAGACACTCTCTIT

CTGTAGGAGACAGAGTCTCCAT AGAGTTCACCCTCACCATCAGCA CCTGAGACTGTCCTGTGCAGCCT AGACAATGCCAAAAACATCCTGTTTC CTGGTGGAGTCTGGGGGAGGCGTGGT ATACTATGGAGACTCCGTGAAGGGCCGA ACACCGTCTCCTCAGGAAATTGTGTTGACA
ATCCAACAGGGCCACTGGCATCCCAGCCAC GATCAAACGACATCCAGATGACCCAGTCTCC TTACAAGATGGAGTCCCATCAAGGTTCAGCGG TICAAGATGGAGTCCCATCAAGGTCAGGTA TGCAGTTGGTGGAGTCTGGGGGAGGCTTGGTA CAAAACATATTATCGAGACTCTGTGAAGGGCCGO CTGGGGCCAGGGGACCACGGTCATCGTCTCCTC.
GGGGCTGGAGTGGGTTCATTTATACGCAAGGATG TAGGTCCCTGGGTTACTTTGACTACTGGGGCCAGG CCAACATAAACCTGGCCAGGCTCCCAGGCTCCTCAT SCGACTGGCCTCCGCTCACTTTCGGCGGAGGGACC AACCAGGGAAAGCCCCCAAGCTCCTCATATATAAGGC CTACTCACTTTCGGCGGAGGGACCAAGGTGGAAATCA GCAGGGGCTGGAGTGGGTCTCAGGCATCAGTGGCAG AAGATAAGGTTCCAGACTATGACGAGGTCGAGATTATG CAGTCGTTATGGCATGCAGTGGGTCCGCCAGGCTCCAC AGCCTGAGAGCTGAGGACACGGCTGTATATTACTGTGCG CAGCCTGAGAGCTGAGGACACGGCTGTATATIACTGTGCG CCTGCAGGGCCAGTCAGAGTCTTGGCAGCTACTTAGCCTC GGCCAGTCAGTCTTTTACGAAGTGGTTGGCCTGGTATCAC CTGATGATTITGCAACTTACTACTGTCAACAATATGATAGT GTTTAGGAGATATGCTTTGAGTTGGGTCCGCCAGGCTCCA CCAGTCCGAGAGTCGAAGACGCGGCCGTITATTACTGTGO GGGTCCCTGAGACTCTCCTGTGTAGCGTCTGGATTCACCT CCAGAGACAATTCCAAAGACACTCTCTTTCTGCAAATGGAC ICTCCTCAGGAAATTGTGTTGACAC AGCCACCCTGTCTTTGTCTCCAGGGGAAAGAGCAACCCTCT

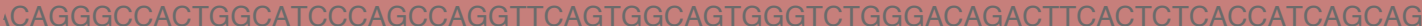
ACGACATCCAGATGACCCAGTCTCCTTCCACCCTGGCTGCATCTGTAGGAGACAGAGTCTCCATCACTTGCCGG GATGGAGTCCCATCAAGGTTCAGCGGCAGTGGGTCTGGGACAGAGTTCACCCTCACCATCAGCAGTCTGCAGCO ITGGTGGAGTCTGGGGGAGGCTTGGTACAGCCGGGGGGGTCCCTGAGACTGTCCTGTGCAGCCTCTGGATTCAO AATATTATCGAGACTCTGTGAAGGGCCGCTTCACCATCTCCAGAGACAATGCCAAAAACATCCTGTTTCTGCAAATGC GCCAGGGGACCACGGTCATCGTCTCCTCAGCAGGTGCAGCTGGTGGAGTCTGGGGGAGGCGTGGTCCAGCCTGG GGAGTGGGTTTCATTTATACGCAAGGATGGAGCTAGTAAATACTATGGAGACTCCGTGAAGGG

ICCTGGGTTACTTTGACTACTGGGGCCAGGGAACCCTGGTCACCGTCTCCTCAGGAAATTGT
ITAAACCTGGCCAGGCTCCCAGGCTCCTCATCTATGATGCATCCAACAGGGCCACTGGCATC

IGGCCTCCGCTCACTTTCGGCGGAGGGACCAAGGTGGAGATCAAACGACATCCAGATGACC

AGGGAAAGCCCCCAAGCTCCTCATATATAAGGCGTCTAATTTACAAGATGGAGTCCCATCAAGE

CACTTTCGGCGGAGGGACCAAGGTGGAAATCAAACGAGGTGCAGTTGGTGGAGTCTGGGGG

GGCTGGAGTGGGTCTCAGGCATCAGTGGCAGTGGAAACAAAACATATTATCGAGACTCTGTG

IAGGTTCCAGACTATGACGAGGTCGAGATTATGGACGTCTGGGGCCAGGGGACCACGGTCATCGi

ITATGGCATGCAGTGGGTCCGCCAGGCTCCAGGCAAGGGGCTGGAGTGGGTTTCATTTATACGCAAGGATGGAGi I AG I /

iAGAGCTGAGGACACGGCTGTATATTACTGTGCGAAAGATAGGTCCCTGGGTTACTTTGACTACTGGGGCCAGGGAACCCTG AGGGCCAGTCAGAGTCTTGGCAGCTACTTAGCCTGGTACCAACATAAACCTGGCCAGGCTCCCAGGCTCCTCATCTATGATG GAGCCTGAAGATTTTGCAGTTTATTACTGTCAGCAGCGTAGCGACTGGCCTCCGCTCACTTTCGGCGGAGGGACCAAGGTGGA 


\section{Can alternative epitope mapping approaches increase the impact of B-cell epitopes in food allergy diagnostics?}

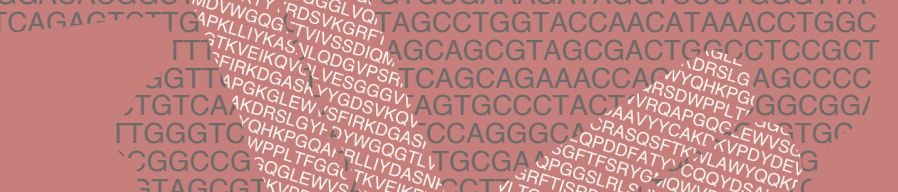

GGCATCAGTGGCAGTGGAAACAAAACATATTATC GGAGGS ITGTTGACACAGTCTCCAGCCACCCTC CCCAGCCAGGTTCAGTGGCAGTGGGT CCAGTCTCCTTCCACCCTGGCTGCATC, GTICAGCGGCAGTGGG TGGACAGA AGGGCCGCTTCACCATCTCCAGAGAC $A$ ;GTCTCCTCAGCAGGTGCAGCTGGTGC CAAGGATGGAGCTAGTAAATACTATG خ्रGGGCCAGGGAACCCTGGTCACCGTC त्र SSEKG TCTCTTTC'WYDE

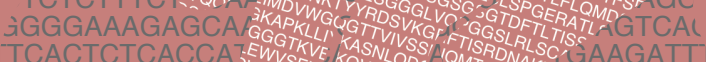

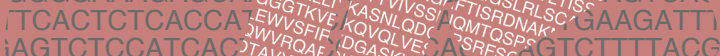

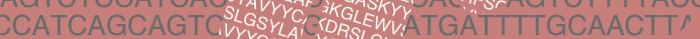
iTGCAGCCTCTG YALSW ¿ATCCTGTTTCT (ITCAAAVT, APPGQG, AGTCCGAGAGTCG/ ìAGGCGTGGTCG

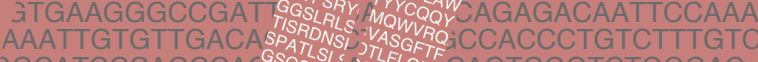

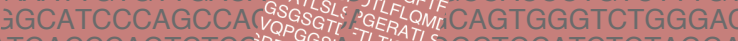

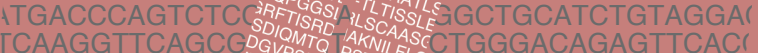
GAGGATTCAGCGDGWTTGE

$$
\text { CTGTGAAGGGCCGCTTCPGG I:TCTCCAGAGACAATGCC }
$$

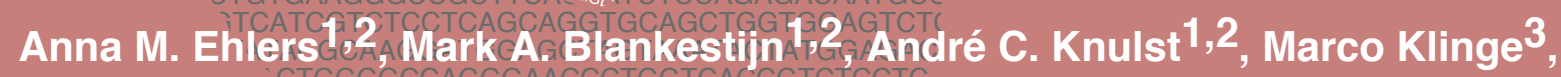

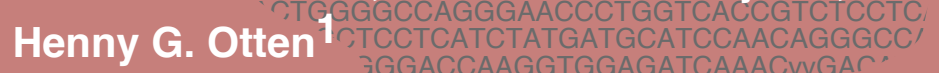
T्रGACCAAGGTGGAGATCAAACVVGA
TATAAGGCGTCTAATTACAAGATr
GAAATCAAACGAGGTGCAGTTr

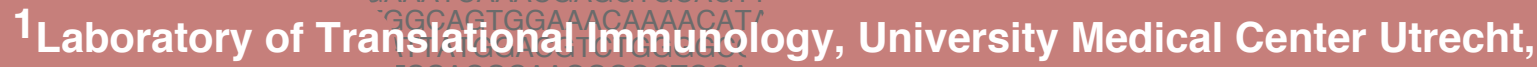
Utrecht University, Utrecht; $A$ The Netherlands A.2Department of Dermatelogy/Allergology, University Medical Center Utrecht, CUUtrecht University (B)

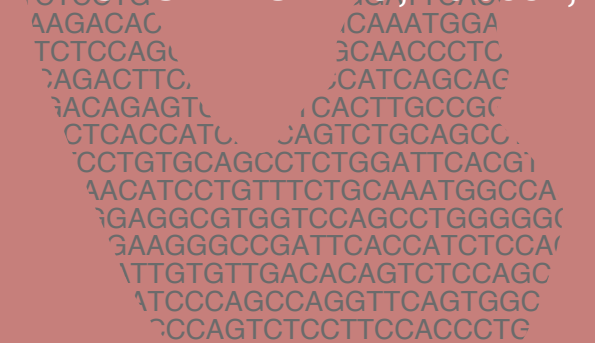




\section{Abstract}

In vitro allergy diagnostics are currently based on detection of specific lgE binding on intact allergens or a mixture thereof. This approach has drawbacks as it may yield false negative and/or false positive results. Thus, we review the impact of known B-cell epitopes of food allergens to predict transience or persistence, tolerance or allergy and the severity of an allergic reaction and to examine new epitope mapping strategies meant to improve serum-based allergy diagnostics. Recent epitope mapping approaches have been worthwhile in epitope identification and may increase the specificity of allergy diagnostics by using epitopes predominately recognised by allergic patients in some cases. However, these approaches did not lead to discrimination between clinically relevant and irrelevant epitopes so far, since the polyclonal serum $\lg \mathrm{E}$ binding epitope spectrum seems to be too individual, independent of the disease status of the patients. New epitope mapping strategies are necessary to overcome these obstacles. The use of patient-derived monoclonal antibodies instead of patient sera for functional characterisation of clinically relevant and irrelevant epitope combinations, distinguished by their ability to induce degranulation, might be a promising approach to gain more insight into the allergic reaction and to improve serum-based allergy diagnostics. 


\section{Introduction}

Food allergy is currently diagnosed by careful history, food challenges, skin prick test (SPT), and measurement of specific $\lg \mathrm{E}$ (slgE). The double-blind placebo-controlled food challenge (DBPCFC) is the gold standard, but it is a costly and burdensome procedure. Both SPT and slgE measurement, using entire foods or single allergenic components, are hampered by false positive test outcomes ${ }^{1}$. This might be related to the presence of both clinically relevant and irrelevant antibodies as it has been shown for serum-based diagnostics of anti-neutrophilic cytoplasmic antibody-associated vasculitis $^{2}$. Serum-based measurement might be improved by defining the epitope specificity, affinity, critical amino acids and antibody isotypes relevant for allergy.

Extensive research has been carried out, especially for peanut ${ }^{3-10}$ and cow's milk ${ }^{11-16}$, to identify the IgE- and IgG4-epitopes of food allergens. Linear epitopes, composed of a continuous amino acid sequence, have been detected by screening patient sera on sequential overlapping peptide libraries or allergen fragments. Conformational epitopes, composed of sterically closed amino acids upon folding, were characterised by phage display technique or mass spectrometry partly in combination with B-cell epitope prediction web tools or software, although the use of these techniques still has to be proven in future studies ${ }^{6,17-21}$.

So far, it is impossible to discriminate between clinically relevant and irrelevant epitopes or combinations using current approaches, and to use these differences as diagnostic or prognostic markers. This review will discuss current knowledge, based on a relatively small number of studies investigating linear as well as conformational epitopes and comparing allergic and tolerant patients, and will propose alternative approaches for epitope mapping, focussing on epitope specificity and how this might impact serumbased allergy diagnostics.

\section{Requirements for effector cell degranulation by FceRI cross-linking}

The major requirement for degranulation is the cross-linking of at least two FceRI re-

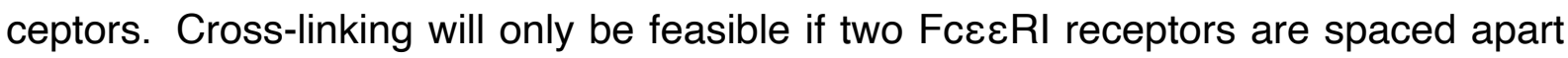
by 50 to $240 \AA$. This range has been defined by using artifical allergens and hence might be somewhat smaller or larger for native allergens ${ }^{22-24}$. Consequently, the distance of two functional IgE-epitopes within one combination is restricted to the required distance of two FceRI receptors. As an example, possible IgE epitope combinations of Ara $\mathrm{h} 2.0201$, based on a 3D model built with the SWISS model web portal, are shown in Figure $1^{25-29}$. Residue distances greater than $35 \AA$, calculated with Chimera $\mathrm{UCSF}^{30}$, were considered as a functional epitope combinations, highlighted on the 3D structure using Schrödinger Release 2018-1 (Maestro, Schrödinger, LLC, New York, 
NY, 2018). Additionally, at least one epitope of the combination must be recognised with high affinity since the cross-linking has to take place for at least 100 seconds. Moreover, at least 1000 cross-links of FcERI receptors on the surface of one effector cell have to take place ${ }^{31-33}$. If all these requirements for FceRI crosslinking are met, the extent of degranulation is regulated by slgE concentration, affinity, the ratio of allergen slgE-antibodies compared to total $\lg \mathrm{E}$, and the specificity and number of epitopes recognised ${ }^{34}$. These requirements suggest that certain epitope/antibody combinations are only present in allergic and not in tolerant patients. However, basophils from 10$20 \%$ of the general population do not respond at all due to low expression of syk and/or SHIP-1 resulting in the inhibition of intracellular signalling ${ }^{35-38}$. Thus, different expression levels might regulate the extent of degranulation.

A

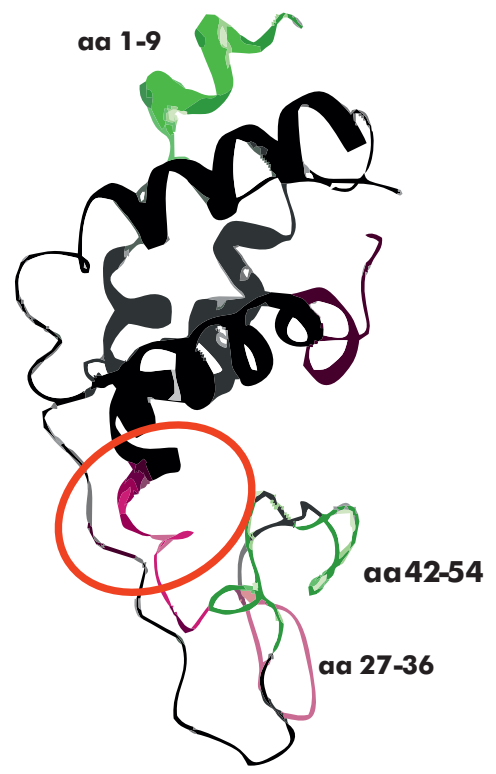

B

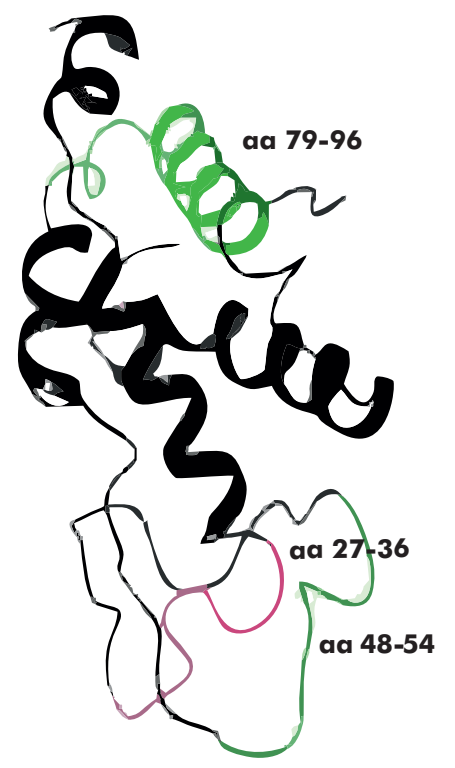

Figure 1: Determination of potentially relevant epitope combinations of the major peanut allergen Ara $\mathrm{h} 2$ The 3D structure of Ara $h 2$ was obtained by Swiss-Model and residues distances were determined by creating a residue distance map. Since the greatest distance was smaller than $50 \AA$, distances between 40 and $45 \AA$ were considered as distances more likely to form relevant epitope combinations (highlighted in green). Distances between 35 and $40 \AA$ were considered as possible epitope combinations but less likely (highlighted in pink). Predicted conformational epitopes by identified mimotopes are surrounded by red circles.; $\boldsymbol{A}$ One possible relevant epitope combination ( $40-45 \AA$ ) regarding the used model is between the described epitope aa 1-9 and the epitope aa 42-54 highlighted in green. Additionally, a combination with the same epitope aa 1-9 and the epitope aa 27-36 highlighted in pink is less likely (35-40 $\AA$ ).; B Another possible relevant epitope combination (40-45 A) regarding the used model is between the described epitope aa 79-96 and the epitope aa 48-54 highlighted in green. Additionally, a combination with the same epitope aa 79-96 and the epitope aa 27-36 highlighted in pink is less likely (35-40 $)$ ). 


\section{Food allergens as antigens: Influence of processing and digestion}

Food allergens are modified by processing during industrial manufacturing or food preparation ${ }^{39}$. After processing, a fraction of these antigens will enter the buccal mucosa unaltered and the remainder will be reprocessed by human digestion before entering the tissue and the blood stream ${ }^{40-42}$.

They can be modified during industrial manufacturing, which can lead to aggregation or modification of polysaccharide structures or appropriate amino acid residues. Certainly, only a portion of the same allergen is affected. One example is the roasting of peanuts, which usually leads, by Maillard reaction, to the modification of amino acids, especially the hydroxylation of prolines. In the study of Bernard et al. ${ }^{43,44}$, the modification of the proline residue located in the DPYSP ${ }^{O H} \mathrm{~S}$ motif of Ara h 2 (aa 49-54 and 61-66 for Ara h 2.0201 and aa 49-54 for Ara h 2.0101) caused an increase in allergenicity. The same increase in allergenicity has been observed for the peanut allergen Ara $h 1^{43}$. Processing effects vary, so for example allergenicity of the hen's egg allergen ovalbumin is decreased upon heat treatment, as shown by patients reacting to raw hen's egg, but not to cooked ones ${ }^{45}$. Modification by heating has also been observed for cow's milk, with allergens a- and B-lactalbumin tending to aggregate upon heat treatment while caseins stay unaffected. However, testing in vitro processed cow's milk allergens showed inconsistent results between in vitro degranulation and the clinical history of the patients, often showing tolerance to heated milk ${ }^{46}$. Such observations of allergenicity changes require further research, to define allergen features that lead to increase or decrease. Knowledge of these features might help to predict the potential allergenicity of proteins even though different conditions of one processing method will have a great impact.

After ingestion, a part of the allergen enters the buccal mucosa and subsequently the bloodstream without being digested; however, as demonstrated in studies with peanut, gastric processing seems to further enhance the uptake and degranulation ${ }^{40,42}$. The influence of digestion on food allergens has been estimated in several studies and is dependent on the allergen structure. Stable proteins, including Ara $\mathrm{h} 2$ and ovalbumin, remain unaffected by low $\mathrm{pH}$ and proteolysis ${ }^{47}$ whereas Ara $\mathrm{h} 1$ and 3 , more labile proteins, are fragmented by pepsin. Upon entering the gut, peptides derived from digestion tend to aggregate due to the basic $\mathrm{pH}$ in the gut ${ }^{48-50}$, which may lead either to shielding of previously accessible epitopes or to the development of new, presumably conformational epitopes. In short, industrial manufacturing, in combination with intestinal processing as well as matrix effects (not discussed here), influences the allergenicity of food proteins potentially by changing epitope profiles even though aggregation can also affect the solubility of the allergen. Precipitated and non-soluble allergen can falsely pretend no IgE binding in in vitro studies. 


\section{Identification of linear and conformational food allergen B-cell epi- topes}

Linear epitopes of several food allergens have been identified, mostly by overlapping peptide libraries, allergen fragments or phage display peptide libraries $9,12,15,51,52$. These approaches were partly coupled with B-cell epitope prediction software or webtools like ABCPred, BepiPred 1.0, and DNAStar Protean ${ }^{21,53}$. Moreover, in the studies of Zheng et al. and Chen et al., the predicted B-cell epitopes were experimentally confirmed with high accordance ${ }^{17,54}$. However, no discrimination can be made between epitopes recognised by $\lg \mathrm{E}, \lg \mathrm{G}$ and $\lg \mathrm{A}$ probably important in the allergy context. So far, prediction models cannot be used without experimental data to result in more accurate diagnosis or immunotherapy since current prediction models do not consider the special requirements of the allergic reaction described above. In comparison to linear epitopes, the detection of conformational epitopes requires more sophisticated techniques, like X-ray crystallography, mass spectrometry or phage display libraries ${ }^{55}$. These phage display libraries can consist of peptides deriving from the allergen of interest or of random peptides. By using random libraries, peptides recognised by allergen specific antibodies have been shown to consist of sequences mimicking a continuous or discontinuous epitope of the allergen; such peptides are called mimotopes and can be mapped on the 3D structure of the allergen by predictive webtools like EpiSearch ${ }^{10,56}$. A large drawback of these approaches is their failure to detect alterations in allergens caused by post-translational modifications or processing and specific antibodies can only be detected against single epitopes and never in combination with others. Additionally, the assignment of mimotopes to surface patches of an allergen is solely based on in silico approaches and thus hampers the reliability of the outcome. Although mimotope mapping has been performed for a few allergens with conclusive functional results $^{10,57,58}$, it still has to be in context with inhibition and mutation studies using the full-length protein. The general limitation is the requirement of a high-resolution structure for the allergen of interest, constraining a broad application of this approach. Admittedly, mass spectrometry can be used to investigate the influence of post-translational modification using native proteins, and X-ray crystallography to detect epitope combinations. Co-crystallization studies have been performed with murine monoclonal IgG antibodies being able to reduce binding of human polyclonal $\lg \mathrm{E}^{59-62}$. Continuatively, co-crystallization has been carried out using monoclonal IgE antibodies generated by combinatorial heavy and light chain libraries of allergic patients. However, it has not been proven weather these antibodies also occur naturally ${ }^{63,64}$. Information from these studies can help in understanding the features being responsible for allergenicity, and in defining critical amino acids more precisely. This knowledge can support the creation of more accurate serum-based diagnostics by modifying critical amino acids recognised 
by clinically non-relevant IgE-antibodies. Moreover, it will give the opportunity to develop hypoallergenic variants for immunotherapy and better (lgE-) epitope prediction tools ${ }^{65}$. However, the co-crystallization of polyclonal serum antibodies bound to the allergen of interest is an almost insuperable bottleneck, making X-ray crystallography a more theoretical approach for conformational epitope mapping. These obstacles might be overcome by human-derived monoclonal lgE-antibodies.

\section{Discrimination between persistence and transience by means of IgE binding epitopes}

Most cow's milk allergic children outgrow their allergy by 3-4 years of age, although $15 \%$ remain allergic. In comparison, HEA arises later in childhood and $34 \%$ of these children will retain a persistent allergy ${ }^{66}$. Persistence has been studied through analysis of the epitope recognition pattern in patient sera. In CMA, persistent allergy was clearly associated with multiple IgE binding epitopes on aS1-, aS2-, k-casein, a- and B-lactalbumin as these were not recognised by $\lg E$ antibodies of children with transient allergy. However, the recognised epitopes do not coincide in different studies ${ }^{12,15,67-69}$. In HEA, four linear IgE binding epitopes of ovomucoid have been associated with persistent allergy since they were not recognised by $\lg E$ antibodies of transient allergic children ${ }^{70,71}$. In short, these data favour the use of epitopes to predict persistence and to discriminate between transience and persistence more precisely.

\section{Discrimination between allergy and tolerance by means of IgE bind- ing epitopes}

For allergies less likely to be outgrown, identification of epitopes/antibodies discriminating between tolerance and allergy is essential to avoid unnecessary food elimination therapies. In cases of peanut allergy, tolerant patients appeared to recognise the same IgE binding epitope spectrum on Ara h 2 (linear epitopes) as allergic patients, although individual allergic patients recognised a higher number of different epitopes with higher $\operatorname{lgE}$ titres $^{72}$. By means of the two key marker epitopes Ara h 2_10 (aa 28-42) and Ara h 2_18 (aa 52-66), allergic patients were correctly diagnosed with a sensitivity of $70 \%$ and a specificity of $60 \%$. Sensitivity and specificity rose to respectively $90 \%$ and 95\% by adding the IgE binding epitopes Ara h 1_16 (aa 46-60) and Ara h 3_140 (aa 418432), which were recognised by few allergic patients ${ }^{72}$. In comparison, the sensitivity and specificity of intact Ara h 2 were defined as respectively $60-100 \%$ and $60-96 \%$, showing no advantage using these two key marker epitopes ${ }^{73}$. Meanwhile, the conformational lgE binding epitope pattern of Gly $\mathrm{m} 4$ showed no correlation with clinical reactivity at all ${ }^{74}$. The impact of linear or conformational epitopes remains restricted in 
terms of ruling out tolerance or confirming allergy, as tolerant patients recognise the same epitope spectrum, although less frequently. In summary, to date, no clear discrimination between allergic and tolerant patients is feasible based on the detection of epitopes.

\section{Severity prediction of an allergic reaction by means of IgE binding epitopes}

Several studies have been performed to predict the severity of an allergic reaction by using explicit or multiple IgE binding epitopes of one or multiple allergenic components. To assess whether the severity of allergic reactions towards peanut is related to the type and number of epitopes recognised, patients with different symptoms (ranging from mild to severe and a slgE level greater than $14 \mathrm{kU} / \mathrm{L}$ ) have been studied ${ }^{9}$. Patients suffering from severe symptoms recognised fewer linear epitopes than patients with mild symptoms, suggesting that specific epitopes may be more relevant than the quantity of epitopes recognised. This may relate to the requirements for effector cell degranulation or suggest a greater relevance for conformational epitopes. However, slgE binding to conformational B-cell epitopes did not contribute to severe symptoms as assessed by mimotopes mapped to the surface of Ara $\mathrm{h} 2$ and $6^{10}$. In CMA, the difference in IgE binding epitope recognition pattern was analysed by including patients allergic to all forms of milk, patients tolerating heated milk, and patients with an outgrown allergy. Patients reactive to all forms of milk had a more diverse IgE and IgG4 epitope recognition pattern, comparable to a study of Sackesen et al. in which patients with persistent CMA reacted to processed milk and patient with transient tolerated processed milk $^{75}$. The IgE binding pattern of patients non-reactive to heated milk was comparable to the recognition pattern of patients who had outgrown their allergy, although the lgG4 binding pattern was comparable to patients being allergic to all forms of milk. Patients tolerating heated milk had the lowest slgG4 level and possessed low affinity lgE antibodies as did the tolerant group. In contrast, when investigated using a competition assay, the allergic group exhibited low and high affinity antibodies ${ }^{76}$. Low IgG4 levels in heated milk tolerating patients suggest a direct class switch from $\mu$ to $\varepsilon$ without a mature germinal centre and thus, less somatic hypermutations and affinity maturation. High affinity antibodies might be generated by sequential class switching from $\mu$ to $\gamma 4$ and to a lesser extent by a subsequent class switch to $\varepsilon^{77}$. In several studies, auxiliary slgG4 levels were similar between tolerant and allergic patients ${ }^{78,79}$. In conclusion, severity prediction can be made regarding the tolerance of processed or non-processed food since transient patients do tolerate processed food and patients with persistence CMA do not, but they recognise specific epitopes not recognised by transient patients. Thus, a more specified dietary advice might be given based on recognised epitopes. 
However, the prediction of symptom severity is not yet feasible and due to a range of host-related factors will probably remain difficult ${ }^{80,81}$. Maximum release and the sensitivity of mast cells and basophils are dependent on intrinsic factors such as the regular exposure to an antigen that influences the production of a histamine-releasing factor interacting with surface-bound IgE. This interaction might lead to a higher extent of degranulation and/or spontaneously release without antigen exposure ${ }^{82}$. Additionally, different miRNAs have been implicated in up- or downregulation of genes involved in key signalling of mast cells (inhibition by miR-155 and miR-223; enhancement by miR-142$3 p$ and miR-221) ${ }^{83-86}$. Moreover, polymorphism of mediator degrading enzymes can influence host-related mediator tolerance thresholds by increasing or decreasing enzyme levels or metabolic capacity. Polymorphism of the histamine degrading enzyme $\mathrm{N}$-methyltransferase was associated with a higher risk of asthma and patients showing SNP His645Asp on the diamine oxidase (DAO) metabolising histamines were prone to develop an allergic reaction at lower slgE levels ${ }^{87-89}$. Further, the polymorphism of platelet-activating factor acetyl hydrolase Thr198 and Val397 leads to lower substrate affinity prolonging the activity of platelet-activating factor ${ }^{90}$, correlated positively with the severity of systemic reactions and anaphylaxis ${ }^{91,92}$. Finally, mast cell priming might be stimulated by medicines like beta-blockers and ACE inhibitors, increasing the severity of an allergic reaction ${ }^{93}$.

\section{Impact of IgG binding epitopes in allergy prediction}

Investigations of the impact of epitopes in allergy prognostic and diagnostic have been mostly based on IgE binding epitopes, although antibodies of a different isotypes, mainly $\operatorname{lgG} 4$, seem to play a role in tolerance induction or retaining tolerance. In several studies, IgG4 increases coincided with decreases in slgE, after outgrowing allergy or successful allergen immunotherapy $(\mathrm{AIT})^{78,79,94}$. No statistically significant association with IgG4 epitopes comparable to IgE epitope pattern was found regarding persistence and transience, or severity of symptoms for the investigated food allergens peanut and cow's milk ${ }^{6,12,72,76,95,96}$. Moreover, conflictive results were obtained regarding the overlap between IgE and IgG4 binding patterns. Caubet et al. 2017 have been shown that transience is associated with similar IgE and IgG4 binding pattern while Savilahti et al. 2010 found similar IgE- and IgG4 binding pattern in patients with transient and persistent $\mathrm{CMA}^{69,97}$. In contrast, ratios of IgE to IgG4 could, in some studies, distinguish between peanut tolerant and allergic patients in children and adults ${ }^{98,99}$, supporting the hypothesis that specific $\lg G 4$ can block IgE binding to definite epitopes. In a study with peanut sensitised children divided by tolerance and allergy, mast cell (LAD2 cells) and basophil degranulation induced by sera from peanut allergic children was inhibited by pre-incubation of the allergen with sera from peanut tolerant children prior to stimula- 
tion. However, degranulation was only partially restored using lgG4-depleted sera ${ }^{99}$. These results can have several explanations:

- sterical hindrance of pre-bound IgE-antibodies from peanut tolerant children

- blocking of IgE binding from allergic children by pre-bound specific IgG4

- the binding of antigen by specific IgG bound by the FcyRIlb (CD32b) receptor on the surface of basophils or mast cells inhibiting degranulation by co-aggregation of the FceRI and FcyRllb ${ }^{100}$

- or a mixture thereof

For further investigation, the stimulation allergen can be pre-incubated with different well-characterised (epitope specificity, affinity) monoclonal $\lg G$ and $\lg E$ antibodies from peanut tolerant patients or a mixture thereof instead of polyclonal sera.

\section{Impact of IgE and IgG binding epitopes in food allergy immunother- apy monitoring}

In contrast to allergy prediction, the impact of $\lg G$, especially $\lg G 4$, binding epitopes in AIT monitoring has been investigated more intensively. In general, patients, irrespective of the AIT outcome, showed an increase in allergen specific lgG4 levels as well as a temporary increase in IL-10 secreting FoxP3 positive Treg cells controlling a class-switch to IgG4 and a decrease in slgE levels ${ }^{101-104}$. In a peanut AIT trial, newly formed IgG4-antibodies had specificities partly identical to identified IgE binding epitopes. Simultaneously, the total slgE decreased, although, as also shown in CMA AIT trials, new IgE specificities were developed ${ }^{94}$. Additionally, patients who discontinued AIT due to adverse reactions showed an increase in quantities and affinity of epitope slgE paired with a greater diversity of recognised IgE and IgG4 binding epitopes with little overlap ${ }^{105}$. Based on IgE binding pattern prior starting AIT, a prediction model for safety (=number of adverse reaction while AIT) and efficacy (= time required to achieve desensitization) was developed. The model included two sets of $16 \mathrm{lg} E$ binding regions of caseins to be associated with safety and efficacy ${ }^{106}$.

In further milk and peanut AIT trials, the induction of a hyporesponsive state of basophils was observed. This state was additionally applicable for hen's egg sensitised patients in the population of peanut allergic patients ${ }^{107,108}$. A possible explanation is the IgG epitope specific co-aggregation of the inhibitory FcyRIIb (CD23b) and FceRI receptor potentially inhibits the PI3k pathway by SHIP-1 activation and subsequently loss of syk expression ${ }^{109}$. This might also explain the risk reduction in high risk infants to develop a peanut allergy by early and regular introduction of peanut consumption as 
shown in the LEAP and LEAP-On study. Infants in the consumption group, compared to the avoidance group, showed higher specific $\operatorname{lgG} 4$ levels and $\lg G 4 / \lg E$ ratios ${ }^{110,111}$. IgG4 binding epitopes do not seem to have a high impact in allergy prediction but do seem to be useful in food AIT monitoring. However, further investigation on IgG- and IgE binding epitope pattern in patients with a positive and negative outcome must be performed.

\section{Limitation of these approaches}

For most IgE binding epitopes, a limited number of studies were performed to pinpoint epitopes to be used as diagnostic markers. These attempts were only partly successful as allergy and tolerance still cannot be predicted by using IgE B-cell epitopes. A fundamental limitation is related to the use of polyclonal sera containing a mixture of antibodies with multiple isotypes recognising diagnostically relevant and irrelevant epitopes. This heterogeneous antibody repertoire in serum was confirmed by mutagenesis studies showing multiple amino acids to be critical for one epitope in $\mathrm{CMA}^{112,113}$. Another essential limitation is the restricted focus on IgE binding epitopes since the role of $\lg G$ or $\lg A$ binding epitopes is almost unknown. Thus, for improving serum-based food allergy diagnostics, human monoclonal antibodies instead of polyclonal patient sera might help to define clinically relevant epitopes and to investigate the role of antibodies with different isotypes, as explained below.

\section{Different epitope mapping approaches may increase the contribu- tion of allergen specific B-cell epitopes}

To overcome the limitations described in the previous paragraphs, requirements for degranulation should be considered. Since cross-linking of at least two FceRI receptors is crucial, an allergic subject must feature IgE antibodies recognising two epitopes with a distinct distance on the cell surface, of which at least one with high affinity ${ }^{22}$. In contrast, two IgE antibodies, with low affinity recognising a relevant epitope combination, or one $\lg E$ antibody recognising one epitope of a relevant epitope combination may occur in a tolerant patient without causing degranulation. Current IgE serum-based diagnostics cannot discriminate between high- and low-affinity antibodies, impeding the discrimination between relevant and irrelevant epitopes or combinations. Instead of polyclonal sera, a more distinct characterisation of specific epitopes might be feasible by using human monoclonal antibodies. This approach may also give precise information about epitope specificity and affinity (illustrated in Figure 2). Furthermore, co-crystallization of an epitope-specific monoclonal antibody or a ScFv with an allergen of interest is significantly more likely to be successful compared to the use of polyclonal sera, which 
offers a chance to detect authentic conformational epitopes.

Since IgE producing B cells in blood are scarce, the first studies with IgE monoclonal antibodies were performed with murine allergen-specific antibodies, originally lgG but subsequently recombinantly expressed as IgE antibodies ${ }^{34}$. In addition, human monoclonal IgE antibodies were obtained by construction of phage display ScFv hybrid libraries with allergic donor-derived epsilon heavy chain and synthetic variable regions for the light chain ${ }^{114,115}$. However, these approaches did not address the whole allergen specific antibody repertoire of one subject. In studies by Hoh et al. and Patil et al. $^{116,117}$, a part of the antibody repertoire was analysed by single cell sequencing of the specific B-cell receptor. The lack of sequenced receptors derived from IgE B cells can be explained by the small number of PBMCs used in these studies ( 1 to $10 \cdot 10^{6}$ cells/experiment) whereas the frequency of Ara $\mathrm{h} 1$ or 2 specific $\mathrm{B}$-cells was determined to be around $0.01 \%$ of the $B$ cell fraction in allergic patients ${ }^{117}$. Due to this low frequency, we recommend the use of a large volume of blood derived from allergic or tolerant donors to increase the number of circulating allergen specific B cells. These B cells can be single cell sorted and expanded in vitro, or the mRNA can be isolated directly ${ }^{118}$. Moreover, specific B cell clones can be obtained by immortalisation and limited dilution, but this approach may cause in vitro class switching or lead to selection bias due to the expansion of the fittest clones. Additionally, we propose, in contrast to Hecker et al. ${ }^{115}$, the isolation of all allergen specific B-cells irrespective of the isotype produced as the role of different allergen-specific isotypes remains unclear. Finally, we recommend the inclusion of tolerant and allergic patients sensitised to the same allergen for the generation of specific monoclonal antibodies. Monoclonal antibody repertoires within these two groups may allow the discovery of differences in epitope specificity and affinity. To ensure the capture of B cells producing antibodies with comparable affinity and epitope specificity as the polyclonal serum repertoire, the plasma of the patients should be saved and analysed for their epitope recognition spectrum and affinity. In addition, competition assays with monoclonal antibodies and serum antibodies can be performed to detect differences in affinity.

Potentially, these findings can contribute not only to improvement of food allergy diagnostics but also monitoring of allergy immunotherapy. This epitope mapping strategy will be restricted by the absence of short and long-living plasma cells in the blood as well as the still discussed presence of $\operatorname{lgE}^{+}$memory $B$ cells ${ }^{77,119-122}$. If re-stimulated IgG memory $B$ cells are the source of class-switched lgE producing cells, instead of IgE memory B cells, the sequence of IgG B cell receptors can be recombinantly expressed as $\lg E$ antibodies and functionally characterised as $\lg G$ and $\lg E$ antibodies. Based on this hypothesis by Aalberse and Platts-Mills $2004, \operatorname{lgE}^{+} \mathrm{B}$ cells class-switched from $\operatorname{lgG}^{+}$memory cells are not able to survive in the germinal centre where affinity maturation takes place and the variable region of the $\lg \mathrm{E}^{+} \mathrm{B}$ cell receptor does not undergo 
further somatic hypermutations ${ }^{77}$. Another aspect to take into account is the expression of the low affinity FcERII (CD23) on non-allergen specific translational B cells in the blood stream ${ }^{123,124}$. The cells can bind allergen specific $\lg \mathrm{E}$ antibodies leading to the selection of B cells irrelevant for allergy. Non-specific antibodies can be excluded through a specificity check, such as performing an ELISA coated with the allergen of interest.

Another approach to enlarging the impact of B cell epitopes in food allergy diagnostics might be the development of improved computational epitope prediction. To exceed the abilities of already available prediction software or webtools for B cell epitopes, computational approaches for predicting allergenic functionality must meet, as described above, the special requirements for degranulation, for example the defined distance between two epitopes. Before using these distances as a basis for prediction, they should be estimated by natural and not, as now, by artificial allergens. Furthermore, new algorithms must consider the potential oligomerization of allergenic proteins, since this can increase the number of possible epitope combinations for triggering effector cell degranulation.
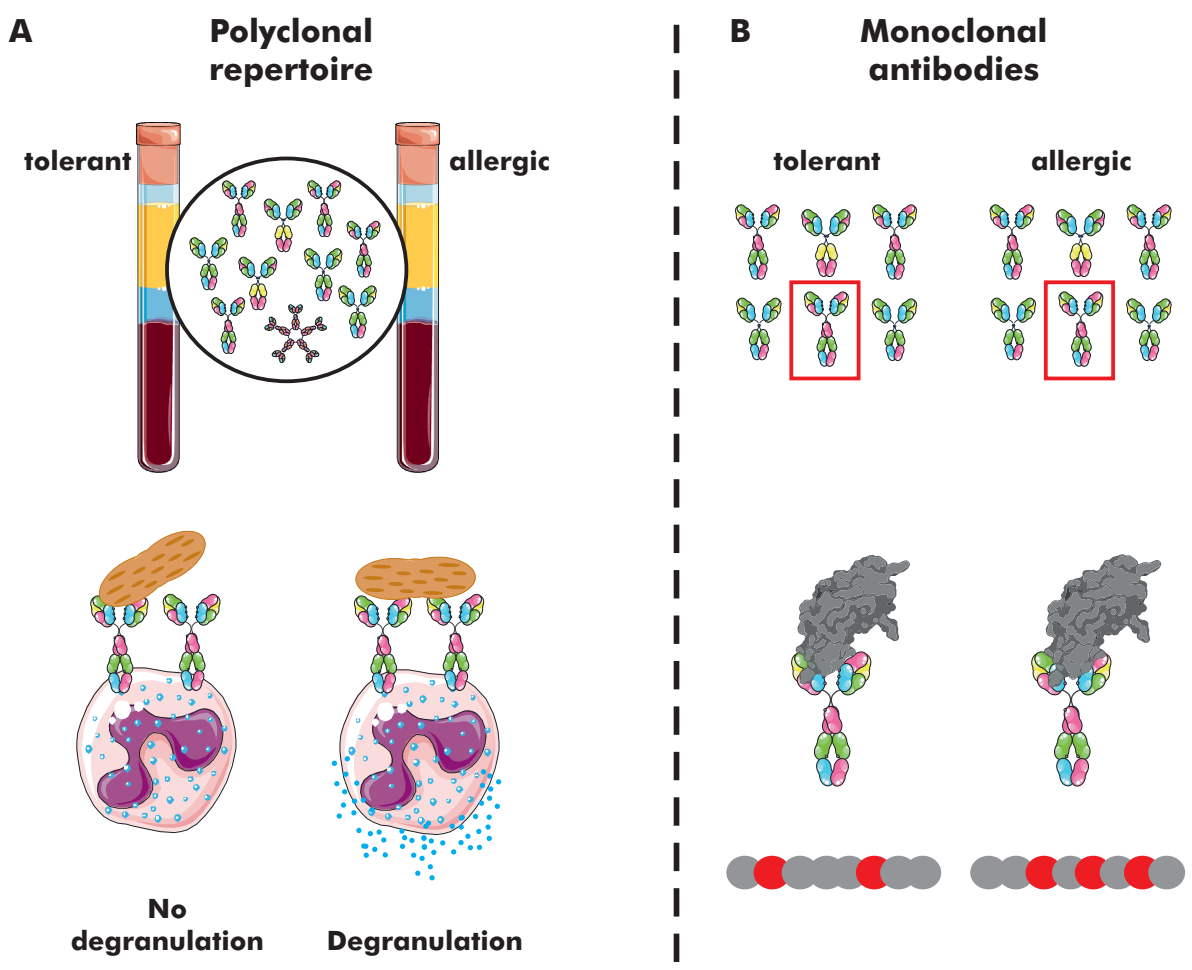

Figure 2: Defining differences in epitope binding pattern of tolerant but sensitised and allergic patients are hampered by similar appearing polyclonal antibody repertoire (Parts of the graphic from Servier Medical Art by Servier)

$\boldsymbol{A}$ The polyclonal repertoires in sera of tolerant but sensitised and allergic patients seem to be similar but only antibodies out of the repertoire from allergic patients are able to induce an allergic reaction; $\boldsymbol{B}$ The use of monoclonal antibodies enables a more extensive characterisation of individual antibodies responsible for the allergic reaction. Tolerant as well as allergic patient can possess antibodies with similar epitope specificities but with different affinity. 


\section{Conclusion}

The use of monoclonal antibodies might be a powerful tool to define the allergen specific antibody repertoire of tolerant and allergic patients more precisely in terms of epitope specificity, affinity and feasible epitope combinations. This will produce more knowledge about the reasons some sensitised patients can tolerate an allergen with no symptoms while others will experience a (severe) allergic reaction. Differences in antibody repertoires can lead to improved slgE measurement by modifying critical amino acids recognised by clinically irrelevant antibodies and thus, preventing false-positive test results and avoiding burdensome food challenges. In addition, the characterisation of allergen specific antibody repertoires during immunotherapy may lead to a better understanding of the underlying mechanism.

\section{Acknowledgements}

We thank dr. Edward F. Knol for critically reviewing the manuscript. This review belongs to a project that is partially funded by EUROIMMUN AG, Lübeck, Germany. 


\section{References}

1. Bird, J. A., Lack, G. \& Perry, T. T. Clinical Management of Food Allergy. J Allergy Clin Immunol Pract 3, 1-11 (2015).

2. Roth, A. J. et al. Epitope specificity determines pathogenicity and detectability in ANCA-associated vasculitis. J Clin Invest 123, 1773-83 (2013).

3. Burks, A. W. et al. Mapping and Mutational Analysis of the IgE-Binding Epitopes on Ara h 1, a Legume Vicilin Protein and a Major Allergen in Peanut Hypersensitivity. Eur J Biochem 245, 334-9 (1997).

4. Shreffler, W. G. et al. Microarray immunoassay: Association of clinical history, in vitro IgE function, and heterogeneity of allergenic peanut epitopes. J Allergy Clin Immunol 113, 776-82 (2004).

5. Stanley, J. S. et al. Identification and Mutational Analysis of the Immunodominant IgE Binding Epitopes of the Major Peanut Allergen Ara h 2. Arch Biochem Biophys 342, 244-53 (1997).

6. Shreffler, W. G., Lencer, D. A., Bardina, L. \& Sampson, H. A. IgE and IgG4 epitope mapping by microarray immunoassay reveals the diversity of immune response to the peanut allergen, Ara h 2. J Allergy Clin Immunol 116, 893-9 (2005).

7. Rougé, P. et al. Mapping and conformational analysis of IgE-binding epitopic regions on the molecular surface of the major Ara $\mathrm{h} 3$ legumin allergen of peanut (Arachis hypogaea). Mol Immunol 46, 1067-75 (2009).

8. Bøgh, K. L. et al. IgE versus IgG4 epitopes of the peanut allergen Ara $\mathrm{h} 1$ in patients with severe allergy. Mol Immunol 58, 169-76 (2014).

9. Otsu, K., Guo, R. \& Dreskin, S. C. Epitope analysis of Ara h 2 and Ara h 6: characteristic patterns of IgE-binding fingerprints among individuals with similar clinical histories. Clin Exp Allergy 45, 471-84 (2015).

10. Chen, X. et al. Conformational lgE epitopes of peanut allergens Ara h 2 and Ara h 6. Clin Exp Allergy 46, 1120-8 (2016).

11. Nakajima-Adachi, $H$. et al. Determinant analysis of $\lg E$ and $\lg G 4$ antibodies and $T$ cells specific for bovine as1-casein from the same patients allergic to cow's milk: Existence of $\alpha$ s1-casein-specific B cells and T cells characteristic in cow'smilk allergy. J Allergy Clin Immunol 101, 660-71 (1998).

12. Chatchatee, P. et al. Identification of IgE- and IgG-binding epitopes on as1casein: Differences in patients with persistent and transient cow's milk allergy. $J$ Allergy Clin Immunol 107, 379-83 (2001). 
13. Heinzmann, A. et al. The Recognition Pattern of Sequential B Cell Epitopes of Beta-Lactoglobulin Does Not Vary with the Clinical Manifestations of Cow's Milk Allergy. Int Arch Allergy Immunol 120, 280-6 (1999).

14. Spuergin, P. et al. Allergenic epitopes of bovine as1-casein recognized by human IgE and IgG. Allergy 51, 306-12 (1996).

15. Järvinen, K.-M. et al. IgE and IgG Binding Epitopes on a-Lactalbumin and BLactoglobulin in Cow's Milk Allergy. Int Arch Allergy Immunol 126, 111-8 (2001).

16. Lisson, M. \& Erhardt, G. Mapping of Epitopes Occurring in Bovine as1-Casein Variants by Peptide Microarray Immunoassay. Methods Mol Biol 1352, 279-96 (2016).

17. Chen, J.-C. et al. Identification of Critical Amino Acids in an Immunodominant IgE Epitope of Pen c 13, a Major Allergen from Penicillium citrinum. PLoS ONE 7, e34627 (2012).

18. Satoh, R. et al. Identification of an IgE-Binding Epitope of a Major Buckwheat Allergen, BWp16, by SPOTs Assay and Mimotope Screening. Int Arch Allergy Immunol 153, 133-40 (2010).

19. Willison, L. N. et al. Conformational epitope mapping of Pru du 6, a major allergen from almond nut. Mol Immunol 55, 253-63 (2013).

20. Guan, X. et al. Epitope mapping of $7 S$ cashew antigen in complex with antibody by solution-phase H/D exchange monitored by FT-ICR mass spectrometry. $J$ Mass Spec 50, 812-9 (2015).

21. Mishra, A., Gaur, S. N., Lavasa, S. \& Arora, N. In vitro assessment of allergenicity features and localization of probable lgE binding regions. Food Chem Toxicol 84, 181-7 (2015).

22. Knol, E. F. Requirements for effective IgE cross-linking on mast cells and basophils. Mol Nutr Food Res 50, 620-4 (2006).

23. Kane, P. M., Holowka, D. \& Baird, B. Cross-linking of lgE-receptor complexes by rigid bivalent antigens greater than $200 \mathrm{~A}$ in length triggers cellular degranulation. J Cell Biol 107, 969-80 (1988).

24. Kane, P. et al. Cross-Linking of IgE-receptor complexes at the cell surface: Synthesis and characterization of a long bivalent hapten that is capable of triggering mast cells and rat basophilic leukemia cells. Mol Immunol 23, 783-90 (1986).

25. Biasini, M. et al. SWISS-MODEL: modelling protein tertiary and quaternary structure using evolutionary information. Nucleic Acids Res 42, W252-8 (2014).

26. Bienert, S. et al. The SWISS-MODEL Repository - new features and functionality. Nucleic Acids Res 45, D313-9 (2016). 
27. Guex, N., Peitsch, M. C. \& Schwede, T. Automated comparative protein structure modeling with SWISS-MODEL and Swiss-PdbViewer: A historical perspective. ELECTROPHORESIS 30, S162-73 (2009).

28. Benkert, P., Biasini, M. \& Schwede, T. Toward the estimation of the absolute quality of individual protein structure models. Bioinformatics 27, 343-50 (2010).

29. Bertoni, M. et al. Modeling protein quaternary structure of homo- and heterooligomers beyond binary interactions by homology. Sci Rep 7, 10480 (2017).

30. Pettersen, E. F. et al. UCSF Chimera: A visualization system for exploratory research and analysis. J Comput Chem 25, 1605-12 (2004).

31. Schweitzer-Stenner, R. \& Pecht, I. Parameters determining the stimulatory capacity of the type I Fce-receptor. Immunol Let 68, 59-69 (1999).

32. DeLisi, C. \& Siraganian, R. P. Receptor cross-linking and histamine release. I. The quantitative dependence of basophil degranulation on the number of receptor doublets. J Immunol 122, 2286-92 (1979).

33. Collins, A. M., Basil, M., Nguyen, K. \& Thelian, D. Rat basophil leukaemia (RBL) cells sensitized with low affinity IgE respond to high valency antigen. Clin Exp Allergy 26, 964-70 (1996).

34. Christensen, L. H. et al. Several distinct properties of the IgE repertoire determine effector cell degranulation in response to allergen challenge. J Allergy Clin Immunol 122, 298-304 (2008).

35. Nguyen, K.-L., Gillis, S. \& MacGlashan, D. W. A comparative study of releasing and nonreleasing human basophils: Nonreleasing basophils lack an early component of the signal transduction pathway that follows IgE cross-linking. J Allergy Clin Immunol 85, 1020-9 (1990).

36. Kepley, C. L. et al. Syk deficiency in nonreleaser basophils. J Allergy Clin Immunol 104, 279-84 (1999).

37. Knol, E. F. et al. Differential activation of human basophils by anti-IgE and formylmethionyl-leucyl-phenylalanine. Indications for protein kinase C-dependent and -independent activation pathways. Eur J Immunol 21, 881-5 (1991).

38. MacGlashan, D. W. Relationship between spleen tyrosine kinase and phosphatidylinositol 5/ phosphatase expression and secretion from human basophils in the general population. J Allergy Clin Immunol 119, 626-33 (2007).

39. Masthoff, L. J. et al. A systematic review of the effect of thermal processing on the allergenicity of tree nuts. Allergy 68, 983-93 (2013).

40. Untersmayr, E. et al. Incomplete digestion of codfish represents a risk factor for anaphylaxis in patients with allergy. J Allergy Clin Immunol 119, 711-7 (2007). 
41. Untersmayr, E. \& Jensen-Jarolim, E. The role of protein digestibility and antacids on food allergy outcomes. J Allergy Clin Immunol 121, 1301-8 (2008).

42. Dirks, C. G. et al. Does absorption across the buccal mucosa explain early onset of food-induced allergic systemic reactions? J Allergy Clin Immunol 115, 1321-3 (2005).

43. Nesbit, J. B. et al. Ara h 1 structure is retained after roasting and is important for enhanced binding to lgE. Mol Nutr Food Res 56, 1739-47 (2012).

44. Bernard, $\mathrm{H}$. et al. Allergenicity of peanut component Ara $\mathrm{h}$ 2: Contribution of conformational versus linear hydroxyproline-containing epitopes. J Allergy Clin Immunol 135, 1267-74.e8 (2015).

45. Stănciuc, N., Banu, I., Turturică, M. \& Aprodu, I. pH and heat induced structural changes of chicken ovalbumin in relation with antigenic properties. Int $J$ Biol Macromol 93, 572-81 (2016).

46. Bloom, K. A. et al. Effect of heat treatment on milk and egg proteins allergenicity. Ped Allergy Immunol 25, 740-6 (2014).

47. Yoshino, K. et al. Peptic digestibility of raw and heat-coagulated hen's egg white proteins at acidic pH range. Int J Food Sci Nutr 55, 635-40 (2004).

48. Khan, I. J., Di, R., Patel, P. \& Nanda, V. Evaluating pH-Induced Gastrointestinal Aggregation of Arachis hypogaea 1 Fragments as Potential Components of Peanut Allergy. J Agricul Food Chem 61, 8430-5 (2013).

49. Van Boxtel, E. L., Koppelman, S. J., van den Broek, L. A. M. \& Gruppen, H. Determination of Pepsin-Susceptible and Pepsin-Resistant Epitopes in Native and Heat-Treated Peanut Allergen Ara h 1. J Agricul Food Chem 56, 2223-30 (2008).

50. Koppelman, S. J., Hefle, S. L., Taylor, S. L. \& de Jong, G. A. H. Digestion of peanut allergens Ara h 1, Ara h 2, Ara h 3, and Ara h 6: A comparative in vitro study and partial characterization of digestion-resistant peptides. Mol Nutr Food Res 54, 1711-21 (2010).

51. Sordet, C. et al. IgE-binding epitopic peptide mapping on a three-dimensional model built for the 13S globulin allergen of buckwheat (Fagopyrum esculentum). Peptides 30, 1021-7 (2009).

52. Lin, J. \& Sampson, H. A. IgE Epitope Mapping Using Peptide Microarray Immunoassay. Methods Mol Biol 1592, 177-87 (2017).

53. Sircar, G. et al. Epitope Mapping of Rhi o 1 and Generation of a Hypoallergenic Variant. J Biol Chem 291, 18016-29 (2016). 
54. Zheng, L.-N. et al. Mapping IgE binding epitopes of major shrimp (Penaeus monodon) allergen with immunoinformatics tools. Food Chem Toxicol 49, 2954-60 (2011).

55. Fedorov, A. A. et al. The molecular basis for allergen cross-reactivity: crystal structure and IgE-epitope mapping of birch pollen profilin. Structure 5, 33-45 (1997).

56. Chen, X. \& Dreskin, S. C. Application of phage peptide display technology for the study of food allergen epitopes. Mol Nutr Food Res 61, 1600568 (2017).

57. Leung, N. Y. H. et al. Screening and identification of mimotopes of the major shrimp allergen tropomyosin using one-bead-one-compound peptide libraries. Cel Mol Immunol 14, 308-18 (2015).

58. Hantusch, B. et al. Mapping of conformational IgE epitopes on Phl p 5 a by using mimotopes from a phage display library. J Allergy Clin Immunol 114, 1294-1300 (2004).

59. Sheriff, S. et al. Three-dimensional structure of an antibody-antigen complex. Proc Natl Acad Sci U S A 84, 8075-9 (1987).

60. Padlan, E. A. et al. Structure of an antibody-antigen complex: crystal structure of the HyHEL-10 Fab-lysozyme complex. Proc Natl Acad Sci U S A 86, 5938-42 (1989).

61. Braden, B. C. et al. Three-dimensional structures of the free and the antigencomplexed Fab from monoclonal anti-lysozyme antibody D44.1. J Mol Biol 243, 767-81 (1994).

62. Li, Y., Li, H., Smith-Gill, S. J. \& Mariuzza, R. A. Three-Dimensional Structures of the Free and Antigen-Bound Fab from Monoclonal Antilysozyme Antibody HyHEL-63. Biochemistry 39, 6296-309 (2000).

63. Niemi, M. et al. Molecular Interactions between a Recombinant IgE Antibody and the B-Lactoglobulin Allergen. Structure 15, 1413-21 (2007).

64. Padavattan, S. et al. High-Affinity IgE Recognition of a Conformational Epitope of the Major Respiratory Allergen Phl p 2 As Revealed by X-Ray Crystallography. J Immunology 182, 2141-51 (2009).

65. Pomés, A. Relevant B Cell Epitopes in Allergic Disease. Int Arch Allergy Immunol 152, 1-11 (2010).

66. Sampson, H. A. \& Scanlon, S. M. Natural history of food hypersensitivity in children with atopic dermatitis. J Ped 115, 23-7 (1989).

67. Vila, L. et al. Role of conformational and linear epitopes in the achievement of tolerance in cow's milk allergy. Clin Exp Allergy 31, 1599-606 (2001). 
68. Järvinen, K.-M. et al. B-cell epitopes as a screening instrument for persistent cow's milk allergy. J Allergy Clin Immunol 110, 293-7 (2002).

69. Caubet, J. C. et al. Natural tolerance development in cow's milk allergic children: IgE and lgG4 epitope binding. Allergy 72, 1677-85 (2017).

70. Holen, E., Bolann, B. \& Elsayed, S. Novel B and T cell epitopes of chicken ovomucoid (Gal d 1) induce T cell secretion of IL-6, IL-13, and IFN-gamma. Clin Exp Allergy 31, 952-64 (2001).

71. Mine, Y. \& Rupa, P. Fine mapping and structural analysis of immunodominant $\lg$ E allergenic epitopes in chicken egg ovalbumin. Protein Eng Design Select 16, 747-52 (2003).

72. Lin, J. et al. A bioinformatics approach to identify patients with symptomatic peanut allergy using peptide microarray immunoassay. J Allergy Clin Immunol 129, 1321-8.e5 (2012).

73. Klemans, R. J. B. et al. Diagnostic accuracy of specific IgE to components in diagnosing peanut allergy: a systematic review. Clin Exp Allergy 45, 720-30 (2015).

74. Husslik, F. et al. The conformational IgE epitope profile of soya bean allergen Gly m 4. Clin Exp Allergy 46, 1484-97 (2016).

75. Sackesen, C. et al. A new Luminex-based peptide assay to identify reactivity to baked, fermented, and whole milk. Allergy 74, 327-36 (2018).

76. Wang, J. et al. Correlation of IgE/lgG4 milk epitopes and affinity of milk-specific $\lg \mathrm{E}$ antibodies with different phenotypes of clinical milk allergy. $J$ Allergy Clin Immunol 125, 695-702.e6 (2010).

77. Aalberse, R. C. \& Platts-Mills, T. A. How do we avoid developing allergy: modifications of the $\mathrm{TH} 2$ response from a B-cell perspective. J Allergy Clin Immunol 113, 983-6 (2004).

78. Ruiter, B. et al. Maintenance of tolerance to cow's milk in atopic individuals is characterized by high levels of specific immunoglobulin G4. Clin Exp Allergy 37, 1103-10 (2007).

79. Ahrens, B. et al. The role of hen's egg-specific $\lg \mathrm{E}$, IgG and $\lg G 4$ in the diagnostic procedure of hen's egg allergy. Allergy 65, 1554-7 (2010).

80. Gieras, A. et al. Molecular determinants of allergen-induced effector cell degranulation. J Allergy Clin Immunol 119, 384-90 (2007).

81. Fewtrell, C. \& Metzger, H. Larger oligomers of IgE are more effective than dimers in stimulating rat basophilic leukemia cells. J Immunol 125, 701-10 (1980). 
82. Sampson, H. A., Broadbent, K. R. \& Bernhisel-Broadbent, J. Spontaneous Release of Histamine from Basophils and Histamine-Releasing Factor in Patients with Atopic Dermatitis and Food Hypersensitivity. N Engl J Med 321, 228-32 (1989).

83. Yamada, Y. et al. miR-142-3p enhances FceRI-mediated degranulation in mast cells. Biochem Biophys Res Commun 443, 980-6 (2014).

84. Wang, Q. et al. Down-Regulation of MicroRNA-223 Promotes Degranulation via the PI3K/Akt Pathway by Targeting IGF-1R in Mast Cells. PLOS ONE 10, e0123575 (2015).

85. Mayoral, R. J. et al. MiR-221 Influences Effector Functions and Actin Cytoskeleton in Mast Cells. PLoS ONE 6, e26133 (2011).

86. Pua, H. H. \& Ansel, K. M. MicroRNA regulation of allergic inflammation and asthma. Curr Opinion Immunol 36, 101-8 (2015).

87. Reber, L. L., Hernandez, J. D. \& Galli, S. J. The pathophysiology of anaphylaxis. J Allergy Clin Immunol 140, 335-48 (2017).

88. Szczepankiewicz, A., Bręborowicz, A., Sobkowiak, P. \& Popiel, A. Polymorphisms of two histamine-metabolizing enzymes genes and childhood allergic asthma: a case control study. Clin Mol Allergy 8, 1 (2010).

89. García-Martín, E. et al. Polymorphisms of histamine-metabolizing enzymes and clinical manifestations of asthma and allergic rhinitis. Clin Exp Allergy 37, 117582 (2007).

90. Kruse, S. et al. The lle198Thr and Ala379Val Variants of Plasmatic Paf-Acetylhydrolase Impair Catalytical Activities and Are Associated with Atopy and Asthma. Am J Human Gen 66, 1522-30 (2000).

91. Vadas, P. et al. Platelet-Activating Factor, PAF Acetylhydrolase, and Severe Anaphylaxis. N Engl J Med 358, 28-35 (2008).

92. Palgan, K. \& Bartuzi, Z. Platelet activating factor in allergies. Int J Immunopath Pharmacol 28, 584-9 (2015).

93. Smith, P. K., Hourihane, J. O. \& Lieberman, P. Risk multipliers for severe food anaphylaxis. World Allergy Organ J 8, 30 (2015).

94. Vickery, B. P. et al. Peanut oral immunotherapy modifies IgE and IgG4 responses to major peanut allergens. J Allergy Clin Immunol 131, 128-34.e3 (2013).

95. Bøgh, K. L. et al. IgE epitopes of intact and digested Ara h 1: A comparative study in humans and rats. Mol Immunol 51, 337-46 (2012). 
96. Flinterman, A. E. et al. Peanut epitopes for IgE and IgG4 in peanut-sensitized children in relation to severity of peanut allergy. J Allergy Clin Immunol 121, $737-$ 43.e10 (2008).

97. Savilahti, E. M. et al. Early recovery from cow's milk allergy is associated with decreasing IgE and increasing IgG4 binding to cow's milk epitopes. J Allergy Clin Immunol 125, 1315-21.e9 (2010).

98. Cerecedo, I. et al. Mapping of the $\lg E$ and $\lg G 4$ sequential epitopes of milk allergens with a peptide microarray-based immunoassay. J Allergy Clin Immunol 122, 589-94 (2008).

99. Santos, A. F. et al. IgG4 inhibits peanut-induced basophil and mast cell activation in peanut-tolerant children sensitized to peanut major allergens. J Allergy Clin Immunol 135, 1249-56 (2015).

100. Kepley, C. L. et al. Co-aggregation of FcyRII with FceRI on Human Mast Cells Inhibits Antigen-induced Secretion and Involves SHIP-Grb2-Dok Complexes. $J$ Biol Chem 279, 35139-49 (2004).

101. Gepp, B. et al. Monitoring the epitope recognition profiles of $\lg E$, IgG1, and IgG4 during birch pollen immunotherapy. J Allergy Clin Immunol 137, 1600-3.e1 (2016).

102. Vickery, B. P. et al. Individualized IgE-based dosing of egg oral immunotherapy and the development of tolerance. Ann Allergy Asthma Immunol 105, 444-50 (2010).

103. Jones, S. M. et al. Clinical efficacy and immune regulation with peanut oral immunotherapy. J Allergy Clin Immunol 124, 292-300.e97 (2009).

104. Wai, C. Y. Y., Leung, N. Y. H., Leung, P. S. C. \& Chu, K. H. Immunotherapy of Food Allergy: a Comprehensive Review. Clin Rev Allergy Immunol 57, 55-73 (2017).

105. Savilahti, E. M. et al. Use of IgE and IgG4 epitope binding to predict the outcome of oral immunotherapy in cow's milk allergy. Ped Allergy Immunol 25, 227-35 (2014).

106. Martínez-Botas, J. et al. Identification of novel peptide biomarkers to predict safety and efficacy of cow's milk oral immunotherapy by peptide microarray. Clin Exp Allergy 45, 1071-84 (2015).

107. Wanich, N., Nowak-Wegrzyn, A., Sampson, H. A. \& Shreffler, W. G. Allergenspecific basophil suppression associated with clinical tolerance in patients with milk allergy. J Allergy Clin Immunol 123, 789-94.e20 (2009). 
108. Thyagarajan, A. et al. Evidence of pathway-specific basophil anergy induced by peanut oral immunotherapy in peanut-allergic children. Clin Exp Allergy 42, 1197-1205 (2012).

109. MacGlashan, D., Moore, G. \& Muchhal, U. Regulation of IgE-mediated signalling in human basophils by CD32b and its role in Syk down-regulation. Clin Exp Allergy 44, 713-23 (2014).

110. DuToit, G. et al. Randomized Trial of Peanut Consumption in Infants at Risk for Peanut Allergy. N Engl J Med 372, 803-13 (2015).

111. DuToit, G. et al. Effect of Avoidance on Peanut Allergy after Early Peanut Consumption. N Engl J Med 374, 1435-43 (2016).

112. Cocco, R. R., Järvinen, K.-M., Sampson, H. A. \& Beyer, K. Mutational analysis of major, sequential IgE-binding epitopes in as1-casein, a major cow's milk allergen. J Allergy Clin Immunol 112, 433-7 (2003).

113. Han, N. et al. Identification of amino acids critical for lgE-binding to sequential epitopes of bovine $\mathrm{k}$-casein and the similarity of these epitopes to the corresponding human K-casein sequence. Allergy 63, 198-204 (2008).

114. Hecker, J. et al. Generation and epitope analysis of human monoclonal antibody isotypes with specificity for the timothy grass major allergen $\mathrm{Phl} \mathrm{p} 5 \mathrm{a}$. Mol Immunol 48, 1236-44 (2011).

115. Hecker, J. et al. An IgE epitope of Bet v 1 and fagales PR10 proteins as defined by a human monoclonal IgE. Allergy 67, 1530- (2012).

116. Patil, S. U. et al. Peanut oral immunotherapy transiently expands circulating Ara h 2-specific B cells with a homologous repertoire in unrelated subjects. J Allergy Clin Immunol 136, 125-34.e12 (2015).

117. Hoh, R. A. et al. Single B-cell deconvolution of peanut-specific antibody responses in allergic patients. J Allergy Clin Immunol 137, 157-67 (2016).

118. Tiller, T. et al. Efficient generation of monoclonal antibodies from single human $B$ cells by single cell RT-PCR and expression vector cloning. J Immunol Methods 329, 112-24 (2008).

119. Wong, K. J. et al. IgE+ B cells are scarce, but allergen-specific B cells with a memory phenotype circulate in patients with allergic rhinitis. Allergy 70, 420-8 (2015).

120. Davies, J. M., Platts-Mills, T. A. \& Aalberse, R. C. The enigma of IgE+ B-cell memory in human subjects. J Allergy Clin Immunol 131, 972-6 (2013).

121. Berkowska, M. A. et al. Human $\lg \mathrm{E}(+) \mathrm{B}$ cells are derived from T cell-dependent and T cell-independent pathways. J Allergy Clin Immunol 134, 688-97.e6 (2014). 
122. Geha, R. S., Jabara, H. H. \& Brodeur, S. R. The regulation of immunoglobulin E class-switch recombination. Nat Rev Immunol 3, 721-32 (2003).

123. Caligaris-Cappio, F. et al. Identification of malignant plasma cell precursors in the bone marrow of multiple myeloma. J Clin Invest 76, 1243-51 (1985).

124. Allman, D. \& Pillai, S. Peripheral B cell subsets. Curr Opin Immunol 20, 149-57 (2008). 
MIUT:

AGCCTCTGGA7

CCTGTTTCTGCAAA

iGCGTGGTCCAGCCTC

AGGGCCGATTCACCATL ,

AATTGTGTTGACACAGTCTCCAGCCALU

'TGGCATCCCAGCCAGGTTCAGTGGCAGTGGG'TC I

$\checkmark$ CAGATGACCCAGTCTCCTTCCACCCTGGCTGCATCTGTAG

AGTCCCATCAAGGTTCAGCGGCAGTGGGTCTGGGACAGAGTTCACL

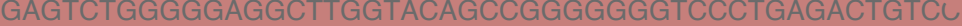

ITATCGAGACTCTGTGAAGGGCCGCTTCACCATCTCCAGAGACAATGCCAAAAAC ¿AGGGGACCACGGTCATCGTCTCCTCAGCAGGTGCAGCTGGTGGAGTCTGGGGGAGG ¿GAGTGGGTTTCATTTATACGCAAGGATGGAGCTAGTAAATACTATGGAGACTCCGTGAAGGG , CCCTGGGTTACTTTGACTACTGGGGCCAGGGAACCCTGGTCACCGTCTCCTCAGGAAATTGTGT CATAAACCTGGCCAGGCTCCCAGGCTCCTCATCTATGATGCATCCAACAGGGCCACTGGCATCCC ACTGGCCTCCGCTCACTTTCGGCGGAGGGACCAAGGTGGAGATCAAACGACATCCAGATGACCCAG

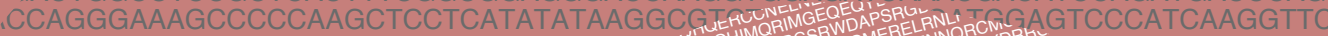

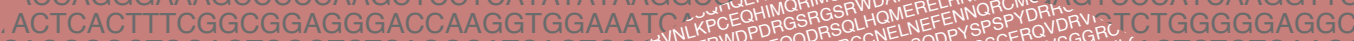

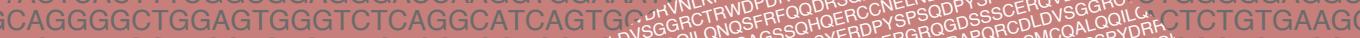

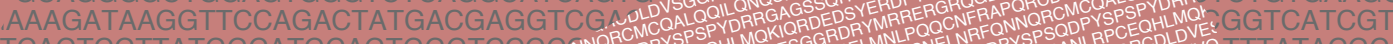

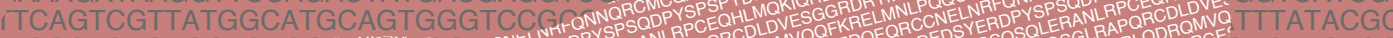

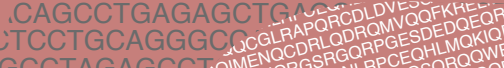

tGCCTAGAGC

GCCTGATGAT

CACGTTTAGG

ATGGCCAGTC

CCATCTCCAGAG

G

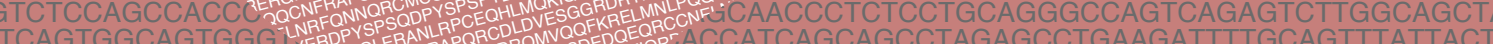

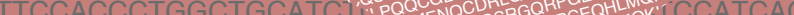

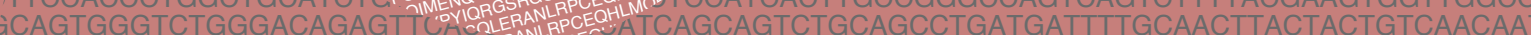

ACAGCCGGGGGGGTCCCTGAGACTGTCCTGTGCAGCCTCTGGATTCACGTTTAGGAGATATGCTTTGAGTTGGGTCCGC ICTTCACCATCTCCAGAGACAATGCCAAAAACATCCTGTTTCTGCAAATGGCCAGTCCGAGAGTCGAAGACGCGGCCGTT TCAGCAGGTGCAGCTGGTGGAGTCTGGGGGAGGCGTGGTCCAGCCTGGGGGGTCCCTGAGACTCTCCTGTGTAGCGTC aATGGAGCTAGTAAATACTATGGAGACTCCGTGAAG TTCTCCAGAGACAATTCCAAAGACACTCTCTTTCT iCCAGGGAACCCTGGTCACCGTCTCCTCAGGAA UTCCTCATCTATGATGCATCCAACAGGGCCAC CATATATAAGGCGTCTAATTTACAAGATGGAGTCC AAGGTGGAAATCAAACGAGGTGCAGTTGGTGGAGTC viGCATCAGTGGCAGTGGAAACAAAACATATTATCGAGA AACGAGGTCGAGATTATGGACGTCTGGGGCCAGGGGACCA GGGTCCGCCAGGCTCCAGGCAAGGGGCTGGAGTGGGTTTCA ?TTGGCAGCTACTTAGCCTGGTACCAACATAAACCTGGCCAGGC

TTTATTACTGTCAGCAGCGTAGCGACTGGCCTCCGCTCACT

CAGGGAAAGCCCCCAAGCT

ACTTTCGGCGGAGGGACC

IGCTGGAGTGGGTCTCAGGC

GGTTCCAGACTATGACGAGG

TGGCATGCAGTGGGTCC

'TGAGGACACGGCTG

AGATTTTGCAG

TTACGAAGTGG ACTTACTACTGT CTTTGAGTTG GAAGACGCGC 'TCTCCTGTGT AAAGACACTC iTCTCCAGGG GAGACAGAG 4CCCTCACCAר AAAAACATCCTG IGGGGAGGCGTGG

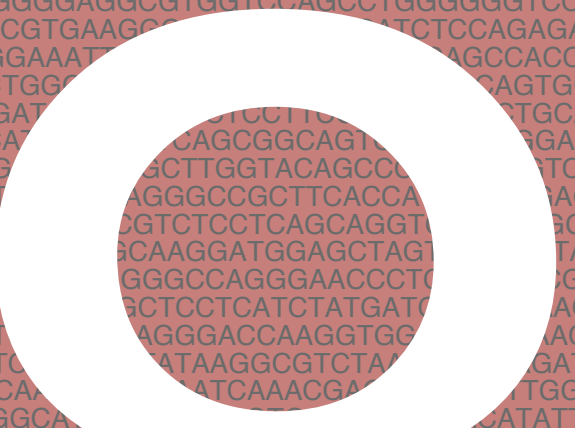
ACCCTGTCTITGTCTCCAGGGGAAAG GGGTCTGGGACAGACTTCACTCTCA CATAGAGAGAGTCTCCAT ACAGAGTTCACCCTCACCATCAGCA CCCTGAGACTGTCCTGTGCAGCCT GACAATGCCAAAAACATCCTGTTTC GTGGAGTCTGGGGGAGGCGTGGT ATGGAGACTCCGTGAAGGGCCGA ITCTCCTCAGGAAATTGTGTTGACA CAGGGCCACTGGCATCCCAGCCAC GACATCCAGATGACCCAGTCTCC TGGAGTCCCATCAAGGTTCAGCGG GTGGAGTCTGGGGGAGGCTTGGTA TTATCGAGACTCTGTGAAGGGCCGC TCCGC

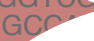
AGGCCAGGGGACCACGGTCATCGTCTCCTC CAGGGGACCACGGTCATCGTCTCCTC,
AGTGGGTTTCATTTATACGCAAGGATG

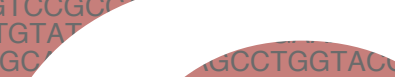
GAGCAGAA ATCAGCAGAA GATAGTGCCCTACTC CAGGCTCCAGGGCAGGGG ATTACTGTGCGAAAGATAAG GGATTCACCTTCAGTCGTTA GCAAATGGACAGCCTGAGAG CAAATGGACAGCCTGAGAG CAACCCTCTCCTGCAGGG ACTTGCCGGGCCAGTCA

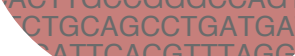

CTGGCCAGGCTCCCAGGCTCCTCAT CGCTCACTTTCGGCGGAGGGACC
CCCCAAGCTCCTCATATATAAGGC IGGAGGGACCAAGGTGGAAATCA GGGTCTCAGGCATCAGTGGCAG ACTATGACGAGGTCGAGATTATG CAGTGGGTCCGCCAGGCTCCAC ACACGGCTGTATATTACTGTGCG AAGTCTTGGCAGCTACTTAGCCTC ITGCAGTTTATTACTGTCAGCAGC CGAAGTGGTTGGCCTGGTATCAC ACTACTGTCAACAATATGATAGT TGAGTTGGGTCCGCCAGGCTCCA AAGACGCGGCCGTTTATTACTGTGC AurumuTCCGTGAAGGGCCGA

GTGTTGACACA ETCTCCTGTGTAGCGTCTGGATTCACCT
CAAAGACACTCTCTTTCTGCAAATGGAC,

ACGACATCCAGATGACCCAGTCTCCTTCCACCCTGGCTGCATCTGTAGGAGACAGAGTCTCCATCACTTGCCGG GATGGAGTCCCATCAAGGTTCAGCGGCAGTGGGTCTGGGACAGAGTTCACCCTCACCATCAGCAGTCTGCAGCC ITGGTGGAGTCTGGGGGAGGCTTGGTACAGCCGGGGGGGTCCCTGAGACTGTCCTGTGCAGCCTCTGGATTCAO ;ATATTATCGAGACTCTGTGAAGGGCCGCTTCACCATCTCCAGAGACAATGCCAAAAACATCCTGTTTCTGCAAATGC GCCAGGGGACCACGGTCATCGTCTCCTCAGCAGGTGCAGCTGGTGGAGTCTGGGGGAGGCGTGGTCCAGCCTGG GGAGTGGGTTTCATTTATACGCAAGGATGGAGCTAGTAAATACTATGGAGACTCCGTGAAGGG

¿CCTGGGTTACTTTGACTACTGGGGCCAGGGAACCCTGGTCACCGTCTCCTCAGGAAATTGTC

ITAAACCTGGCCAGGCTCCCAGGCTCCTCATCTATGATGCATCCAACAGGGCCACTGGCATCr

IGGCCTCCGCTCACTTTCGGCGGAGGGACCAAGGTGGAGATCAAACGACATCCAGATGACC

AGGGAAAGCCCCCAAGCTCCTCATATATAAGGCGTCTAATTTACAAGATGGAGTCCCATCAAGC

CACTTTCGGCGGAGGGACCAAGGTGGAAATCAAACGAGGTGCAGTTGGTGGAGTCTGGGGG

GGCTGGAGTGGGTCTCAGGCATCAGTGGCAGTGGAAACAAAACATATTATCGAGACTCTGTG

IAGGTTCCAGACTATGACGAGGTCGAGATTATGGACGTCTGGGGCCAGGGGACCACGGTCATCGI

ITATGGCATGCAGTGGGTCCGCCAGGCTCCAGGCAAGGGGCTGGAGTGGGTTTCATTTATACGCAAGGATGGAGi I AG I / ¿AGAGCTGAGGACACGGCTGTATATTACTGTGCGAAAGATAGGTCCCTGGGTTACTTTGACTACTGGGGCCAGGGAACCCTG AGGGCCAGTCAGAGTCTTGGCAGCTACTTAGCCTGGTACCAACATAAACCTGGCCAGGCTCCCAGGCTCCTCATCTATGATG GAGCCTGAAGATTTTGCAGTTTATTACTGTCAGCAGCGTAGCGACTGGCCTCCGCTCACTTTCGGCGGAGGGACCAAGGTGGA 


\section{Comparison of two strategies to generate specific human monoclonal antibodies: Which method to choose for which purpose?}
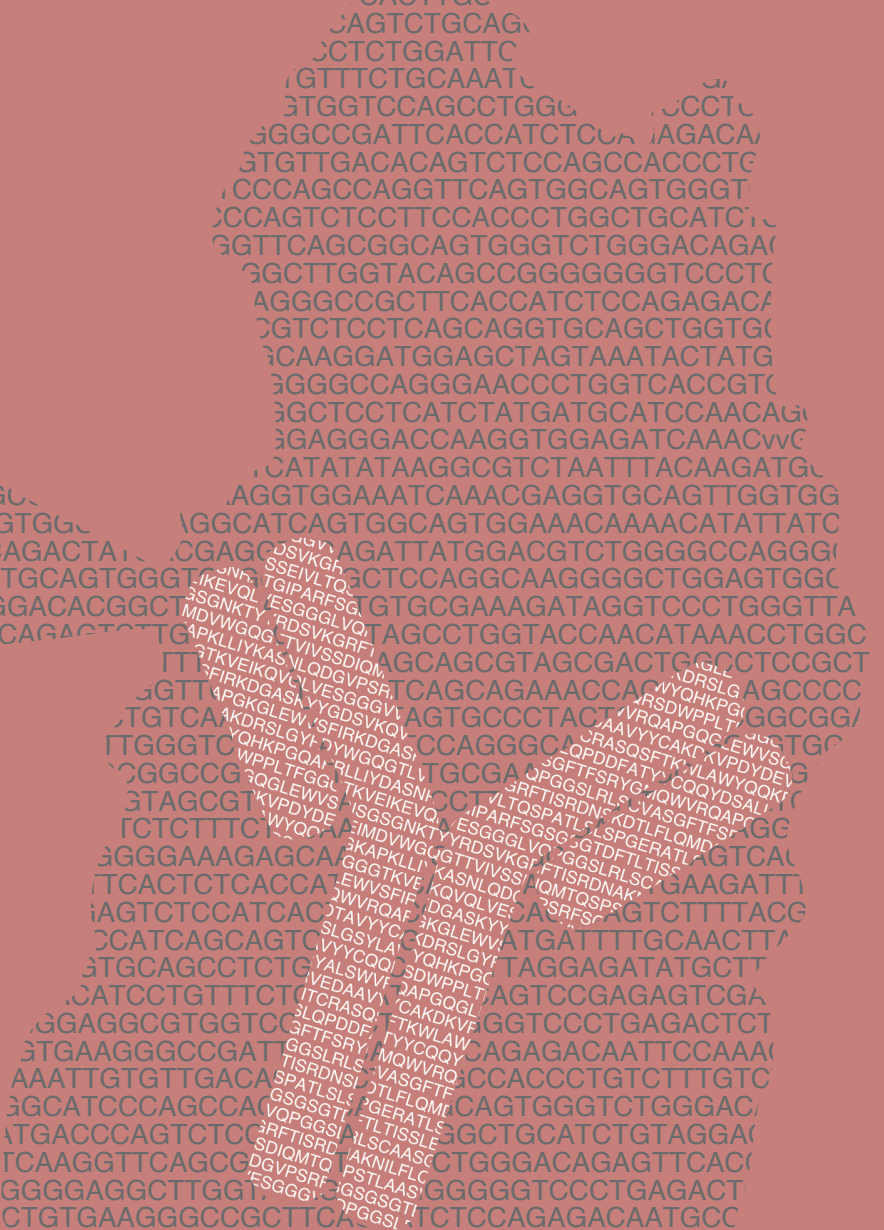

.AGGTGGAAATCAAACGAGGTGCAGTTGGTGG
IGGCATCAGTGGCAGTGGAAACAAAACATATTATC IGGCATCAGTGGCAGTGGAAACAAAACATATTATC
LGAGGUS UGAGGSU,

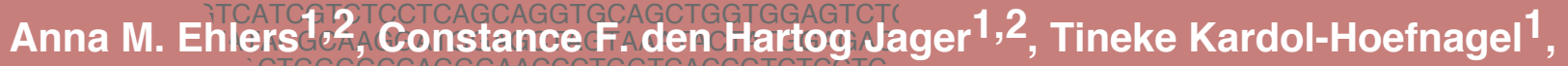

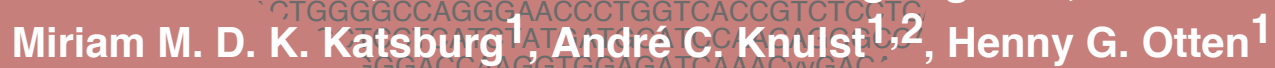

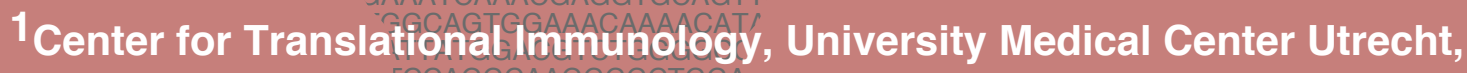
Utrecht University, Netherlands

A.2Department of Dermatelogy/Allergology, University Medical Center Utrecht, iCUtrecht UniversityA Nêtherlands

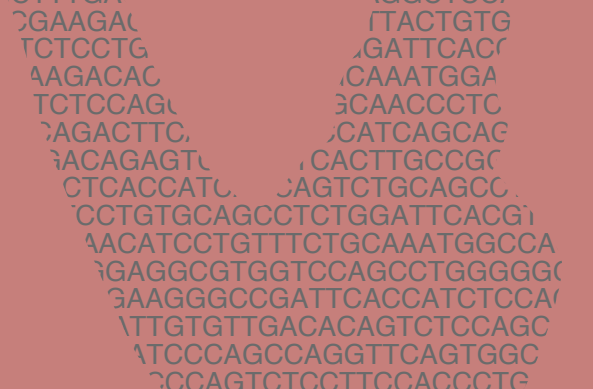




\section{Abstract}

Human monoclonal antibodies (mAbs) are valuable tools to link genetic information with functional features and to provide a platform for conformational epitope mapping. Strategies to generate human mAbs from peripheral blood have been described and used in several studies including single cell sequencing of antigen-binding $B$ cells and the establishment of antigen-specific monoclonal Epstein-Barr Virus (EBV) immortalised lymphoblastoid cell lines (LCLs). However, direct comparisons of these two strategies are scarce. Hence, we sought to set up these two strategies in our laboratory using peanut $2 \mathrm{~S}$ albumins (allergens) and the autoantigen ARHGDIB as antigen targets to directly compare these strategies regarding costs, time expenditure, recovery, throughput and complexity. Regarding single cell sequencing, up to $50 \%$ of corresponding V(D)J gene transcripts were successfully amplified of which $54 \%$ were successfully cloned into expression vectors used for heterologous expression. Seventy-five percent of heterologously expressed $\mathrm{mAbs}$ showed specific binding to peanut $2 \mathrm{~S}$ albumins resulting in an overall recovery of around $25 \%$. In comparison, the establishment of monoclonal EBV-LCLs showed a lower overall recovery of around 16\%. Heterologous expression of a mAb carrying the same variable region as its native counterpart showed comparable concentration-dependent binding abilities. By directly comparing those two strategies, single cell sequencing allows a broad examination of antigen-binding mAbs in a moderate-throughput manner, while the establishment of monoclonal EBV-LCLs is a powerful tool to select a small number of highly reactive mAbs restricted to certain $B$ cell subpopulations. Overall, both strategies, initially set-up for peanut $2 \mathrm{~S}$ albumins, are suitable to obtain human mAbs and they are easily transferrable to other target antigens as shown for ARHGDIB. 


\section{Introduction}

Antibody diversity enables the adaptive immune system to generate a humoral response against virtually any antigen. Gene recombination of variable (V), diversity (D) and joining $(\mathrm{J})$ gene segments for the heavy chain and $\mathrm{V}$ and $\mathrm{J}$ gene segments for the corresponding light chain results in a wide variety of antibodies with distinct specificities. The diversity is even enlarged by imprecision during the $V(D) J$ gene rearrangement process ${ }^{1,2}$. The introduction of somatic hypermutations is an additional tool to increase diversity but more importantly to strengthen antibody's affinity against the respective target ${ }^{3}$.

Disease-related specific antibody repertoires are often studied by comparing specific $B$ cell subpopulations between patients and healthy donors using next generation sequencing ${ }^{4-7}$. This powerful approach, however, does not provide any information about antigen reactivity, affinity and functionality. For this reason, several studies included the generation of human monoclonal antibodies (mAbs) in order to assess their functionality and to map their characteristics to their genetic features ${ }^{8,9}$. Besides identifying genetic features associated with health or disease, human mAbs can support the mapping of conformational epitopes formed by closely located amino acids upon folding ${ }^{9}$. While linear epitopes, comprised of sequential amino acids, can easily be mapped by e.g. peptide microarrays, the mapping of conformational epitopes requires more sophisticated techniques such as mass spectrometry, nuclear magnetic resonance spectroscopy and/or mutation libraries ${ }^{10}$. Since these techniques can hardly be executed with patient serum containing polyclonal antibodies, human mAbs are powerful tools to overcome this obstacle. Moreover, data obtained with mAbs derived from humans are thought to be more easily translatable to clinical research compared with data obtained with e.g. mouse-derived mAbs ${ }^{9,11}$.

The first human mAbs were obtained in the early 90's by phage display technology using single chain or Fab fragment libraries ${ }^{12,13}$. Nearly simultaneously, transgenic animals consisting of human immunoglobulin genes provided an additional tool ${ }^{14}$. These approaches, however, are artificial and cannot represent a complete human antibody repertoire, emphasising the advantage of $m A b s$ generated from human peripheral blood or tissues. The first strategy to generate human mAbs from peripheral blood included the establishment of immortalised B cell lines by Epstein-Barr Virus (EBV) infection followed by limiting dilution cloning ${ }^{15,16}$. More recently, the immortalisation by EBV was partly replaced by $\mathrm{BCl}-6$ overexpression mimicking a germinal centre status accompanied by constant antibody secretion ${ }^{17}$. The most recent technique, however, is single cell sequencing with subsequent heterologous antibody expression ${ }^{18}$.

This study provides a detailed description and comparison of two different strategies to generate human mAbs including single cell sequencing of antigen-binding $B$ cells 
(Method 1) and the establishment of monoclonal EBV-immortalised B cell lines (Method 2). Both methods were used to generate human mAbs against peanut $2 S$ albumins, major allergens in peanut allergy, and anti-Rho guanosine diphosphate dissociation inhibitor 2 (RhoGDI2, alternative 'ARHGDIB'), a non-HLA target potentially involved in graft failure upon kidney transplantation ${ }^{19}$. Method 1 is especially suitable for a broad examination of antigen-binding mAbs due to a less selective process compared with Method 2. Method 2, however, is a powerful tool to select a small number of highly reactive mAbs potentially applicable in the development of treatment strategies. $A$ schematic overview of both methods is shown in Figure 1.

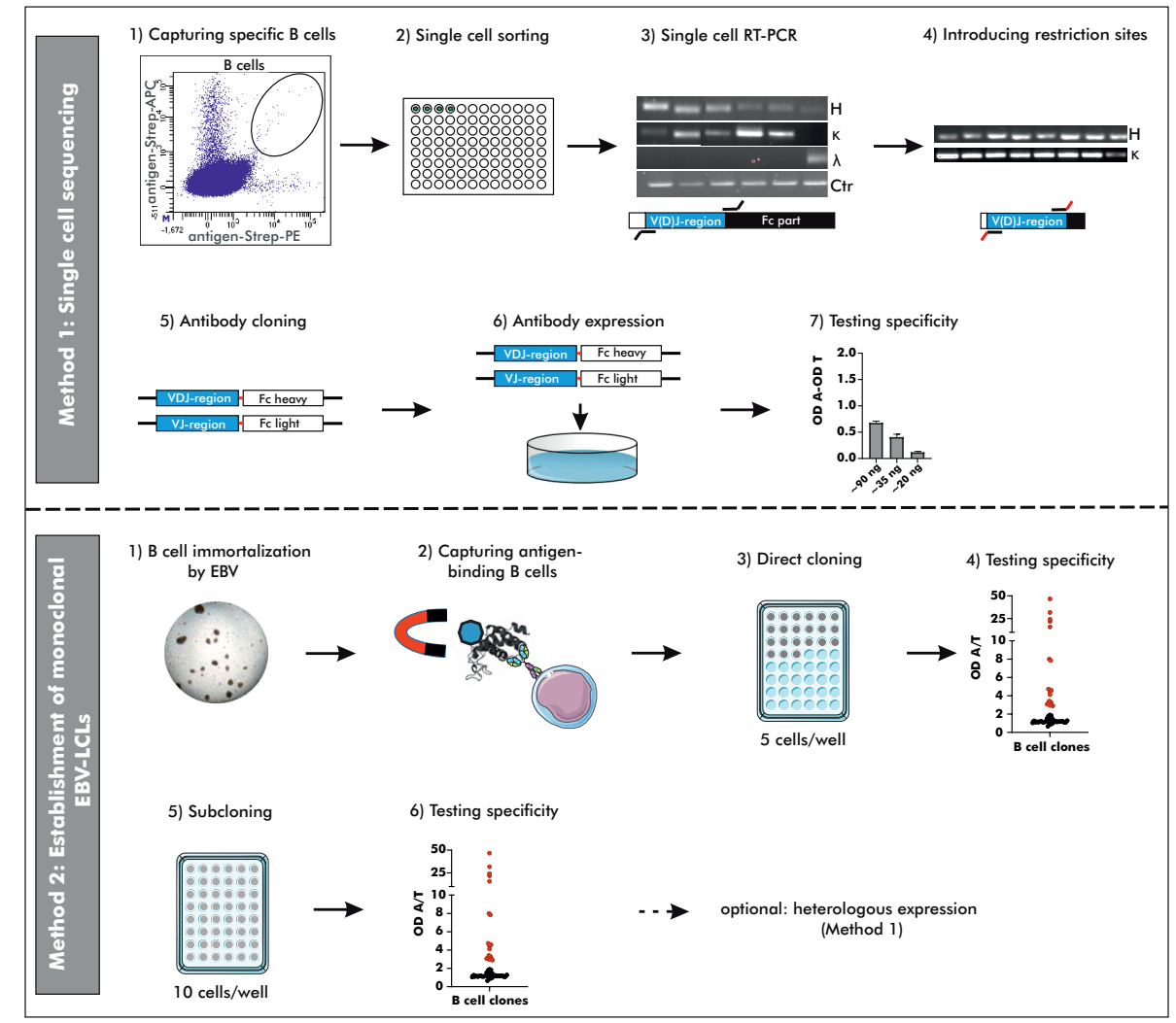

Figure 1: Schematic overview on two different methods to generate human mAbs

Method 1 „Single cell sequencing“ B cells for single cell sorting were captured by double-positive antigen-tetramer staining $(1+2)$. Upon transcription of total $m R N A$ into $c D N A$, the $V(D) J$ gene transcripts of the heavy and corresponding light chain were amplified by reverse transcriptase PCR using multiplex primers (3). The $V(D) J$ gene usage was determined by Sanger sequencing and respective specific simplex primers were used to introduce restriction sites for subsequent cloning (4). Amplified $V(D) J$ gene transcripts were cloned into commercially available pFUSEss-IgH vectors (Invivogen) carrying either the heavy or light chain backbone (5). Vectors with correctly incorporated $V(D) J$ gene transcripts were used for transient mammalian cell transfection (6) and the specificity was examined upon heterologous expression using a direct ELISA (7).

Method 2 „Establishment of monoclonal EBV-LCLs“ Antigen-binding B cells were captured from B cells immortalised by Epstein-Barr Virus (EBV) using antigen-coupled magnetic beads (1+2). Based on theoretical frequency of antigen-binding B cells, cells were directly cloned by seeding 5 cells/well on top of irradiated PBMCs as feeder cells (3). After 4 weeks of culturing, the supernatants were checked for antibodies binding specifically to the antigen of interest (4). B cells with supernatant containing specific antibodies are seeded for an additional round of cloning (5) and the resulting supernatant was screened for specific antibodies (6) after additional 4 weeks of culturing. Monoclonality was checked by Sanger sequencing and the mAbs can be heterologously expressed as described for Method 1. 


\section{Methods}

\section{Method 1: „Single cell sequencing of antigen-binding B cells“}

\section{Antigen-tetramer formation}

The isolated peanut $2 \mathrm{~S}$ albumin fraction or heterologously expressed ARHGDIB were treated with an excess of biotin in accordance with manufacturer's instructions. The excess of biotin resulted on average in four biotin molecules per one molecule protein (EZ-Link ${ }^{T M}$ Sulfo-NHS-Biotin, ThermoFisher Scientific) ${ }^{20}$. Antigen-tetramers were subsequently formed by separately adding streptavidin-PE or streptavidin-APC to the biotinylated $2 S$ albumin fraction or ARHGDIB in a molecular ratio of 1:1 (streptavidin: protein) $)^{21,22}$.

\section{Single-cell sorting of antigen specific B cells}

Blood was drawn into heparin-coated tubes and freshly processed within 24 hours. Peripheral blood mononuclear cells (PBMCs) were obtained by density gradient centrifugation and $B$ cells were, subsequently, negatively enriched from the PBMCs fraction by magnetic beads (B cell isolation kit II, Miltenyi Biotech). Enriched B cells were stained for antigen specificity with CD45-PO (1:40, Life Technologies), CD3-PB (1:160, BD Pharming), CD19-FITC (1:20, BD Pharming), CD14-PE-Cy7 (1:800, ITK Diagnostics BV), CD16- PE-Cy7 (1:1000, BD Pharming) and antigen-tetramers (1:5000 (= 75 pg $2 S$ albumins $\left.\left.\left.{ }^{23}\right)^{1}\right), 1: 500\left(=1.1 \mathrm{ng} \mathrm{ARHGDIB}^{23}\right)^{2}\right)$. Stained B cells were gated for $\mathrm{CD}^{4} 5^{+}, \mathrm{CD}^{-}, \mathrm{CD}^{+} 9^{+}$, antigen tetramers (double positive) ${ }^{+}$and CD14/16 ${ }^{-}$and single cell sorted into 96 wells plates supplemented with $14 \mathrm{mM}$ DTT and $11.2 \mathrm{U}$ RNAse inhibitor in a total volume of $4 \mu \mathrm{l} 0.5 x$ PBS. Plates containing single-sorted B cells were immediately put on dry ice. These plates were stored at $-80{ }^{\circ} \mathrm{C}$ until further processing. While PBMCs from a donor with specific $B$ cells in previous experiments served as positive control, B cells stained with biotin were used as negative control (Ctr 1). The gating was based on the respective negative control 1. An additional negative control (Ctr 2) was added to evaluate the optimal dilution for the antigen-tetramers. This control consisted of a pre-incubation step with non-biotinylated antigen followed by the normal staining protocol described above.

\footnotetext{
$1 \rightarrow 30 \cdot 10^{6}$ antigen-tetramers sufficient for approximately $150-300$ resting $B$ cells
}

${ }^{2} \rightarrow 112 \cdot 10^{6}$ antigen-tetramers sufficient for approximately 500-1000 resting B cells 


\section{Gene amplification of B cell receptors}

$V(D) J$ gene transcripts amplification was performed as previous described with minor modifications ${ }^{18,21,24}$. While keeping the frozen $B$ cells on dry ice, they were supplemented with $1.4 \% \mathrm{NP}-40,3 \mathrm{U}$ RNAse inhibitor and $7 \mu \mathrm{M}$ hexamer primers reaching a total volume of $7.5 \mu$ l. For primer annealing, this mixture was subsequently incubated for one minute at $68{ }^{\circ} \mathrm{C}$ and cDNA transcription was performed in accordance with manufacturer's instruction (SuperScript III, ThermoFisher Scientific). The resulting cDNA was subsequently used as template for the amplification of the heavy chain gene transcript and its corresponding light chain gene transcript. Amplification was accomplished with 1.25 U hot-start DNA polymerase (AmpliTaq Gold, ThermoFisher Scientific), $2.5 \mathrm{mM} \mathrm{MgCl} 2,1 \mathrm{mM}$ dNTP mix and $40 \mathrm{nM}$ multiplex primers (Suppl. Table S1 S3) for 50 cycles. The annealing temperature was set to 62,60 and $58{ }^{\circ} \mathrm{C}$ for heavy, $\mathrm{k}$ and $\lambda$ light chain, respectively.

\section{Sequence analysis of B cell receptors}

Amplified $V(D) J$ gene transcripts were purified by adding $0.5 \mu l$ Exo RI (0.01 U, Bioke) and $1 \mu \mathrm{I}$ FAST-AP ( $1 \mathrm{U}$, ThermoFisher Scientific) and incubating this mixture for $30 \mathrm{~min}$ at $37^{\circ} \mathrm{C}$ followed by $20 \mathrm{~min}$ at $80^{\circ} \mathrm{C}$. Purified heavy chain gene transcripts were Sanger sequenced using $200 \mathrm{nM}$ multiplex reverse or framework (FR) 1 forward primers. Light chain gene transcripts were sequenced using $200 \mathrm{nM}$ of the respective reverse primers. To check the quality of the sequences, they were evaluated using Chromas Lite 2.6.5. Double peaks, potentially resulting from errors in the beginning of the amplification reaction, were aligned to their germline and corrected if plausible. Quality-checked gene sequences were saved as FASTA files and subsequently used for automatic germline alignment using the IgBLAST web interface (reference: IMGT database) ${ }^{25}$. The resulting output was written into a SQLite database using $R$.

\section{Cloning of V(D)J gene transcripts}

To ensure the cloning of the entire $V(D) J$ gene transcript without the introduction of additional amino acids, restriction sites were introduced with specific $V$ gene forward and $\mathrm{J}$ gene reverse primers. This introduction was achieved using $0.5 \mathrm{U}$ Phusion high-fidelity DNA polymerase (New England BioLabs (NEB)) in presence of $1 \mathrm{x}$ reaction buffer, $2.5 \mathrm{mM} \mathrm{MgCl}_{2}, 1 \mathrm{mM}$ dNTP mix and $400 \mathrm{nM}$ respective forward and reverse primer. The annealing temperature of 62,60 and $58{ }^{\circ} \mathrm{C}$ for heavy, $\mathrm{k}$ and $\lambda$ light chain, respectively and the amplification reaction was performed for 30 cycles. Gene products, purified accordingly to manufacturer's instructions (NucleoSpin gel and PCR 
clean up, Macherey Nagel), were digested with $1 \mathrm{U}$ of the respective restriction enzymes Eco RI and NhE I (heavy chain, NEB), BsWi ( $k$ light chain, NEB) or Avr II ( $\lambda$ light chain, NEB). Before cloning the digests into human $\lg \mathrm{H}(\lg E, \lg \mathrm{G} 1, \lg \mathrm{G} 4)$ and $\lg \mathrm{L}(\mathrm{K}$, $\lambda$ ) expression vectors ${ }^{3}$, they were purified as described above and phosphorylated with $1 \mathrm{U}$ T4-polynucleotide kinase (PNK, NEB) for $60 \mathrm{~min}$ at $37{ }^{\circ} \mathrm{C}$ followed by $20 \mathrm{~min}$ at $65{ }^{\circ} \mathrm{C}$. To prevent self-ligation, $1 \mu \mathrm{g}$ of digested and gel-purified parent vector $(1 \mathrm{U}$ respective restriction enzyme) was dephosphorylated with 4 U FAST-AP (ThermoFisher Scientific) for $10 \mathrm{~min}$ at $37^{\circ} \mathrm{C}$ followed by $10 \mathrm{~min}$ at $70{ }^{\circ} \mathrm{C}$. Dephosphorylated vectors were mixed with digests in a molecular ratio of 5:1 and incubated with $1 \cup$ T4 ligase (NEB) for $60 \mathrm{~min}$ at room temperature. The ligated vector was transformed into competent $E$. coli Top10 cells by incubating the mixture for $45 \mathrm{sec}$ at $42{ }^{\circ} \mathrm{C}$ (heat shock). Overnight grown colonies were screened for incorporated $V(D) J$ gene transcripts by PCR using $400 \mathrm{nM}$ forward primers binding to the respective FR2 region and $400 \mathrm{nM}$ reverse primers suitable for the constant part of the respective vector (Suppl. Table S1 - S3) in presence of $2.5 \mathrm{U}$ AmpliTaq polymerase (ThermoFisher Scientific), $2.5 \mathrm{mM}$ $\mathrm{MgCl}_{2}$ and $1 \mathrm{mM}$ dNTP mix. The annealing temperature was set to 63,62 and $61{ }^{\circ} \mathrm{C}$ for heavy, $\mathrm{k}$ and $\lambda$ light chain, respectively and the amplification cycle was repeated for 30 times. Positive clones were grown overnight in $3 \mathrm{ml} \mathrm{LB}$ medium containing either zeocin or blasticidin. Purified vectors (NucleoSpin Plasmid EasyPure, Macherey Nagel) were Sanger sequenced. The correctness was verified by aligning the vector sequence to the first sequence result.

\section{Heterologous expression of monoclonal antibodies}

For heterologous expression of human mAbs, human embryonic kidney (HEK) 293F cells were cultured in FreeStyle 293 expression medium (ThermoFisher Scientific) using $125 \mathrm{ml}$ shaking culture flasks. Exponentially growing cells at a confluence of $80 \%$ and viability of $90 \%$ were transiently transfected with VH and VL expression vectors in a ratio of 2:3 (total $0.5 \mu$ plasmid DNA per $1 \cdot 10^{6}$ cells) using $150 \mathrm{~mm}^{2}$ culture plates and 293fectin ( $2 \mu \mathrm{l} / \mu \mathrm{g}$ plasmid DNA, ThermoFisher Scientific). To ensure a sufficient intake, the expression vectors were supplemented with $0.5 \mu \mathrm{g}$ pAdvantage plasmid (Promega). The supernatant was harvested three days upon transfection and stored at $-20{ }^{\circ} \mathrm{C}$ for further analyses.

\footnotetext{
${ }^{3} \mathrm{hEF} 1-\mathrm{HTLV}$ promotor, IL-2 signal sequence, multiple cloning site upstream the constant regions and zeocin $(\mathrm{IgH})$ or blasticidin $(\mathrm{IgL})$ resistance genes for selection (pFUSEss vector series, Invivogen)
} 


\section{Method 2: "Establishment of monoclonal EBV-LCLs“}

\section{Immortialisation of enriched B cells by Epstein-Barr-Virus}

B cells, isolated from heparin blood as described for Method 1, were immortalised with EBV in presence of the TLR9 agonist CpG 2006 and the immunosuppressive Cyclosporine A to establish lymphoblastoid cell lines $(\mathrm{LCLs})^{15}$. In detail, $1 \mathrm{ml}$ of EBVcontaining supernatant, obtained from growing B-95.8 cells, was added to $5 \cdot 10^{6}$ pelleted $B$ cells and incubated for $60 \mathrm{~min}$ at $37{ }^{\circ} \mathrm{C}$ and $5 \% \mathrm{CO}$. Upon washing with $1 \mathrm{ml}$ PBS, infected B cells were resuspended in $3 \mathrm{ml} \mathrm{RPMI}-1640$ supplemented with $20 \%$

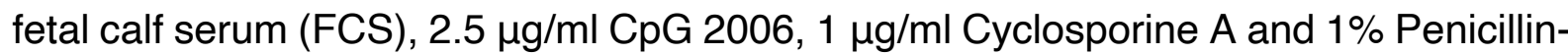
Streptomycin (Pen/Strep). The suspension was cultured at $37{ }^{\circ} \mathrm{C}$ and $5 \% \mathrm{CO} 2$ until visible clusters were formed and the medium changed its colour from red to yellow due to acidification.

\section{Isolation of antigen-binding B cells}

Antigen-binding B cells were isolated from LCLs with an excess of antigen-coupled NHS-activated magnetic beads (ThermoFisher Scientific). The antigen was coupled to the beads in accordance with manufacturer's instruction. For the isolation, LCLs $\left(10 \cdot 10^{6}\right.$ cells) were pelleted and cooled on ice for $60 \mathrm{~min}$. The pellet was subsequently resuspended in $100 \mu \mathrm{l}$ PBS supplemented with $0.5 \%$ bovine serum albumin and $2 \mathrm{mM}$ EDTA accompanied by $30 \mu \mathrm{l}$ of antigen-coupled beads and cooled on ice for another $10 \mathrm{~min}$. This suspension was applied on a magnetic column (MACS Cell Separation Columns, Miltenyi Biotec B.V.) and the separation was achieved in accordance with manufacturer's instruction. The elution fraction was collected as antigen-binding B cells and was used to generate antigen-binding monoclonal LCLs.

\section{Direct cloning}

The number of isolated antigen-binding LCLs was estimated based on the frequency of antigen-binding B cells determined by flow cytometry analysis (around $0.01 \%$ of the $B$ cells - Method 1). The elution fraction of the magnetic separation was mixed with $1 \cdot 10^{6} / \mathrm{ml}$ irradiated PBMCs (35 Gy) suspended in RPMI- 1640 containing $20 \%$ FCS and $1 \%$ Pen/Strep to achieve a concentration of 25 antigen-binding LCLs/ml. $200 \mu$ of this mixture was transferred to one well of a flat-bottom 96 wells plate ( $=5$ cells/well). The cells were allowed to grow for 4 weeks without re-feeding and the plate was tilted after the first week of culturing to keep the cells in close contact after the feeder cells died off. The plate was straightened again after an additional week of culturing to avoid too close contact between steadily proliferating cells ${ }^{16}$. 


\section{Generation monoclonal LCLs by limiting dilution cloning}

LCLs with supernatant containing antibodies specific to the antigen of interest were used for a second round of cloning to generate monoclonal LCLs. To this end, positive LCLs from the direct cloning step were counted and diluted to 50 cells $/ \mathrm{ml}$ in RPMI1640 supplemented with $20 \%$ FCS, $1 \%$ Pen/Strep and $1 \cdot 10^{6} / \mathrm{ml}$ irradiated PBMCs (35 Gy). This mixture was seeded in a volume of $200 \mu \mathrm{l} /$ well in a flat-bottom 96 wells plate (10 cells/well) and incubated for 4 weeks as described above. For defining the optimal seeding density, a range from 0.3 to 10 cells/well were used. LCLs secreting antibodies specific for the antigen of interest were transferred to a $5 \mathrm{~mL}$ round bottom polystyrene test tube and cultured until a visible pellet was observed and the medium colour changed from red to yellow. For further expansion, the LCLs were first transferred to a $25 \mathrm{~cm}^{2}$ culture flask and subsequently to a $75 \mathrm{~cm}^{2}$ culture flask. Expanded LCLs secreting specific mAbs were frozen at $-80{ }^{\circ} \mathrm{C}$ and the supernatant containing $\mathrm{mAbs}$ was stored at $-20^{\circ} \mathrm{C}$. Monoclonality was checked by Sanger Sequencing as described for Method 1.

\section{RNA extraction}

Total RNA was isolated accordingly to manufacturer's instruction (RNA-Bee, BioConnect). Briefly, pelleted LCLs were homogenized in $1 \mathrm{ml}$ RNA-Bee and the RNA was separated from the genomic DNA by adding $200 \mu \mathrm{l}$ of chloroform and spinning for $15 \mathrm{~min}$ at $12.000 \times \mathrm{g}$ and $4{ }^{\circ} \mathrm{C}$. The colourless phase was transferred to $500 \mu \mathrm{l}$ icecold isopropanol and incubated for $10 \mathrm{~min}$ on ice to precipitate RNA. The precipitate was washed with $75 \%$ ethanol and the resulted pellet was resolved in RNAse free water for 15 min at $55^{\circ} \mathrm{C}$. Extracted RNA was either stored at $-80^{\circ} \mathrm{C}$ or immediately used.

\section{cDNA transcription and gene transcript amplification}

RNA was transcripted into cDNA in accordance with manufacturer's instructions (Transcriptor First Strand cDNA Synthesis Kit, Roche). Briefly, $2 \mu \mathrm{l}$ of random hexamer nucleotides $(5 \mu \mathrm{M})$ were mixed with $8 \mu \mathrm{l}$ of RNA template $(1 \mu \mathrm{g})$ and the mixture was incubated for $3 \mathrm{~min}$ at $85^{\circ} \mathrm{C}$. Upon cooling down, $10 \mu \mathrm{l}$ cDNA reaction mixture containing 1X RT-buffer, $5 \mathrm{mM} \mathrm{MgCl}_{2}, 1 \mathrm{mM}$ dNTP mix, $5 \mathrm{U}$ RNA inhibitor, $1.5 \mathrm{U}$ avian myeloblastosis virus (AMV)-RT and $10 \mathrm{mM}$ gelatine was added and incubated for $90 \mathrm{~min}$ at $42{ }^{\circ} \mathrm{C}$. The reaction was stopped by inactivating the AMV-RT for 3 min at $85^{\circ} \mathrm{C}$. CDNA was either stored at $-20{ }^{\circ} \mathrm{C}$ or immediately used for $\mathrm{V}(\mathrm{D}) \mathrm{J}$ gene transcript amplification as described for Method 1. Contrary to Method 1, the primer concentrations were adjusted to $400 \mathrm{nM}$ instead of $40 \mathrm{nM}$. 


\section{Examination of specific antigen-binding}

\section{Specific binding to peanut $2 S$ albumins}

Specificity of native and heterologously produced mAbs to peanut $2 \mathrm{~S}$ albumins was tested using a direct ELISA. Briefly, plates were coated by applying either $0.3 \mu \mathrm{g} / \mathrm{well}$ Ara $\mathrm{h} 2$ and $6^{26}$ or transferrin (negative control) overnight at room temperature. On the following day, the plate was blocked with PBS supplemented with $1 \%$ BSA and $0.1 \%$ Tween-20 (blocking buffer) for $60 \mathrm{~min}$ at room temperature. Subsequently, supernatants were applied upon 1:2 dilution in blocking buffer (EBV LCLs) or in serial dilution ( 1 to $10 \mu \mathrm{g} / \mathrm{ml}$ ) (heterologously expressed mAbs) 60 min at room temperature under continuous shaking. Bound antibodies from EBV-LCLs were detected with ahuman kappa (1:10.000, SouthernBiotech) and a-human lambda antibodies coupled with horse radish peroxidase (1:5000, SouthernBiotech) for $60 \mathrm{~min}$ at room temperature under continuous shaking. Bound heterologously expressed mAbs, on the other hand, were detected by either goat a-human $\lg E(1: 5000, \mathrm{KPL})$ or goat a-human $\lg \mathrm{G}$ (1:2000, Jackson ImmunoResearch) antibodies coupled with horse-radish peroxidase under the same conditions. Visualisation was provided by adding tetramethylbenzidine (TMB) for $15 \mathrm{~min}$ in the dark and the optical density (OD) was measured at $450 \mathrm{~nm}$. Native Abs were considered for further cloning or analysis when the sample OD was at least two times higher than the OD of the negative control. Heterologously expressed mAbs were defined as specific if the sample OD was, upon subtraction of the negative control OD value, at least 1.5 times greater than the OD obtained with culture medium. mAbs with OD values above 1 at a concentration of $10 \mu \mathrm{g} / \mathrm{ml}$ were considered as strong binders, mAbs with OD values between 0.07 and 1 at all concentration steps were considered as moderate binders and mAbs with increased OD values at the highest concentration of $10 \mu \mathrm{g} / \mathrm{ml}$, but no detectable OD at the lowest concentration of $1 \mu \mathrm{g} / \mathrm{ml}$, were considered as weak binders.

\section{Specific binding to ARHGDIB}

Antibody specificity to ARHGDIB was evaluated using ARHGDIB-coupled microspheres diluted in PBS supplemented with 0.1\% BSA (wash buffer) ${ }^{20}$. IgG-coupled microspheres served as positive control whilst empty and transferrin-coupled microspheres served as negative controls. All incubation steps were performed in the dark, at room temperature and with continuous shaking.

For each antibody to be tested, 1500 microspheres consisting of 4 colours, each individually coated, were incubated overnight with $50 \mu$ l undiluted HEK293 (Method 1) or EBV-LCLs supernatant (Method 2) containing the respective mAb. Upon washing with a Bio-Plex Pro Wash station (Bio-Rad), bound mAbs were detected with either an 
1-step (Method 1) or a 2-step procedure (Method 2). For the 1-step procedure, $50 \mu \mathrm{l}$ of 1:50 diluted PE-conjugated goat-a human IgG antibody (Jackson ImmunoResearch) was added and incubated for $30 \mathrm{~min}$. For the 2-step procedure, $50 \mu \mathrm{l}$ of goat a-human kappa (1:100, Southern Biotech) and goat a-human lambda antibody (1:32, Southern Biotech) was added and incubated for $30 \mathrm{~min}$. For the second step, $50 \mu \mathrm{l}$ of 1:100 diluted PE-conjugated donkey a-goat IgG antibody was added and incubated for additional $30 \mathrm{~min}$. For the readout of both procedures, $50 \mu \mathrm{l}$ of washing buffer was added and the median fluorescence intensities (MFI) were measured on a Luminex 200 flow analyser (Luminex Corp) (50 counts, $75 \mu$ sample volume, 90 sec time out).

\section{Recovery rate and cost calculation}

The recovery rate of specific human mAbs was calculated for both methods as followed: Method 1:

recovery $=100 \% \cdot$ amplification efficiency $\cdot$ cloning efficiency $\cdot$ portion specific mAbs

$$
\begin{gathered}
\text { recovery }=100 \% \cdot 0.5 \cdot 0.54 \cdot 0.75 \\
\text { recovery }=20.3 \%
\end{gathered}
$$

Method 2:

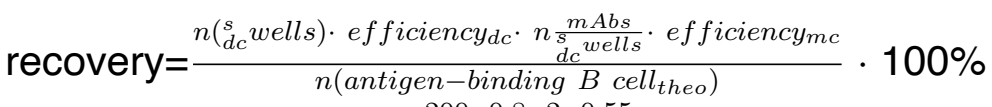

$$
\begin{aligned}
& \text { recovery }=\frac{200 \cdot 0.8 \cdot 2 \cdot 0.55}{1000} \cdot 100 \% \\
& \text { recovery }=17.6 \%
\end{aligned}
$$

$\mathrm{n}\left({ }_{d c}^{s}\right.$ wells $)=$ seeded wells for direct cloning based on theoretically antigen-binding $\mathrm{B}$ cells

efficiency $_{d c}=$ efficiency of direct cloning (approximate 2S albumins and ARHGDIB) efficiency $_{m c}=$ efficiency of the second round of cloning $\frac{m A b s}{s}=$ approximate number of mAbs obtained from one seeded well for direct cloning

Costs for the generation of one single human mAb was calculated by adding up the expenses and correcting it for the recovery rate of the respective strategy. Personal costs were included based on an average salary of a research technician and the approximate working hours needed to produce one single mAb. 


\section{Results}

\section{Method 1: „Single cell sequencing“}

\section{Double tetramer-staining reduced the selection of non-specific CD19+ B cells}

Antigen-binding B cells for subsequent single cell $V(D) J$ gene transcript amplification were detected by flow cytometry using antigen-tetramers formed with fluorophore labelled streptavidin. For the development of an optimal staining protocol, enriched $B$ cells, derived from the same blood bank donor, were stained with 2S albumin-tetramers accompanied by either a single (APC) or two distinct fluorophores (APC and PE). As shown in Figure 2a, B cells single-positive for $2 \mathrm{~S}$ albumin-tetramer binding accounted for $0.1 \%$ of the total $\mathrm{CD} 19^{+} \mathrm{B}$ cell fraction. Both controls - staining with biotin without the antigen (Ctr 1) and blocking with unlabelled antigen (Ctr 2) - showed, however, a comparable percentage of $2 \mathrm{~S}$ albumin-binding B cells (Ctr 1: 0.092\%; Ctr 2: 0.097\%). The subtraction of $\mathrm{Ctr} 2$ resulted in a final percentage of $0.008 \% 2 \mathrm{~S}$ albumin-binding $B$ cells. In comparison, the fraction of double-positive $2 S$ albumin-binding B cells was already reduced to a percentage of $0.004 \%$ without any background staining, indicating the potential of double antigen-tetramer staining for identifying bona fide antigenbinding $B$ cells. For validation purposes, double-tetramer staining was used for the detection of $2 S$ albumin (Figure 2b) or ARHGDIB-binding B cells (Figure 2c) in 6 independent blood bank donors. 2S albumin-binding $B$ cells ranged from 0.002 to $0.007 \%$ whilst ARHGDIB-binding B cells ranged from 0.005 to $0.015 \%$, indicating a good reproducibility of the developed staining protocol. 


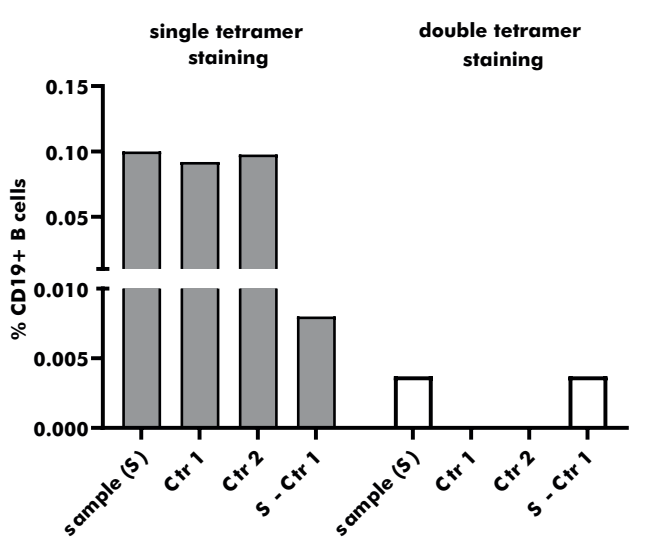

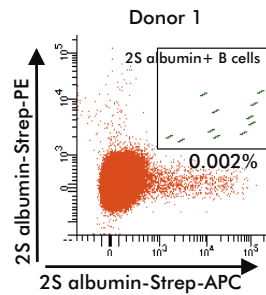

c

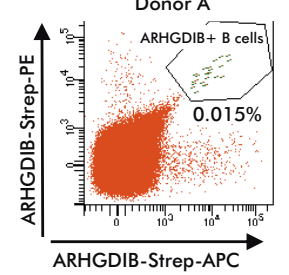

Donor 2

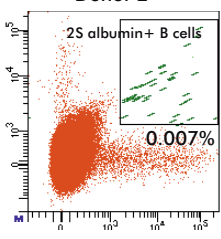

Donor B

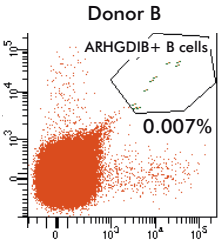

Donor 3
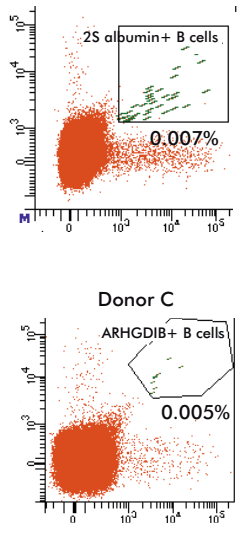

Figure 2: Selection of antigen-binding $B$ cells using antigen-tetramers

A Percentage of $2 S$ albumin-binding B cells in relation to the total $C D 19^{+} B$ cell fraction using single or double-tetramer staining. Control 1 (Ctr 1) represents the staining with only biotin and Control 2 (Ctr 2) is executed by pre-blocking with unlabelled 2S albumin prior normal staining procedure. The final percentage is calculated by subtracting Ctr 2 from the sample.; $\boldsymbol{B}$ and $\boldsymbol{C} F A C S$ plots representing antigenbinding $B$ cells in relation to the total $B$ cell population of independent donors. $2 S$ albumin-binding $B$ cells are shown in $\boldsymbol{B}$ and $A R H G D I B$-binding $B$ cells in $\boldsymbol{C}$.

\section{$V(D) J$ gene transcript amplification efficiency is donor-dependent}

Amplification efficiency upon single cell sorting of 2S albumin-binding B cells was evaluated in 6 independent blood bank donors. Amplification efficiency of the heavy chain VDJ gene transcript from 24 to 50 individual B cells ranged from 29 to $63 \%$ whilst the percentage of successfully amplified $V(D) J$ gene transcripts from the heavy and corresponding light chain was reduced and ranged from 17 to $50 \%$. Although a low amplification efficiency was shown for two donors, most of the donors (4/6) showed sufficient amplification efficiencies of approximately $50 \%{ }^{4}$. Cloning of successfully amplified heavy and corresponding light chain gene transcripts resulted into an overall cloning efficiency of $54 \%$ which may be increased by ordering the not successfully cloned gene sequences commercially ${ }^{5}$. Individual cloning efficiencies were estimated to $76 \%$ for the heavy chain gene transcripts and $71 \%$ for the light chain gene transcripts (78\% kappa and $31 \%$ lambda).

\footnotetext{
${ }^{4}$ Note: We observed higher amplification efficiencies when primer aliquots were stored in $50 \mathrm{mM}$ Tris- $\mathrm{HCl}$ buffer supplemented with $2 \mathrm{mM}$ EDTA instead of RNAse free water.

${ }^{5}$ Note: We observed successful cloning of commercially obtained gene sequences while the cloning of the original gene sequences failed.
} 


\section{Antibodies derived from double-positive tetramer-binding B cells are mostly specific}

The specificity of human mAbs, generated by single cell sequencing, can only be examined upon heterologous expression in the end of the workflow. Overall, 32 heterologously expressed mAbs from 10 different donor carried the variable region of $2 S$ albumin-binding $B$ cells and $1 \mathrm{mAb}$ carried the variable region of an ARHGDIB-binding $B$ cell and their concentrations varied between 0.1 and $10 \mu \mathrm{g} / \mathrm{ml}$. Binding to $2 \mathrm{~S}$ albumins was observed in $75 \%$ (24/32) of all heterologously expressed mAbs. Based on their binding abilities, examined by comparing their achieved OD values at different concentrations, they were roughly categorised into weak $(n=13)$, moderate $(n=8)$ and strong $(n=1)$ binders (Figure $3 a$ and $3 b)$. This variability in target binding indicates that our selection strategy was not restricted to only strongly binding $B$ cells and implicates no selection bias regarding distinct affinities. Our staining protocol can also be adjusted to different antigen targets as the mAb generated from an ARHGDIB-binding $B$ cell showed strong binding to ARHGDIB-coupled microspheres with an ARHGDIB/transferrin ratio greater than 2 (Figure $3 c$ ). 
A

$\lg \mathbf{E}$

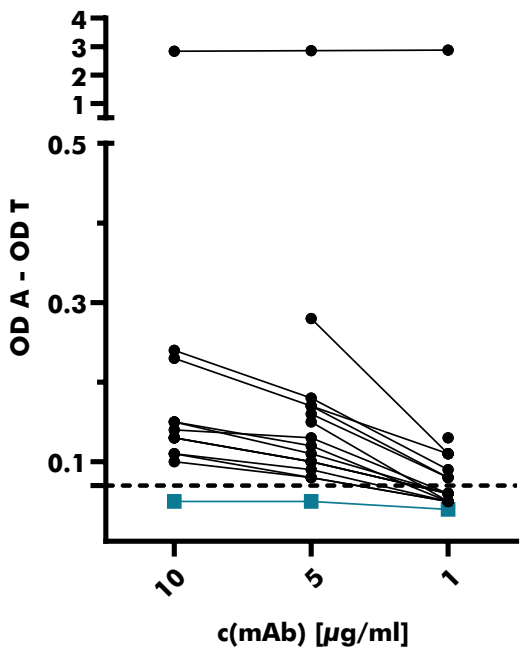

- mAbs
B

$\lg \mathbf{G} 1$

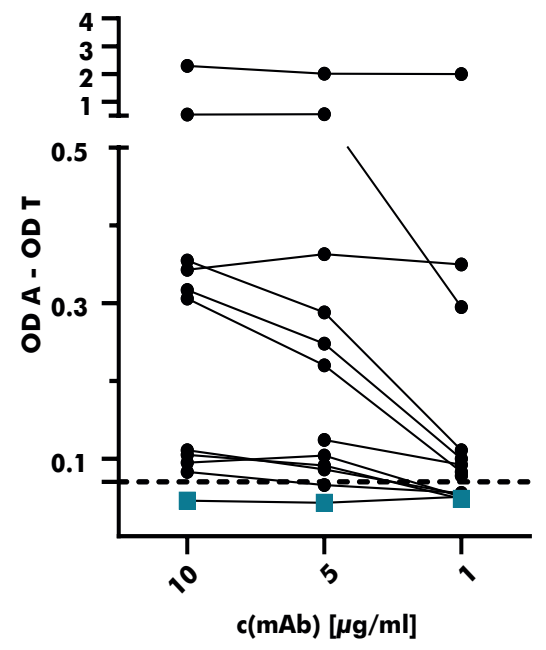

HEK supernatant

C

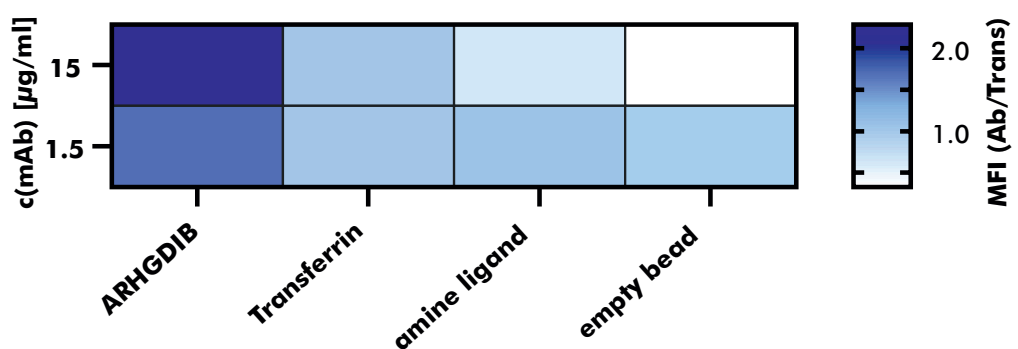

Figure 3: Specificity of heterologously expressed mAbs (Method 1)

$\boldsymbol{A}$ The binding of serial-diluted (1 to $10 \mu \mathrm{g} / \mathrm{ml}$ ) human mAbs (IgE) towards peanut $2 \mathrm{~S}$ albumins was measured using a-human IgE-HRP as detection antibody. Measured OD values were corrected by subtracting the $O D$ value measured for the control antigen (transferrin) and compared to the $O D$ value obtained with HEK supernatant not containing any antibodies (turquoise). The threshold was set to an $O D$ value 1.5 times greater than the $O D$ value obtained with HEK supernatant; $\boldsymbol{B}$ The binding of serialdiluted (1 to $10 \mu \mathrm{g} / \mathrm{ml}$ ) human mAbs (lgG1) towards peanut 2S albumins was measured using a-human IgG1-HRP as detection antibody. The measured $O D$ value was corrected as described for $\boldsymbol{A}$.; $\boldsymbol{C}$ The specificity of the mAb derived from an ARHGDIB-binding B cell was examined using ARHGDIB-coupled microspheres. The median fluorescence intensity (MFI) towards ARHGDIB was evaluated in relation to the MFI towards transferrin as control antigen. 


\section{Method 2: "Establishment of monoclonal EBV-LCLs“}

\section{LCLs isolated with antigen-coupled beads are mostly antigen- specific}

Native Abs can be produced by in vitro culturing of primary B cells secreting polyclonal Abs upon activation. Since in vitro culturing of primary B cells is challenging ${ }^{27}$, especially in the absence of a particular antigen, we chose for the establishment of LCLs by EBV immortalisation. Immortalised LCLs were selected for their binding to either peanut $2 S$ albumins or ARHGDIB. As shown in Figure $4 \mathrm{a}$, almost all polyclonal EBVLCLs $(98.8 \%)$ obtained from the first round of cloning secreted antibodies specifically binding to $2 S$ albumins ( $2 S$ albumin/transferrin ratio $\geq 2$ ) compared to the supernatant of irradiated PBMCs (Ctr). This large number of positive EBV-LCLs (100\%) was confirmed by selecting for $2 \mathrm{~S}$ albumin-binding EBV-LCLs using a second blood bank donor. Although the number of positive clones was reduced for ARHGDIB-binding B cells (Donor 1: $67 \%$, Donor 2: $56 \%$ ), a representative number of EBV-LCLs was selected for a second round of cloning to achieve monoclonality (Figure $4 b)$.

A
Donor 1

$2 \mathrm{~S}$ albumins

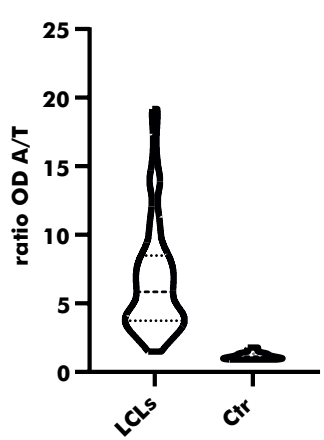

Donor 2

$2 \mathrm{~S}$ albumins

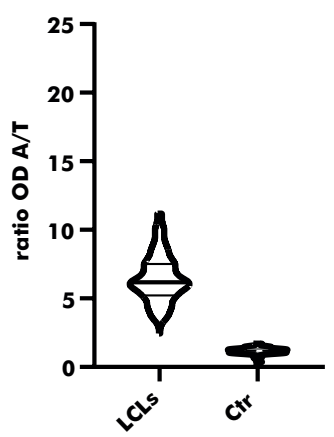

Donor 4 ARHGDIB
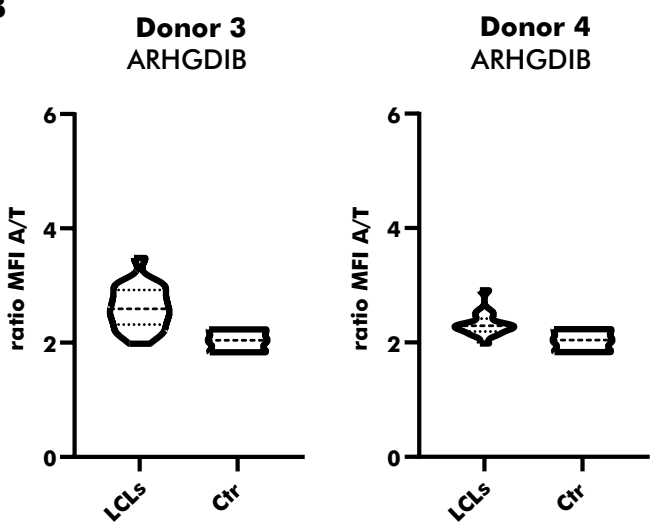

Figure 4: Direct cloning of peanut $2 S$ albumin and ARHGDIB-binding EBV-LCLs A Antibodies secreted by directly cloned EBV-LCLs were screened for their binding to peanut $2 S$ albumins using a direct ELISA. EBV-LCLs secreting antibodies with an $O D$ ratio (2S albumin/transferrin) $\geq 2$ were selected for an additional round of cloning.; B Antibodies secreted by directly cloned EBV-LCLS were screened for their binding to ARHGDIB using antigen-coupled microspheres. EBV-LCLs secreting antibodies with a MFI ratio (ARHGDIB/transferrin) $\geq 2$ were selected for an additional round of cloning.

\section{Optimal seeding density to achieve monoclonal LCL clones}

LCLs positive for antigen-binding were used for an additional round of cloning to achieve LCLs secreting monoclonal instead of polyclonal Abs. EBV-LCLs from the first blood bank donor were used to determine the optimal seeding density. Even though a seeding density of 0.3 cells/well results theoretically in the highest probability of obtaining monoclonal LCLs, only a small number of wells contained LCLs showing proliferation. By increasing the seeding density, the number of LCLs secreting Abs binding to $2 S$ 
albumins rose in accordance with the number of LCLs seeded per well. Despite a high seeding density of 10 cells/well, 55\% of sequenced EBV-LCLs (6/11) showed monoclonality, leading to a compromise between a high number of proliferating EBVLCLs and a reasonable rate of achieved monoclonal EBV-LCLs (Figure 5a). Seeding $2 S$ albumin-binding EBV-LCLs from the second blood bank donor at a density of 10 cells/well resulted in an approximate recovery of 2 EBV-LCLs with specific binding to $2 \mathrm{~S}$ albumins per seeded plate (Figure $5 \mathrm{~b}$ ). A comparable recovery was achieved for ARHGDIB-binding EBV-LCLs from another two independent blood bank donors (Figure $5 c)$.

A

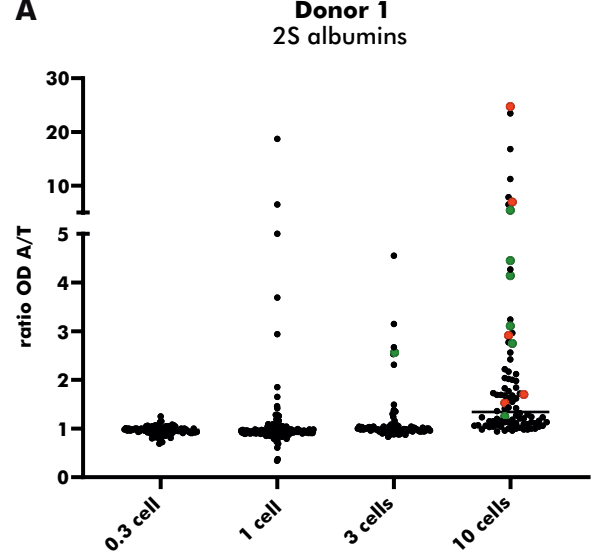

B

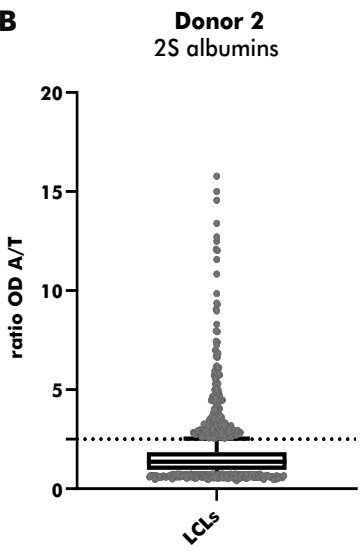

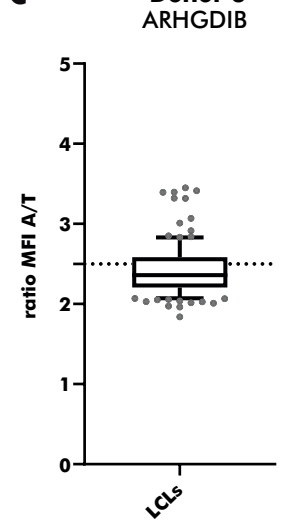

Donor 4 ARHGDIB

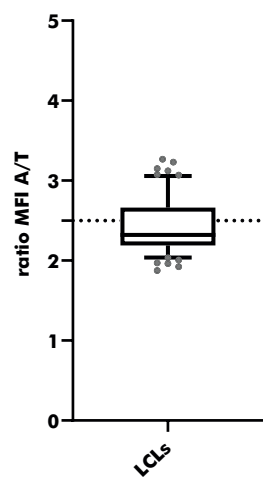

Figure 5: Subcloning of $2 S$ albumin and ARHGDIB-binding EBV-LCLs

$\boldsymbol{A}$ Determination of the optimal seeding density for the second round of EBV-LCLs cloning. EBV-LCLS with specific binding to peanut $2 S$ albumins (ratio $2 S$ albumins/transferrin $\geq 2$ ) were considered for expansion. The $V(D) J$ gene transcript of these EBV-LCLs were Sanger sequenced to check for monoclonality. Monoclonal EBV-LCLs are highlighted in green and polyclonal EBV-LCLs are highlighted in red. $B$ Antibodies secreted by subcloned EBV-LCLs were screened for their binding to peanut $2 S$ albumins. EBV-LCLs with a ratio (2S albumin/transferrin) $\geq 2$ were considered for expansion.; $C$ Antibodies secreted by subcloned EBV-LCLs were screened for their binding to ARHGDIB. EBV-LCLs with a ratio (ARHGDIB/transferrin) $\geq 2.5$ were considered for expansion.

\section{Heterologously expressed mAbs showed comparable binding to $2 S$ albumins}

To validate heterologous expression of mAbs secreted by established EBV-LCLs, one pair of heavy and corresponding light chain variable regions was selected from the pool of $2 \mathrm{~S}$ albumin-specific monoclonal EBV-LCLs. Upon heterologous expression with a vector containing the IgG1 backbone, native and heterologously expressed counterparts were applied on a direct ELISA in a serial dilution as shown in Figure 6. The measured $O D$ values were comparable between the native $\operatorname{lgM} \mathrm{mAb}$ and the heterologously expressed lgG1 mAb (light chain detection), indicating that mAbs from subcloned EBVLCLs can also be heterologously expressed with comparable binding abilities. 

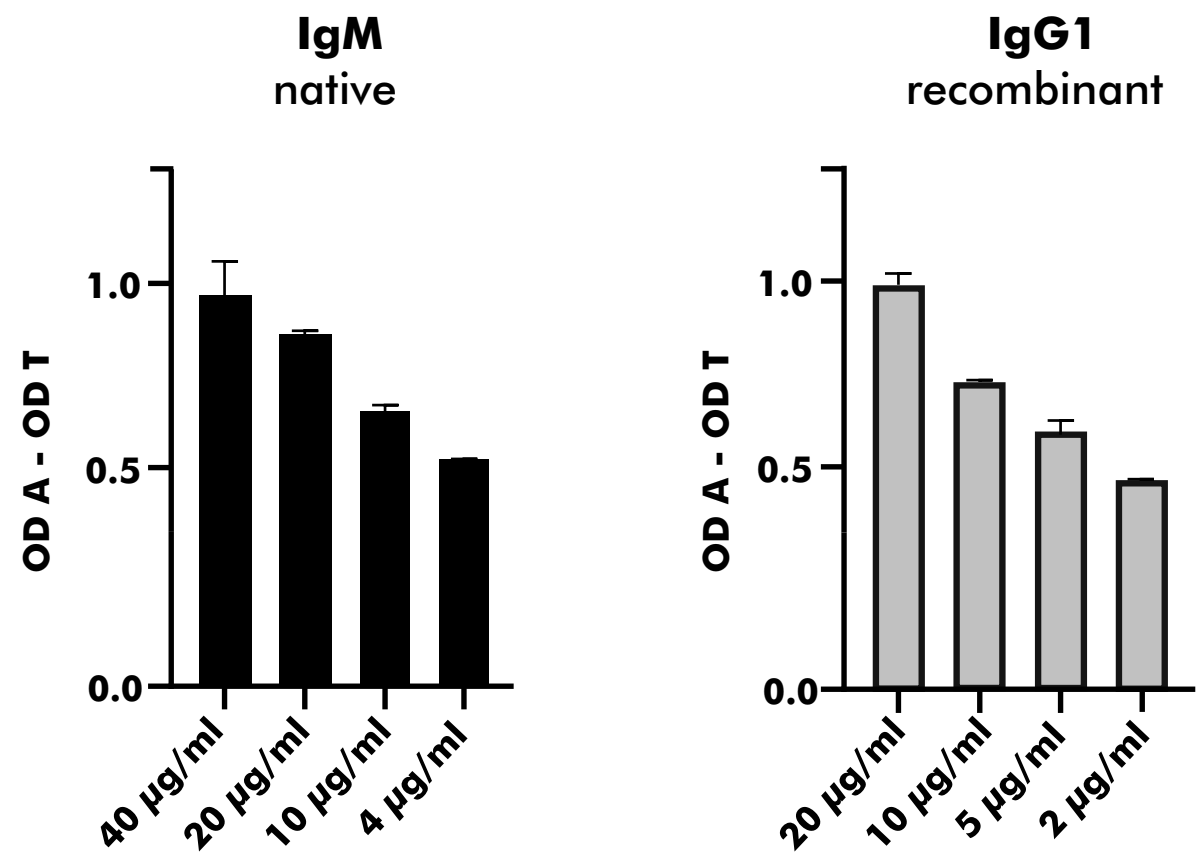

Figure 6: Heterologous expression of a human mAb with specific binding to peanut $2 S$ albumins A $m A b$ carrying the same variable region as native antibodies obtained from a monoclonal $E B V-L C L$ was heterologously expressed with a vector containing the IgG1 backbone. Its binding ability expressed as $O D$ values (corrected for binding to transferrin) was compared to the binding ability of its native counterpart.

\section{Single cell sequencing allows higher throughput}

Both methods described within this chapter are characterised by several up- and downsides influencing the choice which method to use. A comparison regarding costs, expenditure of time, recovery, throughput and complexity is shown in Table 1.

Table 1: Comparison of both methods to generate human mAbs

\begin{tabular}{|c|c|c|}
\hline & Method 1: „Single cell“ & Method 2: „EBV-LCLS“ \\
\hline Costs $^{A}$ & $650-750 €$ & $450 €$ \\
\hline Time & 3 weeks & 12 weeks \\
\hline Adaptation & sorting strategy: 3 weeks & no special adaptation needed \\
\hline Recovery & $20-29 \%{ }^{B}$ & $18 \%{ }^{\mathrm{C}}$ \\
\hline Throughput & +++ & + \\
\hline Compexity & +++ & +++ \\
\hline \multicolumn{3}{|c|}{${ }^{A}$ Costs per one single human mAb } \\
\hline \multicolumn{3}{|c|}{$\begin{array}{l}\text { B Cloning efficiency of } 54 \% \text { to } 76 \% \text { (might be achieved by ordering gene sequences to increase cloning } \\
\text { efficiency) }\end{array}$} \\
\hline
\end{tabular}


Without considering any further characterisation, the generation of one single human mAb can add up to $750 €$. The establishment of monoclonal EBV-LCLs, however, is generally less expensive (400 - 500€) compared twith the generation by single sequencing (650 - 750€) resulting from expensive reagents required for single cell amplification. On the other hand, the workflow of single cell sequencing shortens the required time from 12 weeks including long culturing periods to only 3 weeks. Even though both methods showed comparable recovery rates in our experiments, the establishment of monoclonal EBV-LCLs reaches easily a capacity threshold limiting the overall throughput and leading to random selection of clones to proceed with. Hence, the establishment of monoclonal EBV-LCLs is a suitable tool to generate a limited number of human mAbs, especially due to the ability to screen for binding, functionality and neutralisation capacity throughout the workflow. Single cell sequencing is, however, a more suitable tool for a broader examination of antigen-specific B cells, since a much higher throughput can be achieved. Unfortunately, specificity and functionality can only be examined in the end of the workflow. Both methods are extremely laborious and highly complex.

\section{Discussion}

Studying human-derived mAbs offers the possibility to link genetic information to functional features, making them indispensable in modern molecular biology research. In this study, we directly compared two distinct strategies to generate (highly) specific mAbs from peripheral blood of human donors, i.e.: single cell sequencing and the establishment of monoclonal EBV-LCLs. While single cell sequencing is a suitable tool to generate a large panel of mAbs with distinct binding features, the establishment of monoclonal EBV-LCLs provides the possibility to screen for specificity and functionality throughout the workflow. Both strategies, initially set-up for mAbs specifically directed against peanut $2 S$ albumins, were easily adaptable to other antigen targets as shown for ARHGDIB.

Corresponding $\mathrm{V}(\mathrm{D}) \mathrm{J}$ gene transcripts were successfully amplified from up to $50 \%$ of all single cell sorted $2 S$ albumin-binding $B$ cells. The overall recovery of $2 S$ albuminspecific human mAbs, however, was reduced to around $20 \%$ when cloning efficiency (54\%) and proportion of mAbs with proven specificity $(75 \%)$ was taken into consideration. The cloning efficiency may be increased by ordering gene sequences without cloning success commercially. Overall, our amplification efficiency (up to 50\%) corresponds to the work of Tiller and colleagues who described an amplification efficiency of up to $60 \%{ }^{18}$. Moreover, a comparable overall recovery of $27 \%$ has been shown for single cell sequencing of antigen-specific B cells from guinea pigs ${ }^{28}$. Increased amplification efficiencies of 90 to even $100 \%$ have been described for performing a com- 
parable amplification protocol in triplicates ${ }^{29}$. However, such an approach will simultaneously increase the probability of amplification errors, potentially hampering gene analysis and antibody cloning.

Regarding the establishment of antigen-specific monoclonal EBV-LCLs, the overall recovery was estimated to around $18 \%$. This is in accordance with cloning efficiencies of around $15 \%$ observed for limiting dilution approaches upon EBV immortalisation ${ }^{16}$. However, we lack the information of what proportion of antigen-specific B cells were initially immortalised by EBV. To overcome this limitation, Fraussen and colleagues described in their protocol the immortalisation of 50 cells/well upon sorting the desired $B$ cell subpopulation ${ }^{30}$. However, we were not able to immortalise such small numbers of B cells successfully in our laboratory.

Both strategies are characterised by their own strengths and limitations. Single cell sequencing, on the one hand, enables the execution in a moderate-throughput manner and the selection of antigen-specific B cells is not restricted to certain subpopulations as EBV immortalisation is restricted to CpG-activated memory B cells ${ }^{15}$. These advantages make the single cell sequencing platform a suitable tool for a broad examination of antigen-binding $B$ cells and their corresponding mAbs. The establishment of monoclonal EBV-LCLs, on the other hand, provides continuous screening for specific binding and functionality throughout the workflow, resulting in an easy selection of mAbs with high affinity towards their targets and making this strategy a powerful tool in therapeutic research. Moreover, this strategy enables the comparison of heterologously expressed mAbs with their natural counterparts, allowing the identification of potential structure alterations by post-translational modifications during heterologous expression ${ }^{31,32}$. An additional advantage of generating human mAbs by establishing monoclonal EBV-LCLs is their limited need for expensive reagents.

In conclusion, both strategies - single cell sequencing and establishment of monoclonal EBV-LCLs - are able to generate (highly) antigen-specific human mAbs and they are easily adaptable to other target antigens. The recommended method to choose is dependent on the research question to explore as both strategies have their own strengths and limitations. 


\section{References}

1. Vandyk, L. \& Meek, K. Assembly of IgH CDR3: Mechanism, Regulation, and Influence on Antibody Diversity. Int Rev Immunol 8, 123-33 (1992).

2. Gough, N. M. \& Bernard, O. Sequences of the joining region genes for immunoglobulin heavy chains and their role in generation of antibody diversity. Proc Natl Acad Sci U S A 78, 509-13 (1981).

3. Mishra, A. K. \& Mariuzza, R. A. Insights into the Structural Basis of Antibody Affinity Maturation from Next-Generation Sequencing. Front Immunol 9, 117 (2018).

4. O'Connell, A. E. et al. Next Generation Sequencing Reveals Skewing of the $\mathrm{T}$ and B Cell Receptor Repertoires in Patients with Wiskott-Aldrich Syndrome. Front Immunol 5, 340 (2014).

5. Hansen, A., Dörner, T. \& Lipsky, P. E. Use of Immunoglobulin Variable-Region Genes by Normal Subjects and Patients with Systemic Lupus Erythematosus. Int Arch Allergy Immunol 123, 36-45 (2000).

6. Cameron, E. M. et al. Potential of a unique antibody gene signature to predict conversion to clinically definite multiple sclerosis. J Neuroimmunol $\mathbf{2 1 3}, \mathbf{1 2 3 - 3 0}$ (2009).

7. Pantazes, R. J. et al. Identification of disease-specific motifs in the antibody specificity repertoire via next-generation sequencing. Sci Rep 6, 30312 (2016).

8. Titcombe, P. J. et al. Pathogenic Citrulline-Multispecific B Cell Receptor Clades in Rheumatoid Arthritis. Arthritis Rheumatol 70, 1933-45 (2018).

9. Wilson, P. C. \& Andrews, S. F. Tools to therapeutically harness the human antibody response. Nat Rev Immunol 12, 709-19 (2012).

10. Abbott, W. M., Damschroder, M. M. \& Lowe, D. C. Current approaches to fine mapping of antigen-antibody interactions. Immunology 142, 526-35 (2014).

11. Rajan, S. et al. Recombinant human B cell repertoires enable screening for rare, specific, and natively paired antibodies. Commun Biol 1, 5 (2018).

12. McCafferty, J., Griffiths, A. D., Winter, G. \& Chiswell, D. J. Phage antibodies: filamentous phage displaying antibody variable domains. Nature $348,552-4$ (1990).

13. Rondot, S., Koch, J., Breitling, F. \& Dübel, S. A helper phage to improve singlechain antibody presentation in phage display. Nat Biotechnol 19, 75-8 (2001).

14. Lonberg, N. Human Monoclonal Antibodies from Transgenic Mice. Therapeutic Antibodies 181, 69-97 (2008). 
15. Traggiai, E. et al. An efficient method to make human monoclonal antibodies from memory B cells: potent neutralization of SARS coronavirus. Nat Med 10, 871-5 (2004).

16. Steinitz, M. Production of Human Monoclonal Antibodies by the Epstein-Barr Virus Method. Methods Mol Biol 1060, 111-22 (2014).

17. Kwakkenbos, M. J. et al. Generation of stable monoclonal antibody-producing B cell receptor-positive human memory B cells by genetic programming. Nat Med 16, 123-8 (2009).

18. Tiller, T. et al. Efficient generation of monoclonal antibodies from single human $B$ cells by single cell RT-PCR and expression vector cloning. $J$ Immunol Methods 329, 112-24 (2008).

19. Kamburova, E. G. et al. Antibodies against ARHGDIB are associated with longterm kidney graft loss. Am J Tansplantat 19, 3335-44 (2019).

20. Kamburova, E. G. et al. Development and Validation of a Multiplex Non-HLA Antibody Assay for the Screening of Kidney Transplant Recipients. Front Immunol 9, 3002 (2018).

21. Patil, S. U. et al. Peanut oral immunotherapy transiently expands circulating Ara $\mathrm{h} 2$-specific $\mathrm{B}$ cells with a homologous repertoire in unrelated subjects. J Allergy Clin Immunol 136, 125-34.e12 (2015).

22. Franz, B., May, K. F., Dranoff, G. \& Wucherpfennig, K. Ex vivo characterization and isolation of rare memory $B$ cells with antigen tetramers. Blood 118, 348-57 (2011).

23. Yang, J. \& Reth, M. Oligomeric organization of the B-cell antigen receptor on resting cells. Nature 467, 465-9 (2010).

24. Hoh, R. A. et al. Single B-cell deconvolution of peanut-specific antibody responses in allergic patients. J Allergy Clin Immunol 137, 157-67 (2016).

25. Ye, J., Ma, N., Madden, T. L. \& Ostell, J. M. IgBLAST: an immunoglobulin variable domain sequence analysis tool. Nucl Acids Res 41, W34-40 (2013).

26. Koppelman, S. J. et al. Purification and immunoglobulin E-binding properties of peanut allergen Ara h 6: evidence for cross-reactivity with Ara h 2. Clin Exp Allergy 35, 490-7 (2005).

27. Kumar, A., Guido, E. C., Liu, R.-S. \& Saedi, M. S. Long-term culture of primary $B$ cells and in vitro expression of an exogenous gene. Immunol Let 47, 193-7 (1995).

28. Lei, L. et al. Antigen-Specific Single B Cell Sorting and Monoclonal Antibody Cloning in Guinea Pigs. Front Microbiol 10, 672 (2019). 
29. Guselnikov, S. V. et al. A simple way to increase recovery of the expressed VH and VL genes in single-sorted human B cells. BioTechniques 67, 184-7 (2019).

30. Fraussen, J. et al. A novel method for making human monoclonal antibodies. $J$ Autoimmun 35, 130-4 (2010).

31. Jefferis, R. Posttranslational Modifications and the Immunogenicity of Biotherapeutics. J Immunol Res 2016, 1-15 (2016).

32. Tokmakov, A. A. et al. Multiple Post-translational Modifications Affect Heterologous Protein Synthesis. J Biol Chem 287, 27106-16 (2012). 


\section{Supplementary}

Table S1: Primers for heavy chain gene transcripts, restriction site underlined

\begin{tabular}{lc}
\hline Primer & Sequence \\
\hline \multicolumn{2}{l}{ Heavy chain primer forward } \\
\hline Single cell RT-PCR & \\
5' L-Vh1 & ACAGGTGCCCACTCCCAGGTGCAG \\
5' L-Vh3 & AAGGTGTCCAGTGTGARGTGCAG \\
5' L-Vh4/6 & CCCAGATGGGTCCTGTCCCAGGTGCAG \\
5' L-Vh5 & CAAGGAGTCTGTTCCGAGGTGCAG \\
& \\
Introduction restriction sites \\
5' EcoRI_VH1 & ATATTGAATTCGCAGGTGCAGCTGGTGCAG \\
5' EcoRI_VH1/5 & ATATTGAATTCGGAGGTGCAGCTGGTGCAG \\
5' EcoRI_VH1-18 & ATATTGAATTCGCAGGTTCAGCTGGTGCAG \\
5' EcoRI_VH1-24 & ATATTGAATTCGCAGGTCCAGCTGGTACAG \\
5' EcoRI_VH3 & ATATTGAATTCGGAGGTGCAGCTGGTGGAG \\
5' EcoRI_VH3-23 & ATATTGAATTCGGAGGTGCAGCTGTTGGAG \\
5' EcoRI_VH3-33 & ATATTGAATTCGCAGGTGCAGCTGGTGGAG \\
5' EcoRI_VH3-9 & ATATTGAATTCGGAAGTGCAGCTGGTGGAG \\
5' EcoRI_VH4 & ATATTGAATTCGCAGGTGCAGCTGCAGGAG \\
5' EcoRI_VH4-34 & ATATTGAATTCGCAGGTGCAGCTACAGCAGTG \\
5' EcoRI_VH4-39 & ATATTGAATTCGCAGCTGCAGCTGCAGGAG \\
5' EcoRI_VH6-1 & ATATTGATTCGCAGGTACAGCTGCAGCAG \\
\end{tabular}

Sanger sequencing

5' Vh1-FR1_(1-2)

GGCCTCAGTGAAGGTCTCCTGCAAG

5' Vh2-FR1_(2-5)

GTCTGGTCCTACGCTGGTGAAACCC

5'Vh3-FR1_(3-7)

CTGGGGGGTCCCTGAGACTCTCCTG

5' Vh4-FR1_(4-4)

CTTCGGAGACCCTGTCCCTCACCTG

5' Vh5-FR1_(5-51)

CGGGGAGTCTCTGAAGATCTCCTGT

5'Vh6-FR1_(6-1)

TCGCAGACCCTCTCACTCACCTGTG

Colony screening

5' Vh1-FR2_(1-2)

5' Vh2-FR2_(2-5)

5' Vh3-FR2_(3-7)

5' Vh4-FR2_(4-4)

5' Vh5-FR2_(5-51)

5' Vh6-FR2_(6-1)

5' Vh7-FR2_(7)

CTGGGTGCGACAGGCCCCTGGACAA
TGGATCCGTCAGCCCCCAGGGAAGG
GGTCCGCCAGGCTCCAGGGAA
TGGATCCGCCAGCCCCCAGGGAAGG
GGGTGCGCCAGATGCCCGGGAAAGG
TGGATCAGGCAGTCCCCATCGAGAG
TTGGGTGCGACAGGCCCCTGGACAA

Heavy chain primer reverse

Single cell RT-PCR

3' $\mathrm{CH} 1$ Ig $\mathrm{A}^{\mathrm{A}}$

AGCCCTGGACCAGGCA 


\begin{tabular}{|c|c|}
\hline 3' CH1_lgE ${ }^{A, B}$ & GAAGACGGATGGGCTCTGT \\
\hline 3' $\mathrm{CH}$ 1_lgG ${ }^{\mathrm{A}, \mathrm{B}}$ & GGAAGGTGTGCACGCCGCTG \\
\hline $3^{\prime} \mathrm{CH} 1 \_\mathrm{Ig} \mathrm{M}^{\mathrm{A}}$ & GGGAATTCTCACAGGAGACG \\
\hline \multicolumn{2}{|c|}{ Introduction restriction sites } \\
\hline 3' Nhel_JH1/2/4/5 & ATGCTAGCTGAGGAGACGGTGACCAG \\
\hline 3' Nhel_JH3 & ATGCTAGCTGAAGAGACGGTGACCATT \\
\hline ' Nhel JH6 & ATGCTAGCTGAGGAGACGGTGACCGTC \\
\hline
\end{tabular}


Table S2: Primers for kappa light chain gene transcripts, restriction site underlined

\begin{tabular}{|c|c|}
\hline Primer & Sequence \\
\hline \multicolumn{2}{|c|}{ Kappa light chain primer forward } \\
\hline \multicolumn{2}{|l|}{ Single cell $R T-P C R$} \\
\hline $5^{\prime} \mathrm{L}-\mathrm{V} \mathrm{K} 1 / 2$ & ATGAGGSTCCCYGCTCAGCTGGTGG \\
\hline 5' L-Vk3 & СТСTTССТССТGСTACTCTGGCTCCCAG \\
\hline $5^{\prime}$ L-Vk4 & АTTTCTCTGTTGCTCTGGATCTCTG \\
\hline \multicolumn{2}{|c|}{ Introduction restriction sites } \\
\hline 5'EcoRI_Vk1-5 & ATATTGAATTCAGACATCCAGATGACCCAGTC \\
\hline 5' EcoRI_Vk1-9 & ATATTGAATTCAGACATCCAGTTGACCCAGTCT \\
\hline 5'EcoRI_Vk1D-43 & ATATTGAATTCAGCCATCCGGATGACCCAGTC \\
\hline 5' EcoRI_Vк2-24 & ATATTGAATTCAGATATTGTGATGACCCAGAC \\
\hline 5' EcoRI_Vk2-28 & ATATTEAATTCAGATATTGTGATGACTCAGTC \\
\hline 5' EcoRI_Vк2-30 & ATATTGAATTCAGATGTTGTGATGACTCAGTC \\
\hline 5'EcoRI_Vк3-11 & ATATTGAATTCAGAAATTGTGTTGACACAGTC \\
\hline 5' EcoRI_Vk3-15 & ATATTGAATTCAGAAATAGTGATGACGCAGTC \\
\hline 5' EcoRI_Vк3-20 & ATATTGAATTCAGAAATTGTGTTGACGCAGTCT \\
\hline 5' EcoRI_Vk4-1 & ATATTGAATTCAGACATCGTGATGACCCAGTC \\
\hline
\end{tabular}

Colony screening

5' $\mathrm{V} 1 \mathrm{kf} / 6$

TCAAGGTTCAGCGGCAGTGGATCTG

5 ' Vk2f GGCCTCCATCTCCTGCAGGTCTAGTC

5' $\mathrm{V}$ К $3 f$ CCAGGCTCCTCATCTATGATGCATCC

5' Vk4_int CAACTGCAAGTCCAGCCAGAGTGTTTT

5' Vk5_int CCTGCAAAGCCAGCCAAGACATTGAT

5' Vk7_int GACCGATTTCACCCTCACAATTAATCC

Kappa light chain primer reverse

Single cell RT-PCR

3' CK $494^{A, B}$

GTGCTGTCCTTGCTGTCCTGCT

Introduction restriction sites

3' BsiWI_Jk1/4

ATCGTACGTTTGATYTCCACCTTGGTC

3' BsiWI_Jk2 ATCGTACGTTTGATCTCCAGCTTGGTC

3' BsiWI_Jk3 ATCGTACGTTTGATATCCACTTTGGTC

3'BsiWI_Jk5 ATCGTACGTTTAATCTCCAGTCGTGTC

A Sanger Sequencing

${ }^{B}$ Colony screening 
Table S3: Primers for lambda light chain gene transcripts, restriction site underlined

\begin{tabular}{|c|c|}
\hline Primer & Sequence \\
\hline \multicolumn{2}{|c|}{ Lambda light chain primer forward } \\
\hline \multicolumn{2}{|c|}{ Single cell $R T-P C R$} \\
\hline $5^{\prime} L-V \lambda 1$ & GGTCCTGGGCCCAGTCTGTGCTG \\
\hline $5^{\prime} \mathrm{L}-\mathrm{V} \lambda 2$ & GGTCCTGGGCCCAGTCTGCCCTG \\
\hline $5^{\prime} L-V \lambda 3$ & GCTCTGTGACCTCCTATGAGCTG \\
\hline $5^{\prime} L-V \lambda 4 / 5$ & GGTCTCTCTCSCAGCYGTTGCTG \\
\hline $5^{\prime} L-V \lambda 6$ & GTTCTTGGGCCAATTTTATGCT \\
\hline 5' L-V $\lambda 7$ & GGTCCAATTCYCAGGCTGTGGTG \\
\hline 5' L-V 18 & GAGTGGATTCTCAGACTGTGGTG \\
\hline \multicolumn{2}{|c|}{ Introduction restriction sites } \\
\hline 5'EcoRI_V $\lambda 1$ & ATATTGAATTCGCAGTCTGTGCTGACKCAG \\
\hline 5' EcoRI_V $\lambda 2$ & АTATTGAATTCGCAGTCTGCCCTGACTCAG \\
\hline 5'EcoRI_V $\lambda 3$ & ATATTGAATTCGTCCTATGAGCTGACWCAG \\
\hline 5' EcoRI_V $\lambda 4 / 5$ & ATATTGAATTCGCAGCYTGTGCTGACTCA \\
\hline 5' EcoRI_V $\lambda 6$ & ATATTGAATTCGAATTTTATGCTGACTCAG \\
\hline 5' EcoRI_V $\lambda 7 / 8$ & ATATTGAATTCGCAGRCTGTGGTGACYCAG \\
\hline
\end{tabular}

Colony screening

5' V $\lambda 1 / 2$ int

ATTCTCTGGCTCCAAGTCTGGC

5'V $\vee \lambda 3$ int

GGATCCCTGAGCGATTCTCTGG

Lambda light chain primer reverse

Single cell RT-PCR

$3^{\prime} \mathrm{C} \lambda$ A,B CACCAGTGTGGCCTTGTTGGCTTG

Introduction restriction sites

3'Avrll_J 1

ATTCCTAGGACGGTGACCTTGGT

3' Avrll_J $2 / 3$

ATTCCTAGGACGGTCAGCTTGGT

3'Avrll_J 6

ATTCCTAGGACGGTCACCTTGGT

3' Avrll_J $\lambda 7-1$

ATTCCTAGGACGGTCAGCTGGGT

3' Avrll_J 7-2

ATTCCTAGGGCGGTCAGCTGGGT

A Sanger Sequencing

B Colony screening 
MIUT:

AGCCTCTGGA7

CCTGTTTCTGCAAA

iGCGTGGTCCAGCCTC

AGGGCCGATTCACCATL ,

AATTGTGTTGACACAGTCTCCAGCCALU

UTGGCATCCCAGCCAGGTTCAGTGGCAGTGGG'TC I

$\checkmark$ CAGATGACCCAGTCTCCTTCCACCCTGGCTGCATCTGTAG

AGTCCCATCAAGGTTCAGCGGCAGTGGGTCTGGGACAGAGTTCACL

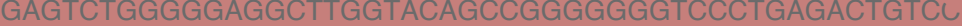

ITATCGAGACTCTGTGAAGGGCCGCTTCACCATCTCCAGAGACAATGCCAAAAAC ¿AGGGGACCACGGTCATCGTCTCCTCAGCAGGTGCAGCTGGTGGAGTCTGGGGGAGG ¿GAGTGGGTTTCATTTATACGCAAGGATGGAGCTAGTAAATACTATGGAGACTCCGTGAAGGG , CCCTGGGTTACTTTGACTACTGGGGCCAGGGAACCCTGGTCACCGTCTCCTCAGGAAATTGTGT CATAAACCTGGCCAGGCTCCCAGGCTCCTCATCTATGATGCATCCAACAGGGCCACTGGCATCCC ACTGGCCTCCGCTCACTTTCGGCGGAGGGACCAAGGTGGAGATCAAACGACATCCAGATGACCCAG

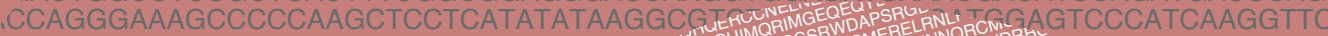

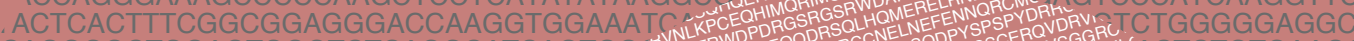

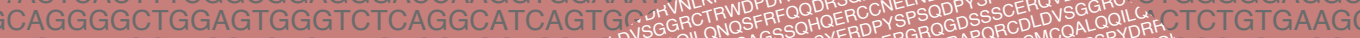

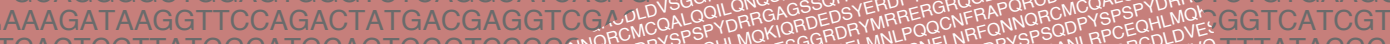

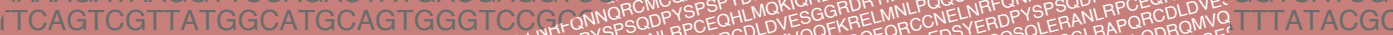

TCCTGCAGGGCGGLRAORO tGCCTAGAGC

\section{GCCTGATGAT}

\section{CACGTTTAGG}

\section{ATGGCCAGTCE}

\section{CCATCTCCAGAG}

GTCTCCAGCCACCE

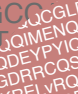

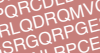

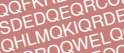

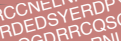

\section{Q}

\section{Q
QDRQMPG}

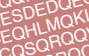

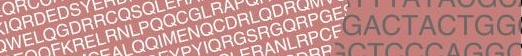

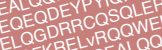

QQMENQRCCDE

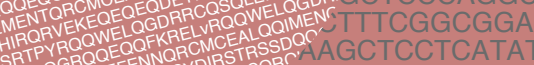

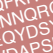
DEALQS QQQRCCDPCEE STTPYRQQQEQQFKRELMCE

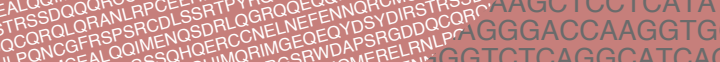

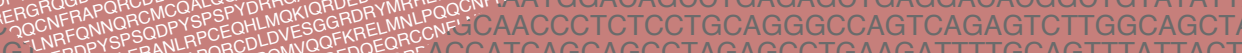
GCAGCCTAGAGCCTGA iCAGTGGGTCTGGGACAGAGTTUDYOA ERAN TCAGCAGGTGCAGCTGGACAATGCCAAAAACATCCT GTITCTGCAAATGGCCAGTCCGAGAGTCGAAGACGCGGCCGTT AATGGAGCTAGTAAATACTATGGAGACTCCGTGAAGRTGGCAGCCTGGGGGGTCCCTGAGACTCTCCTGTGCTCTTTCT CCAGGGAACCCTGGTCACCGTCTCCTCAGGAAAT TCCTCATCTATGATGCATCCAACAGGGCCACTG GAGGGACCAAGGTGGAGATCAAACGACATCCAG . CATATATAAGGCGTCTAATTTACAAGATGGAGTCC AGCATCAGTGGCAGTGGAAACAAAACATATTATCGAGAC AACGAGGTCGAGATTATGGACGTCTGGGGCCAGGGGACC ज̈GGTCCGCCAGGCTCCAGGCAAGGGGCTGGAGTGGGTTTC GGCTGTATATTACTGTGCGAAAGATAGGTCCCTGGGTTACTT TTGGCAGCTACTTAGCCTGGTACCAACATAAACCTGGCCAG TTIATTACTGTCAGCAGCGTAGCGACTGGCCTCCGCTCAC CAGGGAAAGCCCCCAA ACTTTCGGCGGAGGG 7GCTGGAGTGGGTCT GGTTCCAGACTATGA TGGCATGCAGTGGG CTGAGGACACGGCTC AGTCAGAGTCTTGGC AGATTTTGCAGTTTAT TTACGAAGTGGTTGGC ACTTACTACTGTCAACAA CTTTGAGTTGGGTCCGCC 'GAAGACGCGGCCGTTTATTA 'TCTCCTGTGTAGCGTCTGGATTCACCTTCAGTCGTT AAAGACACTCTCTTTCTGCAAATGGACAGCCTGAGA iTCTCCAGGGG GAGACAGAGT 4CCCTCACCAT TGTCCTGTGCA AAAAACATCCTG ìGGGGAGGCGTGG

unum TCCGTGAAGGGCCGA

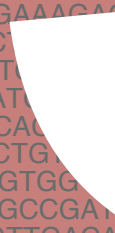
CAACCCTCTCCTGCAGG ATCAGCAGCCTAGA

\section{TTTGGTACAGC} GGGCGCTTCACCA CGTCTCCTCAGCAGGT GCAAGGATGGAGCTAGT GGGGCCAGGGAACCCTG GGCTCCTCATCTATGATGC GGAGGGACCAAGGTGGAG TATATAAGGCGTCTAAT GGAAATCAAACGAGGTC AGTGGCAGTGGAAACAA GAGATTATGGACGTCT

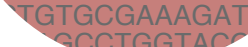
CAGGAAATTGTGTTGACA

GTCTITGTCTCCAGGGGAAAGAGCAACCCTCT ACGACATCCAGATGACCCAGTCTCCTTCCACCCTGGCTGCATCTGTAGGAGACAGAGTCTCCATCACTTGCCGG GATGGAGTCCCATCAAGGTTCAGCGGCAGTGGGTCTGGGACAGAGTTCACCCTCACCATCAGCAGTCTGCAGCD TTGTGGAGTCTGGGGGAGGCTTGGTACAGCCGGGGGGGTCCCTGAGACTGTCCTGTGCAGCCTCTGGATTCAC ¿ATATTATCGAGACTCTGTGAAGGGCCGCTTCACCATCTCCAGAGACAATGCCAAAAACATCCTGTTTCTGCAAATGQ GCCAGGGGACCACGGTCATCGTCTCCTCAGCAGGTGCAGCTGGTGGAGTCTGGGGGAGGCGTGGTCCAGCCTGG GGAGTGGGTTTCATITATACGCAAGGATGGAGCTAGTAAATACTATGGAGACTCCGTGAAGGG

¿CCTGGGTTACTTTGACTACTGGGGCCAGGGAACCCTGGTCACCGTCTCCTCAGGAAATTGTऽ

ITAAACCTGGCCAGGCTCCCAGGCTCCTCATCTATGATGCATCCAACAGGGCCACTGGCATCr

IGGCCTCCGCTCACTTTCGGCGGAGGGACCAAGGTGGAGATCAAACGACATCCAGATGACCC AGGGAAAGCCCCCAAGCTCCTCATATATAAGGCGTCTAATTTACAAGATGGAGTCCCATCAAGC CACTTTCGGCGGAGGGACCAAGGTGGAAATCAAACGAGGTGCAGTTGGTGGAGTCTGGGGG GGCTGGAGTGGGTCTCAGGCATCAGTGGCAGTGGAAACAAAACATATTATCGAGACTCTGTG

\AGGTTCCAGACTATGACGAGGTCGAGATTATGGACGTCTGGGGCCAGGGGACCACGGTCATCGI

ITATGGCATGCAGTGGGTCCGCCAGGCTCCAGGCAAGGGGCTGGAGTGGGTTTCATTTATACGCAAGGATGGAGiL I AG I ¿AGAGCTGAGGACACGGCTGTATATTACTGTGCGAAAGATAGGTCCCTGGGTTACTTTGACTACTGGGGCCAGGGAACCCTG AGGGCCAGTCAGAGTCTTGGCAGCTACTTAGCCTGGTACCAACATAAACCTGGCCAGGCTCCCAGGCTCCTCATCTATGATG GAGCCTGAAGATTTTGCAGTTTATTACTGTCAGCAGCGTAGCGACTGGCCTCCGCTCACTTTCGGCGGAGGGACCAAGGTGGA 


\section{Distinction between peanut allergy and tolerance by characterization of B-cell receptor repertoires}

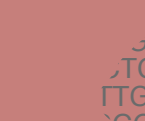

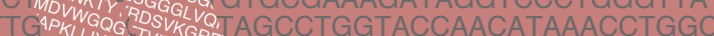

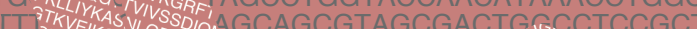

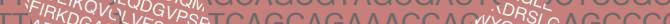

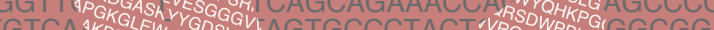

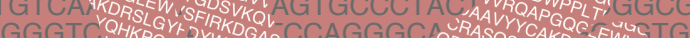

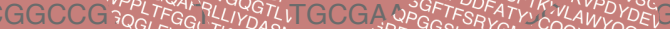

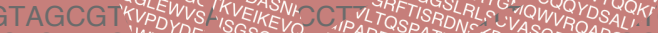
CTCTTTC'WY Y $_{D E}$ IGGGAAAGAGCAf हैaA $K W_{\text {G }}$ GGT

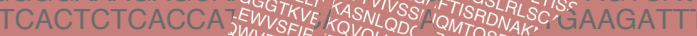

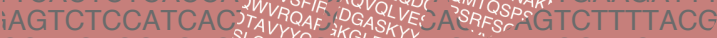

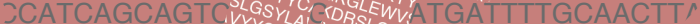
iTGCAGCCTCTG raLSQ ATCCTGTTTCT (TEAAV, PAPGOL, AGTCCGAGAGTCG/ IGAGGCGTGGTCG

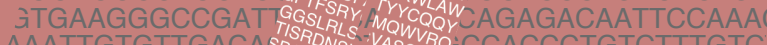
AAATTGTGTTGACA IGCATCCCAGCCACVQPG

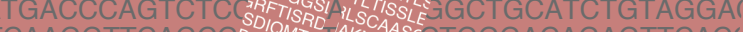
ICAAGGTTCAGCGDG MPS TAKNMACCTGGGACAGAGTTCAC submitted

Anna M. Ehierster, Constance FA den Hartog Jager 1,2, André C. Knulst 1,2, Henny G. Otten 1 TCCTCATCTATGATGCATCCAACAGGGCC T्रGGACCAAGGTGGAGATCAAACVVGA
TATAAGGCGTCTAATTTACAAGATr

AGGTGCAGTTr

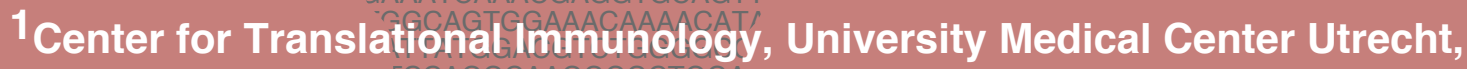
Utrecht University, Netherlands

A 2 Department of Dermatology/Ailergology, University Medical Center Utrecht, iCUfiecht University

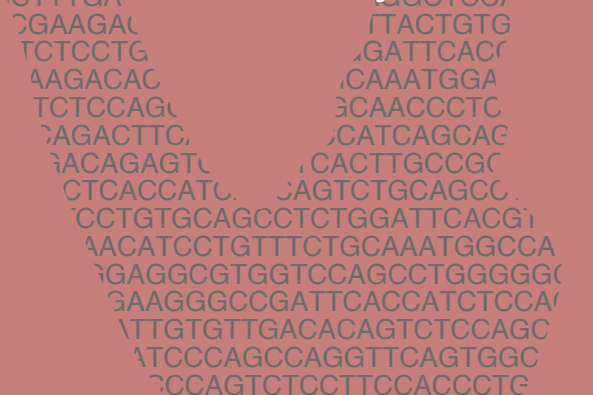




\section{Abstract}

Specific IgE against a peanut $2 S$ albumin (Ara $\mathrm{h} 2$ or 6 ) is the best predictor of clinically relevant peanut sensitization. However, slgE levels of peanut allergic and those of peanut sensitized but tolerant patients partly overlap, highlighting the need for improved diagnostics to prevent unnecessary food restrictions. By single-cell sequencing of $2 S$ albumin-binding $B$ cells, we explored differences in $V(D) J$ gene transcripts encoding for 2S albumin-specific monoclonal antibodies from 6 peanut allergic, 6 peanut sensitized but tolerant, and 5 non-atopic donors. In the allergic donors, $89 \%$ of heavy chain gene transcripts consisted of VH3 family genes, compared with only $54 \%$ in sensitized but tolerant and $63 \%$ of non-atopic donors. Additionally, certain HCDR3 sequence motifs were associated with allergy or tolerance upon hierarchical clustering of their Levenshtein distances. These sequence motifs may support the diagnosis of patients with suspected peanut allergy and sensitization to Ara h 2 and/or 6. 


\section{Introduction}

Food allergy affects around $2.5 \%$ of the European population ${ }^{1}$. Symptoms of food allergic reactions can range from mild oral itching to life-threatening anaphylaxis. Even though anxiety for unexpected allergic reactions can have a great negative impact on patients' quality of life, elimination diets and prescription of epinephrine auto-injectors are still the main treatment options in daily practice. To ensure precise dietary advices, there is a strong need for accurate, preferably minimally invasive, diagnostic strategies. Current food allergy diagnostics comprise careful history, skin prick tests, measuring specific $\lg \mathrm{E}$ (slgE) and double-blind placebo-controlled food challenges (DBPCFC) as the gold standard. However, DBPCFCs are burdensome for the patient, costly and require dedicated hospital facilities ${ }^{2,3}$. On the other hand, current technologies to measure slgE detect both clinically relevant and irrelevant sensitization, potentially leading to incorrect diagnosis and consequently unnecessary food restrictions ${ }^{4,5}$. Clinically relevant peanut sensitization is associated with slgE against the major, partly crossreactive, peanut allergens belonging to the $2 S$ albumin family, Ara $h 2$ and 6 . In previous studies in adults, $100 \%$ positive predictive values for slgE against Ara $\mathrm{h} 2$ and 6 were found using slgE positivity thresholds of respectively $1.75 \mathrm{kU} / \mathrm{l}$ and $1.8 \mathrm{kU} / \mathrm{l}$. Specific lgE levels below these thresholds, however, overlapped between allergic and tolerant subjects, preventing precise diagnosis ${ }^{6,7}$.

The occurrence of clinically irrelevant sensitization to Ara h 2 and 6 might be explained by differences in peanut (Ara h 2 and 6) specific antibody repertoires. These differences may include the presence of non-lgE antibodies blocking the binding of clinically relevant epitopes by slgE of tolerant patients. Moreover, differences may also be based on antibody affinity and/or epitope recognition patterns. So far, no clear differences between allergic and tolerant subjects were observed by epitope mapping approaches ${ }^{8}$. These evaluations may have been hampered by the use of patient sera consisting of polyclonal IgE antibodies. Sera of allergic subjects usually contain mixtures of antibodies recognizing both clinically relevant and irrelevant epitopes. On the other hand, sera from tolerant subjects might contain antibodies recognizing clinically relevant epitopes with insufficient affinity for successful FceRI receptor crosslinking, as well as antibodies recognizing clinically irrelevant epitopes ${ }^{9}$. Hence, deep analysis of monoclonal antibodies (mAbs) from specific $B$ cells may provide more insights into differences in specific antibody repertoires between allergic and tolerant subjects.

To this end, we analyzed gene sequences encoding the variable region of peanut $2 S$ albumin specific mAbs from 6 allergic and 6 sensitized but tolerant adults. In particular, sequence motifs of the HCDR3 region, the most important region for recognizing antigens ${ }^{10}$, were hierarchically clustered. Clustering of HCDR3 regions resulted in four motifs exclusively present in allergic donors and three motifs associated with tolerance. 


\section{Methods}

\section{Patient selection}

Blood samples $(100 \mathrm{ml})$ were drawn from allergic $(n=6)$ and tolerant $(n=6)$ adults sensitized to Ara $\mathrm{h} 2$ and/or 6 (ImmunoCAP $\geq 0.1 \mathrm{kU} / \mathrm{I}^{11}$ ). Their diagnoses were confirmed by DBPCFCs or in the case of two tolerant subjects, by convincing history of tolerated peanut ingestion, as evaluated by a trained physician. Tolerant patients consumed at least small amounts of peanuts $\leq 2$ weeks before study inclusion. Five non-atopic donors (total $\mathrm{lg} \mathrm{E} \leq 100 \mathrm{lU} / \mathrm{ml}$ ) served as reference. The study, conducted in accordance with the declaration of Helsinki, was ethically approved (number 17-945) by the medical ethical committee of the University Medical Center Utrecht and informed consent was given by all participants.

\section{Isolation of peanut $2 \mathrm{~S}$ albumins}

Roasted, salted, and ground peanuts were soaked in $50 \mathrm{mM}$ sodium citrate buffer $(\mathrm{pH} 5.5)$ and incubated overnight at $4{ }^{\circ} \mathrm{C}$. The filtrate was applied onto a cation ion exchange column (Capto S, GE Healthcare) and the pooled fraction, containing proteins with molecular masses between 10 and $25 \mathrm{kDa}$, was separated by size exclusion (Superdex 75 pg, GE Healthcare). Enclosed proteins were analyzed by mass spectrometry, as described previously ${ }^{12}$, and by inhibition assays.

Inhibition assays were performed with a concentration range of $0-100 \mu \mathrm{g} / \mathrm{ml}$ peanut 2S albumin fraction and the commercially available EUROLINE (EL) DPA-Dx Peanut 1 strip (EUROIMMUN AG) including the seed storage proteins Ara h 2, 6 and 7 . Briefly, $1: 11$ in washing buffer diluted sera were pre-incubated with the respective concentration of the peanut $2 S$ albumin fraction for $30 \mathrm{~min}$ at room temperature. Pre-incubated sera were applied onto EL strips and incubated overnight at room temperature. Bound IgE antibodies were detected by applying a-human IgE conjugate coupled with alkaline phosphatase for $60 \mathrm{~min}$ at room temperature. Visualization was achieved by applying nitro-blue tetrazolium/5-bromo-4-chloro-3'-indolyphosphate substrate for $10 \mathrm{~min}$ and specific lgE levels were evaluated as EUROLINE (EL)-intensities. Inhibition was calculated as the percentage of remaining EL-intensities compared to the EL-intensities without pre-incubation with the peanut $2 \mathrm{~S}$ albumin fraction. Results of the MS analysis and inhibition experiments are shown in the Suppl. Table S1 and Figure S1.

\section{Allergen-tetramer formation}

An excess of biotin was added to the isolated peanut $2 \mathrm{~S}$ albumin fraction resulting on average in four biotin molecules per one molecule protein (EZ-Link ${ }^{T M}$ Sulfo-NHS- 
Biotin, ThermoFisher Scientific). Allergen-tetramers were formed by separately adding streptavidin-R-phycoerythrin or streptavidin-Allophycocyanin (ThermoFisher Scientific) to the biotinylated $2 S$ albumin fraction in a molecular ratio of 1:1 (streptavidin: protein $)^{13,14}$.

\section{Single-cell sorting of allergen specific B cells}

Blood drawn into heparin-coated tubes was freshly processed within 24 hours and peripheral blood mononuclear cells (PBMCs) were isolated by density gradient centrifugation. B cells were negatively enriched from the PBMCs fraction by magnetic beads (B cell isolation kit II, Miltenyi Biotec) and they were stained for allergen specificity with CD45-Pacific Orange (1:40, ThermoFisher Scientific), CD3-Pacific Blue (1:160, BD Biosciences), CD19-Fluorescein isothiocyanate (1:20, BD Biosciences), CD14-Rphycoerythrin/cyanine dye 7 (1:800, Sony Biotechnology), CD16-R-phycoerythrin/ cyanine dye 7 (1:1000, BD Biosciences) and allergen-tetramers $\left(75 \mathrm{pg} \rightarrow 30 \cdot 10^{6}\right.$ antigentetramers sufficient for approximately $150-300$ resting $B$ cells $\left.{ }^{15}\right)$. Cells gated for CD45 ${ }^{+}$, $\mathrm{CD}^{-}, \mathrm{CD}^{+}{ }^{+}$, allergen tetramers (double positive) ${ }^{+}$and $\mathrm{CD} 14 / 16^{-}$were single cell sorted into 96 well plates supplemented with $14 \mathrm{mM}$ DTT and 11.2 U RNAse inhibitor (N8080119, ThermoFisher Scientific) in 0.5x PBS. Sorted cells were immediately put on dry ice and stored at $-80{ }^{\circ} \mathrm{C}$ until further processing. PBMCs from a donor who showed specific $B$ cells in previous experiments served as positive control and staining with only biotin served as negative control. The gating was based on the negative control (example FACS plot is shown in the Suppl. Figure S2).

\section{Gene amplification of B cell receptors}

B cell receptors were amplified as previously described with minor modifications ${ }^{13,16,17}$. Briefly, frozen B cells were put on dry ice, supplemented with 1.4\% NP-40, 3 U RNAse inhibitor (N8080119, ThermoFisher Scientific) and $7 \mu \mathrm{M}$ hexamer primers and subsequently incubated for one minute at $68^{\circ} \mathrm{C}$ for primer annealing. cDNA transcription was performed accordingly to manufacturer's instruction (SuperScript III, ThermoFisher Scientific) and cDNA was used as a template for amplifying the variable gene transcript of the heavy and the corresponding light chain. Amplification was performed with $1.25 \mathrm{U}$ hot-start DNA polymerase (AmpliTaq Gold, ThermoFisher Scientific) and $40 \mathrm{nmol}$ multiplex primers for 50 cycles and the annealing temperature was set to 62,60 and $58{ }^{\circ} \mathrm{C}$ for heavy, $\mathrm{k}$ and $\lambda$ light chain, respectively. 


\section{Sequence analysis of B cell receptors}

Enzyme purified heavy chain amplification products were sequenced (Sanger sequencing, Macrogen) with either multiplex reverse primers or framework (FR) 1 forward primers (isotype determination) while the light chain amplification products were all sequenced with the respective reverse primers. All sequences were checked for their quality using Chromas Lite 2.6.5, and double peaks, potentially resulting from amplification errors in the beginning of the amplification reaction, were aligned and corrected for the germline if plausible. Quality-checked gene sequences, saved as FASTA files, were used for automatic germline alignment using the IgBLAST web interface (reference: IMGT database $)^{18}$ and the output was automatically written into a SQLite database using $R$, version 3.6.3. For further evaluation, sequences were considered if the sequence was valid and declared as 'productive'.

\section{Cloning of gene products from single cells}

Restriction sites were introduced with specific $\mathrm{V}$ gene forward primers and $\mathrm{J}$ gene reverse primers, ensuring that the entire variable region is cloned without any additional amino acids, using 0.5 U Phusion high-fidelity DNA polymerase (NEB). The amplification was performed for 30 cycles with an annealing temperature of 62,60 and $58{ }^{\circ} \mathrm{C}$ for heavy, $\mathrm{k}$ and $\lambda$, respectively, and purified gene products were digested with the respective restriction enzymes Eco RI and NhE I (heavy chain), BsWi ( $\mathrm{k}$ light chain) or Avr II ( $\lambda$ light chain). Before cloning these digests into human $\lg H(\lg E, \lg G 1, \lg G 4)$ and $\lg L$ $(\kappa, \lambda)$ expression vectors ( $p F U S E s s$ vector series, Invivogen), they were purified and phosphorylated (T4-PNK, NEB). All vectors contained an hEF1-HTLV promotor, an IL2 signal sequence, a multiple cloning site upstream the constant regions and zeocin $(\mathrm{lgH})$ or blasticidin $(\mathrm{lgL})$ resistance genes for selection. To prevent self-ligation, the digested parent vector was dephosphorylated (parent vector, FAST-AP, ThermoFisher Scientific) before the digests were added in a molecular ratio of 5:1 (T4 ligase, NEB). Heat-shock transformation was performed by adding the ligated vector to competent $E$. coli Top 10 cells and incubating them for $45 \mathrm{sec}$ at $42^{\circ} \mathrm{C}$. Grown colonies were screened for correctly incorporated variable regions by PCR, using forward primers binding to the respective FR2 region and reverse primers suitable for the constant part of the vector. Positive clones were Sanger sequenced and the correctness was checked by aligning the vector sequence with the first sequence result.

\section{Heterologous expression of monoclonal antibodies}

Human embryonic kidney (HEK) 293F cells were cultured in FreeStyle 293 expression medium (ThermoFisher Scientific) using $125 \mathrm{ml}$ shaking culture flasks. Exponentially 
growing cells ( $80 \%$ confluence, $90 \%$ viability) were transiently transfected with $\mathrm{VH}$ and VL expression vectors in a ratio of $2: 3$ (total $0.5 \mu \mathrm{g}$ plasmid DNA per $1 \cdot 10^{6}$ cells) in $150 \mathrm{~mm}$ culture plates using $293 \mathrm{fectin}(2 \mu \mathrm{l} / \mu \mathrm{g}$ plasmid DNA, ThermoFisher Scientific). Three days upon transfection, the supernatant was harvested and stored at $-20{ }^{\circ} \mathrm{C}$ for further analyses.

\section{Determination allergen specificity}

Specificity of heterologously expressed mAbs was tested using a direct ELISA. Coating was performed overnight at room temperature with either $0.3 \mu \mathrm{g} /$ well Ara $\mathrm{h} 2 / 6^{19}$ or transferrin (negative control). Upon blocking (PBS + 1\% BSA + 0.1\% Tween-20), serial-diluted thawed (1x) supernatant was applied for one hour at room temperature and bound mAbs were detected by goat a-human $\lg E(1: 5000, \mathrm{KPL})$ or a-human $\lg \mathrm{G}$ (1:2000, Jackson ImmunoResearch) antibodies coupled with horse-radish peroxidase. Visualization was provided by adding tetramethylbenzidine (TMB) for $15 \mathrm{~min}$ in the dark and the OD was measured at $450 \mathrm{~nm}$. The cut-off levels were determined for IgE and IgG1 mAbs individually and were defined as the blank value (HEK293F cells supernatant) plus three times its standard deviation. A mAb was defined as specific when the sample had an OD greater than the set cut-off levels upon subtraction of the negative control (transferrin) OD value. The negative control OD value was defined as the OD value of the mAb upon subtraction of its blank value.

\section{Specificity of patient serum and inhibition experiments}

ELISA to measure serum slgA and inhibition experiments were performed as described in the previous paragraph with the following modifications:

1. Patients' serum samples were 1:10 diluted in blocking buffer before application (duplicates, same sample)

2. Detection of slgA binding by goat a-human $\lg A$ (1:2000, Southern Biotech) coupled with horse-radish peroxidase and incubation for $60 \mathrm{~min}$ at room temperature

3. Determination of optimal dilution for slgE inhibition experiments by applying serum samples in a dilution series $(1: 10,1: 20,1: 50,1: 100)$; optimal serum dilution for inhibition experiments was defined as the first dilution showing lowered OD values in relation to the dilution yielding the maximum value or maintaining a plateau phase

4. Additional incubation step prior to serum application with the respective $m A b$ in a concentration-dependent manner ( 1 to $100 \mathrm{ng} / \mathrm{ml}$ ) and incubation for $60 \mathrm{~min}$ at room temperature 


\section{Indirect BAT}

Functionality of $2 \mathrm{~S}$ albumin-specific mAbs were evaluated by indirect basophil activation test (BAT) ${ }^{20}$. Briefly, isolated PBMCs were treated with $13.4 \mathrm{mM}$ lactic acid buffer to strip off receptor-bound $\mathrm{IgE}$. Receptors were re-loaded with human $\mathrm{mAbs}$ expressed as $\mathrm{lgE}$, supplemented with $10 \mu \mathrm{g} / \mathrm{ml}$ heparin and $8 \mathrm{mM}$ EDTA and incubated for $90 \mathrm{~min}$ at $37^{\circ} \mathrm{C}$. After extensive washing, re-loaded cells were cultured overnight in RPMI supplemented with $10 \%$ fetal calf serum and $1 \mathrm{ng} / \mathrm{ml} \mathrm{IL}-3$ at $37^{\circ} \mathrm{C}$. Dose-dependent stimulation was performed with a mixture of native Ara h 2/6 and $1 \mathrm{ng} / \mathrm{ml} \mathrm{IL}-3(1 \mu \mathrm{g} / \mathrm{ml}$ to $5 \mathrm{ng} / \mathrm{ml})$. RPMI + $1 \mathrm{~g} / \mathrm{ml} \mathrm{IL-3,} 100 \mathrm{ng} / \mathrm{ml}$ C5a (R and D Systems, 2037-C5), 1 $\mu \mathrm{g} / \mathrm{ml}$ a-human $\operatorname{lgE}$ (Vector Laboratories, BA-3040-.5) and $10 \mu \mathrm{M}$ fMLP (Sigma Aldrich, F3506) served as negative and positive controls, respectively. Stimulated cells were stained for IL-3 activated basophils with CD123-Fluorescein isothiocyanate (1:400, BioLegend), CD63R-phycoerythrin (1:800, Sanbio B.V.), CD203c-Allophycocyanin (1:160, Sony Biotechnology) and CD193-eridinin-Chlorophyll-Protein/Cyanine5.5 (1:160, Sony Biotechnology) and basophils were defined as $\mathrm{CD} 203 \mathrm{c}^{+} \mathrm{CD} 123^{+} \mathrm{CD} 193^{+}$. Basophil activation was calculated as the percentage of $\mathrm{CD}^{+} 3^{+}$basophils and the threshold was set to $5 \%$ $\mathrm{CD}^{+} 3^{+}$cells. All measurements were performed in duplicates and the gating strategy is shown in the Suppl. Figure S2.

\section{Data analyses}

Comparisons of continuous data between allergic and tolerant patients were statistically analyzed with the two-sided non-parametric Mann-Whitney-U-test. Correction for multiple testing was obtained by Bonferroni correction. Comparison of continuous data between allergic, tolerant and non-atopic donors was statistically evaluated using the non-parametric Kruskal-Wallis test combined with Dunn's multiple testing correction. Categorical comparisons were performed with the Chi Square test. Descriptive analysis consisted of isotype distribution, mutational status and $\mathrm{VH}$ usage. Moreover, related clones were defined as sequences resulted from identical $\mathrm{VH}$ and $\mathrm{JH}$ genes and HCDR3 length and related clones were aligned using ClustalW2 ${ }^{21}$. Sequence motifs were identified by calculating Levenshtein distances (R package 'factoextra') of HCDR3 regions combined with hierarchical clustering (default unsupervised clustering in $R$ ). HCDR3 sequences with five or fewer differences were defined as one motif. Physical properties of the HCDR3 regions were calculated using the BRepertoire interface ${ }^{22}$. For the latter analysis, non-redundant HCDR3 amino acid sequences were used. 


\section{Results}

\section{IgE levels overlapped between allergic and tolerant donors}

In order to study differences in antibody repertoires between peanut allergic and peanut sensitized ( $\lg E)$ but tolerant patients, blood was drawn from peanut allergic $(n=6$, age: 27-41) and tolerant ( $n=6$, age: 27-63) donors sensitized to Ara $\mathrm{h} 2$ and/or 6 ( $\geq 0.1 \mathrm{kU} / \mathrm{l}$ ImmunoCAP). Specific lgE levels to Ara $\mathrm{h} 2$ ranged from 1.0 to $72 \mathrm{kU} / \mathrm{l}$ in the allergic and from 0 to $1.7 \mathrm{kU} / \mathrm{l}$ in the tolerant group. Specific lgE levels to Ara $\mathrm{h} 6$ were lower compared to slgE levels to Ara $\mathrm{h} 2$ and ranged from 0 to $13 \mathrm{kU} / \mathrm{l}$ in the allergic and from 0 to $0.9 \mathrm{kU} / \mathrm{l}$ in the tolerant group, suggesting a more important role of Ara h 2. Monosensitization to either Ara h 2 or 6 was detected in both groups, although it occurred more often within the tolerant group (allergic: Pt 01, tolerant: Pt 02, 03, 07) as shown in Table 1.

Table 1: Patient characteristics

\begin{tabular}{|c|c|c|c|c|c|c|c|}
\hline Patient & Sex & Age & Last reaction & $\begin{array}{c}\text { Severity } \\
\text { (prov) }\end{array}$ & $\begin{array}{c}\text { Ara h } 2 \\
{[\mathrm{kU} / \mathrm{l}]}\end{array}$ & $\begin{array}{c}\text { Ara h } 6 \\
{[k U / l]}\end{array}$ & $\begin{array}{c}\text { Total IgE } \\
{[\mathrm{kU} / \mathrm{l}]}\end{array}$ \\
\hline
\end{tabular}

\begin{tabular}{|c|c|c|c|c|c|c|c|}
\hline \multicolumn{8}{|c|}{ Allergic group } \\
\hline Pt 05 & $f$ & 34 & 3 years & severe & 1.0 & 1.4 & 625 \\
\hline Pt 09 & $f$ & 29 & 1 year & moderate & 2.4 & 5.3 & 807 \\
\hline Pt 01 & $f$ & 39 & 1.5 years & moderate & 3.3 & 0 & 14.5 \\
\hline Pt 04 & $\mathrm{~m}$ & 27 & 7 years & moderate & 8.2 & 1.7 & 417 \\
\hline Pt 10 & $f$ & 27 & 3 years & severe & 23 & 7.9 & 252 \\
\hline Pt 06 & $\mathrm{~m}$ & 41 & 2 years & moderate & 72 & 13.1 & 83.3 \\
\hline \multicolumn{8}{|c|}{ Tolerant group } \\
\hline Pt 02 & $\mathrm{~m}$ & 45 & $N / A$ & $\mathrm{~N} / \mathrm{A}$ & 0 & 0.9 & 14.2 \\
\hline Pt 07 & $f$ & 29 & N/A & N/A & 0.2 & 0 & 250 \\
\hline Pt 08 & $\mathrm{~m}$ & 36 & $\mathrm{~N} / \mathrm{A}$ & $\mathrm{N} / \mathrm{A}$ & 0.6 & 0.2 & 98.3 \\
\hline Pt 11 & $\mathrm{~m}$ & 27 & $\mathrm{~N} / \mathrm{A}$ & $\mathrm{N} / \mathrm{A}$ & 1.3 & 0.8 & $>1000$ \\
\hline Pt 03 & $\mathrm{~m}$ & 63 & $N / A$ & $N / A$ & 1.7 & 0 & 208 \\
\hline Pt 12 & $f$ & 31 & $N / A$ & $N / A$ & 2.3 & 0.3 & $>1000$ \\
\hline
\end{tabular}




\section{Frequency of peanut $2 S$ albumin-binding B cells was significantly higher in allergic donors}

As a first step, the frequencies of $2 \mathrm{~S}$ albumin-binding $\mathrm{B}$ cells were compared between peanut allergic patients, peanut sensitized but tolerant patients, and non-atopic controls. 2S albumin-binding B cells, double-positive for allergen-tetramer staining, were defined as putatively specific, and their frequency was expressed as percentage of total $\mathrm{B}$ cells acquired from the respective sample. The frequency ranged from $0.0004 \%$ to $0.0164 \%$ across all included donors. While the frequency in allergic patients (median: $0.01 \%, 95 \% \mathrm{Cl}: 0.005-0.164)$ was only slightly increased compared to the tolerant patients (median: $0.006 \%, 95 \% \mathrm{Cl}: 0.0016-0.014$ ), the frequency was significantly elevated compared with non-atopic controls (median: $0.002 \%, 95 \% \mathrm{Cl}: 0.0004-0.004$, $\left.\mathrm{p}=0.008{ }^{* *}\right)$ ). Those $2 \mathrm{~S}$ albumin-binding B cells were single-cell sorted and served as source for the amplification of the heavy and corresponding light chain gene transcripts. No correlation was found between the frequency of $2 S$ albumin-binding $B$ cells and the number of $2 S$ albumin-binding $B$ cells from which the heavy and the corresponding light chain gene transcripts were successfully amplified and sequenced. A high number of $\mathrm{V}(\mathrm{D}) \mathrm{J}$ gene transcripts were successfully amplified from the B cells of patients 4 and $6-$ both allergic - while none or only one $V(D) J$ gene transcript was successfully amplified from $B$ cells of patient 2 (tolerant) and 9 (allergic), respectively. For both of these patients, only a small number of $2 \mathrm{~S}$ albumin-binding B cells were sorted (Pt 2: 8, Pt 9: 12), which may exclude amplification failure as a reason for obtaining such a low number of successfully amplified gene transcripts. Frequencies, number of sorted $2 \mathrm{~S}$ albuminbinding $B$ cells and successfully amplified corresponding $V(D) J$ gene transcripts are shown in Figure $1^{1}$. Taken together, those successfully sequenced gene transcripts - 280 heavy chain gene transcripts, 221 light chain gene transcripts - provide a good basis for further analyses.

\footnotetext{
${ }^{1}$ Sorted B cells/amplified corresponding V(D)J gene transcripts - allergic: Pt 1 (32/49), Pt 4 (63/192), Pt 5 (8/96), Pt 6 (61/192), Pt 9 (1/12), Pt 10 (19/96); tolerant: Pt 2 (0/8), Pt 3 (7/108), Pt 7 (13/40), Pt 8 (7/36), Pt 11 (36/144), Pt 12 (17/72); non-atopic: NA-1 (21/50), NA-2 (14/32), NA-3 (7/36), NA-4 (8/48), NA-5 $(12 / 24)$
} 


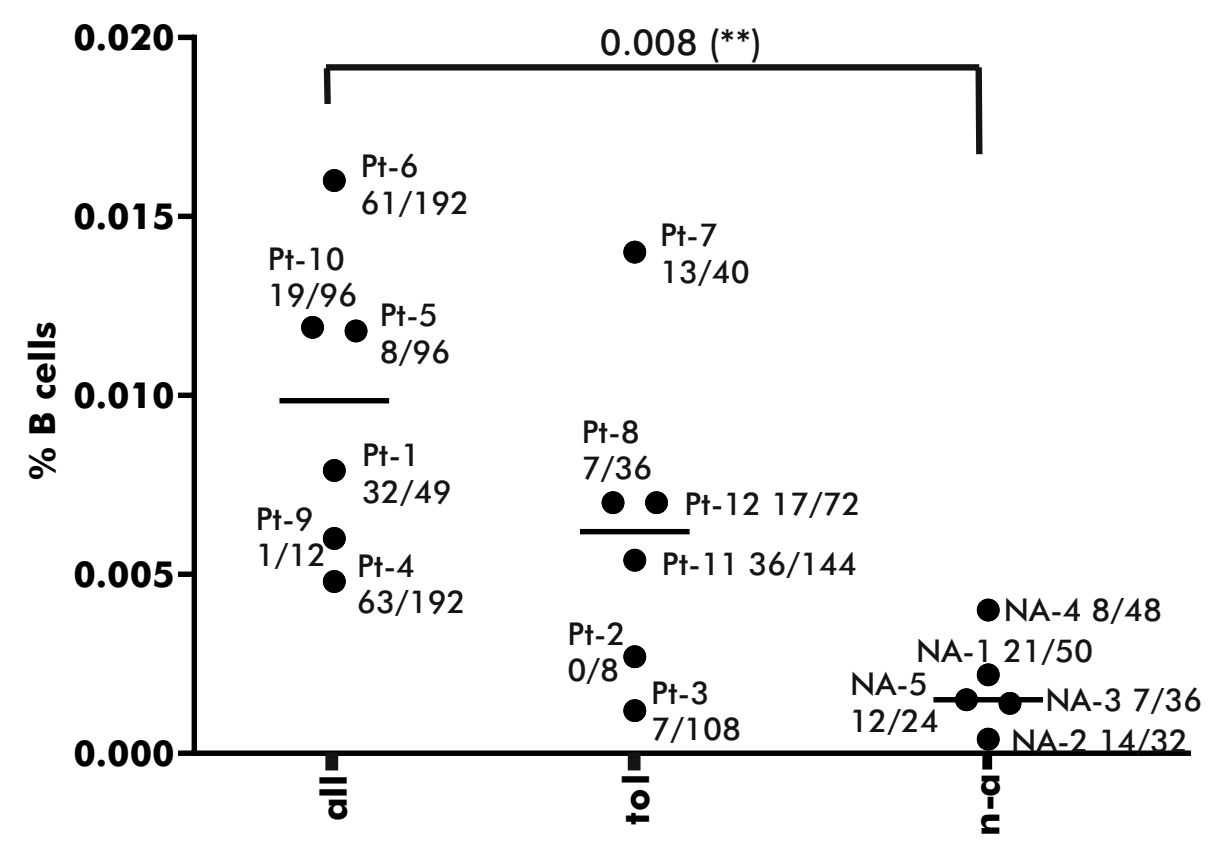

Figure 1: Frequency of $2 S$ albumin-binding $B$ cells

Frequency of $2 S$ albumin-binding $B$ cells, double-positive for allergen-tetramer ( $P E$ and $A P C)$ staining, expressed as percentage of the total $B$ cell number acquired from the respective sample, indicated with the median. Each frequency is labelled with the respective patient number and the number of successfully amplified corresponding heavy and light $V(D) J$ gene transcripts in relation to sorted B cells. Statistical significance was proven with the Kruskal-Wallis test and corrected for multiple testing using the Dunn's multiple comparison test (adjusted $p$-value); all = allergic, tol = tolerant, $n$-a = non-atopic

\section{S albumin-binding B cells show specific binding to Ara $h 2$ and 6}

In order to verify the specificity of identified $2 S$ albumin-binding B cells, 42 different sets of heavy and corresponding light chain gene transcripts were heterologously expressed as IgE or IgG1 mAbs. The majority of these mAbs (81\%, 34/42), mostly derived from allergic and tolerant patients, showed specific binding to Ara h 2 and 6 at $10 \mu \mathrm{g} / \mathrm{ml}$ or lower in a concentration-dependent manner (Figure 2a). These heterologously expressed mAbs originated from $2 S$ albumin-binding $\operatorname{lgM}^{+}(41 \%, 14 / 34), \operatorname{lgG} 1^{+}(3 \%, 1 / 34), \lg G 2^{+}$ $(23 \%, 8 / 34), \lg \mathrm{E}^{+}(3 \%, 1 / 34), \lg \mathrm{A} 1^{+}(18 \%, 6 / 34)$ and $\lg \mathrm{A} 2^{+}(9 \%, 3 / 34) \mathrm{B}$ cells. While the two mAbs with relatively high optical density (OD) values originated from $\operatorname{lgG} 1^{+}$ and $\lg A 1^{+} \mathrm{B}$ cells, mAbs with relatively low $\mathrm{OD}$ values originated predominately from $\operatorname{lgM}^{+} \mathrm{B}$ cells. About half of the specific mAbs $(58 \%, 11 / 19)$ tested for separate binding to Ara $\mathrm{h} 2$ or 6 recognized both allergens, and had comparable OD values. Eight mAbs exclusively recognized either Ara h 2 or 6 (21\%, 4/19 Ara h 2 and 21\%, 4/19 Ara $h$ 6) (Figure 2b). The remaining antibodies without specific binding to Ara $h 2$ or 6 at $10 \mu \mathrm{g} / \mathrm{ml}$ originated predominately from IgM+B cells of non-atopic controls $(88 \%$, $7 / 8$ ). These data confirm the specificity of most selected $2 S$ albumin-binding B cells and support the relevance of the gene analyses described in the following paragraphs. 


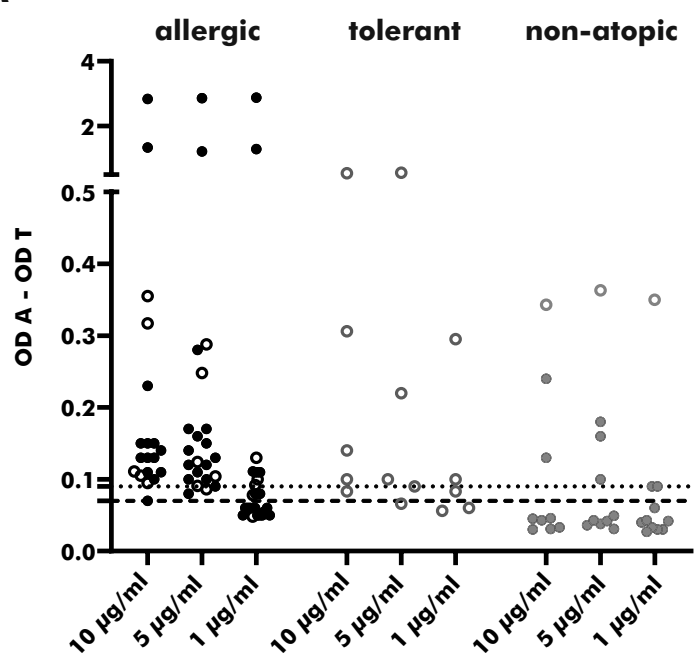

C

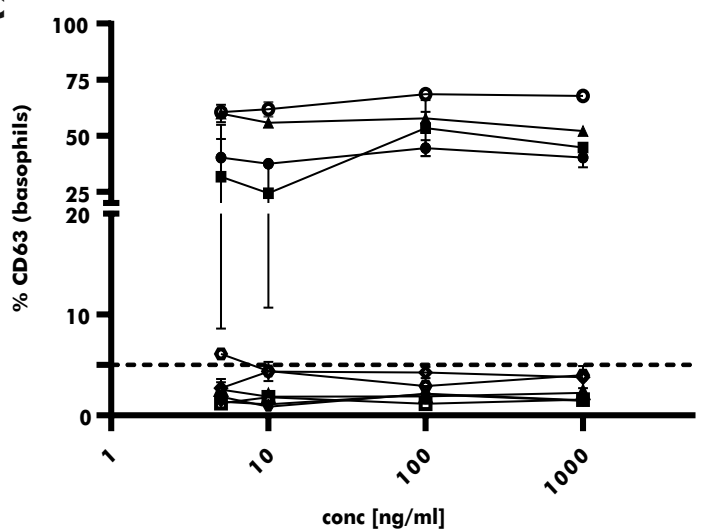

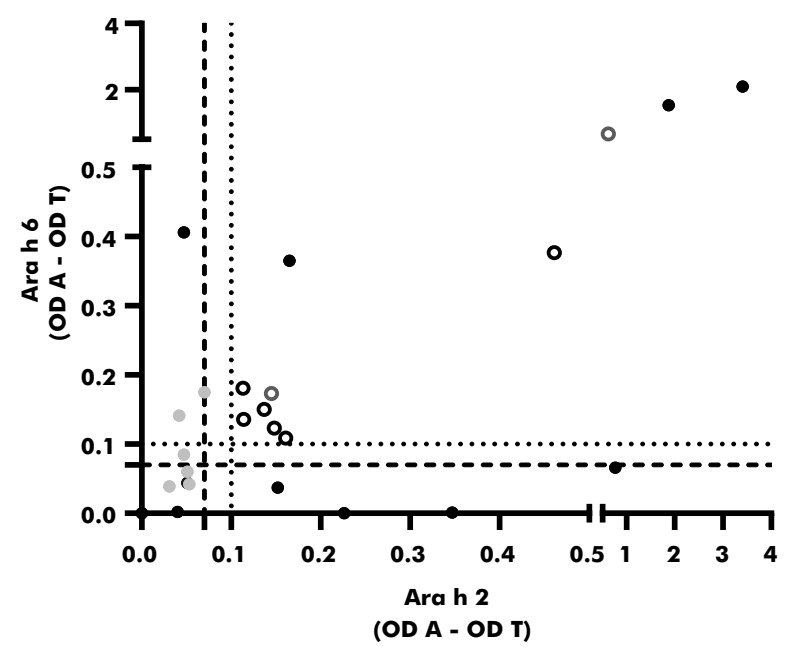

Figure 2: Specificity and functionality of heterologously expressed mAbs

A Concentration-dependent ( 1 to $10 \mu \mathrm{g} / \mathrm{ml}$ ) binding of heterologously expressed $\mathrm{mAbs}(n=42)$ to Ara $h 2$ and 6 defined as $O D$ values corrected for their binding to transferrin; B Binding of heterologously expressed mAbs to Ara $h 2$ and $6(n=24)$ at $10 \mu \mathrm{g} / \mathrm{ml}$ defined as $O D$ values corrected for their binding to transferrin; filled dots indicate mAbs expressed as IgE and open dots mAbs expressed as IgG1, bold dash line is the cut-off for IgE mAbs and non-bold dash line the cut-off for IgG1 antibodies; black = allergic, grey = tolerant, light grey = non-atopic; $\boldsymbol{C}$ Upregulation of CD63 upon loading of human basophils with $\mathrm{mAb}$ mixture and subsequent stimulation with Ara $h 2$ and 6 in a concentration-dependent manner (5 to $1000 \mathrm{ng} / \mathrm{ml}$ ); the dots indicate the mean of duplicate measurements and their range; Mix $1=P t 6-2$, Mix 2 = Pt 6-2 + Pt 6-17, Mix 3 = Pt 6-2 + Pt 3-2 + Pt 6-11, Mix 4 = Pt 6-17, Pt 3-2, Pt 6-11, Mix $5=$ Pt 1-32, Pt 6-11, Pt 6-6, Pt 6-8, Mix $6=$ NA 1-10 + Pt 4-28 + Pt 4-34 + Pt 6-2, Mix $7=$ Pt NA 1-10 + Pt 4-28 + Pt 4-34, Mix $8=$ Pt 1-32 + Pt 6-15 + Pt 6-21, stripped basophils before loading, Rituximab expressed as IgE; dash line: threshold level set to $5 \%$

\section{Selected $2 S$ albumin-specific mAbs can induce degranulation}

As IgE B cell memory is potentially stored in $\lg G 1^{+}$memory $B$ cells and to a lesser extent in $B$ cells belonging to another isotype, the functionality of mAbs originated from non- $\lg E$ $2 S$ albumin-binding $B$ cells were tested for their functionality ${ }^{23-25}$. To determine mAb functionality, stripped human basophils were loaded with mixtures of randomly selected mAbs (expressed as $\mathrm{lgE}$ ) and subsequently stimulated with Ara h 2 and 6 (Figure 2c and Suppl. Figure S4). Stimulated basophils showed individual CD63 upregulation 
upon stimulation when loaded with mixtures containing the mAb Pt 6-2 (Mix 1, 2, 3 and 6). Such responses were already achieved at low concentrations (5 $\mathrm{ng} / \mathrm{ml} \mathrm{Ara} \mathrm{h}$ 2/6). Moreover, the overall CD63 upregulation induced by Mix 3 and 6 was even higher than the CD63 upregulation induced by the a-lgE control at all allergen concentrations, pointing to an additive effect by using mAb Pt 6-2 (Mix 1) in combination with additional mAbs. The functionality of mAb Pt 6-2 and the observed additive effect confirm the selection of specific and functional B cells with the FACS sorting strategy presented in this study.

\section{Allergic and tolerant donors possess more class-switched 2S albumin-binding $B$ cells than non-atopic controls}

All successfully sequenced heavy chain gene transcripts were initially analyzed for isotype distribution, as class-switching is mostly accompanied by somatic hypermutation maturation upon antigen challenge. Overall, IgM was the most dominant isotype across all groups. Notably, its prevalence was much lower in allergic (47\%) and tolerant $(53 \%)$ donors than in the non-atopic reference group (94\%), indicating more antigenchallenged and matured specific $B$ cells originated from allergic and tolerant patients. Moreover, the prevalence of IgA2-expressing B cells was larger increased in the tolerant group (20\%), while a comparable distribution was observed in allergic and nonatopic donors (2\%), as shown in Figure $3 a$. This finding suggests potential protection by specific $\lg A$ in tolerant patients, as $\lg A$ is generally able to prevent mucosal antigen crossing ${ }^{26}$. Regarding IgE, only a small number of $\mathrm{V}(\mathrm{D}) \mathrm{J}$ gene transcripts derived from $\operatorname{lgE}^{+} \mathrm{B}$ cells were successfully amplified (allergic: 1 ; tolerant: 2 ), possibly due to the extreme low abundance $\left(0.002\right.$ to $1 \%$ ) of $\operatorname{lgE}^{+} B$ cells within the circulation ${ }^{27}$. Even though slightly higher proportions $\operatorname{lgG} 3^{+}$(around $1 \%$ ) and $\operatorname{lgG} 4^{+} \mathrm{B}$ cells (around $0.75 \%$ ) are known ${ }^{28,29}$, no $\mathrm{V}(\mathrm{D}) \mathrm{J}$ gene transcripts derived from $\operatorname{lgG} 3^{+}$and $\lg \mathrm{G}^{+} \mathrm{B}$ cells were amplified. Overall, the individual isotype distribution varied for each patient, e.g. 2S albumin-binding $B$ cells of the allergic patient 4 were predominantly $\lg ^{+} B$ cells, while those of patient 1 were predominantly $\lg ^{+} B$ cells with a shift to class-switched $B$ cells in peanut allergic and tolerant patients. This indicates that the $2 S$ albumin-binding $B$ cells from allergic and tolerant patients are more often matured and antigen-challenged B cells compared with the non-atopic control group. 

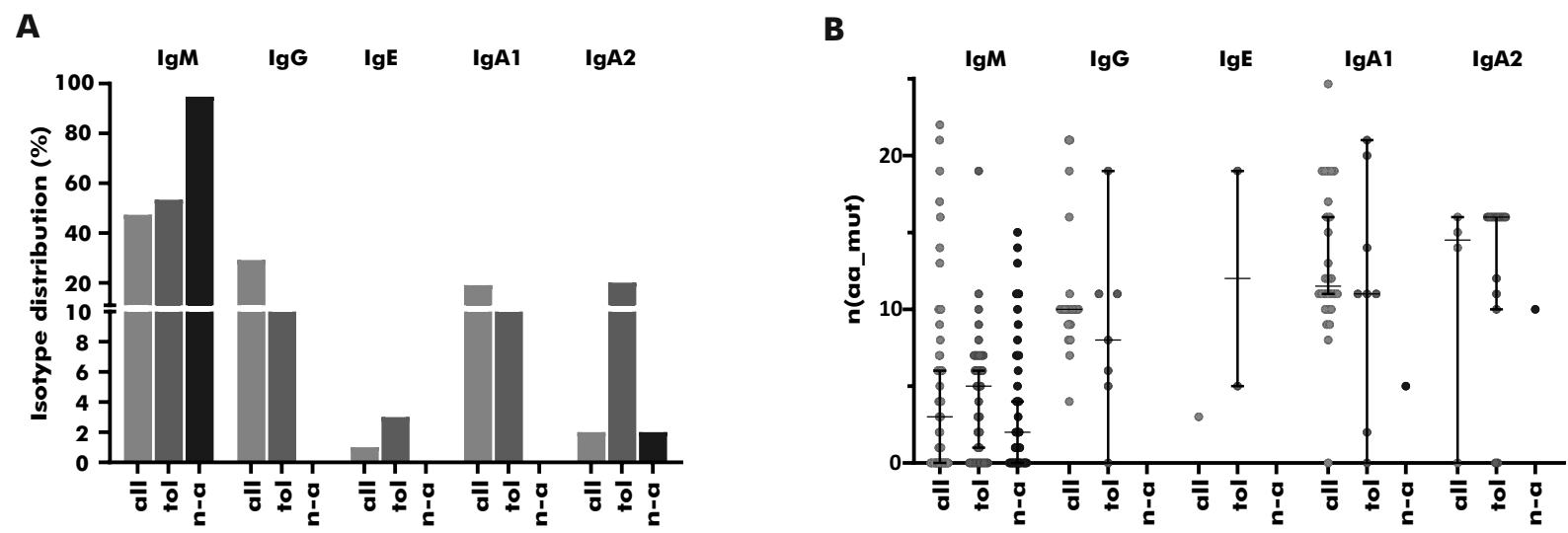

Figure 3: Descriptive gene analysis

Descriptive gene lineage analysis of successfully sequenced heavy chain gene transcripts defined as productive (allergic $n=151$; tolerant $n=68$; non-atopic, $n=51$ ), irrespectively of successfully sequenced corresponding light chain gene transcript;A Isotype distribution split for allergic (all), tolerant (tol) and nonatopic ( $n$-a) donors; $\boldsymbol{B}$ Numbers of non-silent mutations expressed with the median (95\% Cl) and split for allergic (all), tolerant (tol) and non-atopic ( $n-a)$ donors

\section{$\operatorname{lgM}^{+}$B cells from allergic donors partly show strong maturation by somatic hypermutations}

The introduction of non-silent (mutations on amino acid level) somatic hypermutations can increase target specificity and affinity during $B$ cell maturation. Hence, non-silent mutations are an indication of antigen-challenged and matured B cells. As shown in Figure $3 b$, the median number of non-silent mutations was increased in VDJ heavy chain gene transcripts of class-switched $B$ cells and was the highest for $\lg A 1^{+}$and $\lg A 2^{+} \mathrm{B}$ cells ( $\lg \mathrm{A} 1$ : 5-11 mutations, IgA2: 10-16 mutations). The number of non-silent mutations in VDJ gene transcripts of IgM expressing $B$ cells, on the other hand, ranged from 0 to more than 10 in all study groups. Of note, a small number of IgM expressing $B$ cells, mostly originating from the allergic group, consisted of highly mutated heavy chain gene transcripts with mutation numbers comparable to the other isotypes, pointing to potential maturation of $2 \mathrm{~S}$ albumin-binding $\operatorname{lgM}^{+} \mathrm{B}$ cells by antigen challenge. 


\section{VH3 family genes are predominately used in gene transcripts of al- lergic donors}

While the variable region of the heavy chain gene transcript consists of recombined $\mathrm{V}, \mathrm{D}$ and $\mathrm{J}$ genes, the light chain lacks the $\mathrm{D}$ gene component. The $\mathrm{V}$ gene accounts for most nucleotides of the variable region and contributes, besides $\mathrm{D}$ and $\mathrm{J}$ genes, to the CDR3 region. Hence, the $\mathrm{V}$ gene lineage of the heavy chain can greatly affect $B$ cell receptor specificity and affinity. In line with $B$ cell repertoires described in literature $^{30}$, VH3 family genes were observed to dominate across all groups, with a shift to higher proportions (89\%, mainly IGHV3-30, VH3-23 and VH3-72) in allergic donors $\left(X^{2}(2, \mathrm{n}=183)=23.67, \mathrm{p}<.0001\right)$. This significant difference was still present upon neglecting redundant sequences from the same donor, excluding a bias by proliferation of certain 2S albumin-binding B cells. In turn, IGHV4 genes accounted for a larger part of incorporated $\mathrm{VH}$ genes in tolerant $(21 \%)$ donors than in allergic ones (7\%) (Table 2). Overall, $\mathrm{VH}$ family gene usage differs between allergic and tolerant patients sensitized to Ara h 2/6, supporting the hypothesis of differences in allergen specific antibody repertoires between allergic and tolerant patients.

Table 2: Comparison of the VH family gene usage between the present study, healthy controls and Ara $h$ 2-related datasets (OIT trails)(transcripts from allergic, $n=120$; tolerant, $n=63$; non-atopic, $n=52$ )

\begin{tabular}{lccccccc}
\hline VH family & Ghraichy $^{\text {A31 }}$ & Goldstein $^{\text {32 }}$ & Patil $^{13}$ & Hoh $^{16}$ & Allergic & Tolerant & Non-atopic \\
\hline VH1 [\%] & $5-18$ & $15-18$ & 23 & 18 & 2 & 21 & 3.8 \\
VH2 [\%] & 2 & 4 & - & - & - & - & - \\
VH3 [\%] & $40-65$ & $46-50$ & 62 & 60 & $89^{\text {B }}$ & 54 & 63 \\
VH4 [\%] & $25-35$ & $22-25$ & 15 & 15 & 6.3 & 21 & 29 \\
VH5 [\%] & $1-2$ & $5-7$ & - & - & 1.3 & 2.9 & 3.8 \\
VH7 [\%] & $0.5-1$ & $0.05-1$ & - & - & 1.3 & - & -
\end{tabular}

A Age group between 25-40 years used for comparison, most closely related to the average age of the study population; data for different subsets were combined

B dominant usage of $\mathrm{VH} 3$ family genes in all individuals 


\section{$\lg \mathrm{E}^{+} \mathrm{B}$ cell is largely clonally related to an $\lg \mathrm{G} 1+\mathrm{B}$ cell in allergic patients}

In total, three VDJ gene transcripts were successfully amplified from $\lg \mathrm{E}^{+} \mathrm{B}$ cells, representing the smallest isotype group when disregarding IgG3 and IgG4. Where two VDJ gene transcripts of $\lg ^{+} \mathrm{B}$ cells derived from one tolerant donor were not closely related to another amplified VDJ gene transcript derived from the same donor, the VDJ gene transcript of one $\operatorname{lgE}^{+} \mathrm{B}$ cell originating from an allergic donor (Pt 10) was nearly identical to the gene transcript of an $\operatorname{lgG} 1^{+} \mathrm{B}$ cell derived from an unrelated allergic donor (Pt 04). These two sequences differed only in one silent mutation within the FR4 region and one non-silent mutation within the FR2 region, pointing towards clonal relationship between $\lg \mathrm{E}^{+}$and $\operatorname{lgG} 1^{+} \mathrm{B}$ cells and conserved clones between unrelated donors (Figure 4a). This finding supports the hypothesis that the $\lg E$ memory is stored in $\lg G 1^{+} \mathrm{B}$ cells and to lesser extent in B cells of another isotype ${ }^{24,25}$.

A

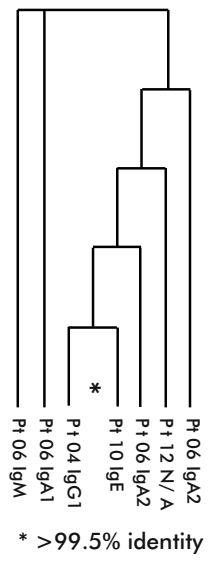

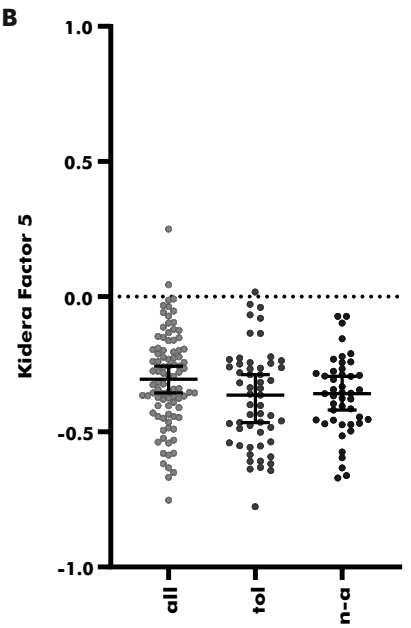

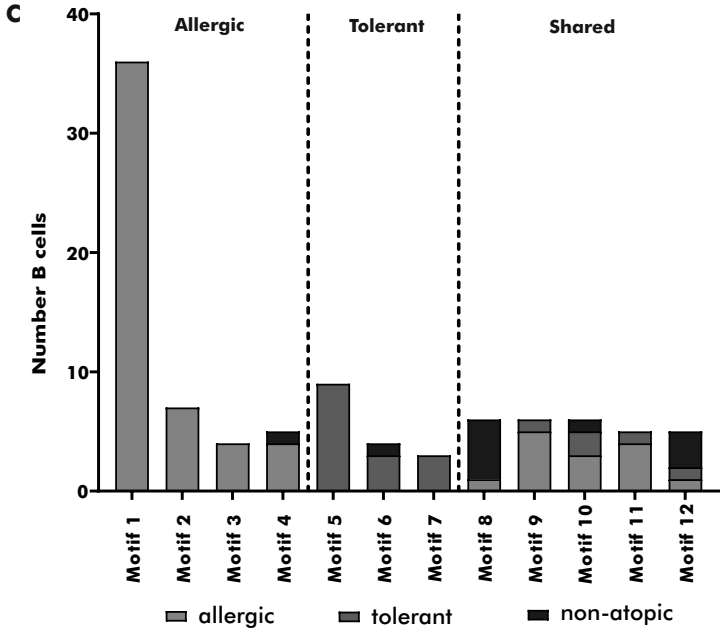

Figure 4: IgE-related clonotypes and motif analysis of HCDR3 regions

A Clonotypes were defined as identical $V$ gene, $J$ gene and HCDR3 length and related clones were aligned using ClustalW2. The IgE mAb of Pt 10 was highly related to an IgG1 antibody of Pt 4 . B Tendency of non-redundant HCDR3 regions (allergic $n=88$; tolerant $n=55$; non-atopic, $n=48$ ) to form double-bended structures on amino acid level expressed as Kidera factor 5 in allergic (all), tolerant (tol) and non-atopic ( $n-a)$ donors (median and 95\% Cl). Correction for multiple measurements (Bonferroni) resulted in no statistically significant difference. More physicochemical properties of the HCDR3 region are shown in the Suppl. Figure S5 and S6. $C$ HCDR3 sequence motifs (allergic $n=151 ;$ tolerant $n=$ 68; non-atopic, $n=51$ ) were analyzed by Levenshtein distances and hierarchical clustering (detailed description in the method section) and sequences with distances $\geq 5$ were grouped into one motif. More detailed description of the motifs is presented in Table 3 


\section{HCDR3 sequence motifs are related to allergy or tolerance}

As HCDR3 regions have a great impact on antigen binding, their characteristics were more deeply analyzed. Detailed analysis of physiochemical properties, such as amino acid distribution, aliphatic index, Boman index and the 10 Kidera factors, are shown in Suppl. Figure $\mathbf{S 5}$ and $S 6^{22}$. The Kidera factor 5, expressing the preference to form double-bended structures, was significantly higher for the HCDR3 region of allergic donors compared with that of tolerant donors $(p=0.0226$, non-significant upon Bonferroni correction, Figure 4b). Nevertheless, the mean Boman index, describing the theoretical ability to bind proteins, was comparable between HCDR3 regions of allergic, tolerant, and non-atopic donors. For deeper analysis, HCDR3 sequence motifs associated with peanut allergy and/or tolerance, were evaluated by Levenshtein distances ( $\geq 5$ differences defined as replacement, deletion or insertion) and hierarchical clustering. Levenshtein distances were favored over clonal relationship analysis because of individual genetic variation regarding haplotype diversity, single nucleotide polymorphisms (SNPs), gene copy number and preferred gene lineage ${ }^{33}$. For this analysis, all successfully amplified and sequenced heavy chain gene transcripts were used irrespectively of successfully sequenced corresponding light chain gene transcripts. Overall, four unique HCDR3 sequence motifs were associated with peanut allergy and three with peanut tolerance (Figure 4c, Table 3). The most dominant motif was 'CARDSSALEIYNRFDPW' (motif 1), which was derived from 36 different $B$ cells belonging to three different allergic donors (Pt $1(n=32)$, Pt $5(n=3)$, Pt $6(n=1))$ including 32 nearly identical $B$ cells of Pt 1 (monoclonal proliferation). This motif was formed by VH3-30, DH3-3 and $\mathrm{JH} 5$ genes. Besides motifs exclusively related to either allergy or tolerance, five motifs were shared between allergic, tolerant, and non-atopic donors. It must be mentioned that one motif (CARNVFDGYWLVYW) associated with tolerance was only found in Pt 11 and no motif was shared between all allergic or tolerant donors. Heterologously expressed mAbs corresponding to these motifs showed specific binding to Ara $\mathrm{h} 2$ or 6 , supporting the relevance of the identified motifs and their potential role in diagnosing patients with suspected peanut allergy. 
오

$\frac{\sqrt{2}}{\mathrm{D}}$

ก

气ุ

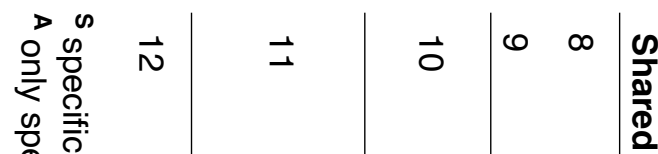

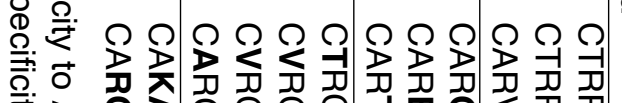

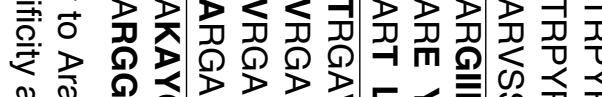

ڤొ

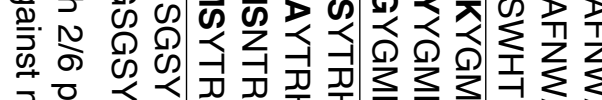

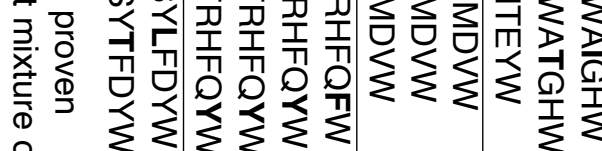

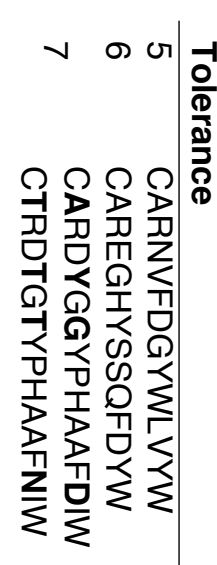

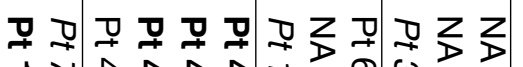

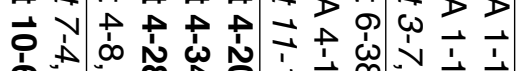

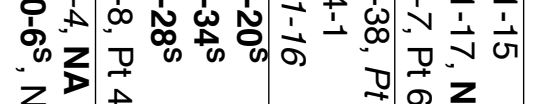

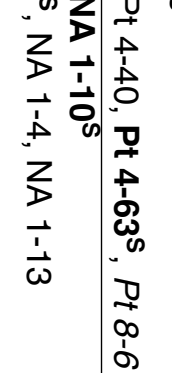

무모어구 $\dot{\omega} \approx \dot{\omega}=$

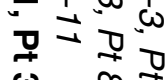

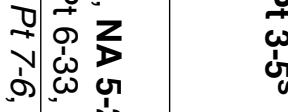
꾸 꿍

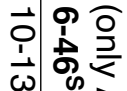

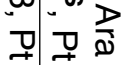

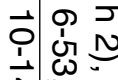
$\frac{\vec{A}}{\mathrm{~A}}$

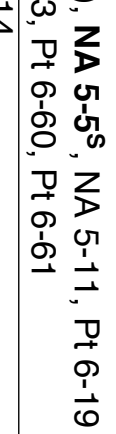

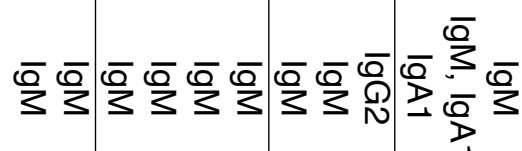

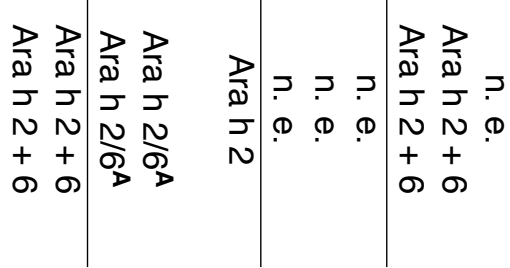

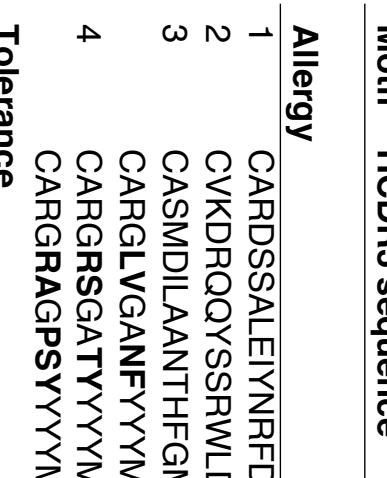

굴 ฉ 工) ग्गे के 내에 要 จุ ఠ 공

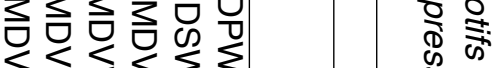
$\sum \sum \sum \sum \sum$ 무놈ㄲㅁㅁㅁㅁ

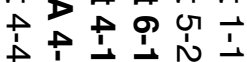
जु유

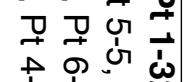
突 꾸 象 N 성무 무 0
7
1 우의 is o जि. 꼬 구 \begin{tabular}{ll}
0 \\
1 \\
1 \\
1 & 1 \\
\hline
\end{tabular} प्र $\stackrel{1}{\infty}$ ญ ป ถ क Q ? ปั 2. के กิ ํำ क्ष ริ ญे

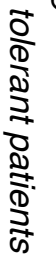




\section{Discussion}

Specific IgE levels to Ara $\mathrm{h} 2$ and 6 between 0.1 and $1.8 \mathrm{kU} / \mathrm{l}$ overlap between peanut allergic and tolerant subjects ${ }^{6,7}$, risking inaccurate diagnosis, and therefore indicating the need for new diagnostic strategies. In the present study, we observed a preferential usage of $\mathrm{VH} 3$ family genes in peanut $2 \mathrm{~S}$ albumin-specific $\mathrm{B}$ cells from peanut allergic patients. Additionally, we identified $2 S$ albumin binding B cells carrying HCDR3 sequence motifs either related to peanut allergy or tolerance. This finding may lead towards new diagnostic strategies able to discriminate between allergy and tolerance in sensitized patients with suspected peanut allergy.

Despite a large number of amplified heavy chain VDJ gene transcripts ( $n=280)$, only three of them belonged to IgE class-switched B cells. This low abundance may be potentially explained by dominant class-switching to $\lg E$ in the tissue ${ }^{23}$, low BCR surface expression of plasmablast-like $\lg ^{+} \mathrm{B}$ cells ${ }^{34-36}$ and the extreme low abundance of $\lg ^{+}$ $B$ cells within the circulation ${ }^{27}$. Nevertheless, the relevance of the $B$ cells sorted within the present study is supported by the close relationship between one heavy chain gene transcript from an $\operatorname{lgE}^{+} \mathrm{B}$ cell and a gene transcript from an $\operatorname{lgG} 1^{+} \mathrm{B}$ cell derived from an unrelated peanut allergic donor. Additionally, some generated mAbs were able to slightly inhibit patient-derived serum IgE-binding to Ara h 2 and 6 (Suppl. Figure S3). Correspondingly, there is evidence that human $\operatorname{lgE}^{+} \mathrm{B}$ cells are predominately plasmablasts or plasma cells generated by sequential class-switching from lgG1+ $\mathrm{B}$ cells ( $Y$ switch region remnants) and that the humoral $\lg E$ memory is contained in $\operatorname{lgG} 1^{+}$ memory $B$ cells ${ }^{25}$. Further evidence for this theory was obtained by clonal relationship analyses, since $\lg \mathrm{E}^{+} \mathrm{B}$ cells were dominantly clonally related to $\lg \mathrm{G} 1^{+} \mathrm{B}$ cells, but also, to a lesser extent, to $B$ cells of other isotypes ${ }^{23,24,34}$. In contrast, IgE memory in mice has been shown to be at least partly embedded in $\lg ^{+}$memory $B$ cells ${ }^{37}$.

By contrast to the low number of $\operatorname{lgE}^{+} \mathrm{B}$ cells, $\operatorname{lgM}^{+} \mathrm{B}$ cells represented the largest isotype group, as detected in respectively 47,53 and $94 \%$ of allergic, tolerant and nonatopic donors. Additionally, $\operatorname{lgM}^{+} \mathrm{B}$ cells from non-atopic donors, all specific for Ara $\mathrm{h} 2$ and/or 6 , shared HCDR3 motifs (motif $4,8,10$ ) with $\operatorname{lgM}^{+}, \operatorname{lgG} 2^{+}$and $\lg A 1^{+} \mathrm{B}$ cells from allergic donors. Clonal relationships between $\lg A / G / M^{+}$and $\lg E^{+} B$ cells have been described for $B$ cells derived from gut tissues by Hoh and colleagues ${ }^{23,38}$. Combining the findings from the present study with the finding of Hoh and colleagues leads to the suggestion that non-atopic donors can potentially possess non-lgE antibodies with required specificity or affinity to theoretically induce an allergic reaction.

Moreover, tolerant donors showed a higher proportion of $\lg \mathrm{A}^{+} \mathrm{B}$ cells and tended to have higher specific IgA serum levels than allergic donors (Suppl. Figure S3). Allergenspecific $\lg A$ serum levels have been shown to be increased in peanut allergic subjects undergoing oral or sublingual immunotherapy compared with their baseline levels. 
Moreover, salivary IgA levels have been closely associated with the degree of tolerance induction confirmed by DBPCFCs, pointing to a protective role of specific IgA against mucosal allergen absorption ${ }^{26,39}$. Such a protective role is supported by increased intestinal permeability in the absence of $\lg A$ in mice ${ }^{40}$. Taken together, these findings suggest a potential protective effect of allergen-specific IgA in the mucosa of sensitized but tolerant patients.

Regarding gene lineage, VH3 family genes were significantly more often used $(p<.0001)$ in heavy chain gene transcripts of peanut $2 S$ albumin-specific $B$ cells from allergic donors $(89 \%)$ than in those from tolerant $(54 \%)$ and non-atopic donors $(63 \%)$. Previous studies on $\mathrm{VH}$ gene usage of heavy chain gene transcripts in healthy donors showed $\mathrm{VH} 3$ family gene usage in 40 to $65 \%$ of the B cells, which was comparable to the usage in the tolerant and non-atopic groups in our study (Table 2) ${ }^{31,32}$. These findings suggest a shift towards $\mathrm{VH} 3$ family gene usage in $2 \mathrm{~S}$ albumin specific $\mathrm{B}$ cells from allergic donors. Contrary to our findings, other datasets of Ara $\mathrm{h} 2$ specific B cells did not observe a similar shift in VH family gene usage ${ }^{13,16}$. These datasets, however, were generated from patients undergoing peanut oral immunotherapy with a focus on different time points during immunotherapy. This fact hampers the comparison between the present and previous Ara h 2-related datasets. Moreover, conflicting results regarding preferred $\mathrm{VH}$ gene usage were described for different food and respiratory allergies, with a shift to VH3 usage for a-alpha Gal antibodies and a-grass pollen Phl p 6 and 11 antibodies ${ }^{41,42}$, indicating that the preferred $\mathrm{VH}$ family gene usage may be allergen dependent. Moreover, the preferred usage might depend on the status of allergy or tolerance and can potentially be used for diagnostic purposes upon validation in a larger patient cohort. A validation study will also provide information about the number of detected $2 S$ albumin-binding $B$ cells is sufficient for a diagnostic workflow.

Besides differences in VH family gene usage, certain HCDR3 sequence motifs were associated with either peanut allergy or tolerance and appear to have the ability to discriminate between those two groups. The main HCDR3 sequence motif 'CARDSSALEIYNRFDPW' was associated with peanut allergy and derived from recombined VH3-30, DH3-3 and JH5 genes. Comparably, VH3-30*18 was incorporated in the VDJ gene transcript of clonally related IgE $+B$ cells specific for Ara $\mathrm{h} 2$ in the study of Croote and colleagues ${ }^{34}$. Additionally, a highly similar HCDR3 region (CAREGYESSGFDYW) to motif 6 (CAREGHYSSQFDYW), associated with tolerance, has been described for peanut allergic subjects undergoing oral immunotherapy ${ }^{16}$. Oral immunotherapy may shape the antibody repertoire towards repertoires present in tolerant subjects. These comparisons support the potential of HCDR3 motifs in diagnosing peanut allergy and tolerance.

In conclusion, the dominant usage of $\mathrm{VH} 3$ family genes and the identification of HCDR3 sequence motifs related to either peanut allergy or tolerance may potentially lead to the 
development of new diagnostic strategies for subjects with suspected peanut allergy and sensitization to Ara h 2 and/or 6 . Validation of these HCDR3 sequence motifs in a larger patient cohort may be achieved using next-generated sequencing approaches, potentially combined with the sorting strategy of $2 S$ albumin-binding B cells presented in this study. Next-generation sequencing allows high-throughput and can be more easily implemented in diagnostic routine ${ }^{43,44}$.

\section{Acknowledgments}

We would like to thank all participants for their efforts and dedication in this study. Additionally, we would like to thank Anouska Michelsen-Huisman, Anne van Dijk, Ans Lebens and Mehran Alizadeh Aghdam for their support in the patient inclusion and Michael Drefahl, Rowena Melchers and Miriam Katsburg for their technical support. For critical discussion and reading of the manuscript, we would like to thank Melanie Abram, Edward Knol and Femke van Wijk. For financial support, we want to acknowledge EUROIMMUN AG, Lübeck, Germany. 


\section{References}

1. Lyons, S. A. et al. Food Allergy in Adults: Substantial Variation in Prevalence and Causative Foods Across Europe. J Allergy Clin Immunol Pract 7, 19208.e11 (2019).

2. Muraro, A. et al. EAACI Food Allergy and Anaphylaxis Guidelines: diagnosis and management of food allergy. Allergy 69, 1008-25 (2014).

3. Yanagida, N. et al. Risk Factors for Severe Reactions during Double-Blind Placebo-Controlled Food Challenges. Int Arch Allergy Immunol 172, 173-82 (2017).

4. Portnoy, J. M. Appropriate Allergy Testing and Interpretation. Mol Med 108, 33943 (2011).

5. Yu, W., Freeland, D. M. H. \& Nadeau, K. C. Food allergy: immune mechanisms, diagnosis and immunotherapy. Nat Rev Immunol 16, 751-65 (2016).

6. Klemans, R. J. B. et al. Ara h 2 Is the Best Predictor for Peanut Allergy in Adults. J Allergy Clin Immunol Pract 1, 632-8.e1 (2013).

7. Kansen, H. M. et al. Accurate prediction of peanut allergy in one third of adults using a validated Ara $h 2$ cut-off in EAACI (London, 2020).

8. Ehlers, A. M. et al. Can alternative epitope mapping approaches increase the impact of B-cell epitopes in food allergy diagnostics? Clin Exp Allergy 49, 17-26 (2018).

9. Bucaite, G. et al. Interplay between Affinity and Valency in Effector Cell Degranulation: A Model System with Polcalcin Allergens and Human Patient-Derived IgE Antibodies. J Immunol 203, 1693-700 (2019).

10. Xu, J. L. \& Davis, M. M. Diversity in the CDR3 Region of VH Is Sufficient for Most Antibody Specificities. Immunity 13, 37-45 (2000).

11. Martinet, J. et al. Diagnostic Value of Antigen-Specific Immunoglobulin E Immunoassays against Ara h 2 and Ara h 8 Peanut Components in Child Food Allergy. Int Arch Allergy Immunol 169, 216-22 (2016).

12. Scharf, M. et al. A Spectrum of Neural Autoantigens, Newly Identified by HistoImmunoprecipitation, Mass Spectrometry, and Recombinant Cell-Based Indirect Immunofluorescence. Front Immunol 9, 1447 (2018).

13. Patil, S. U. et al. Peanut oral immunotherapy transiently expands circulating Ara $\mathrm{h} 2$-specific $\mathrm{B}$ cells with a homologous repertoire in unrelated subjects. J Allergy Clin Immunol 136, 125-34.e12 (2015). 
14. Franz, B., May, K. F., Dranoff, G. \& Wucherpfennig, K. Ex vivo characterization and isolation of rare memory $B$ cells with antigen tetramers. Blood 118, 348-57 (2011).

15. Yang, J. \& Reth, M. Oligomeric organization of the B-cell antigen receptor on resting cells. Nature 467, 465-9 (2010).

16. Hoh, R. A. et al. Single B-cell deconvolution of peanut-specific antibody responses in allergic patients. J Allergy Clin Immunol 137, 157-67 (2016).

17. Tiller, $\mathrm{T}$. et al. Efficient generation of monoclonal antibodies from single human $\mathrm{B}$ cells by single cell RT-PCR and expression vector cloning. J Immunol Methods 329, 112-24 (2008).

18. Ye, J., Ma, N., Madden, T. L. \& Ostell, J. M. IgBLAST: an immunoglobulin variable domain sequence analysis tool. Nucl Acids Res 41, W34-40 (2013).

19. Koppelman, S. J. et al. Purification and immunoglobulin E-binding properties of peanut allergen Ara h 6: evidence for cross-reactivity with Ara h 2. Clin Exp Allergy 35, 490-7 (2005).

20. Santos, A. F. et al. IgG4 inhibits peanut-induced basophil and mast cell activation in peanut-tolerant children sensitized to peanut major allergens. J Allergy Clin Immunol 135, 1249-56 (2015).

21. Larkin, M. A. et al. Clustal W and Clustal X version 2.0. Bioinformatics 23, 29478 (2007).

22. Margreitter, C. et al. BRepertoire: a user-friendly web server for analysing antibody repertoire data. Nucl Acids Res 46, W264-70 (2018).

23. Hoh, R. A. et al. Origins and clonal convergence of gastrointestinal lgE+ B cells in human peanut allergy. Sci Immunol 5, eaay4209 (2020).

24. Looney, T. J. et al. Human B-cell isotype switching origins of IgE. J Allergy Clinical Immunol 137, 579-86.e7 (2016).

25. Saunders, S. P., Ma, E. G. M., Aranda, C. J. \& de Lafaille, M. A. C. Non-classical B Cell Memory of Allergic IgE Responses. Front Immunol 10, 715 (2019).

26. LaHood, N. et al. Ara h 2 Specific IgA B Cell Repertoire Matures During Peanut Oral Immunotherapy. J Allergy Clin Immunol 145, AB181 (2020).

27. Jiménez-Saiz, R. et al. Human BCR analysis of single-sorted, putative lgE+ memory B cells in food allergy. J Allergy Clin Immunol 144, 336-9.e6 (2019).

28. Karagiannis, P. et al. Elevated IgG4 in patient circulation is associated with the risk of disease progression in melanoma. Oncolmmunology 4, e1032492 (2015). 
29. Marsh-Wakefield, F. et al. IgG3+ B cells are associated with the development of multiple sclerosis. Clin Translat Immunol 9, e01133 (2020).

30. Brezinschek, H. P. et al. Analysis of the human VH gene repertoire. Differential effects of selection and somatic hypermutation on human peripheral CD5(+)/lgM+ and CD5(-)/lgM+ B cells. J Clin Invest 99, 2488-501 (1997).

31. Ghraichy, M. et al. Maturation of the Human B-Cell Receptor Repertoire with Age. SSRN Electronic Journal doi: 10.2139/ssrn.3518535, pre-print (2020).

32. Goldstein, L. D. et al. Massively parallel single-cell B-cell receptor sequencing enables rapid discovery of diverse antigen-reactive antibodies. Commun Biol 2 , 304 (2019).

33. Watson, C. T., Glanville, J. \& Marasco, W. A. The Individual and Population Genetics of Antibody Immunity. Trends Immunol 38, 459-70 (2017).

34. Croote, D., Darmanis, S., Nadeau, K. C. \& Quake, S. R. High-affinity allergenspecific human antibodies cloned from single lgE B cell transcriptomes. Science 362, 1306-9 (2018).

35. Karnowski, A. et al. Inefficient processing of mRNA for the membraneform of IgE is a genetic mechanism to limit recruitment of IgE-secreting cells. Eur J Immunol 36, 1917-25 (2006).

36. Yang, Z., Sullivan, B. M. \& Allen, C. D. C. Fluorescent In Vivo Detection Reveals that $\lg E+B$ Cells Are Restrained by an Intrinsic Cell Fate Predisposition. Immunity 36, 857-72 (2012).

37. Talay, O. et al. IgE+ memory $B$ cells and plasma cells generated through a germinal-center pathway. Nat Immunol 13, 396-404 (2012).

38. Iweala, O. I. \& Burks, A. W. IgE producers in the gut expand the gut's role in food allergy. Nat Rev Gastroenterol Hepatol 17, 384-6 (2020).

39. Kulis, M. et al. Increased peanut-specific $\lg A$ levels in saliva correlate with food challenge outcomes after peanut sublingual immunotherapy. J Allergy Clin Immunol 129, 1159-62 (2012).

40. Strait, R. T. et al. Ingested allergens must be absorbed systemically to induce systemic anaphylaxis. J Allergy Clin Immunol 127, 982-9.e1 (2012).

41. Wang, L., Radic, M. Z. \& Galili, U. Human anti-Gal Heavy Chain Genes. Prefential Use of VH3 and the Presence of Somatic Mutations. J Immunol 155, 127685 (1995).

42. Persson, H., Sadegh, M. K., Greiff, L. \& Ohlin, M. Delineating the specificity of an IgE-encoding transcriptome. J Allergy Clin Immunol 120, 1186-92 (2007). 
43. Hung, S. S. et al. Assessment of Capture and Amplicon-Based Approaches for the Development of a Targeted Next-Generation Sequencing Pipeline to Personalize Lymphoma Management. J Mol Diagn 20, 203-14 (2018).

44. Cummings, C. A. et al. The Role of Next-Generation Sequencing in Enabling Personalized Oncology Therapy. Clin Trans/ Sci 9, 283-92 (2016). 


\section{Supplementary}

All DNA sequences are available at the NCBI genbank under the accession numbers MW271045 - MW271525.

Table S1: Mass spectrometry analysis of the 2S albumin fraction from roasted peanut italic: $M S / M S$ analysis

\begin{tabular}{|c|c|c|c|c|c|}
\hline No. & $\begin{array}{c}\text { Protein } \\
\text { (Accession Number) }\end{array}$ & Cut-Off & Protein Score & $\begin{array}{c}\text { Sequence } \\
\text { Coverage [\%] }\end{array}$ & $\begin{array}{c}\text { Molecular } \\
\text { Mass [kDa] }\end{array}$ \\
\hline 1 & $\begin{array}{c}\text { Ara h } 2.01 \text { allergen } \\
\text { (ACN62248.1) }\end{array}$ & $80 / 51$ & $49 / 39$ & $32 / 16$ & 19.0 \\
\hline 2 & $\begin{array}{c}\text { Ara h } 2.01 \text { allergen } \\
\text { (ACN62248.1) }\end{array}$ & $80 / 51$ & $93 / 56$ & $51 / 16$ & 19.0 \\
\hline \multirow[t]{2}{*}{3} & $\begin{array}{cc}\text { Ara h } 6 & \\
(\text { Q647G9.1) } & 80 / 48\end{array}$ & $103 / 70$ & $71 / 17$ & 17.5 & \\
\hline & $\begin{array}{c}\text { Ara h } 7.0301 \\
\text { (AAU21496.1) }\end{array}$ & 80 & 64 & 37 & 18.8 \\
\hline 4 & $\begin{array}{c}\text { Ara h } 6 \\
\text { (Q647G9.1) }\end{array}$ & $80 / 48$ & $54 / 43$ & $39 / 8$ & 17.5 \\
\hline 5 & $\begin{array}{c}\text { Ara h } 6 \\
\text { (Q647G9.1) }\end{array}$ & 49 & 14 & 8 & 17.5 \\
\hline
\end{tabular}

A

Ara h 2

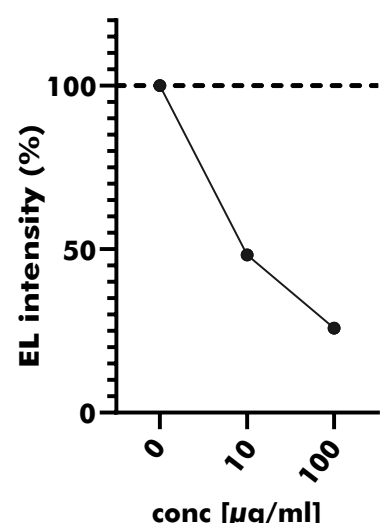

B

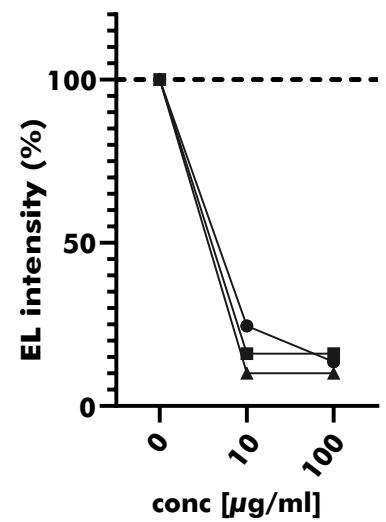

C

Ara h 7

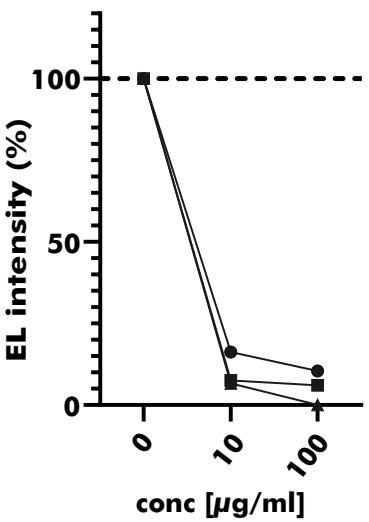

Figure S1: Inhibition of the peanut $2 S$ albumin fraction with Ara h 2, 6 and 7 Inhibition of serum binding to $\boldsymbol{A}$ Ara $h$ 2, B Ara $h 6$ and $\boldsymbol{C}$ Ara $h 7$ in concentration-dependent manner $(0-100 \mu \mathrm{g} / \mathrm{ml}$ peanut $2 S$ albumin fraction) with the isolated peanut $2 S$ albumin fraction confirming the present of these allergens in the $2 S$ albumin fraction. Dots: serum with increased slgE titers for Ara $h$ 2, 6 and 7, Squares: serum with increased slgE titers to Ara $h 6$ and 7, Triangle: serum with increased slgE titers to Ara $h 6$ and 7 

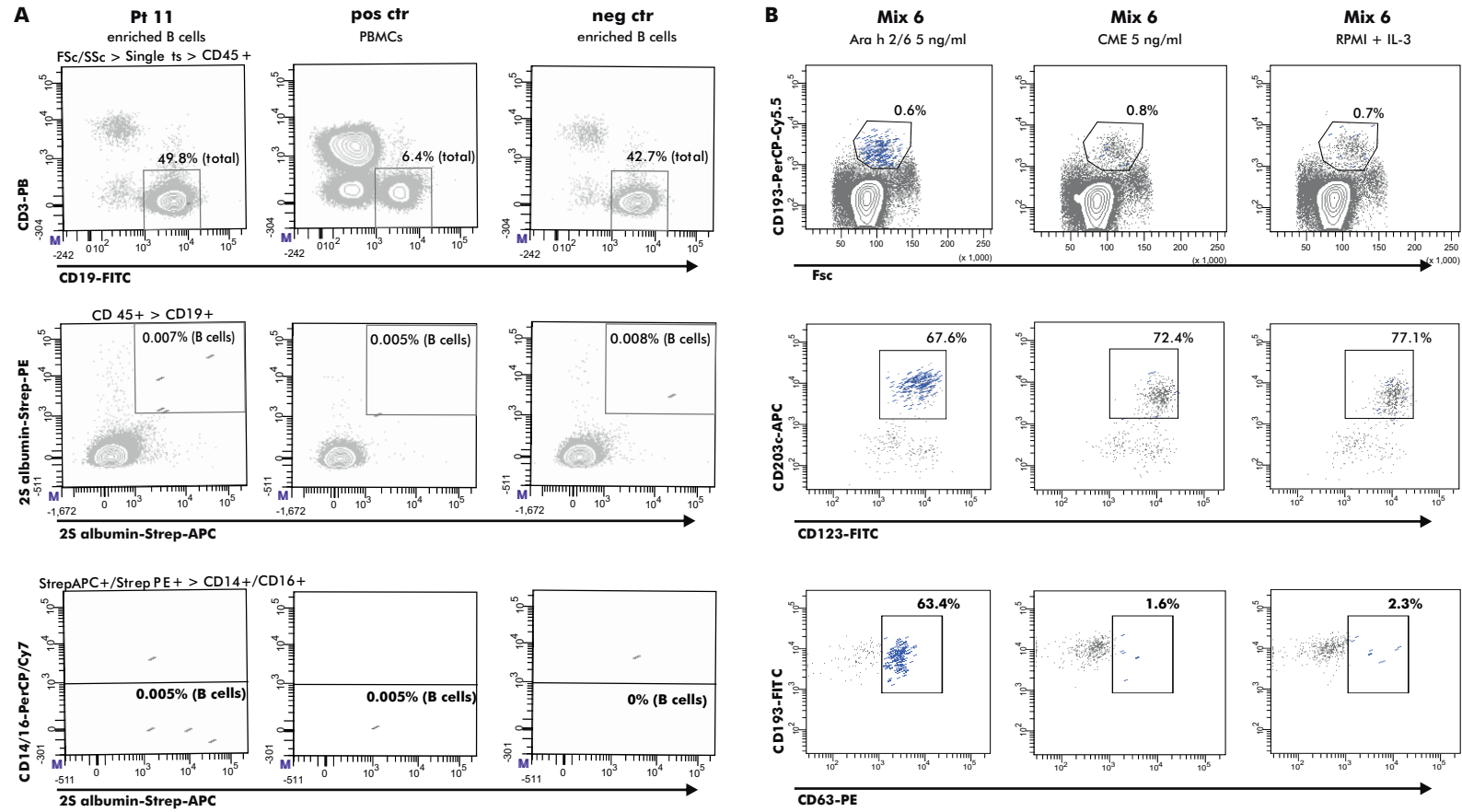

Figure S2: FACS gating strategies for the selection of 2 S albumin-specific B cells and indirect basophils activation test

$\boldsymbol{A}$ Selection of 2S albumin-binding B cells - the lowest gate panel was used for sorting; $\boldsymbol{B}$ Indirect basophil activation test; $C M E=$ cow's milk extract (control)

A

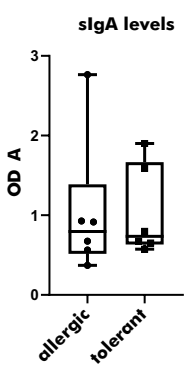

B
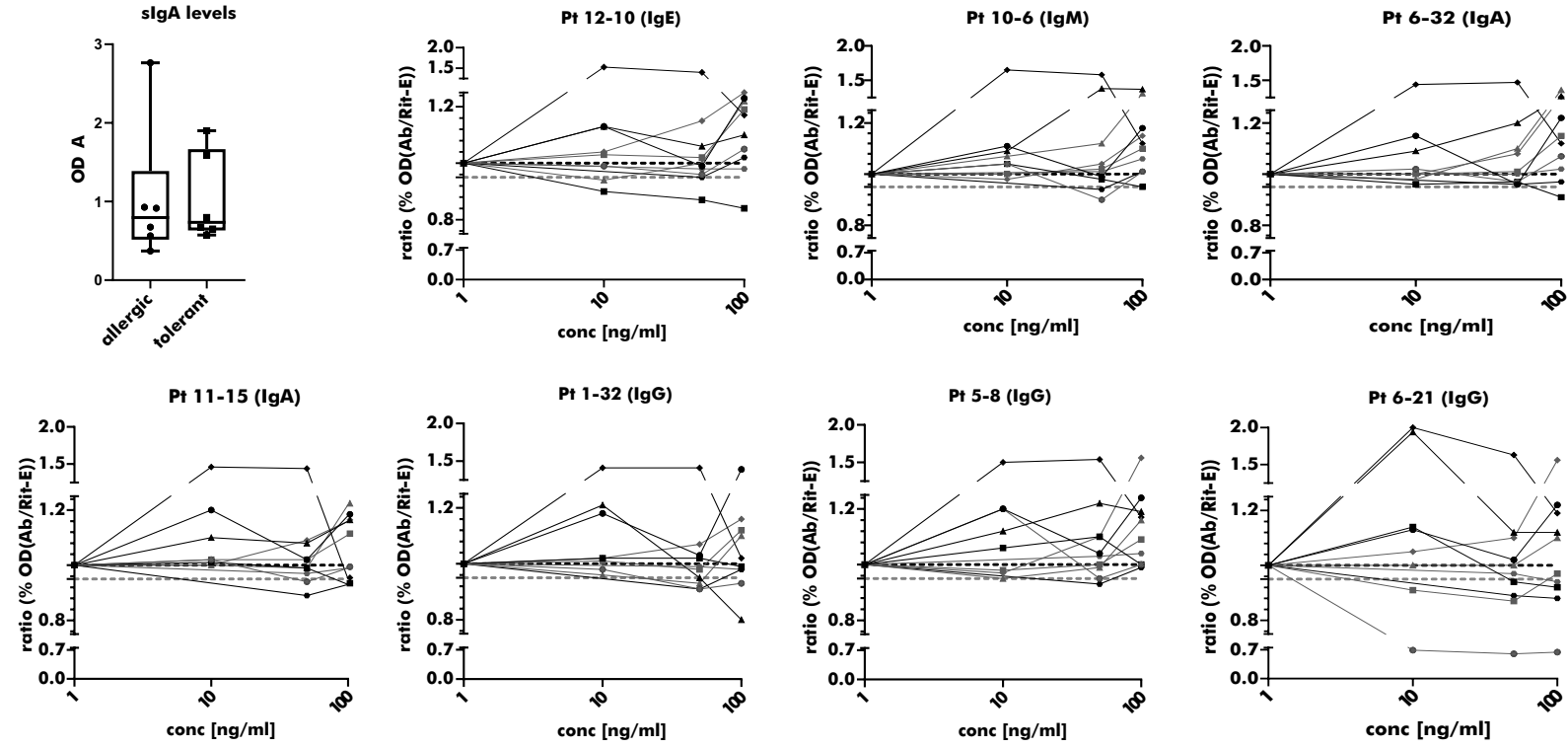

Figure S3: Binding of specific $\lg A$ to peanut $2 S$ albumins and inhibition of slgE binding to $2 S$ albumins by $2 S$ albumin-specific mAbs

A Specific IgA levels against Ara $h 2$ and 6 were measured in patients' serum samples, each dot is the mean of duplicate measurements, box plots shows the interquartile range of measured slgA levels; $\boldsymbol{B}$ Inhibition of slgE binding from patients' serum samples by pre-incubation with 7 distinct $2 S$ albuminspecific mAbs expressed as lgG1 in a dose-dependent manner (1 to $100 \mathrm{ng} / \mathrm{ml}$, mean of duplicates, the original isotype is displayed in brackets); inhibition is the remaining OD signal of the serum without preincubation and is displayed as the ratio of the inhibition by the respective mAb and Rituximab (negative control) expressed as IgG1; the critical range of 0 to $5 \%$ inhibition is highlighted by two dash lines 
Mix 1

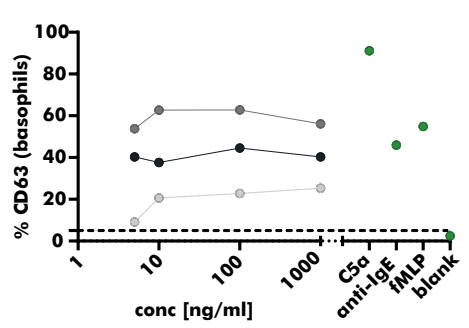

$\operatorname{Mix} 4$

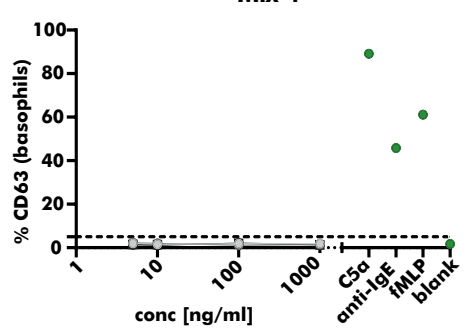

$\operatorname{Mix} 7$

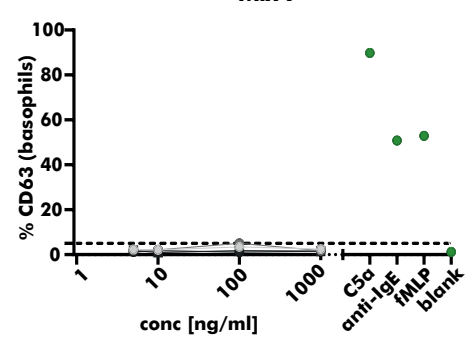

Mix 2

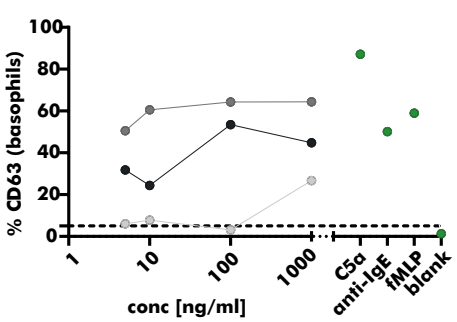

Mix 5

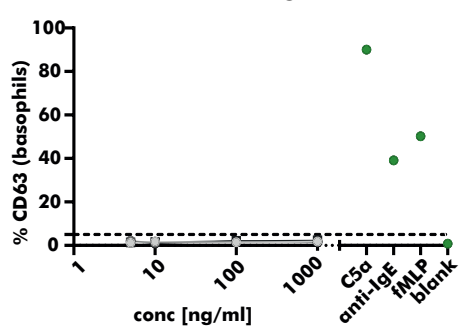

Mix 8

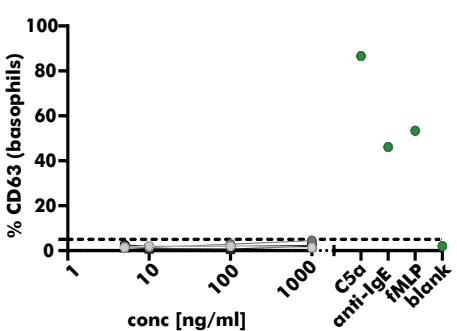

conc $[\mathrm{ng} / \mathrm{ml}]$

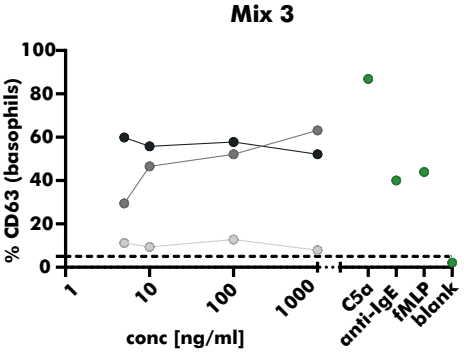

Mix 6

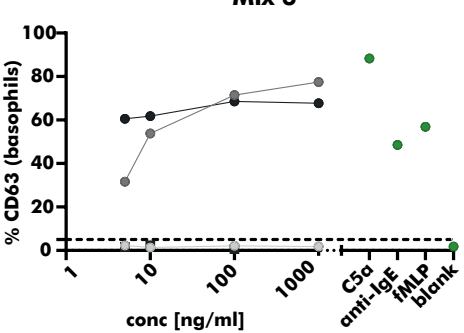

Mix 9

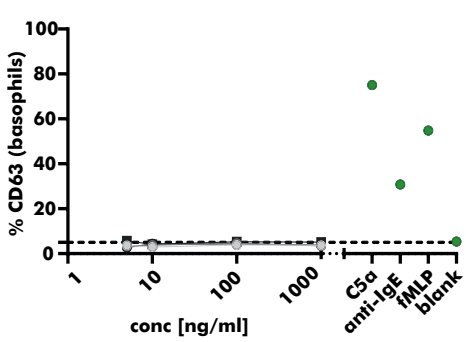

- Ara h 2

- Ara h 6

- Ara h 2/6

Figure S4: Indirect basophil activation tests with $2 S$ albumin-specific mAbs including all controls Upregulation of CD63 upon loading of human basophils with mAb mixture and subsequent stimulation with Ara $h 2$ and 6 in a concentration-dependent manner ( 5 to $1000 \mathrm{ng} / \mathrm{ml}$ ); the dots indicate the mean of duplicate measurements; Mix $1=$ Pt 6-2, Mix $2=$ Pt 6-2 + Pt 6-17, Mix $3=$ Pt 6-2 + Pt 3-2 + Pt 6-11, Mix 4 = Pt 6-17, Pt 3-2, Pt 6-11, Mix $5=$ Pt 1-32, Pt 6-11, Pt 6-6, Pt 6-8, Mix $6=$ NA 1-10 + Pt 4-28 + Pt 4-34 + Pt 6-2, Mix $7=$ Pt NA 1-10 + Pt 4-28 + Pt 4-34, Mix $8=$ Pt 1-32 + Pt 6-15 + Pt 6-21, stripped basophils before loading, Rituximab expressed as IgE; dash line: threshold level set to $5 \%$ 
Physical properties of the HCDR3 regions were calculated using the BRepertoire web server 1 . For this analysis, only non-redundant HCDR3 amino acid sequences (allergic $n=88$; tolerant $n=55$; non-atopic, $n=48$ ) were used.

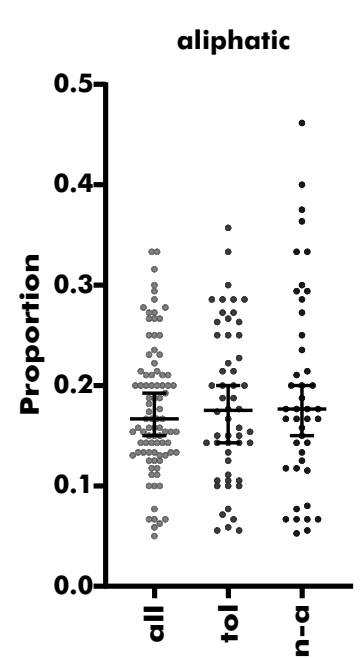

basic

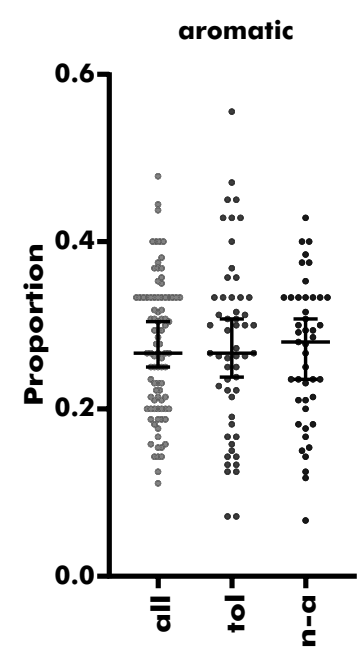

acidic

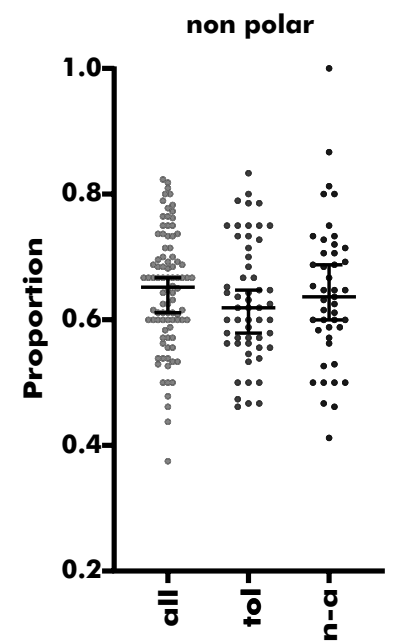

Aliphatic index

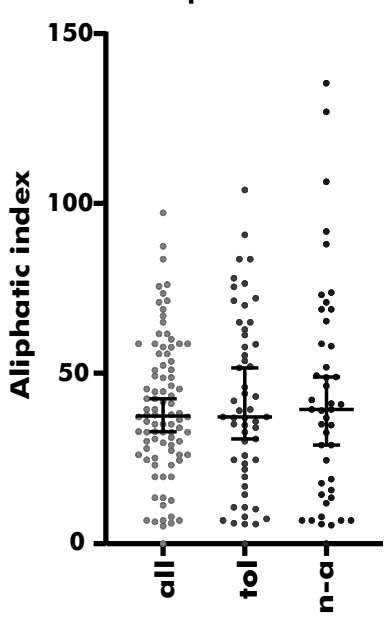

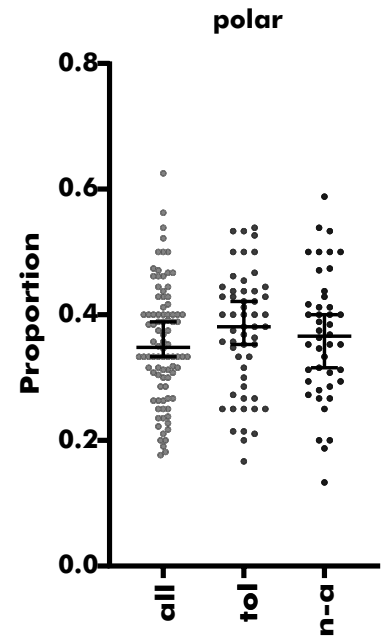

Boman index

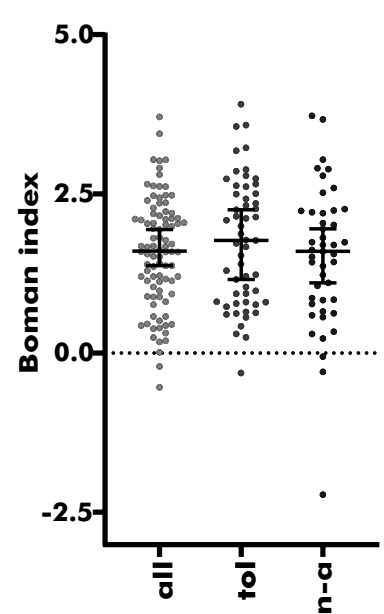

Figure S5: Proportion of amino acids within HCDR3 regions

Proportion of amino acids are categorized into different groups (aliphatic, aromatic, non-polar, polar, basic and acidic); calculation of the aliphatic index and the Boman index; Boman indices $\geq 2.48$ indicate high probability for protein-protein interactions, all sample sets show the median with the $95 \%$ confidence interval; all = allergic, tol = tolerant, $n$-a = non-atopic 

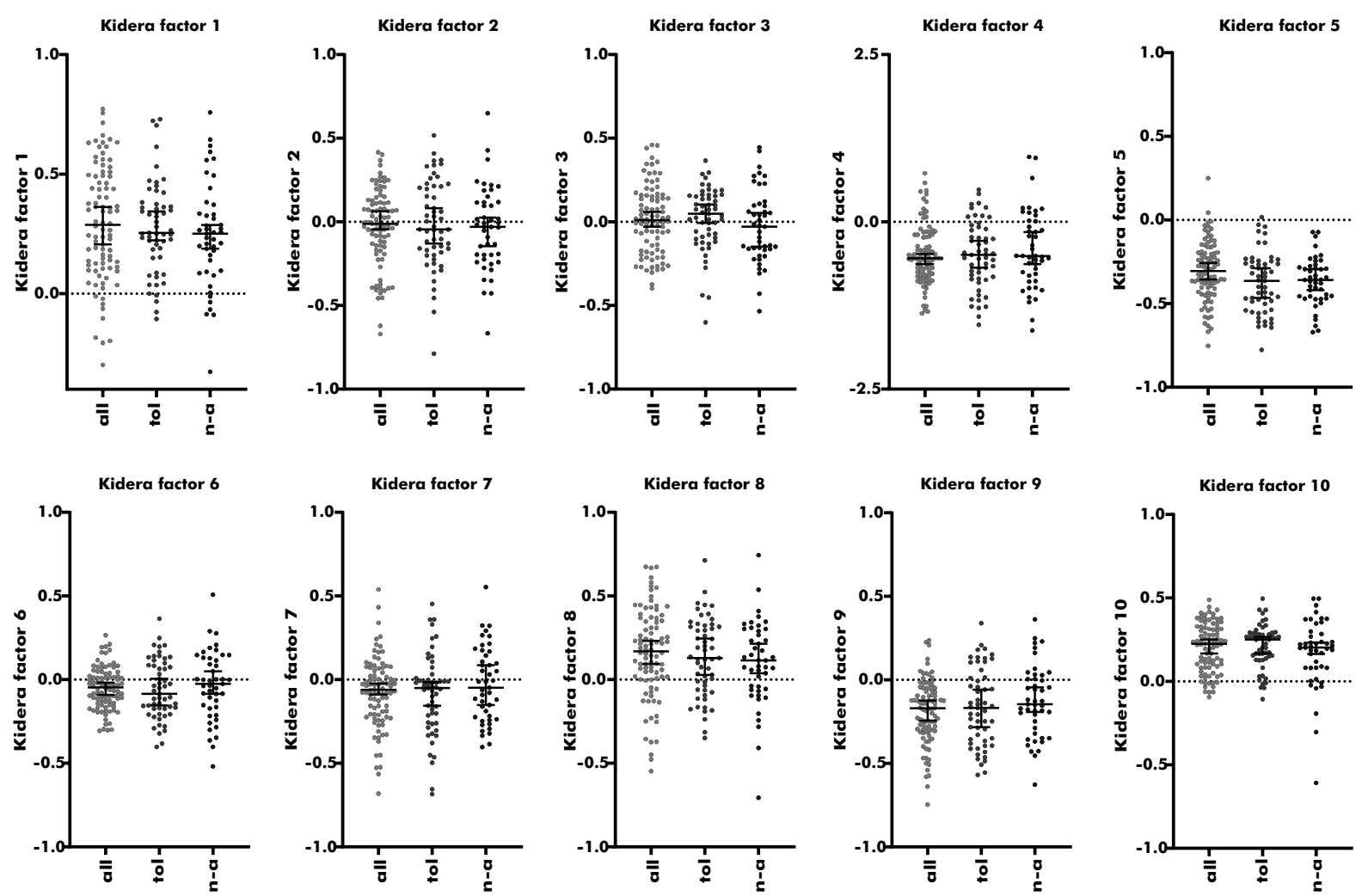

Figure S6: Properties of HCDR3 regions expressed as Kidera factors (KF) 1-10

KF1: Helix/bend preference, KF2: side chain size, KF3: extended structure preference, KF4: Hydrophobicity, KF5: double-bend preference , KF6: partial specific volume, KF7: flat extended preference, KF8: occurrence in alpha region, KF9: pK-C, KF10: surrounding hydrophobicity, all sample sets show the median with the $95 \%$ confidence interval; all = allergic, tol = tolerant, $n$-a = non-atopic 
(1)

CAGCCTCTGGAT

IGCGTGGTCCAGCCTC

AGGGCCGATTCACCATC

AATTGTGTTGACACAGTCTCCAG

UTGGCATCCCAGCCAGGTTCAGTGGCAGTGGG̈TC I

CAGATGACCCAGTCTCCTTCCACCCTGGCTGCATCTGTAG

AGTCCCATCAAGGTTCAGCGGCAGTGGGTCTGGGACAGAGTTCACL

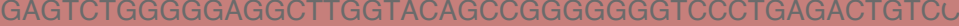

ITATCGAGACTCTGTGAAGGGCCGCTTCACCATCTCCAGAGACAATGCCAAAAACh ¿AGGGGACCACGGTCATCGTCTCCTCAGCAGGTGCAGCTGGTGGAGTCTGGGGGAGG iGAGTGGGTTTCATTTATACGCAAGGATGGAGCTAGTAAATACTATGGAGACTCCGTGAAGGG ,CCTGGGTTACTTTGACTACTGGGGCCAGGGAACCCTGGTCACCGTCTCCTCAGGAAATTGTGT

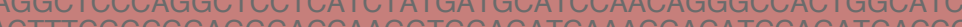
CCAGGGAAAGCCCCCAAGCTCCTCATATATAAGGCGT ERUNG

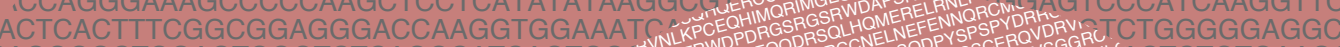

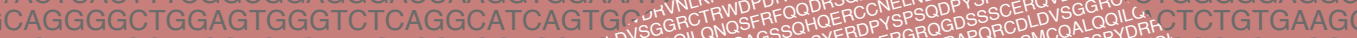

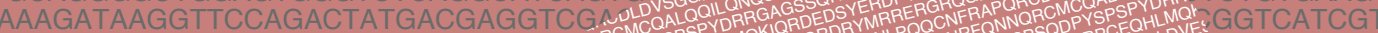

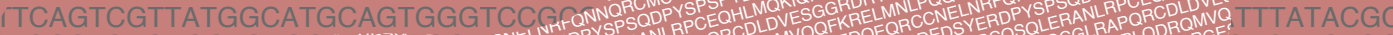

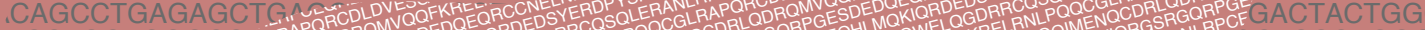
:TCCTGCAGGGCGGGLRAPROD tGCCTAGAGCCT

\section{GCCTGATGAT}

CACGTTTAGG

ATGGCCAGTC

CCATCTCCAGAG

SPSOP

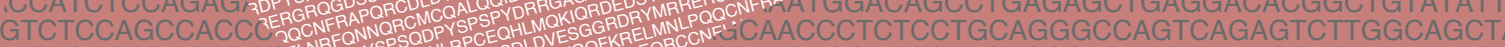

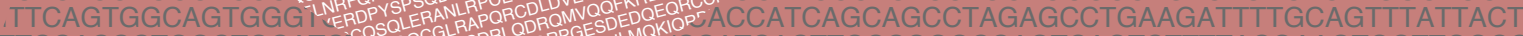

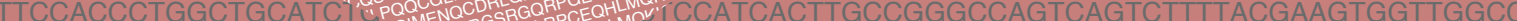

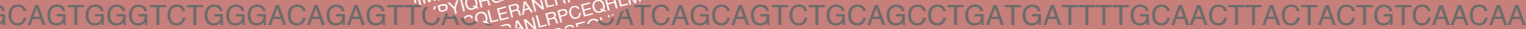
ACAGCCGGGGGGGTCCCTGAGACTGTCCTATGCAGCCTCTGGATTCACGTTTAGGAGATATGCTTTGAGTTGGGTCCGC ICTTCACCATCTCCAGAGACAATGCCAAAAACATCCTGTTTCTGCAAATGGCCAGTCCGAGAGTCGAAGACGCGGCCGTT
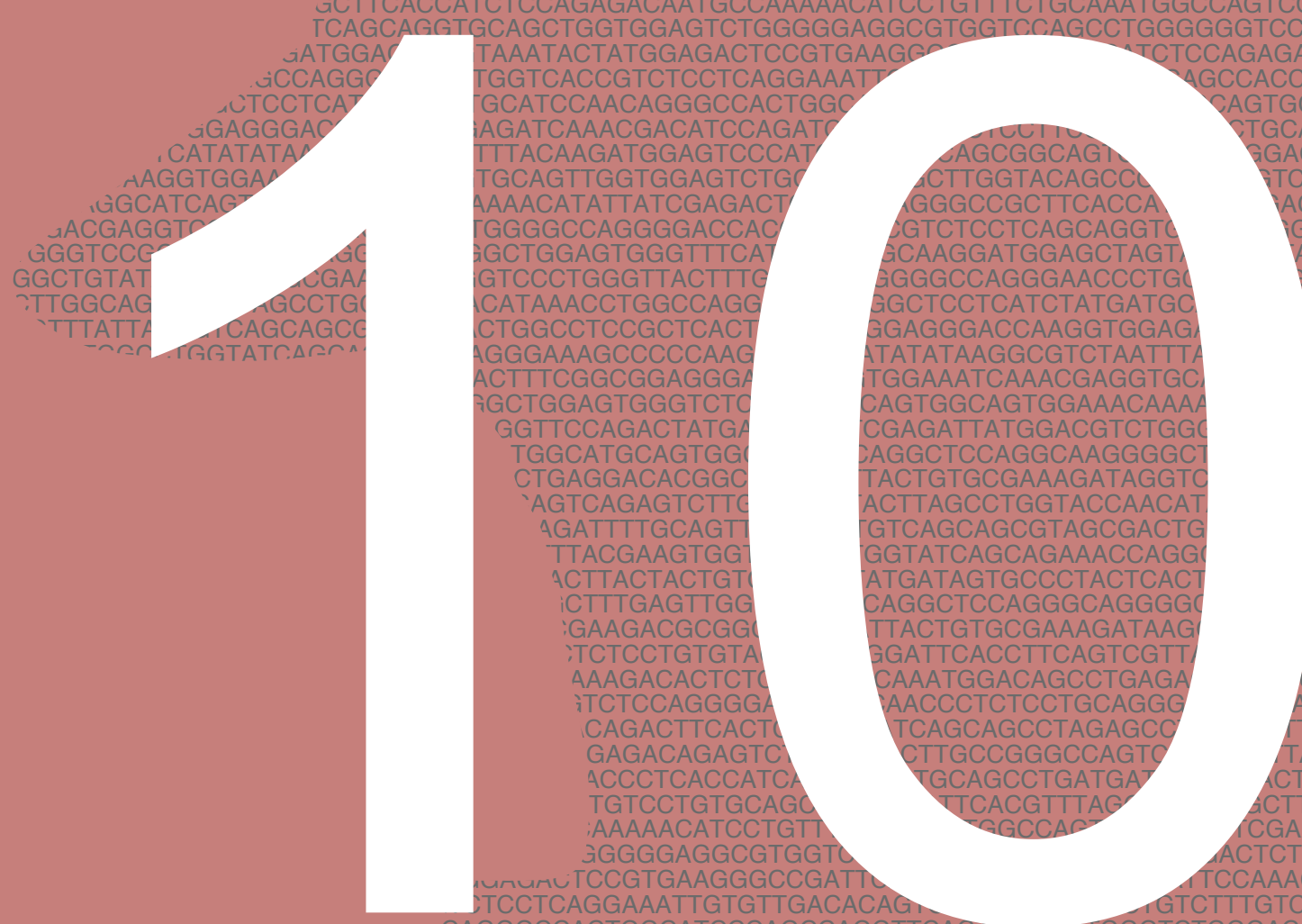
AGAGACAATTCCAAAGACACTCTCTTTCT ACCCTGTCTITGTCTCCAGGGGAAAG GGGTCTGGGACAGACTTCACTCTCA GACAGAGTTCACCCTCACCATCAGCA CCCTGAGACTGTCCTGTGCAGCCT GACAATGCCAAAAACATCCTGTTTC TTGGAGTCTGGGGGAGGCGTGGT ITGGAGACTCCGTGAAGGGCCGAT CTCCTCAGGAAATTGTGTTGACA AGGGCCACTGGCATCCCAGCCAC GACATCCAGATGACCCAGTCTCC GAGTCCCATCAAGGTTCAGCGG GGAGTCTGGGGGAGGCTTGGTA TCGAGACTCTGTGAAGGGCCGC GGACCACGGTCATCGTCTCCTC GGTTTCATTTATACGCAAGGATG TTACTTTGACTACTGGGGCCAGG GCCAGGCTCCCAGGCTCCTCAT GCTCACTTTCGGCGGAGGGACC GCTCACTITCGGCGGAGGGACC GGAGGGACCAAGGTGGAAATCA GGGTCTCAGGCATCAGTGGCAG ACTATGACGAGGTCGAGATTATG CAGTGGGTCCGCCAGGCTCCAC ACACGGCTGTATATTACTGTGCG AGTCTTGGCAGCTACTTAGCCTC TGCAGTTTATTACTGTCAGCAGC ACGAAGTGGTTGGCCTGGTATCAC TACTACTGTCAACAATATGATAGT TTGAGTTGGGTCCGCCAGGCTCCA AAGACGCGGCCGTITATTACTGTGC CTCCTGTGTAGCGTCTGGATTCACCT GCTCAGGAAATTGTGTTGACAC TTGTCTCACTCTCTITCTGCAAATGGAC,

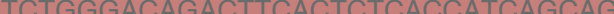
ACGACATCCAGATGACCCAGTCTCCTTCCACCCTGGCTGCATCTGTAGGAGACAGAGTCTCCATCACTTGCCGG GATGGAGTCCCATCAAGGTTCAGCGGCAGTGGGTCTGGGACAGAGTTCACCCTCACCATCAGCAGTCTGCAGCC ITGGTGGAGTCTGGGGGAGGCTTGGTACAGCCGGGGGGGTCCCTGAGACTGTCCTGTGCAGCCTCTGGATTCAC ¿ATATTATCGAGACTCTGTGAAGGGCCGCTTCACCATCTCCAGAGACAATGCCAAAAACATCCTGTTTCTGCAAATGC GCCAGGGGACCACGGTCATCGTCTCCTCAGCAGGTGCAGCTGGTGGAGTCTGGGGGAGGCGTGGTCCAGCCTGG GGAGTGGGTTTCATTTATACGCAAGGATGGAGCTAGTAAATACTATGGAGACTCCGTGAAGGG

¿CCTGGGTTACTTTGACTACTGGGGCCAGGGAACCCTGGTCACCGTCTCCTCAGGAAATTGTC

ITAAACCTGGCCAGGCTCCCAGGCTCCTCATCTATGATGCATCCAACAGGGCCACTGGCATCr

rGGCCTCCGCTCACTTTCGGCGGAGGGACCAAGGTGGAGATCAAACGACATCCAGATGACCC

CACGAAGCCCCAAGCTCCTCATATATAAGGCGTCTAATTTACAAGATGGAGTCCCATCAAG

GGCTGGAGTGGGTCTCAGGCATCAGTGGCAGTGGAAACAAAACATATTATCGAGACTCTGTG

IAGGTTCCAGACTATGACGAGGTCGAGATTATGGACGTCTGGGGCCAGGGGACCACGGTCATCGi

ITATGGCATGCAGTGGGTCCGCCAGGCTCCAGGCAAGGGGCTGGAGTGGGTTTCATTTATACGCAAGGATGGAGi I AG I

iAGAGCTGAGGACACGGCTGTATATTACTGTGCGAAAGATAGGTCCCTGGGTTACTTTGACTACTGGGGCCAGGGAACCCTG AGGGCCAGTCAGAGTCTTGGCAGCTACTTAGCCTGGTACCAACATAAACCTGGCCAGGCTCCCAGGCTCCTCATCTATGATG GAGCCTGAAGATTTTGCAGTTTATTACTGTCAGCAGCGTAGCGACTGGCCTCCGCTCACTTTCGGCGGAGGGACCAAGGTGGA 


\section{General discussion}

GGC,

TCTCL

AGCGG

TTGGTAC

CTCCTCAG

AAGGATGGA

GGCCAGGGA

TCCTCATCTAT

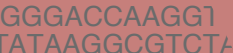

GAAATCAAACGAC

'TGGCAGTGGAAA!

AGATTATGGACGT

GGCTCCAGGCAAG

ACTGTGCGAAAGATAL

ACTTAGCCTGGTACCAAC,

GTCAGCAGCGTAGCGACTGG

TTGGTATCAGCAGAAACCAGGGAA,

TATGATAGTGCCCTACTCACTTTCGGL

CCAGGCTCCAGGGCAGGGGCTGGAGTGG

ГATTACTGTGCGAAAGATAAGGTTCCAGACTA

GGATTCACCTTCAGTCGTTATGGCATGCAGTG

GCAAATGGACAGCCTGAGAGCTGAGGACACGGC

GCAACCCTCTCCTGCAGGGCCAGTCAGA $\cong T \cap T$ TG

CCATCAGCAGCCTA

GTCTGCAGCCTr

CTGGATTCACGT

TTGCAAATGGCC.

CAGCCTGGGGG

TCACCATCTCCAG

CAGTCTCCAGCCAC
GGTTCAGTGGCAGTG

TTCCACCCTGGCTGCA

CAGTGGGTCTGGGACA

CAGCCGGGGGGGTCCC

TTCACCATCTCCAGAGAC

AGCAGGTGCAGCTGGTGC

GAGCTAGTAAATACTATG

GAACCCTGGTCACCGTC

CTATGATGCATCCAAC

AAGGTGGAGATr"

GTCTAATTTA

AACGAGGTGC

TGGAAACAA

GGCAAGGG'

AAAGATAC

AGTACCA

GTAGCC

CAGAAACL.

GCCCTACTCAL

GGGCAG!

GAAAGA

TCAGTC

AGCCT

CCTA

GCCA

GTTT

(1)

ar

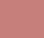

ITT

IAL

'TC,

ITAT,

ICTTAC

CTTTGA

כGAAGAL

TCTCCTG

AAGACAC

TCTCCAG

:AGACTTC,

CTCACCAT

CCTGTGCAGCCTCTGGATTCACG

AACATCCTGTTTCTGCAAATGGCCA

iGAGGCGTGGTCCAGCCTGGGGG

IAAGGGCCGATTCACCATCTCCA'

ITTGTGTTGACACAGTCTCCAGC

ITCCCAGCCAGGTTCAGTGGC

.CCAGTCTCCTTCCACCCTG

AGCAGCG

ATCAGCA

GGCTCCA

GATTCACS

AAATGGA

XCAACCCTC

CATCAGCA

CACTTGCCG

GATC

AGTCTGCAG

¿CTCTGGATTC

GTGGTCCAGCCTGG

GGCGATTCACCATCTCUn AGACA

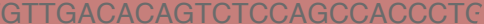

CCCAGCCAGGTTCAGTGGCAGTGGGT

:CCAGTCTCCTTCCACCCTGGCTGCATC '

त्रGCTTGGTACAGCCGGGGGGGTCCCTC

AGGGCCGCTTCACCATCTCCAGAGAC

GTCTCCTCAGCAGGTGCAGCTGGTG

CAAGGATGGAGCTAGTAAATACTATG

GGGCCAGGGAACCCTGGTCACCGTC

i्GCTCCTCATCTATGATGCATCCAACAGi

GAGGACCAAGGTGGAGATCAAACvvৎ

作 GGCATCAGTGGCAGTGGAAACAAAACATATTATC CGAGEUS AGATTATGGACGTCTGGGGCCAGGGI

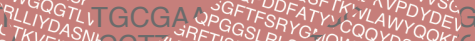

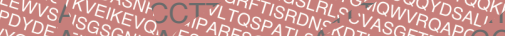

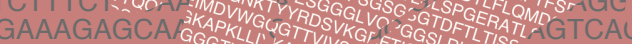

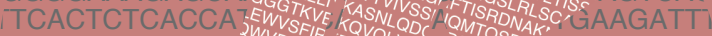

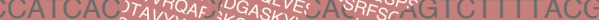

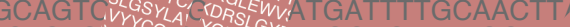

作

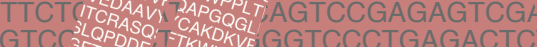

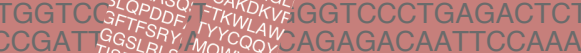
徒 作

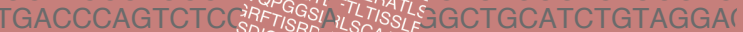
Q GGGGAGGCTTGG':SGAT

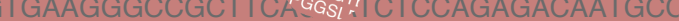

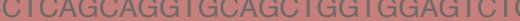

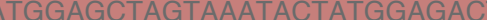

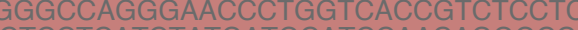

CACATAGATGCATCCAACAGGGCC

作 TCGTCCTr 


\section{General discussion}

Food allergy is an IgE-mediated, multifaceted clinical manifestation with symptoms reaching from mild oral itching to generalised life-threatening conditions like anaphylaxis. Even though food allergies can have a great impact on patients' quality of life, the cure of food allergies remains an unsolved issue. In daily practice, the only commonly used treatment options are elimination diets although the clinical implementation of oral immunotherapies is starting to take place ${ }^{1}$. To give precise dietary advice, to ensure a healthy and balanced diet and to prevent unnecessary food avoidance, there is a need for accurate, preferably minimally invasive, diagnostic strategies. Food allergy diagnosis is generally based on careful history and complementary in vivo (SPT) or in vitro (slgE) diagnostics. However, food allergy diagnosis by burdensome and costly oral food challenges requiring dedicated hospital facilities is still the gold standard. Numbers of those food challenges may be reduced by the development of highly accurate in vitro diagnostics.

Instead of measuring slgE levels against a whole food allergen extract containing many different allergenic and non-allergenic proteins, component-resolved diagnostics (CRD) provide the possibility to measure slgE levels against individual allergenic components in a simplex or multiplex manner ${ }^{2}$. Specific lgE binding against single components such as peanut Ara $\mathrm{h} 2$, hazelnut Cor a 14 or cashew nut Ana o 3, all belonging to the $2 \mathrm{~S}$ albumin family, has been shown to be useful in stratifying patient's risk for (severe) allergic reactions ${ }^{3-6}$. However, slgE measurements to certain foods/allergens are still hampered by lacking sufficient sensitivity due to non-detectable clinically relevant sensitisation („false-negative“) or specificity by detecting clinically irrelevant sensitisation (,false-negative“) (Figure 1, indicated by the text highlighted in red). 


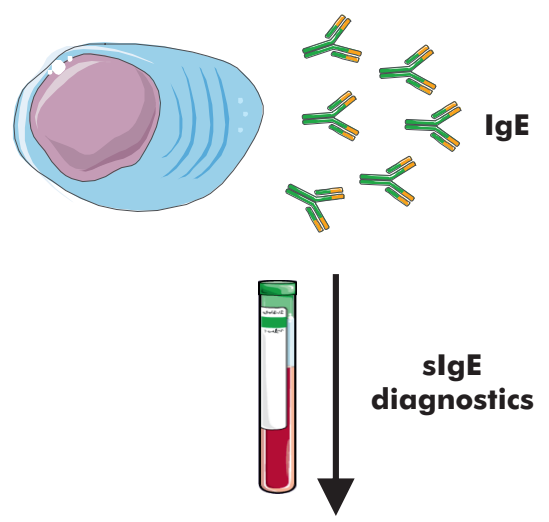

negative test outcomes

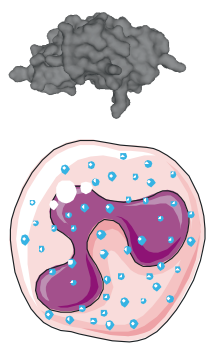

no clinically relevant sensitisation

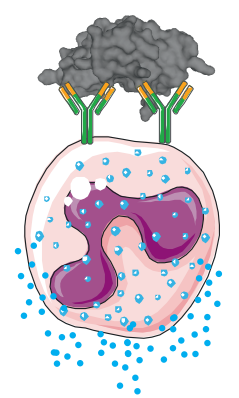

non-detectable clinically relevant sensitisation

„false-negative"

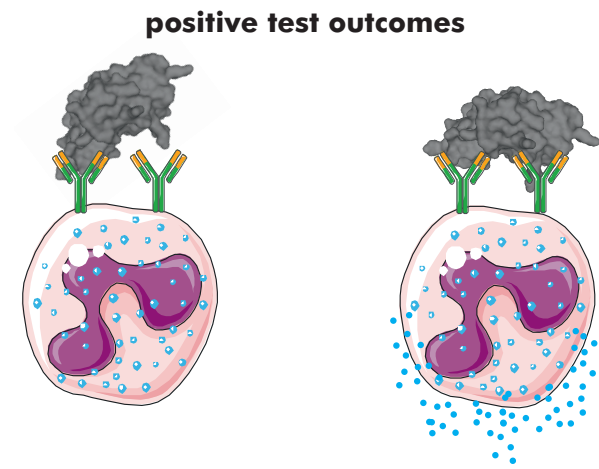

clinically irrelevant sensitisation relevant sensitisation „false-positive"

Figure 1: Current drawbacks of slgE measurements

The research presented in this thesis focused on the development of possible strategies to overcome the present disadvantages of slgE measurements in order to improve their clinical application in the future. 


\section{Part 1: Preventing (,false-negative“) slgE testing: non- detectable clinically relevant sensitisation}

A proportion of allergic patients, ranging from $18 \%$ for peanut to $30 \%$ for sesame seed $^{7-9}$, are characterised by non-detectable clinically relevant sensitisation (referred to as „false-negative“) using conventional slgE diagnostics. Possible explanations for those false-negative test outcomes and potential solutions (a to $\mathrm{c}$ ) are summarised in Figure 2:

A The allergenic component recognised by clinically relevant slgE is currently unknown

$\rightarrow$ Identification of novel allergenic components potentially belonging to a protein family with known allergenic potential in order to stratify patients at risk $k^{4,10}$ (chapter 2)

$\rightarrow$ Studying slgE binding to hydrophobic proteins (e.g. oleosins) present in the lipid phase of the respective food source but potentially absent in commercially available, aqueous-based food extracts ${ }^{11,12}$ (chapter 3)

B Incorrect protein folding or incorrect post-translational modifications prevent binding of clinically relevant slgE

$\rightarrow$ Disruption of protein folding during manufacturing may be prevented by optimised expression systems and purification strategies ${ }^{13}$

$\rightarrow$ Co-factors such as lipids may be essential for the correct folding of the protein by e.g. binding to the hydrophobic cavity of novel or already known allergenic components (future directive chapter 3$)^{14}$

$\rightarrow$ Simulation of food processing or gastrointestinal ingestion in vitro may change protein folding in such a way that it more closely mimics the in vivo situation ${ }^{15,16}$

C Clinically relevant sensitisation cannot be serologically measured despite surfacebound slgE on mast cells and basophils; this may be caused by 1) slgE levels under the detection limit ${ }^{17}$ or 2 ) high slgG levels saturating the binding sites and hence, preventing binding by slgE

$\rightarrow$ performing basophil activation test ex vivo 
D The underlying mechanism of the allergic reaction is non- $\lg E$ mediated characterised by late-onset and exclusive gastrointestinal manifestations - this explanation is less likely because patients with false-negative outcomes are mostly characterised by early onset and gastrointestinal manifestations are accompanied by additional immediate symptoms such as respiratory or cardiovascular indications ${ }^{18,19}$

E Allergic symptoms are related to IgG-mediated anaphylaxis triggered by macrophages or neutrophils - this explanation is less likely because its existence has so far only been proven in mice. Additionally, at least 100 fold higher antigendoses are required compared to IgE-mediated reactions which can be hardly be achieved by systemically absorption of food antigens ${ }^{20-22}$

\section{Potential explanations}

A Missing component

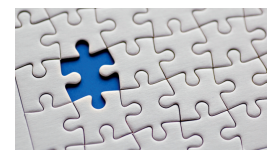

B Incorrect folding

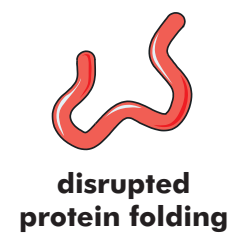

Non-detectable sensitisation despite effector cell-bound slgE

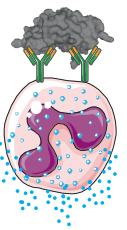

[1]

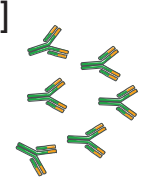

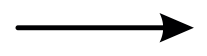

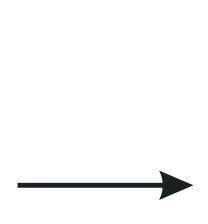

Optimised expression systems and purfication strategies

Identification of novel components

\section{chapter 2}
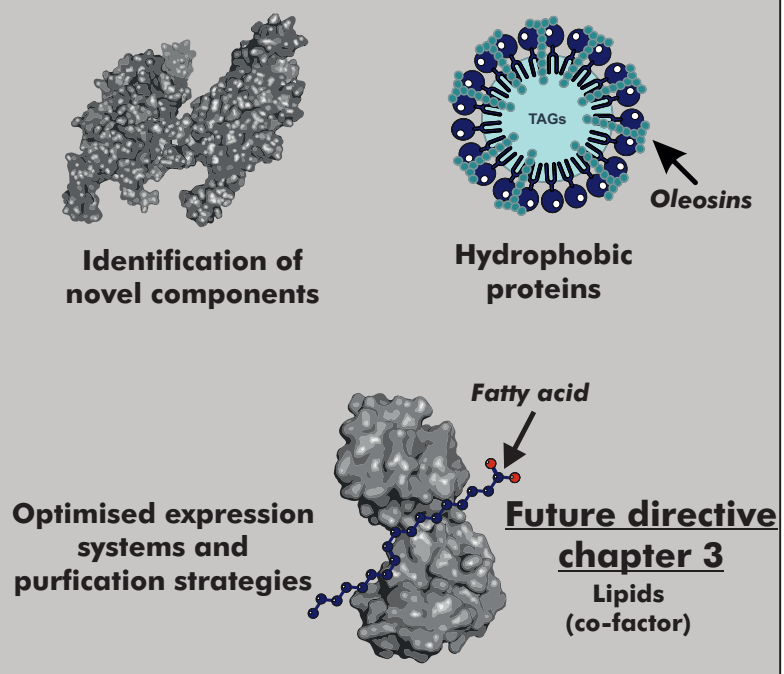

Hydrophobic proteins chapter 3

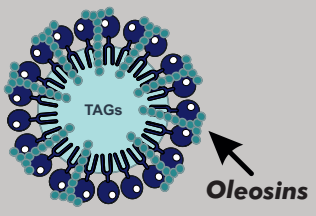

Oleosins

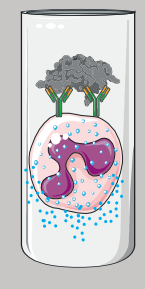

ex vivo
Basophil activation test

Figure 2: Summary of potential explanations (left side) of „false-negative" slgE testing in allergic subjects and their potential solutions (right side) 


\section{Seed storage proteins are important single components for slgE diagnostics}

Specific IgE binding to seed storage proteins including $2 S$ albumins, $11 S$ and $7 S$ globulins (vicilins) is known for its association with clinically relevant sensitisation and the risk for severe allergic reactions to seeds, legumes, and tree nuts. The diagnostic value of slgE measurements against seed storage proteins has been extensively studied for peanut and to a lesser extent for tree nuts such as walnut and hazelnut ${ }^{23}$. The peanut 2S albumins Ara h 2 (AUC: 0.85 in adults; 0.94 in children) and 6 (AUC: 0.85 in adults; 0.98 in children) are known as highly valuable single components for the prediction of peanut allergy in Northern Europe ${ }^{9,24,25}$. Besides $2 S$ albumins, slgE binding to vicilins, especially recombinant (excluding the presence of cross-reactive carbohydrate determinants (CCDs)) Jug r 2 (walnut), has also been associated with clinically relevant sensitisation, although the evidence is less strong than the evidence for $2 \mathrm{~S}$ albumins. Moreover, specific IgE binding to the walnut vicilin Jug $r 6$ has been more often accompanied by rather mild reactions. This latter vicilin showed greater cross-reactivity to vicilins from other tree nuts and legumes ${ }^{10,26-28}$. In chapter 2 , vicilins from macadamia nut, an allergen source with increasing relevance, have been identified as the first single allergenic components for CRD in macadamia nut allergy. Specific IgE binding to these vicilins was only detected in patients experiencing moderate to severe symptoms upon ingestion. Next to vicilins, we hypothesise the discovery and characterisation of additional single allergenic components from macadamia nut. These components might be 2S albumins and 11S globulins as Jug r 1 (2S albumin) and Jug r 4 (11S globulin) partly inhibited slgE binding to macadamia nut extract ${ }^{29}$. In comparison to other tree nuts and peanut, a $2 S$ albumin from macadamia nut might serve as an additional marker for clinically relevant sensitisation to macadamia nut or even replace our newly identified vicilin markers if these components are implemented in CRD in the future. However, we expect that the vicilin from macadamia nut will be a marker for macadamia nut allergy in a subpopulation of macadamia nut allergic patients. This hypothesis is supported by the potential of vicilins to induce even severe allergic reactions, although vicilins are more often responsible for cross-reactivity between legumes or tree nuts and rather mild food allergies $^{26,30,31}$.

\section{Lipophilic allergens in component-resolved diagnostics}

Even though a large number of allergic patients shows slgE binding against known single allergenic components, there is still a remaining subpopulation without detectable sensitisation. This subpopulation ranges from $18 \%$ for peanut to $30 \%$ for sesame seed $^{7-9}$. Undetectable sensitisation in those patients may be explained by an $\lg E$ re- 
sponse against lipophilic proteins absent in commercially available food extracts. The lack of lipophilic proteins such as oil-body associated proteins (OAPs) in food extracts is caused by defatting steps prior extract preparation ${ }^{32}$. OAPs such as oleosins from peanut, hazelnut and sesame seed have been recognised by slgE from allergic patients with partly false-negative slgE testing, suggesting an important role of these proteins for $\mathrm{CRD}^{7,11,12}$. Hence, in chapter 3 we studied the diagnostic value of slgE binding to sesame oleosins in sesame allergic patients with negative testing to aqueous extract or known hydrophilic single components (e.g. 2 S albumin Ses i 1) ${ }^{7}$. Contrary to our exspectations, slgE binding to native and heterologously expressed sesame oleosins was completely absent in sesame allergic patients with false-negative testing. Additionally, sesame oleosins were recognised with rather low slgE levels by only $15 \%$ of allergic and tolerant patients with detectable sensitisation. In contrast to previous studies, we developed an improved purification protocol for OAPs including hydrophobic interaction chromatography as a last purification step. This protocol possesses the strength to separate even traces of seed storage proteins from the OAPs fraction as shown by mass spectrometry and western blot analyses. This improved purification strategy of native OAPs and heterologous expression of oleosins offered the possibility to exclusively study the role of oleosins in slgE diagnostics. The use of hydrophobic interaction chromatography, however, could have disrupted lipids potentially essential for recognition of OAPs by slgE. Lipids may support correct and natural folding of OAPs by stabilising their long hydrophobic core ${ }^{14}$. Moreover, lipids might also play a critical role in the recognition of known allergenic components by slgE in a subset of allergic patients ${ }^{33}$. Currently, the exact role of hydrophobic proteins is not completely understood. Future research should focus on their recognition in the context of lipid availability while ensuring the absence of other known allergenic components. Overall, we hypothesise that lipids are important for the recognition by slgE of the responsible allergens in patients without detectable sensitisation using conventional testing.

\section{Future directive: Lipids may contribute to the recognition of single allergenic components}

The interaction between lipids and allergens is an emerging field of research and lipids are involved in several processes including the activation of the innate immune system $^{34}$. In the context of sensitisation, lipids can be presented to invariant natural killer T cells (NKT cells) by the non-polymorphic MHC class 1 molecule CD1 expressed on several antigen-presenting cells. Although lipids, such as LPS binding to different tolllike receptors (TLRs), generally induce a Th1 response, small amounts of LPS binding to TLR4 in presence of the respective inhalant allergen have been able to induce a Th2 response ${ }^{14}$. This special involvement in the sensitisation process has also been 
shown for polar lipids from Brazil Nut as these were essential for inducing an $\lg E$ and $\lg \mathrm{G}$ response to the recombinant $2 \mathrm{~S}$ albumin Ber e 1 in mice. Moreover, lipids from cypress pollen induced proliferation of human derived NKT cells ex vivo. Notably, these lipids were also recognised by slgE from cypress pollen allergic patients, indicating a direct involvement in recognition by $\operatorname{slg}^{35,36}$. On the protein level itself, lipids can protect allergens from digestion in the gastrointestinal tract or function as an allergen carrier through the epithelium as hypothesised for oleosins ${ }^{37}$. This protective role of lipids suggests a combined presentation of allergens and lipids to immune cells triggering an IgE response. Nevertheless, lipids can also have a direct effect on allergens and their allergenic properties. A large panel of allergens is able to bind lipids via their hydrophobic cavities closely located to their surface or via electrostatic/hydrophobic interactions ${ }^{14,38,39}$. These interactions between lipids and proteins can greatly influence the folding of the protein. Hence, new conformational epitopes are potentially formed or previous covered linear epitopes become accessible. Conformational changes by lipid binding has been most intensively studied for non-specific lipid transfer proteins (nsLTPs), known as markers for severe allergic reactions in the Mediterranean area. Their binding by slgE and their ability to induce basophil degranulation were enhanced in presence of oleic acid. This effect was mainly pronounced for the peach nsLTP Pru p 3 and to a lesser extent for the nsLTPs from walnut (Jug $r$ ) and hazelnut $(\text { Cor a } 8)^{26,33,40}$. These data suggest that it might be beneficial to study the impact of lipids on allergen recognition by $\mathrm{lgE}$ for other known lipid-binding allergens including (sesame) oleosins. Preliminary (unpublished) experiments of our research group with the lipid phase of peanut showed slgE binding by sera from peanut allergic patients without detectable slgE sensitisation to peanut (18\%) using conventional testing ${ }^{9}$. We therefore assume that recombinant allergen-lipid complexes formed in vitro with the responsible lipid ligands may offer improved clinical application of CRD in the future. However, the fact that lipid-protein complexes might not be as easy to handle needs to be taken into consideration. This may require the development of an adjusted platform for testing these complexes in vitro compared to the platforms currently commercially available. 


\section{Part 2: Preventing „false-positive“ slgE testing: clini- cally irrelevant sensitisation}

The detection of clinically irrelevant sensitisation to single components associated with (severe) allergic reactions (referred to as „false-positive“) complicates the interpretation of positive slgE testing in approximately $10 \%$ of sensitised but tolerant patients ${ }^{41,42}$. This may lead to inaccurate diagnosis and consequently dietary advices accompanied by unnecessary food avoidance.

False-positive slgE testing implicates the lack of degranulation induction upon allergen exposure in vivo ${ }^{1}$ despite bound slgE on the surface of effector cells via high affinity $\mathrm{Fc} R \mathrm{RI}$ receptors. This phenomenon might be explained by certain antibody-related requirements identified for degranulation induction (Figure 3, left side). IgE-mediated degranulation can only be induced by cross-linking of at least two, but preferably more, Fce $\mathrm{RI}$ receptors and consequently by the recognition of at least two different epitopes on the surface of multivalent allergens ${ }^{43,44}$. The required spacing between these epitopes has been estimated to a distance between 5 and $24 \mathrm{~nm}(50-240 \AA$ ) using artificial antigens. A larger spacing also appeared to be beneficial for the natural allergen Phl $p 7$ [a] ${ }^{45,46}$. However, approximately one third of all food allergens, including $2 S$ albumins and PR-10 proteins, are even smaller than $10 \mathrm{~nm}$ and thus the requested spacing may only be achieved by aggregation events ${ }^{47}$. Additionally, at least one of the lgE antibodies has to bind with high affinity to its binding site ${ }^{46}$. Notably, the required affinity is reduced for recognition of multivalent allergens due to increasing avidity [b] ${ }^{45,48}$. Besides requirements for sufficient cross-linking, blocking antibodies ( $\operatorname{lgG}$ or $\lg A$ ) can hinder the binding of clinically relevant epitopes by surface-bound $\lg E[c]^{22,49,50}$. Moreover, cross-linking of $F_{c} \varepsilon R I$ receptors with inhibitory FcyRllb receptors, loaded with allergenspecific IgG, can lead to attenuated degranulation. This attenuation is achieved by inhibitory interaction of phosphorylated SHIP-1 (Src homology 2 containing inositol 5' polyphosphatase 1 ) with the FcERI signalling cascade $[d]^{51}$. The latter mode of action has only been confirmed for human basophils, while human skin mast cells lack this inhibitory receptor ${ }^{52,53}$.

Another potential explanation of false-positive slgE testing is related to distinct glycosylation patterns of IgE antibodies (Fc part or variable region) between allergic and sensitised but tolerant patients [e]. Glycosylation of variable regions is achieved by the introduction of $\mathrm{N}$ - or O-glycosylation sites during somatic hypermutation maturation (SHM). These glycosylations, mostly located in CDR regions, can affect antibody's

\footnotetext{
${ }^{1}$ Remark: Positive SPT or BAT results contradicting clinical diagnosis might be explained by high allergen concentrations not reflecting the concentration present in vivo upon ingestion, potentially triggering non-specific stimulation (in chapter 9, we observed non-specific stimulation of basophils loaded with peanut $2 \mathrm{~S}$ albumin-specific mAbs upon stimulation with high concentration $(1 \mu \mathrm{g} / \mathrm{ml})$ of cow's milk extract). Concentration threshold might be patient dependent.
} 
affinity to its target ${ }^{54,55}$. On the other hand, distinct glycosylation patterns of the Fc part have been shown to influence the binding of $\operatorname{lgG}$ antibodies to Fcy receptors in an antigen-specific manner by altering affinities of their interactions. While sialylation of IgG antibodies has been associated with anti-inflammatory traits, sialylation of IgE antibodies was more likely in peanut allergic subjects than in non-atopic controls ${ }^{56-59}$. Notably, impaired sialylation of lgG was induced by persistent antigen exposure ${ }^{60}$. This may explain why continuous antigen (e.g. peanut) exposure can have protective effects on developing a clinically relevant allergy by sensitised but tolerant children ${ }^{61}$. However, it is poorly understood how the information on antigen-specific glycosylation patterns is stored in the respective $B$ cells.

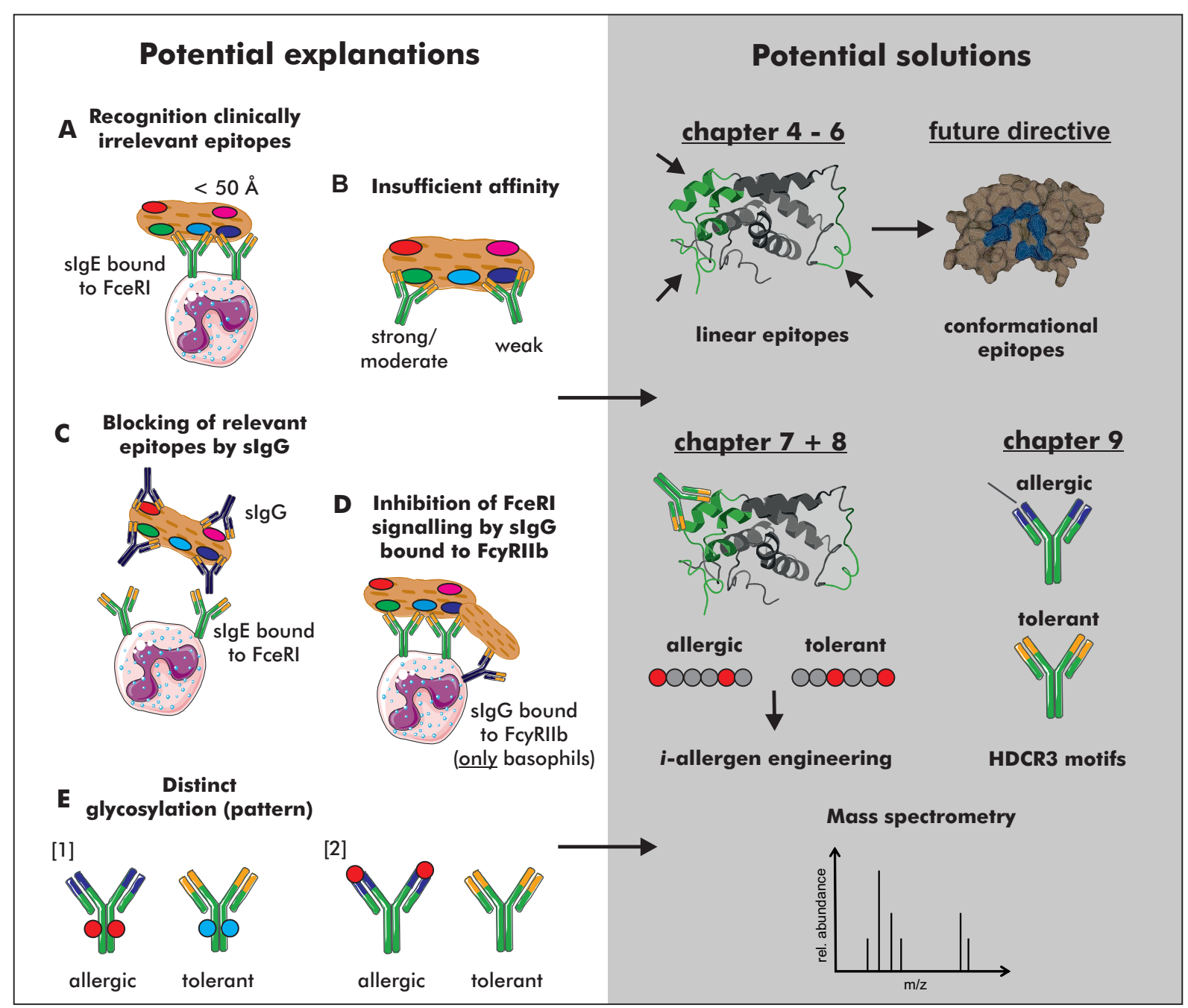

Figure 3: Summary of potential explanations (left side) of „false-positive“ slgE testing in sensitised but tolerant subjects and their potential solutions (right side); clinically relevant epitopes are highlighted in green and blue

These potential explanations support the hypothesis of differences in specific antibody repertoires; one supporting the development of allergic reactions in allergic patients and one lacking this ability in sensitised but tolerant patients. To detect such differ- 
ences, the focus has to be changed from slgE binding to entire (single) components towards specific epitopes. Potential strategies to address distinct antibody repertoires are shown on the right side of Figure 3:

- Studying linear epitope recognition patterns using polyclonal patient sera (chapter 4-6)

- Combining linear epitope recognition patterns with the recognition of conformational epitopes to complete the picture (future directive)

- Polyclonal sera comprise a complex antibody mixture preventing high resolution epitope mapping ( $\rightarrow$ epitope dissimilarities on amino acid level); high resolution epitope mapping can be achieved by using antigen-specific human monoclonal antibodies (mAbs) (chapter 7 and 8)

- High resolution epitope mapping may support the engineering of single components ( $i$-allergens) recognised by slgE from allergic but not from sensitised but tolerant patients (chapter $\mathbf{7}$ and 8 )

- Evaluating differences of specific antibody repertoires on antibody level: Sequences of the variable regions, especially of the HCDR3 region, may have unique features such as amino acid motifs or increased numbers of glycosylation sites associated with either allergy or tolerance (chapter 9)

- Evaluating differences in glycosylation patterns using mass spectrometry analysis or evaluating gene/protein expression levels of enzymes involved in antibody glycosylation of antigen-specific B cells

The focus on antibody-related explanations may not entirely unravel the complex picture of why slgE sensitisation is not always attended by clinical symptoms. Polymorphisms in promotor regions, epigenetics or miRNA control mechanisms have great influence on gene and protein expression ${ }^{62,63}$. Individual gene/protein expression levels may increase or decrease the susceptibility of effector cells to extrinsic stimuli like antigen exposure. Moreover, these changes may alter the production of pre-formed mediators and/or de novo formed mediators released during degranulation. On the other hand, the susceptibility of the surrounding environment towards released mediators may influence the occurrence and severity of clinical symptoms, e.g. levels of PAF-AH (platelet activation factor-acetyl hydrolase, an enzyme degrading the pro-inflammatory mediator PAF) have been inversely correlated with levels of PAF. Subsequently, levels of PAF-AH were also associated with less severe allergic reactions ${ }^{64,65}$. All these latter explanations, however, are not able to elucidate the reason why polysensitisation can be partly clinically relevant and partly irrelevant in the same patient. 


\section{Impact of slgE binding to linear epitopes on diagnosing food aller- gies}

Linear epitopes can be directly exposed on the surface of allergens or they can become accessible upon food processing or proteolysis in the gastrointestinal tract, similarly important for food allergy sensitisation and effector cell stimulation. In the last decades, linear epitopes derived from several allergens such as peanut Ara $\mathrm{h} 2$ and hen's egg Gal $d 1$ have been intensively studied regarding their recognition by slgE. Overall, broad recognition of several linear epitopes (degree of IgE clonality) seems to be associated with allergy and partly with severity of clinical symptoms upon exposure ${ }^{66}$. Defined sets of linear epitopes, however, have only be described for a limited number of food allergens. Recognition of these sets have mainly been described as prognostic markers for e.g. persistence of cow's milk and hen's egg allergy in children ${ }^{67-69}$. In chapter 4, we identified three potential peptide markers for the discrimination between hen's egg allergic and tolerant adults similarly sensitised to the major hen's egg allergen Gal $\mathrm{d} 1$. Specific IgE binding to at least one of these three epitopes largely increased the specificity compared to slgE binding against the full-length allergen and may therefore serve as an additional diagnostic tool in diagnosing hen's egg allergic adults in the future. Comparably, slgE binding to peptide markers of Ara $\mathrm{h} 2$ has been shown to slightly increase diagnostic accuracy in peanut sensitised children compared to slgE binding to full-length Ara $\mathrm{h} 2^{70}$. In contrast, slgE binding to linear epitopes of Ara $\mathrm{h} 7$ isoforms, which are also 2S albumins like Ara h 2 and 6, provided no basis for elucidating their divergent potencies to induce degranulation (chapter 5 and 6). This observation emphasises the importance of conformational epitopes in recognising Ara $h$ 7. Similarly, recognition of full-length Ara $\mathrm{h} 6$ and to a lesser extent of Ara $\mathrm{h} 2$ were not completely explained by slgE binding to linear epitopes ${ }^{71-73}$. The greater importance of linear epitopes for recognising Ara h 2 compared to Ara h 6 and 7 may be explained by an extremely long loop carrying important linear epitopes already accessible without disrupting its 3D structure ${ }^{74}$. Unlike linear epitopes, conformational epitopes are formed upon correct folding of the allergen, suggesting that correctly folded food allergens are presented to the immune system during sensitisation and effector cell stimulation. This presentation might be dependent on lasting allergen stability due to a high number of disulphide bridges. Moreover, such presentation may also be achieved by intact allergen crossing over the buccal mucosa which additionally may explain the early onset of many food allergic reactions ${ }^{75}$. Overall, the nature of epitopes (linear vs conformational) being important for recognition by slgE appears to be allergen-specific, as their importance already appears to differ for the three peanut $2 S$ albumins. Additionally, each food allergen shows a distinct susceptibility to processing and digestion, potentially masking epitopes or making other epitopes accessible. We therefore suggest 
additionally considering slgE binding to conformational epitopes as well as to linear epitopes, although it has been suggested that especially linear epitopes are important in food allergy due to food processing and digestion.

\section{Clinical application of slgE binding to epitopes in food allergy diag- nosis}

As mentioned in the previous paragraph, allergy has been mostly associated with a broad recognition of linear epitopes. Even though broad recognition patterns increase the probability of binding epitope combinations with sufficient spacing, assessment of such panels would require microarrays covering the entire sequence of an allergen ${ }^{66}$. Moreover, no cut-off levels (= minimal number of recognised linear epitopes) have yet been defined. Such cut-off levels would facilitate clinician's diagnosis when using sigE binding to linear epitopes as a diagnostic tool. Defined sets of linear epitopes, however, provide a larger clinical applicability. These sets are not only easier to interpret, they are also easier to implement in already commercially available diagnostic platforms. For clinical implementation, we still lack clinical studies on technological applicability, diagnostic accuracy, additional diagnostic value and cost comparison to currently available conventional testing ${ }^{66}$. Specific IgE binding to conformational epitopes as a diagnostic tool, however, will require the development of more sophisticated in vitro platforms than required for linear epitopes since the 3D structure of the epitopes has to be conserved. A potential solution might be the translation of conformational epitopes into linear peptides mimicking the structure of the conformational epitope $e^{76,77}$.

\section{High resolution epitope mapping with human monoclonal antibo- dies to define clinically relevant epitopes}

Normally, epitope mapping in food allergy research is performed with polyclonal patient sera. Even though polyclonal patient sera are easily accessible for in vitro research, they contain a complex mixture of distinct antibodies. This mixture may hamper the definition of single clinically relevant epitopes for in vitro diagnostics. As discussed in our review (chapter 7), specific antibodies present in sera of sensitised but tolerant patients can theoretically recognise clinically relevant epitopes with lower affinity than required for sufficient cross-linking. Antibodies in sera from allergic patients, on the other hand, may recognise clinically irrelevant epitopes as well as the ones responsible for successful cross-linking. Moreover, blocking antibodies ( $\lg \operatorname{or} \lg \mathrm{A})$ may be present in sera of sensitised but tolerant patients preventing slgE binding to clinically relevant epitopes. To overcome these limitations, we proposed the use/generation of antigenspecific human mAbs derived from allergic and sensitised but tolerant patients. This 
tool provides a less complex matrix and offers the possibility to perform high resolution epitope mapping on amino acid level - same amino acid sequence but distinct critical amino acids. Additional advantages of human mAbs are the possibility to connect genetic features with specific allergen binding and their use for conformational epitope mapping requiring sophisticated techniques not compatible with complex polyclonal patient sera ${ }^{78}$.

In chapter 8, we set up and compared two pipelines for the generation of human mAbs derived from peripheral blood. One strategy entailed B cell immortalisation by EBV and limited dilution, while the other strategy was characterised by single cell sequencing and heterologous antibody expression in mammalian cells. The major advantage of single cell sequencing is the broad examination of antigen-binding mAbs due to a less selective process compared to EBV immortalisation combined with limited dilution. However, both techniques solely rely on BCR expression of antigen-specific $B$ cells for selection. Hence, plasma blasts and IgE $+B$ cells with limited surface expression are hard to detect and plasma cells are nearly completely excluded from selection ${ }^{79,80}$. In vaccine research, plasma cells were selected 3-5 days after vaccination without staining for antigen binding. Most of the plasma cells were specific for the target antigen, since the boost greatly enlarged the plasma cell compartment for plasma cells recognising one specific antigen ${ }^{81-83}$. This interesting approach is, unfortunately, limited in allergy research, as the last time point of stimulation is hard to define (unknown ingestion). Moreover, the choice of the antigen for B cell selection is especially critical in food allergy research. The antigen is presented in a mixture of intact and partly digested conditions. Additionally, food processing may have influenced the folding of the antigen presented to the immune system. Overall, human mAbs are a great step forward towards defining clinically relevant epitopes and will provide new insights on antibodies produced by allergic and sensitised but tolerant patients.

\section{Allergen engineering to prevent the binding of clinically irrelevant slgE}

Human mAbs generated for defining differences in antibody repertoires of allergic and tolerant patients sensitised to Ara h 2 and 6 (chapter 9) may be beneficial for high resolution epitope mapping as discussed in the previous paragraph. By combining the exact binding sites of these antibodies with their potency to induce degranulation, epitopes can be categorised into clinically relevant and irrelevant ones. This knowledge offers the possibility to develop engineered/improved allergens for slgE diagnostics (Figure 4). Improved allergens may be achieved by site-directed mutations within clinically irrelevant epitopes to prevent slgE binding from sensitised but tolerant patients. Moreover, exact site-directed mutations within clinically relevant epitopes may also prevent 
slgE binding from tolerant patients as only critical amino acids may differ. The use of such engineered/improved allergens ( $i$-allergens) in CRD may dramatically reduce the measurement of clinically irrelevant sensitisation, facilitating the extrapolation of in vitro diagnostics to relevant dietary restrictions in the future.
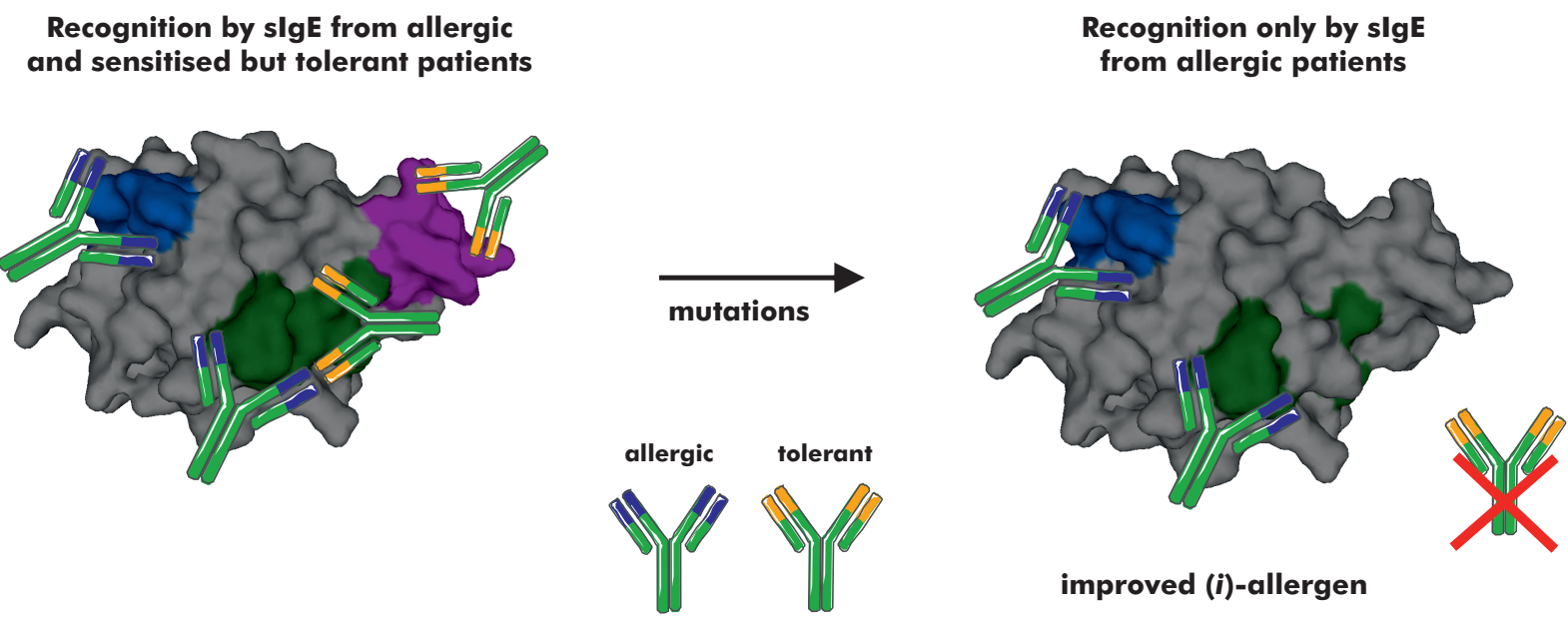

Figure 4: Site-directed mutations of clinically irrelevant epitopes can support the development of improved (i)-allergens by preventing slgE binding from sensitised but tolerant patients; clinically relevant epitopes are highlighted in green and blue; antibodies from allergic patients possess a blue variable region and antibodies from tolerant patients possess a yellow variable region

\section{Genetic fingerprints as a step towards a new diagnostic strategy}

One major advantage of human mAbs is the straightforward combination of genetic features with antigen specificity, providing a powerful platform to study genetic features associated with health or disease. In chapter 9, HCDR3 region motifs of human mAbs directed against the peanut allergens Ara $\mathrm{h} 2$ and 6 were partly associated with allergy or tolerance despite overlapping slgE levels against these components. This finding presents a first step towards the development of novel, non-slgE based in vitro diagnostic strategies to discriminate between allergy and tolerance in case of clinically irrelevant sensitisation. A new diagnostic strategy may combine our selection procedure for Ara h 2 and 6 specific B cells from peripheral blood (FACS) with high-throughput next generation sequencing. High-throughput next generation sequencing offers a less laborious platform than Sanger sequencing of single-sorted cells. Comparable approaches are already used for cancer diagnostics ${ }^{84}$. 


\section{Future perspectives of food allergy in-vitro diagnostics: Preventing ,individualised diagnostics“}

The nature of each allergen potentially responsible for sensitisation and subsequently for the allergic reaction is unique. Hence, each allergen requires individual strategies to diagnose their associated food allergies. The in vitro diagnostics of animal-related allergies such as hen's egg have already shown promising results using epitope-based approaches. This approach, however, seems to be more limited for plant-derived allergens such as peanut, since no specific sets of epitopes could be defined. For peanut with its major allergens Ara h 2 and 6 , classical CRD is already a highly valuable tool to identify patients at risk. However, tolerant patients with increased slgE levels to Ara $\mathrm{h} 2$ and inconclusive history may experience diet limitations accompanied by a negative impact on their quality of life. In this case, the development of engineered allergens ( $i$-allergens) will offer the chance to minimise these clinically irrelevant test outcomes. Despite potentially higher costs, an alternative to $i$-allergens for CRD, in case of development failure, may be the analysis of HCDR3 regions derived from antibodies recognising Ara h 2 and 6 (Figure 5). Overall, the way to improve the diagnostics for a certain allergen has to be adjusted to its nature, and the best approach will differ between allergens.

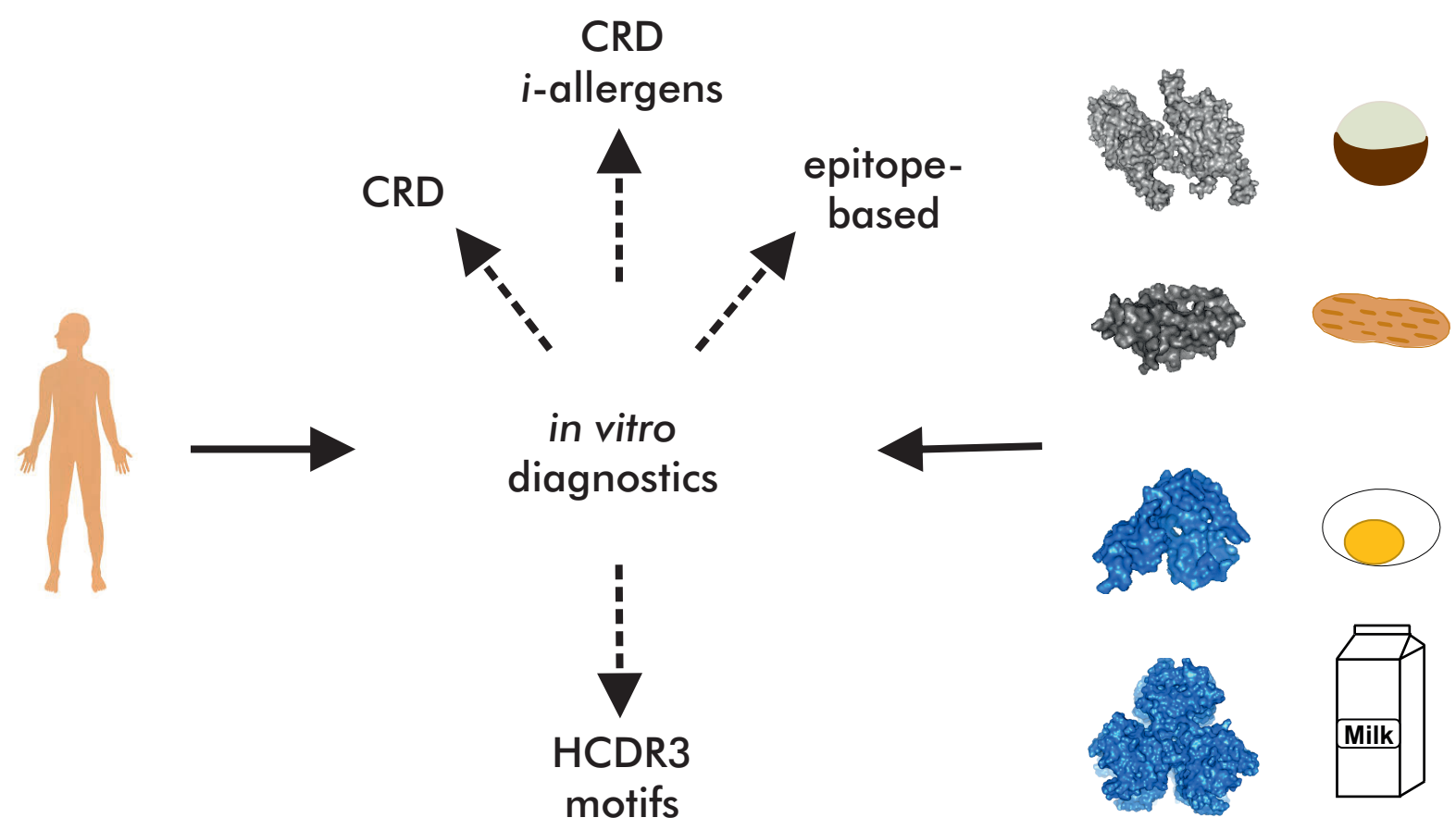

Figure 5: Individualised in vitro diagnostics strategies for unique allergens, indicating that each allergen needs the development of its own optimised in vitro diagnostic strategy 
The benefit of developing the best in vitro diagnostic strategy for each individual allergen should be estimated before starting a long-lasting and costly development. Regarding Bet v 1-related food allergies, it might be highly interesting to predict the food sources which can or cannot be eaten. However, the rather mild character of these allergies provide the possibility to safely test the respective food sources at home. Nevertheless, sensitisation to Gly m 4, the PR-10 protein from soy, is partly associated with even severe reactions, indicating the benefit of developing an epitope-based or $i$-allergen approach for this particular PR-10 protein ${ }^{85}$. Moreover, accurate diagnostic strategies are primarily required for allergens often hidden in a large panel of foods while allergens like kiwi are more easily avoidable.

Despite the promising future perspectives of these in vitro diagnostic strategies, they are limited in predicting the severity of an allergic reaction. To achieve allergy combined with severity prediction, the individualised approaches discussed in the thesis should be combined with diagnostics focussing on severity prediction. However, these tools are not yet developed and may be based on epigenetic features, biomarkers related to effector cells (gene expression, miRNAs) or glycosylation patterns of IgE antibodies $^{56,86,87}$.

\section{Concluding remarks}

Overall, the results presented in this thesis provide a basis for developing in vitro diagnostic strategies preventing „false-negative" and „false-positive" slgE testing. The exact development strategy is dependent on the unique nature of the allergen, e.g. „falsepositive" results to Ara $\mathrm{h} 2$ and 6 may be prevented by analysing HCDR3 patterns while slgE binding to linear epitopes of Gal d 1 appear to be promising for discriminating between hen's egg allergy and tolerance in adults. Overall, individually improved in vitro diagnostics on allergen level may potentially reduce the required number of burdensome food challenges in the future. 


\section{Main findings of the presented thesis}

\section{Preventing „false-negative“ slgE testing: non-detectable clinically relevant sensitisation}

- Specific IgE binding to macadamia nut vicilins may support the stratification of macadamia nut allergic patients experiencing moderate to severe symptoms (chapter 2)

- Highly purified native and heterologously expressed sesame oleosins were not recognised in sesame allergic adults with false-negative slgE testing. Hence, slgE binding to sesame oleosins has no additional value in diagnosing sesame allergic adults (chapter $\mathbf{3}$ )

- Our purification strategy of native oleosins, potentially disrupting lipids, pinpoints to a potential role of lipids in allergen recognition. The evaluation of this role might be a future directive of research to minimise the number "false-negative“ test outcomes

\section{Preventing „false-positive“ slgE testing: non-detectable clinically relevant sensitisation}

- Specific IgE measurement against linear epitopes of Gal d 1 (aa 30-41, aa 3950 , aa 84-95) supports the discrimination between hen's egg allergic and tolerant adults similarly sensitised to full-length Gal d 1. Hen's egg allergic adults recognising these epitopes suffered mainly from objective symptoms (chapter 4).

- The isoform Ara h 7.0201 has comparable potency to induce degranulation compared to the other peanut 2S albumins Ara $\mathrm{h} 2$ and 6, while the other isoforms Ara h 7.0101 and Ara h 7.0301 showed lower potencies (chapter 5). Lower potency of the latter isoforms was not explained by linear epitope recognition patterns, leading to the assumption that conformational epitopes play a critical role in this context (chapter 6)

- We assume that the use of specific human mAbs supports the identification of clinically irrelevant epitopes and can be used for conformational epitope mapping requiring sophisticated techniques (chapter $\mathbf{7}$ and $\mathbf{8}$ )

- Certain HCDR3 region motifs of peanut 2S albumin-specific mAbs were either associated with peanut allergy or tolerance. This observation provides a first step towards the development of a novel diagnostic strategy for discriminating between allergy and tolerance on antibody level (chapter 8 and 9) 


\section{References}

1. Pajno, G. B. et al. EAACI Guidelines on allergen immunotherapy: IgE-mediated food allergy. Allergy 73, 799-815 (2017).

2. Sato, S., Yanagida, N. \& Ebisawa, M. How to diagnose food allergy. Curr Opinion Allergy Clin Immunol 18, 214-21 (2018).

3. Lange, L. et al. Ana o 3-specific lgE is a good predictor for clinically relevant cashew allergy in children. Allergy 72, 598-603 (2016).

4. Klemans, R. J. B. et al. Ara $\mathrm{h} 2$ Is the Best Predictor for Peanut Allergy in Adults. J Allergy Clin Immunol Pract 1, 632-8.e1 (2013).

5. Masthoff, L. J. N. et al. Sensitization to Cor a 9 and Cor a 14 is highly specific for a hazelnut allergy with objective symptoms in Dutch children and adults. $J$ Allergy Clin Immunol 132, 393-9 (2013).

6. Dodig, S. \& Čepelak, I. The potential of component-resolved diagnosis in laboratory diagnostics of allergy. Biochem Med (Zagreb) 28, 020501 (2018).

7. Leduc, V. et al. Identification of oleosins as major allergens in sesame seed allergic patients. Allergy 61, 349-56 (2006).

8. Ballmer-Weber, B. K. et al. IgE recognition patterns in peanut allergy are age dependent: perspectives of the EuroPrevall study. Allergy 70, 391-407 (2015).

9. Kansen, H. M. et al. Accurate prediction of peanut allergy in one third of adults using a validated Ara $h 2$ cut-off in EAACI (London, 2020).

10. Teuber, S. S. et al. Identification and cloning of a complementary DNA encoding a vicilin-like proprotein, Jug $r$ 2, from English walnut kernel (Juglans regia), a major food allergen. J Allergy Clin Immunol 104, 1311-20 (1999).

11. Zuidmeer-Jongejan, L. et al. Oil body-associated hazelnut allergens including oleosins are underrepresented in diagnostic extracts but associated with severe symptoms. Clin Transl Allergy 4, 4 (2014).

12. Schwager, C. et al. Peanut oleosins associated with severe peanut allergy - importance of lipophilic allergens for comprehensive allergy diagnostics. $J$ Allergy Clin Immunol 140, 1331-8.e8 (2017).

13. Baneyx, F. \& Mujacic, M. Recombinant protein folding and misfolding in Escherichia coli. Nature Biotechnol 22, 1399-1408 (2004).

14. Bublin, M., Eiwegger, T. \& Breiteneder, H. Do lipids influence the allergic sensitization process? J Allergy Clin Immunol 134, 521-9 (2014).

15. Masthoff, L. J. et al. A systematic review of the effect of thermal processing on the allergenicity of tree nuts. Allergy 68, 983-93 (2013). 
16. Untersmayr, E. \& Jensen-Jarolim, E. The role of protein digestibility and antacids on food allergy outcomes. J Allergy Clin Immunol 121, 1301-8 (2008).

17. Gómez, E. et al. Role of the basophil activation test in the diagnosis of local allergic rhinitis. J Allergy Clin Immunol 132, 975-6.e5 (2013).

18. Anvari, S., Miller, J., Yeh, C.-Y. \& Davis, C. M. IgE-Mediated Food Allergy. Clin Rev Allergy Immunol 57, 244-60 (2019).

19. Ruffner, M. A. \& Spergel, J. M. Non-IgE-mediated food allergy syndromes. Ann Allergy Asthma Immunol 117, 452-4 (2016).

20. Finkelman, F. D., Khodoun, M. V. \& Strait, R. Human IgE-independent systemic anaphylaxis. J Allergy Clin Immunol 137, 1674-80 (2016).

21. Escribese, M. M. et al. Alternative Anaphylactic Routes: The Potential Role of Macrophages. Fron Immunol 8, 515 (2017).

22. Strait, R. T. et al. Ingested allergens must be absorbed systemically to induce systemic anaphylaxis. J Allergy Clin Immunol 127, 982-9.e1 (2012).

23. Ho, M. H.-K., Wong, W. H.-S. \& Chang, C. Clinical Spectrum of Food Allergies: a Comprehensive Review. Clin Rev Allergy Immunol 46, 225-40 (2012).

24. Kukkonen, A. K. et al. Ara h 2 and Ara 6 are the best predictors of severe peanut allergy: a double-blind placebo-controlled study. Allergy 70, 1239-45 (2015).

25. Kansen, H. M. et al. A diagnostic flowchart for pediatric peanut allergy using Ara $h 2$ is accurate, safe and reduces costs in EAACI (London, 2020).

26. Dubiela, $P$. et al. Jug $r 6$ is the allergenic vicilin present in walnut responsible for IgE cross-reactivities to other tree nuts and seeds. Sci Rep 8, 11366 (2018).

27. Lauer, I. et al. Hazelnut (Corylus avellana) vicilin Cor a 11: molecular characterization of a glycoprotein and its allergenic activity. Biochem $J \mathbf{3 8 3}, 327-34$ (2004).

28. Pastorello, E. A. et al. Lipid transfer protein and vicilin are important walnut allergens in patients not allergic to pollen. J Allergy Clin Immunol 114, 908-14 (2004).

29. Teuber, S. S. \& Peterson, W. R. IgE-binding Proteins in Macadamia Nut Crossreact with Walnut. J Allergy Clin Immunology 125, AB222 (2010).

30. Wensing, M. et al. Patients with anaphylaxis to pea can have peanut allergy caused by cross-reactive IgE to vicilin (Ara h 1). J Allergy Clin Immunol 111, 420-4 (2003).

31. Sanchez-Monge, R. et al. Vicilin and convicilin are potential major allergens from pea. Clin Exp Allergy 34, 1747-53 (2004). 
32. David, N. A., Penumarti, A., Burks, A. W. \& Slater, J. E. Food allergen extracts to diagnose food-induced allergic diseases. Ann Allergy Asthma Immunol 119, 101-7 (2017).

33. Dubiela, P. et al. Enhanced Pru p 3 lgE-binding activity by selective free fatty acid-interaction. J Allergy Clin Immunol 140, 1728-31.e10 (2017).

34. Thomas, W. R. Innate affairs of allergens. Clin Exp Allergy 43, 152-63 (2013).

35. Mirotti, L. et al. Lipids are required for the development of Brazil nut allergy: the role of mouse and human iNKT cells. Allergy 68, 74-83 (2013).

36. Agea, E. et al. Human CD1-restricted T cell recognition of lipids from pollens. $J$ Exp Med 202, 295-308 (2005).

37. Jappe, U. et al. Lipophilic Allergens, Different Modes of Allergen-Lipid Interaction and Their Impact on Asthma and Allergy. Fronti Immunol 10, 122 (2019).

38. Mogensen, J. E. et al. The Major Birch Allergen, Bet v 1, Shows Affinity for a Broad Spectrum of Physiological Ligands. J Biol Chem 277, 23684-92 (2002).

39. Moreno, F. J., Mackie, A. R. \& Mills, E. N. C. Phospholipid Interactions Protect the Milk Allergen a-Lactalbumin from Proteolysis during in Vitro Digestion. J Agricul Food Chem 53, 9810-6 (2005).

40. Dubiela, P. et al. Impact of lipid binding on the tertiary structure and allergenic potential of Jug $r$ 3, the non-specific lipid transfer protein from walnut. Sci Rep 9, 2007 (2019).

41. Blankestijn, M. A. et al. Specific lgE to Jug $r 1$ has no additional value compared with extract-based testing in diagnosing walnut allergy in adults. $J$ Allergy Clin Immunol 139, 688-90.e4 (2017).

42. Blankestijn, M. A. et al. Specific lgE to peanut $2 S$ albumin Ara $\mathrm{h} 7 \mathrm{has}$ a discriminative ability comparable to Ara h 2 and 6. Clin Exp Allergy 48, 60-5 (2017).

43. Christensen, L. H. et al. Several distinct properties of the IgE repertoire determine effector cell degranulation in response to allergen challenge. J Allergy Clin Immunol 122, 298-304 (2008).

44. Gieras, A. et al. Molecular determinants of allergen-induced effector cell degranulation. J Allergy Clin Immunol 119, 384-90 (2007).

45. Bucaite, G. et al. Interplay between Affinity and Valency in Effector Cell Degranulation: A Model System with Polcalcin Allergens and Human Patient-Derived IgE Antibodies. J Immunol 203, 1693-700 (2019).

46. Knol, E. F. Requirements for effective IgE cross-linking on mast cells and basophils. Mol Nutr Food Res 50, 620-4 (2006). 
47. Schöll, I. et al. Dimerization of the Major Birch Pollen Allergen Bet v 1 Is Important for its In Vivo IgE-Cross-Linking Potential in Mice. J Immunol 175, 6645-50 (2005).

48. Handlogten, M. W., Kiziltepe, T., Alves, N. J. \& Bilgicer, B. Synthetic Allergen Design Reveals the Significance of Moderate Affinity Epitopes in Mast Cell Degranulation. ACS Chem Biol 7, 1796-1801 (2012).

49. Santos, A. F. et al. IgG4 inhibits peanut-induced basophil and mast cell activation in peanut-tolerant children sensitized to peanut major allergens. J Allergy Clin Immunol 135, 1249-56 (2015).

50. Jiménez-Saiz, R. \& Patil, S. U. The Multifaceted B Cell Response in Allergen Immunotherapy. Curr Allergy Asthma Rep 18, 66 (2018).

51. Malbec, O. et al. Trans-inhibition of activation and proliferation signals by Fc receptors in mast cells and basophils. Sci Signal 9, ra126 (2016).

52. Zhao, W. et al. FcyRlla, Not FcyRllb, Is Constitutively and Functionally Expressed on Skin-Derived Human Mast Cells. J Immunol 177, 694-701 (2006).

53. Cassard, L., Jönsson, F., Arnaud, S. \& Daëron, M. Fcy Receptors Inhibit Mouse and Human Basophil Activation. J Immunol 189, 2995-3006 (2012).

54. Van de Bovenkamp, F. S. et al. Adaptive antibody diversification through Nlinked glycosylation of the immunoglobulin variable region. Proc Natl Acad Sci U $S$ A 115, 1901-6 (2018).

55. Van de Bovenkamp, F. S. et al. Variable Domain N-Linked Glycans Acquired During Antigen-Specific Immune Responses Can Contribute to Immunoglobulin G Antibody Stability. Front Immunol 9, 740 (2018).

56. Shade, K.-T. C. et al. Sialylation of immunoglobulin $\mathrm{E}$ is a determinant of allergic pathogenicity. Nature 582, 265-70 (2020).

57. Shade, K.-T., Conroy, M. E. \& Anthony, R. M. IgE Glycosylation in Health and Disease. Curr Top Microbiol Immunol 423, 77-93 (2019).

58. Epp, A. et al. Sialylation of IgG antibodies inhibits IgG-mediated allergic reactions. J Allergy Clin Immunol 141, 399-402.e8 (2018).

59. Anthony, R. M. \& Ravetch, J. V. A Novel Role for the IgG Fc Glycan: The Antiinflammatory Activity of Sialylated IgG Fcs. J Clin Immunol 30, S9-14 (2010).

60. Irvine, E. B. \& Alter, G. Understanding the role of antibody glycosylation through the lens of severe viral and bacterial diseases. Glycobiology 30, 241-53 (2020).

61. DuToit, G. et al. Effect of Avoidance on Peanut Allergy after Early Peanut Consumption. N Engl J Med 374, 1435-43 (2016). 
62. Jaenisch, R. \& Bird, A. Epigenetic regulation of gene expression: how the genome integrates intrinsic and environmental signals. Nat Gen 33, 245-54 (2003).

63. Mitchison, N. A. Polymorphism in regulatory gene sequences. Genome Biol 2, comment2001.1-6 (2000).

64. Perelman, B., Adil, A. \& Vadas, P. Relationship between platelet activating factor acetylhydrolase activity and apolipoprotein B levels in patients with peanut allergy. Allergy Asthma Clin Immunol 10, 20 (2014).

65. Gill, P., Jindal, N. L., Jagdis, A. \& Vadas, P. Platelets in the immune response: Revisiting platelet-activating factor in anaphylaxis. J Allergy Clin Immunol 135, 1424-32 (2015).

66. Sánchez-Ruano, L., de la Hoz, B. \& Martínez-Botas, J. Clinical utility of microarray B-cell epitope mapping in food allergies: A systematic review. Ped Allergy Immunol 31, 175-85 (2020).

67. Järvinen, K.-M. et al. Specificity of IgE antibodies to sequential epitopes of hen's egg ovomucoid as a marker for persistence of egg allergy. Allergy 62, 758-65 (2007).

68. Chatchatee, P. et al. Identification of lgE- and IgG-binding epitopes on as1casein: Differences in patients with persistent and transient cow's milk allergy. $J$ Allergy Clin Immunol 107, 379-83 (2001).

69. Järvinen, K.-M. et al. B-cell epitopes as a screening instrument for persistent cow's milk allergy. J Allergy Clin Immunol 110, 293-7 (2002).

70. Santos, A. F. et al. IgE to epitopes of Ara $\mathrm{h} 2$ enhance the diagnostic accuracy of Ara h 2-specific lgE. Allergy 75, 2309-18 (2020).

71. Otsu, K., Guo, R. \& Dreskin, S. C. Epitope analysis of Ara h 2 and Ara h 6: characteristic patterns of IgE-binding fingerprints among individuals with similar clinical histories. Clin Exp Allergy 45, 471-84 (2015).

72. Chen, $\mathrm{X}$. et al. Conformational lgE epitopes of peanut allergens Ara $\mathrm{h} 2$ and Ara h 6. Clin Exp Allergy 46, 1120-8 (2016).

73. Breiteneder, H. Mapping of conformational lgE epitopes of food allergens. Allergy 73, 2107-9 (2018).

74. Gruber, P., Becker, W.-M. \& Hofmann, T. Influence of the Maillard Reaction on the Allergenicity of rAra h 2, a Recombinant Major Allergen from Peanut (Arachis hypogaea), Its Major Epitopes, and Peanut Agglutinin. J Agricul Food Chem 53, 2289-96 (2005). 
75. Dirks, C. G. et al. Does absorption across the buccal mucosa explain early onset of food-induced allergic systemic reactions? J Allergy Clin Immunol 115, 1321-3 (2005).

76. Goede, A., Jaeger, I. S. \& Preissner, R. SUPERFICIAL - Surface mapping of proteins via structure-based peptide library design. BMC Bioinformatics 6, 223 (2005).

77. Demolombe, V. et al. Benchmarking the PEPOP methods for mimicking discontinuous epitopes. BMC Bioinformatics 20, 738 (2019).

78. Abbott, W. M., Damschroder, M. M. \& Lowe, D. C. Current approaches to fine mapping of antigen-antibody interactions. Immunology 142, 526-35 (2014).

79. Benckert, J. et al. The majority of intestinal $\lg A+$ and $\lg G+$ plasmablasts in the human gut are antigen-specific. J Clin Invest 121, 1946-55 (2011).

80. Wilson, P. C. \& Andrews, S. F. Tools to therapeutically harness the human antibody response. Nat Rev Immunol 12, 709-19 (2012).

81. Smith, K. et al. Rapid generation of fully human monoclonal antibodies specific to a vaccinating antigen. Nat Prot 4, 372-84 (2009).

82. Whittle, J. R. R. et al. Broadly neutralizing human antibody that recognizes the receptor-binding pocket of influenza virus hemagglutinin. Proc Natl Acad Sci U $S$ A 108, 14216-21 (2011).

83. Wrammert, J. et al. Rapid cloning of high-affinity human monoclonal antibodies against influenza virus. Nature 453, 667-71 (2008).

84. Cummings, C. A. et al. The Role of Next-Generation Sequencing in Enabling Personalized Oncology Therapy. Clin Transl Sci 9, 283-92 (2016).

85. Berneder, M. et al. Allergen Chip Diagnosis for Soy-Allergic Patients: Gly m 4 as a Marker for Severe Food-Allergic Reactions to Soy. Int Arch Allergy Immunol 161, 229-33 (2013).

86. Molderings, G. J. Transgenerational transmission of systemic mast cell activation disease-genetic and epigenetic features. Transl Res 174, 86-97 (2016).

87. Krajewski, D. et al. Epigenetic Regulation via Altered Histone Acetylation Results in Suppression of Mast Cell Function and Mast Cell-Mediated Food Allergic Responses. Front Immunol 9, 105.2 (2018). 
GCAGTCTL

JAGCCTCTGGAT

CCTGTTTCTGCAAA.

iGCGTGGTCCAGCCTC

AGGGCCGATTCACCATL

AATTGTGTTGACACAGTCTCCAGCCALU

UTGGCATCCCAGCCAGGTTCAGTGGCAGTGGG'TC I

$\because$ CAGATGACCCAGTCTCCTTCCACCCTGGCTGCATCTGTAG

AGTCCCATCAAGGTTCAGCGGCAGTGGGTCTGGGACAGAGTTCACL

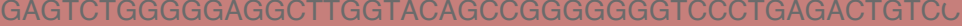

ITATCGAGACTCTGTGAAGGGCCGCTTCACCATCTCCAGAGACAATGCCAAAAAC ¿AGGGGACCACGGTCATCGTCTCCTCAGCAGGTGCAGCTGGTGGAGTCTGGGGGAGG iGAGTGGGTTTCATTTATACGCAAGGATGGAGCTAGTAAATACTATGGAGACTCCGTGAAGGG CCCTGGGTTACTTTGACTACTGGGGCCAGGGAACCCTGGTCACCGTCTCCTCAGGAAATTGTGT CCAGGGAAACCCCCAAGCTCCTCATATATAAGGCGUTUUNG

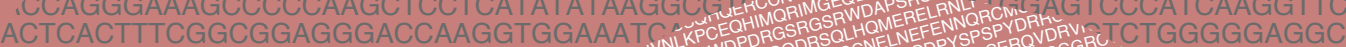

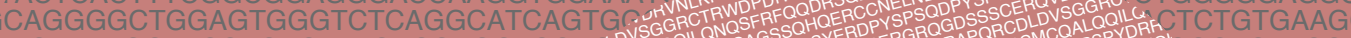

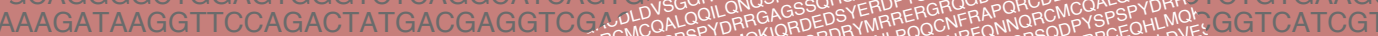

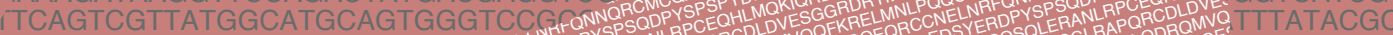
CAGCCTGAGAGC

:TCCTGCAGGGCGCGLRAPROR

tGCCTAGAGC

GCCTGATGAT

CACGTTTAGG

ATGGCCAGTCE

CCATCTCCAGAG

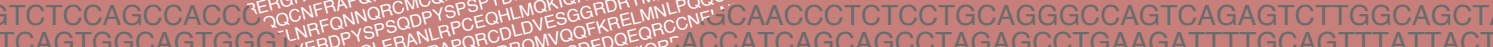

APQ ODROM ESDEDOKIOPACCATCAGCAGCCTAGAGCCTGAAGATITITGCAGTTTATTACT

FATCACTITCCGGGCCAGTCAGTCTITTACGAAGTGGTTGGCC

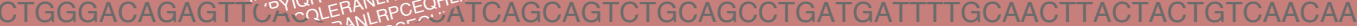

ACAGCCGGGGGGGTCCCTGAGACTGTCCTGTGCAGCCTCTGGATTCACGTTTAGGAGATATGCTTTGAGTTGGGTCCGQ GCTTCACCATCTCCAGAGACAATGCCAAAAACATCCTGTTTCTGCAAATGGCCAGTCCGAGAGTCGAAGACGCGGCCGTT

UTCCTCATCTATGATGCATCCAP

aGAGGGACCAAGGTGGAGATCP

. CATATATAAGGCGTCTAATTTP

HAGGTGGAAATCAAACGAGGTG

ACGAGGTCGAGATTATGGACC

GGGTCCGCCAGGCTCCAGGC

GGCTGTATATTACTGTGCGAAAG

TTGGCAGCTACTTAGCCTGGTP

TTATTACTGTCAGCAGCGTA TCAGCAGGTGCAGCTGGTG

AATGGAGCTAGTAAATACTA

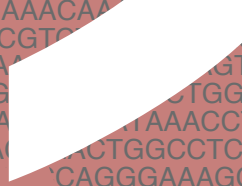

CAGGGAAAG

7GCTGGAG
GGTTCCA

TGGCAT

CTGAGG

AGTCA

AGATT

ACTTA

¿CTT

:GAA

:TCT

AAA

CA

GA

Annumi

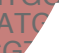

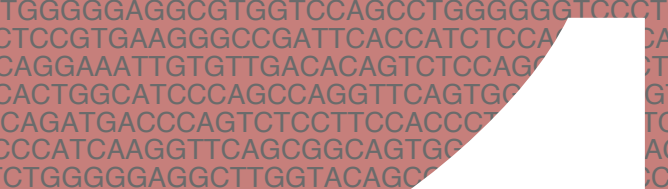

TGAGACTCTCCTGTGTAGCGTC ATTCCAAAGACACTCTCTTICT GTCTTTGTCTCCAGGGGAAAGA TCTGGGACAGACTTCACTCTCA CTGTAGGAGACAGAGTCTCCAT GAGTTCACCCTCACCATCAGCA TGAGACTGTCCTGTGCAGCCTC CAATGCCAAAAACATCCTGTTTC GGAGTCTGGGGGAGGCGTGGT GGAGACTCCGTGAAGGGCCGA GGAGACTCCGTGAAGGGCCGA
TTCCTCAGGAAATTGTGTTGACA TTCCTCAGGAAATTGTGTTGACA
GGGCCACTGGCATCCCAGCCAC ACATCCAGATGACCCAGTCTCC GAGTCCCATCAAGGTTCAGCGG GAGTCTGGGGGAGGCTTGGTA 作 CGAGACTCTGTGAAGGGCCGC
GGACCACGGTCATCGTCTCCTC GGACCACGGTCATCGTCTCCTC, G GTITATTTATACGCAAGGATG TACTTTGACTACTGGGGCCAGG GCCAGGCTCCCAGGCTCCTCAT CTCACTITCGGCGGAGGGACC CCAAGCTCCTCATATATAAGGQ GAGGGACCAAGGTGGAAATCA GGTCTCAGGCATCAGTGGCAG CTATGACGAGGTCGAGATTATG CAGTGGGTCCGCCAGGCTCCAC CACGGCTGTATATTACTGTGCG CACGGCTGTATAT ACTGTGCG AGTCTTGGCAGCTACTTAGCCTC TGCAGTITATTACTGTCAGCAGC GAAGTGGTTGGCCTGGTATCAC ACTACTGTCAACAATATGATAGT GAGTTGGGTCCGCCAGGCTCCA ACGCGGCCGTITATTACTGTGC TGTGTAGCGTCTGGATTCACCT CACTCTCTTTCTGCAAATGGAC CAGGGGAAAGAGCAACCCTCT GTTGACACAGTCTCCAGCCACCCTGTC ACGACATCCAGATGACCCAGTCTCCTTCCACCCTGGCTGCATCTGTAGGAGACAGAGTCTCCATCACTTGCCGG GATGGAGTCCCATCAAGGTTCAGCGGCAGTGGGTCTGGGACAGAGTTCACCCTCACCATCAGCAGTCTGCAGCC TGGTGGAGTCTGGGGGAGGCTTGGTACAGCCGGGGGGGTCCCTGAGACTGTCCTGTGCAGCCTCTGGATTCAC ¿ATATTATCGAGACTCTGTGAAGGGCCGCTTCACCATCTCCAGAGACAATGCCAAAAACATCCTGTTTCTGCAAATGC GCCAGGGGACCACGGTCATCGTCTCCTCAGCAGGTGCAGCTGGTGGAGTCTGGGGGAGGCGTGGTCCAGCCTGG GGAGTGGGTTTCATITATACGCAAGGATGGAGCTAGTAAATACTATGGAGACTCCGTGAAGGG

¿CCTGGGTTACTTTGACTACTGGGGCCAGGGAACCCTGGTCACCGTCTCCTCAGGAAATTGTГ

ITAAACCTGGCCAGGCTCCCAGGCTCCTCATCTATGATGCATCCAACAGGGCCACTGGCATCr

IGGCCTCCGCTCACTTTCGGCGGAGGGACCAAGGTGGAGATCAAACGACATCCAGATGACCC

AGGGAAAGCCCCCAAGCTCCTCATATATAAGGCGTCTAATTTACAAGATGGAGTCCCATCAAGC

CACTTTCGGCGGAGGGACCAAGGTGGAAATCAAACGAGGTGCAGTTGGTGGAGTCTGGGGG

GGCTGGAGTGGGTCTCAGGCATCAGTGGCAGTGGAAACAAAACATATTATCGAGACTCTGTG,

AAGGTTCCAGACTATGACGAGGTCGAGATTATGGACGTCTGGGGCCAGGGGACCACGGTCATCG I

1TATGGCATGCAGTGGGTCCGCCAGGCTCCAGGCAAGGGGCTGGAGTGGGTTTCATTTATACGCAAGGATGGAGi I AG I

¿AGAGCTGAGGACACGGCTGTATATTACTGTGCGAAAGATAGGTCCCTGGGTTACTTTGACTACTGGGGCCAGGGAACCCTG AGGGCCAGTCAGAGTCTTGGCAGCTACTTAGCCTGGTACCAACATAAACCTGGCCAGGCTCCCAGGCTCCTCATCTATGATGC GAGCCTGAAGATTTTGCAGTTTATTACTGTCAGCAGCGTAGCGACTGGCCTCCGCTCACTTTCGGCGGAGGGACCAAGGTGGA 


\section{Nederlandse samenvatting}

GGC,

TCTCL

AGCGG

TTGGTAC

CTCCTCAG

AAGGATGGA

GGCCAGGGA

TCCTCATCTAT

GGGACCAAGG 7 ,
CATAAGGCGTCT/

GAAATCAAACGAC

TTGGCAGTGGAAA:

AGATTATGGACGT

GGCTCCAGGCAAG

ACTGTGCGAAAGATAL

ACTTAGCCTGGTACCAAC,

GTCAGCAGCGTAGCGACTGG

TTGGTATCAGCAGAAACCAGGGA

TATGATAGTGCCCTACTCACTTTCGGL

CCAGGCTCCAGGGCAGGGGCTGGAGTGG

TATTACTGTGCGAAAGATAAGGTTCCAGACTA

GGATTCACCTTCAGTCGTTATGGCATGCAGTGG

GCAAATGGACAGCCTGAGAGCTGAGGACACGGC

CCATCAGCAGCCTG

CACTTGCCGGGCC

GTCTGCAGCCTr

CTGGATTCACG

TCAAATGGCC.

CAGCCTGGGGG

TCACCATCTCCAG

CAGTCTCCAGCCAC
GGTTCAGTGGCAGTG

TTCCACCCTGGCTGCA

CAGTGGGTCTGGGACA

CAGCCGGGGGGGTCCC

TTCACCATCTCCAGAGAC

AGCAGGTGCAGCTGGTGC

GAGCTAGTAAATACTATG

GAACCCTGGTCACCGTC

CTATGATGCATCCAAC

AAGGTGGAGATC

GTCTAATTTA

AACGAGGTGC

TGGAAACAA

AGCAAGGG'

AAAGATAC

AGTACCA

GTAGCC

CAGAAACL.

GCCCTACTCAL

GGGCAC

TCAGTC

AGCCT

CCTG

CCTA

GCC

TGA

GTT

acc

ar

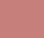

3Ci

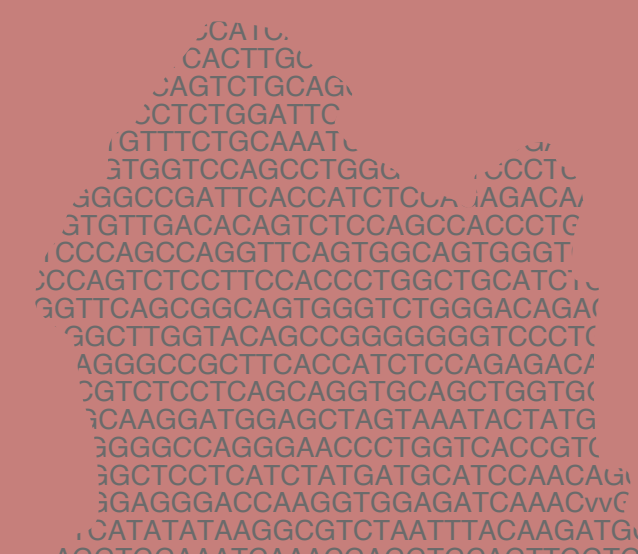

:CCAGTCTCCTTCCACCCTGGCTGCATC í

TGCTTGGTACAGCCGGGGGGGTCCCTC

作

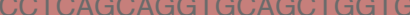

GGGCCAGGGAACCCTGGTCACCGTC

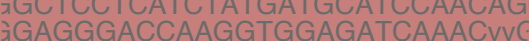

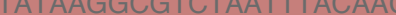
IGGCATCAGTGGCAGTGGAAACAAAACATATTATC ¿GAGNUS

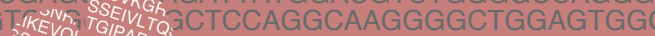

ESGE SA GTGCGAAAGATAGGTCCCTGGGTTA

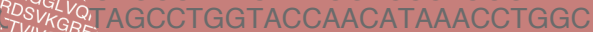

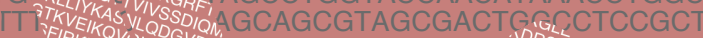

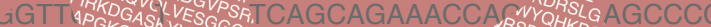
,TGTCA KOD RE W W

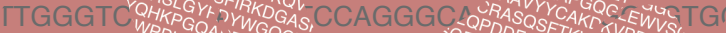

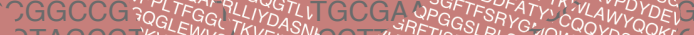

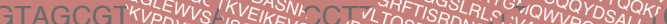
TCTCTTTCWMDE

iGGGAAAGAGCA/GGG

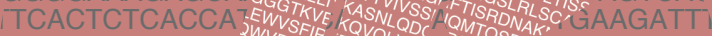

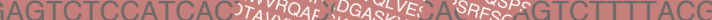

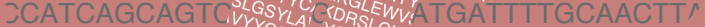

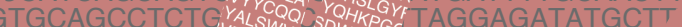

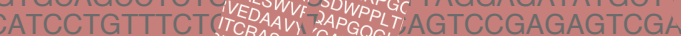

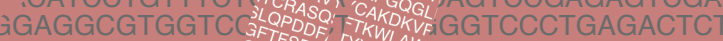

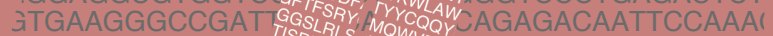

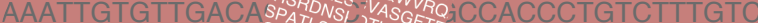

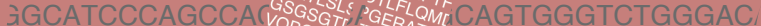

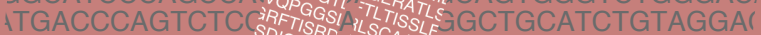
ICAAGGTTCAGCGDOMTO TAKNAASCCTGGGACAGAGTTCACr

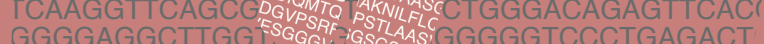
CTGTGAAGGGCCGCTTCRGGS :TCTCCAGAGACAATGCC

ITCATCGTCTCCTCAGCAGGTGCAGCTGGTGGAGTCT(

ATACGCAAGGATGGAGCTAGTAAATACTATGGAGAC

STGGGGCCAGGGAACCCTGGTCACCGTCTCCTC

$\sim$,TCCTCATCTATGATGCATCCAACAGGGCC' IGGACCAAGGTGGAGATCAAACVVGAr

TATAAGGCGTCTAATTTACAAGAT

GAAATCAAACGAGGTGCAGTTr

GGCAGTGGAAACAAAACAT

ITTATGGACGTCTGGGGCC

CCAGGCAAGGGGCTGGA TGCGAAAGA ${ }^{-1}$ TGTCCCTr

AGCAGCG

ATCAGCA

IATAGTG

GGCTCCA

GATTCACI

CAAATGGA

CAAATGGA

¿CAACCCTC

,CATCAGCAC

CACTTGCCGC

IACAGAGT

CTCACCATL

CTCTGGATTCACG:

CATATCTGTTCTGCAAATGGCCA

ACAT

GAGGCGTGGTCCAGCCTGGGGG

TAAAGGGCCGATTCACCATCTCCA

ITTGTGTTGACACAGTCTCCAGC

TTCCAGCCAGGTTCAGTGGC

CCAGTCTCCTTCCACCCTG 


\section{Nederlandse samenvatting}

De incidentie van voedselallergieën groeit wereldwijd nog flink. In Europa heeft gemiddeld drie procent van de bevolking last van een voedselallergie. Bij een voedsel allergische reactie reageert ons immuunsysteem op ongevaarlijke eiwitten die in onze voeding zitten. Deze eiwitten, ook wel allergenen genoemd, die door ons immuunsysteem als gevaarlijk beschouwd worden, komen in Europa vooral voor in pinda, hazelnoot, kippenei, koemelk, fruit (perzik, appel), vis en schaaldieren. Door de herkenning van deze allergenen door ons immuunsysteem, worden afweerstoffen, zo genaamde antilichamen, aangemaakt die tot verschillende subgroepen behoren - bij allergische patiënten worden daarnaast nog antilichamen van het type $E(\lg E)$ aangemaakt. $\lg E$ dat een bepaald allergeen herkent, wordt specifiek IgE genoemd.

\section{Diagnose van een voedselallergie}

De diagnose van voedselallergie kan opgesplitst worden in vier verschillende onderdelen: vraaggesprek (anamnese), huidtest, bloedonderzoek (Box 1) en de dubbelblinde voedselprovocatie (Box 2). Bij binnenkomst van een patiënt met verdenking op voedselallergie wordt allereerst gevraagd naar het optreden van klachten veroorzaakt door bepaalde voedingsmiddelen en hoe snel deze klachten ontstaan. Na het vraaggesprek volgt meestal een huidtest, de zo genaamde skin prik test. Bij de skin prik test wordt een druppel met het extract van het verdachte voedingsmiddel op de huid aangebracht, waarna de huid wordt aangeprikt door de druppel heen. Indien er galbulten op de huid ontstaan, is dat een bewijs voor aanwezigheid van IgE antilichamen tegen het betreffende voedingsmiddel. Als de anamnese en resultaten van de skin prik test uiteen lopen, wordt aanvullend bloedonderzoek uitgevoerd. Daarbij wordt het bloed onderzocht op aanwezigheid van specifiek $\lg \mathrm{E}$ tegen het verdachte voedingsmiddel of een bepaald voedingseiwit/allergeen. Helaas kunnen zowel de skin prick test als het bloedonderzoek resultaten opleveren die in tegen scheid staan met de uitslag van een dubbelblinde provocatie, waarbij kleine hoeveelheden van het verdachte voedingsmiddel worden gegeven (Box 2):

1. De test toont geen specifieke IgE antilichamen, terwijl er wel sprake is van een bevestigde voedselallergie (fout-negatieve test uitslag)

2. De test toont specifieke IgE antilichamen, terwijl er geen sprake is van een bevestigde voedselallergie (fout-positieve test uitslag) 
Box 1: Bloedonderzoek - Bepalen van specifiek IgE

Als IgE antilichamen tegen een bepaald eiwit/allergeen gericht zijn, noemen we het specifiek IgE. Specifiek IgE dat in het bloed van een patiënt zit, kan met een bloedtest aangetoond worden. Bij zo'n bloedtest wordt patiënten serum - de vloeistof die over blijt naar het stollen van bloed - aan het allergeen blot gesteld en vervolgens gemeten hoeveel IgE antilichamen het allergeen binden. Specifiek IgE antilichamen kunnen worden gemeten tegen een hele mix van allergenen afkomstig van een voedingsbron (extract) of tegen een bepaald voedingseiwit/allergeen (component). In de daagse routine worden zulke testen van bedrijven aangeleverd.

Box 2: Duppelblinde voedselprovocatie

Bij een voedselprovocatie neemt de patiënt het verdachte voedingsmiddel in, te beginnen met een hele kleine hoeveelheid, die verstopt zit in bijv. een stukje koek. Tijdens de voedselprovocatie wordt de dosis in kleine stappen verhoogd, waarbij tussen iedere dosering tenminste 30 minuten gewacht wordt om te zien of er klachten ontstaan. Ter controle wordt op de ene dag een koek met het verdachte voedingsmiddel gegeven en op de andere dag een koek zonder het voedingsmiddel (placebo). Hoewel deze methode als gouden standaard beschouwd wordt, zitten er ook nadelen aan. Zo kan een voedselprovocatie alleen in een gespecialiseerde unit in het ziekenhuis plaatsvinden, wat duur is en getraind personeel vereist, maar ook kan de patiënt onaangename klachten ervaren.

Het doel van dit proefschrift was om de diagnose van een voedselallergie op basis van bloedtesten te verbeteren en daarmee het aantal benodigde, belastende voedselprovocaties te verminderen.

\section{Fout-negatieve test uitslagen}

Een deel van de voedsel allergische patiënten - 18\% van de pinda- en $30 \%$ van de sesamzaad allergische patiënten - heeft geen aantoonbaar specifiek IgE in het bloed. Een verklaring voor het optreden van fout-negatieve test uitslagen is de afwezigheid van het allergeen waartegen de patiënt specifiek lgE heeft aangemaakt, in de huidige door bedrijven geleverde bloedtesten.

In hoofdstuk 2 hebben we nog niet geïdentificeerde allergenen van macadamia noten bestudeerd. Momenteel is slechts een klein aantal patiënten met een voedselallergie allergisch voor macadamia noten. Er wordt echter verwacht dat deze aantallen gaan stijgen, aangezien de consumptie van macadamia noten flink groeit. In ons onderzoek hebben we een nieuw allergeen kunnen aantonen, behorend tot een groep eiwitten genaamd vicilinen. Eiwitten behorend tot de viciline groep zijn ook voor andere voedingsmiddelen (bijv. pinda) als allergenen bekend. Dit nieuwe allergeen wordt vooral herkend door patiënten met matig tot ernstige klachten en kan daarom belangrijk zijn bij het identificeren van allergische patiënten met een verhoogd risico op ernstige klachten. 
De huidige bloedtesten die door bedrijven kunnen worden geleverd, bevatten vooral allergenen die in water oplosbaar zijn. Daarentegen zijn eiwitten die niet in water, maar in vet oplosbaar zijn meestal afwezig. Er zijn echter wel in vet oplosbare eiwitten, ook wel oleosines genoemd, die als allergenen bekend zijn. Specifiek IgE tegen deze allergenen kunnen niet met behulp van de huidige bloedtesten aangetoond worden. Om de rol van deze eiwitten beter in kaart te brengen, hebben we in hoofdstuk 3 oleosines uit sesamzaad en hun binding door specifiek IgE onderzocht. In tegenstelling tot onze verwachting konden we bijna geen specifiek IgE tegen sesam oleosines aantonen. Indien er wel specifiek IgE tegen sesam oleosines aantoonbaar was, hadden deze patiënten ook aantoonbaar specifiek lgE tegen bekende water oplosbare allergenen. Deze resultaten wijzen erop dat sesam oleosines geen bijdrage kunnen leveren aan verbetering van de huidige bloedtesten voor sesamzaad.

\section{Fout-positieve test uitslagen}

Het aantal fout-positieve test uitslagen is afhankelijk van het allergeen waartegen specifiek IgE bepaald wordt. Fout-positieve specifiek IgE metingen tegen pinda extract meestal gebruikt in de huidige bloedtesten - komen vaker voor (rond 30-35\%) dan foutpositieve specifiek IgE metingen tegen het stabiele pinda allergeen Ara $\mathrm{h} 2$ (Box 3). Hoewel specifiek IgE tegen Ara h 2 in ongeveer $90 \%$ van patiënten een pinda allergie aantoont, kan ongeveer $10 \%$ van de patiënten met specifiek IgE tegen Ara $\mathrm{h} 2$ pinda eten zonder klachten te ontwikkelen. Als de diagnose uitsluitend gebaseerd zou zijn op deze test, zou dit tot een verkeerde diagnose en onnodige dieet restricties leiden. Het optreden van fout-positieve test uitslagen kan mogelijk deels verklaard worden door verschillen tussen IgE antilichamen van allergische en tolerante patiënten. Hierbij hebben we vooral gekeken naar verschillen waar de $\lg \mathrm{E}$ antilichaam precies aan het allergeen kan binden. In hoofdstuk 4 en $\mathbf{6}$ hebben we naar verschillende IgE herkenningspatronen gekeken - de herkenning van lineaire epitopen (Box 4).

Box 3: Benaming van allergenen

De benaming van allergenen bestaat uit de Latijnse naam van de bron en een doorlopend getal. Hier een voorbeeld voor de benaming van een allergenen afkomstig van pinda:

Latijnse naam: Arachis hypogaea $\rightarrow$ Ara $\boldsymbol{h} 2$ 
Box 4: Lineaire en conformationele epitopen

Als een antilichaam aan een allergeen bindt dan vindt dat op een specifieke plek van het eiwit plaats. Deze specifieke bindingslocaties van antilichamen op allergenen worden epitopen genoemd. Ze bestaan uit keten van kleine bouw blokken: aminozuren. Zij kunnen in twee verschillende categorieën ingedeeld worden:

1. Lineaire epitopen: deze bestaan uit aminozuren die in de keten op elkaar volgen

2. Conformationele epitopen: De lineaire keten van aminozuren komt meestal gevouwen voor, zodat aminozuren dicht bij elkaar in de buurt kunnen komen zonder in de lineaire keten naast elkaar te zitten. Als een antilichaam aminozuren herkent die door de gevouwen structuur bij elkaar in de buurt komen, noemen we dit een driedimentionaal of conformationeel epitoop.

Hoofdstuk 4 was gericht op de epitopen van de hoofd allergenen van kippenei, met als doel om fout-positieve testen voor kippenallergie bij volwassenen te voorkomen. Kippenei allergie komt vaak voor bij kinderen en deze ,groeien er meestal overheen“. Echter komt kippenei allergie in mindere mate ook voor bij volwassenen en ontstaat deels ook pas op volwassen leeftijd. Uit ons onderzoek is gebleken dat een aantal volwassenen niet allergisch is voor kippenei, ondanks verhoogd specifiek lgE tegen kippenei extract en de hoofdallergenen Gal d 1 en 3 . Dit is een aanwijzing daarvoor dat meten van specifiek IgE tegen het complete allergeen Gal d 1 en 3 niet gebruikt kan worden om een kippenallergie bij volwassenen te voorspellen hoewel specifiek IgE tegen Gal d 1 wel aanwijzing is voor een kippenei allergie bij kinderen. Door het bestuderen van de IgE herkenningspatronen konden we drie lineaire epitopen van Gal d 1 identificeren die met voorrang herkend worden door specifiek IgE van kippenei allergische patiënten met vooral ernstige klachten. Daarmee kan diagnostiek gericht op deze drie epitopen bijdragen aan het verminderen van rond $90 \%$ fout-positieve testuitslagen bij kippenei allergie diagnostiek van volwassenen.

Naast Ara h 2 bevat pinda nog twee allergenen uit dezelfde eiwitfamilie: Ara h 6 en 7. Door het bepalen van specifiek IgE tegen deze allergenen kan een pinda allergie net zo goed voorspelt worden. Ara $\mathrm{h} 7$ is erbij bijzonder omdat drie verschillende Ara $\mathrm{h} 7$ isovormen (Box 5) in pinda voorkomen. Door in het laboratorium te bestuderen in hoeverre deze isovormen de cellen die voor de allergische reactie verantwoordelijk zijn, kunnen activeren, konden we in hoofdstuk 5 laten zien dat isovorm 2 de grootste potentie daarvoor heeft. Deze potentie is vergelijkbaar met de potenties van Ara $\mathrm{h} 2$ en 6.

Naar aanleiding van de resultaten in hoofdstuk 5 hebben we in hoofdstuk 6 naar een verklaring gezocht waarom de isovorm 2 een grotere potentie heeft dan de andere Ara h 7 isovormen. Deze vraag hebben we net als in hoofdstuk 4 geprobeerd te beantwoorden door het bestuderen van de specifiek IgE binding aan lineaire epi- 
topen. Dit verklaarde het verschillende gedrag van de Ara $\mathrm{h} 7$ isovormen echter niet. Daarbij viel op dat er over het algemeen minder lineaire epitopen door specifiek IgE herkend werden dan verwacht. Specifiek IgE van sommige patiënten herkende zelfs niet één lineaire epitoop. Deze resultaten suggereren dat conformationele epitopen bij de herkenning van Ara $\mathrm{h} 7$ isovormen waarschijnlijk een grotere rol spelen dan lineaire epitopen. Dit wijst erop dat het niet voldoende is om lineaire epitopen te bestuderen en dat in de toekomst meer onderzoek naar conformationele epitopen gedaan moet worden.

Box 5: Isovormen

In pinda komt het pinda allergeen Ara $h 7$ in drie verschillende vormen voor. leder vorm bestaat uit bijna dezelfde bouw blokken maar wijst wel verschillen in vergelijking met de andere vormen op. Deze soort gelijke eiwitten noemen we isovormen en de isovormen van Ara $h 7$ dragen de volgende namen: Ara h 7.0101, Ara h 7.0201 en Ara h 7.0301.

Voor de studies naar de herkenning van lineaire epitopen in hoofdstuk $\mathbf{4}$ en $\mathbf{6}$ hebben we patiënten sera gebruikt die een mix van IgE antilichamen bevatten die verschillende epitopen herkennen. In hoofdstuk 7 hebben we geconstateerd dat door het gebruik van patiënten sera mogelijk relevante epitopen niet duidelijk of zelfs helemaal niet geïdentificeerd kunnen worden. Dit kan veroorzaakt worden doordat:

- serum van allergische patiënten zowel lgE antilichamen kan bevatten die epitopen herkennen die voor het optreden van een allergische reactie relevant zijn, als IgE antilichamen die epitopen herkennen die niet van belang zijn. Dit bemoeilijkt de identificatie van relevante epitopen.

- serum van tolerante patiënten antilichamen kan bevatten die ogenschijnlijk relevante epitopen herkennen. Deze epitopen zijn echter toch niet relevant, omdat het binden door het antilichaam waarschijnlijk niet sterk genoeg is om de cellen die voor een allergische reactie van belang zijn, te activeren.

Een mogelijke oplossing is om deze antilichamen afzonderlijk van elkaar te bekijken. Dit hebben we gedaan door de extractie van DNA uit een B cel die éen specifiek antilichaam aanmaakt. Deze DNA-informatie kan vervolgens gebruikt worden om het specifieke antilichaam dat deze $\mathrm{B}$ cel normaalgesproken maakt te produceren in het laboratorium. Als deze in het laboratorium geproduceerde antilichamen allemaal de DNA informatie uit éen $B$ cel bevatten, worden ze monoklonaal genoemd.

In hoofdstuk 8 hebben we op basis van de literatuur twee methodes ontwikkeld om monoklonale antilichamen in het laboratorium te produceren:

1. Door witte bloedcellen die antilichamen maken (de B cellen) te infecteren met het Epstein-Barr virus, waardoor ze onsterfelijk gemaakt worden. Deze B cellen kunnen vervolgens zo opgegroeid worden dat éen kweek slechts dochters van éen 
bepaalde B cel bevat. Als de B cellen in deze kweek antilichamen aanmaken, kunnen deze een type antilichamen aanmaken (monoclonale antilichamen) die voor verder onderzoek gebruikt worden. Deze monoklonale antilichamen kunnen dan op basis van hun binding aan een specifiek allergeen zoals het pinda allergeen Ara h 2 geselecteerd worden.

2. B cellen bezitten een receptor op hun oppervlakte die dezelfde DNA-informatie, dus dezelfde specificiteit, bezit als de antilichamen die door deze B cellen gemaakt worden. Daardoor kunnen we B cellen op basis van hun specificiteit selecteren. Door éen $\mathrm{B}$ cel te selecteren weten we dat de DNA-informatie van deze B cel gebruikt is voor het maken van die betreffende monoklonale antistof.

Aan beide methodes zitten voor- en nadelen. De eerste methode heeft als nadeel dat hij gebruikt kan worden om slechts een klein aantal verschillende monoklonale antilichamen te produceren, terwijl het voordeel is dat deze methode goedkoper is dan de tweede methode. De tweede methode heeft echter als voordeel dat hij voor een bredere selectie van monoklonale antilichamen gebruikt kan worden. Voor ons patiënten studie in hoofdstuk 9 hebben ervoor gekozen methode 2 te gebruiken. De opbouw van een antilichaam staat in Box 6 beschreven.

Box 6: Antilichaam opbouw

Een antilichaam bestaat uit twee zware en twee lichte ketens. Deze ketens omvatten telkens een constant gedeelte en een variabel gedeelte. Het variabel gedeelte bepaalt welk eiwit/allergeen herkent wordt. Het zware gedeelte is anderzijds ervoor verantwoordelijk op welke manier de lichaam op de herkenning van een eiwit gaat reageren.

Het variabel gedeelte bestaat uit drie verschillende genen: $V$, D en J genen. Tijdens de ontwikkeling van een $B$ cel worden deze genen willekeurig uit een pool (65 V genen, $27 \mathrm{D}$ genen, $6 \mathrm{~J}$ genen) geselecteerd en met elkaar gecombineerd. Dit leidt tot heel veel

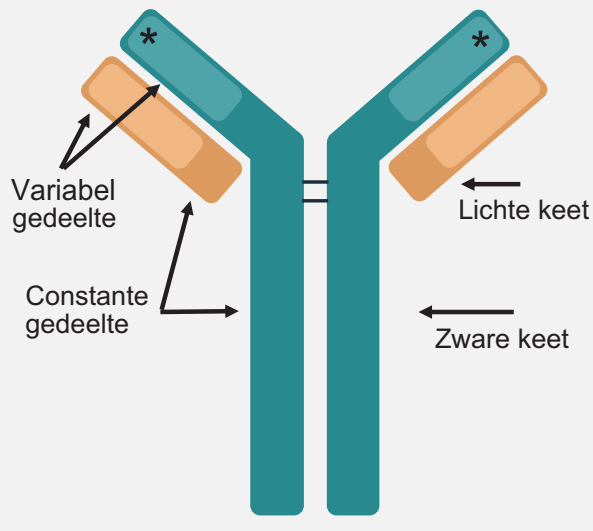
mogelijke combinaties die het herkennen van bijna oneindig verschillende eiwitten waarborgt. Voor het herkennen van een eiwit is de regio tussen het einde van het $V$ gen en het begin van het $J$ gen naar vertaling in aminozuren - meest belangrijk.

Om na te gaan of met behulp van deze monoklonale antilichamen de diagnostiek kan worden verbeterd, hebben we in hoofdstuk $9 \mathrm{~B}$ cellen van pinda allergische en pinda tolerante patiënten verkregen die allemaal specifiek IgE hadden tegen de pinda allergenen Ara h 2 en 6 . Deze B cellen werden gebruikt om monoklonale antilichamen in het laboratorium te produceren. Door het bestuderen van hun DNA-informatie konden we belangrijke verschillen tussen pinda allergische en tolerante patiënten aantonen: 
- Het variabel gedeelte van antilichamen afkomstig van allergische patiënten werd veel vaker door één bepaalde groep $\mathrm{V}$ genen gevormd.

- De regio die naar het vertalen in aminozuren het meest belangrijk is voor het binden aan het allergeen, heeft deels een unieke genetische opbouw als de antilichaam afkomstig is van een pinda allergische patiënt.

Deze resultaten wijzen erop dat er DNA-patronen bestaan die onderscheid kunnen maken tussen pinda allergische en pinda tolerante patiënten. Als er een eenvoudigere techniek ontwikkeld zou worden voor methode 2 , kunnen bovenstaande resultaten bijdragen aan de ontwikkeling van een bloedtest die een beter onderscheidt maakt tussen pinda allergische en niet pinda allergische patiënten.

De resultaten van dit proefschrift vormen een solide basis voor de ontwikkeling van verbeterde of nieuwe bloedtesten die fout-negatieve en fout-positieve test uitslagen kunnen verminderen of zelfs voorkomen. Afhankelijk van het allergeen bestaan er verschillende mogelijkheden om dit doel te bereiken: bijvoorbeeld de bepaling van specifiek IgE tegen lineaire epitopen voor het kippenei allergeen Gal d 1 of het evalueren van antilichaam DNA-patronen voor de pinda allergenen Ara h 2 en 6. Ongeacht de strategie die uiteindelijk voor een bepaald allergeen gebruikt wordt/moet worden, worden bloedtesten betrouwbaarder en kunnen zo het aantal vereiste voedselprovocaties in de toekomst verminderen. Daarnaast zijn de herkenning van het nieuwe macadamia noot allergeen en ook van de lineaire epitopen van Gal d 1 waarschijnlijk in staat om mensen met een verhoogd risico op een ernstige reactie te voorspellen. 
GCIUICTL

AGCCTCTGGA7

CCTGTTTCTGCAAA

iGCGTGGTCCAGCCTC

AGGGCCGATTCACCATL,

AATTGTGTTGACACAGTCTCCAGCCALL

'TGGCATCCCAGCCAGGTTCAGTGGCAGTGGG'TC I

CAGATGACCCAGTCTCCTTCCACCCTGGCTGCATCTGTAG

AGTCCCATCAAGGTTCAGCGGCAGTGGGTCTGGGACAGAGTTCACL

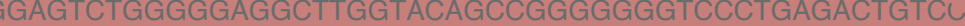

ITATCGAGACTCTGTGAAGGGCCGCTTCACCATCTCCAGAGACAATGCCAAAAACh ¿AGGGGACCACGGTCATCGTCTCCTCAGCAGGTGCAGCTGGTGGAGTCTGGGGGAGG ¿GAGTGGGTTTCATTTATACGCAAGGATGGAGCTAGTAAATACTATGGAGACTCCGTGAAGGG , CCCTGGGTTACTTTGACTACTGGGGCCAGGGAACCCTGGTCACCGTCTCCTCAGGAAATTGTGT CATAAACCTGGCCAGGCTCCCAGGCTCCTCATCTATGATGCATCCAACAGGGCCACTGGCATCCC ACTGGCCTCCGCTCACTTTCGGCGGAGGGACCAAGGTGGAGATCAAACGACATCCAGATGACCCAG

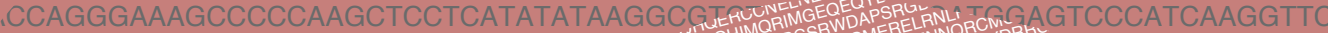

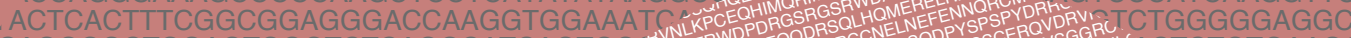

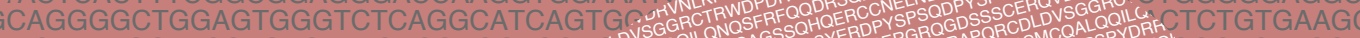

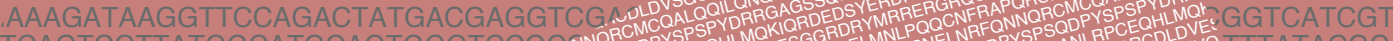

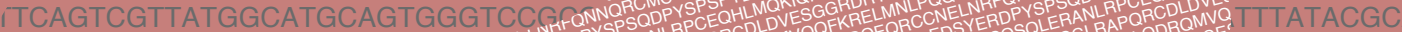

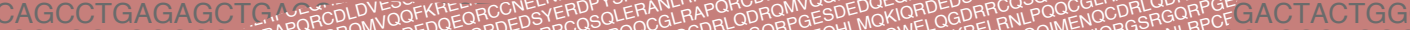
:TCCTGCAGGGCGCGLRAORODRO

tGCCTAGAGCCT

GCCTGATGATH

CACGTTTAGGE

ATGGCCAGTC

;TGGGGGGTCC

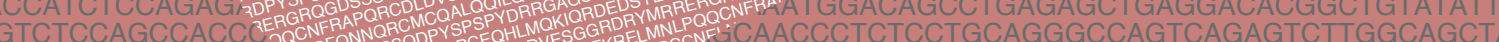

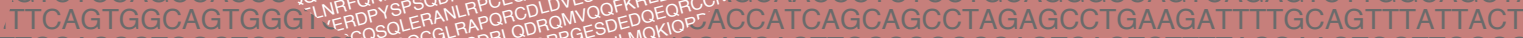

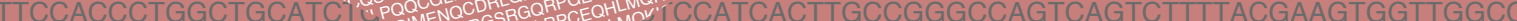

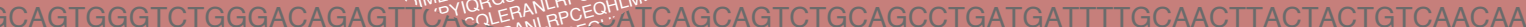

ACAGCCGGGGGGGTCCCTGAGACTGTCCTGTGCAGCCTCTGGATTCACGTTTAGGAGATATGCTTTGAGTTGGGTCCG ICTTCACCATCTCCAGAGACAATGCCAAAAACATCCTGTTTCTGCAAATGGCCAGTCCGAGAGTCGAAGACGCGGCCGTT

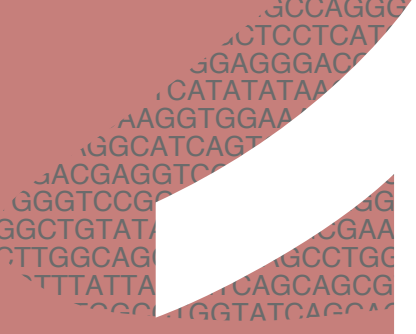
AAATACTATGGAGACTC
GGTCACCGTCTCCTCAG
GCATCCAACAGGGCCAC
AGATCAAACGACATCCAG
TTACAAGATGGAGTCCC
GCAGTTGGTGGAGTCT
AAACATATTATCGAGAC
GGGGCCAGGGGACCA GCTGGAGTGGGTTTCA

GTCCCTGGGTTACTT

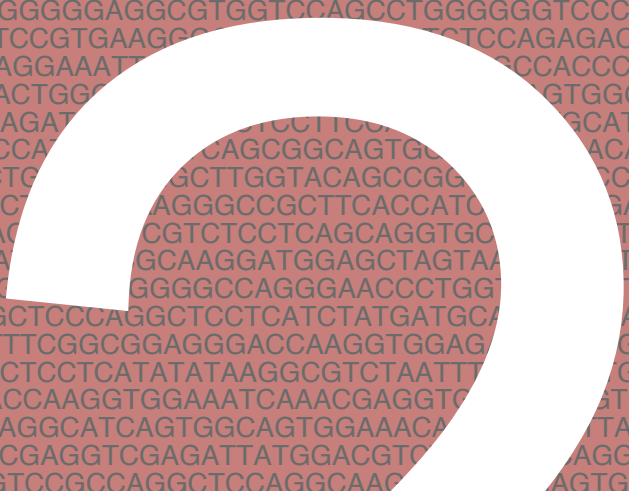

TTGCCTCCGCTCACTTTCGGCGGAGGGACCAAGGTGGAG

CTTTCGGCGGAGGGACCAAGGTGGAAATCAAACGAGGTV

GCTGGAGTGGGTCTCAGGCATCAGTGGCAGTGGAAA

GGTTCCAGACTATGACGAGGTCGAGATTATGGACGT

TGGCATGCAGTGGGTCCGCCAGGCTCCAGGCA

CTGAGGACACGGCTGTATATTACTGTGCGA

AGATTTTGCAGTTTATTACTGTCAGCAG

TTACGAAGTGGTTGGCCTGGTATCA
ICTTACTACTGTCAACAATATGATA ACTTACTACTGTCAACAATA
CTTTGAGTTGGGTCCGCC

:GAAGACGCGGCCGTTTAT

:TCTCCTGTGTAGCG

AAAGACACTCTCT

iTCTCCAGGGGAA

GAGACAGAGTC

ACCCTCACCA

TGTCCTGTGC

;AAAAACATC

TCCGTGAAG

MnUm TCCGTGAAG

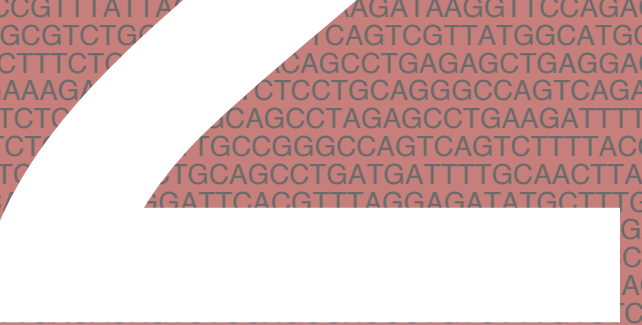

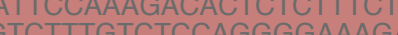
GGTCTGGGACAGACTTCACTCTCA TCTGTAGGAGACAGAGTCTCCAT AGAGTTCACCCTCACCATCAGCA ACAATGCCAAAAACATCCTGTTTd GGAGTCTGGGGGAGGCGTGGT GGAGACTCCGTGAAGGGCCGAT TCCTCAGGAAATTGTGTTGACA GGGCCACTGGCATCCCAGCCAC ACATCCAGATGACCCAGTCTCC AGTCCCATCAAGGTTCAGCGG GAGTCTGGGGGAGCTTGGTA (1) ACGAGACTCT GT GAAGGGCCGC GGGGACCACGGTCATCGTCTCCTC, ATGGTTTCATTTATACGCAAGGATG 作 AGGTCCAGGCTCC ACCTCCGCTCACTTTCGGCGGAGGGACC AGGC CTTTCGGCGGAGGGACCAAGGTGGAAATCA GGCTGGAGTGGGTCTCAGGCATCAGTGGCAG AGGTTCCAGACTATGACGAGGTCGAGATTATG
AGC ATGACGAGGTCGAGATTATG 作 GCAGTTIATTACTGTCAGCAGC LAGTGGTTGCCTGGTATCAC AGTTGGGTCCAATATGATAGT ACGCGGCCGTITATTACTGTGC TGTGTAGCGTCTGGATTCACCT CACTCTCTTTCTGCAAATGGAC CAGGGGAAAGAGCAACCCTCT

ACGACATCCAGATGACCCAGTCTCCTTCCACCCTGGCTGCATCTGTAGGAGACAGAGTCTCCATCACTTGCCGG

GATGGAGTCCCATCAAGGTTCAGCGGCAGTGGGTCTGGGACAGAGTTCACCCTCACCATCAGCAGTCTGCAGCO ITGGTGGAGTCTGGGGGAGGCTTGGTACAGCCGGGGGGGTCCCTGAGACTGTCCTGTGCAGCCTCTGGATTCAC AATATTATCGAGACTCTGTGAAGGGCCGCTTCACCATCTCCAGAGACAATGCCAAAAACATCCTGTTTCTGCAAATGC GCCAGGGGACCACGGTCATCGTCTCCTCAGCAGGTGCAGCTGGTGGAGTCTGGGGGAGGCGTGGTCCAGCCTGG GGAGTGGGTTTCATTTATACGCAAGGATGGAGCTAGTAAATACTATGGAGACTCCGTGAAGGG

¿CCTGGGTTACTTTGACTACTGGGGCCAGGGAACCCTGGTCACCGTCTCCTCAGGAAATTGTГ

ITAAACCTGGCCAGGCTCCCAGGCTCCTCATCTATGATGCATCCAACAGGGCCACTGGCATC

IGGCCTCCGCTCACTTTCGGCGGAGGGACCAAGGTGGAGATCAAACGACATCCAGATGACC

AGGGAAAGCCCCCAAGCTCCTCATATATAAGGCGTCTAATTTACAAGATGGAGTCCCATCAAGC

CACTTTCGGCGGAGGGACCAAGGTGGAAATCAAACGAGGTGCAGTTGGTGGAGTCTGGGGG

GGCTGGAGTGGGTCTCAGGCATCAGTGGCAGTGGAAACAAAACATATTATCGAGACTCTGTG

IAGGTTCCAGACTATGACGAGGTCGAGATTATGGACGTCTGGGGCCAGGGGACCACGGTCATCGi

ITATGGCATGCAGTGGGTCCGCCAGGCTCCAGGCAAGGGGCTGGAGTGGGTTTCATTTATACGCAAGGATGGiAGi I AG I

iAGAGCTGAGGACACGGCTGTATATTACTGTGCGAAAGATAGGTCCCTGGGTTACTTTGACTACTGGGGCCAGGGAACCCTG AGGGCCAGTCAGAGTCTTGGCAGCTACTTAGCCTGGTACCAACATAAACCTGGCCAGGCTCCCAGGCTCCTCATCTATGATG GAGCCTGAAGATTTTGCAGTTTATTACTGTCAGCAGCGTAGCGACTGGCCTCCGCTCACTTTCGGCGGAGGGACCAAGGTGGA 


\section{Zusammenfassung in deutscher Sprache}

IGCi

TCTCL

AGCGG

TTGGTAL

CTCCTCAG

AAGGATGGA

GGCCAGGGA

TCCTCATCTAT

GGGACCAAGG 7 ,
CATAAGGCGTCT/

GAAATCAAACGAG

TTGGCAGTGGAAA

AGATTATGGACGT

GGCTCCAGGCAAG

ACTGTGCGAAAGATAL

ACTTAGCCTGGTACCAAC,

GTCAGCAGCGTAGCGACTGG

TTGGTATCAGCAGAAACCAGGGA

TATGATAGTGCCCTACTCACTTTCGGL

CCAGGCTCCAGGGCAGGGGCTGGAGTGG

IATTACTGTGCGAAAGATAAGGTTCCAGACTA

GGATTCACCTTCAGTCGTTATGGCATGCAGTGG

GCAACCCTCTCCTGCAGGGCCAGTCACAЛT?T

CCATCAGCAGCCTA'

CACTTGCCGGGCr
GTCTGCAGCCTr

CTGGATTCACG

TTGCAAATGGCC.

CCAGCCTGGGGG

TCACCATCTCCAG

CAGTCTCCAGCCAC

TTCCACCCTGGCTGCA

CAGTGGGTCTGGGACA

CAGCCGGGGGGGTCCC

TTCACCATCTCCAGAGAC

AGCAGGTGCAGCTGGTGC

GAGCTAGTAAATACTATG

GAACCCTGGTCACCGTC

CTATGATGCATCCAAC

AAGGTGGAGATr

GTCTAATTTA

AACGAGGTGC

TGGAAACAA

GGCAAGGG'

AAAGATAC

IGTACCA

GTAGCG

CAGAAACL.

IGGGCAG!

GAAAGA

TCAGTC

AGCCT

CCTG

CCTA

GCC

TGA

GTT

(n)

ar
IGC

'TC,

IAT,

ITACL

ICTTAC

-GAAGAC

TCTCCTG

AAGACAC

TCTCCAGL

AGACTTC

iACAGAGTL

CCTGTGCAGCCTCTGGATTCACG

AACATCCTGTTTCTGCAAATGGCCA

iGAGGCGTGGTCCAGCCTGGGGG

तAAAGGGCCGATTCACCATCTCCA

ITTGTGTTGACACAGTCTCCAGC

ITCCCAGCCAGGTTCAGTGGC

CCAGTCTCCTTCCACCCTG

AGCAGCG

ATCAGCA

GGCTCCA

GATTCACI

AAATGGA

ICAACCCTC

,CATCAGCA

CACTTGCCG

AGTCTGCAG

¿CTCTGGATTC

GTTCTGCAAATL

UCCTL

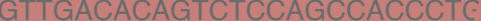

CCCAGCCAGGTTCAGTGGCAGTGGGT

:CCAGTCTCCTTCCACCCTGGCTGCATC :

TGCTTGGTACAGCCGGGGGGGTCCCTC

4GGGCCGCTTCACCATCTCCAGAGAC

GTCTCCTCAGCAGGTGCAGCTGGTG

CAAGGATGGAGCTAGTAAATACTATG

AGGGCCAGGGAACCCTGGTCACCGTC

iGCTCCTCATCTATGATGCATCCAACAGi

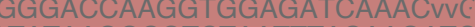
GGCATCAGTGGCAGTGGAAACAAAACATATTATC GAGG N

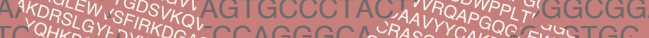

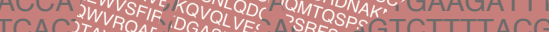

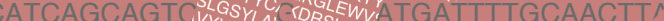

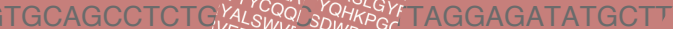

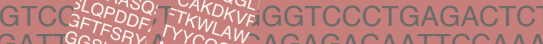

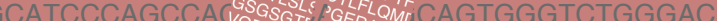

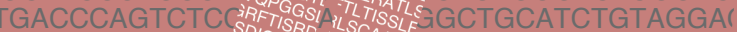

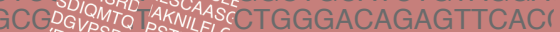
GGGGAGGCTTGG'

A

C.

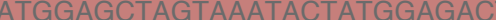

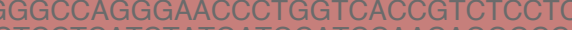

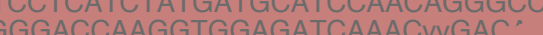

作

作 GCAAAGA T्रGTCCCT 


\section{Zusammenfassung in deutscher Sprache}

Nahrungsmittelallergien nehmen weltweit stetig zu. In Europa leiden durchschnittlich drei Prozent der Bevölkerung an einer Nahrungsmittelallergie. Dabei reagiert das menschliche Immunsystem auf sonst harmlose Eiweiße (Proteine), die in Nahrungsmitteln enthalten sein können. Diese als gefährlich eingestuften Proteine, auch Allergene genannt, kommen in Europa hauptsächlich in Erdnüssen, Haselnüssen, Hühnereiern, Kuhmilch, Früchten, Fisch und Krustentieren vor. Bei der Erkennung dieser Allergene durch das menschliche Immunsystem werden Antikörper verschiedener Untergruppen gegen das jeweilige Allergen hergestellt - bei Allergikern wird zusätzlich auch die Untergruppe der sogenannten Antikörper vom Typ $\mathrm{E}$ ( $\lg \mathrm{E})$ hergestellt. IgE, das ein bestimmtes Allergen erkennt, wird spezifisches IgE genannt.

\section{Diagnose einer Nahrungsmittelallergie}

Die Diagnose einer Nahrungsmittelallergie kann in vier Bereiche eingeteilt werden: Befragung (Anamnese), Hauttest, Bluttest (Box 1) und doppelblinde Nahrungsmittelprovokation (Box 2). Ein Patient mit Verdacht auf eine Nahrungsmittelallergie wird bei dem ersten Besuch bei einem Allergologen zu den Beschwerden, die durch bestimmte Lebensmittel verursacht werden, befragt. Hierbei wird zusätzlich ergründet, wie schnell diese Beschwerden auftreten. Nach der Befragung wird in der Regel ein Hauttest, der sogenannte Skin Prick Test, durchgeführt. Bei diesem wird ein Tropfen eines Extrakts, das aus dem vermuteten Lebensmittel gewonnen wird, auf die Haut aufgetragen. Anschließend wird die Haut durch den Tropfen hindurch angestochen. Das Auftreten von Hautschwellungen ist ein Hinweis auf das Vorhandensein von IgE-Antikörpern gegen das betreffende Lebensmittel. Im Falle einer Diskrepanz zwischen Anamnese und Testergebnis werden zusätzliche Bluttests durchgeführt. Dabei wird das Blut auf spezifisches lgE gegen das vermutete Lebensmittel oder ein bestimmtes Lebensmittelprotein/Allergen untersucht. Der Skin Prick Test als auch der Bluttest können zu Ergebnissen führen, die dem Ergebnis einer doppelblinden Nahrungsmittelprovokation, bei der kleine Portionen des verdachten Lebensmittels verabreicht werden, widersprechen (Box 2):

1. Es können mit dem Bluttest keine spezifischen IgE-Antikörper nachgewiesen werden, obwohl eine bestätigte Nahrungsmittelallergie vorliegt (falsch-negatives Testergebnis)

2. Es können mit dem Bluttest spezifische IgE-Antikörper nachgewiesen werden, obwohl keine bestätigte Nahrungsmittelallergie vorliegt (falsch-positives Testergebnis). 
Box 1: Bluttest - Bestimmung von spezifischem lgE

IgE-Antikörper, welche gegen ein bestimmtes Protein/Allergen gerichtet sind, werden als spezifische IgE bezeichnet. Spezifisches IgE, das sich im Blut eines Patienten befindet, kann mit einem Bluttest nachgewiesen werden. Hierbei wird das Patientenserum - die Flüssigkeit, die bei der Blutgerinnung zurückbleibt - mit dem Allergen zusammengebracht und anschließend gemessen, wie viele IgE-Antikörper an das Allergen gebunden haben. Die Bestimmung des spezifischen IgEs kann mit einer Mischung aus mehreren Allergenen, die aus derselben Nahrungsquelle stammen (Extrakt) oder mit einem bestimmten Nahrungsmittelprotein/Allergen (Komponente) durchgeführt werden. In der täglichen Routine werden Tests verwendet, die kommerziell hergestellt sind.

Box 2: Doppelblinde Nahrungsmittelprovokation

Bei einer Nahrungsmittelprovokation nimmt der Patient das vermeintlich allergieauslösende Lebensmittel zu sich, wobei mit einer sehr kleinen Menge begonnen wird. Diese kann beispielsweise in einem Stück Kuchen versteckt sein. Während der Provokation wird die Dosis in kleinen Schritten erhöht, wobei zwischen jeder Dosis mindestens 30 Minuten gewartet wird, um festzustellen, ob Beschwerden auftreten. Zur Kontrolle wird an einem Tag ein Kuchen, der das vermeintliche Nahrungsmittel enthält, verabreicht und an einem weiteren Tag ein Kuchen ohne das verdächtige Nahrungsmittel (Placebo). Obwohl diese Methode als Goldstandard angesehen wird, weist sie auch weitreichende Nachteile auf. Beispielsweise kann eine Nahrungsmittelprovokation nur auf einer dafür spezialisierten Station im Krankenhaus durchgeführt werden. Dies führt zu hohen Kosten und erfordert geschultes Personal. Auch können hierbei unangenehme Beschwerden als auch lebensbedrohliche Symptome auftreten

Ziel dieser Arbeit war die Verbesserung der Diagnose von Nahrungsmittallergien mit Hilfe von Bluttests, um die Anzahl der erforderlichen und belastenden Nahrungsmittelprovokationen zu verringern.

\section{Falsch-negative Testergebnisse}

Einige Patienten mit einer Nahrungsmittelallergie - 18\% der Erdnuss- und 30\% der Sesamsamen-Allergiker - haben kein nachweisbares spezifisches IgE im Blut. Eine Erklärung für das Auftreten falsch-negativer Testergebnisse ist das Fehlen des betreffenden Allergens in den Bluttests, die kommerziell hergestellt werden.

In Kapitel 2 wurde die Macadamianuss auf noch nicht bekannte Allergene hin untersucht. Bislang ist nur eine kleine Anzahl von Nahrungsmittelallergikern allergisch gegen Macadamianüsse. Es wird jedoch erwartet, dass die Zahl der MacadamianussAllergiker wachsen wird, da der Konsum von Macadamianüssen kontinuierlich zunimmt. Im Verlauf der Arbeit wurde ein neues Macadamia-Allergen nachgewiesen, das zu einer Gruppe von Proteinen gehört, die Viciline genannt werden. Proteine der Vicilin-Gruppe sind auch als Allergene für andere Nahrungsmittel (z. B. Erdnüsse) bekannt. Diese neuen Allergene werden hauptsächlich von Patienten mit mittelschweren bis schweren Beschwerden erkannt und können daher wichtig sein, um allergische Patienten mit 
einem erhöhten Risiko auf schwerwiegende Verläufe zu identifizieren.

Bluttests, die momentan kommerziell erhältlich sind, beinhalten hauptsächlich wasserlösliche Allergene. Im Gegensatz dazu fehlen Proteine, die nicht wasser-, sondern fettlöslich sind. Es gibt jedoch fettlösliche Proteine, z.B. sogenannte Oleosine, die bereits als Allergene identifiziert sind. Spezifisches IgE gegen diese Allergene kann daher nicht mit den derzeit zur Verfügung stehenden Bluttests nachgewiesen werden. In Kapitel 3 wurden Oleosine aus Sesamsamen auf ihre Bindung durch spezifisches IgE hin untersucht, um das Potenzial dieser Proteine für die Allergiediagnostik besser zu verstehen. Entgegen der Erwartungen konnte kaum spezifisches IgE gegen Oleosine aus Sesamsamen nachgewiesen werden. War spezifisches IgE gegen diese Oleosine nachweisbar, so hatten diese Patienten auch nachweisbares spezifisches IgE gegen bekannte wasserlösliche Allergene. Diese Ergebnisse zeigen, dass Oleosine aus Sesamsamen nicht dazu beitragen können, die aktuellen Bluttests für Sesam-Allergiker zu verbessern.

\section{Falsch-positive Testergebnisse}

Die Anzahl der falsch-positiven Testergebnisse hängt von dem Allergen ab, gegen das das spezifische IgE bestimmt wird. Falsch-positive spezifische IgE-Messungen gegen Erdnussextrakt - meistens in momentan erhältlichen Bluttests verwendet - sind häufiger (etwa 30-35\%) als falsch-positive spezifische lgE-Messungen gegen das stabile Erdnussallergen Ara h 2 (Box 3). Obwohl spezifisches IgE gegen Ara $\mathrm{h} 2$ bei etwa $90 \%$ der Patienten auf eine Erdnuss-Allergie hinweist, können etwa $10 \%$ der Patienten mit spezifischem lgE gegen Ara h 2 Erdnüsse essen, ohne Beschwerden zu entwickeln. Würde die Diagnose ausschließlich auf diesem Test basieren, würde dies zu einer Fehldiagnose und unnötigen diätetischen Einschränkungen führen. Das Auftreten falsch-positiver Testergebnisse kann möglicherweise durch Unterschiede bei lgE-Antikörpern zwischen allergischen und toleranten Patienten erklärt werden. In der vorliegenden Arbeit wurde bezüglich möglicher Unterschiede betrachtet, auf welche Weise IgE-Antikörper an Allergene binden. In den Kapiteln 4 und $\mathbf{6}$ haben wir verschiedene Erkennungsmuster von spezifischen IgE untersucht, insbesondere die Erkennung von linearen Epitopen (Box 4).

Box 3: Namensgebung von Allergenen

Die Namen der Allergene bestehen aus dem lateinischen Namen der Quelle und einer fortlaufenden Nummer. Hier ein Beispiel für den Namen eines Allergens aus Erdnuss:

Lateinischer Name: Arachis hypogaea $\rightarrow$ Ara h 2 
Box 4: Lineare und konformationelle Epitope

Wenn ein Antikörper an ein Allergen bindet, findet dies an einer bestimmten Stelle des Proteins statt. Diese spezifischen Bindungsstellen von Antikörpern werden Epitope genannt. Sie bestehen aus einer Kette kleiner Bausteine, den sogenannten Aminosäuren und können in zwei verschiedene Kategorien unterteilt werden:

1. Lineare Epitope bestehen aus Aminosäuren, die in der Kette aufeinander folgen.

2. Konformationelle Epitope: Die lineare Kette, die aus Aminosäuren besteht, kann so gefaltet sein, dass Aminosäuren nahe beieinander liegen, ohne dass sie in der linearen Kette nebeneinander vorkommen. Wenn ein Antikörper Aminosäuren erkennt, die in der linearen Kette weiter voneinander entfernt sind und die aufgrund der gefalteten Struktur nun nahe beieinander liegen, wird dies als dreidimensionales oder konformationelles Epitop bezeichnet.

In Kapitel 4 wurden Epitope der Hauptallergene von Hühnereiern untersucht, um zukünftig falsch-positive Testergebnisse in Bezug auf Hühnerei-Allergien bei Erwachsenen zu vermeiden. Eine Hühnerei-Allergie tritt bei Kindern häufig auf und wächst sich normalerweise aus. Zuweilen kann eine fortdauernde Hühnerei-Allergie aus dem Kindesalter jedoch auch bei Erwachsenen auftreten oder sie entwickelt sich erst im Erwachsenenalter. Es konnte gezeigt werden, dass eine Reihe von Erwachsenen trotz eines erhöhten spezifischen IgE gegen Hühnereiextrakt und gegen die Hauptallergene Gal d 1 und 3 nicht allergisch auf Hühnerei reagieren. Dies ist ein Hinweis darauf, dass das spezifische IgE gegen das Allergen Gal d 1 und 3 nicht verwendet werden kann, um eine Hühnerei-Allergie bei Erwachsenen vorherzusagen, obwohl spezifisches lgE gegen Gal d 1 auf eine solche bei Kindern hinweist. Durch die nähere Betrachtung der IgE-Erkennungsmuster konnten drei lineare Epitope von Gal d 1 identifiziert werden, die vorrangig von spezifischem IgE bei Hühnerei-Allergikern mit vornehmlich schweren Beschwerden erkannt werden. Dies bedeutet, dass eine Diagnostik, die auf spezifisches IgE gegen diese drei Epitope basiert, zu einer Verringerung der falschpositiven Testergebnisse bei der Diagnose von Hühnerei-Allergien im Erwachsenenalter um etwa $90 \%$ beitragen kann.

Zusätzlich zu Ara h 2 besitzt die Erdnuss noch zwei Allergene aus derselben Proteinfamilie (2S Albumine): Ara h 6 und 7. Durch die Bestimmung des spezifischen IgEs gegen diese Allergene kann eine Erdnuss-Allergie ebenso zuverlässig vorhergesagt werden. Die Erdnuss besitzt drei verschiedene Ara h 7-Isoformen (Box 5). Es wurde untersucht, inwieweit diese Isoformen Zellen aktivieren können, die für die allergische Reaktion verantwortlich sind. Es zeigte sich in Kapitel 5, dass die Isoform 2 das größte Potenzial aufzeigt, vergleichbar mit den Potentialen von Ara h 2 und 6.

Basierend auf den Ergebnissen in Kapitel 5 wurde in Kapitel 6 untersucht, warum die Isoform 2 ein größeres allergenes Potenzial im Vergleich zu den anderen Ara h 7- 
Isoformen besitzt. In Analogie zu Kapitel 4 wurde hierzu die Bindung von spezifischen $\lg \mathrm{E}$ an lineare Epitope analysiert. Jedoch konnten nicht die unterschiedlichen Potenziale der Ara h 7-Isoformen erklärt werden. Auffällig war, dass weniger lineare Epitope von spezifischem IgE erkannt wurden als erwartet. Es zeigte sich sogar, dass spezifisches IgE einiger Patienten keine linearen Epitope erkannte. Diese Ergebnisse legen nahe, dass konformationelle Epitope bei der Erkennung von Ara h 7-Isoformen eine größere Rolle spielen als lineare Epitope. In Zukunft sollten daher neben den linearen Epitopen auch konformationelle Epitope eine größere Beachtung finden.

Box 5: Isoformen

In Erdnüssen kommt das Erdnussallergen Ara $h 7$ in drei verschiedenen Formen vor. Jede Form besteht aus fast den gleichen Bausteinen, weist jedoch gewisse Unterschiede zu den anderen Formen auf. Diese ähnlichen Proteine werden als Isoformen bezeichnet; die Isoformen von Ara $h 7$ besitzen die Namen Ara h 7.0101, Ara h 7.0201 und Ara h 7.0301.

Für die Untersuchungen zur Erkennung von linearen Epitopen in den Kapiteln 4 und 6 wurden Patientenseren verwendet. Patientenseren enthalten für gewöhnlich eine Mischung von IgE-Antikörpern. Diese IgE-Antikörper können eine Reihe von verschiedenen Epitopen erkennen. In Kapitel 7 wurde die Theorie aufgestellt, dass bei der Verwendung von Patientenseren potenziell relevante Epitope nicht eindeutig oder gar nicht identifiziert werden können. Dies kann folgendermaßen erklärt werden:

- Das Serum von allergischen Patienten kann sowohl IgE-Antikörper enthalten, die für die Auslösung einer allergischen Reaktion relevante Epitope erkennen, als auch IgE-Antikörper, die nicht relevante Epitope erkennen. Diese Mischung erschwert die Identifizierung der relevanten Epitope.

- Das Serum toleranter Patienten kann Antikörper enthalten, die scheinbar relevante Epitope erkennen. Diese Epitope sind jedoch eigentlich irrelevant, da die Bindung durch den Antikörper wahrscheinlich nicht stark genug ist, um Zellen, die für eine allergische Reaktion verantwortlich sind, zu aktivieren.

Eine mögliche Lösung besteht darin, diese Antikörper separat zu betrachten. Dazu kann DNA aus einer B-Zelle - die Produktionsstelle von identischen Antikörpern - extrahiert werden. Diese DNA-Information kann dann verwendet werden, um den spezifischen Antikörper, den die B-Zelle normalerweise im Körper bildet, im Labor herzustellen. Wenn Antikörper alle dieselbe definierte DNA-Information einer B-Zelle tragen, werden sie als monoklonal bezeichnet.

In Kapitel 8 wurden basierend auf der Literatur zwei Methoden entwickelt, um monoklonale Antikörper herzustellen: 
1. B-Zellen können durch die Infektion mit dem Epstein-Barr-Virus unsterblich gemacht werden. Diese B-Zellen können danach im Labor so gezüchtet werden, dass Kulturen aus identischen Kopien einer bestimmten B-Zelle bestehen. Diese identischen B-Zellen können anhand ihrer gebildeten monoklonalen Antikörper anschließend so ausgewählt werden, dass diese ein Protein von Interesse erkennen - in unserem Falle das Erdnuss-Allergen Ara h 2.

2. B-Zellen besitzen auf ihrer Außenhülle einen Rezeptor, der identisch zu dem Antikörper ist, der durch die B-Zelle hergestellt wird. Das bedeutet, dass dieser Rezeptor die gleiche DNA-Information trägt und das gleiche Bindungsverhalten wie die von diesen B-Zellen hergestellten Antikörper aufweist. Durch Auswahl dieser bestimmten B-Zelle konnte gezeigt werden, dass die DNA-Informationen aus dieser B-Zelle verwendet werden können, um damit monoklonale Antikörper herzustellen.

Beide Methoden besitzen Vor- und Nachteile. Das erste Verfahren hat den Nachteil, dass damit nur eine begrenzte Anzahl verschiedener monoklonaler Antikörper hergestellt werden kann. Dennoch ist dieses Verfahren kostengünstiger im Vergleich zum zweiten Verfahren, das den Vorteil hat, für eine größere Auswahl monoklonaler Antikörper verwendet werden zu können. Für die Patientenstudie in Kapitel 9 wurde Methode 2 verwendet. Der Aufbau eines Antikörpers ist in Box 6 dargestellt.

Box 6: Aufbau eines Antikörpers

Ein Antikörper besteht aus zwei schweren und zwei leichten Ketten. Diese Ketten umfassen jeweils einen konstanten und einen variablen Teil. Der variable Teil bestimmt, welches Protein/Allergen erkannt wird. Der schwere Teil hingegen ist dafür verantwortlich, wie der Körper auf die Erkennung eines Proteins reagiert.

Der variable Teil besteht aus verschiedenen Abschnitten, der durch 3 Gene codiert wird: V-, D- und J-Genen. Während der Entwicklung einer B-Zelle werden diese Gene zufällig aus einem Pool ausgewählt (65 V-Gene, 27 D-Gene, $6 \mathrm{~J}$-Gene) und miteinander kombiniert. Dies führt $z u$ vielen möglichen Kombinationen, die die Erkennung

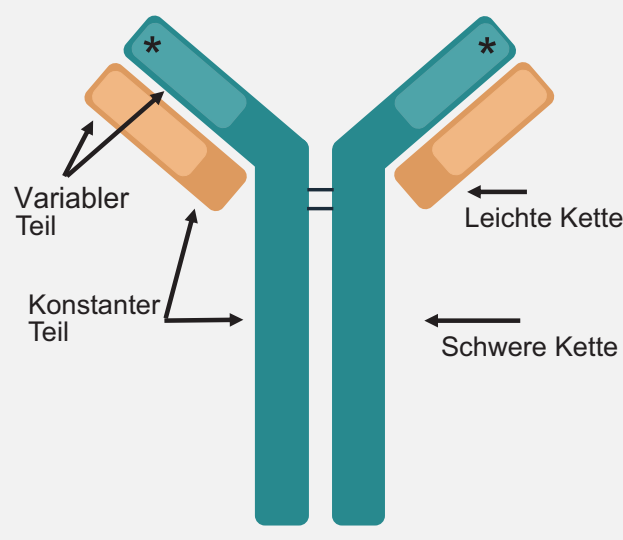
von nahezu unendlich vielen, unterschiedlichen Proteinen gewährleisten. Für die Proteinerkennung ist der in Aminosäuren übersetzte Bereich zwischen dem Ende des V-Gens und dem Beginn des J-Gens am wichtigsten.

In Kapitel 9 wurden B-Zellen aus dem Blut von Patienten mit Erdnuss-Allergie und solchen mit Erdnuss-Toleranz isoliert, die spezifisches IgE gegen die Erdnuss-Allergene 
Ara h 2 und 6 produzierten. Diese B-Zellen wurden verwendet, um monoklonale Antikörper herzustellen und um zu untersuchen, ob mit Hilfe dieser monoklonalen Antikörper die Diagnostik von Erdnuss-Allergien verbessert werden kann. In Analysen der DNA-Informationen aus diesen B-Zellen wurden Unterschiede zwischen erdnussallergischen und -toleranten Patienten festgestellt:

- Der variable Teil der Antikörper von Allergikern besitzt viel häufiger V-Gene (Box 6), die einer bestimmten Gruppe angehören.

- Die Region, die nach der Übersetzung in Aminosäuren für die Bindung an das Allergen am wichtigsten ist, weist teilweise einzigartige DNA-Informationen auf, wenn der Antikörper von einem Erdnuss-Allergiker stammt.

Diese Ergebnisse zeigen, dass DNA-Muster existieren, die sich zwischen ErdnussAllergikern und toleranten Patienten, die Erdnüsse essen können, unterscheiden. Wenn eine Methode, die technisch einfacher ist als die hier verwendete entwickelt würde, könnten die zuvor genannten Ergebnisse zur Entwicklung eines Bluttests beitragen, der besser zwischen Patienten mit und ohne Erdnuss-Allergie unterscheidet.

Die Ergebnisse dieser Arbeit bieten eine hochwertige Grundlage für die Entwicklung verbesserter und neuer Bluttests, mit denen falsch-negative und falsch-positive Testergebnisse reduziert oder sogar verhindert werden können. Je nach Allergen gibt es verschiedene Möglichkeiten, um dieses Ziel zu erreichen wie zum Beispiel die Bestimmung von spezifischem IgE gegen lineare Epitope für das Hühnerei-Allergen Gal d 1 oder die Auswertung von Antikörper-DNA-Mustern für die Erdnuss-Allergene Ara h 2 und 6. Unabhängig von der Strategie, die letztendlich für ein bestimmtes Allergen angewendet wird bzw. werden sollte, können Bluttest zuverlässiger werden. Dies kann die Anzahl der erforderlichen Nahrungsmittelprovokationen in der Zukunft verringern. Darüber hinaus ist es wahrscheinlich, dass durch die Bestimmung von spezifischen IgE gegen das Macadamianuss-Allergen sowie gegen die linearen Epitope von Gal d 1 Patienten mit einem erhöhten Risiko auf eine schwere allergische Reaktion identifiziert werden können. 
MIUGTCT

AGCCTCTGGA7

CCTGTTTCTGCAAA

¿GCGTGGTCCAGCCTC

AGGGCCGATTCACCATL ,

AATTGTGTTGACACAGTCTCCAGCCALL

TTGGCATCCCAGCCAGGTTCAGTGGCAGTGGGTC I

$\because C A G A T G A C C C A G T C T C C T T C C A C C C T G G C T G C A T C T G T A G L$

AGTCCCATCAAGGTTCAGCGGCAGTGGGTCTGGGACAGAGTTCACL

FAGTCTGGGGGAGGCTTGGTACAGCCGGGGGGGTCCCTGAGACTGTCL

ITATCGAGACTCTGTGAAGGGCCGCTTCACCATCTCCAGAGACAATGCCAAAAACh ¿AGGGGACCACGGTCATCGTCTCCTCAGCAGGTGCAGCTGGTGGAGTCTGGGGGAGGu iGAGTGGGTTTCATITATACGCAAGGATGGAGCTAGTAAATACTATGGAGACTCCGTGAAGGG ICCCTGGGTTACTTTGACTACTGGGGCCAGGGAACCCTGGTCACCGTCTCCTCAGGAAATTGTGT .CATAAACCTGGCCAGGCTCCCAGGCTCCTCATCTATGATGCATCCAACAGGGCCACTGGCATCCC ACTGGCCTCCGCTCACTTTCGGCGGAGGGACCAAGGTGGAGATCAAACGACATCCAGATGACCCAG

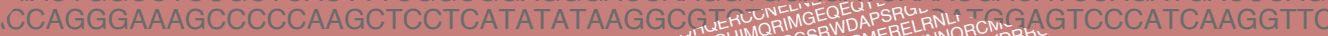

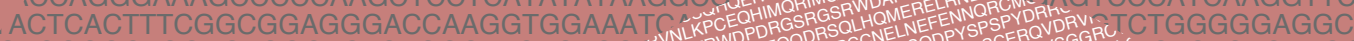

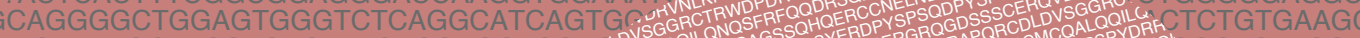

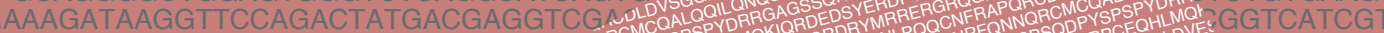

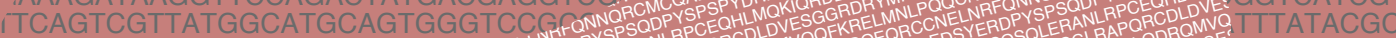

:TCCTGCAGGGCSCGLAPQRORDQN

GCCTAGAGCCTOMUNQC

;GGGCCAGTCA

GACGTATGATT

ATGGCCAGTC

;CTGGGGGGTC

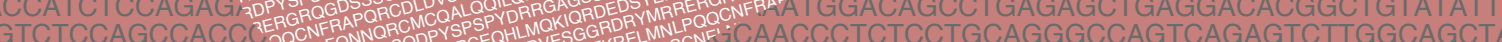

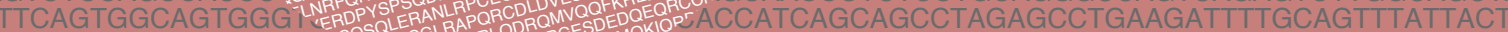
TTCCACCCTGGCTGCATC' "PQQC NOCDRRGQRPGEOHLW' CCATCACTTGCCGGGCCAGTCAGTCTTTTACGAAGTGGTTGGCC CAGTGGGTCTGGGACAGAGTT CONORLERANLPCEOHLWCAGCAGTCTGCAGCCTGATGATITTGCAACTTACTACTGTCAACAA

ACAGCCGGGGGGGTCCCTGAGACTGTCCTGTGCAGCCTCTGGATTCACGTTTAGGAGATATGCTTTGAGTTGGGTCCGC GCTTCACCATCTCCAGAGACAATGCCAAAAACATCCTGTTTCTGCAAATGGCCAGTCCGAGAGTCGAAGACGCGGCCGTI
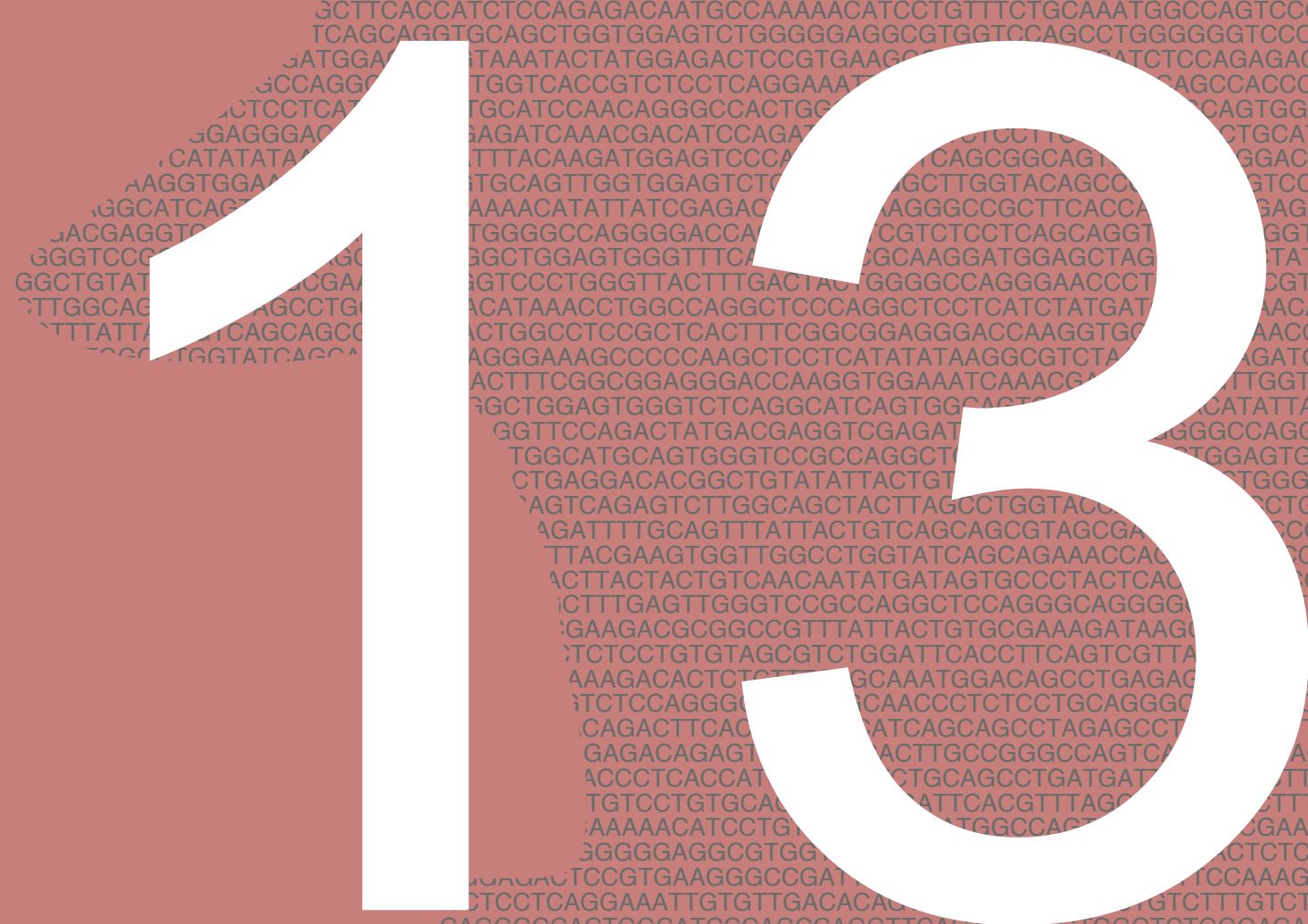
(1) TGGGTCTGGGACAGACTTCACTCTCA CATCTGTAGGAGACAGAGTCTCCAT ACAGAGTTCACCCTCACCATCAGCA CCTGAGACTGTCCTGTGCAGCCT COCATCCTTTC (1) ATCTCCTCAGGAAATTGTGTTGACA CAGGGCCACTGGCATCCCAGCCA CGACATCCAGATGACCCAGTCTCC ATGGAGTCCCATCAAGGTTCAGCGG GTGGAGTCTGGGGGAGGCTTGGTA TATCGAGACTCTGTGAAGGGCCGO TTGGGTTTCATTTATACGCAAGGATG GTTACTTTGACTACTGGGGCCAGG GCCAGGCTCCCAGGCTCCTCAT ACTCACTTTCGGCGGAGGGACC GAGGGACCAAGGTGGAAATCAA GGTCTCAGGCATCAGTGGCAG ACTATGACGAGGTCGAGATTATG CAGTGGGTCCGCCAGGCTCCAC ACACGGCTGTATATTACTGTGCG AGTCTTGGCAGCTACTTAGCCTC TGCAGTTTATTACTGTCAGCAGC GAAGTGGTTGGCCTGGTATCAC ACTACTGTCAACAATATGATAGT GAGTTGGGTCCGCCAGGCTCCA AGACGCGGCCGTITATTACTGTGC TCCTGTGTAGCGTCTGGATTCACCT ICTCAGGAAATTGTGTTGACA ACACTCTCTIICTGCAAATGGAC

ACGACATCCAGATGACCCAGTCTCCTTCCACCCTGGCTGCATCTGTAGGAGACAGAGTCTCCATCACTTGCCGG

GATGGAGTCCCATCAAGGTTCAGCGGCAGTGGGTCTGGGACAGAGTTCACCCTCACCATCAGCAGTCTGCAGCD ITGGTGGAGTCTGGGGGAGGCTTGGTACAGCCGGGGGGGTCCCTGAGACTGTCCTGTGCAGCCTCTGGATTCAC ;ATATTATCGAGACTCTGTGAAGGGCCGCTTCACCATCTCCAGAGACAATGCCAAAAACATCCTGTITCTGCAAATGQ GCCAGGGGACCACGGTCATCGTCTCCTCAGCAGGTGCAGCTGGTGGAGTCTGGGGGAGGCGTGGTCCAGCCTGG GAGTGGGTTTCATTTATACGCAAGGATGGAGCTAGTAAATACTATGGAGACTCCGTGAAGGG

ICCTGGGTTACTTTGACTACTGGGGCCAGGGAACCCTGGTCACCGTCTCCTCAGGAAATTGTГ

ITAAACCTGGCCAGGCTCCCAGGCTCCTCATCTATGATGCATCCAACAGGGCCACTGGCATCr

IGGCCTCCGCTCACTTTCGGCGGAGGGACCAAGGTGGAGATCAAACGACATCCAGATGACCC AGGGAAAGCCCCCAAGCTCCTCATATATAAGGCGTCTAATTTACAAGATGGAGTCCCATCAAGE CACTTTCGGCGGAGGGACCAAGGTGGAAATCAAACGAGGTGCAGTTGGTGGAGTCTGGGGG^

GGCTGGAGTGGGTCTCAGGCATCAGTGGCAGTGGAAACAAAACATATTATCGAGACTCTGTG,

\AGGTTCCAGACTATGACGAGGTCGAGATTATGGACGTCTGGGGCCAGGGGACCACGGTCATCGI

1TATGGCATGCAGTGGGTCCGCCAGGCTCCAGGCAAGGGGCTGGAGTGGGTTTCATTTATACGCAAGGATGGAGiL I AG I iAGAGCTGAGGACACGGCTGTATATTACTGTGCGAAAGATAGGTCCCTGGGTTACTTTGACTACTGGGGCCAGGGAACCCTG ,AGGGCCAGTCAGAGTCTTGGCAGCTACTTAGCCTGGTACCAACATAAACCTGGCCAGGCTCCCAGGCTCCTCATCTATGATGC GAGCCTGAAGATTTTGCAGTTTATTACTGTCAGCAGCGTAGCGACTGGCCTCCGCTCACTTTCGGCGGAGGGACCAAGGTGGA 


\section{Appendices}

GGC,

TCTCL

AGCGG

TTGGTAC

AAGGATGGA

GGCCAGGGA

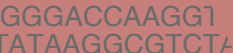

GAAATCAAACGAC

TTGGCAGTGGAAAI

AGATTATGGACGT

GGCTCCAGGCAAG

ACTTAGCCTGGTACCAAC,

GTCAGCAGCGTAGCGACTGG

TTGGTATCAGCAGAAACCAGGGAA,

TATGATAGTGCCCTACTCACTTTCGGL

CCAGGCTCCAGGGCAGGGGCTGGAGTGG

ГATTACTGTGCGAAAGATAAGGTTCCAGACTA
CTCCTCAG

TCCTCATCTAT

ACTGTGCGAAAGATAL

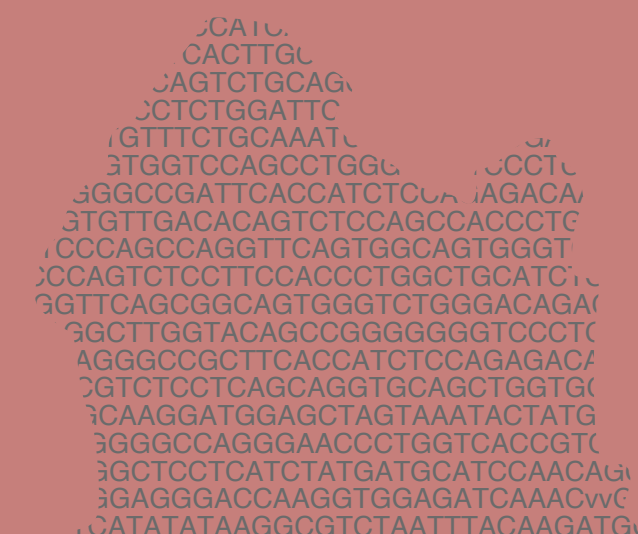

CACTTGL

AGTCTGCAG

CTCTGGATTC

GTTCTGCAAATL

GCCATTCACCATCTCU AGACA

作

CCAGCCAGGTTCAGTGGCAGTGGG

CCAGTCTCCTTCCACCCTGGCTGCATC '

GCTTGGTACAGCCGGGGGGTCCCTC

AGGGCGCTTCACCATCTCCAGAGAC

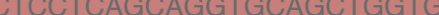

IGGGCCAGGGAACCCTGGTCACCGTC

TGAGGACCAAGGTGGAGATCAAACVVG

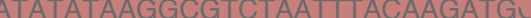
AGGTGGAAATCAAACGAGGTGCAGTTGGTGG IGGCATCAGTGGCAGTGGAAACAAAACATATTATC . EGAGGUs, AGATTATGGACGTCTGGGGCCAGGGI

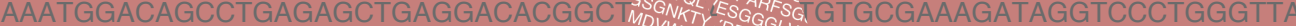

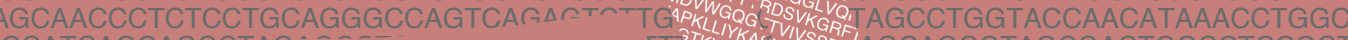
CCATCAGCAGCCTA' CACTTGCCGGGC GTCTGCAGCCTr

CTGGATTCACGT

TGCAAATGGCC

CCAGCCTGGGGG

TCACCATCTCCAG

CAGTCTCCAGCCAC
GGTTCAGTGGCAGTG

TTCCACCCTGGCTGCA

CAGTGGGTCTGGGACA

CAGCCGGGGGGGTCCC

TTCACCATCTCCAGAGAC

AGCAGGTGCAGCTGGTGC

GAGCTAGTAAATACTATG

GAACCCTGGTCACCGTC

CTATGATGCATCCAAC

AAGGTGGAGATr"

GTCTAATTTA

AACGAGGTGC

TGGAAACAA/

GACGTCTG

AACAAGGA

GGTACCA

GTAGCC

CAGAAACL

GCCCTACTCAL

GGGCAG! $3 \mathrm{C}^{\circ}$

GAAAGA

TCAGTC

AGCCT

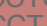

CCTA

GCCF

GTA7

(n)

ar

ITT

AAL

IAT,

ITACL

ICTTAC

:CTTTGA

כGAAGAC

TCTCCTG

AAGACAC

TCTCCAGL

iACAGAGT

CTCACCATL

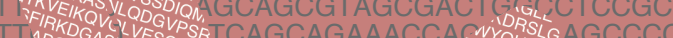
IGT

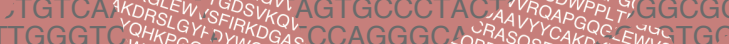

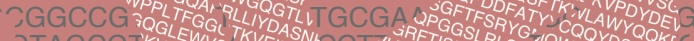

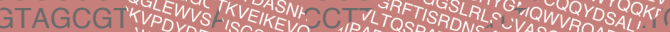

TCTCTTTCWDY

WAT

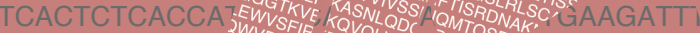

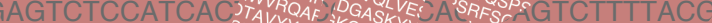

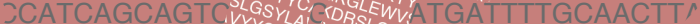

iTGCAGCCTCTGYALCOL YOHKOY TAGGAGATATGCTT

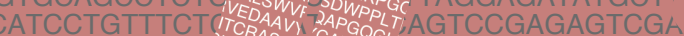

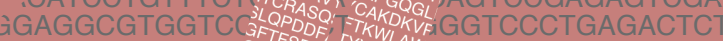

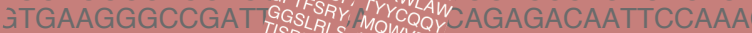
AAATTGTGTTGACA SPATNS,

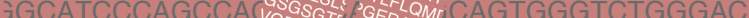
TGACCCAGTCTCCDDGGSA OL TIATLSGCTGCATCTGTAGGAI

TTACCCAGTCTCSDIOSRD is SCA

TCAAGGTCAGCGES

GGGGAGGCTTGG

CTGTGAAGGGCCGCTTC $\$$ GGI:TCTCCAGAGACAATGCC

(TCATCGTCTCCTCAGCAGGTGCAGCTGGTGGAGTCTC

ATACGCAAGGATGGAGCTAGTAAATACTATGGAGAC

STGGGGCCAGGGAACCCTGGTCACCGTCTCCTC

$\sim$ STCCTCATCTATGATGCATCCAACAGGGCC

GGGACCAAGGTGGAGATCAAACVVGAГ"

TATAAGGCGTCTAATTTACAAGAT

TAAATCAAACGAGGTGCAGTT

GGCAGTGGAAACAAAACAT

TTATGGACGTCTGGGGCC

CCAGGCAAGGGGCTGGA

TGCGAAAGA ${ }^{-1}$ TGTCCCTr

AGCAGCG

ATCAGCA

IATAGTG

GGCTCCA

ITACTGTG

GATTCACr

CAAATGGA

İCAACCCTC

CATCAGCAC

CACTTGCCGC
AGTCTGCAGCC

CCTGTGCAGCCTCTGGATTCACG i

AACATCCTGTTTCTGCAAATGGCCA

iGAGGCGTGGTCCAGCCTGGGGG

IAAGGGCCGATTCACCATCTCCA!

ITTGTGTTGACACAGTCTCCAGC

ITCCCAGCCAGGTTCAGTGGC

?CCAGTCTCCTTCCACCCTG 


\section{Abbreviations}

\begin{tabular}{ll} 
aa & amino acid \\
AIT & Allergen immunotherapy \\
AMV & Avian myeloblastosis virus \\
AP & Alkaline phosphatase \\
AUC & Area under the curve \\
BAT & Basophil activation test \\
CCDs & Cross-reactive carbohydrate determinants \\
CDR & Complementarity-determining region \\
CMA & Cow's milk allergy \\
CME & Cow's milk extract \\
CRD & Component resolved diagnostics \\
CPE & Crude peanut extract \\
DAO & Diamine oxidase \\
DBPCFC & Double-blind placebo-controlled food challenge \\
DOPE & Discrete Optimized Protein Energy \\
EBV & Epstein Barr Virus \\
EL & EUROLINE \\
FCS & Fetal calf serum \\
FR & Frame work \\
HEA & Hen's egg allergy \\
HEK & Human embryonic kidney cells \\
HIC & Hydrophobic interaction chromatography \\
i-allergen & improved allergen \\
IEF & Isoelectric focussing \\
IMGT & International ImMunoGeneTics information system \\
IPG strip & Immobilised pH gradient strip \\
IQR & Interquartile range \\
LCLs & Lymphoblastoid Cell Lines \\
mAb & monoclonal antibody \\
MA & Macadamia nut allergic \\
MFI & Mean fluorescence intensity \\
\hline HE &
\end{tabular}




\begin{tabular}{|c|c|}
\hline MT & Macadamia nut tolerant \\
\hline NA & Nut allergic \\
\hline NKT cells & Natural killer T cells \\
\hline nsLTP & non-specific Lipid transfer protein \\
\hline NT & Nut tolerant \\
\hline OAPs & Oil-body associated proteins \\
\hline OAS & Oral allergy syndrome \\
\hline OD & Optical density \\
\hline PAF & Platelet-activating factor \\
\hline PAF-AH & Platelet-activating factor acetylhydrolase \\
\hline PBMCs & Peripheral blood mononuclear cells \\
\hline PC & Positive control \\
\hline $\begin{array}{l}\text { PHYRE } \\
\text { pl }\end{array}$ & $\begin{array}{l}\text { Protein Homology/AnalogY Recognition Engine } \\
\text { isoelectric point }\end{array}$ \\
\hline PMF & Peptide mass fingerprinting \\
\hline PNK & Polynucleotide Kinase \\
\hline RU & Response unit \\
\hline SDS-PAGE & $\begin{array}{l}\text { sodium dodecyl sulfate polyacrylamide gel elec- } \\
\text { trophoresis }\end{array}$ \\
\hline SHIP-1 & $\begin{array}{l}\text { Src homology } 2(\mathrm{SH} 2) \text { domain containing inositol } \\
\text { polyphosphate } 5 \text {-phosphatase } 1\end{array}$ \\
\hline SHM & Somatic hypermutation \\
\hline $\operatorname{slg} \mathrm{E}$ & specific lgE \\
\hline $\operatorname{sig} G$ & specific lgG \\
\hline SNP & Single nucleotide polymorphism \\
\hline SPT & Skin prick test \\
\hline TLR & Toll-like receptor \\
\hline TMB & $3,3^{\prime}, 5,5^{\prime}$-Tetramethylbenzidine \\
\hline TMHMM & $\begin{array}{l}\text { Transmembrane helix prediction hidden Markov } \\
\text { model }\end{array}$ \\
\hline UMCU & University Medical Center Utrecht \\
\hline VDJ & recombined variable, diversity, joining genes \\
\hline VLAP & Vicilin-like anti-microbial peptides \\
\hline WB & Western Blot \\
\hline
\end{tabular}




\section{Dankwoord}

The way from starting as a PhD student towards the finish - with its ups and downs cannot be walked alone. Thus, I would like to thank all who supported me during this exciting time and the following ones in particular.

Om te beginnen zou ik graag de studiedeelnemers bedanken, zonder jullie had mijn studie nooit tot stand kunnen komen. Bedankt voor jullie interesse en bereidheid om mee te doen.

Beste André, bedankt voor je betrokkenheid en enthousiasme voor mijn, lab-gerelateerd, onderzoek. Je hebt je heel waardevolle inzichten uit de kliniek met mij gedeeld waardoor ik mijn lab onderzoek weer vanuit een andere invalshoek kon bekijken. Daarnaast heb je er altijd op gelet dat ik genoeg ruimte kreeg om mijzelf verder te ontwikkelen.

Beste Henny, bedankt dat je me - samen met Waltraud - de kans hebt gegeven om mijn promotieonderzoek bij het UMC Utrecht uit te voeren. Daarbij gaf je me de vrijheid om veel zelf uit te zoeken en mijn onderzoek op basis van mijn eigen overtuiging te plannen en uit te voeren, ook toen je niet helemaal geloofde dat we mRNA uit één cel zouden kunnen isoleren. Daarnaast is jouw reactietijd op mails echt bewonderenswaardig. Ons complementaire ritme daar werkte natuurlijk wel goed mee.

Liebe Waltraud, ohne deinen Einsatz und deine Unterstützung wäre es mir nicht möglich gewesen meine Doktorarbeit am UMC Utrecht zu schreiben. Auch während meiner Doktorarbeit warst du immer für mich erreichbar und du hast mit deiner Genauigkeit auch noch die letzte Ungenauigkeit in meinen Manuskripten gefunden. Vielen Dank!

Beste Edward, je was altijd bereikbaar voor me om even over mijn project te sparren of om op een andere manier aan mijn onderzoek bij te dragen, bedankt daarvoor. Daarnaast waardeer ik jouw grote hoeveelheid enthousiasme en kennis en de bereidheid die met mij te delen.

Beste leden van de leescommissie, Prof. dr. G.J.P.H. Boons, Prof. dr. J. Garssen, Prof. dr. G.F. Houben, Prof. dr. L. Koenderman en Prof. dr. A. Scheffold, hartelijk dank voor de tijd die jullie hebben genomen om dit proefschrift te beoordelen en deel te nemen aan mijn verdeging. Herzlichen Dank für die genommene Zeit, um meine Doktorarbeit zu beurteilen und um an meiner Disputation teilzunehmen. 
Beste Stans, waar moet ik beginnen? Zonder jou was ik nu nog steeds bezig met pipetteren en PCR'en. We delen de liefde voor grote experimenten, voor 10 samples beginnen we niet eens met kloneren. Je was altijd bereid om samen met mij deze grote experimenten, ieder op ons eigen eiland, uit te voeren. Maar ook buiten het lab was je er altijd voor een theetje of koffietje en een gesprek, waarbij het niet uit maakte waar het over ging. Heel erg bedankt voor deze dierbare tijd en voor het feit dat je mijn paranimf wilt zijn!

Beste Anne en Anouska, jullie ondersteuning bij het rekruteren van studie deelnemers was onmisbaar! Alleen door jullie inzet kon ik uiteindelijk het beoogde aantal tolerante patiënten in mijn studie includeren, wat veel waardevolle data opleverde. Daarnaast wil ik ook graag Thuy-My bedanken: jouw hulp bij het categoriseren van patiënten was heel waardevol even als jouw input op mijn manuscripten.

Beste Dana, Kim, Marieke, Miriam en Natalie: Thank you for all your effort and valuable input during your internships!

Verder zou ik graag al mijn collega's van de dermatologie bedanken. In het begin stond ik voor de grote klus om METC goedkeuring te verkrijgen maar Ischa manoeuvreerde me daar doorheen. Bedankt daarvoor! Daarnaast zou ik graag Mark Blankestijn willen bedanken - Aan de start van mijn promotie was je er altijd voor iedere kleine, maar ook grote vraag bereikbaar, wat me erg hielp om de oriëntatie te behouden. Mehran, je bent onmisbaar. Of je nu met mij op en neer naar Twente rijdt om kort bloed te prikken, of mij een Netflix account aanbiedt, niets was je te gek! Je was er ook altijd voor een leuk praatje, hoe vreemd het praatje ook was. Mijn flexplek kamergenoten Celeste, Daphne, Fleur, Hannah, Lieneke, Mark, Michelle, Roselie en Sarah: dank voor jullie gezelligheid, het luisteren naar mijn verhalen als het goed maar ook als het minder goed ging en de leuke borrels. Jantine en Miranda, dank voor jullie hulp rondom mijn promotietraject en Kitty, Heike en Astrid voor jullie inzichten tijdens de acute ontsteking overleggen, maar zeker ook daarbuiten.

Beste (voormalige) collega's van de Otten groep, ik had het erg naar mijn zin de aflopende vier jaar bij jullie in de groep. Tineke, onze gespreken over monoklonale antilichamen in het kader van niertransplantatie vond ik heel inspirerend. Bram, bedankt voor je dierbare input op mijn METC-voorstel en voor de vele leuke gespreken op de G.02 kamer. Laura, jouw doelgerichtheid is heel bewonderenswaardig en ik heb erg van onze etentjes genoten. Rowena, bedankt voor je gezelligheid en je moleculairbiologische inzichten. Ze waren heel waardevol voor het opzetten van onze single cell 
sequencing strategie. Voor alle heel veel leuke en interessante werkbesprekingen wil ik graag Danial, Daniel, Elena, Kevin,Leon, Loes, Saskia en Tammo bedanken.

Dear (former) colleagues from the $\mathrm{CTI}$, it was a pleasure to be part of this special lab community with so many funny, but also serious, chats at the lab or while having a cup of tea. Kim, a special thanks to you for so many sport sessions at Olympos with all the great conversations. Vania, I loved our odd times in the lab after everyone else already went home (they were also earlier in the morning). Susi, ich habe es sehr genossen einen deutschen Weggefährten links neben mir sitzen zu haben und es war schon etwas traurig als du nach Luxemburg gegangen bist. Kaylee, I love your passion for climbing and I always enjoyed our chats in the meanwhile. And... I will never forget our Eifel-Trip with the special dancefloor!

Beste Laura, mijn paranimf, ik wil je enorm bedanken voor de vele klimsessies waar je meer dan eens naar mijn verhalen hebt geluisterd. Bij jou kon en kan ik alles kwijt. Ik ben heel erg blij dat je mijn paranimf wilt zijn!

Liebe Kollegen von Euroimmun, ich möchte mich herzlich für Eure Unterstützung bedanken. Ich habe immer sehr gerne mit Euch zusammengearbeitet, ob nun vor oder während meiner Promotion. Bettina, du hast mir unermüdlich Feedback auf meine Manuskripte gegeben und du warst immer für mich erreichbar, ungeachtet was das Problem war. Melanie, unsere vielen Gespräche, wissenschaftlich oder auch privat, habe ich immer sehr genossen und sie haben auch die zahlreichen Kongresse immer wieder bereichert. Marco und Frithjof, unsere gemeinsamen Inkubationen in Utrecht waren legendär. Eva, Madlen und Steffi, vielen Dank für die gute Zusammenarbeit an den verschiedenen Teil-Projekten und die guten Gespräche, die wir nebenbei noch geführt haben.

Many thanks to all the LN residents, from the remaining one to all the residents I met here: Alex, Aisha, Anne, Carlo, Casper, Elena, Isabell, João, Lisa, Magda, Matteo, Mirthe, Saskia, Soraya, Stefano, Tom and Tessa. You all gave me the feeling to be a part of a special house community and you showed me how „German “ I am ;). I will always remember the big brunches, lunches and dinners with a big smile.

Liebe Antje, Christina, Franzi, Karina, Lisa, Lisa und Maria, vielen Dank, dass ihr in jeder Lebenslage für mich da seid. Ihr wart stets interessiert am Fortgang meiner Doktorarbeit, was auch zu interessanten, wissenschaftlichen Diskussionen führte. Aber natürlich auch für die vielen Stunden, in denen ihr dafür gesorgt habt, dass nicht an die Doktorarbeit gedacht habe. 
Liebe Mama und lieber Papa, vielen Dank, dass ihr immer für mich da wart und seid, ihr an mich glaubt und mich immer unterstützt. Das ist unersetzlich. Auch meinen Geschwistern, Cathi, Claus und Joey, möchte danken, dass sie immer darauf bedacht waren, dass ich mich auch mit anderen Dingen beschäftigt habe.

Lieber Micha, ohne dich wäre ich wahrscheinlich nun immer noch mit der Datenbank beschäftigt. Auch für allen anderen technischen Input bin ich dir sehr dankbar. Aber ich möchte mich nicht nur bezüglich deines technischen Inputs bedanken, sondern auch für deine unglaubliche Unterstützung in den letzten vier Jahren. Danke, dass du immer für mich da bist und mir jegliche Freiheit gibst, um meine Träume zu verwirklichen. 


\section{List of publications}

\section{Published}

Hayen SM, Ehlers AM, den Hartog Jager CF, Garssen J, Knol EF, Knulst AC, Suer W, Willemsen LEM, Otten HG. 2S protein Ara $\mathrm{h}$ 7.0201 has unique epitopes compared to other Ara $\mathrm{h} 7$ isoforms and is comparable to $2 S$ proteins Ara $\mathrm{h} 2$ and 6 in basophil degranulation capacity. Clin Exp Allergy 2018; 48(7): 890-7, doi: 10.1111/cea.13134

Ehlers AM, Blankestijn MA, Knulst AC, Klinge M, Otten HG. Can alternative epitope mapping approaches increase the impact of B-cell epitopes in food allergy diagnostics? Clin Exp Allergy 2019; 49(1): 17-26, doi: 10.1111/cea.13291

Ehlers AM, Rossnagel M, Brix B, Blankestijn MA, Le TM, Suer W, Otten HG, Knulst AC. Sesame oleosins are minor allergens. Clin Transl Allergy 2019; 9: 32, doi: 10.1186/s13601-019-0271-x

Ehlers AM, Klinge M, Suer W, Weimann Y, Knulst AC, Besa F, Le TM, Otten HG. Ara $\mathrm{h} 7$ isoforms share many linear epitopes: Are 3D epitopes crucial to elucidate divergent abilities? Clin Exp Allergy 2019; 49(11):1512-9, doi:10.1111/cea.13496

Hogenkamp A, Ehlers AM, Garssen J, Willemsen LEM. Allergy Modulation by N-3 Long Chain Polyunsaturated Fatty Acids and Fat Soluble Nutrients of the Mediterranean Diet. Front Pharmacol 2020; 11: 1244, doi: 10.3389/fphar.2020.01244

Ehlers AM, Otten HG, Wierzba E, Flügge U, Le TM, Knulst AC, Suer W. Detection of specific IgE against linear epitopes from Gal d 1 has additional value in diagnosing hen's egg allergy in adults. Clin Exp Allergy 2020; 50(12): 1415-23, doi: 10.1111/cea.13730

Ehlers AM, Rohwer S, Otten HG, Brix B, Le TM, Suer W, Knulst AC. IgE-binding to vicilin-like antimicrobial peptides is associated with systemic reactions to macadamia nut. Clin Transl Allergy 2020, 10: 55, doi: 0.1186/s13601-020-00364-5

Kansen HM, van Erp FC, Knulst AC, Ehlers AM, Lyons, SA, Knol EF, Meijer Y, Otten HG, van de Ent CK, Le TM. Accurate prediction of peanut allergy in one-third of adults using a validated Ara h 2 cutoff. J Allergy Clin Immunol Pract 2020, S22132198(20)31245-9, doi: 10.1016/j.jaip.2020.11.024 In press 


\section{In preparation}

Ehlers AM, den Hartog Jager CF, Knulst AC, Otten HG. Distinction between peanut allergy and tolerance by characterization of $\mathrm{B}$-cell receptor repertoires. Authorea Preprints; 2020. doi: 10.22541/au.160588804.43147170/v1 submitted 2020

Ehlers AM, den Hartog Jager CF, Kardol-Hoefnagel T, Knulst AC, Otten HG. Comparison of two pipelines to generate specific human monoclonal antibodies: Which method to choose for which purpose? submitted 2020 


\section{Curriculum vitae}

Anna Ehlers was born on the $11^{\text {th }}$ of December 1990 in Itzehoe, Germany. She graduated from High School in 2009 at the Alexander-van-Humboldt-College in Neumünster. In the same year, she started the bachelor program 'Chemical and Environmental Engineering' at the University of Applied Sciences in Lübeck. After graduating with her bachelor thesis entitled 'Substantial utilization of cacao pod husks' in 2013, she started the Master program 'Biochemical Engineering' at the University of Applied Sciences in Lübeck. She graduated cum laude in 2015 after completing her master thesis entitled 'Method development for the isolation of oil body-associated proteins and their characterisation', which she conducted at EUROIMMUN AG in Lübeck. Directly after her graduation, she started as a scientist at EUROIMMUN AG working on the isolation and production of native and recombinant proteins for in vitro allergy diagnostics.

In October 2016, Anna moved to the Netherlands and started as a PhD student at the University Medical Center Utrecht under the supervision of Prof. dr. André Knulst and dr. Henny Otten. During her PhD, she evaluated different possibilities to improve in vitro food allergy diagnostics in order to prevent test outcomes contradicting clinical diagnosis. The results are presented and discussed in this thesis. 